\title{
CHARACTERISING EVOKED POTENTIAL SIGNALS USING WAVELET TRANSFORM SINGULARITY DETECTION
}

A thesis submitted in fulfillment of the requirements

for the degree of Doctor of Philosophy

Conor McCooey

B.E.

School of Electrical and Computer Engineering Science, Engineering and Technology Portfolio RMIT University August 2007 


\section{DECLARATION}

The following statements are certified as true by the $\mathrm{PhD}$ candidate:

a) The work enclosed in this thesis is entirely the work of the candidate alone except where due acknowledgement has been made.

b) The work has not been submitted previously, in whole or in part, to qualify for any other academic award.

c) The content of the thesis is the result of work which has been carried out since the official commencement date of the approved research program.

d) Any editorial work carried out by a third party is acknowledged.

e) All ethics procedures and guidelines have been followed.

Candidate Signature:

Candidate Name:

Conor McCooey

Date: 


\section{ACKNOWLEDGEMENTS}

I would like to thank my supervisor Assoc. Prof. Dinesh Kant Kumar for his trust, assistance and belief throughout this research. I would also like to thank my partner Kathy for her patience, support and perseverance throughout the rollercoaster ride that life has thrown our way through the course of this research.

This thesis is dedicated to the memory of my father Brendan McCooey who took great pride in its inception and followed its progress carefully. I only wish he could have seen its completion. 
"Singularity is almost invariably a clue."

The Adventures of Sherlock Holmes (1892)

"The Boscombe Valley Mystery"

Sir Arthur Conan Doyle. 


\section{TABLE OF CONTENTS}

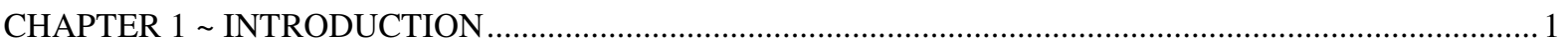

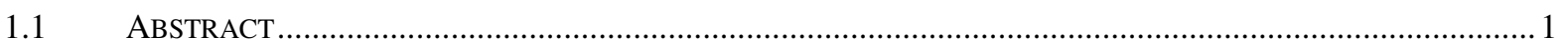

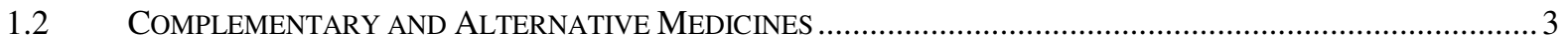

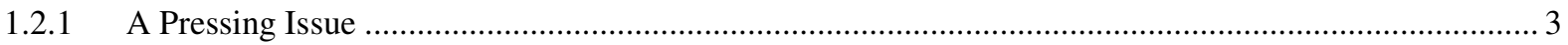

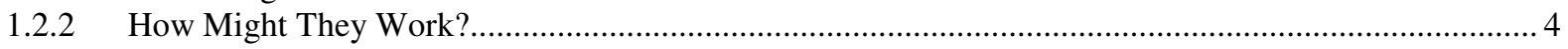

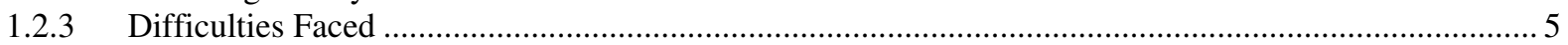

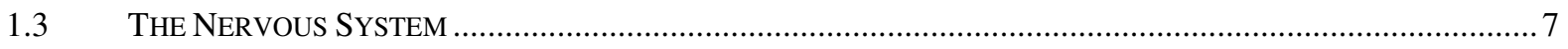

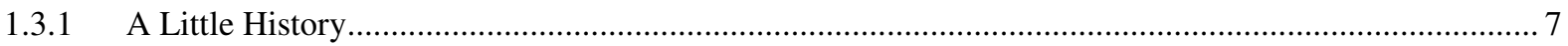

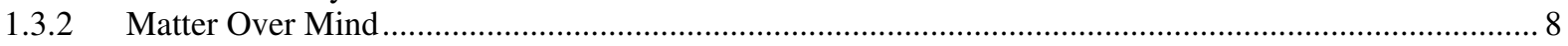

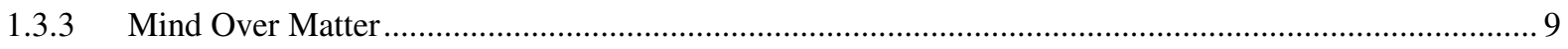

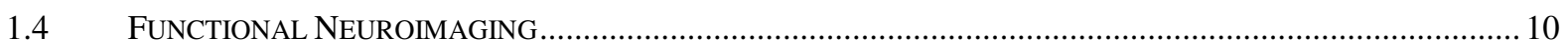

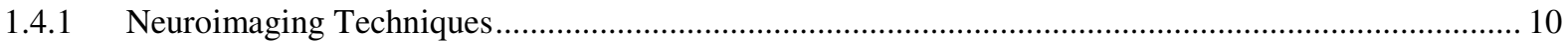

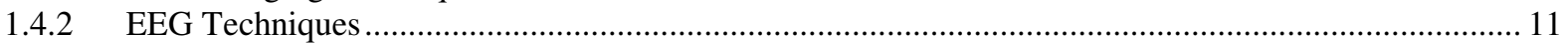

1.4.3 Evoked Potentials \& Averaging ………………................................................................... 12

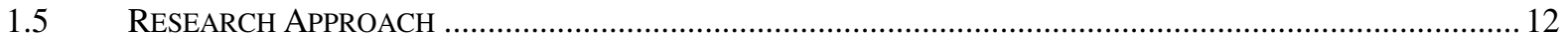

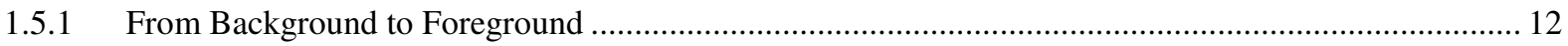

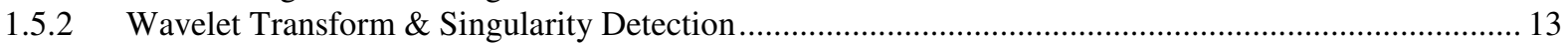

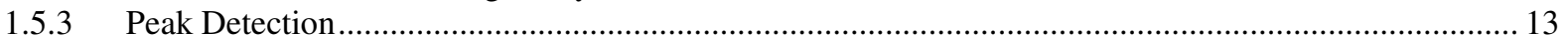

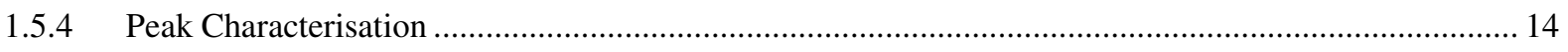

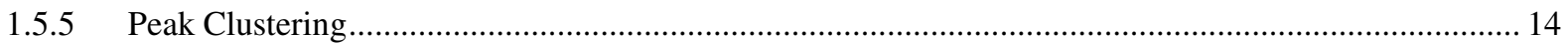

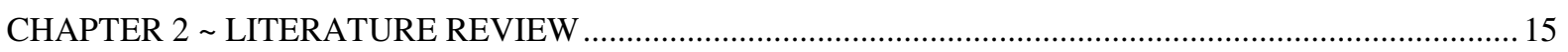

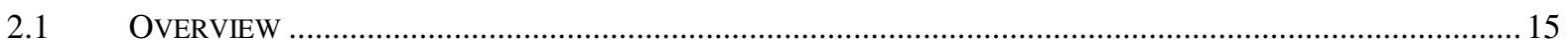

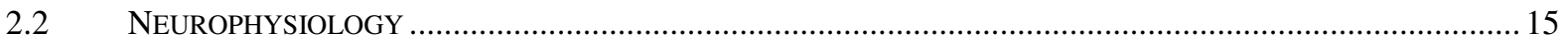

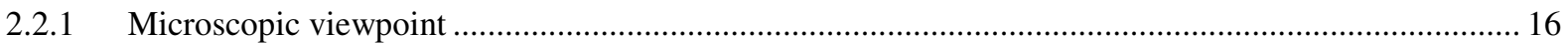

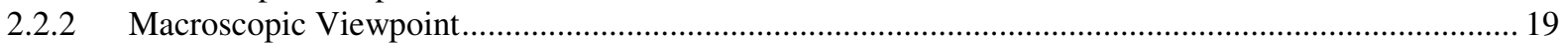

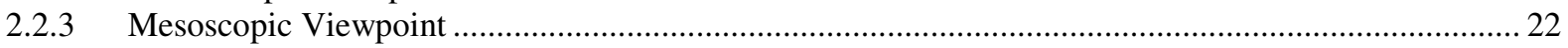

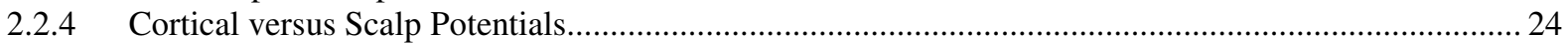

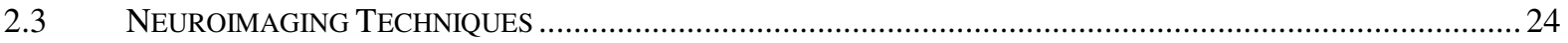

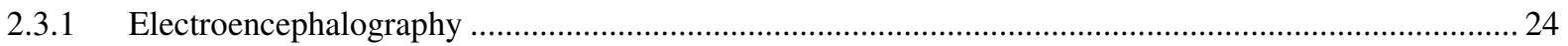

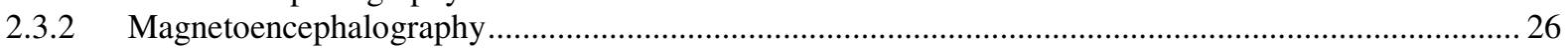

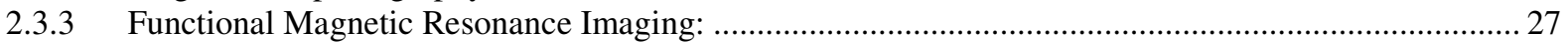

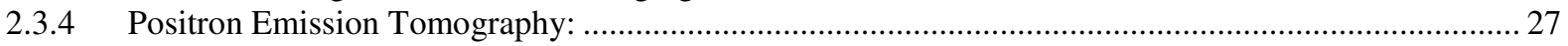

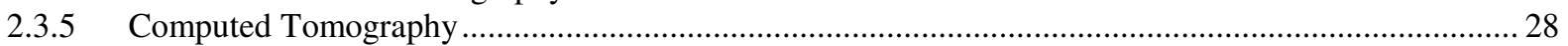

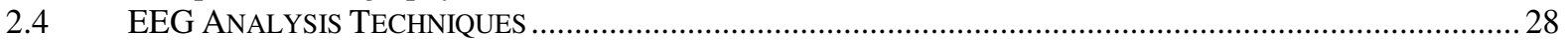

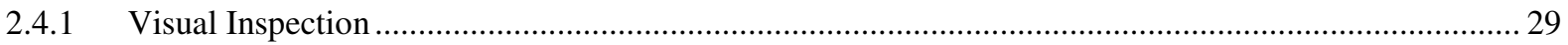

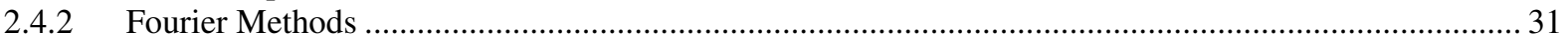

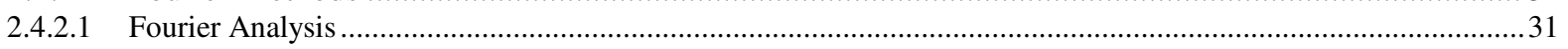

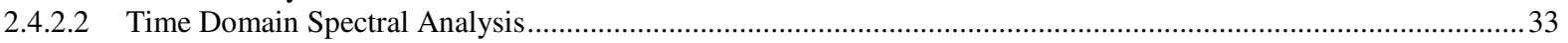

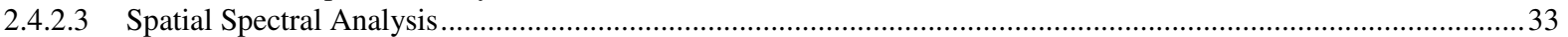

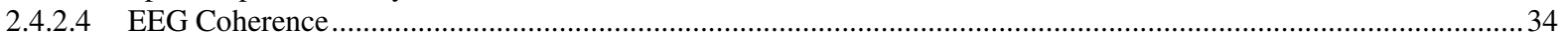

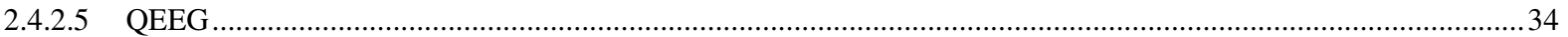

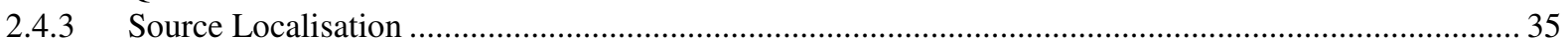

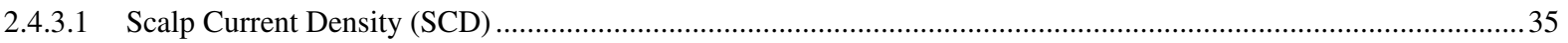

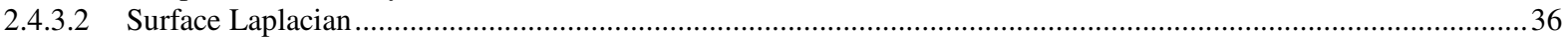

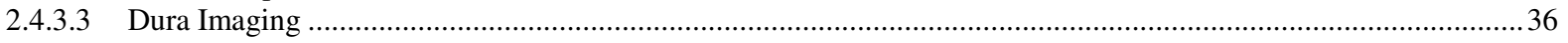

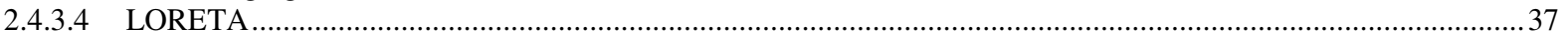

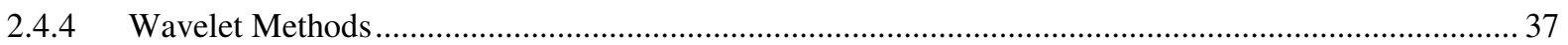

2.4.4.1 Wavelet Filtering and Denoising ........................................................................................................... 38

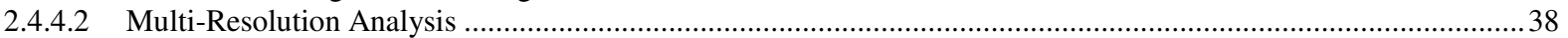

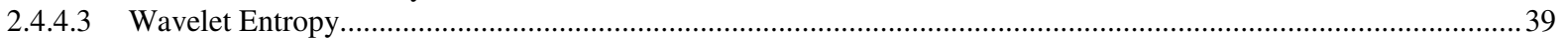

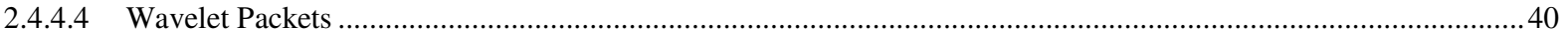

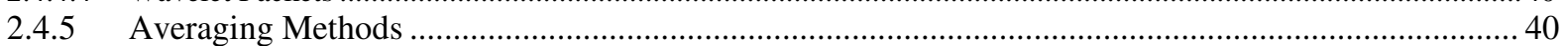

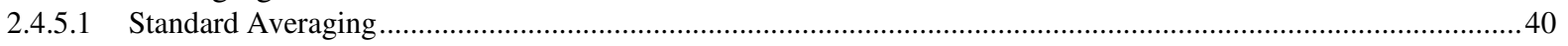

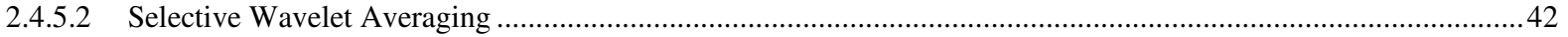

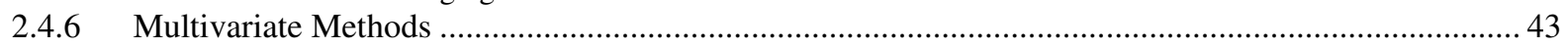

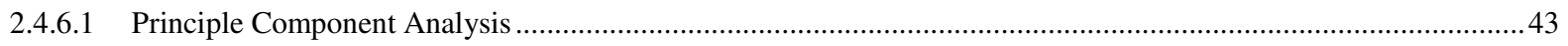




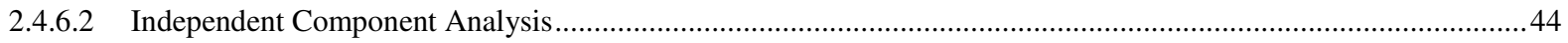

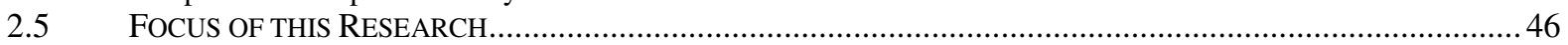

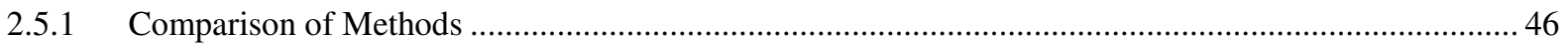

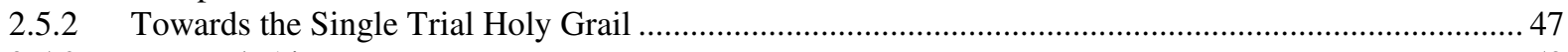

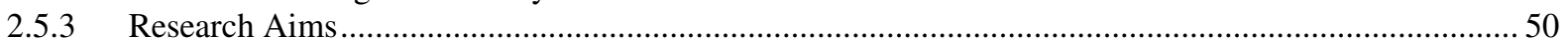

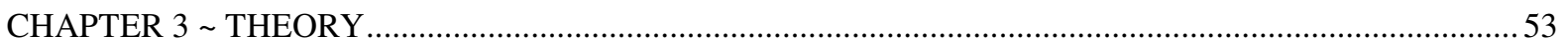

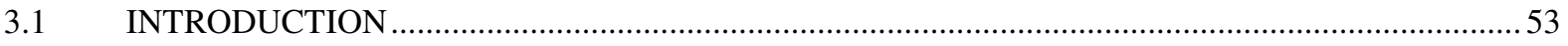

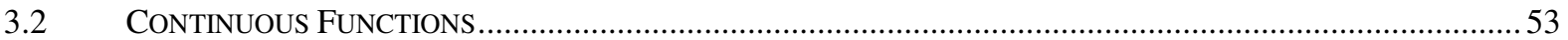

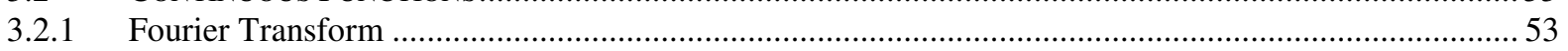

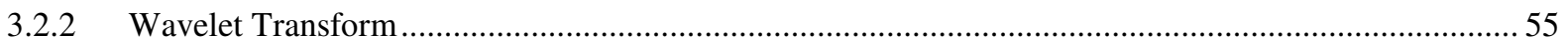

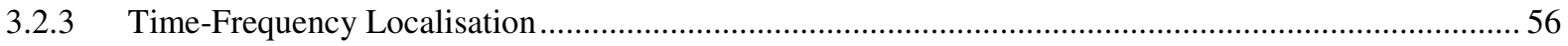

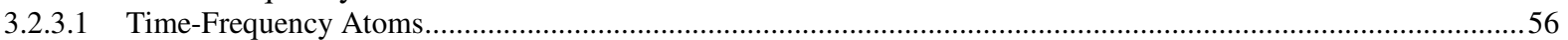

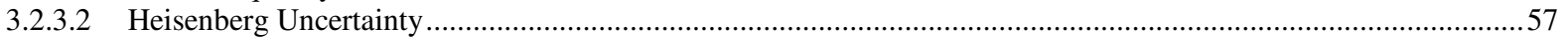

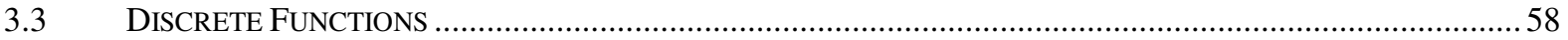

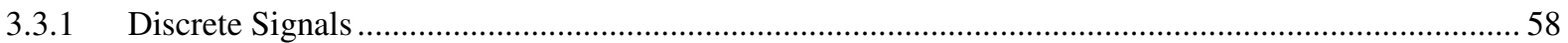

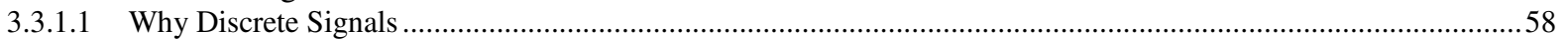

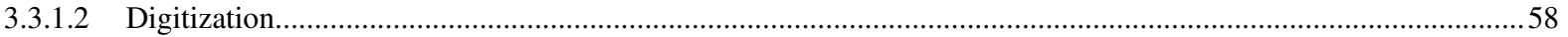

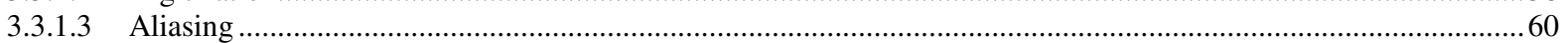

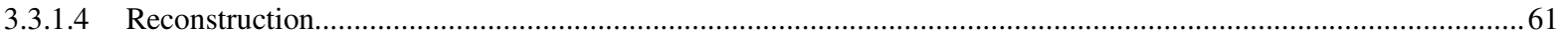

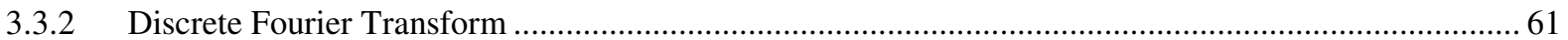

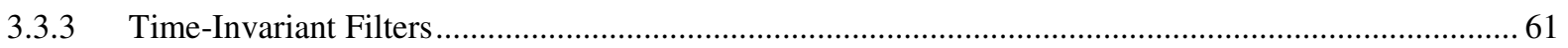

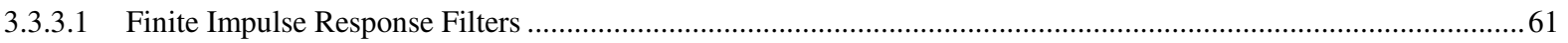

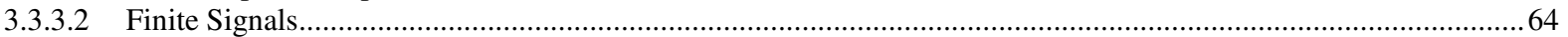

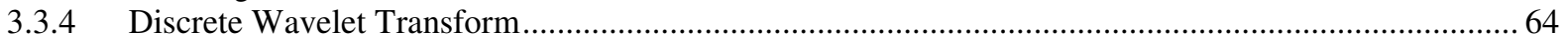

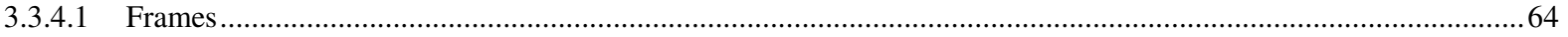

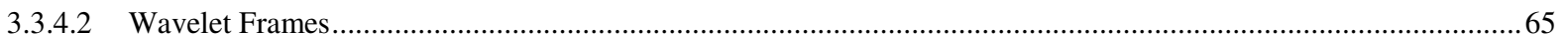

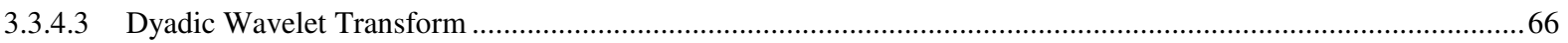

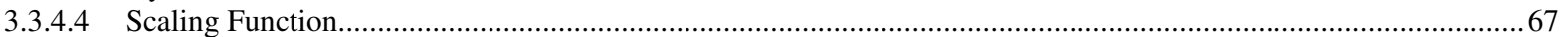

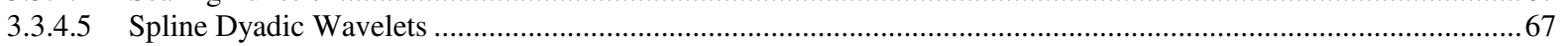

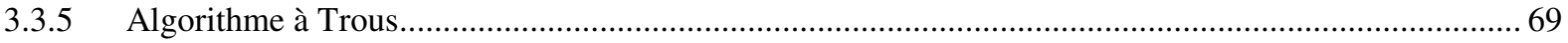

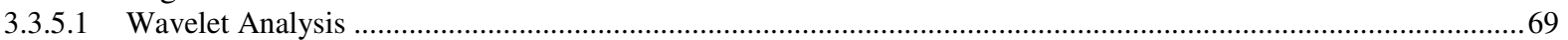

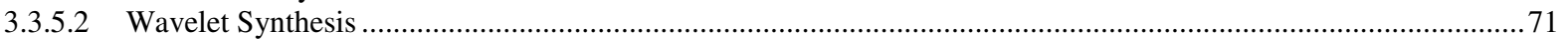

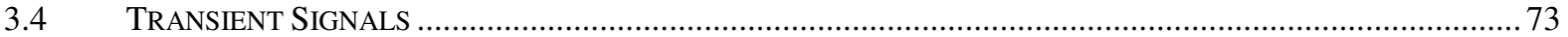

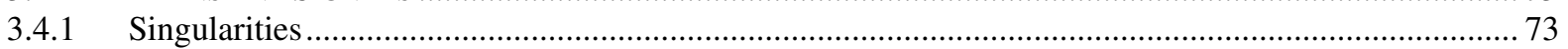

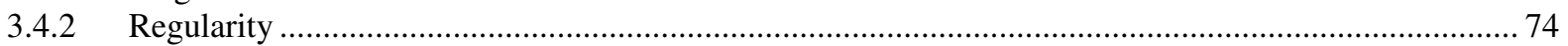

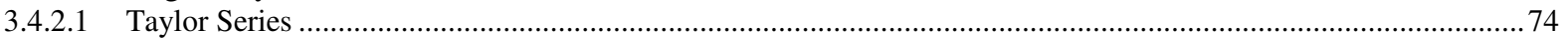

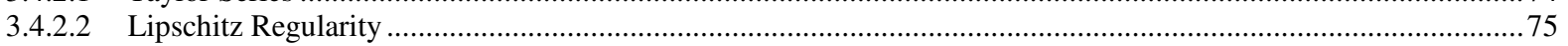

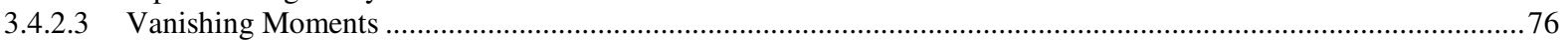

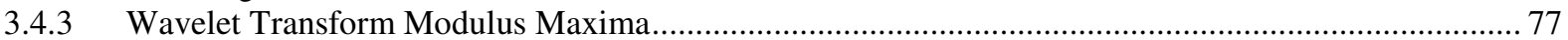

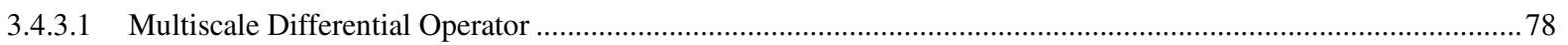

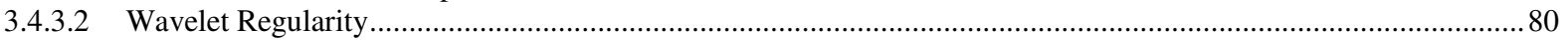

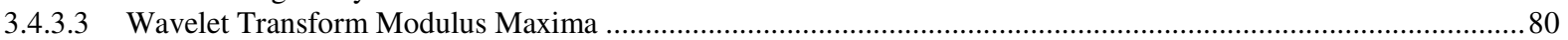

3.4.4 Reconstruction from Modulus Maxima Coefficients ......................................................... 81

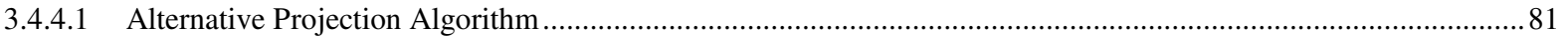

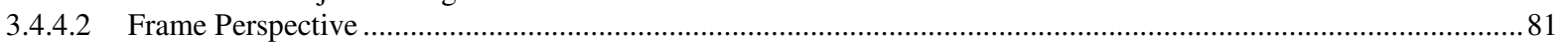

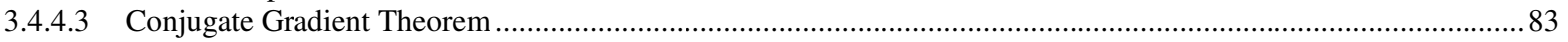

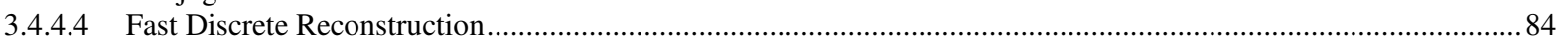

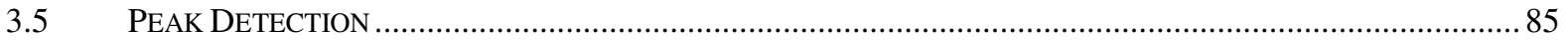

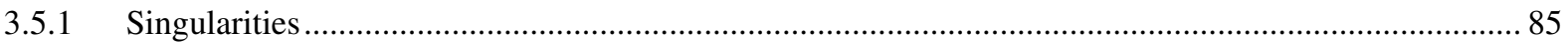

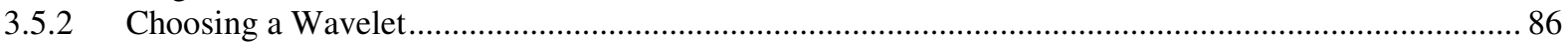

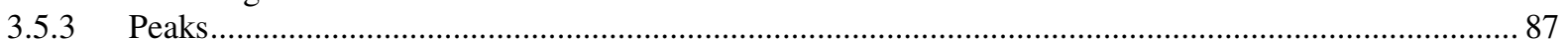

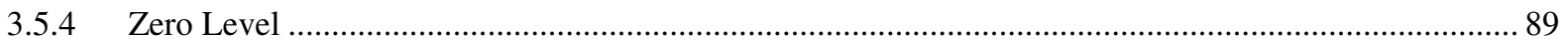

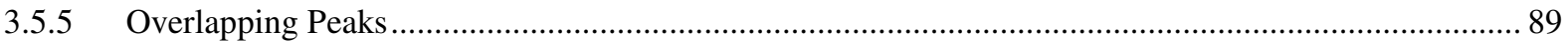

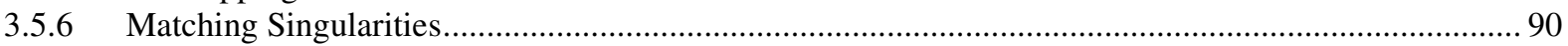

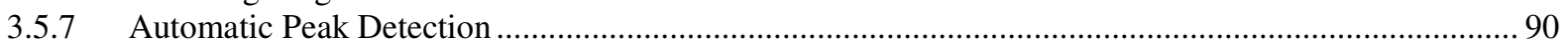

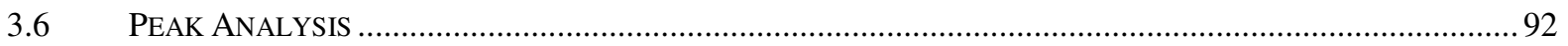

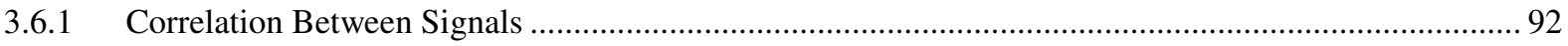

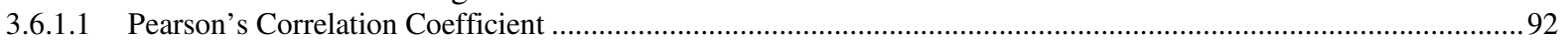

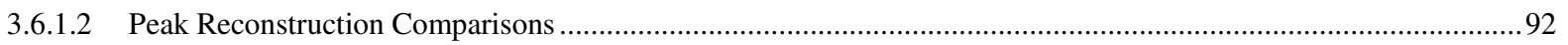

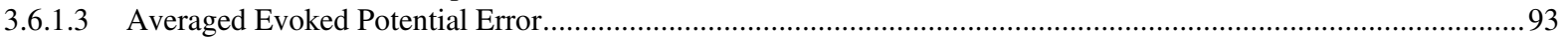




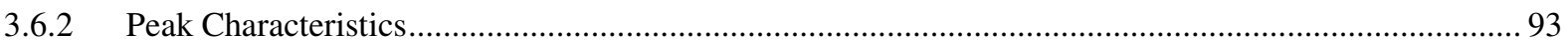

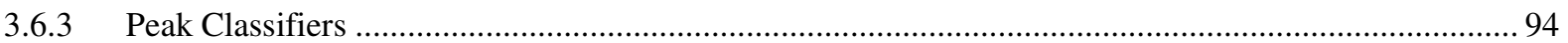

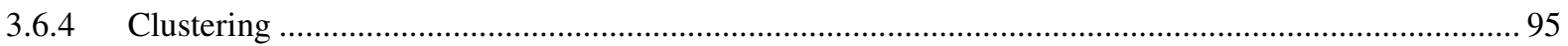

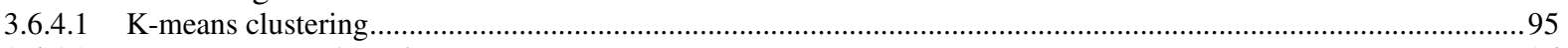

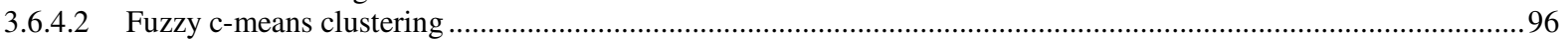

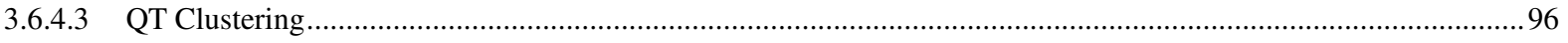

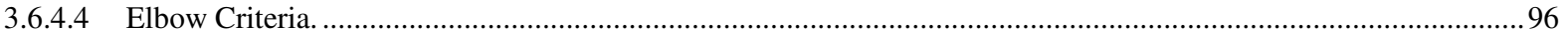

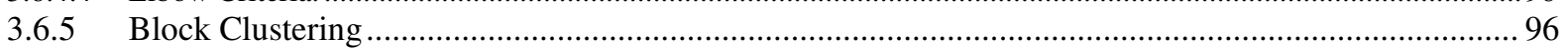

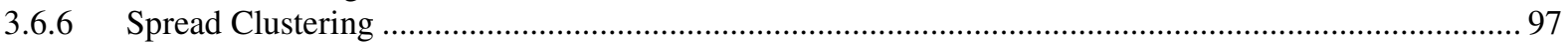

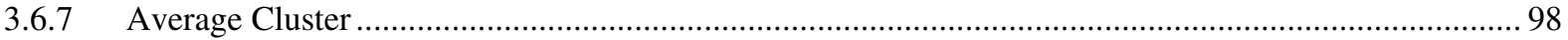

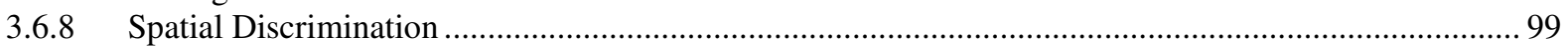

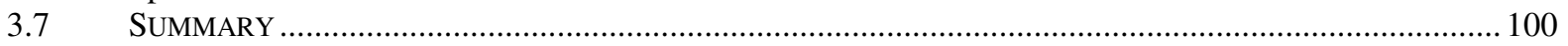

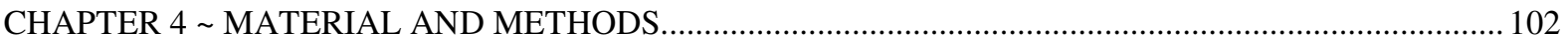

OVERVIEW

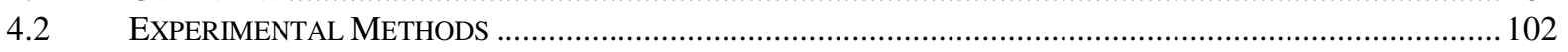

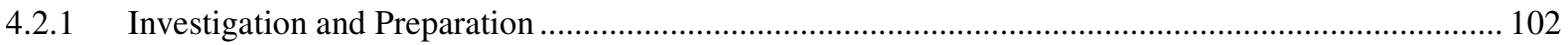

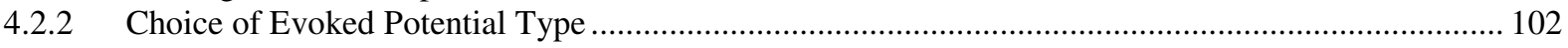

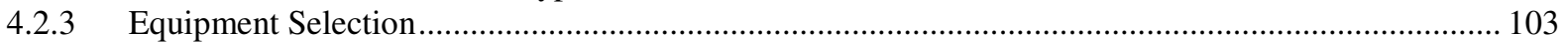

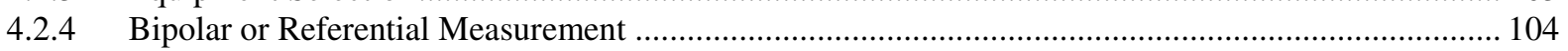

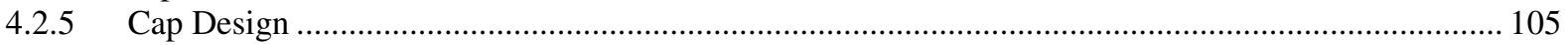

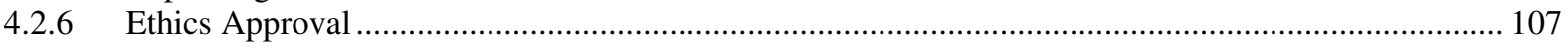

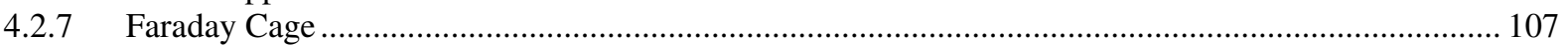

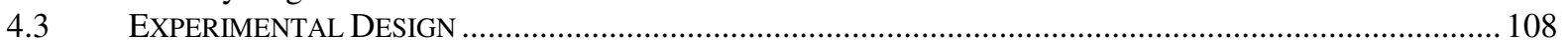

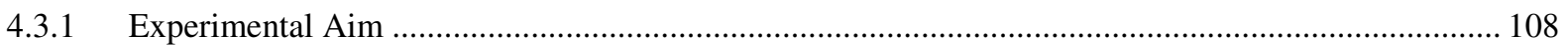

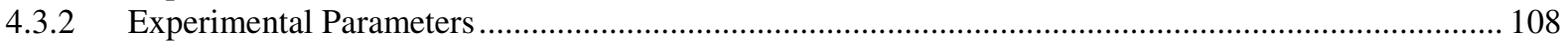

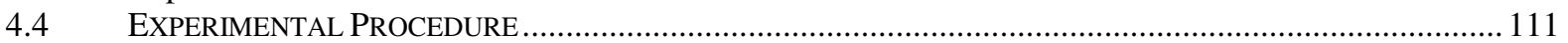

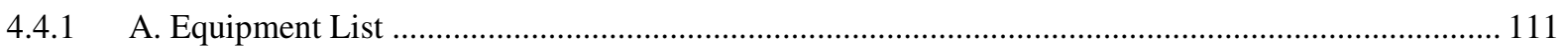

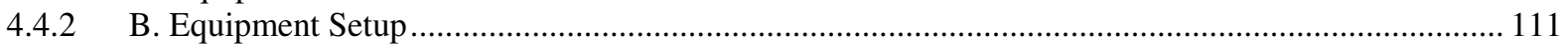

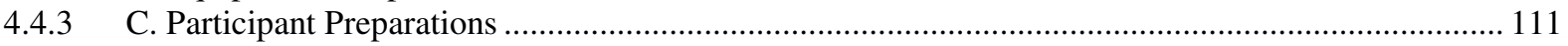

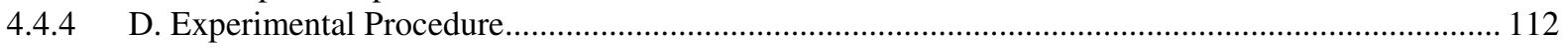

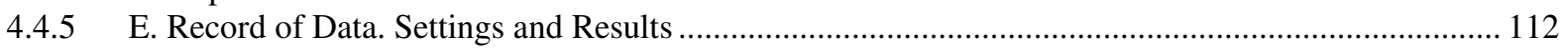

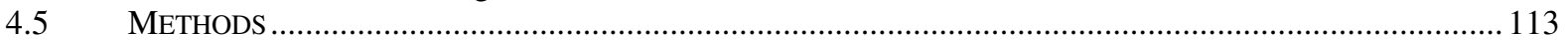

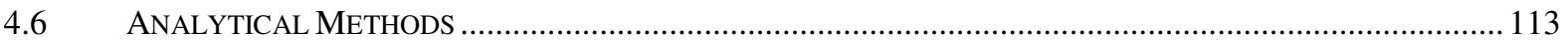

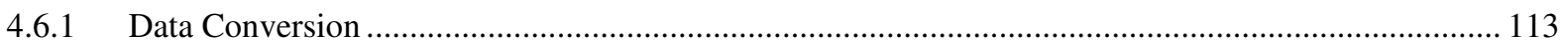

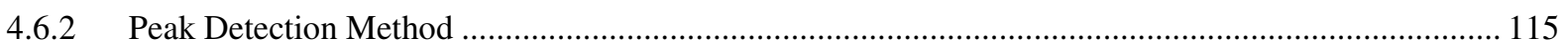

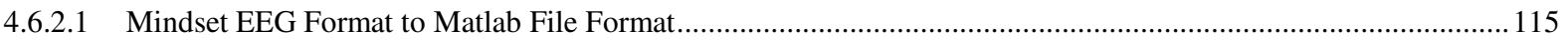

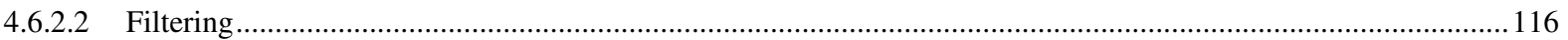

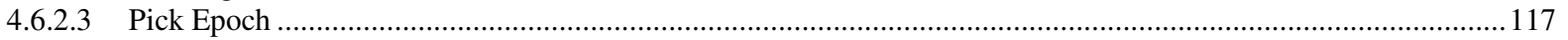

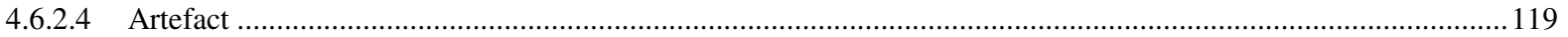

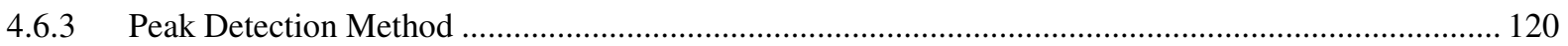

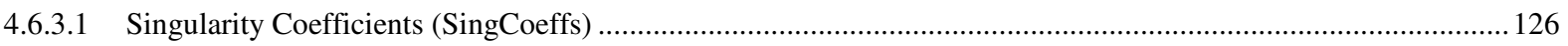

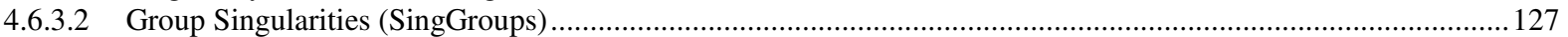

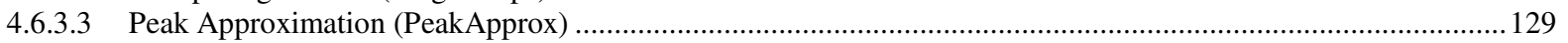

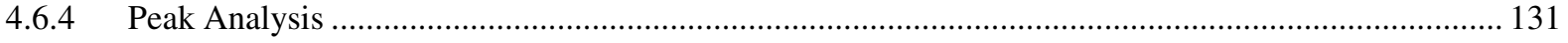

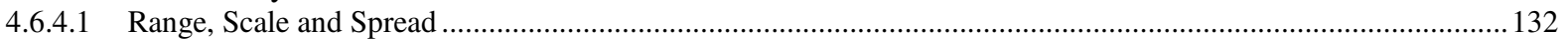

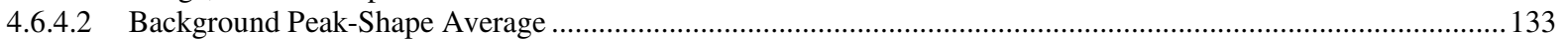

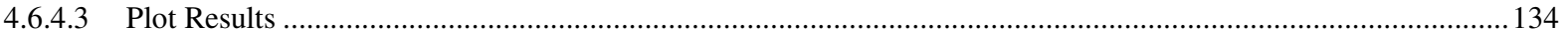

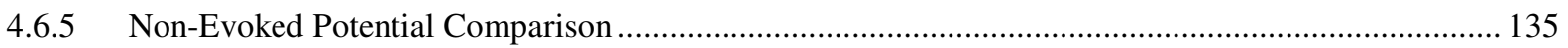

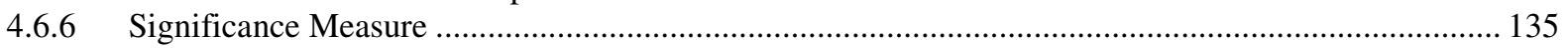

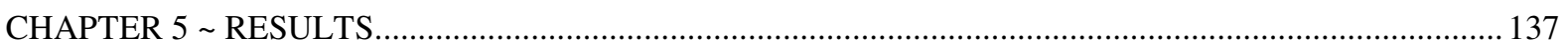

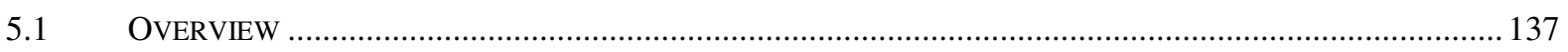

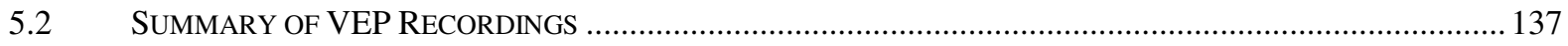

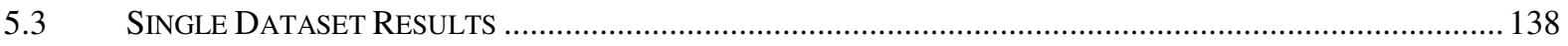

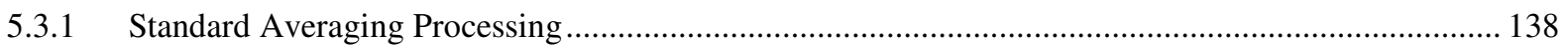

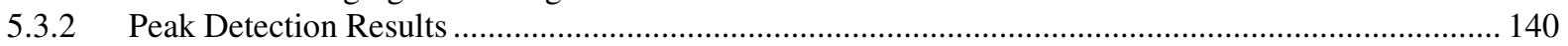

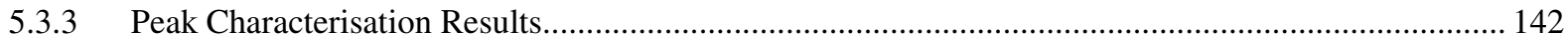

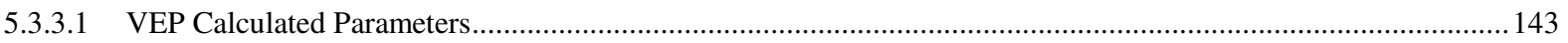

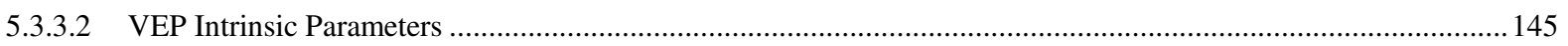




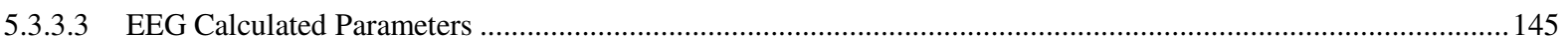

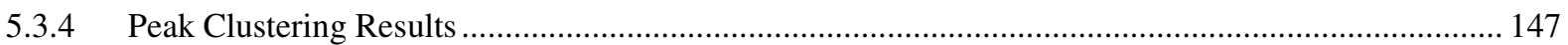

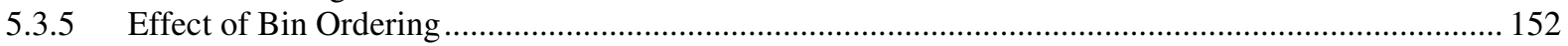

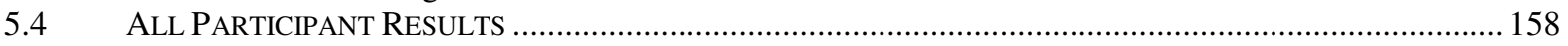

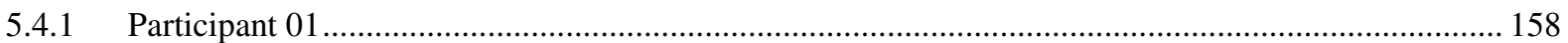

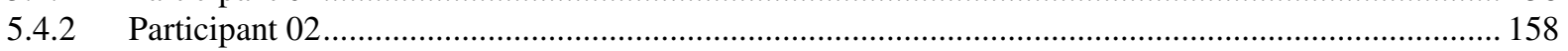

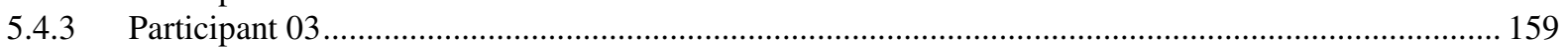

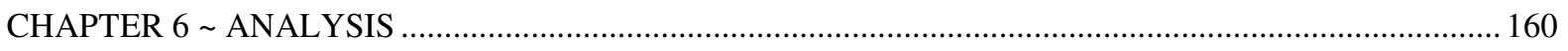

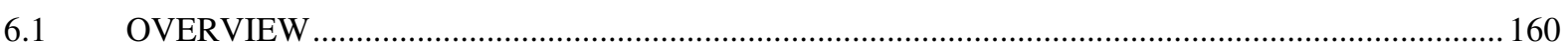

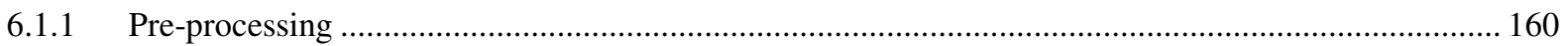

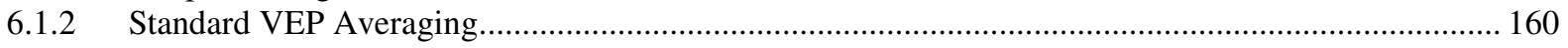

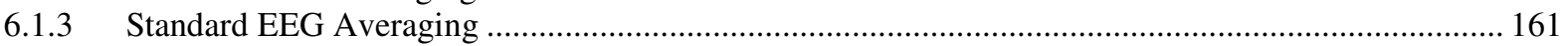

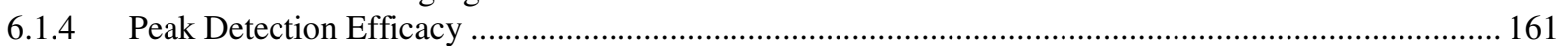

6.1.5 Peak Characterisation - Calculated Time Position ................................................................. 161

6.1.6 Peak Characterisation - Calculated Peak Width...................................................................... 162

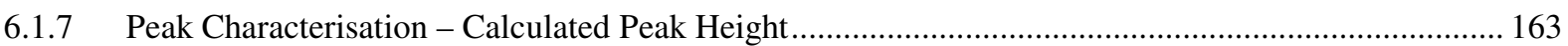

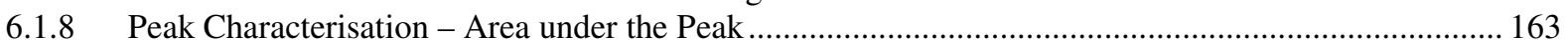

6.1.9 Peak Characterisation - Interrelatedness .......................................................................... 163

6.1.10 Peak Clustering - Mean Concentration Matrices .................................................................. 165

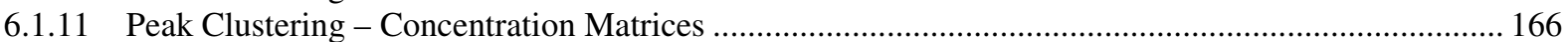

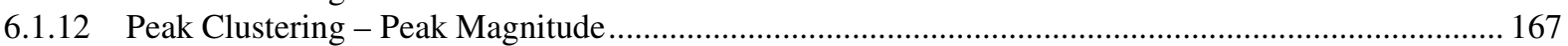

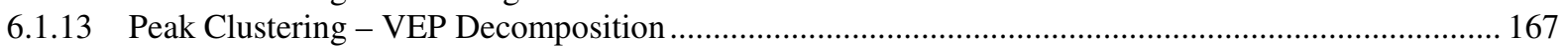

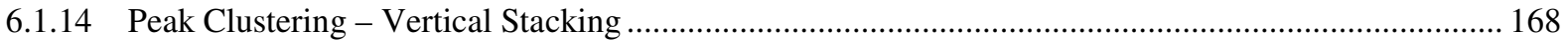

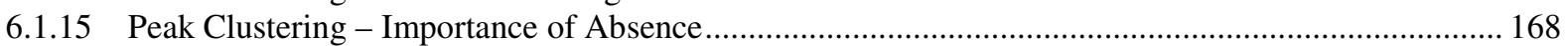

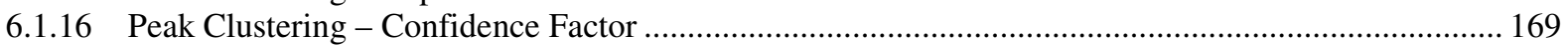

6.1.17 Peak Clustering - Pearson Correlation Coefficient Trends .......................................................... 169

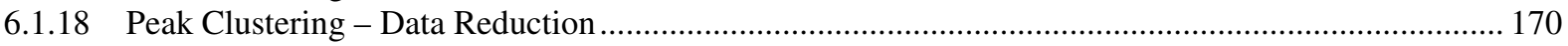

6.1.19 Peak Clustering - P100 and N150 Components ..................................................................... 171

6.1.20 Peak Clustering - Cross-dataset Correlations............................................................................ 172

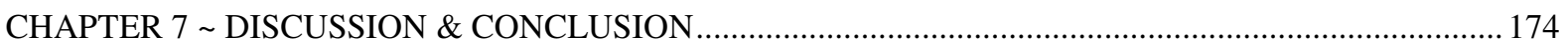

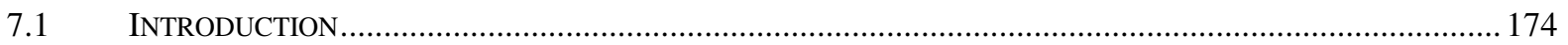

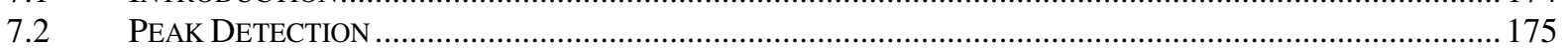

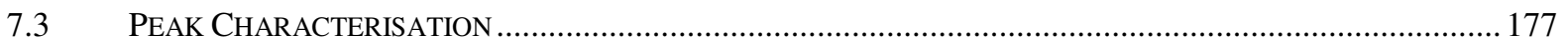

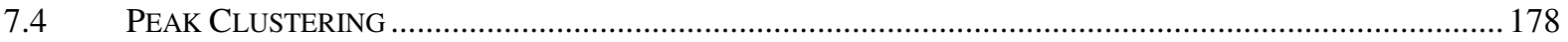

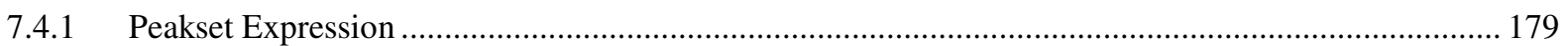

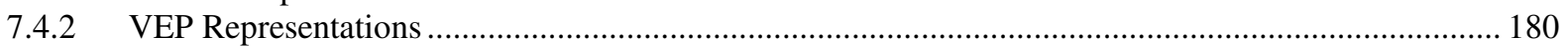

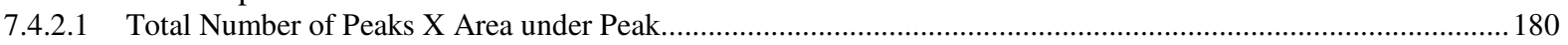

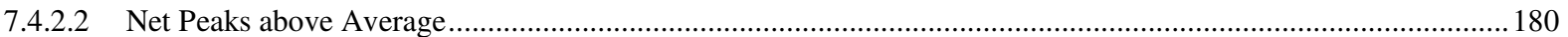

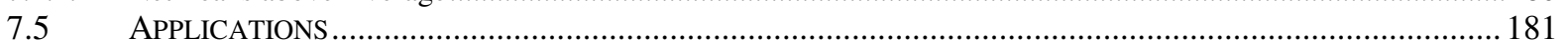

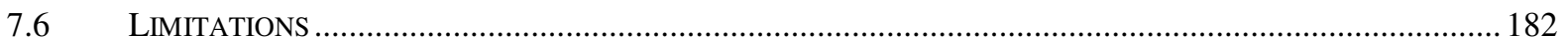

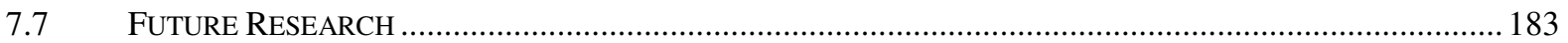

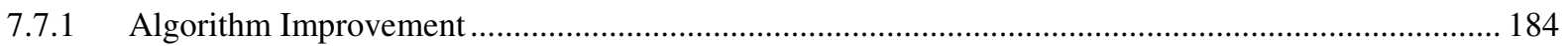

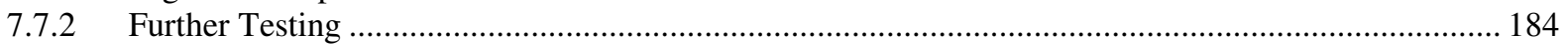

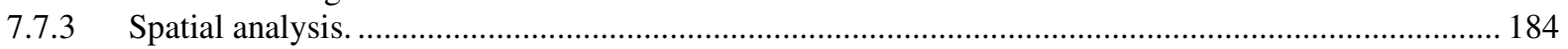

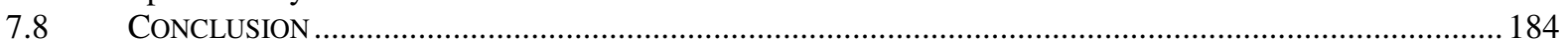

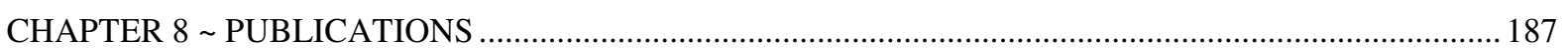

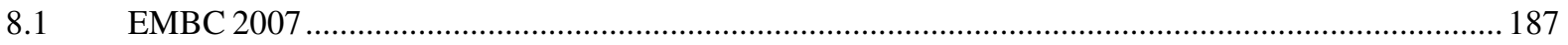

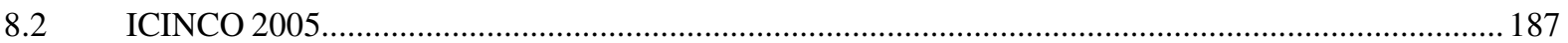

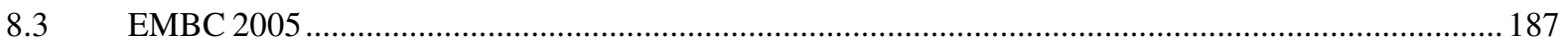

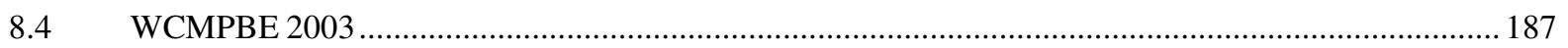

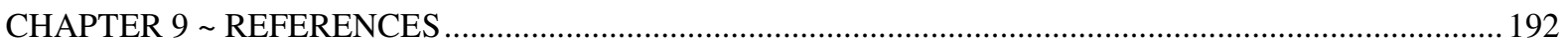

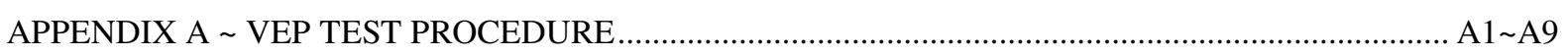

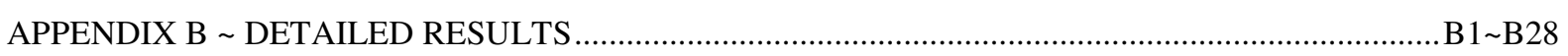

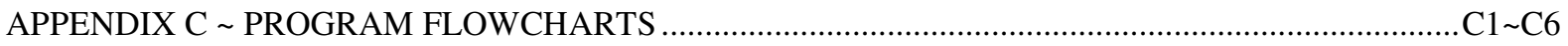


APPENDIX D PUBLISHED PAPERS

D1 D22

APPENDIX E ORIGINAL DATA AND SOFTCOPIES.

..E1 



\section{CHAPTER 1 INTRODUCTION}

\subsection{ABSTRACT}

actile therapies such as reflexology and acupuncture pose a scientific
conundrum. Although, they are ancient medical techniques, predating nearly all
current medical treatments, to this day very little is understood about how, or even if, they work. The majority of the western scientific and medical world is sceptical, yet the public embraces these modalities in ever increasing numbers. These dichotomies make it an area worthy of study and this research investigates innovative scientific methods which may eventually lead to these modalities being better understood.

For both modalities, stimulation (either tactile pressure or a needle piercing the surface of the skin) is applied to a precise location on the body and it is believed that this 'activates' a particular organ elsewhere in the body. Complex acupuncture and reflex pressure point maps have been developed over the centuries that directly relate stimulation of a bodily point to an organ or disease elsewhere in the body. One theory regarding how reflexology and acupuncture work, suggests that stimulation of the nervous system leads to activation of a particular organ. If this is the case then the brain is an obvious thoroughfare through which such messages could be sent. Therefore, this research begins with the question; can a localised, single stimulation of sensory nervous tissue in the body be detected in the brain?

Currently, the answer is no. Of all brain scanning techniques available today, only Electroencephalography (EEG) offers the temporal resolution (in the order of milliseconds) to pick out single events. However, EEG signals from the brain are so tiny (in the order of microvolts) and so shrouded in ongoing background EEG activity that many repeated stimulations are necessary to detect a signal - a technique known as averaging of Evoked Potentials (EP). Also, the spatial resolution of EEG is poor, although advances in high resolution EEG are helping to mitigate this drawback. This research looks at EPs and investigates ways in which a localised, single stimulation may be detected. Evoked Potentials are studied rather than acupuncture or reflexology directly because evoked potentials offer more efficient and controllable stimuli than these modalities. Once developed for evoked potentials, these techniques may then be applied to investigate therapeutic modalities such as acupuncture and reflexology in future research. 
The analysis of Evoked Potentials is a rich area of research study and various different approaches are discussed in more detail in Chapter 2. It is noted that these different techniques tend to emphasize features in one domain at the expense of features in other domains. In this way the Fourier Transform will yield excellent spectral information but all temporal information is lost in the process. The identifiable domains described in this research are temporal (time), spectral (frequency/scale), spatial (space) and epoch (multiple events). The epoch domain is not a truly independent domain since it provides a repetition of the event and so is made up of time, frequency and spatial components. However, it does add to the overall information content and analysis techniques will impact how information may be observed in the epoch domain.

Useful information may be detected in each of these domains. The extent of this research is to examine the temporal, spectral and epoch domains. It does not extend to including the spatial domain; although it is possible to include spatial domain analysis in future research. From this approach, a proposition is suggested. Is it possible to detect features across different domains, a true multi-domain analysis, and yet retain features in other domains at the same time? The aim of this research is to use multi-domain analysis of electroencephalographic evoked potential data to elicit a better understanding of evoked potential data. This research attempts to steer a course along a path where detecting the response of the brain to a localised, single stimulation to the body is the ultimate destination.

The implications for making real strides along such a path go far beyond a mechanism for better understanding complementary therapies. It could form a foundation for new braincomputer interface (BCI) techniques. This is a fast developing area of research where signals from the brain are interpreted to allow control over external devices. Decomposing brain signals into constituent parts is of foremost importance in this field of study. It is only by separating components and identifying those associated with sense or motor control that genuine and reliable communication channels can be opened between brain and computer. For example, some Brain-Computer Interface applications utilize averaged evoked potentials to interpret intention from a subject. By reducing the number of averages necessary, it is possible to speed up the interface reaction time.

It is proposed that investigation in this area may also elucidate the area of mesoscopic brain dynamics. The microscopic view of the brain encompasses the field of neuroanatomy - the function and behaviour of individual neurons and other cells in the 
nervous system. The behaviour of individual cells is well understood. At the other end of the scale, macroscopic brain dynamics investigates broad areas of the brain as one and how coordinated groups act together. Functional Magnetic Resonance Imaging is one technology that provides very useful information in this domain. However, there is no unifying theory to explain behaviour at both scales - i.e. how neurons organise themselves into the complex processing required by the brain is not understood. Thus investigation in the intermediate mesoscopic brain dynamic field is most important. Decomposing detected peaks is one level down from the existing macroscopic view.

A broader discussion of this topic is presented in the rest of the introduction chapter and the research questions arising are posed. Methods of identifying and characterising the peaks and troughs of evoked potentials are investigated and the Discrete Dyadic Wavelet Transform is chosen. By using this transform to characterise each peak, a dataset is decomposed into a set of discrete peaks. Each peak is describable in any domain and hence may be analysed in different domains. There is also an inherent traceability where any observation may be traced back to particular peaks. The complexity of analysing across multiple domains requires some simplification and so grouping of similar type peaks is performed. The analytical problem of examine massive amounts of data in multiple domains is transformed into a much simpler classification problem. A clustering analysis technique is employed to better isolate commonly observed evoked potentials components. The results provide additional information about evoked potential signals which is useful to clinicians. This is achieved by providing a breakdown of the components that make up an evoked potential.

\subsection{COMPLEMENTARY AND ALTERNATIVE MEDICINES}

\subsubsection{A Pressing Issue}

Tactile complementary and alternative medicine (CAM) therapies such as reflexology and acupuncture are becoming more prevalent in modern western society. It is estimated that over 8 million U.S. adults have had acupuncture treatment, with over 2 million receiving acupuncture treatment in 2001 alone [8]. These therapies generally predate modern conventional medicine $(\mathrm{CM})$ techniques yet despite a great wealth of knowledge into the exact methods involved and how to apply them; they have lagged conventional medicine in understanding of the mechanisms by which they may work. Studies in allied techniques such as chiropractic care and massage treatment report very high patient 
satisfaction [9]. Practise of CAM therapies has grown significantly mostly outside the conventional health care system based on patient demand. Private health insurance funds have, in recent years, helped to bring such modalities into the mainstream by offering coverage of such services. This coverage tends to be based on meeting customer expectations rather than scientific proof of the efficacy of the modality. There is also evidence to suggest that high-technology medical equipment and high development cost pharmacological drugs, which are favoured in the western medical system, do not necessary yield the best patient outcomes ${ }^{[14]}$. The follow-on suggestion is that perhaps more low-tech, low-cost complementary and alternative solutions could have a larger role to play.

While much research continues to try to draw CAM and CM together, with an emphasis on clinical trials; very little is understood regarding how these techniques actually work. As the use of these techniques becomes more widespread, the lack of broad acceptance within western conventional medicine becomes more of an issue.

\subsubsection{How Might They Work?}

Acupuncture is by far the most studied and accepted CAM modality. There are considerably less formal studies into reflexology and other tactile therapies. The purpose of this research is to identify techniques that may be applied in the future to acupuncture, reflexology and perhaps other tactile therapies.

Many studies offer no rationale as to how acupuncture may work and those that do tend of offer a brief hypothesis which is not tested in the rest of the study [10]. The dominant theory regarding how acupuncture may work is described by the National Center for Complementary and Alternative Medicine (NCCAM) which is part of the National Institute of Health in the United States of America.

"Preclinical studies have documented acupuncture's effects, but they have not been able to fully explain how acupuncture works within the framework of the Western system of medicine that is commonly practiced in the United States. It is proposed that acupuncture produces its effects through regulating the nervous system, thus aiding the activity of pain-killing 
biochemicals such as endorphins and immune system cells at specific sites in the body. In addition, studies have shown that acupuncture may alter brain chemistry by changing the release of neurotransmitters and neurohormones and, thus, affecting the parts of the central nervous system related to sensation and involuntary body functions, such as immune reactions and processes that regulate a person's blood pressure, blood flow, and body temperature." [11]

A recent review of acupuncture clinical trial groups acupuncture theory into five major theories [10]:

1. Acupuncture stimulates the release of neurochemicals usually endogenous opioids,

2. Acupuncture has a segmented effect known as "gate theory". This theory postulates that pain is blocked by sensory input due to acupuncture,

3. Acupuncture has a modulating effect on the autonomic nervous system,

4. Acupuncture has a local effect on tissues, nerve or immune function,

5. Acupuncture can cause specific functional effects in the brain.

The nervous system is strongly favoured as the mechanism by which acupuncture may work. The human nervous system provides the sensory hardware and communications network that is capable of recognising both endogenous pain and exogenous stimulation and also, triggering a corrective response in another area of the body.

\subsubsection{Difficulties Faced}

It is notoriously difficult to prove the efficacy of complementary and alternative medicine techniques and invariably most formal trials in the past have suffered in some deficiency such as a compromised test procedure or lack of a viable placebo. Those trials that appear of sufficient scientific standard don't usually offer glowing proof of these methods. In a nutshell, practitioners and patients appear much more convinced than the mainstream scientific community.

The placebo response or placebo effect is described as a positive therapeutic effect in response to a sham therapy or inactive medication. This effect has been observed for hundreds of years and in 1955 Henry Beecher published 'The Powerful Placebo' which 
brought it to the forefront or modern scientific research. Since this time it has become the mainstay of modern clinical trials by virtue of the fact that most clinical trials split subjects into a test group and a control group. The test group will receive the treatment under trial; the control group will receive an apparently identical treatment but without the active agent. Hence if a pharmacological drug is under trial they will receive a sugar pill instead. Only if the results for the test group are significantly better than the control group can the treatment be classified as effective. The implication from this rationale is that the sham treatment is expected to demonstrate some patient amelioration and only if the genuine treatment is significantly higher than this can a genuine therapeutic effect of the test treatment be asserted.

There are many studies proving the presence of a placebo response. For example, one study showed that depressed patients who responded to placebo treatment exhibited significant changes in pre-frontal cerebral blood flow [12]. While another review paper argues that up to $75 \%$ of the effectiveness of anti-depressant medication may be attributable to the placebo response instead of the treatment itself [13].

Understanding the body's placebo response is important since it can be used to effect improvement in a subjects underlying condition. In the majority of cases there is no intermediate measurable quantity between the therapeutic treatment and the subjective response of the patient. Developing an intermediate detection technique would allow an objective measure to be taken and perhaps assist in delineating between true therapeutic improvement and a placebo response.

Since the interaction between therapeutic effect and placebo effect is difficult to separate, the trend in western communities is more and more towards acceptance of complementary therapies even without formal scientific acceptance.

Reflexology and acupuncture are based on the premise that stimulation of particular localised areas of the body causes organs located elsewhere to be beneficially affected. How is this possible and what pathways might be implicated? It is acknowledged that the philosophy attached to these techniques usually employed generic 'energy shift' descriptions outside conventional scientific understanding. However, this study particularly wanted to try to understand these techniques within conventional scientific wisdom and so it is hypothesised that the brain may the sensory conduit through which such messages may be sent. Is it possible to apply a particular stimulation to the body 
such as from reflexology or acupuncture and observe a response from the brain? If so, can the response from the brain be linked to any currently known brain processing function associated with the organ expected to be affected from reflexology or acupuncture methods? These are the follow-on questions that arise from this research.

\subsection{THE NERVOUS SYSTEM}

\subsubsection{A Little History}

The earliest recorded mention of the brain was recorded in the Edwyn Smyth Surgical Papyrus written in the $17^{\text {th }}$ century BC and credited to the Egyptian high priest Imhotep. The hieroglyphic for brain (Fig 1.1) occurs eight times in the

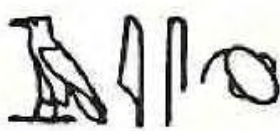

Fig. 1.1: Hieroglyphic for the word brain (c. $1700 \mathrm{BC}$ ) [1] description of the symptoms, diagnosis and prognosis of two patients with compound skull fractures. The first detailed view on the nature of the brain is recorded by the ancient Greeks. It was Alcmaeon in the $5^{\text {th }}$ century BC who first considered the brain to be the place where the mind was located. He is recorded as the first to link the brain to the sense organs. In the $4^{\text {th }}$ century BC Hippocrates declared the brain to be the seat of intelligence. Aristotle meanwhile believed the heart was the seat of intelligence and the brain was a cooling mechanism for the blood. He reasoned that humans were more rational than animals because their larger brain helped cool their hot-headedness.

In the $2^{\text {nd }}$ century $\mathrm{BC}$, Herophilis together with Erasistratus made great advances in the medical understanding of the brain. The former recognised the brain as the centre of the nervous system and distinguished nerves from blood vessels and motor and sensory nerves. Interestingly, Herophilis is credited with being one of the first scientists, in the modern sense of the word. He introduced experimental method to medicine using empirical evidence for his findings. As such his legacy lives on in the scientific rigour applied in this thesis [1].

Recorded history regarding the brain from the ancient Greeks up to the time of Descartes in the $17^{\text {th }}$ century focuses on the so-called mind-body problem. That is the delineation between the physical brain and the higher cognitive processes assigned to the mind. It was Galvani in the $18^{\text {th }}$ century who introduced the electrical theory of the brain. He electrically stimulated the sciatic nerve of a frog causing it to move. The techniques of observing responses in the brain to particular stimuli have evolved greatly in the 
intervening years, yet a huge mass of research continue to revolve around this very topic. Stimulus and response experiments remain at the heart of our search to understand ourselves.

\subsubsection{Matter Over Mind}

The nervous system is divided into the Central Nervous System (CNS) made up of the brain and the spinal cord and the Peripheral Nervous System (PNS) made up of all nerve tissue outside the brain and spinal cord. The PNS is further divided into the Somatic Nervous system which sensory signals to the CNS and conducts motor signals from the CNS to the skeletal muscles and the Autonomic Nervous system which regulates the internal organs of the body relaying sensory information from the organs to the CNS and motor signals from the CNS to the organs. Fig. 1.2 graphically represents some of the higher level divisions of the nervous system. The path highlighted in blue illustrates the path of interest in this research. This is the path taking sensory information from the outside world and sending this information to the brain. It includes the 5 typical senses of sight, hearing, smell, taste and touch. Acupuncture and Reflexology fit under the sense of touch, known as the somatosensory system 


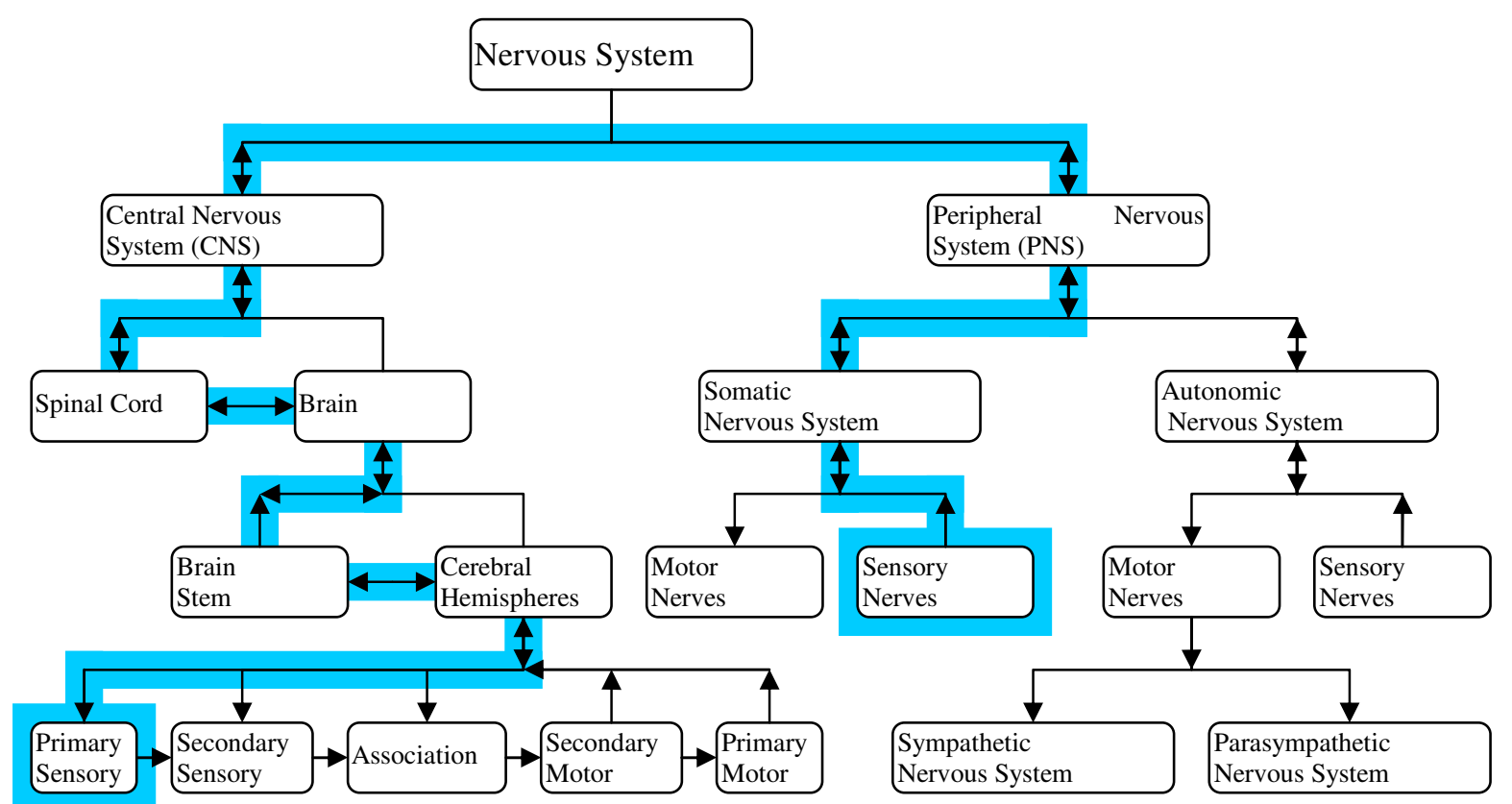

Fig. 1.2: Divisions of the Nervous System. Boxes indicate hierarchical nomenclature given to the separate parts. Arrows indicate the direction of flow of information. For example, the information flow for a Somatosensory Evoked Potential is illustrated by the blue shading. In this case, an electrical stimulus is detected by the sensory nerves in the somatic nervous system, typically in the hard or foot, and travels along neural pathways until it reaches the primary sensory cortex of the brain. Evoked Potentials are measured at this point. There are several items of note in this situation. There is an aggregation of sensory information along this path particularly once the signal enter the brain which makes a 1 to 1 mapping of stimulus and cortex response more difficult. Secondly, the arrival of the observable response at the primary sensory cortex is not the endpoint of the signal. This information may be relayed to the secondary sensory area and further integrated with other sensory information in the association area and if a physical response is then initiated the motor area is activated and a new signal is sent along the motor path back to the relevant organ. (Adapted from [3])

\subsubsection{Mind Over Matter}

Consider the advancement of our species and the planet we inhabit; complex societies, engineering feats, scientific understanding, creative arts, and even wilful destruction. The common thread in all these facets is the human brain and one can only marvel at its omnipotent influence on our lives and our planet. In comparing our physiology with other creatures in the animal kingdom, it is our brain which sets us apart. Our achievements as a species by any measure are outstanding. The correlation between mind and brain is not a simple matter. Physical aspects and simpler functions tend to be ascribed to the brain. Complex, higher reasoning tends to be ascribed to the mind. Are they the same? It is clear that if the answer is not known now, then the only way it will be found is by more advanced study of the brain and its functions. We must use the brain to study the brain to understand the brain. Since the first semi-quantitative measurement of brain activity by Hans Berger in 1924, the workings of the brain have been gradually unravelled and our understanding improved. However, this one organ still retains more 
secrets as to its understanding than any other organ in the body due to its massive complexity for which our technology and comprehension cannot yet match.

\subsection{FUNCTIONAL NEUROIMAGING}

\subsubsection{Neuroimaging Techniques}

In order to understand brain activity, it is first necessary to measure it in some way. Functional neuroimaging describes a range of techniques that measure brain activity and then relate it to certain mental functions. Electroencephalography (EEG) measures tiny electrical fields detectable on the surface of the scalp. A pair of EEG electrodes creates a loop which measures the potential difference between these electrodes. It is believed that EEG is mostly detecting coherent firing of post synaptic potentials in many nerve cells located in the cerebral cortex [6]. EEG is the technique that most directly measures nerve cell activity as both nerve cells are electrical conductors. Magnetoencephalography (MEG) measures the magnet field that is associated with the electrical field produced by groups of nerve cells. It has a similar good temporal resolution and poor spatial resolution as EEG.

Functional Magnetic Resonance Imaging (fMRI) uses a rotating magnetic field and radio waves to detect changes in blood flow. When nerve cells are activated, they use oxygen. This in turn triggers blood to flow to replenish the deoxygenated blood. This is known as the hemodynamic response. fMRI can detect this blood flow. As it is an indirect measure of nerve activity there is an intrinsic delay between activity and observed response which limits the temporal resolution of this device to the order of seconds. However, it has excellent spatial resolution to the order of $1-2 \mathrm{~mm}$ and can provide highly detailed structural and functional images of the brain and its activity. Other techniques such as Computed Tomography (CT) and Positron Emission Tomography (PET) use different principles but provide similar results to fMRI i.e. excellent spatial resolution but poor temporal resolution.

Each of the currently available techniques varies in principle, resolution and level of invasiveness. A comparison is illustrated in Table 1.1 with a more detailed discussion on the analytical aspects provided in Chapter 2. Currently there is no method to overcome the temporal limitations of fMRI, PET and CT techniques although there are gradual incremental improvements being developed. The spatial limitation of EEG can to some 
extent be overcome by using a high density array of electrodes. This yields an interpreted set of discrete measurements in space which may be interpolated to yield an improved spatial resolution. Higher density MEG is more difficult to achieve due to the high cost of MEG electrodes and their bulkiness. Therefore, in this research, it was decided that EEG would provide the most suitable measurement technique from which to investigate the brains response to single stimuli.

\begin{tabular}{|l|l|l|l|l|}
\hline Technique & Principle & Spatial Resolution & Temporal Resolution & Invasive \\
\hline EEG & Electric Field & $\begin{array}{l}\text { Poor but improving } \\
\sim 2-3 \mathrm{~cm}\end{array}$ & $\begin{array}{l}\text { Excellent } \\
\sim 1 \mathrm{~ms}\end{array}$ & $\begin{array}{l}\text { Subject can be } \\
\text { mobile and remote }\end{array}$ \\
\hline MEG & Magnetic Field & Poor & $\begin{array}{l}\text { Excellent } \\
\sim 1 \mathrm{~ms}\end{array}$ & $\begin{array}{l}\text { Laboratory only, } \\
\text { stationary subject }\end{array}$ \\
\hline fMRI & $\begin{array}{l}\text { Changes in } \\
\text { Blood flow }\end{array}$ & $\begin{array}{l}\text { Excellent } \\
\sim 2-3 \mathrm{~mm}\end{array}$ & $\begin{array}{l}\text { Poor but improving } \\
\sim 1-2 \mathrm{~s}\end{array}$ & $\begin{array}{l}\text { Laboratory only, } \\
\text { stationary subject }\end{array}$ \\
\hline PET & $\begin{array}{l}\text { Radioactive } \\
\text { Emissions }\end{array}$ & Poor & Poor $\sim 1-2 \mathrm{~s}$ & $\begin{array}{l}\text { Lab only, stationary } \\
\text { \& ingested dye }\end{array}$ \\
\hline CT & X-ray & Good & Poor & $\begin{array}{l}\text { Laboratory only, } \\
\text { stationary subject }\end{array}$ \\
\hline
\end{tabular}

Table 1.1: Brief comparison of the major neuroimaging techniques, their ability to resolve in both the space and time domain and the level of invasiveness for the subject.

\subsubsection{EEG Techniques}

There are many analytical techniques associated with EEG studies. In Electric Fields of the Brain, Paul Nunez discusses the appropriateness of using our own predictions in relation to dynamic measures of complex systems such as the brain.

“... By transforming raw data in ways suggested by our guesses about EEG properties, we attempt to establish new relationships between the transformed data and brain state that would be difficult or impossible with only the raw data." [6]

From the myriad of analysis techniques just a few are discussed here as being perhaps the most utilised or illuminating of the dynamic properties of the brain. These are Spectral Analysis, Averaging, Coherence, Principle Component Analysis (PCA), Independent Component Analysis (ICA) and Dipole Source Modelling. These methods are described and compared in chapter 2. Essentially, they all transform data into a single domain or two domains at most - i.e. temporal, spectral, epoch or spatial, at the expense of the data contained in the other domain. Loss of data is critical in complex systems such as the brain. Thus this research focuses on retaining as much information across domains as possible. By developing techniques to retain and then cross-analyse this information, correlations between individual responses to individual events may be investigated. In acknowledging that this goal, if achievable, would be a major scientific advancement, it is 
also a goal in this research to determine how far along this path it is possible to go and to highlight new insights observed along the way.

\subsubsection{Evoked Potentials \& Averaging}

Cortical electric potentials that could be related to particular brain events are of most interest in this research; hence it was decided to study Evoked Potentials. Evoked Potentials are a type of Electroencephalograph (EEG) response, where the response is not triggered endogenously by the natural processes of the brain but exogenously by an external trigger. Typical external triggers include visual, auditory or somatosensory stimuli. These stimuli are presented to a subject at a known instant in time and the EEG response is then related to the stimuli. Even with such precise time scale for the input stimuli, in virtually all cases, it is still impossible to clearly discern any individual brain wave pattern which directly correlates to a single stimulus. Again, the complexity of the brain function means a transformation method is applied in order to observe a direct link.

For evoked Potentials, the simplest and most effective transform is to average the response in the time domain. So instead of a single stimulus being presented to a subject, perhaps 500 stimuli are presented, then the individual responses for these 500 stimuli are added together and divided by the number of stimuli yielding a response which has a characteristic response over certain response time areas due to the additive effect of the coherent response of the brain caused by the presence of the stimuli. While this method gives a very good guide to the integrity of the neurological pathways between sensory organ and the brain, it is a cumulative response to 500 stimuli and not due to a single stimulus. Also, there is no traceability to show the effect of individual responses to the overall response i.e. it is not an invertible transform.

\subsection{RESEARCH APPROACH}

\subsubsection{From Background to Foreground}

Having outlined some of the background to this area of research, here we focus on the techniques utilised in the research in order to elucidate the problem of single sample evoked potentials. 


\subsubsection{Wavelet Transform \& Singularity Detection}

A wavelet is a time domain wave which is localised, oscillatory and with zero average. The Wavelet Transform compares a time scale waveform to a particular wavelet and highlights areas of similarity in both the temporal and spectral domain. The output of this transform is a set of coefficients along the time axis for each scale factor. Coefficient values are larger where the signal more closely approximates the particular scale of the wavelet at that particular time. Equally, where there is little or no 'fit', coefficient values tend towards zero. It is this specificity in both the temporal and spectral domain which makes the Wavelet Transform most attractive for the purposes of this research. The Wavelet Transform is a relatively modern analytical technique that has been used in EEG research before. Typically it is applied as a means of noise reduction or spike detection.

In this research, it is the singularity detection property of the Wavelet Transform that is most relevant. Singularity Detection in the Wavelet Transform was first described by Stephane Mallat in 1989 [14]. A singularity is a mathematical term to describe a sharp, irregular transition within a signal. It may also be applied to peaks and troughs typical of EEG signals. By choosing a wavelet that was the derivative of a smoothing function, such as the quadratic spline wavelet of degree two, Mallat and Hwang [14] proved that the evolution of the local maxima of the wavelet transform modulus across scales could be used to detect local singularities. The detail components, taken from the "algorithme à trous" method, yield a set of coefficients for each scale. At a point of singularity, there is a linear relationship between $\log _{2}$ of the modulus maxima response and its associated scale $j$ - a concept known as Lipschitz regularity. By selecting only the modulus maxima values that fitted the linear relationship, Mallet and Hwang simplified the large set of wavelet coefficients into a much smaller discrete set of coefficients grouped per singularity.

This is a powerful step forward in the characterisation of a signal into a group of singularities. Mallet and Hwang did not further analyse individual singularities but did demonstrate an inverse discrete wavelet transform that showed that the selected singularity coefficients still approximated the original signal well.

\subsubsection{Peak Detection}

This research takes this concept one step further and postulates that if a group of discrete singularities can be used to characterize a signal, then it may be possible to automatically 
group these singularities into singularity sets that characterize each individual peak. By analyzing in terms of peaks we retain both the temporal and spectral aspects of the signal and can then summate these peaks to recover the original signal. Moreover, this characterization of separate individual discrete peaks can be analysed in novel way in both the temporal and spectral (and epochal) domain. The process of characterizing a signal into a set of peaks constitutes a major portion of this research. A set of algorithms were developed in the Matlab environment. These algorithms automatically characterize a signal into a set of discrete peaks using the singularity detection wavelet transform methodology. This algorithm had to be robust and accurately characterize the original signal. The large size of the datasets also meant that it was necessary for the algorithm to perform automatically.

\subsubsection{Peak Characterisation}

Having characterized the EEG signal into set of peaks, it then remains to identify meaningful ways to classify this data and several methods are investigated. Discrete independent parameters were identified that could uniquely define each detected peak. Typical parameters include peak time location, peak width, peak height, area under the peak and the number of similar peaks across epochs. The parameters chosen encode aspects from different domains.

\subsubsection{Peak Clustering}

A novel clustering technique was applied to a subset of these parameters in order to identify areas of higher parameter concentration. In the results section, it is demonstrated that these high concentration areas are directly related to the components of evoked potential. They also represent a discrete and meaningful decomposition of the evoked potential response. The evoked response is decomposed into a discrete group of peaksets. Peaksets are locations where similar peak shapes overlap in sufficient numbers to be elevated above the average expected concentration at that location. By identifying these peaksets and using them to reconstruct an approximation of the evoked response, a new expression for evoked potentials is generated; one which provide neurological experts significant extra details regarding the evoked response. 


\section{CHAPTER 2 LITERATURE REVIEW}

\subsection{OVERVIEW}

hapter 1 described how an initial concept of trying to understand acupuncture and reflexology evolved into examining how to detect individual neural signals in the brain. This chapter elaborates on this idea and reviews various approaches to addressing this problem. This process gives perspective to the work from which further advances are investigated.

There are four major sections within this chapter. The first (Section 2.2) describes our understanding of the mechanics or physiology of the functioning brain and, in particular, its interaction with the senses. Here the basics of electricity in the body are explained and special focus is given to the topic of Visual Evoked Potentials (VEP). Readers familiar with this area may wish to skim-read or skip this section.

The next section (Section 2.3) summarizes the current brain imaging techniques available and evaluates their suitability with regard to detecting single event responses to known stimuli. From this review it becomes clear that Electroencephalography currently offers the best combination of excellent temporal resolution and adequate and improving spatial resolution with which to approach this topic. This leads to the next section (Section 2.4); a review of the main methods of EEG analysis. Applicability to the area of identifying single trial evoked potentials is highlighted. This forms the crux of the body of knowledge that currently exists and how it related to this research.

The last section (Section 2.5) investigates ideas to extend the boundaries of understanding in these areas and focuses attention on the areas that concern this research. Out of this discussion, relevant research questions are posed that form the basis this doctoral study and which are elaborated upon in the remaining chapters.

\subsection{NEUROPHYSIOLOGY}

When considering how the brain functions, it is first necessary to ask from what perspective you wish to view. The massive complexity of the brain prevents any simple model from fully describing its functions completely. The neuron is the basic building block for all brain activity. For the purposes of this review, processes within the brain are 
broken into three viewpoints and related to the neuron in each viewpoint. These viewpoints form the microscopic, mesoscopic and macroscopic view.

\subsubsection{Microscopic viewpoint}

This nerve cell or neuron is the essential building block of the entire central and Peripheral nervous system. Fig. 2.1 illustrates the main components of the neuron which are $[3,15]$ :

soma: the cell body and metabolic centre of the neuron. At the centre is the nucleus which contains the genetic material.

dendrites: numerous short branch-like strands extending from the soma whose primary purpose is to receive data from other neurons.

axon: single long fibre that conducts neural signals from the cell body to other parts of the nervous system. The axon hillock is formed where the axon meets the cell body and aggregates all the received action potentials. The axon ends at the pre-synaptic terminal which is connected to the dendrite(s) of another neuron via the synaptic cleft.

To understand the functioning of a neuron, one must examine the electrochemical properties of the neuron. A voltage difference is observed across the cell membrane of all cells. Neurons are excitable which means that the cell membrane can produce electrochemical impulses and conduct them along the membrane [16].

The cell membrane encloses the nerve cell. The cell membrane is formed by a lipid bilayer of specialized cells. Cells in each layer have a head made from fatty acids which is hydrophilic and a tail made from hydrocarbon chains which is hydrophobic. These cells are aligned in two layers of cells where the cells are orientated tail-to-tail. Macromolecular pores (ion channels) within the cell membrane then allow ions such as sodium, potassium and chloride to flow through the membrane leading to the bioelectric phenomena of neurons. Most of these ion channels in the dendrite and cell body are chemically gated while those in the axon are voltage gated.

At rest, the permeability of cell membrane to the $\mathrm{K}+$ ion is greatest and so $\mathrm{K}+$ diffuses out of the neuron into the extracellular space. The anionic proteins are unable to diffuse also and this leads to a voltage gradient across the cell membrane. The voltage gradient balances the chemical gradient preventing further $\mathrm{K}+$ diffusion out of the neuron and leads to the equilibrium potential or resting potential for the neuron which is of the order 
of $-75 \mathrm{mV}$ [16]. This equilibrium potential or transmembrane potential is defined as the potential of the inner surface relative to the outer surface of the membrane [15].

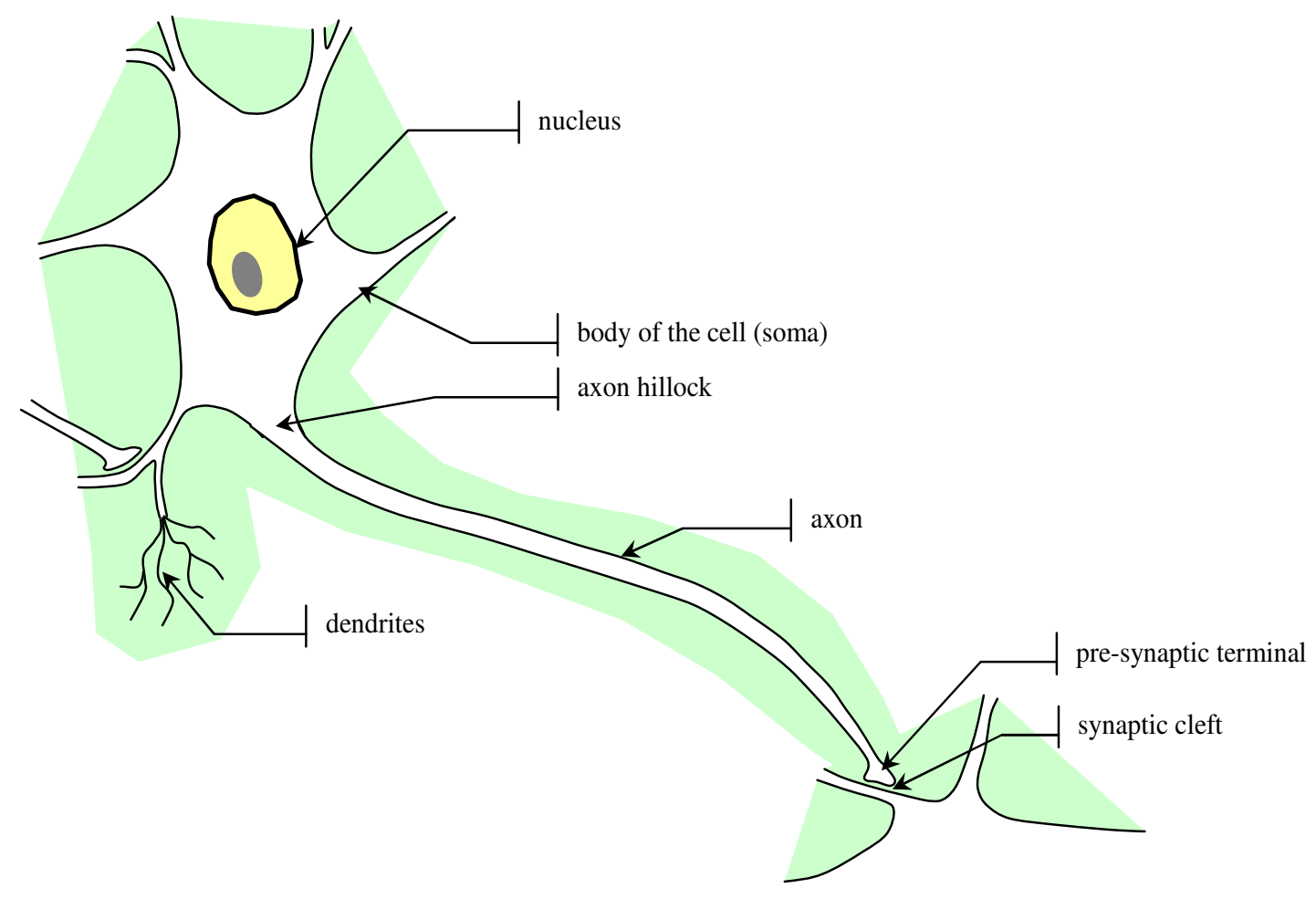

Fig. 2.1: Nerve Cell or Neuron. The three main parts are illustrated - the soma, dendrites and axon. Connection from one neuron to another is shown. In this way millions of millions of neurons are interconnected to form the neurology of the brain.

There are certain normal fluctuations around this level due to internal cell potentials, synaptic potentials and generator and receptor potentials. Receptors in the dendrites and to some extent the cell body are activated by other neurons and sensors. These potentials conduct through the soma and yield a cumulative potential signal at the axon hillock. However, it is only if the transmembrane potential at the axon hillock rises above the threshold voltage that a characteristic electric impulse is produced. This is called the nerve impulse or action potential. The size of the action potential is not related to the size of the stimulus across the transmembrane potential. It follows the all-or-nothing rule. If the threshold is reached then the nerve impulse is produced ('all'). If the threshold is not reached then there is no nerve impulse ('nothing').

Activation occurs because once the transmembrane potential rises above the threshold of about $20 \mathrm{mV}$ above its resting level, the sodium $(\mathrm{Na}+)$ and potassium $(\mathrm{K}+)$ ionic permeabilities of the membrane change. Fast moving Na+ ions flood into the nerve cell through the macromolecular pores (ionic channels), increasing the transmembrane potential to an absolute value $>0 \mathrm{mV}$. Then as time progresses the slower moving $\mathrm{K}+$ 
ions exit the nerve cell through these same pores and reduce the transmembrane potential back to the resting potential. Over time, the concentrations of $\mathrm{Na}+$ and $\mathrm{K}+$ return to their equilibrium concentrations without any resting

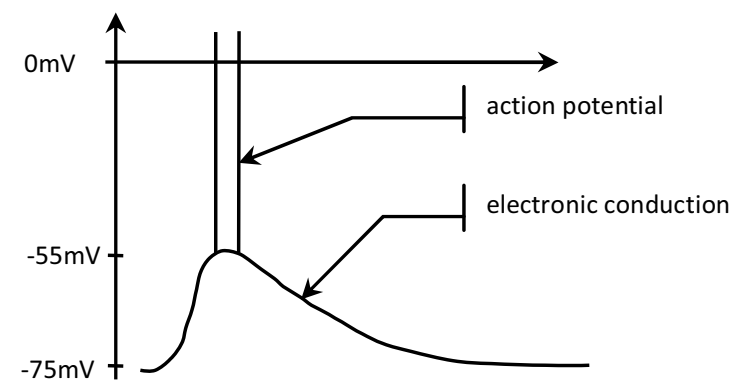
potential variation.

Fig. 2.2: Excitatory post-synaptic potentials produced by synaptic transmission. Threshold is reached so action Action potentials propagate along the potentials are present.

axon membrane in a similar fashion to how it was generated. Depolarization of membrane adjacent to the activated membrane occurs due to the regenerative opening of sodium channels with a resultant action potential. A refractory period before which reactivation is possible prevents bi-directional propagation of action potentials. Hence action potentials are first seen in the root of the axon near the soma and propagate along the axon until it reaches the pre-synaptic terminal. Once the action potential reaches the synapse, it causes depolarization of the nerve terminal. Ionic channels open allowing the entry of a chemical transmitter $(\mathrm{Ca}+)$ into the nerve terminal. These $\mathrm{Ca}+$ ions fuse with the vesicles in the membrane causing the vesicles to open up and release neurotransmitter into the synaptic cleft. The neurotransmitter crosses the synaptic cleft and binds to the receptors one the post-synaptic membrane. The post-synaptic membrane is typically located on the dendrite of the next neuron in the chain. Binding to the receptor causes the opening of the membrane ionic channels generating a postsynaptic activation potential.

Receptor activation of the nerve may be excitatory or inhibitory. Excitatory behaviour is characterized by a change in potential inside the cell relative to the outside positively and so represents a decrease in the normally negative resting potential. These are known as excitatory post-synaptic potentials (EPSP), refer to Fig. 2.2. Inhibitory behaviour is characterized by a change in potentials inside the cell relative to the outside negatively and so represents an increase in the normally negative resting potential. These are known as inhibitory post-synaptic potentials (IPSP).

Inhibitory and excitatory synapses potentials cause local current sources and sinks respectively. The local current source generates an electric field and forms a closed loop system in order to satisfy current conservation. This is achieved by creating a current sink at the dendrites for every source at the soma in a single neuron. This source-sink 
combination with a specific separation is directly analogous with a dipole source in a uniform conductor. This process is pictorially described in Fig. 2.3.

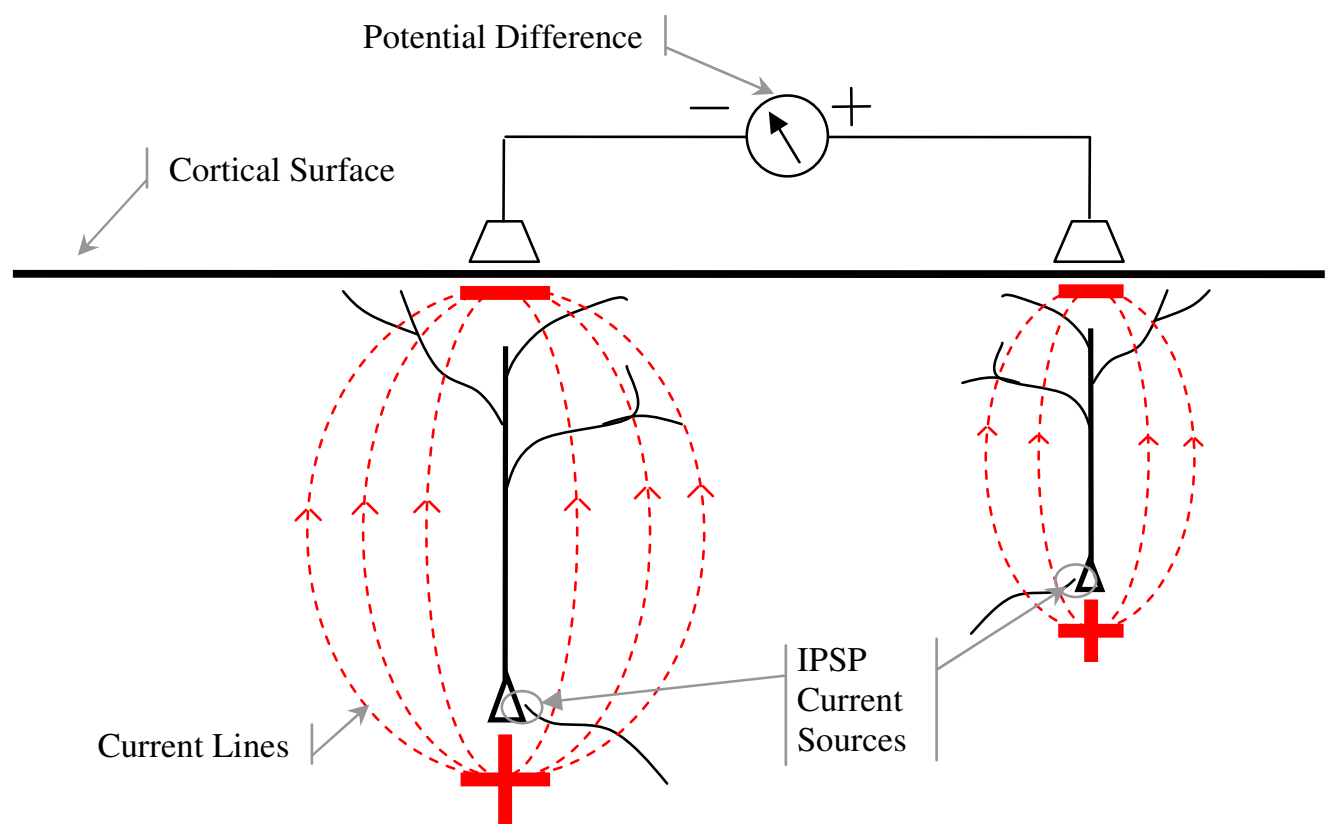

Fig. 2.3: Synaptic potentials (IPSP) give rise to current source (or sink) at the soma whose counterpart becomes a current sink (or source) at the dendrites. Current conservation is preserved. The potential difference at two points on the cortical surface measure the relative difference in current field strength in the neighbourhood of the electrode. Adapted from [6].

This review of neuronal generation gives a brief description of the electrical properties and function of the most basic building block in a functioning brain - the neuron. We get a sense of the flow of information in terms of transitory electrical pulses. Individual neuronal activity can be measured by applying microelectrodes to specific neurons in the brain. However, it is not possible to directly or consistently relate this activity to the macroscopic patterns observed generally over the surface of the scalp.

\subsubsection{Macroscopic Viewpoint}

The very first electrical measurements observed from the human brain were taken by Hans Berger in 1929. He called this study Electroencephalography (EEG) and for nearly 80 years scientists have probed the limits of this technique to try to understand the workings of the brain in ever more refined terms.

EEG potentials are believed to be mostly generated in the cerebral cortex of the brain [6]. This is the convoluted surface of the brain whose surface area has increased to such a degree that it folds in upon itself to the extent that much of the human cerebral cortex is invisible from external examination and allows the surface area to grow to about 1600- 
$4000 \mathrm{~cm}^{2}$. The cerebrum or cerebral cortex extends about $2-5 \mathrm{~mm}$ into the brain surface and is made up of soma, dendrites and unmyelinated axons and known as grey matter due to the grey colour of unmyelinated axons. Beneath this is white matter or sub-cortical brain which includes soma, dendrites and myelinated axons. Myelinated axons are white in colour.

The way that neurons are arranged in the brain is not random. If, for example, neurons formed a mish-mash spaghetti shaped arrangement then it is likely that measuring EEG at the surface of the skull would be fruitless and potential differences from individual neurons would cancel out due to the random nature of the arrangement. However, this is not the case. In the cerebral cortex, neurons are organized in a linear fashion with the dendritic arborization near the surface and the soma and axon hillock in the deeper layers. This creates a columnar organization perpendicular to the cerebral cortex surface. This arrangement means that when local dendritic populations fire, a measurable and cumulative electrical potential is observable on the surface of the cortex. This signal may be measured with micro electrodes on the cortex surface and is known as Electrocorticography. When measured at the scalp, it is called Electroencephalography.

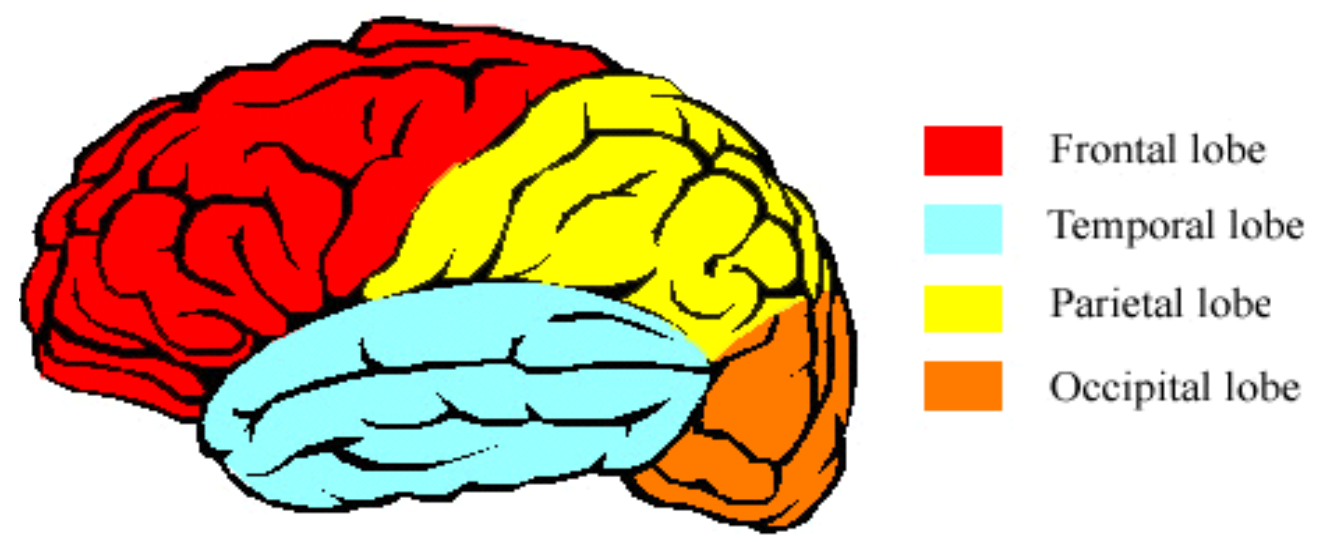

Fig. 2.4: Functional areas of left side of cerebral cortex. Equivalent areas for the right side are not shown. These areas are essentially empirically chosen based on the architecture of the brain (position of the clefts or gyri) and observation (correlation with observed sensory or cognitive processes). Adapted from [4].

The cerebral cortex is divided in empirical areas called lobes which are mirrored on each side of the brain, refer to Fig. 2.4. Across the cortex surface, certain functional processes have been linked to particular areas of the brain. Hence the occipital area of the cortex is linked with the processing of visual information from the eyes.

To illustrate the pathways followed from organ to brain and onwards, let us describe the flow of information that occurs in the human visual system, refer to Fig. 2.5. Light enters the eye and strikes the retina at the back of the eyeball. The visual receptors in the retina 


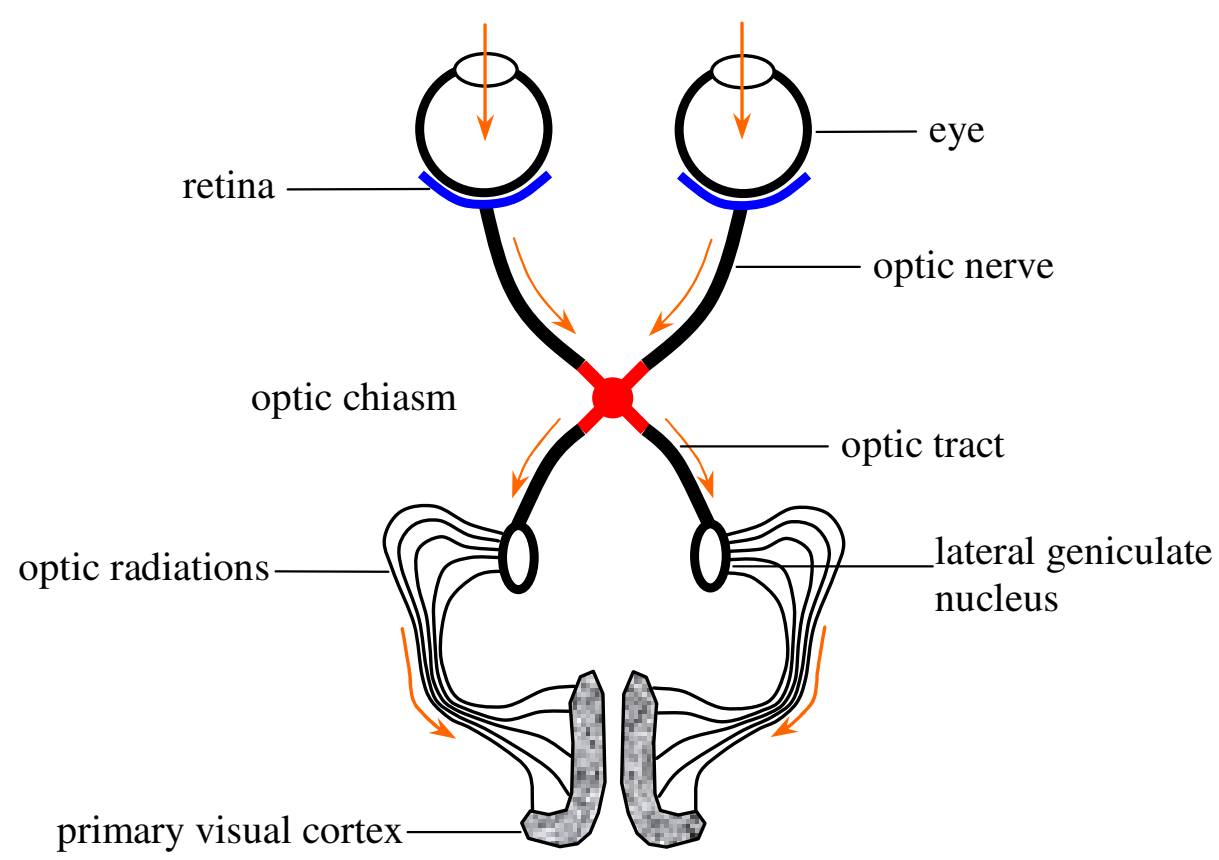

Fig. 2.5: Sensory pathway for the human visual system from eye to primary visual cortex in the occipital area of the brain. Adapted from [3].

are stimulated by light and produce signals conducted through the optic nerve to the optic chiasm where signals from the optic nerve from the other eye meet. Half the optic nerve axons cross over to the other side of the brain and half do not. These nerve bundles now known as the optic tract. They enter either side of the brain at the lateral geniculate nucleus. These thalamic nuclei relay the visual information from the optic tract to the primary visual cortex via the optic radiations.

The primary visual cortex makes up most of the occipital area of the brain although much of the cortex is hidden from view in the longitudinal fissure. It is described as the gateway of visual input from the thalamus to the cerebral cortex [3]. From the primary visual cortex, visual signals are distributed to other associated areas of the cortex, known as secondary visual cortex.

Each secondary area contains a complete retinotopic map of the contralateral visual field and is associated with processing different features of the visual system such as colour, form or motion [3]. Secondary visual cortex areas are located in the pre-striate cortex and the infero-temporal cortex and generally follow either the dorsal route or ventral route respectively. 


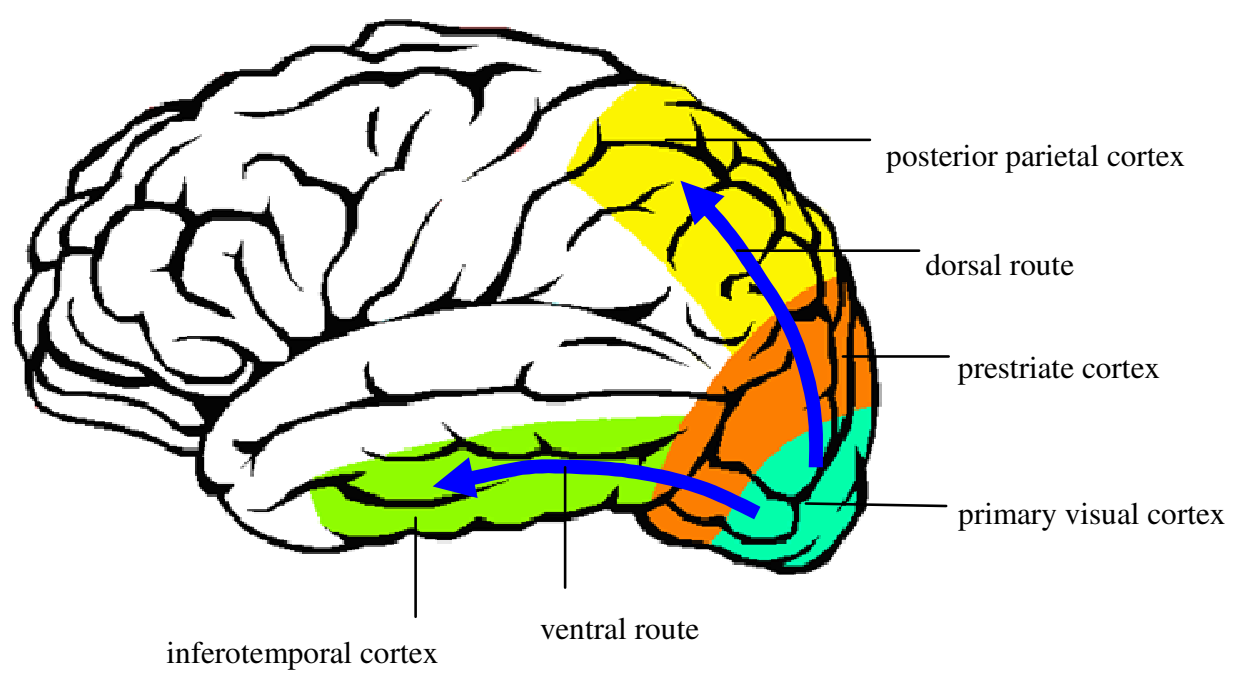

Fig. 2.6: Apparent dispersion pattern for processing of visual information. Adapted from [3, 4].

The dorsal route then leads to the posterior parietal cortex which is an area of association cortex that processes both somatosensory and auditory input as well as visual input - refer to Fig. 2.6. It is believed the dorsal route deals with perception of location and motion where things are going and the ventral route deals with perception of colour and form what things are.

By this example, we get a high-level overview of the pathways involved in processing visual information. The detail of exactly how these processes operate is not well understood particularly at the cortex surface. It is possible to design experiments and demonstrate a particular area of association cortex is responding to some visual stimulus but this is by cause and effect without understanding the processes along the way that produce this response or indeed the set of interactions that have to occur to make this happen [17].

\subsubsection{Mesoscopic Viewpoint}

In this section we consider the role of the neuron within the architecture of the brain at a local level. We are looking at more than one neuron but less than a whole brain. We are heading towards the theory of what are the generators that cause EEG signals. It is clear that EEG does not represent a measure of all brain function but measures some of the activity of a particular portion of the brain at a particular time. It is important to focus on what activity (and, in particular, what is meant by activity) is believed to be measured. Only then is it possible to postulate a theory by which our experiments may be tested. 
Earlier we discussed two mechanisms that cause neurons to be observed as electrically active, namely action potentials and post-synaptic potentials. There are other mechanisms but these are not believed to contribute towards observed EEG signals [16]. There is believed to be in the order of $10^{10}$ discrete neurons in the brain and for every major cortical neuron there are perhaps $10^{4}$ to $10^{5}$ synapses transmitting to other cortical neurons. In examining the contribution of both action potential and synaptic potential, the vast majority of signals observed using EEG seem to be caused by synaptic potentials. Using microelectrodes and $\mathrm{ECoG}$, it is possible to confirm individual neurons exhibit dipole pair behaviour [6]. Using volume conduction models to predict the equivalent observed potentials at a distance from the cortex surface, i.e. at the scalp, we would expect the potential to fall off in inverse square proportion to the distance. So the observed potential at the scalp $1 \mathrm{~cm}$ from the cortex would be approximately one hundredth of the cortical potential. The observed scalp potential is actually one-half to one-fifth of the cortical potential that is much higher than would be expected. The reason for this increase is that the source of the potential is not a single dipole potential but a parallel and synchronous set of many neuronal dipoles. The overall cortical potential in the area covered by the synchronous set of neurons is the sum of the individual dipole potentials. It is said that the minimum area of cortex required to fire synchronously in order for an observed EEG response to be detected at the scalp is $6 \mathrm{~cm}^{2}$ [16].

The concept of cell assemblies was introduced by Donald Hebb in 1949. The phrase cell assembly denotes "a diffuse cell group capable of acting briefly as a single structure" [6]. By this we allow for cooperative behaviour within the cell assemblies without describing the interaction mechanisms. This is described by Nunez [6]:

“Cell assemblies are pictured as embedded within synaptic and action potential fields. Electric and magnetic fields (EEG and MEG) provide large-scale short-time measures of the modulations of the synaptic and action potential field around their background levels. These synaptic fields are analogous to common physical fields.... Dynamic brain behaviours are conjectured by many Neuroscientists to result from the interaction of neurons and assemblies of neurons that form at multiple spatial states ..."

It is precisely this cooperative behaviour together with the perpendicular orientation of cortical neurons to the scalp which leads to EEG measurements being observed at all. 


\subsubsection{Cortical versus Scalp Potentials}

Electrocorticography (ECoG) employs intracranial electrodes to measure the potentials arising at the surface of the brain as described in the previous section. This highly invasive procedure requires the brain to be exposed and so is usually restricted to subjects already undergoing brain surgery for some pre-diagnosed brain condition. ECoG potentials are particularly localized as they are able to measure both membrane potentials (intracellular) and neuronal potentials (extracellular). While useful for single neuronal understanding, this scale is too local for observing overall brain processes. A comparison may be drawn in putting your eye as close as possible to a television screen as possible and focusing on a single screen pixel. You may be able to definitely identify the colour of that pixel but in doing so, you cannot observe the overall picture on the screen.

ECoG is not a feasible technique for large scale studies due to the ethical implications involved. In requiring a readily usable technique to measure potentials from the brain, it is necessary to "move away from the television screen", so to speak and observe the potentials arising on the surface of the scalp rather than the surface of the neocortex.

In moving the detecting electrode from cortex surface to scalp surface you have moved from the micro-meso scale to the meso-macro scale. The potentials observed represent not individual neurons or small groups of neurons physically located under the electrode but at the scalp they represent the cumulative current field strength due to the probably numerable cell assembly groups firing in the area of the scalp electrode. The upside is that coherent cell assembly firing is potentially more useful than individual neuronal firing. The downside is that the process of the current field strength through the layers of skin, bone and cerebrospinal fluid reduce and smear the detected signal with summation and cancellation with other detected cell assemblies adding to the complexity of the observed signals.

\subsection{NEUROIMAGING TECHNIQUES}

\subsubsection{Electroencephalography}

The electroencephalogram (EEG) is a record of the oscillations of brain electric potential recorded from electrodes on the human scalp [6]. Electroencephalograph signals are tiny non-stationary electrical potentials that are emitted from the brain and can be detected on 
the surface of the scalp. From their first detection, brain electric potentials have been easy to detect but difficult to interpret due to the complexity of the brain and it's functioning. Numerous mathematical analysis techniques have been used over the last 80 years and there is no doubt that many meaningful interpretations of brain electric potentials have been developed. However, the basic correlation of a single brain-wave peak to a specific brain event has remained elusive [18-22].

There are in the order of a million million neurons in the brain and groups of these neurons firing coherently can be measured. The purpose of measuring such signals is to be able to correlate the signals to particular brain function and so gleam an objective measure of brain performance. Primarily, this process has developed empirically. At the microscopic level, the action of neurons and synapses in generating electric potentials is well understood. However, at the macroscopic level, how the brain integrates all the neurons and synapses together to form a coherent efficient machine is much more nebulous. Other electrical systems in the body are more fully understood in terms of cell (microscopic) to organ (macroscopic) activity. Hence, the Electrocardiograph (ECG) signals measured are explainable by the cell electrochemical properties and the physiology of the heart. The heart is essentially a mono-functional system and the electrical signal of interest is much easier to detect and isolate. The complexity of the brain makes it much more difficult to isolate individual events. In the brain relationships between scalp potentials and function have evolved empirically. Hence a typical auditory evoked potential response will contain at least 15 reproducible components in humans. An abnormal delay of any component may indicate reduced axon propagation speed in the auditory nerve demyelination (indicating multiple sclerosis) [6]. Many such correlations have been identified and used.

As neurological activity is electrical in nature, EEG is the only neuroimaging technique that directly measures this activity. The time resolution of the measured activity is excellent down to sub millisecond level. However, the spatial resolution is poor as a separate channel (or electrode) is required for each measurement point. EEG is only truly an imaging technique when multi-channel or high-density EEG is measured. The spectral properties of EEG signals are well understood leading to four major types of continuous sinusoidal activity to be identified - labelled alpha, beta, theta and delta activity. 
Evoked Potentials (EP) and Event Related Potentials (ERP) are sub-classes of EEG. Both relate repeated controlled stimuli to the EEG response and endeavour to identify a 1:1 relationship between stimuli and response. Evoked Potentials are concerned with the direct physiological response to exogenous sensory stimulation such as visual, auditory or somatosensory stimulation. Event Related Potentials endeavour to identify a secondary endogenous cognitive response to a particular stimulation. Hence, in a typical oddball paradigm experiment, a subject would be requested to observe a changing checkerboard pattern and when a particular pattern is onscreen, they are asked to count the number of times that it appears. The cognitive counting exercise may be measured with the EEG data. EP and ERP require many repetitions and processing with averaging in order to separate the stimulus response from the background ongoing EEG signal.

EEG requires precise setup in the application of electrodes to the scalp. However, once applied, it is quite robust and can be recorded on patients in their normal everyday environment.

\subsubsection{Magnetoencephalography}

Magnetoencephalography (MEG) measures extremely small magnetic fields produced by electrical activity in the brain [6]. During synaptic transmission between neurons, ionic currents flow. Applying Maxwell's equations, any electric current will have an associated orthogonally orientated magnetic field. It is this field that is measured by MEG [1]. Approximately 50,000 concurrent active neurons are required to fire in order to be detected by MEG. Although EEG and MEG are measuring different manifestations of the same phenomena, they yield different data. MEG and EEG are preferentially sensitive to different cortical sources. Thus EEG is more sensitive to sources in cortical gyri, while MEG is more sensitive to sources in cortical sulci [6].

MEG has a similar high temporal resolution a low spatial resolution as EEG. The superconducting quantum interference devices (SQUIDs) required for measuring these tiny magnetic fields make MEG a very expensive technique to use, particularly when compared to EEG. MEG signals are not distorted by the intervening head layers. However this benefit is somewhat negated by the fact that MEG sensors are located about twice as far as EEG sensors. MEG is not invasive but the subject must be still and precisely locate within a large piece of equipment in a laboratory environment. 


\subsubsection{Functional Magnetic Resonance Imaging:}

Active nerve cells consume oxygen in the form of haemoglobin in red blood cells. The response to this is a flow of blood in the around the deoxygenated blood. This is called the hemodynamic response. Functional Magnetic Resonance Imaging (fMRI) produces high quality slices of brain structure using a combination of magnetic fields and radio waves without the use of ionisation radiation (X-rays) or radioactive tracers. A large cylindrical magnet creates a magnetic field around the head of the patient. Radio waves transmitted and received inside the chamber have a unique radio frequency from which a detailed picture of the medium (in this case the brain) is computed. It is the paramagnetic property of oxygenated and deoxygenated blood that is being measured.

fMRI is highly sensitive to blood flow with a resolution of about 2-3mm limited by the spatial response of the hemodynamic response to neural activity. However, the temporal response is of the order of seconds due to the nature of the property being measured. The hemodynamic response takes a few seconds to occur. Evoked Potential studies demonstrate a response time of the order of several hundred milliseconds. Therefore fMRI is not suitable as a measure of the unique response to a single event.

\subsubsection{Positron Emission Tomography:}

Positron Emission Tomography (PET) works by detecting the emission of positrons from radioactive metabolically active chemicals (called radiotracers) injected into the bloodstream. Sensors in the PET scanner detect the radioactivity as the compound accumulates in different regions of the brain [1]. The data gathered is typically converted into a 2-dimensional slice or with more advanced processing a 3-dimensional image. Different tracers can show blood flow, oxygen and glucose metabolism in the tissues of the brain, reflecting a measure of the brain activity. PET scans have improved resolution over CT scans but are inferior to fMRI scans. The temporal resolution is still in the order of seconds and so it is not suitable for measuring responses in the order of milliseconds. The use of radiotracers means this technique is invasive. Also, the radioactive decay is rapid and so testing is limited to short tasks. PET scanning is still widely used for the diagnosis of brain disease such as tumours, strokes and dementia where great change in brain metabolism is apparent. 


\subsubsection{Computed Tomography}

Computed Tomography is a brain scanning technique where a series of $\mathrm{X}$-rays are taken axially around a subject and these X-ray slices are used to generate a 3-dimensional representation of the various structures of the body. Separation of different bodily tissue is observed by the ability of different tissue to block the X-ray beam. It has a great advantage over traditional x-rays in that data can be viewed at any axial angle or in 3dimensions. CT scans are very good at measuring anatomical features but they are not applicable in measuring brain activity.

\subsection{EEG ANALYSIS TECHNIQUES}

So far the process of understanding brain activity from single neuron potentials to EEG scalp potentials has been described. Relating the macroscopic measurements to either localized brain function or cognitive understanding is a vast body of brain research. The complexity of the brain and its working have necessitated the transformation and effective simplification of information detected in order to pick out one perceived relevant facet which can be meaningfully interpreted. Here we describe some common EEG analysis techniques and draw attention to their merits and drawbacks with regard to the problem described here, namely the detection of single event features in the brain.

A summary of the different approaches together with the associated techniques is illustrated in Fig. 2.8. It should be noted that some techniques do overlap different approaches and that in such cases the most applicable approach for the technique is chosen. Fig. 2.8 provides a hierarchical view of these techniques. There then follows a detailed discussion of these methods with specific cited articles. At the end of this section a domain view of these techniques is presented which emphasizes the perspective of these techniques in terms of the temporal, spectral, spatial and reiterative (or epoch) domains. This is shown in Fig. 2.10.

At the end of the discussion for each technique, a representative formula is presented to illustrate the relationship between the input variables and output variables of the examined domains for each transformation technique. This notation is expressed in the form, 
The domain variables are $f$ for frequency, $t$ for time, $s$ for space and $e$ for epoch. Therefore the input signal $(x)$ which is a function of some subset of these domain variables has a characteristic transformation $(T)$ applied to it yielding an output $(y)$ which will again be a function of a probably different subset of the same domain variables. The transformation, $\mathrm{T}$ is not necessarily a simple relationship but this notation is intended as an illustrative mechanism to assert the input domain variables to the described technique, together with the output domain variables. This yields a powerful comparative mechanism for examining the interrelations between techniques in terms of output domain variables. The variables of the output, $y$ correspond to the domain intersects in Fig. 2.10.

\subsubsection{Visual Inspection}

The mainstay of interpretation of EEG data in clinical situations has been the visual interpretation of the data by Electroencephalographers. This is based on experience and acute visual inspection skills. The data is presented in strip form - a time domain representation of the variation in potential difference between active and reference electrode. Over the years, meaningful interpretation has been applied to certain patterns. This process is exceedingly hard to automate as it is based on empirical observation and experience rather than theoretical laws which may be programmed into a computer. The intuitive analyse performed by skilled Electroencephalographers is really temporal and spectral domain analysis.

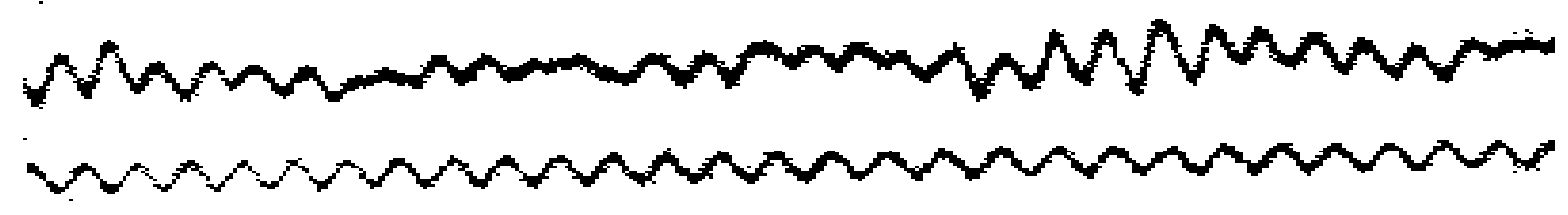

Fig. 2.7: Sample of stripchart EEG display. The horizontal axis is time and the vertical axis is Potential difference in $\mu \mathrm{V}$. Two separate recording channels are shown. This is taken from Hans Berger's original EEG recordings published in 1929 and reprinted in 1969 [23]. 


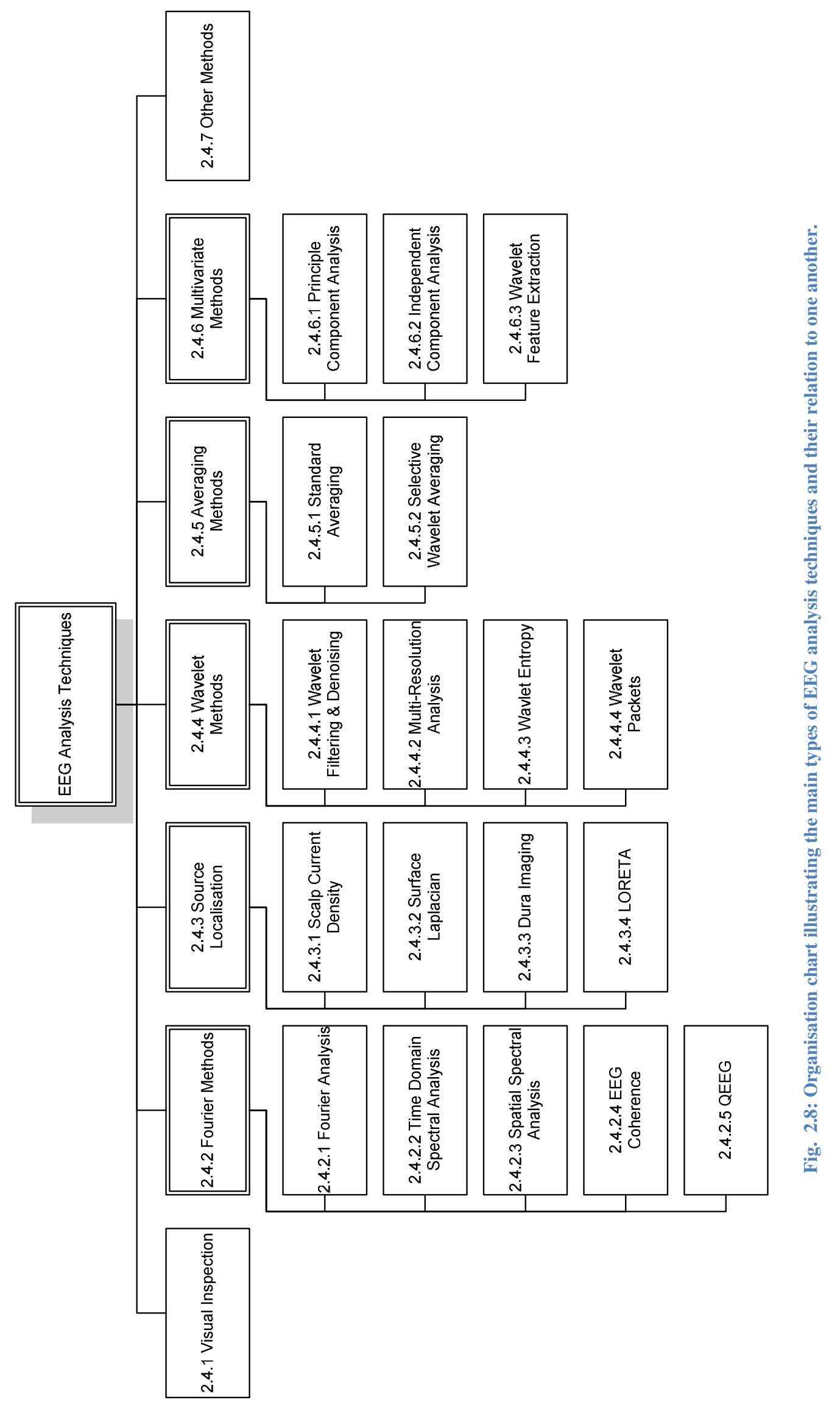


Visual inspection is important in the understanding of seizure disorders. From experience, different patterns of spikes and sharp waves at particular location have come to define different seizure pathologies and so helped to diagnose the type of seizure involved. EEG is also invaluable in pinpointing the position of anomalous activity. These methods are very good for diagnosis of a particular patient. However, very little if any understanding is gained about what is actually happening to a person during a seizure activity.

Visual inspection is important for distinguishing between genuine EEG and extraneous artefact. While quicker automated techniques have now been developed for artefact identification, clinical neurophysiologists still observe valuable insights directly from the stripchart.

\subsubsection{Fourier Methods}

\subsubsection{Fourier Analysis}

From an early stage of EEG study, it was observed in the stripchart that certain repetitive cyclical near-sinusoidal patterns were present. Fourier analysis can be employed to quantify and describe these patterns. A crude forerunner of Fourier analysis consisted of counting the number of zero crossing in certain parts of the stripchart [6]. However, as digital data acquisition improved and analysis techniques became easier to implement by computers, the Fourier Transform was used to represent the EEG time series as a sum of sine waves with different discrete frequencies. The Fourier transform is essentially a frequency filter of EEG signals. The most widely used implementation is the Fast Fourier Transform (FFT).

In order to calculate the FFT of a signal, a finite interval is required. It is the range of frequencies within this interval that forms the output of the FFT. In mathematical terms, the input, $x$ is a function of time, $t$ the output, $y$ or amplitude spectrum is a function of frequency, $f$ and the epoch domain, $e$ and spatial domain, $s$ are constant. If we let $T$ represent the fast Fourier transform, then we may express this relation as,

$$
y(f)=T x(t) \text { with e.s constant }
$$

Fourier analysis is the most widely used analytical method applied to EEG data. From early on, certain frequency patterns were observed in the amplitude spectrum. These 
(a)

\begin{tabular}{|l|l|}
\hline Band & $\begin{array}{l}\text { Range } \\
(\mathrm{Hz})\end{array}$ \\
\hline Delta $\delta$ & $1-4$ \\
\hline Theta $\theta$ & $5-9$ \\
\hline Alpha $\alpha$ & $9-13$ \\
\hline Beta $\beta$ & $14-20$ \\
\hline Gamma $\gamma$ & $>20$ \\
\hline
\end{tabular}

(b)

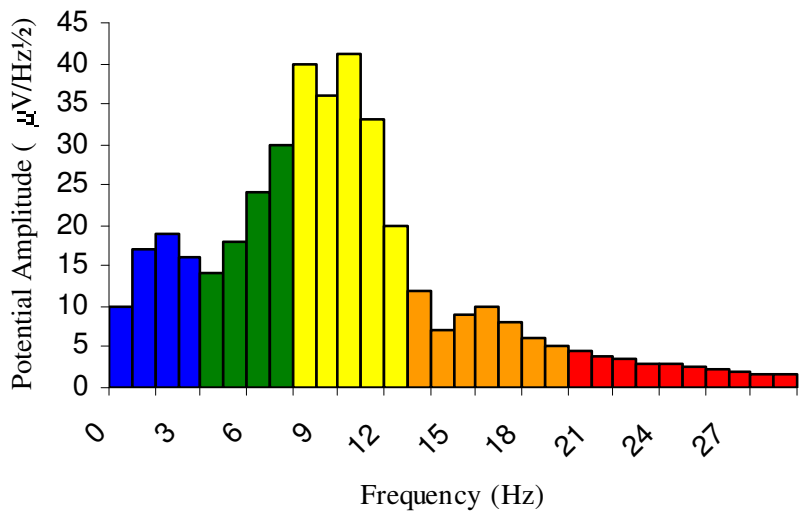

Fig. 2.9: (a) EEG frequency band names and ranges. (b) A sample output of FFT (Fast Fourier Transform) of typical ongoing EEG activity. Frequency bands are colour coded. Note the presence of the alpha rhythm at about $10 \mathrm{~Hz}$.

patterns tended to be localized and showed a fair degree of repeatability across subjects. Out of these empirical observations certain frequency band were grouped and labelled as shown in Fig. 2.9(a). These bands must be interpreted carefully for actual EEG is composed of multiple EEG components which may be seen in Fig. 2.9(b).

Interpretation of the amplitude spectrum must always be relative to some normative standard. Typically, any well designed study shall observe a sample of typical EEG activity, then provide some interaction which is hope will cause typical EEG pattern to shift in some way. Then by measuring the frequency strength under the new conditions, a significant shift between the typical EEG and the 'interaction' EEG may be observed. The reasoning is then that the shift in frequency spectrum power gives a measure of the interaction. Statistical analysis of the results must be carried out to confirm that the shift is statistical significant and not the results of some anomalous or spurious effect.

This type of analysis has been particularly useful in many areas. Polysomnography or the study of EEG during sleep is well suited to this type of analysis. Examining the frequency spectrum gives a good measure of frequency content over a period of time and not instantaneously. In sleep studies you are concerned with the shifting EEG patterns over the course of the sleeping period which is probably a number of hours. The particular changes from second to second are not so important but broader shifts over the course of many minutes or even hours are much more significant and can come to define the type of sleep being observed based on comparisons of other previous norms. Therefore sleep studies have defined different sleep stages by the relative content of the above frequency band. For example, deeper stages of sleep are characterized by slower 
wave activity, "Stage 4 sleep is characterized by predominance of slow activity in the delta range" [16].

\subsubsection{Time Domain Spectral Analysis}

Experimental EEG may be viewed as one realisation of a random process (or stochastic process) [6]. Spectral analysis permits the statistical properties of oscillations in different frequency bands to be examined. The amplitude spectrum is obtained by applying the FFT to a single EEG epoch. However this one realisation is insufficient to represent the stochastic process itself. By taking an ensemble of $\mathrm{K}$ observations, we may make a statistical estimate of the power spectrum (also known as the power spectral density function) of an EEG signal. One important assumption in spectral density is that the underlying process is stationary. This implies that the power spectrum is invariant to shifts in time. Therefore, this analysis is more suited to detecting longer term brain states such as eyes closed or different sleep stages as opposed to the shorter term brain responses such as the responses to external stimuli that form the basis of this research.

$$
y(f, e)=T x(t, e) \text { with } s \text { constant }
$$

\subsubsection{Spatial Spectral Analysis}

Spatial spectral analysis is identical to temporal spectral analysis with the time domain EEG signal replaced by an EEG signal taken along an equidistant array of EEG electrodes. An epoch of time sequence data is replaced by an epoch of 1-dimensional sequential electrodes. The sampling rate is replaced by the inter-electrode distance. The power spectral density is then calculated across many such epochs to give a representation of the underlying stochastic process in the brain. The representation of domains for this technique includes a measure of the spatial frequency (denoted $s_{f}$ ) which is a measure of the rate of change of EEG signals across electrodes.

$$
y\left(s_{f}, e\right)=T x(s, e) \text { with } f \text { not calculated }
$$

Again it is worth noting that the output is a density function which is an aggregate of many epochs and so the individual epoch data is not extractable from the final output. This technique has been used to estimate an ideal inter-electrode distance of $1 \mathrm{~cm}$ for capturing non-local EEG components located on gyri [24]. 


\subsubsection{EEG Coherence}

Coherence is the linear correlation coefficient representing an estimate of the amount of phase synchronisation between any two data channels [6]. Scalp recorded EEG coherence and covariance are large scale measures of functional interrelations between pairs of neocortical regions [25]. They can often be correlated to cognitive, behavioural or sensorial activity. Covariance is calculated in the time domain and is usually applied to evoked or event-related potentials. The cross-spectrum is a similar metric but calculated in the frequency domain. The coherence between two data channels is calculated by normalising the square magnitude of the cross-spectrum by the power spectrum of each channel. EEG data is divided into subsets of epoch data recorded in different location and then compared together to determine whether a spectral synchrony is present. It is this synchrony that is used to demonstrate the link between the two mechanisms being studied. Coherence is relatively sensitive to the relative phase between the two examined channels.

Again, this method employs the Fourier Transform allows frequency domain characteristics to be investigated but discarding the temporal domain data. Individual peaks are lost in the transform and what becomes important is the population rather than the individual. Increasing the number of epochs increases the confidence in any observed coherence between the channels. Spatial analysis is introduced in that 2 channels are compared; however, this binary spatial analysis (denoted $s_{z}$ ) is highly restrictive in that a separate complete analysis is required for every pair of electrode locations under investigation.

$$
y\left(f, e, s_{2}\right)=T x(t, e)
$$

\subsubsection{QEEG}

Quantitative EEG is measured by calculating various power measurements across different band of the frequency response for a single epoch of EEG data. One particular measure called cordance has been shown to have a better relationship to underlying cerebral perfusion than more traditional power measurements [26]. It has been used in studies to compare the general state of EEG week-to-week over the course of a treatment [12]. The spatial resolution is limited to selected electrodes or local groups of electrodes and is not an imaging technique as such. Also, the lack of specificity in relation to individual events makes it an unsuitable technique for this research. 


\subsubsection{Source Localisation}

In section 2.2 we discussed the origins of EEG potentials in terms of dipole pairs. In uniform conductor electric field theory, this phenomenon may be described using volume conduction modelling. Calculating the electric field given the dipole source strength and vector is known as the forward problem. Trying to estimate the source given the electric field is known as the inverse problem. In EEG studies, we are primarily concerned with the inverse problem since we measure the field using EEG and wish to determine its source. The solution to the inverse problem is non-unique - there are multiple possible sources that can generate any one observed field. This is due to the fact that the known field is on the surface only (scalp) and not throughout the volume concerned (cortex). Hence additional constraints are necessary to provide a particular solution. Examples of these constraints, which may be based on reasonable physiological interpretations or utilize independent anatomical data (e.g. MRI or PET) include [6]:

a) Limiting the number of dipole sources to one or a few.

b) Assuming all sources are located in neocortical tissue of more specifically crowns of cortical gyri.

c) Applying spatial smoothness criteria to the source [27].

d) Applying temporal smoothness criteria to the source.

Source localisation is concerned with fitting a measured field distribution to a particular model - a model which has assumptions and generalizations. It provides a 'whole-head' model or broad spatial view but with a broad spatial resolution. Within this field there are a number of analytical techniques that have been developed.

\subsubsection{Scalp Current Density (SCD)}

The collective firing of cortical generators with a perpendicular orientation to the scalp surface generates a directional current source. Since the head is ostensibly an enclosed space, the current source cannot penetrate the scalp surface and so an equal and opposite current sink if generated elsewhere inside the head. This has lead to the theory of representing current generators as dipoles. At the scalp surface there is an associated electric field emanating from the scalp surface. EEG represents a measure of this electric field at the scalp surface. Studies have shown that modelling dipole source and using a 3 
concentric spherical shell head model leads to realistic EEG field measurements [28] [29].

This technique requires known recorded EEG from multiple scalp sites, assumed scalp geometry and a mapping from the scalp to the scalp current density plane. SCD is the second spatial derivative of scalp potential. The $m^{\text {th }}$ degree surface spline is used to generate a smooth surface constrained through known points. The higher the degree, the smoother the surface. Spatiotemporal source analysis techniques such as Brain Electrical Source Analysis (BESA) perform source localisation and source imaging. Spectral information is not used. I provides excellent spatial presentation of data and genuine imaging with the ability to present different time sequences of the spatial information [30].

$$
y(t, s, e)=T x(t) \text { with e constant }
$$

\subsubsection{Surface Laplacian}

The surface Laplacian of scalp potential (or second spatial derivative in two surface coordinates) is an estimate of current density entering (or exiting) the scalp through a local region of the skull [6]. Only the outer volume shape must be specified. To obtain good spatial resolution, high resolution EEG sampling, of 64-128 electrodes, is required. It can be shown that the surface Laplacian algorithm acts as a band-pass spatial filter that tends to isolate effects due to sources localized in the superficial cortex [6]. Typically resolution is in the range of $2-3 \mathrm{~cm}$ for surface tangential directions.

$$
y(t, s)=T x(t, e) \text { with e constant }
$$

\subsubsection{Dura Imaging}

An application of Poisson's equation is Dura Imaging where measured scalp potentials are projected onto the dura surface. Therefore, with some assumptions, the potential on the inner surface of the skull can be estimated directly from the scalp potential, given a volume conductor model of the skull [6]. Dura imaging and surface Laplacians estimate different physical quantities - dura potentials and radial skull current density, respectively.

$$
y(t, s)=T x(t, e) \text { with e constant }
$$




\subsubsection{LORETA}

Low Resolution Electromagnetic Tomography (LORETA) [27] computes a current distribution throughout the whole head. It does not assume a limited number of dipolar point sources nor a distribution on a given known surface. In order to determine a unique solution, it does assume neighbouring neurons are simultaneously and synchronously activated. It gives a genuine 3-D picture (expressed as a series of 2-D slices) of possible dipole sources although the spatial resolution is limited. LORETA is applied to evoked potentials data but individual epochs are not extractable from the analysis, hence it is another estimation algorithm where analysis is performed on a population rather than individual

$$
y(f, s)=T x(t, e)
$$

\subsubsection{Wavelet Methods}

The origins of Wavelet theories began in the field of quantum mechanics with physicist Gabor [31] in 1946. He "defined elementary time-frequency atoms as waveforms that have minimum spread in the time-frequency plane" [32]. He decomposed signals over such elementary atomic waveforms and demonstrated they are closely related to how we perceive sound. Nearly 40 years later and working in the fields of theoretical physics and reflection seismology respectively, Grossmann and Morlet [33] formalised the continuous wavelet transform. By the early 1990's a variety of Wavelet Transform techniques had been developed by Daubechies [34], Mallat [14] [5], Cvetkovic and Vetterli [35].

During the 1990's, many researchers began to apply these techniques to biomedical application. Unser's review [36] describes the main wavelet techniques; filter banks, multi-scale matched filters, time-frequency localisation, bases and perception models. He then describes the biomedical areas in which there techniques are applied; ECG, EEG, CT, MRI, PET and fMRI. Early studies concentrated on comparisons between the Fourier transform and the wavelet transform and highlighted areas where the wavelet transform gave better results. However Unser also describes the challenge which forms the basis of this research,

"The benefit of these techniques is a possible reduction of the number of trials, the ultimate goal being to extract the ERP using no averaging at all." [36] 
Unser uses the term ERP to refer to Evoked Related Potentials although they more typically refer to Event Related Potentials. Event Related Potentials are scalp potentials stimulated by a cognitive event in the brain while Evoked (Related) Potentials are scalp potentials stimulated a direct external stimulus to a sensory organ in the body. The papers referred to by Unser are described in more detail in the following sections.

In 1999, an entire edition of journal Brain and Language was devoted to presenting the latest research into the application of wavelet techniques for neuroelectric signals. In his introduction Samar [37] outlines the value of single trail ERPs.

"The detection of single trial ERPs permit the dynamic assessment of changes in the cognitive state of individuals and allows for response-based in contrast to stimulus based averaging. Single-trial ERP detection therefore has many potential applications in research, human performance, and clinical settings." [37]

\subsubsection{Wavelet Filtering and Denoising}

Some early wavelet analysis techniques applied this transform as a direct comparison for Fourier methods. Thakor et al [38] found that certain decomposition bands provided a better detection method for the absence of SEP in the case of cerebral hypoxia than comparable Fourier methods. Bertrand et al [39] describe how use of the wavelet transform permits implementation of a time-varying linear filter to selectively filter out high frequency noise from the later components of Mid-Latency Auditory Evoked Potentials while retaining the wanted higher frequency early components. Quian Quiroga [40] describes a similar time-varying filtering scheme using a subset of averaged evoked potentials wavelet detail coefficients to detect particular temporal and spectral signal components of individual evoked potential epochs. In this way individual trials that more represent the averaged signal can be selected and processed separately. This area overlaps with the selective averaging area. These filtering and denoising methods are similar to the Fourier methods but with an added temporal perspective.

$$
y(f, t)=T x(t) \text { with } e_{.} \text {s constant }
$$

\subsubsection{Multi-Resolution Analysis}

One of the first paper to examine Evoked Potentials using the Wavelet Transform formalism was that of Bartnik, Blinkowska and Durka [21]. They took the multiresolution analysis (MRA) decomposition method that Mallat [14] (described more fully 
in Chapter 3) which was implemented for image processing applications and applied it to Evoked Potentials signals. Their theory made 3 assumptions; firstly that EPs are characterised by a deterministic repeatable pattern, secondly that EEG is stochastic in nature and thirdly that EEG and EP are independent. These assumptions have been challenged and indeed the variability of the EP pattern was demonstrated by John [41]. Bartnik et al applied multi-resolution analysis to two sets of EEG data. Both were 1 second in duration and the first included the period of response to an auditory evoked potential (EEG+EP) and the second was 1second EEG data immediately after this period (EEG only). The MRA process breaks down these epochs into measures of eight different scales over a dyadic sequence. By performing correlation analysis and discriminant analysis between the two sets at each MRA level, they were able to identify canonical correlations with the discrimating variable. Run over all measured epochs this provided a classification process where 34 out of 40 epochs correctly identified the evoked potential signal. As the output only uses 2 scale levels to calculate the single evoked potentials, it provided only a crude approximation of the Evoked Potential. It was an important paper because it demonstrated that single evoked potential signal could be used in analysis. However, it also proved that multiple epoch of the EEG samples would still be necessary, to provide confirmation and repeatability of the results.

It is the first of the techniques described to retain both a temporal and spectral viewpoint.

$$
y(f, t, e)=T x(t, e)
$$

\subsubsection{Wavelet Entropy}

EEG may be envisaged as ensembles of generators producing spontaneous and event driven oscillations which are ongoing [42]. An external stimulus triggers cortical generators in a coherent fashion. This transition from a less ordered to a more ordered state may be described by the concept of entropy. In information theory, entropy is a measure of the order and disorder in a dynamic system [43]. Entropy methods have been applied to EEG using the Fourier Transform [44]. However the lack of temporal resolution with the Fourier Transform is a major drawback. Entropy is low in monofrequency signals and high is multi-frequency signals. Wavelet entropy studies have shown quantitatively that after sensory stimulation, there occurs a highly ordered state [42] [45]. This state is transient and localized in time. This regularity has been further pinpointed to occurring in the theta frequency range. The frequency resolution is limited 
to the common frequency bands (alpha, gamma, delta, beta, and theta) rather than discrete frequency resolution. Also spatial resolution is not continuous but rather a wavelet entropy figure at on site may be compared to that at another site.

$$
y(f, t, s)=T x(t, e, s)
$$

\subsubsection{Wavelet Packets}

Wavelet Packets analysis represents a broader form of the discrete wavelet transform where the detail component may be decomposed as well as the approximation component. Hence better choice of resolution of higher frequencies is possible. Raz, Dickerson and Turetsky describe the assumptions of the nature of evoked responses [46].

"The single-channel wavelet packet model is based on assumptions similar to, but less restrictive than, those required by peak analysis and PCA. First, we assume that the EPs arise from a small set of fixed set of anatomical sources and that the electrical current at each source is conducted through the head to the recording electrode. This assumption implies that the data are a linear combination of a small number of component waveforms. While this is clearly a simplifying approximation to real brain function, it has proved to be a useful one ..... Second, we assume that different EP components represent different brain processes and thus respond differently to changes in the experimental conditions. Third, we assume that each EP component is localized in time and frequency. Considerable empirical evidence is available to support this assumption."

This viewpoint is carried into this research. In this paper a fourth assumption is stated that some, possibly vague, prior information on the time and frequency of each EP component is available. This is a considerable drawback of this and some other methods - that some approximation of what you are looking for is a necessary starting point. It

$$
y(t)=T x(f, t, e)
$$

\subsubsection{Averaging Methods}

\subsubsection{Standard Averaging}

Averaging is one of the simplest EEG techniques first introduced by Dawson in 1954

[47]. Repeated stimulus response epochs are aligned in relation to the stimulus onset and 
added linearly in time and then divided by the number of epochs. A response linked to the stimulus will hopefully occur with identical latency in each epoch and so will add to the average signal. However background EEG which is stochastic or random in nature will tend to average towards zero as more and more epochs are added to the average response. By definition, an Evoked Potential signal is the averaged EEG response to multiple repeated trials. The individual trials are explicitly stated, such as single trial evoked potentials signal.

In practice, the stimulus response is not always present in every epoch and is subject to a certain amount of variation [48] [49]. Some of the factors of variability associated with evoked potentials and thus associated with averaging are [50]:

Non-Evoked Potential variability: Factors of variability that arise but are not due to the stimulus we wish to measure are:

- On-going EEG activity: As background EEG signal are the same order of magnitude as the evoked potential signal, summation of all ongoing activity may obliterate the evoked potential for a particular epoch.

Intra-individual variability: Between each consecutive trial on a single individual, considerable variation is observed.

- Changes in attention and alertness: EPs vary with the alertness of the subject.

- Habituation: As more stimuli are presented to the subject, the measured response may decrease over time as the subject becomes habituated to the stimuli. This can be more of a problem in Event-Related Potentials as we are dealing with late cortical peaks associated with a cognitive response to the stimuli as opposed to Evoked Potentials which mostly deal with an earlier physiological response.

- Heart Rate and Blood Pressure: Changes in these parameters may affect the evoked response.

- Time between Averages: sequential average tests performed on the one day will show less variability over averaging tests performed over the course of weeks or months.

- Age: A person's evoked potential response will vary considerably over their life cycle with most variability in infant years which will settle to a more stable level in adult years with some higher variability in older years occurring. 
Inter-individual variability: Further variability between results is found when averaged EPs from different subjects is observed.

- Non-brain physiology: Factors such as sex, body and proportions as well as peripheral sensory receptors and neuroanatomy will vary the EP.

- Brain physiology: Spatial orientation of the structures that cause the evoked potential is a very important variability factor.

- Skull physiology: skull and skin thickness, capacitance and resistance also introduce variability.

Laboratory variability: The highly sensitive nature of the EP readings means that different laboratories with different equipment and different sources of noise will introduce a further variability factor.

However, despite these variability mechanisms, a typical averaged response is commonly seen in Visual Evoked Potentials, Somatosensory Evoked Potentials and Auditory Evoked Potentials, amongst others. The epoch domain is an input to this method but not an output. Hence the averaged output yields excellent temporal information but no spatial, spectral or epoch information.

$$
y(t)=T x(t, e)
$$

\subsubsection{Selective Wavelet Averaging}

Some techniques have been investigated to selectively choose the epochs that form the evoked potential. One such technique selects epochs that look similar to the expected evoked potentials [19]. In this case multi-resolution analysis is applied to both the evoked potential response and the individual epochs that make up this response. Epochs whose wavelet coefficients match or are similar to those of the averaged response are kept while those that do not match are discarded. A new and de-noised averaged evoked potential signal is calculated from the remaining epochs. In another paper investigating selective averaging of ERP's, 28\% of target sweeps (epochs) did not contain the characteristic P300 component [22]. In this paper the authors demonstrate that by characterising the P300 component using wavelet coefficients, it is possible to separate epochs which contained the P300 response from those that did not. This was confirmed by averaging both the epochs with the response and the remaining epochs without the response. One drawback with this approach is that you need the final wave-shape in 
order to detect individual wave-shapes in each epoch. If the wave-shape is more complex like a summation of two or more peaks then this approach will not distinguish different sub-peaks. The results from this approach lead to enhanced EPs with slightly more defined results and some noise reduction. However, the amount of additional new information obtained is questionable. They do suggest that an intermediate analysis of evoked potential epochs prior to averaging can be constructive. This is relevant to the work of our research.

$$
y(t)=T x(t, e)
$$

\subsubsection{Multivariate Methods}

One of the major difficulties with representing multiple variable (or domain) data is that the human brain cannot visualise data in more than three dimensions. Mathematical techniques are not restricted in this way. However, as it is a person who must interpret the results, it still becomes necessary to reduce the dimensional view to at most 3 dimensions. Multivariate statistics describes a range of techniques that reduce the number of dimensions to an interpretable amount yet also attempt to retain the perspective and insight of the original data.

\subsubsection{Principle Component Analysis}

Principle Component Analysis (PCA) is a linear, multivariate data-reduction technique that was first proposed in the 1960's [51]. It works by grouping and identifying unique variance patterns for a given set of EP or ERP waveforms. The variance structure is decomposed into components (known as factor loadings) with associated weights (known as factor scores). These are interpreted as observational definitions of ERP components [52]. Throughout the eighties PCA found much favour in the analysis of EP and ERP waveforms. However, by the end of the eighties several studies questioned PCA as an appropriate technique for EP/ERP detection [53] [54]. However, one of the early limitations of PCA was the processing power of computers at the time. In the modern era, this limitation has been overcome and researchers are again looking at PCA for EP/ERP analysis [52, 55].

$$
y(f, s)=T x(t)
$$




\subsubsection{Independent Component Analysis}

Independent Component Analysis is a more recent technique that separates multi-channel data into activation maps due to temporally independent stationary sources [56]. The classical example to demonstrate the use of ICA is the cocktail party problem where many conversations are occurring at one time in one room [57]. People have become very adept at being able to focus on one particular conversation and filtering out background other conversations. However, separating these multi-channel signals electronically is quite a different matter. Place two microphones in different parts of the room recording the same hubbub of conversation and by assuming the recordings are statistically independent; it is possible to get an estimate of separate conversations in the room. This is Independent Component Analysis. EEG has some similarity to the cocktail problem in that there are many interfering signals occurring simultaneously in a stochastic manner which are spatial separate. Hence ICA has been applied to decompose these signals and localise the source of the signals. ICA has been very effective at separating artefacts from EEG and MEG data. Methods such as FastICA can separate stationary and quasi-stationary components with EEG signals $[58,59]$. However, despite the obvious presence of certain regular frequency components with EEG signals, overall EEG is considered non-stationary. The localisation achieved in ICA is in the spatial and spectral domain.

$$
y(f, s)=T x(t)
$$




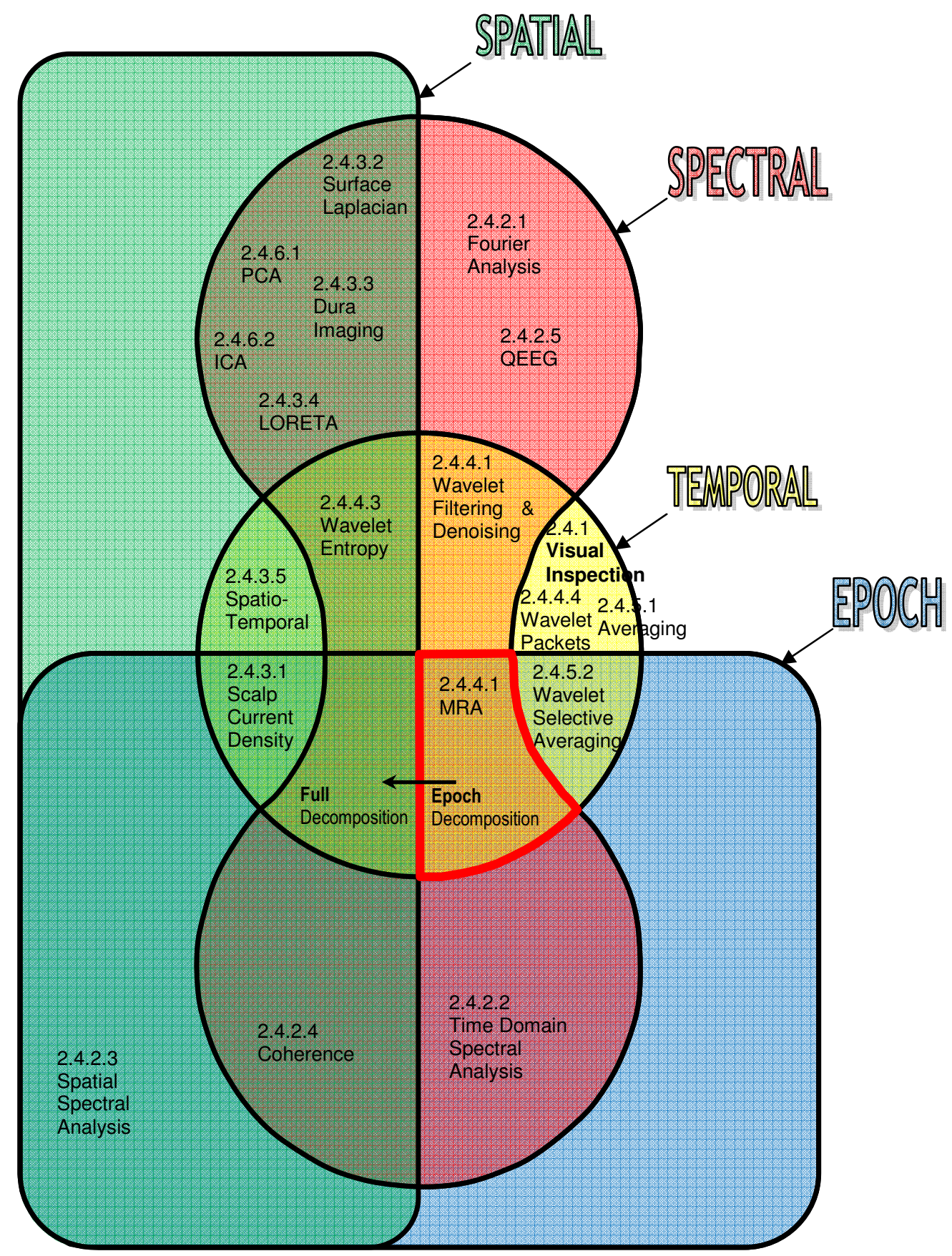

Fig. 2.10: This Edwards Venn diagram presents 4 different domains in which analysed EEG output may be viewed. The typical well known domains of time (temporal), frequency (spectral) and space (spatial) are joined by the 'Reiterative domain' which is denoted as epoch domain. This is not a true domain type; rather it represents the technique of measuring a repeated EEG event and the additional test data to pinpoint the event. The additional data is still in the temporal-spectral-spatial domain; however, for the purposes of the above illustration, it may be viewed as a domain. 


\subsection{FOCUS OF THIS RESEARCH}

\subsubsection{Comparison of Methods}

Each of the methods described in the previous section transforms the original EEG data by some expressed technique and so elicits some new observation. Even in the case of the basic stripchart, although trained Electroencephalographers can interpret the peaks and troughs often without additional calculation or visual aids, the techniques they are using are merely manual observational transforms which focus on certain aspects and disregard others.

The important aspect from the point of view of this research is to examine the variables of each particular technique. The variables within any technique define the domains in which analysis occurs. For example, standard EP averaging requires a dataset in the time domain and multiple epochs of data. Hence the input variables to this technique are time and epoch. However, the output variable is time alone as the epoch data has been lost. Therefore the output of the averaging technique has a single domain - with variability in the temporal domain alone.

Fig. 2.10 pictorially represents the different EEG methods and the domains in which their output operates. There are 4 output domains indicated and so an Edwards Venn Diagram is utilized to describe all the possible combinations that could occur. Note that a particular technique will fit in a particular domain only if the output of that technique is variable in that domain. Variability in any one domain implies that the output of the technique yields a range of values across the particular output domain from which interpretation at a particular point (or points) may then be inferred. There are usually additional domains that provide variability at the input side.

Three of the domains indicated are intuitive i.e. the temporal, spectral and spatial domains. EEG has excellent resolution in the time domain (greatly surpassing MRI and PET) but poor resolution in the spatial domain (inferior to MRI and PET). High resolution EEG has improved spatial domain over the last few years with the advent of a reliable 100-200 electrode headcap. However, this is still not near MRI or PET standard.

The fourth domain is the reiterative domain, labelled as 'epoch'. It is not a true domain. It represents additional discrete EEG samples taken which are believed to characterize or represent the same EEG event under examination. The most common examples are evoked potentials or event-related potentials where a repeated stimulus triggers the same 
EEG response which may be observed by averaging many EEG trials together. The repeated trials yield a new set of temporal-spectral-spatial EEG data however, it is the trial-to-trial variable which is of interest and so this is described as the 'epoch' domain in Fig. 2.10. It is interpreted here as a domain because it presents another perspective by which additional information regarding the nature of EEG may be investigated. Therefore, existing EEG methods are labelled in the appropriate segments of Fig. 2.10 depending on the domain(s) that there output operates in.

One important insight to observe is that there is no one best method of analysis for EEG signals. The type of method to use really depends on the question you are interesting in answering. At the highest level, one may argue that all these methods are concerned with understanding the meaning and workings of the brain. However, this unified broad aim of all researchers breaks down into many different possible questions once a more specific view is taken. Some of the questions that the methods described here seek to answer include: What is the source of EEG signals inside the brain? Are there inherent resonance patterns in the brain? How long does it take messages to get sent from the senses to the brain? Can we understand the cognitive messages within the brain? Are there common brain patterns that foretell a person's behaviour and nature? It is also apparent that the field of EEG analysis is shared between many scientific disciplines from Biomedical Engineering to Clinical Neuroscience, from Cognitive Psychology and the Medical Profession.

\subsubsection{Towards the Single Trial Holy Grail}

Different techniques emphasize different domains at the expense of other domains (refer to Fig. 2.10). It is apparent that useful information may be obtained in all domains and so this research aims to study the interaction of different domains with the goal of extracting novel useful EEG by maximizing the retained inter-domain information.

The question raised in this research is: can a localised, single stimulation of sensory nervous tissue in the body be detected in the brain? It is clear that this question has been asked before and various different approaches have been investigated. Evoked potentials and event-related potentials offer the best method for identifying single-trial activity since the origin, timing and nature of the stimulus is well known. The classical averaging algorithm emphasizes data in a single domain - the time domain. It is an excellent and simple method for observing the evoked potential signal across multiple epochs. It is not 
possible to detect single-trial responses with this method, only multiple trial responses. Nevertheless, most single-trial approaches begin with the averaging method and add another domain from which to garner additional data from which single-trial information is hoped to be detected. These double-domain methods retain the temporal domain variability but add either spectral, spatial of epoch domain information. They also introduce exclusivity to the analysis where, for example, a spectral-temporal analysis will not be able to analyse any spatial or epoch information.

Fig. 2.11 illustrates this idea graphically. The various approaches attempted by researchers from which to attain single-trial EEG are shown. Each method shows merit in moving towards a single-trial EEG understanding but none are there yet as indicated by Rodionov et al [60]:

"Recent studies have shown that background activity and single trial response are interrelated with each other .... Consequently, it would be advantageous to use the same methods for processing and analysing both aspects (responses and background) of the recorded activity. However, the problem of differentiation of single trial evoked responses from the background EEG activity has yet to be solved, and reliable methods for the analysis of EP and background variability are needed."

The approach by these authors examines characteristics of the "positive and negative time deflections", called peaks in our research, into time and amplitude distributions. Examination of these distributions leads to recognizing time locations where the number of positive peaks is significantly greater than the number of negative peaks. Although the authors use peak characteristics to segregate background EEG from evoked response, in rebuilding the evoked response from a reduced dataset, they use the entire epoch.

Standard averaging is the mainstay of the temporal approach to EP analysis. This singular domain approach cannot achieve single-trial EEG results. Efforts in this field have focussed on noise separation, filtering and selective averaging techniques. There is no doubt that standard averaging detects synchronous stimulus response activity. The latency of this response is consistent enough to allow interpretation in a clinical setting $[61,62]$. The assumption that the stimulus response is stationary amongst background stochastic process has been strongly questioned [63, 64]. 
"More specifically, each time a stimulus is applied, the specific response of the brain or evoked potential changes. It therefore behoves the investigator to take into account this behaviour of the data." [64]

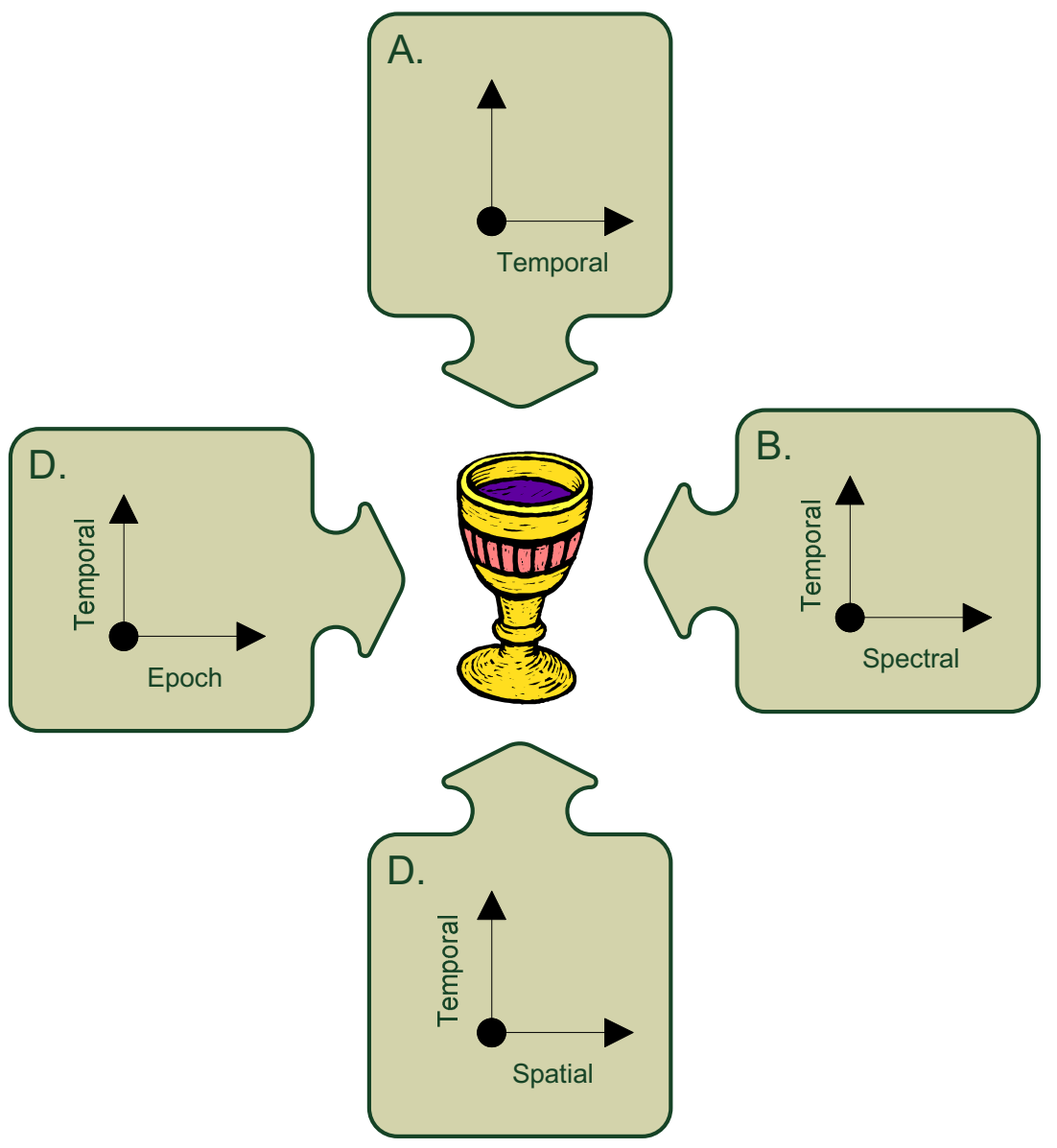

Fig. 2.11: An illustration of the different approaches to reaching the goal of understanding single-trial EEG. Each approach which emphasizes a different domain has merit and so it is observed that each domain is seen to contribute to reaching this goal. Traditionally, the exclusivity of each method i.e. one domain is emphasized at the expense of another has limited this progression. It follows that developing a method that allows analysis in multiple domains may have merit and is worth investigating.

Several papers apply methods that overlap in this research. Arikidis et al [65] developed a time-frequency analysis method based on examining the progression of wavelet modulus maxima coefficients across scales. This was used to differentiate between neuropathy and myopathic abnormalities in the electromyogram (EMG) interference pattern (IP). This theory is based on singularity detection which was developed by Stephane Mallat and is discussed in greater depth in the next chapter [32]. The advantage of this technique is that is can retain a perspective in both the temporal and spectral domains which is important in our application. 


\subsubsection{Research Aims}

Each of the approaches outline in Fig. 2.11 emphasizes a domain to the exclusion of other domains. If each domain can provide useful information towards a single-trial evoked potential EEG understanding, then it is worth attempting a multi-domain approach where as many domains as possible are retained in the analysis. This is the focus of this research:

- Can a multi-domain single-trial evoked potential EEG analysis technique be identified and developed?

- How close to a single-trial evoked potential EEG understanding does this technique bring us?

In order to describe an evoked potential signal in multiple domains, it is first necessary to decompose it into its lowest level constituent parts. At the level of EEG recording, the lowest level this signal can be decomposed into is its set of peaks and troughs. Each peak or trough may and usually does represent summed cortical activity, however that is at the sub-EEG detection level. Hence, this research looks to decompose evoked potential data into discrete sets of peaks and troughs. It is apparent that for multi-domain representations to be most effective that inevitability is an important property to retain throughout the analysis. The method of decomposing EP signals into sets of peaks is discussed in Chapter 3 in full detail. For the moment, let us examine the possible benefits of such a representation and its applicability in this research.

An individual peak can be uniquely characterised in all of the domains of interest:

Temporal: It has a unique time location centred about the peak maximum

Spectral: Typically, spectral properties refer to the frequency of stationary signals. EEG and EP signals are generally non-stationary with some semi-stationary subcomponents. An individual peak has a breadth or scale that, if repeated, would be consistent with it frequency value. Hence the breadth of the peak is characteristic of the frequency (or scale) of the signal.

Spatial: It is apparent that the location of the EEG electrode uniquely defines the position of the signal relative to other recording sites.

Epoch: It is also equally apparent that the particular epoch or reiteration number is also uniquely defined in a particular signal. 
Therefore, it is observed that a set of peaks decomposed from an EP signal can uniquely represent both the original EP signal and still retain a characterisation in the four domains discussed. This is the first major step towards a new analysis technique.

In Fig. 2.10 the bolded labels refer to those new methods that are developed in this study. This research focuses on the area labelled as Epoch Decomposition. A natural extension of the techniques devised for this research is to apply them to Spatial Decomposition. The combination of the two methods has the potential to offer new insights into the single stimulus response paradigm for EEG while retaining perspective in all four domains. This has been labelled 'Full Decomposition' and is located in the red highlighted segment representing the intersection of all 4 domains in Fig. 2.10. 


\section{CHAPTER 3 THEORY}

\subsection{INTRODUCTION}

his chapter details the theoretical basis of the research undertaken. It is divided
into five major sections. Firstly, a discussion of the Fourier transform and the
wavelet transform is given for continuous functions. Although the real life signals dealt with in this research are discrete, it is more intuitive to describe the theory using continuous functions first and then, the second major section describes how these concepts translate to the discrete signal domain. Once these basics are in place, we look at the third major section which is transient signal analysis. These first three sections describes theoretical concepts already published, however this review is necessary to outline the concepts on which the novel aspects of this research are based.

For readers already familiar with these well known concepts, they may skip to the fourth major section which describes the peak detection method devised during the course of this research. By the end of this section a proposed novel peak detection technique has been outlined as a means of decomposing data into constituent parts or peaks. It is demonstrated that this decomposition is an extension of singularity detection and multiresolution wavelet analysis theory described by Mallat. The peak detection method yields a new representation of evoked potential data but in itself does not provide particular insight.

The fifth and last major section describes the techniques to be employed to analyse these peak detected signals which potentially provides new insight into this particular class of signal. A novel clustering method is described and compared to standard clustering techniques.

Finally, a summary of the important concepts raised in this chapter is provided.

\subsection{CONTINUOUS FUNCTIONS}

\subsubsection{Fourier Transform}

The Fourier Transform decomposes a function into a continuous spectrum of its frequency components, and the inverse transform synthesizes a function from its spectrum of frequency components [1]. A signal $f$ is decomposed into a sum of sinusoidal eigenvectors $\left\{e^{i \omega t}\right\}_{\omega \in \mathbf{M}}$ : 


$$
f(t)=\frac{1}{\mathbf{2} \pi} \int_{\omega}^{+\infty} f(\omega) e^{i \omega t} d \omega
$$

If $f$ has finite energy then the theory of Fourier integrals proves that the amplitude $f(\omega)$ of each sinusoidal wave $e^{i \omega t}$ is the Fourier transform of $f:$ [32]

$$
\hat{f}(\omega)=\int_{-\infty}^{+\infty} f(\tilde{t}) e^{-i \omega t} d t
$$

For linear time-invariant signal processing, the Fourier Transform has become the most important technique because sinusoidal waves $e^{i \omega t}$ are eigenvectors of time-invariant operators and a linear time-invariant operator $\boldsymbol{L}$ is entirely specified by the eigenvalues $\tilde{h}(\omega)$ :

$$
\nabla \omega \in \mathbf{R}, \quad L e^{i \omega t}=\widehat{h}(\omega) e^{i \omega t}
$$

Applying the operator to $\mathrm{f}$ and inserting the eigenvector expression gives:

$$
\boldsymbol{L} f(t)=\frac{1}{2 \pi} \int_{\infty}^{+\infty} \hat{f}(\omega) \hat{h}(\omega) e^{i \omega t} d \omega
$$

The operator $\boldsymbol{L}$ is a frequency filter of $f-$ attenuating or amplifying each sinusoidal component $e^{i \omega t}$ of $f$ by $\widehat{h}(\omega)$. $\widehat{h}(\omega)$ is then the transfer function. Depending on its characteristics it may act as a low, high or band-pass filter. The Fourier transform is a powerful tool for stationary signal processing. However for transient phenomena, it is more difficult to apply.

Gabor developed the Windowed Fourier Transform to address this difficulty in 1946 [31]. A real and symmetric window $g(t)=g(-t)$ is translated by $u$ and modulated by the frequency $\omega$ :

$$
g_{u_{n} \omega}(t)=e^{i \omega t} g(t-u)
$$

By then normalising $\|g\|=\mathbf{1}$ so that $\left\|g_{u, \omega}\right\|=\mathbf{1}$ for any $(u, \omega) \in \mathbf{R}^{\mathbf{2}}$, the windowed Fourier transform also called the short time Fourier transform $(S)$ of $f \in L^{2}(\mathbb{R})$ is given by:

$$
S f(u, \omega)=\left\langle f, g_{u, \omega}\right\rangle=\int_{\omega}^{+\infty} f(t) g(t-u) e^{-i \omega z} d t
$$

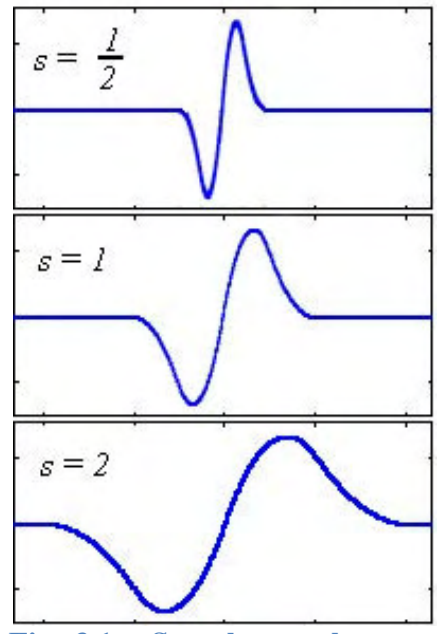

Fig. 3.1 : Sample wavelet - a quadratic spline with a scale of 1 (middle). The same wavelet with scale factor of $1 / 2$ (top). Same wavelet with scale factor 
The multiplication by $g(t-u)$ localizes the Fourier integral in the neighbourhood of $t=u$.

\subsubsection{Wavelet Transform}

As discussed in Chapter 2, in order to characterise individual transient signals such as EEG, it is not sufficient to transform a signal into a single domain. The time domain yields no data regarding the scale or breadth of individual peaks while frequency domain analysis (Fourier transform) yields no data regarding the relative position of transients in the time domain. The wavelet transform provides a suitable starting point for examining both the temporal and spectral properties of a signal simultaneously.

A wavelet is a time domain wave which is localised, oscillatory and with zero average. Fig. 3.1 shows a sample wavelet - the quadratic spline [32]. A wavelet $\psi(t)$ may be expressed in terms of a scale or dilation factor, $s$ which when varied compresses or expands the wavelet shape and a translation factor, $u$ that physically shifts the wavelet along the axis (in this case time axis). Wavelets are observed to have similar properties to the peaks we wish to detect insofar as they have large amplitude changes over narrow time changes.

Integrating a wavelet function, $\psi(t)$, over the whole time, $t$, axis range equals zero (the zero average condition):

$$
\int_{-\infty}^{+\infty} \psi(t) d t=0
$$

The wavelet function is then dilated by the scale parameter $\mathrm{s}$ and translated by $\mathrm{u}$ as follows:

$$
\psi_{u, s}(t)=\frac{1}{\sqrt{s}} \psi\left(\frac{\mathbf{t}-\mathbf{u}}{\mathbf{s}}\right)
$$

The nomenclature for the wavelet transform was first formalised by Morlet and Grossman [33]. The continuous wavelet transform (CWT), $W$ of $f(t) \in L^{2}(\mathbf{R})$ at translation $u$ and scale $s$ may be defined in terms of the inner product of $f$ and $\psi$.

$$
\begin{array}{ll}
W f(u, s)=\left(f, \psi_{u, g}\right)=\int_{-\infty}^{+\infty} f(t) \psi_{u, g}{ }^{*}(t) d t & \\
& \\
=\int_{-\infty}^{+\infty} f(t) \frac{1}{\sqrt{s}} \psi^{*}\left(\frac{t-u}{s}\right) d t & \text { Eq. } 3.9
\end{array}
$$


This wavelet integral expression measures the variation of $f$ in the neighbourhood of $u$, whose size is proportional to $s$. The wavelet transform yields a matrix of coefficients in terms of $u$ and $s$ which are used to characterise the signal. The wavelet transform may be rewritten as a convolution product as follows:

$$
W f(u, s)=\int_{-\infty}^{+\infty} f(t) \frac{1}{\sqrt{s}} \psi^{*}\left(\frac{t-u}{s}\right) d t=f * \bar{\psi}_{s}(u)
$$

With $\bar{\psi}_{s}(t)=\frac{1}{\sqrt{s}} \psi^{*}\left(\frac{-t}{s}\right)$

The convolution product computes the wavelet transform with dilated bandpass filters. The integral is said to characterise the nature of the signal in terms of position and size. This is an advance on the Fourier integral which characterised the nature of the signal in terms of size only. The next section compares the time-frequency localization properties of the windowed Fourier transform and the wavelet transform.

\subsubsection{Time-Frequency Localisation}

\subsubsection{Time-Frequency Atoms}

A linear time-frequency transform correlates the signal with a family of waveforms that are well concentrated in time and in frequency and known as time-frequency atoms [32]. Using Mallet's notation, consider a general family of time-frequency atoms $\left\{\phi_{\gamma}\right\}_{\gamma \in \Gamma}$ where $\gamma$ might be a multi-index parameter. We suppose that $\phi_{y} \in L^{2}(\mathbb{E})$ and that $\left\|\phi_{r}\right\|=\mathbf{1}$. The corresponding linear time-frequency transform of $f \in L^{z}(\mathbb{R})$ is defined by

$$
T f(\gamma)=\int_{-\infty}^{+\infty} f(t) \phi_{\gamma}^{*}(t) d t=\left\langle f, \phi_{\gamma}\right\rangle
$$

Applying the Parseval formula to this expression proves

$$
T f(\gamma)=\frac{1}{2 \pi} \int_{-\infty}^{+\infty} \hat{f}(\omega) \hat{\phi}_{\gamma}^{*}(\omega) d \omega
$$

If $\phi_{\gamma}(t)$ is nearly zero when $t$ is outside the neighbourhood of an abscissa $u$, then $\left\langle f, \phi_{\gamma}\right\rangle$ depends only one the values of $f$ in this neighbourhood. Similarly, if $\hat{\phi}_{\gamma}(\omega)$ is negligible 
for $\omega$ far from the frequency abscissa $\xi$, then the above equation proves that $\left\langle f, \phi_{\gamma}\right\rangle$ reveals the properties of $\hat{f}$ in the neighbourhood of $\xi$ [32].

\subsubsection{Heisenberg Uncertainty}

There is a trade-off between resolution in the time and frequency domains which is underpinned by the Heisenberg uncertainty principle. In quantum physics, this principle is very important in describing the uncertainty between the momentum and position of a free particle. One example in the temporal-spectral domain is the dirac function which has a unique location in time, but when Fourier transformed has energy uniformly spread over all frequencies. If you scale a function in time to reduce the time spread, you will find that when the function is Fourier transformed, its frequency spread is dilated by the same scale factor. What you gain in one domain, you lose in the other domain. More generally, it can be shown that the temporal variance and the frequency variance of $f \in L^{2}(\Re)$ satisfies [66],

$$
\sigma_{t}^{2} \sigma_{\omega}^{2} \geq \frac{1}{4}
$$

where $\sigma_{t}^{2} \sigma_{\omega}^{2}$ is the variance. Essentially there is an inverse relationship between the energy spread in each domain. The narrower the energy spread in the time domain, the broader it is in the frequency domain and vice-versa.

The windowed Fourier transform localizes both in the time domain, $u$ and the frequency domain, $\xi$. It can be shown that the time spread around both $u$ and $\xi$ expressed in terms of their variances $\sigma_{t}^{2}$ and $\sigma_{\omega}^{2}$ is independent of both $u$ and $\xi$ [32]. This may be visually described in the time-frequency plane using Heisenberg boxes which plot a box centred at a particular time-frequency position with box widths determined by the $\sigma_{t}$ and $\sigma_{\omega}$ parameters. For the windowed Fourier Transform, the size of the Heisenberg boxes are independent of the position of $(u, \xi)$, which means that a windowed Fourier transform has the same resolution across the time-frequency plane.

In the case of the wavelet transform, it may be shown that the location and size of the Heisenberg boxes are scaled the scale factor, s. Smaller scales decrease the time spread but increase the frequency support, which is shifted to higher frequencies [32]. 


\subsection{DISCRETE FUNCTIONS}

\subsubsection{Discrete Signals}

\subsubsection{Why Discrete Signals}

Time varying analogue signals carry information within the peaks and troughs that characterize its nature. In natural systems such as EEG data, the generators underlying the observed signals are complex and not fully determinable. Thus the ability to apply meaningful interpretations to such signals constitutes a complex signal processing conundrum and is the subject of much study.

The prevalence of analogue signal processing has waned in recent years as digital signal analysis methods have taken over. Traditionally analogue signals could be analysed using electronic circuits more quickly than digital algorithms analysed using microprocessors [32]. However, once the operational performance of a microprocessor improves to the point where the data can be digitized and analysed in real time, then digital signal processing has taken over for that application. Digital processing is more precise and more flexible. Examples of this evolutionary process are the transition from Long Play Records to Compact Discs and the conversion to Digital Television. In EEG analysis, this transition began in the 1980's when pen and trace records were gradually replaced with digital amplifiers. However it is only really in the last decade that microprocessor speeds have increased dramatically to the point where EEG signal processing can achieve far more complex analyses.

\subsubsection{Digitization}

In order to apply computer methods to analyse analogue real world signals, it is first necessary to digitize the signal into a sequence of discrete points. Fig. 3.2 illustrates the digitization process where an analogue signal is represented by a set of discrete periodic samples. 


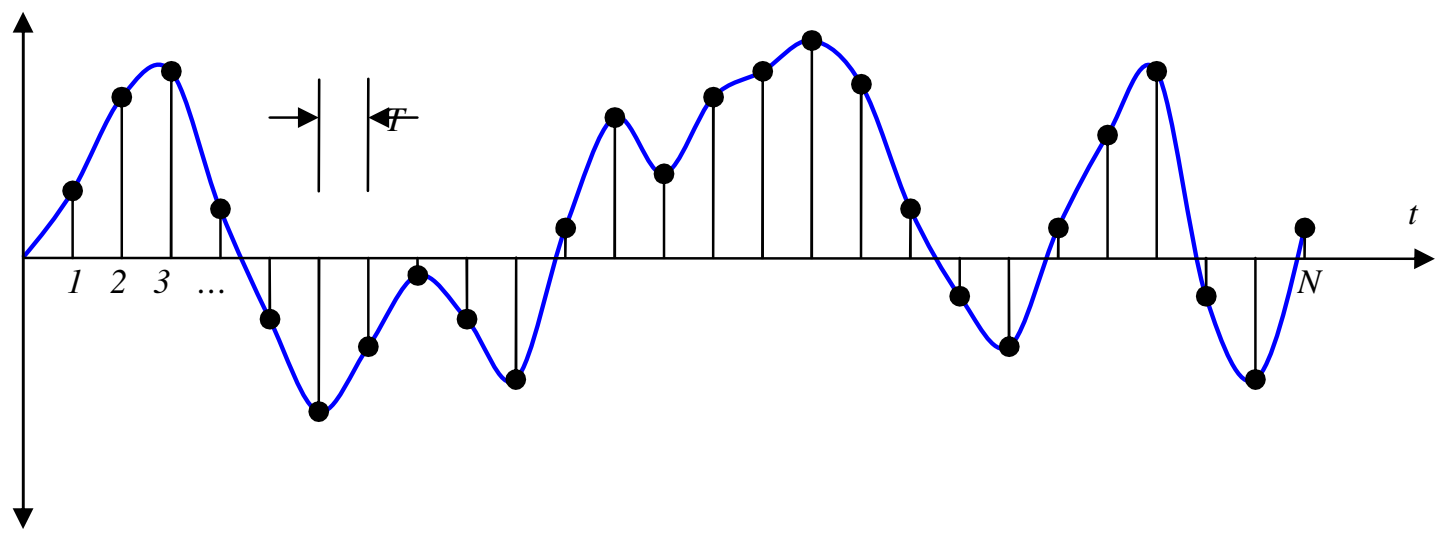

Fig. 3.2: Continuous irregular analogue signal $f(-)$ ) is discretized into a set of $N$ samples ( 9 ). The sample rate, $r$, in Hertz is related to the sampling interval, $T$, via $\mathrm{r}=1 / \mathrm{T}$.

Mathematically, an analogue signal $f$ is discretized into a set of sample values $\{f(n T)\}_{n \in Z}$ measured at intervals $T$. By interpolating these samples an approximation of $f(t)$ at any $t \in \mathfrak{R}$ is found. It is possible to express a discrete representation $f_{d}$ of an analogue signal $f$ as a weighted sum of Dirac functions $\delta(t-n T)$ uniformly sampled at $n T$ expressed as follows [32]:

$$
f_{d}(t)=\sum_{n=-\infty}^{+\infty} f(n T) \delta(t-n T)
$$

The Fourier transform of $\delta(t-n T)$ is $e^{-n T \omega}$ so the Fourier transform of $f_{d}$ is a Fourier series

$$
\hat{f}_{d}(\omega)=\sum_{n=-\infty}^{+\infty} f(n T) e^{-i n T \omega}
$$

Since each Dirac is zero outside each $t=n T, f_{d}$ may be rewritten as multiplication of the function with a dirac comb $c(t)$,

$$
f_{d}(t)=f(t) \sum_{n=-\infty}^{+\infty} \delta(t-n T)=f(t) c(t)
$$

The Fourier transform of $f_{d}(t)$ is then given by,

$$
\hat{f}_{d}(\omega)=\frac{1}{2 \pi} \hat{f} * \hat{c}(\omega)
$$

The Poisson formula proves the Fourier transform of a Dirac comb is given by

$$
\hat{c}(\omega)=\frac{2 \pi}{T} \sum_{k=-\infty}^{\infty} \delta\left(\omega-\frac{2 \pi k}{T}\right)
$$


Substituting eq 3.18 into eq 3.17 yields,

$$
\hat{f}_{d}(\omega)=\frac{1}{T} \sum_{k=-\infty}^{\infty} \hat{f}\left(\omega-\frac{2 \pi k}{T}\right)
$$

This expression shows sampling $f$ at intervals $T$ is the same as making its Fourier Transform $2 \pi / T$ periodic by summing all its translations. This leads to the ShannonWhittaker sampling theorem which states, if the support of $\hat{f}$ is included in $[-\pi / T, \pi / T]$ then,

$$
f(t)=\sum_{n=-\infty}^{\infty} f(n T) h_{T}(t-n T)
$$

with $h_{T}(t)=\frac{\sin \left(\frac{\pi t}{T}\right)}{\frac{\pi t}{T}}=\operatorname{sinc}\left(\frac{\pi t}{T}\right)$

Imposing the condition that support of $\mathrm{f}$ is included in $[-\pi / T, \pi / T]$, guarantees that $\mathrm{f}$ has no extreme variations between consecutive samples, and therefore may be recovered with a smooth interpolation. The sinc function, $\mathrm{h}_{\mathrm{T}}$, is an ideal low pass filter in the frequency domain and it acts to remove the periodic frequency domain repetitions introduced by the discretizing process. The discretizing and forward and inverse Fourier transform flow is illustrated in Fig. 3.3.

\subsubsection{Aliasing}

If frequencies higher than $[-\pi / T, \pi / T]$ are present in the signal then overlapping of periodic Fourier transform means that the original signal will not be recoverable. This phenomenon is known as aliasing. In accordance with the Nyquist criterion, the sampling rate must be at least twice the highest frequency of interest within the signal to prevent aliasing. Aliasing occurs when a higher frequency signal component is digitized as a lower frequency signal component due to under-sampling of the signal. The filtering of $f$ by $h_{T}$ prevents aliasing occurring by removing any frequency larger than $\pi / T$. In this way, we observe that an analogue to digital filter is composed of a low-pass filter with band $[-\pi / T, \pi / T]$ followed by uniform sampling at intervals $\mathrm{T}$.

Scalp voltages are continuous varying time signals localised in space. Electroencephalographic equipment measures these voltages at a discrete specified 
sampling rate. This discrete time varying signal is the input for all EEG signal processing methodologies.

\subsubsection{Reconstruction}

Mallet demonstrates that the Whittaker sampling theorem can be interpreted as signal decomposition in an orthogonal basis in order to yield a sufficient condition for reconstructing a signal from its samples.

Taking eq 3.21 then $\left\{h_{T}(t-n T)\right\}_{n \in Z}$ is an orthogonal basis of the space $\boldsymbol{U}_{T}$ of functions whose Fourier transforms have a support included in $[-\pi / T, \pi / T]$. If $f \in U_{T}$ then

$$
f(n T)=\frac{1}{T}\left\langle f(t), h_{T}(t-n T)\right\rangle
$$

\subsubsection{Discrete Fourier Transform}

It can be shown that the following family is an orthogonal basis of the space of signals of period $\mathrm{N}^{[17]}$,

$$
\left\{e_{k}[n]=\exp \left(\frac{i 2 \pi k n}{N}\right)\right\}_{0 \leq k<N}
$$

Expressing eq 3.4 for discrete signals of period $\mathrm{N}$ gives

$$
\langle f, g\rangle=\sum_{n=0}^{N-1} f[n] g^{*}[n]
$$

The definition of the discrete Fourier transform of $f$ is,

$$
\hat{f}[k]=\left\langle f, e_{k}\right\rangle=\sum_{n=0}^{N-1} f[n] \exp \left(\frac{-i 2 \pi k n}{N}\right)
$$

\subsubsection{Time-Invariant Filters}

\subsubsection{Finite Impulse Response Filters}

Classical discrete signal processing algorithms are mostly based on time-invariant linear operators [32]. An operator is time-invariant if a delay to its inputs produces an equal delay at the output. Stated mathematically, for samples values, $f[n]$, across a normalised sampling interval, $T=1$, a linear discrete operator, $L$, is time-invariant if the input $f[n]$ delayed by $p \in Z, f_{p}[n]=f[n-p]$ produces an output delayed by $p$ : 


$$
L f_{p}[n]=L f[n-p]
$$

The discrete Dirac is demoted by:

$$
\delta[n]= \begin{cases}1 & \text { if } n=0 \\ 0 & \text { if } n \neq 0\end{cases}
$$

Eq. 3.29

Any discrete signal $f[n]$ may be expressed as a sum of shifted discrete Diracs:

$$
f[n]=\sum_{p=-\infty}^{\infty} f[p] \delta[n-p]
$$

Eq. 3.30 

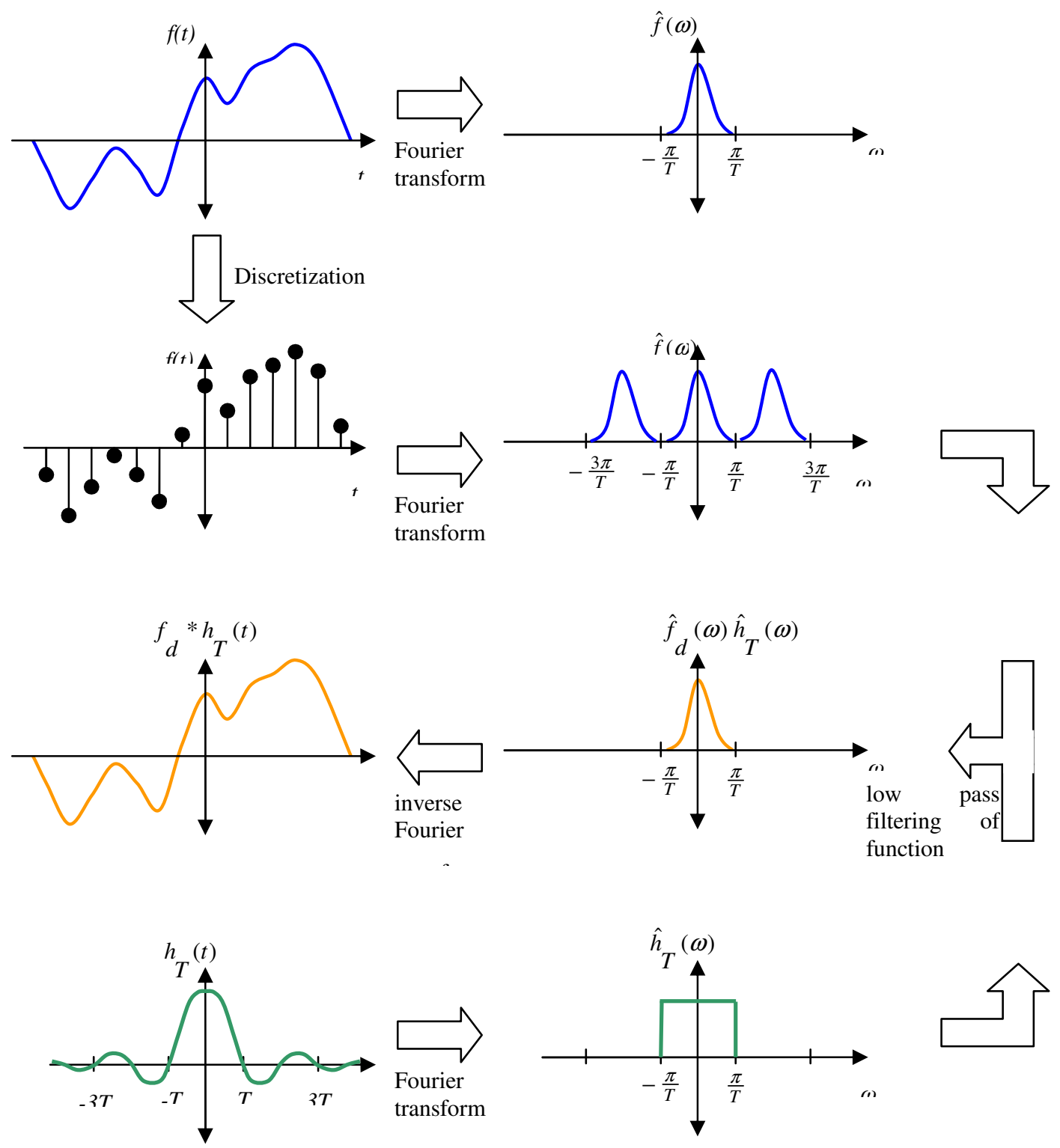

Fig. 3.3: Graphical illustration of the Fourier transform and the inverse Fourier transform for a discrete signal. $h[n]=L \delta[n]$ is called the discrete impulse response. Since the operator $\mathrm{L}$ is linear and time-invariant, it follows that,

$$
L f[n]=\sum_{p=-\infty}^{\infty} f[p] h[n-p]=f * h[n]
$$

Hence a discrete linear time-invariant operator if computed with a discrete convolution. Eq. 3.31 may be calculated with a finite number of operations if $h[n]$ has finite support. These are Finite Impulse Response (FIR) filters. Infinite impulse response (IIR) filters are calculated with a finite number of operations and are expressed as a recursive equation. 


\subsubsection{Finite Signals}

So far, the signals examined have been infinite, defined for all $n \in Z$. However, practically, our discrete signal is known over a finite range $0 \leq n<N$. As we are dealing with convolutions, there will then be border effects at $n=0$ and $n=N-1$. The approach used to solve this problem is to extend the function of $\mathrm{N}$ samples by making it periodic such that,

$$
f[n]=\tilde{f}[n \bmod N] \quad, \quad h[n]=\tilde{h}[n \bmod N]
$$

where $\tilde{f}$ and $\tilde{h}$ are signals of $\mathrm{N}$ samples. The circular convolution operator, $L$, of two such signals, both with period $\mathrm{N}$, is defined over their period [32]:

$$
L f[n]=f \otimes h[n]=\sum_{p=0}^{N-1} f[p] h[n-p]=\sum_{p=0}^{N-1} f[n-p] h[p]
$$

The output of Eq. 3.33 is also a signal of period N. The eigenvectors of a of a circular convolution operator are the discrete complex exponentials

\subsubsection{Discrete Wavelet Transform}

\subsubsection{Frames}

Frame theory provides a formalism to analyse the completeness, stability and redundancy of linear discrete signal representations [32]. A frame is a family of vectors $\left\{\phi_{n}\right\}_{n \in \Gamma}$ that are said to characterise any signal $f$ from its inner products $\left\{\left\langle f, \phi_{n}\right\rangle\right\}_{n \in \Gamma}$. In other words, one can recover a vector $f$ in a Hilbert space $\boldsymbol{H}$ from its inner products with the frame vectors.

The definition for a frame is as follows: The sequence $\left\{\phi_{n}\right\}_{n \in \Gamma}$ is a frame of $\boldsymbol{H}$ if there exists two constants $A>0$ and $B>0$ such that for any $f \in \boldsymbol{H}$

$$
A\|f\|^{2} \leq \sum_{n \in \Gamma}\left|\left\langle f, \phi_{n}\right\rangle\right|^{2} \leq B\|f\|^{2}
$$

Where $\mathrm{A}=\mathrm{B}$ the frame is said to be tight. If the frame condition is satisfied then the frame operator $U$ is defined by

$$
\forall n \in \Gamma \quad, \quad U f[n]=\left\langle f, \phi_{n}\right\rangle
$$


When the frame vectors are normalised $\left\|\phi_{n}\right\|=1$, the redundancy of the frame bounds is measured by A and B. If $\left\{\phi_{n}\right\}_{n \in \Gamma}$ are linearly independent then it has been proved that

$$
A \leq 1 \leq B
$$

A frame is an orthonormal basis if and only if $\mathrm{A}=1=\mathrm{B}$.

\subsubsection{Wavelet Frames}

By sampling the time and scale parameters of a continuous wavelet transform, wavelet frames are constructed. A real continuous wavelet transform $f$ is generated by taking its inner product with a wavelet as in eq 3.7 ,

$$
W f(u, s)=\left\langle f, \psi_{u, s}\right\rangle
$$

and $\psi$ is a real wavelet and

$$
\psi_{u, s}(t)=\frac{1}{\sqrt{s}} \psi\left(\frac{t-u}{s}\right)
$$

Imposing $\|\psi\|=1$ implies that $\left\|\psi_{u, s}\right\|=1$. Constructing a frame required us to cover the time-frequency plane with Heisenberg boxes of the corresponding discrete wavelet family. A wavelet $\psi_{u, s}$ has energy in time that is centred at $u$ over a domain proportional to $s$. Over positive frequencies, its Fourier transform, $\hat{\psi}_{u, s}$ has a support centred at a frequency $\eta / s$ with a spread proportional to $1 / s$. Let us sample s along an exponential sequence $\left\{a^{j}\right\}_{j \in Z}$ with a sufficiently small dilation step $a>1$. The time translation $\mathrm{u}$ is sampled uniformly at intervals proportional to the scale $a_{j}$. Refer to Fig. 3.4 for a graphical representation of the Heisenberg boxes. Thus the wavelet is expressed as,

$$
\psi_{j, n}(t)=\frac{1}{\sqrt{a^{j}}} \psi\left(\frac{t-n u_{0} a^{j}}{a^{j}}\right)
$$

To express the necessary conditions for $\psi, a$ and $u_{0}$ so that $\left\{\psi_{j, n}\right\}_{(j, n) \in Z^{2}}$ is a frame of $\boldsymbol{L}^{2}$ $(\mathbb{B})$, we suppose $\psi$ is real, normalised and satisfies the admissibility condition that,

$$
C_{\psi}=\int_{0}^{+\infty} \frac{|\hat{\psi}(\omega)|^{2}}{\omega} d \omega<+\infty
$$


Then Daubechies [34] proved that the frame bounds must satisfy,

$$
\begin{aligned}
& A \leq \frac{C_{\psi}}{u_{0} \log _{e} a} \leq B \\
& \forall \omega \in \Re-\{0\}, A \leq \frac{1}{u_{0}} \sum_{j=-\infty}^{\infty}\left|\hat{\psi}\left(a^{j} \omega\right)\right|^{2} \leq B
\end{aligned}
$$

The frame is an orthonormal basis is and only if,

$$
A=B=\frac{C_{\psi}}{u_{0} \log _{e} a}=1
$$

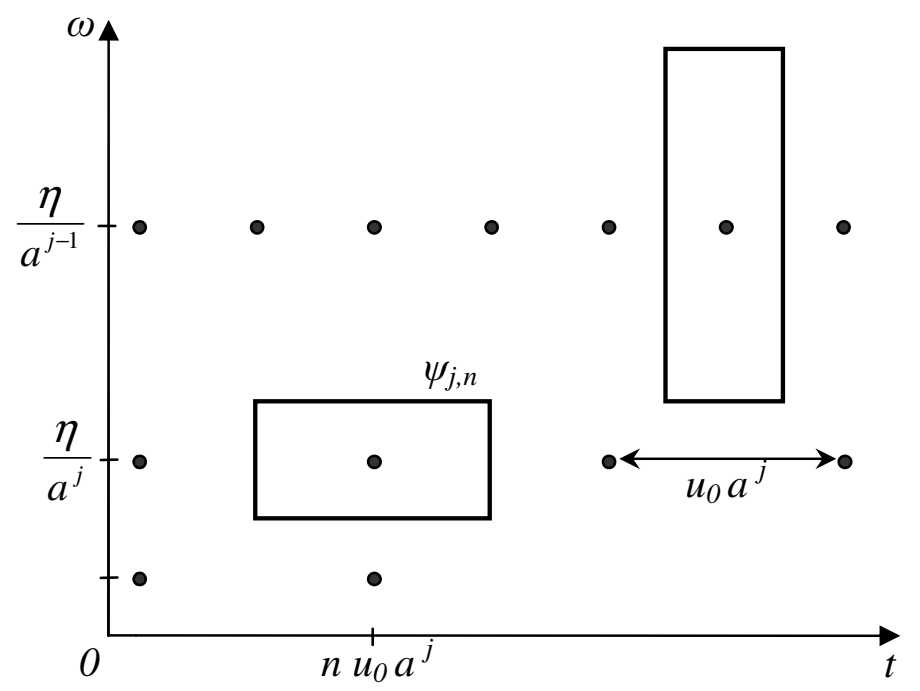

Fig. 3.4: Heisenberg boxes for the wavelet frame $\psi_{j, n}$ scaled by $s=a^{j}$. The time width is proportional to $a^{j}$ and the frequency width is proportional to $a^{-j}$. In order to cover the time-frequency plane completely with Heisenberg boxes, $u_{0}$ and $a$ must be sufficiently small.

\subsubsection{Dyadic Wavelet Transform}

It is important for pattern recognition applications that signal descriptors are translation invariant, i.e. patterns are translated by the process but not modified. The continuous wavelet transform is a translation invariant representation as described previously, however uniform sampling of the translation parameter undermines this property. The dyadic wavelet transform is a translation-invariant wavelet representation where scale $s$ is discretized but not the translation parameter $u$. The scale is sampled across a dyadic sequence $\left\{2^{j}\right\}_{j \in \mathbf{z}}$ which simplifies the numerical calculations. The dyadic wavelet transform of $f \in \boldsymbol{L}^{2}(\mathbf{R})$ is thus defined as, 


$$
\begin{aligned}
& W f\left(u, 2^{j}\right)=\int_{-\infty}^{\infty} f(t) \frac{1}{\sqrt{2^{j}}} \psi\left(\frac{t-u}{2^{j}}\right) d t=f * \bar{\psi}_{2^{j}}(u) \\
& \bar{\psi}_{2^{j}}(t)=\psi_{2^{j}}(-t)=\frac{1}{\sqrt{2^{j}}} \psi\left(\frac{-t}{2^{j}}\right)
\end{aligned}
$$

\subsubsection{Scaling Function}

When $W f(u, s)$ is known only for $s<s_{0}$, we need a 'complement of information' corresponding to $W f(u, s)$ for $s>s_{0}$ in order to recover the original function $f$. This is achieved by defining the scaling function $\phi$ as an 'aggregation of wavelets at scales larger than 1 [32]. The modulus of the Fourier transform is defined by

$$
|\hat{\phi}(\omega)|^{2}=\int_{1}^{\infty}|\hat{\psi}(s \omega)|^{2} \frac{d s}{s}=\int_{\omega}^{\infty} \frac{|\hat{\psi}(\xi)|^{2}}{\xi} d \xi
$$

The complex plane of $\hat{\phi}(\omega)$ can be chosen arbitrarily. It may be verified that $\|\phi\|=1$ and the admissibility condition is derived

$$
\lim _{\omega \rightarrow 0}|\hat{\phi}(\omega)|^{2}=C_{\psi}
$$

The scaling function may be interpreted as the impulse response of a low pass filter. Using the following notation for $\phi_{s}(t)$,

$$
\phi_{s}(t)=\frac{1}{\sqrt{s}} \phi\left(\frac{t}{s}\right) \text { and } \bar{\phi}_{s}(t)=\phi_{s}^{*}(-t)
$$

then the low frequency approximation of $f$ at the scale $s$ is,

$$
L f(u, s)=\left\langle f(t), \frac{1}{\sqrt{s}} \phi\left(\frac{t-u}{s}\right)\right\rangle=f * \bar{\phi}_{s}(u)
$$

\subsubsection{Spline Dyadic Wavelets}

A box spline of degree $m$ is a translation of $m+1$ convolutions of $\boldsymbol{1}_{[0,1]}$ with itself [32]. It is centred at $t=\frac{1}{2}$ if $m$ is even and at $t=0$ if $m$ is odd. Its Fourier transform is given by, 


$$
\hat{\phi}(\omega)=\left(\frac{\sin \left(\frac{\omega}{2}\right)}{\frac{\omega}{2}}\right)^{m+1} \exp \left(\frac{-i \varepsilon \omega}{2}\right) \text { with } \varepsilon= \begin{cases}1 & \text { if } m \text { is even } \\ 0 & \text { if } m \text { is odd }\end{cases}
$$

so the FIR filter $\hat{h}(\omega)$ is given by

$$
\hat{h}(\omega)=\sqrt{2} \frac{\hat{\phi}(2 \omega)}{\hat{\phi}(\omega)}=\sqrt{2}\left(\cos \frac{\omega}{2}\right)^{m+1} \exp \left(\frac{-i \varepsilon \omega}{2}\right)
$$

We can choose a wavelet which has one vanishing moment by $\hat{g}(\omega)=\boldsymbol{O}(\omega)$ in the neighbourhood of $\omega=0$. An example of this would be,

$$
\hat{g}(\omega)=-i \sqrt{2} \sin \frac{\omega}{2} \exp \left(\frac{-i \varepsilon \omega}{2}\right)
$$

The Fourier transform of the resulting wavelet is,

$$
\hat{\psi}(\omega)=\frac{1}{\sqrt{2}} \hat{g}\left(\frac{\omega}{2}\right) \hat{\phi}\left(\frac{\omega}{2}\right)=\frac{-i \omega}{4}\left(\frac{\sin \left(\frac{\omega}{4}\right)}{\frac{\omega}{4}}\right)^{m+2} \exp \left(\frac{-i \omega(1+\varepsilon)}{4}\right)
$$

This is a first derivative of a box spline of degree $\mathrm{m}+1$ centred at $t=\frac{1+\varepsilon}{4}$. The expressions for the case of $m=2$ are known as the quadratic spline wavelet. Fig 3.6 illustrates the quadratic spline wavelet and scaling function. In order to design dual scaling functions $\tilde{\phi}$ and dual wavelet functions $\tilde{\psi}$ which are quadratic splines, we choose $\hat{\tilde{h}}=\hat{h}$. Consequently, $\phi=\tilde{\phi}$ and using the reconstruction condition, yields,

$$
\hat{\tilde{g}}(\omega)=\frac{2-|\hat{h}(\omega)|^{2}}{\hat{g}^{*}(\omega)}=-i \sqrt{2} \exp \left(\frac{-i \omega}{2}\right) \sin \frac{\omega}{2} \sum_{n=0}^{m}\left(\cos \frac{\omega}{2}\right)^{2 n}
$$

It turns out that a discrete dyadic wavelet transform can be computed efficiently with a fast filter bank algorithm if the wavelet has appropriate properties. The quadratic spline wavelet is one such appropriately designed wavelet. The filter coefficients may be calculated from their transfer functions given in Eq. 3.51, Eq. 3.52 and Eq. 3.54 and are shown in Table 3.1. 


\begin{tabular}{|l|l|l|l|l|}
\hline $\mathrm{n}$ & $h[n]$ & $\tilde{h}[n]$ & $g[n]$ & $\tilde{g}[n]$ \\
\hline-2 & & & & $-\frac{1}{32 \sqrt{2}}$ \\
\hline-1 & $\frac{1}{8 \sqrt{2}}$ & $\frac{1}{8 \sqrt{2}}$ & & $-\frac{7}{32 \sqrt{2}}$ \\
\hline 0 & $\frac{3}{8 \sqrt{2}}$ & $\frac{3}{8 \sqrt{2}}$ & $-\frac{1}{2 \sqrt{2}}$ & $-\frac{22}{32 \sqrt{2}}$ \\
\hline 1 & $\frac{3}{8 \sqrt{2}}$ & $\frac{3}{8 \sqrt{2}}$ & $\frac{1}{2 \sqrt{2}}$ & $\frac{22}{32 \sqrt{2}}$ \\
\hline 2 & $\frac{1}{8 \sqrt{2}}$ & $\frac{1}{8 \sqrt{2}}$ & & $\frac{7}{32 \sqrt{2}}$ \\
\hline 3 & \multicolumn{5}{|l|}{} & $\frac{1}{32 \sqrt{2}}$ \\
\hline
\end{tabular}

The time domain shape of the quadratic spline wavelet and quadratic spline scaling function are illustrated in Fig. 3.5. Note that the overall shape of the wavelet is similar to the typical shape of features in EEG data. Wavelets that are similar to the shapes contained in the analysed signal yield better wavelet transform coefficients.
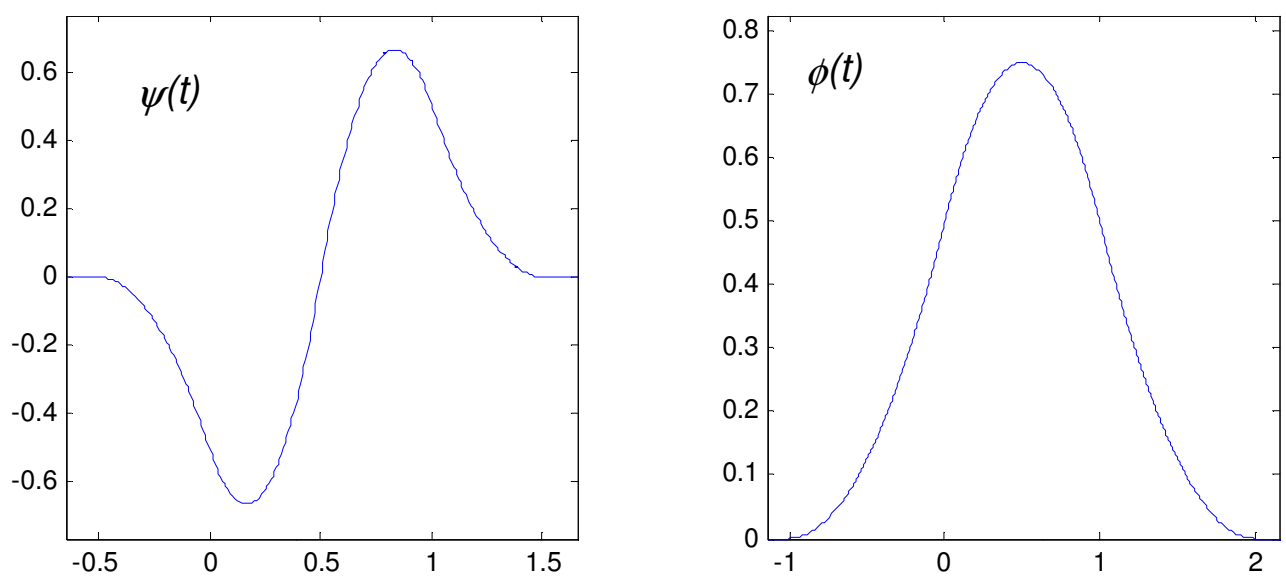

Fig. 3.5: Quadratic spline wavelet,

$\psi(t)$

and scaling function,

$\phi(t)$. (adapted from

\subsubsection{Algorithme à Trous}

\subsubsection{Wavelet Analysis}

The output of the wavelet transform is a set of coefficients dependant on translation, $u$ and scale $s\left(=2^{j}\right)$, which give a measure of how the properties of the chosen wavelet 'fit' the function at the particular translation and scale. Mallat [32] describes a practical implementation of the dyadic wavelet transform using a filter bank algorithm called the "algorithme à trous". It generates a detail component (similar to a low pass filter) at each $2^{j}$ scale up to the maximum calculated scale, together with a remaining approximation component. 
Take a continuous time signal $f(t)$ which may be sampled $N$ time at a sampling spacing of $N^{1}$ over the interval $[0,1]$. The dyadic wavelet transform of this signal may only be calculated at scales $1>2^{j} \geq N^{-1}$. For simplicity of the filter bank algorithm, the distance between samples may be set to 1 by letting

$$
f(t)=\dot{f}\left(N^{-1} t\right)
$$

Applying a change of variable to the dyadic wavelet transform integral show that,

$$
W \dot{f}\left(u, 2^{j}\right)=\frac{1}{\sqrt{N}} W f\left(N u, N 2^{j}\right)
$$

This allows us to concentrate on calculating the dyadic wavelet transform of $f(t)$ as we can easily derive $\dot{f}(t)$ from $f(t)$. Now we may express samples $\mathrm{a}_{0}[\mathrm{n}]$ of the input discrete signal are not equal to the equivalent value at $n$, that is $f(n)$, but are equal to a local average of $f$ in the neighbourhood of $t=n$. This local average representation is typical of actual real life digital signal acquisition for EEG or other digital systems. Therefore, the samples are written as averages of $f(t)$ now weighted by the scaling kernels thus:

$$
a_{0}[n]=\langle f(t), \phi(t-n)\rangle=\int_{-\infty}^{+\infty} f(t) \phi(t-n) d t
$$

For any $j \geq 0$, we denote

$$
a_{j}[n]=\left\langle f(t), \phi_{2^{j}}(t-n)\right\rangle \text { with } \phi_{2^{j}}(t)=\frac{1}{\sqrt{2^{j}}} \phi\left(\frac{t}{2^{j}}\right)
$$

and compute the dyadic wavelet coefficients for $\mathrm{j}>0$ over the integer grid,

$$
d_{j}[n]=W f\left(, 2^{j}\right)=\left\langle f(t), \psi_{2^{j}}(t-n)\right\rangle
$$

Now take any filter $x[n]$ and denote by $x_{j}[n]$ the filters obtained by inserting $2^{j}-1$ zeros between each sample of $x[n]$. Its Fourier transform becomes $\hat{x}\left(2^{j} \omega\right)$. By inserting zeros in the filter, we are effectively creating 'holes'. In French this is 'trous', hence the name algorithme à trous. Now let $\bar{x}_{j}[n]=x_{j}[-n]$. Using this notation, Mallet proved that the dyadic wavelet transform may be computed from a digital filter cascade using convolution formulae [32]:

For any $\mathrm{j}>0$, 


$$
\begin{aligned}
& a_{j+1}[n]=a_{j} * \bar{h}_{j}[n] \\
& d_{j+1}[n]=a_{j} * \bar{g}_{j}[n]
\end{aligned}
$$

The dyadic wavelet transform of the original discrete signal $a_{0}$ is defined as the set of wavelet coefficients us to a scale $2^{J}$ plus the remaining low-frequency information $a_{j}$ :

$$
\left[\left\{d_{j}\right\}_{1 \leq j \leq J}, a_{J}\right]
$$

An example of a typical representation of the dyadic wavelet transform is illustrated in Fig. 3.9 which shows the original signal and the detail coefficients $d_{j}$ and the lowfrequency approximation coefficients $a_{J}$. This representation is calculated using Mallat's filter bank algorithm of Eq. 3.60 and Eq. 3.61. A block diagram of this filter bank representation is illustrated in Fig. 3.6.
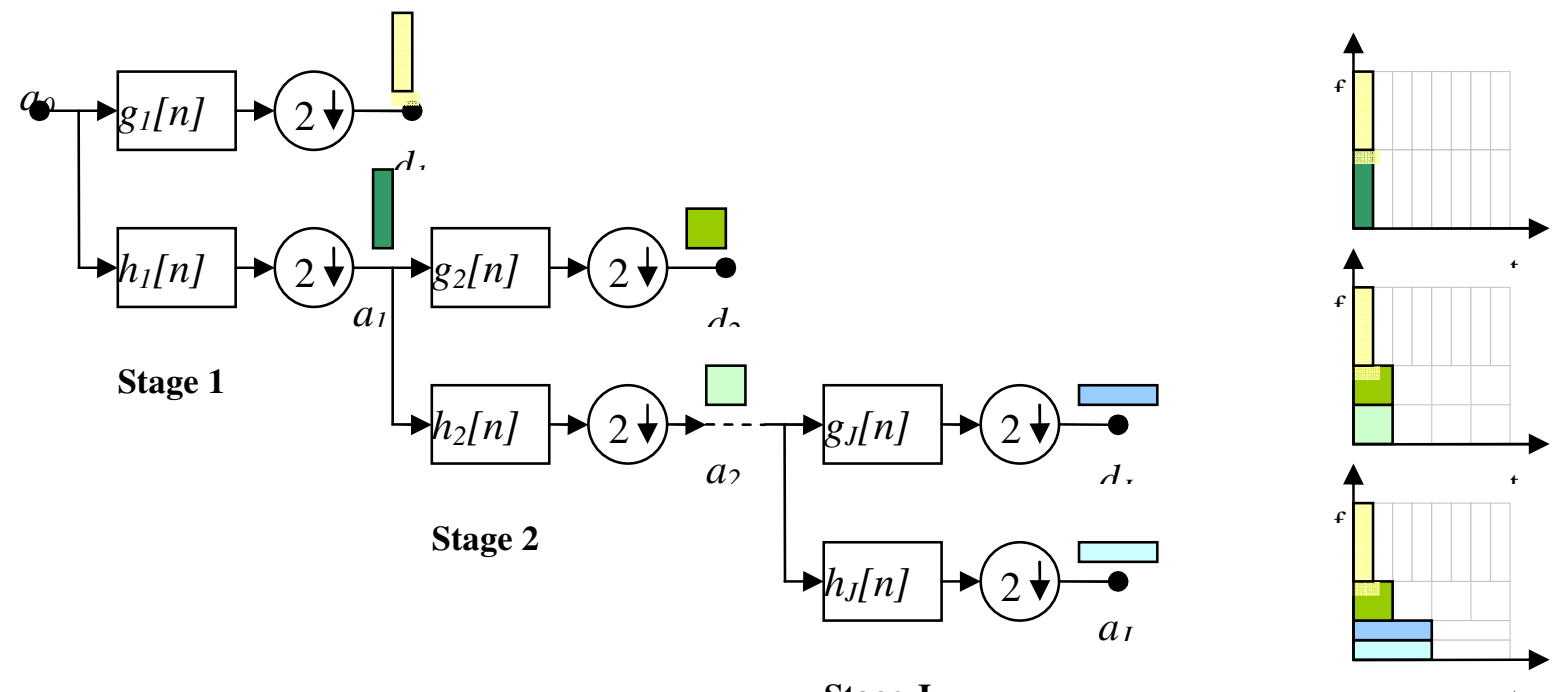

Stage J

Fig. 3.6: Filter bank representation of the discrete dyadic wavelet transform analysis illustrating how applying appropriate filters progressively decomposes the input signal $\left(a_{0}\right)$ into detail $\left(d_{j}\right)$ and approximation $\left(a_{j}\right)$ components. (adapted from [7])

\subsubsection{Wavelet Synthesis}

The detail and approximation coefficients of eq 3.48 and eq 3.49 may be recombined to synthesize the original signal $a_{0}$. This formula was also derived by Mallat,

$$
a_{j}[n]=\frac{1}{2}\left(a_{j+1} * \tilde{h}_{j}[n]+d_{j+1} * \tilde{g}_{j}[n]\right)
$$


Assuming the input signal is of finite sample size $N$, the convolutions of eq 3.50 are replaced by circular convolutions. The maximum scale $2^{J}$ is then limited to $N$, and for $J=\log _{2} N$ it may be verified that $a_{J}[n]$ is constant and equal to

$$
a_{J}[N]=\frac{1}{\sqrt{N}} \sum_{n=0}^{N-1} a_{0}[n]
$$

The original discrete signal $a_{0}$ may be recovered by using Mallat's filter bank algorithm of Eq. 3.63. A block diagram of its filter bank representation is illustrated in Fig. 3.7.

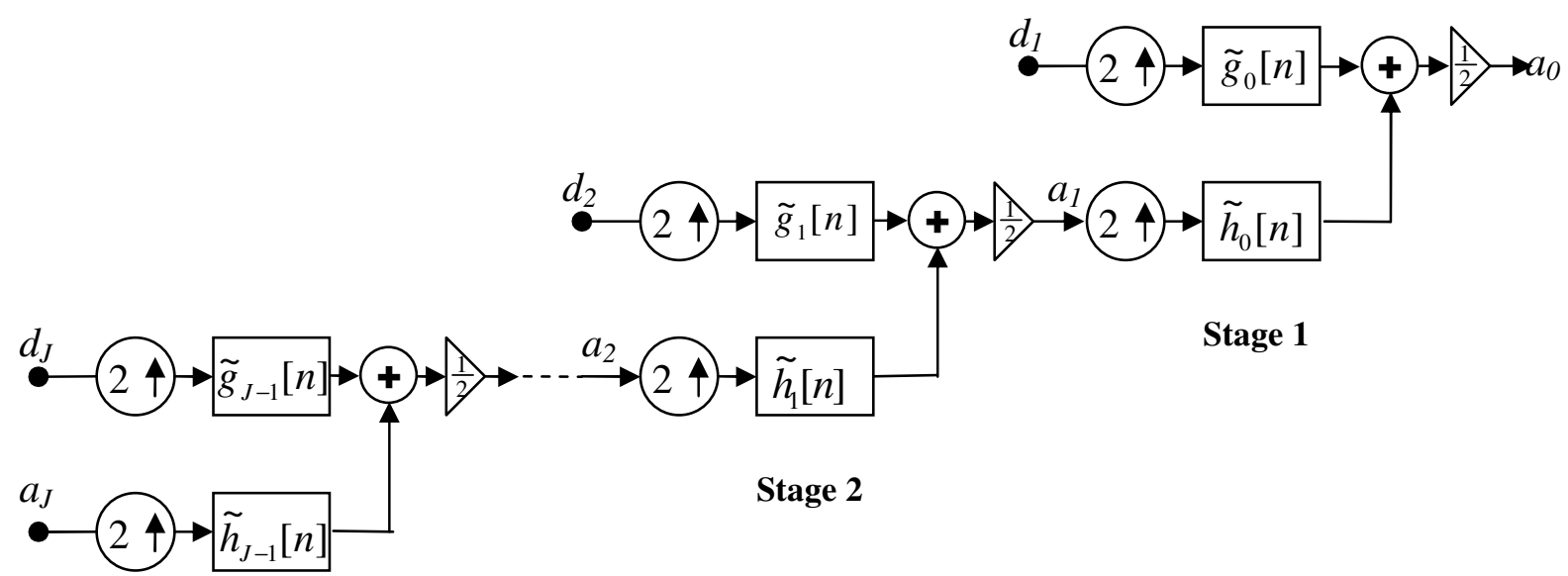

\section{Stage J}

Fig. 3.7: Filter bank representation of the discrete dyadic wavelet transform analysis illustrating how applying appropriate filters progressively decomposes the input signal $\left(a_{0}\right)$ into detail $\left(d_{j}\right)$ and approximation $\left(a_{j}\right)$ components Adapted from [7].

By choosing a wavelet that was the derivative of a smoothing function, such as the quadratic spline wavelet of degree 2, Mallat and Hwang proved that the evolution of the local maxima of the wavelet transform modulus across scales could be used to detect local singularities [5]. The detail components, taken from the "algorithme à trous" method, yield a set of coefficients for each scale. At a point of singularity, there is a linear relationship between $\log _{2}$ of the modulus maxima response and its associated scale $j$. By selecting only the modulus maxima values that fitted the linear relationship, Mallet and Hwang simplified the large set of wavelet coefficients into a much smaller discrete set of coefficients grouped per singularity. This is a powerful step forward in the characterisation of a signal into a group of singularities. Mallet and Hwang did not further analyse individual singularities but did demonstrate an inverse discrete wavelet transform that showed that the selected singularity coefficients still approximated the original signal well. 


\subsection{TRANSIENT SIGNALS}

\subsubsection{Singularities}

Analysing a signal often involves transforming it using a particular mathematical technique to accentuate an observation in a particular domain. Hence information present in the form of rhythm or resonance would typically be analysed in frequency domain. Once example of a signal suited to frequency domain analysis would be speech signal which are audible in the 20-20,000 Hertz range. Other signals contain information in the bias of the signal which may vary over time such as a simple thermocouple device. Still other signals contain information in the sharp, irregular transitions that occur within the signal. Sharp, irregular transitions within a signal are known as singularities. It is to this third class of signal that the methods described herein are applied. It should be stated at this early point that EEG signals are better described as smooth irregular transitions than sharp irregular transitions and that the term singularity is still applied. It will be shown that the theory is applicable in both cases.

In signal processing, identifying these transitions is very important. In both seismic data and image processing, these transitions are associated with edge detection. Hence the edges can be discerned from the smooth areas. In EEG analysis, this is not sufficient. The nature of EEG is that there are continuous irregular transitions which need to separate from each other without any real smooth areas in-between. This makes the signal processing goal not just one of edge detection but multiple edge detection and classification.

According to Mallet and Hwang [5], "Singularities and irregular structures often carry the most important information in signals". In EEG, these singularities record the firing of neurotransmitters in the brain close the recording electrode. In images, singularities occur at the boundaries of structures within the image. Still other areas of application include radar detection and fingerprinting.

Mallet and Hwang [5] describe a singularity detection method using the discrete wavelet transform (DWT). Firstly, the DWT is performed on the digitized signal. Then at each scale of the wavelet transform, the modulus maxima values are kept but all non-maxima points are set to zero. They then showed that the trend of the coefficient values for the maxima values could be used to characterise the singularities present in the signal. Finally, Mallet and Hwang describe an inverse DWT algorithm which gives a good 
approximation of the original signal. This demonstrated that the vast majority of the information contained in the signal could be characterised by the sum of the singularities. This paper is the starting point for the theory outlined herein. Here, we take the modulus maxima coefficients and further process then into discrete singularity sets. These singularity sets are compared to identify distinct peaks within the data. The goal is to describe each of the important peaks within a signal using the modulus maxima coefficients only.

Scalp electroencephalographic signals have been shown to contain useful information regarding the functioning of the brain [6]. The basic EEG signal is made up of undulating electrical signal comprising a summation of all electrical activity detected at a particular scalp position at a particular time. This research is concerned with detecting a one-to-one correlation between a sensory stimulus and the response within the brain detectable via EEG.

\subsubsection{Regularity}

The output of the wavelet transform is set of coefficients based on the dilation and scale parameters of $u$ and $s$ respectively. Since the wavelet has zero average and assuming it is real, a wavelet with a particular value of $u$ and $s$ will yield a high wavelet transform value, if the function $\mathrm{f}$ has a similar shape to this particular wavelet. In this way a multiscale zooming procedure can be developed. Sharp signal variations create large amplitude wavelet coefficients across multiple scales. These sharp signal variations may be detected by tracing the local maxima of the wavelet transform across scales. For the purposes of this study, singularities, irregular structures, edges and sharp transitions will all be termed as singularities. In strict mathematical parlance, a singularity refers to a point at which a function is not defined, irregular or discontinuous. Many EEG peaks, although sharply varying, are continuous and so are not technically singularities. However, following standard mathematical notation in describing this theory, they will be lumped under the same umbrella term of singularities.

\subsubsection{Taylor Series}

The Taylor series yields an expression for function about a point expressed in terms of a differential polynomial series expansion. A one-dimensional Taylor series is an expansion 
of a real function $f(t)$ about a point $t=v$ where $f$ is $m$ times differentiable in the region $[v$ $h, v+h]$. Let $p_{v}$ represent the Taylor series polynomial in the neighbourhood of $v$ [33],

$$
p_{v}=\sum_{k=0}^{m-1} \frac{f^{(k)}(v)}{k !}(t-v)^{k}
$$

Eq. 3.65

The approximation error function between the original function $f$ and the Taylor series $p_{v}$ is represented by

$$
\varepsilon_{v}(t)=f(t)-p_{v}(t)
$$

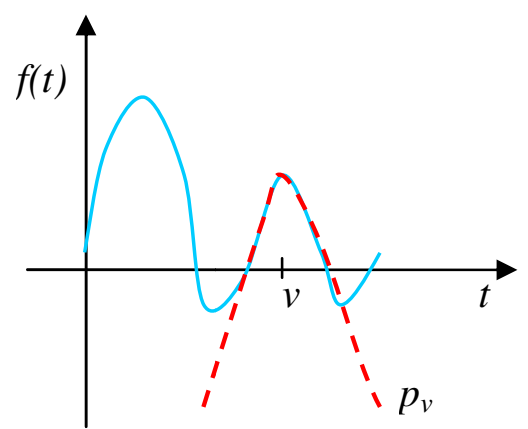

The Taylor formula states that $m^{\text {th }}$ order differentiability

Fig. 3.8: A Taylor series expansion $p_{v}$ representing a function $f$ at point $v$. of $f$ in the neighbourhood of $v$ yields an upper bound on the error when $t$ tends to $v$.

$$
\forall t \in[v-h, v+h] \quad, \quad\left|\mathcal{E}_{v}(t)\right| \leq \frac{|t-v|^{m}}{m !} \sup _{u \in[v-h, v+h]}\left|f^{m}(u)\right|
$$

\subsubsection{Lipschitz Regularity}

The wavelet transform can highlight local features in a signal by observing the pattern of detail components across different scales. Our aim is to characterise EEG signals and so an important first step is to characterise the regularity of the basic EEG signal. Mallet describes how the notion of Lipschitz regularity may be used to this end.

"Lipschitz exponents provide uniform regularity measurements over time intervals, but also at any point $v$. If $f$ has a singularity at $v$, which means that it is not differentiable at $v$, then the Lipschitz exponent at $v$ characterizes this behaviour." [32]

The Lipschitz regularity refines the upper bound of eq 3.54 with non-integer exponents. The conditions underlying Lipschitz exponents (also known as Hölder exponents) for a function are outlined herein [32]:

Definition:

A function is pointwise Lipschitz $\alpha>0$ at $v$, if there exists $K>0$, and a polynomial $p_{v}$ of degree $m=\lfloor\alpha\rfloor$ such that

$$
\forall t \in \mathbb{R}, \quad\left|f^{\prime}(t)-p_{v}(t)\right| \leq K|t-v|^{\alpha}
$$


A function $f$ is uniformly Lipschitz $\alpha$ over $[a, b]$ if it satisfies eq 3.55 for all $v \in[a, b]$, with a constant $K$ that is independent of $v$.

The Lipschitz regularity of $f$ at $v$ or over $[a, b]$ is the sup of $\alpha$ such that $f$ is Lipschitz $\alpha$.

Pointwise Lipschitz exponents vary from one abscissa to the next. However, uniform Lipschitz exponents give a better indicator of regularity as they are constant over an interval. Table 3.2 illustrates some interpretation of different uniform Lipschitz exponents.

\begin{tabular}{|l|l|}
\hline $\begin{array}{l}\text { Uniform Lipschitz } \\
\text { Exponent }\end{array}$ & Interpretation \\
\hline$\alpha>m$ & $f$ is $m$ times continuously differentiable in the neighbourhood of $v$. \\
\hline $0 \leq \alpha<1$ & $\begin{array}{l}p_{v}(t)=f(v) \text { and the Lipschitz condition of eq 3.55 becomes } \\
\forall t \in \mathbb{R}, \quad|f(t)-f(v)| \leq K|t-v|^{\alpha}\end{array}$ \\
\hline$\alpha<1$ & $f$ is not differentiable at $v$ and $\alpha$ characterises the singularity type. \\
\hline$\alpha=0$ & $f$ is bounded but discontinuous at $v$. \\
\hline \multicolumn{1}{|c|}{ Table 3.2: Various Lipschitz exponent values and their interpretation in terms of regularity. }
\end{tabular}

Now the Fourier transform is well suited to measuring the minimum global regularity of functions. But it cannot measure the local regularity of $f$ at a particular point $v$ from the decay of $|\hat{f}(\omega)|$ at high frequencies $\omega$. However, the wavelet transform yields a first relation between the differentiability of $\mathrm{f}$ and its wavelet transform decay at fine scale because wavelets are well localized in time.

\subsubsection{Vanishing Moments}

We define the $n$-th moment of a wavelet $\psi$ to be:

$$
M_{n}\{\psi\}=\int_{-\infty}^{+\infty} x^{n} \psi(t) d x
$$

If $M_{\mathrm{o}}\{\psi\}$ then $\psi$ is said to have one vanishing moment. All wavelets have at least one vanishing moment by their definition (eq. 3.5):

$$
M_{0}\{\psi\}=\int_{-\infty}^{+\infty} x^{0} \psi(x) d x=\int_{-\infty}^{+\infty} \psi(x) d x=0
$$

If $M_{\mathbf{0}}\{\psi\}=M_{\mathbf{1}}\{\psi\}=\mathbf{0}$, then $\psi$ has 2 vanishing moments. It then follows that for all $i=1,2, . ., n-1$ if all $M_{i}[\psi]=\mathbf{0}$ then $\psi$ has $n$ vanishing moments. It can also be shown that if $\psi$ has $n$ vanishing moments then it is orthogonal to any polynomial of degree less than $n$. 


$$
\left\langle a_{n-1} x^{n-1}+\cdots+a_{1} x+a_{0}, \psi\right\rangle=0
$$

We have seen the Lipschitz regularity equation approximate a function $f$ with a polynomial $p_{v}$ in the neighbourhood of some $v$.

$$
f(t)=p_{v}(t)+\varepsilon_{v}(t) \text { with }\left|\varepsilon_{v}(t)\right| \leq K|t-v|^{\alpha}
$$

By using a wavelet with the orthogonality property of Eq. 3.71, we may suppress the polynomial $p_{v}$ yielding an expression for the regularity $\alpha$. To do this let us use a wavelet that has $n>\alpha$ vanishing moments.

$$
\int_{-\infty}^{\infty} t^{k} \psi(t) d t=0 \text { for } 0 \leq k<n
$$

Eq. 3.71 states that a wavelet with $n$ vanishing moments is orthogonal to polynomials of degree $n-1$ and since $\alpha<n$, the polynomial $p_{v}$ has degree at most $n-1$. It follows,

$$
W p_{v}(u, s)=\int_{-\infty}^{\infty} p_{v}(t) \frac{1}{\sqrt{s}} \psi\left(\frac{t-u}{s}\right) d t=\left\langle p_{v}, \psi_{u, s}\right\rangle=0
$$

Furthermore since $f=p_{v}+e_{v}$ it follows,

$$
W f(u, s)=W \varepsilon_{v}(u, s)
$$

\subsubsection{Wavelet Transform Modulus Maxima}

The local Lipschitz regularity of a signal $f$ at $v$ depends on the decay at fine scales of $|W f(u, s)|$ in the neighbourhood of $v[32]$. However, it is not necessary to measure the decay directly in the time-space plane $(u, s)$. Mallat describes how the decay of $|W f(u, s)|$ may be controlled from its local maxima values.

The term modulus maxima describes any point $\left(u_{0}, s_{0}\right)$ such that $|W f(u, s)|$ is locally maximum at $u=u_{0}$. Therefore, it is implied that,

$$
\frac{\partial W f\left(u_{0}, s_{0}\right)}{\partial u}=0
$$

There is no local maxima when $|W f(u, s)|$ is constant because eq. 3.52 refers to a strict local maximum in either the right or left neighbourhood of $\mathrm{u}_{0}$. The term maxima line is 
used to describe any connected curve $s(u)$ in the scale-space plane $(u, s)$ along which all points are modulus maxima.

\subsubsection{Multiscale Differential Operator}

Mallat [5] describes the detection and measurement of singularities through the multiscale differential operator formalism. This operator predates wavelet theory but may be employed here to give a concise understanding of singularity detection. Multiscale transforms were developed for edge detection in computer vision theory and the wavelet transform is one such multiscale transform.

Take a smoothing function, any real function, $\theta(x)$ such that,

$$
\theta(x)=O\left(\frac{1}{1+x^{2}}\right)
$$

and whose integral is non-zero. This smoothing function may be thought of as the impulse response to a low pass filter. It turns out that where the smoothing function is a Gaussian function, some very meaningful results occur. The scalable smoothing function is then denoted as a Gaussian of the following form,

$$
\theta_{s}(x)=\frac{1}{s} \theta\left(\frac{x}{s}\right)
$$

Let $f(x)$ be any real function in $\boldsymbol{L}^{2}(\mathbf{R})$. When $f(x)$ is smoothed by $\theta_{s}(x)$, local sharp variation points of $f(x)$ may be detected through the wavelet transform as follows. Let $\psi^{l}(x)$ and $\psi^{2}(x)$ be the two wavelets defined by,

$$
\psi^{1}(x)=\frac{d \theta(x)}{d t} \text { and } \psi^{2}(x)=\frac{d^{2} \theta(x)}{d t^{2}}
$$

Next take the wavelet transform of $\mathrm{f}$ with respect to the above wavelets,

$$
\begin{aligned}
& W^{1} f(s, x)=f * \psi_{s}^{1}(x) \text { and } W^{2} f(s, x)=f * \psi_{s}^{2}(x) \\
& W^{1} f(s, x)=f *\left(s \frac{d \theta_{s}}{d t}\right)(x)=s \frac{d}{d x}\left(f * \theta_{s}\right)(x) \\
& W^{2} f(s, x)=f *\left(s^{2} \frac{d^{2} \theta_{s}}{d t^{2}}\right)(x)=s^{2} \frac{d^{2}}{d x^{2}}\left(f * \theta_{s}\right)(x)
\end{aligned}
$$


Hence the wavelet transforms $W^{l}$ and $W^{2}$ are proportional respectively to the first and second derivatives of $f(x)$ smoothed by $\theta_{s}(x)$. Fig. 3.9 gives a graphical representation of what this means. For a fixed scale $s$, by convolving the smoothing function with the function $f(x)$, singularity points in $f(x)$ now become points of inflection in the convolved function. The wavelet transform of this convolved function is given by its derivative using Eq. 3.81 and so points of inflection in the convolved function become local maxima in $W^{l}$. The wavelet transform of the second derivative of the smoothing function is then the second derivative of the convolved function and the points of inflection become zero crossings.

Singularities can be located by examining the wavelet transform local maxima of a signal where the wavelet is a derivative of a Gaussian or they may also be located by examining the zero crossings of the wavelet transform of a signal where the wavelet is a second derivative of a Gaussian function. In practical application, the former approach is advantageous because it may be used to discriminate sharp transition points and slow variation points. This is not possible with the latter approach. The reason for this is that the modulus maxima and modulus minima in $W^{l} f(s, x)$ correlate to sharp transition and slow transition points respectively. Hence, the two types of inflection points can be

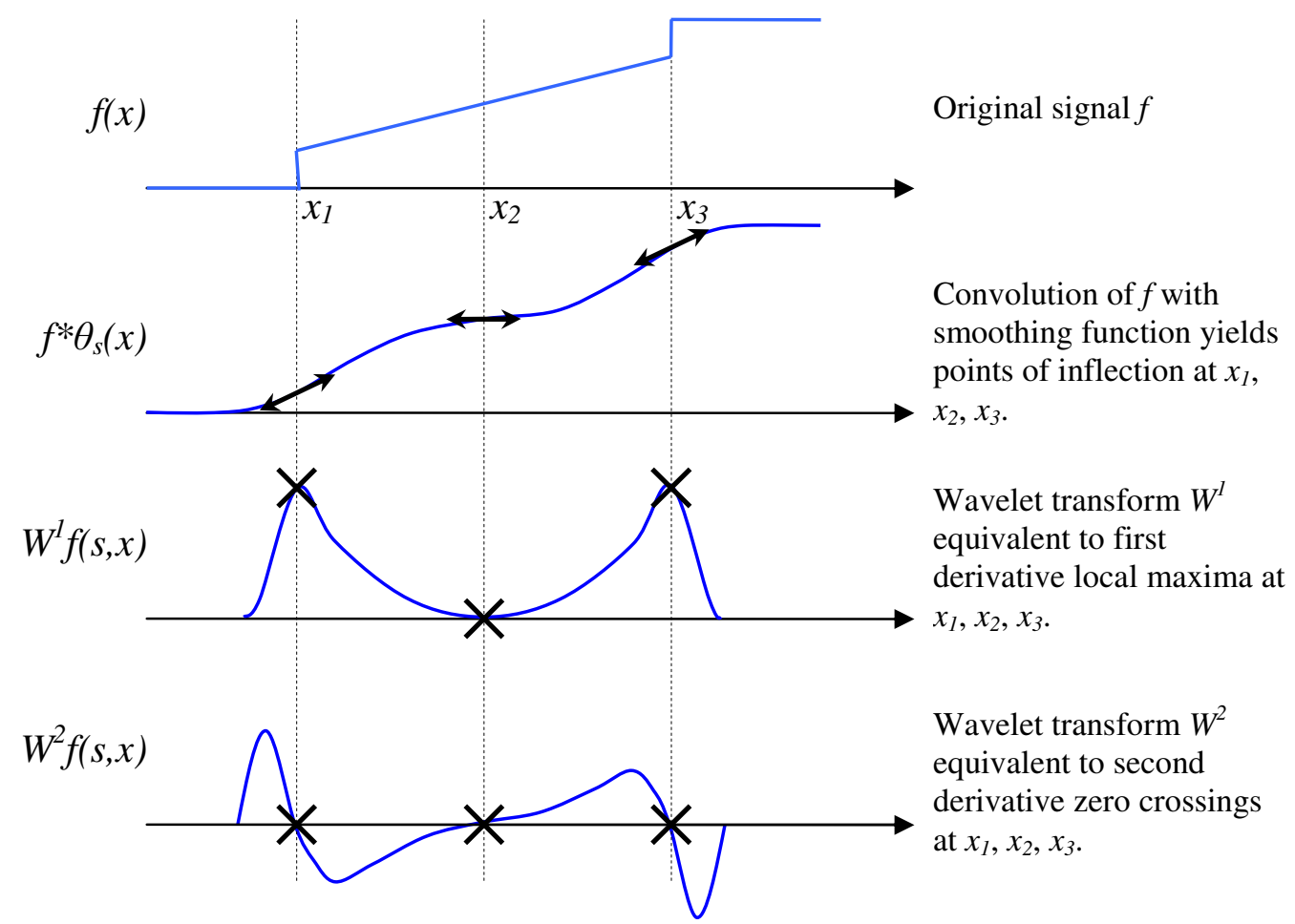

Fig. 3.9: Gaussian multiscale differential operator applied to a sample signal $f(x)$. [5] 
distinguished by examining the extrema of $\left|W^{1} f(s, x)\right|$ to see if they are maximal or minimal. This discrimination is not possible using the zero-crossings of $W^{2} f(s, x)$. From this, Mallet showed that the values of the modulus maxima often characterize the Lipschitz exponents of the signal irregularities.

\subsubsection{Wavelet Regularity}

The following theorem proved in [32] proves that a wavelet with $n$ vanishing moments can be written as an $n^{\text {th }}$ order derivative of a function $\theta$. It is supposed that $\psi$ has a fast decay which means that there exists $C_{m}$ where $m$ is any decay component and $m \in \mathbb{N}$, such that,

$\forall t \in \Re,|\psi(t)| \leq \frac{C_{m}}{1+|t|^{m}}$

Theorem: A wavelet $\psi$ with a fast decay has $n$ vanishing moments if and only if there exists $\theta$ with a fast decay such that,

$\psi(t)=(-1)^{n} \frac{d^{n} \theta(t)}{d t^{n}}$

Eq. 3.84

Consequently,

$$
W f(u, s)=s^{n} \frac{d^{n}}{d t^{n}}\left(f * \bar{\theta}_{s}\right)(u) \quad \text { with } \bar{\theta}_{s}(t)=\frac{1}{\sqrt{s}} \theta\left(\frac{-t}{s}\right)
$$

Moreover, $\psi$ has no more than $n$ vanishing moments if and only if $\int_{-\infty}^{+\infty} \theta(t) d t \neq 0$

\subsubsection{Wavelet Transform Modulus Maxima}

Mallat precisely defines what is meant by the local maxima of the wavelet transform modulus [32]. Let $W f(s, x)$ be the wavelet transform of a function $f(x)$,

We call local extremum any point $\left(s_{0}, x_{0}\right)$ such that $\frac{\partial W f\left(s_{0}, x\right)}{\partial x}$ has a zero-crossing at $x=x_{0}$, when $x$ varies. 
We call modulus maximum any point $\left(s_{0}, x_{0}\right)$ such that $\left|W f\left(s_{0}, x\right)\right|<\left|W f\left(s_{0}, x_{0}\right)\right|$ when $x_{0}$ belongs to either a right or a left neighbourhood of $x_{0}$, and $\left|W f\left(s_{0}, x\right)\right| \leq\left|W f\left(s_{0}, x_{0}\right)\right|$ when $x_{0}$ belongs to the other side of the neighbourhood of $x_{0}$.

We call maxima line, any connected curve in the scale space $(s, x)$ along which all points are modulus maxima.

The strict local maximum condition prevents recurrent series of zeros from being interpreted as a meaningful minimum.

\subsubsection{Reconstruction from Modulus Maxima Coefficients}

\subsubsection{Alternative Projection Algorithm}

Having identified that singularities can be characterised by their modulus maxima coefficients, it remains to investigate whether these maxima coefficients (both detail and approximation values) can be reconstructed back into the original signal. Once this is achieved then the modulus maxima coefficient representation becomes a very powerful tool for manipulating the signal as singularities may then be selectively chosen depending on the application required.

The dyadic wavelet transform $\left\{W f\left(u, 2^{j}\right)\right\}_{j \in Z}$ is a complete a stable representation which means that it has a bounded left inverse. The dyadic adaptive sampling technique produces a translation invariant representation of the signal. Mallet and Zhong developed the alternative projection algorithm to reconstruct a signal approximation from its wavelet maxima values [67]. Other approaches have been developed [68-70] but in all approaches exact reconstruction is not possible [71, 72]. However, the error in the reconstructed signal (relative mean-square error) is of the order of $1 \%$ and so the alternative projection algorithm gives a good representation of the original signal [68].

\subsubsection{Frame Perspective}

The most concise and quickest description of the alternative projection algorithm is using the frame perspective introduced in section 3.3.5.2 [32]. Frame coefficients are expressed as:

$$
U f[n]=\left\langle f, \phi_{n}\right\rangle
$$


and the pseudo inverse condition $\tilde{U}^{-1}=\left(U^{*} U\right)^{-1} U^{*}$ is put into,

$$
\begin{aligned}
& f=\tilde{U}^{-1} U f \\
& f=\left(U^{*} U\right)^{-1} U^{*} U f \\
& f=L^{-1} L f \\
& L f=U^{*} U f=\sum_{n \in \Gamma}\left\langle f, \phi_{n}\right\rangle \phi_{n}
\end{aligned}
$$

where $\boldsymbol{L}$ is a frame symmetrical operator. For each dyadic scale $2^{j}$, the positions of the local maxima $\left\{u_{j, p}\right\}_{p}$ for the wavelet transform $\left|W f\left(u, 2^{j}\right)\right|$ are known. Therefore we know the values of the wavelet transform,

$$
W f\left(u_{j, p}, 2^{j}\right)=\left\langle f, \psi_{j, p}\right\rangle \text { with } \psi_{j, p}(t)=\frac{1}{\sqrt{2^{j}}} \psi\left(\frac{t-u_{j, p}}{2^{j}}\right)
$$

Setting $\psi^{\prime}$ to be the derivative of $\psi$ then,

$$
\psi_{j, p}^{\prime}(t)=\frac{1}{\sqrt{2^{j}}} \psi^{\prime}\left(\frac{t-u_{j, p}}{2^{j}}\right)
$$

Now we know that $W f\left(u, 2^{j}\right)$ has a local extremum at $u=u_{j, p}$ (modulus maxima condition) then,

$$
\frac{\partial W f\left(u_{j, p}, 2^{j}\right)}{\partial u}=-\frac{1}{\sqrt{2^{j}}}\left\langle f, \psi_{j, p}^{\prime}\right\rangle=0
$$

The reconstructing algorithm must then recover a function $\tilde{f}$ such that

$$
W \tilde{f}\left(u_{j, p}, 2^{j}\right)=\left\langle\tilde{f}, \psi_{j, p}\right\rangle=\left\langle f, \psi_{j, p}\right\rangle
$$

and $\left\langle\tilde{f}, \psi_{j, p}^{\prime}\right\rangle=\left\langle f, \psi_{j, p}^{\prime}\right\rangle=0$

Eq. 3.68 imposes the condition that the derivative of $\tilde{W f}\left(u, 2^{j}\right)$ goes to zero at $u=u_{j, p}$. It turns out that it is sufficient for the norm $\|\tilde{f}\|$ must be minimized in order to meet the modulus maxima condition. The reconstruction algorithm recovers the function $\tilde{f}$ of minimum norm that satisfies Eq. 3.67 and Eq. 3.68 [32]. The signal $\tilde{f}$ of minimum 
norm that satisfies these equations is the orthogonal projection $P_{V} f$ of $f$ on the space $V$ generated by the wavelets $\left\{\psi_{j, p}, \psi_{j, p}^{\prime}\right)_{j, p}$. This is a basis (or redundant frame of $\boldsymbol{V}$ ) in discrete calculations because there are a finite known number of maxima. Expressing the symmetrical operator $\boldsymbol{L}$ as a function of this wavelet basis in $\boldsymbol{V}$ we get,

$$
\begin{aligned}
& \tilde{f}=L^{-1} L f=L^{-1} g \\
& g=L \tilde{f}=\sum_{j, p}\left(\left\langle\tilde{f}, \psi_{j, p}\right\rangle \psi_{j, p}+\left\langle\tilde{f}, \psi_{j, p}^{\prime}\right\rangle \psi_{j, p}^{\prime}\right) \\
& g=L \tilde{f}=\sum_{j, p}\left\langle f, \psi_{j, p}\right\rangle \psi_{j, p}
\end{aligned}
$$

\subsubsection{Conjugate Gradient Theorem}

A conjugate gradient algorithm may be employed to recover $\tilde{f}$ from the frame coefficients with a pseudo-inverse. The conjugate gradient algorithm computes $f=L^{-1} g$ with a gradient descent along orthogonal directions with respect to the norm induced by the symmetric operator $L$ [32]:

$$
\|f\|_{L}^{2}=\|L f\|^{2}
$$

Eq. 3.96

The conjugate gradient theorem was described by Gröchenig [73]. By setting the following initial conditions,

$$
\mathrm{f}_{0}=0, \mathrm{r}_{0}=\mathrm{p}_{0}=\mathrm{g}, \mathrm{p}_{-1}=0
$$

and defining the following functions for $n \geq 0$,

$$
\begin{aligned}
& \lambda_{n}=\frac{\left\langle r_{n}, p_{n}\right\rangle}{\left\langle p_{n}, L p_{n}\right\rangle} \\
& f_{n+1}=f_{n}+\lambda_{n} p_{n} \\
& r_{n+1}=r_{n}-\lambda_{n} L p_{n} \\
& p_{n+1}=L p_{n}-\frac{\left\langle L p_{n}, L p_{n}\right\rangle}{\left\langle p_{n}, L p_{n}\right\rangle} p_{n}-\frac{\left\langle L p_{n}, L p_{n-1}\right\rangle}{\left\langle p_{n-1}, L p_{n-1}\right\rangle} p_{n-1}
\end{aligned}
$$

and setting $\sigma=\frac{\sqrt{B}-\sqrt{A}}{\sqrt{B}+\sqrt{A}}$ then Gröchenig proved by induction that 


$$
\left\|f-f_{n}\right\|_{L} \leq \frac{2 \sigma^{n}}{1+\sigma^{2 n}}\|f\|_{L} \text { and hence } \tilde{f}=\lim _{n \rightarrow \infty} f_{n}=f
$$

This theorem recovers the approximation signal $\tilde{f}$ by an iterative process of inverting the frame symmetrical operator $\boldsymbol{L}$ with an exponential convergence. The minimisation of $\|\tilde{f}\|$ recovers a function such that $W \tilde{f}\left(u, 2^{j}\right)$ is nearly locally maximum at $u=u_{j, p}$ and fewer operations are needed with the reduced frame operator - for $n=10$ iterations a relative mean square error is of the order of $10^{-2}$ [32]. Because $\tilde{f} \neq f$, increasing the number of iterations does necessarily improve this error figure. Each iteration is implemented with the "algorithme a trous" requires $\mathrm{O}\left(\mathrm{Nlog}_{2} N\right)$ calculations.

\subsubsection{Fast Discrete Reconstruction}

Signal approximation may be recovered from its wavelet maxima representation using the alternate projection algorithm introduced by Mallet and Zhong [67]. Meyer [74] and Berman [75] have proved that exact reconstruction is not possible but numerical experiments that the relative mean-square error is of the order of $10^{-2}$.

The implementation of this algorithm is described from the frame perspective. At each $2^{j}$, the positions $\left\{u_{j, p}\right\}_{p}$ of the local maxima of $\left|W f\left(u, 2^{j}\right)\right|$ and the values,

$$
W f\left(u_{j, p}, z^{j}\right)=\left\langle f, \psi_{j, p}\right\rangle
$$

with $\psi_{j, p}(t)=\frac{1}{\sqrt{2}^{j}} \psi\left(\frac{t-u_{j, p}}{\mathbf{2}^{j}}\right)$

Letting $\psi^{\prime}$ be the derivative of $\psi$ and since $W f\left(u, 2^{j}\right)$ has a local extremum at $u=u_{j, p}$

$$
\frac{\partial W f\left(u_{j, p}, 2^{j}\right)}{\partial u}=-2^{-j}\left(f \cdot \psi_{j, p}^{\prime}\right)=0
$$

We wish to reconstruct a function $\tilde{f}$ that gives a good approximation of the original function $f$ such that,

$$
W f\left(u_{j, p}, 2^{j}\right)=\left(f, \psi_{j, p}\right)=\left(f \cdot \psi_{j, p}\right)
$$

and $\left\langle\tilde{f}, \psi_{j, p}^{\prime}\right\rangle=\left\langle f, \psi_{j, p}^{\prime}\right\rangle=0$

Eq. 5.105 imposes the condition that the derivative of $W f\left(u, 2^{j}\right)$ vanishes at $u=u_{j, p}$. This condition is further enforced by minimizing $\|\tilde{f}\|$. The signal $\tilde{f}$ of minimum norm 
that satisfies Eq. 5.104 and Eq. 5.105 is the orthogonal projection $P_{V} f$ of $f$ on the space $\mathrm{V}$ generated by the wavelets $\left\{\psi_{j, p}, \psi_{j, p}^{\prime}\right\}_{j, p}$. In discrete calculations the number of maxima is finite and $\left\{\psi_{j, p}, \psi_{j, p}^{\prime}\right\}_{j, p}$ is a finite family and so is a basis or a redundant frame of $\mathrm{V}$. The conjugate gradient theorem is applied to recover $\tilde{f}^{f}$ from the frame coefficients with a pseudo-inverse. About 10 iterations are usually sufficient to recover an approximation of $f$ with a small mean square error.

\subsection{PEAK DETECTION}

\subsubsection{Singularities}

The theory of singularities was developed mostly by Mallat and is employed to characterise a signal by identifying and pinpointing the areas of change in any given signal. Typical applications for this methodology include signal denoising where selectively choosing certain singularities and discarding others allows you separate noise from signal [76, 77]. Taken together, the remaining singularities can then be inverse wavelet transformed back to the original signal. The research presented here differs from such an approach by taking singularities (which may be used to locate areas of change) and categorizing them into groups that can be formed into peaks. Hence grouped sub sets of singularities form peaks. These peaks can then be inverse transformed into a separate time domain peak. This is the first stage of this research: to develop an automatic peak detection algorithm to accurately and repeatedly separate a time varying EEG signal into a set of peaks. Once this stage is reached, it is possible to analyse these separated peaks in ways which are discussed later.

The first step in this process is to characterise the evoked potential signals. The word 'characterise' can have many interpretations. In this case, we are asserting that information in the evoked potential is contained in the transitory parts of the signal. From visual examination, it is seen that both EEG and evoked potential signal are made up of overlapping peaks and so characterisation of this signal, means identification and location in time of all the signal variations. Singularities in the case of evoked potential are continuous smooth changes in potential. The modulus maxima discrete wavelet transform method yields a two-dimensional set of detail coefficients whose trend across scales is interpreted to identify the location of the singularities. Decisions are then made about the significance of these singularities (i.e. are they signal or noise?) and so a small 
set of detail coefficient values and their time locations completely characterise that particular singularity.

\subsubsection{Choosing a Wavelet}

In the case of singularity detection, the choice of wavelet is important and calculating the modulus maxima discrete wavelet transform of a signal yields singularities that are detected by observing a specific pattern in the transform result. Namely that as the scale parameter increases the modulus maxima increase along the maxima line. This pattern is dependent on the wavelet used in the transformation. We are using the quadratic spline wavelet implemented in a filter bank arrangement.

Let us take a quadratic spline wavelet, $\psi$ which has vanishing moment $n$, exactly equal to 1 and a compact support. There exists $\theta$ of compact support such that $\psi=(-1)^{n} \theta^{(n)}$ with

$\int_{-\infty}^{+\infty} \theta(t) d t \neq 0$. In this case $\theta$ is a Gaussian function and wavelet transform may be expressed as,

$$
W f(u, s)=s \frac{d}{d t}\left(f * \bar{\theta}_{s}\right)(u)
$$

Because $\psi$ has one vanishing moment, wavelet modulus maxima are the maxima of the first order derivative of $f$ smoothed by $\bar{\theta}_{s}$. Fig. 3.10 illustrates this process using a step function which is the simplest transition state in any signal. Hummel, Poggio and Yuille proved that for a Gaussian function, a modulus maxima $\left(x_{0}, u_{0}\right)$ belonging to a maxima line is guaranteed to propagate towards finer scales without any interruption.

So in calculating the Discrete Wavelet Transform where the wavelet is a quadratic spline, the output is a matrix of coefficients in the scale-time plane which characterise well the areas of change within the original signal. Fig. 3.12 illustrates just such a process for a sample signal. In real EEG signals, there is considerable more overlap and noise which interferes with signal. Hence it is very important to understand the mechanisms that define where the maxima are likely to occur. 


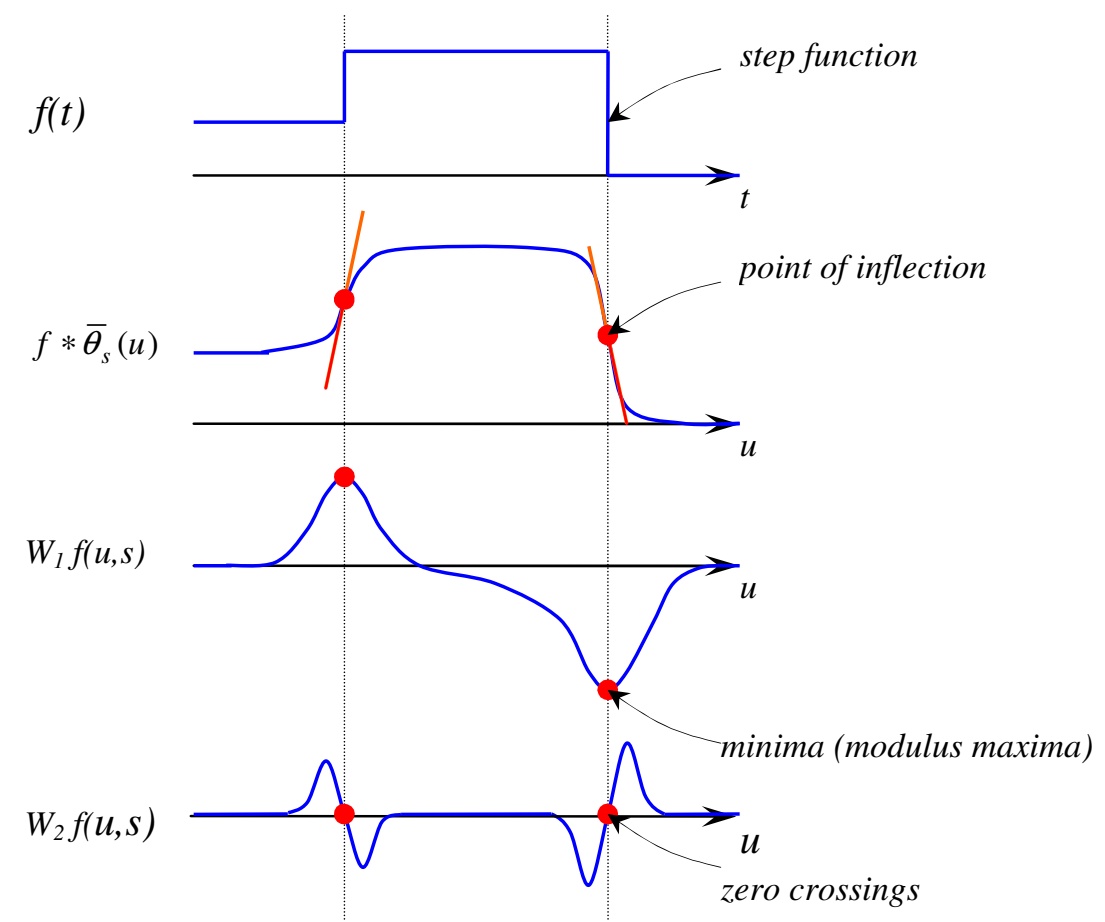

Fig. 3.10: Wavelet transform of a sample step function signal with a quadratic spline multiscale differential operator illustrating the relationship between wavelet transform coefficients and the Gaussian $(\theta)$ differentiation.

\subsubsection{Peaks}

This research further develops the 'discrete set of coefficients grouped per singularity'. Singularities characterise areas of large magnitude change in the signal over a short period i.e. points where there is a large slope. The peak of a signal may be thought of as (usually) a pair of singularities that record a simple shift away from and back to a particular baseline.

Note that at this early stage, no assumption is made that individual peaks have any significance per se. Rather that we are going to separate the signal into peaks and investigate the relation of peaks to each other in order to better understand the underlying mechanisms and try to identify particular correlations in the data.

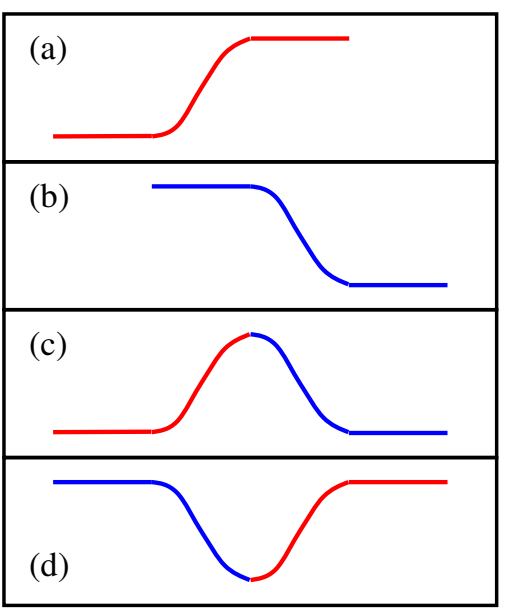

Fig. 3.11: examples of singularities and peaks, (a) positive singularity, (b) negative singularity, (c) a positive peak and (d) a negative peak. 
Arbitrarily but intuitively, we call a positive singularity those singularities that chart an increase in electric potential. It follows that we call a negative singularity those singularities that chart a decrease in electric potential.
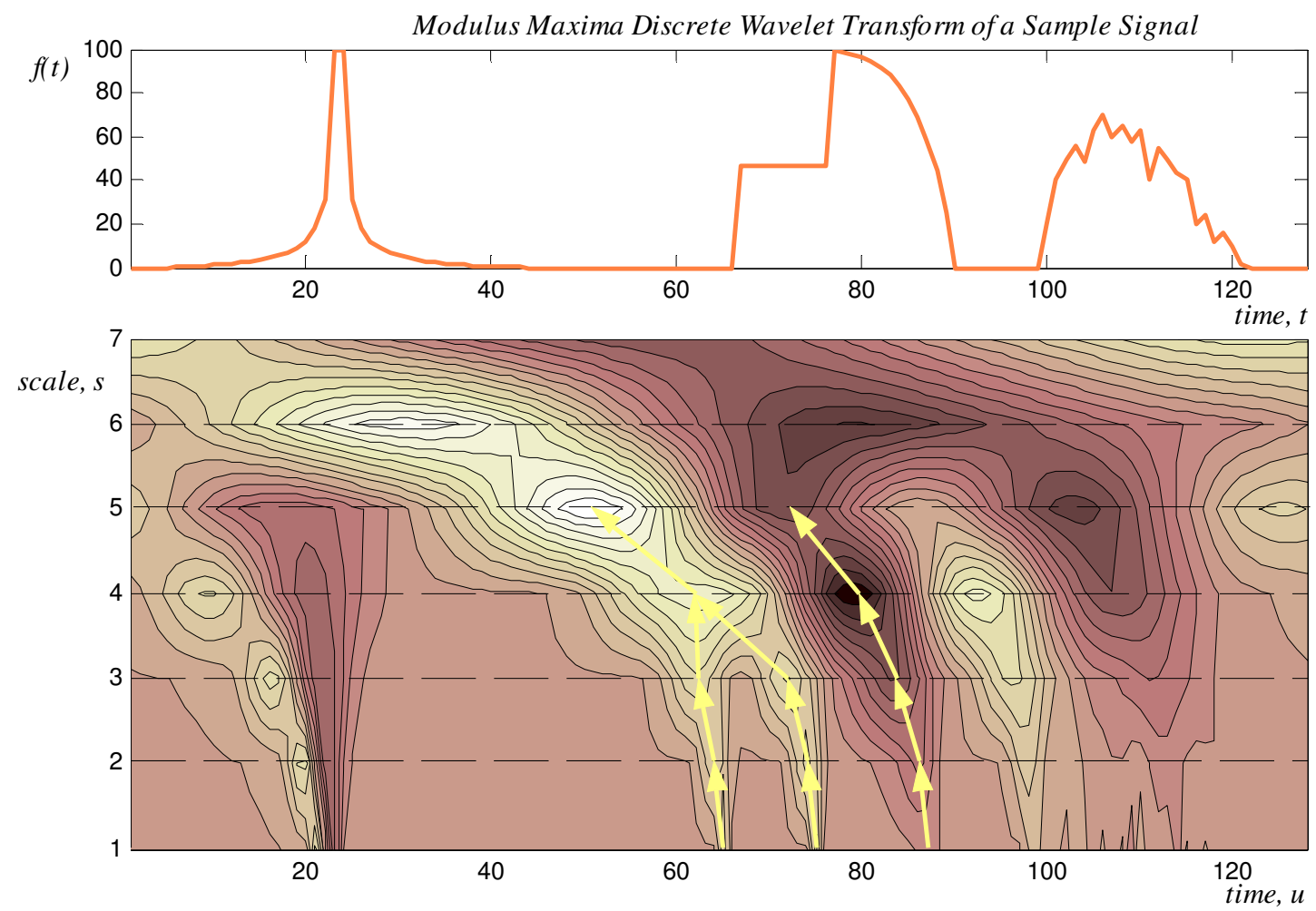

Fig. 3.12: The upper graph shows a sample signal and the lower graph illustrates its modulus maxima discrete wavelet transform. Coefficients are calculated for dyadic scale levels from 1 to 7 along the dotted lines. Positive singularities have positive coefficients (tending towards white) and negative singularities have negative coefficients (tending towards black). The yellow arrows which chart the maxima lines indicate how modulus maxima values propagate from one scale to the next. As the scale increases and becomes coarser, there is more interaction between adjacent singularities

It is apparent from examination of Fig. 3.11 and Fig. 3.12 that a positive singularity has positive modulus maxima coefficients and a negative singularity has negative modulus maxima coefficients. We describe a simple peak as being a combination of a positive and negative singularity together. The order of selection is important as a positive singularity followed by a negative singularity yield a positive peak while conversely, a negative singularity followed by a positive singularity yields a negative peak.

In both Fig. 3.12 it is possible to identify how singularities can be combined in pairs to form what we will call peaks. Essentially a positive set of maxima coefficients and a negative set of maxima coefficients together form a peak. 


\subsubsection{Zero Level}

Singularities, as defined here, are reference free in that they are not tied to the zero voltage line. Our data is tied to the zero line as the electroencephalograph data is given as reference between two specific points. In effect since we are not measuring an absolute voltage, the zero voltage line is not an absolute reference. It is a relative parameter by which all individual measurements are compared. The important point is that all measurements are made and remain referred to this single reference standard.

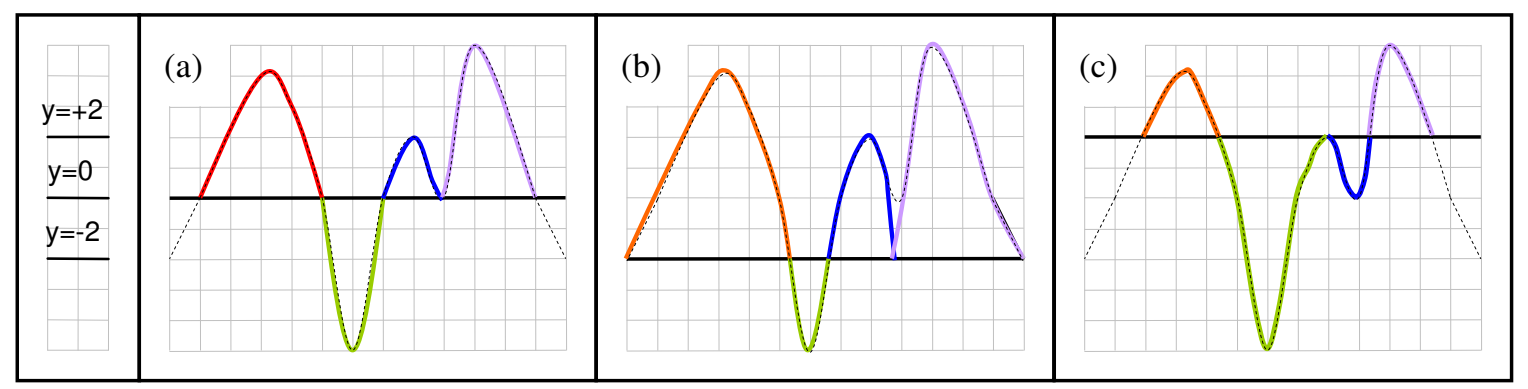

Fig. 3.13: The effect of different reference levels on the peak shape, size and location. Moving the reference level (y-axis), in most cases makes peaks bigger or smaller however, the blue peak in (b) has changed from a positive peak in (b) to a negative peak at a slightly different location in (c).

A particular difficulty with characterising peaks from a signal where the zero level is purely a reference level is how to deal with peaks that generally do not begin and finish on the zero line. Fig. 3.13 shows how the nature of peaks varies considerably with the position of the zero level. The approach taken in this research is that once pre-processing of the signal is complete the zero volt line will be the absolute reference point for all following calculations. For the most part, positive peaks shall then occur above this zero level and negative peaks shall occur below the zero volt level. There will be some exceptions to this rule as discussed in the next section.

\subsubsection{Overlapping Peaks}

Due to the nature of EEG and evoked potentials signal, there is an abundance of overlapping and interfering peaks which may have a non-unique separation solution. For example, a peak shape shown in Fig. 3.14(a) may be interpreted in two ways; either as a pair of adjacent positive peaks shown in Fig. 3.14(b) or as a single large peak with a negative smaller peak at its centre as illustrated in Fig. 3.14(c). Without any additional information such as a signal from an adjacent site, both cases are valid interpretations although obviously only one is accurate. Factors such as this must be borne in mind when analysis of peaks is performed. 

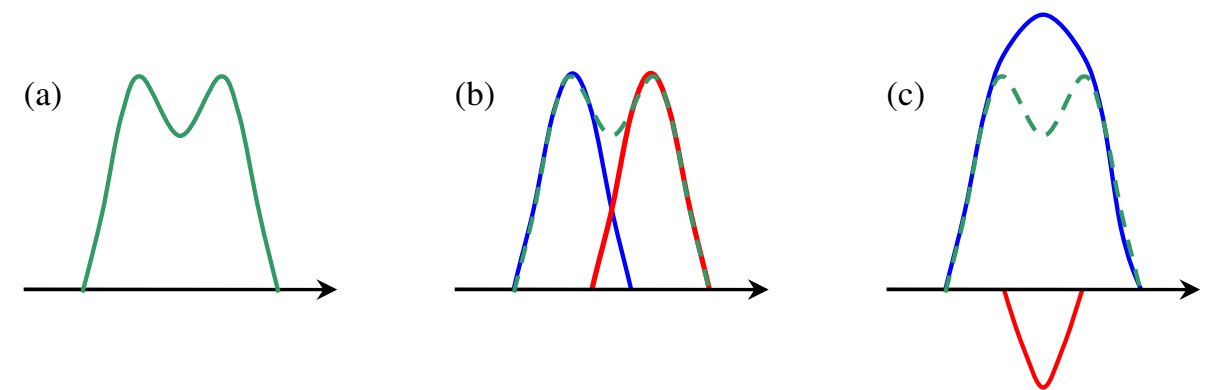

Fig. 3.14: Example of how a single feature from a signal (a) can be interpreted validly as two distinct types of peak (b) and (c). In (b) and (c) the distinct red and blue peaks add together to form the same green peak as in (a).

\subsubsection{Matching Singularities}

The process of identifying singularities is much simpler than matching them into pairs as a precursor to peak separation. The modulus maxima coefficients at low scales give a very good localised position for the singularity. However in order to fully characterise each singularity all maxima must be followed from low to high scales. The higher scale modulus maxima values by their nature interact with adjacent peaks and so the maxima value at higher scales may arise from more than one lower scale maxima line. These overlapping maxima lines must be separated in order to separate the peaks within the signal. In order to solve difficulties relating to separating maxima values at higher scales we shall employ the Lipschitz exponent method to characterise the peaks across scales and endeavour to characterise 'best fit' peaks.

\subsubsection{Automatic Peak Detection}

Since EEG and evoked potential signals yield a large amount of data, it is not feasible to manually perform all the calculations necessary to choose peaks. This process must be automated. Automation itself is a difficult process of encoding the rules, which may often appear simple to a human, into a repetitive and generic algorithm. The flow for the automatic peak detection algorithm is outline in Fig. 3.15. 


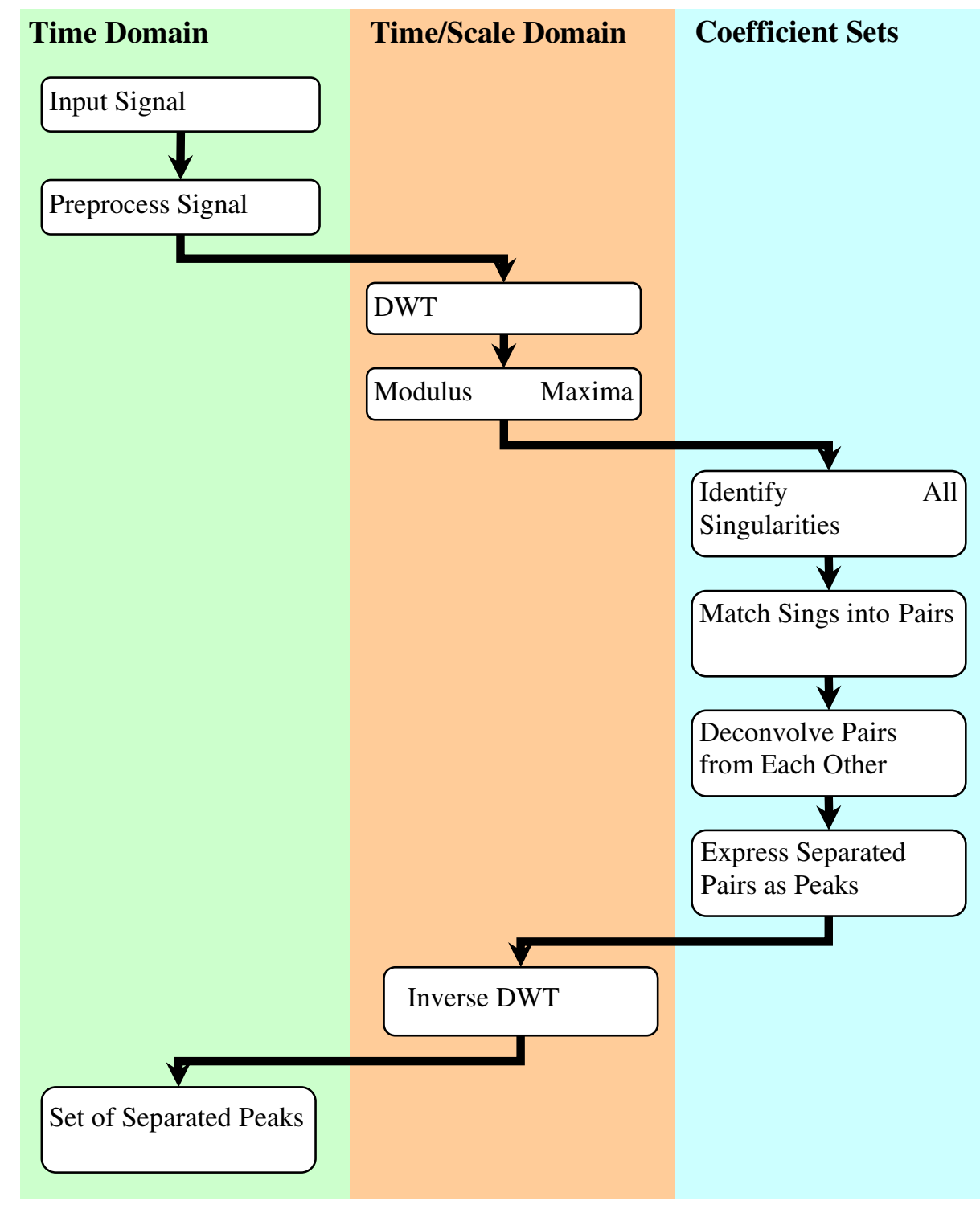

Fig. 3.15: Flowchart outlines steps required to take an input signal and separate it into a constituent set of peaks. Three state domains are identified in which processing occurs dependent on the relevant step. 


\subsection{PEAK ANALYSIS}

\subsubsection{Correlation Between Signals}

\subsubsection{Pearson's Correlation Coefficient}

In determining the performance of the peak detection algorithm it is important to consider ways of comparing signals analysed by different methods. On such method is the Pearson Correlation Coefficient (or Pearson's product moment correlation) named after English statistician Karl Pearson. It yields a measure of the least squares fitting between two sets of data. For two discrete functions, $x$ and $y$, with $N$ points each, the correlation coefficient, $r$, is defined as follows:

$$
r=\frac{\sum_{i=1}^{N}\left(x_{i}-x\right)\left(y_{i}-y\right)}{\sqrt{\sum_{i=1}^{N}\left(x_{i}-x\right)^{2} \sum_{i=1}^{N}\left(y_{i}-y\right)^{2}}}
$$

where $\bar{x}$ and $\bar{y}$ are the mean across all $N$ points. The range of values that can be taken on by $\mathrm{r}$ is from -1 to +1 , where +1 represents a perfect match and occurs only where $x=y$. The closer the fit between the two functions, the higher the correlation coefficient. In practise, correlation coefficients above 0.95 represent a very close match between the signals under comparison.

\subsubsection{Peak Reconstruction Comparisons}

Peak detection decomposes a signal into a set of logical discrete features. Several analysis techniques are employed to examine different aspects of the resultant decomposition. Firstly, we recombine the set of peaks and compare it to the original signal. It is important to compare these two signals as the set of peaks purports to accurately represent the original signal. Given an input EEG signal with T samples, $f(t)$, let $\left[g_{k}(t)\right]_{k=1, p}$ be the set of peak signals with $P$ peaks, which form the output of the peak detection algorithm described above. Then it follows that summing all $g_{k}(t)$ forms a representation of $f^{(t)}$ as follows,

$$
g(t)=\sum_{k=1}^{P} g_{k}(t)
$$

The original signal $f(t)$ and reconstructed signal $g(t)$ are then compared using the Pearson correlation coefficient of Eq. 3.106. It is expected that the primary observable 
difference between the two signals is a certain amount high frequency denoising caused by the selection of peaks that are deemed significant. Mallat's method of selecting modulus maxima coefficients that propagate to larger scales has the follow-on effect that those modulus maxima that do not propagate do not get selected. Higher frequency components within a signal will have this characteristic and so tend not to be selected. Hence, it is expected that the peak detected signal should resemble a denoised version of the original signal.

\subsubsection{Averaged Evoked Potential Error}

Next this process is extended across an entire set of averaged EEG data to compare the evoked potential signal based on the set of original data and compare it to the evoked potential signal based on the set of decomposed peak signals. For a set of $J$ EEG data epochs $\left.f_{i}(t)\right]_{i=1, J}$ the evoked potential response $f_{s p}$ is the average of the set of EEG epochs and $\theta_{s p}$ is the equivalent evoked potential response for the decomposed peak signal $\left\{g_{i, k}(t)\right\}_{i=1, J, k=1, P}$ as follows,

$$
f_{s p}=\frac{\sum_{t=1}^{J} f_{i}(t)}{J} \text { and } g_{s p}=\frac{\sum_{i=1}^{J} \sum_{k=1}^{P} f_{k, i}(t)}{J}
$$

Again we examine the Pearson correlation coefficient between these two evoked potential signals which should be as close to 1 as possible.

This process will at least confirm that evoked potential signal present in the original signal is still present in the dataset of extracted peaks. This analysis verifies that the information of the original signal is 'similar' to the information in the peak detected signal.

The averaging process has a tendency to cancel out spurious noise and high frequency components due to the random nature of such effects. Hence, it is expected that the averaged reconstructed peak signal will more closely approximate the averaged signal than was the case for individual epoch comparisons (3.6.1.2).

\subsubsection{Peak Characteristics}

Having extracted individual peaks, the challenge is now to analyse these peaks in a manner which allows the evoked potential components to be separated from the ongoing background EEG. In examining a typical peak, as in Fig. 3.16, it is clear that three independent properties may be identified that characterise a peak. These three properties 
are latency, width and height as labelled in Fig. 3.16. Peaks are also broken down into positive or negative peaks and analysis may be performed separately and then combined at the end. It is not possible to uniquely define all these parameters for all peaks without first performing the peak detection algorithm to separate peaks. Original EEG signal may well detect most latencies and heights as the peak zenith stands out from overlapping peaks but the width measure is immersed in overlapping adjacent peaks.

The latency parameter is a peak measure in the temporal domain. The width is an indirect peak measure of the scale or frequency domain. The set of peaks is taken not just across one evoked response, but over a set of

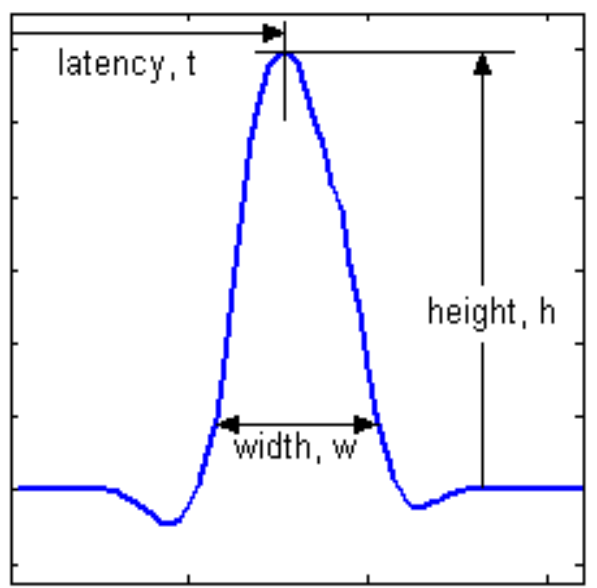

Fig. 3.16: Graphical illustration of the three main discriminant variables of latency, width (or scale) and height. evoked response epochs. Hence the epoch domain is utilised. Thus by developing an analysis technique that incorporates the data extracted from each peak and using all peaks, we attempt to gain insights in the temporal, spectral and epoch domains. This is a statistical classification problem and some approaches are described below,

\subsubsection{Peak Classifiers}

We have proposed in section 3.5 that EEG signals may be transformed into a discrete set of separable peaks. The data has been transformed in such a way that it can now be viewed from a new perspective. While these features can be viewed as a subset of the original signal, it is not appropriate to interpret them as the basic building block of EEG features. The peaks identified although separated as much as possible within the constraints of the data available, are still composites of the observed EEG activity and so any particular peak could still represent a summing of two or more smaller peaks coincident (or semi-coincident) in time. It is appropriate to search for similar repeated features within the dataset that may indicate a particular peak is indeed a direct evoked potential response instead of background EEG signal or some other composite of peaks.

Given that EEG data has been decomposed into separable peaks, we now look for novel ways to investigate the data. Let us use the parameters discussed in section 3.6.2 to classify each peak for each EEG signal of each epoch. Examining an individual peak we 
note the important information which succinctly describes each peak. The three characteristics which are most visually obvious are the time of peak event (latency), the magnitude of the peak event (height) and the breadth of peak event (width). Another measure which will be recorded is the Lipschitz regularity of both the rise and fall of the peak.

\subsubsection{Clustering}

Clustering is a classification technique where objects are partitioned into groups based on some similar trait. Often this trait is proximity. There are two types of clustering hierarchical or partitional. Hierarchical clustering iteratively uses previously defined clusters to determine further cluster refinement. Partitional clustering determines all the clusters at once. Partitional clustering is most appropriate for the type of clustering under investigation here. There are a number of partitional clustering types that we shall examine further.

\subsubsection{1 $\mathrm{K}$-means clustering}

A set number of clusters are chosen. These clusters are randomly located within the state space and the Euclidian distance from each object to the centroid of each cluster is calculated. The object is assigned to the nearest cluster centroid and this process is repeated for all objects in the set. The centroid is calculated as the average position of all point in the cluster. This process is repeated until predetermined convergence criteria are met. This method is quick and simple to implement, however it is not always repeatable as the result is dependent on the location of the initial random cluster location. A graphical example of k-means clustering is illustrated in Fig. 3.18.

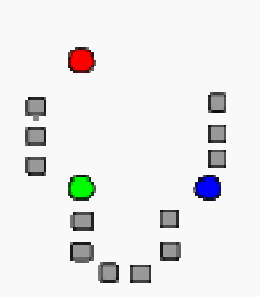

Shows the initial randomized centroids and a number of points.
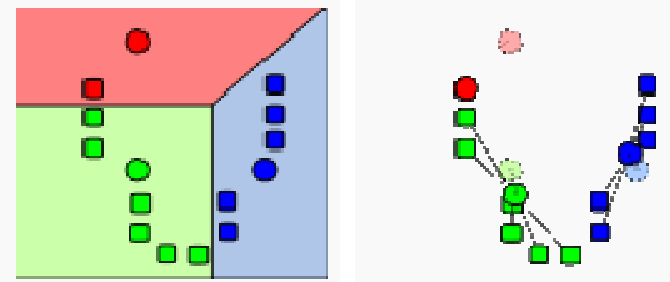

Points are associated Now the centroids are with the nearest moved to the center of until a suitable level of centroid.

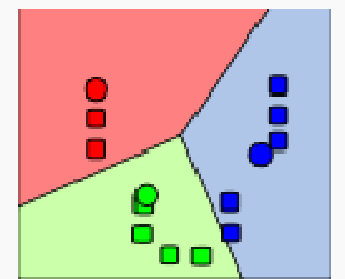

Steps $2 \& 3$ are repeated convergence has been reached. their

clusters.

Fig. 3.17: An example of k-means clustering. It is apparent that the clustering is somewhat dependent on the initial starting point. 


\subsubsection{Fuzzy c-means clustering}

Another problem with K-means clustering is that each object is uniquely assigned to a particular cluster even though it may be almost equidistant to another cluster. Fuzzy cmeans clustering overcomes this problem by assigning a measure of 'belonging' to each cluster determined by the Euclidian distance. However it is still yields different results for different cluster starting positions.

\subsubsection{QT Clustering}

Quality Threshold (QT) clustering is another variation of partitional clustering which was developed for gene clustering [78]. In QT clustering a maximum threshold is ascribed to the cluster. Thus points are added to a cluster until the cluster threshold exceeds the maximum threshold. This process is repeated for other points generating other clusters. The candidate cluster with the most points is then selected as the true cluster and all points within that cluster are removed from the set and the process is repeated to find the next highest concentration true cluster and so on. This method requires more computational power however, it does not require an initial number of clusters to be set and it is repeatable when re-run [1].

\subsubsection{Elbow Criteria.}

A general rule for determining the number of clusters to select is given by the elbow criteria. Typically the first few clusters will contain much more information that the latter clusters. The number of clusters chosen is determined by the point where adding additional clusters does not add significantly to the information gained. The clustering techniques described in this section do not maximise the output of the EEG results. Hence, two modified clustering techniques were investigated in this research. They are essentially a variation on QT clustering. The first technique is called 'block' clustering and the second technique is 'contour' clustering.

\subsubsection{Block Clustering}

Once the identified measures are calculated for each and every peak, it is possible to ascribe these parameters into a 3 dimensional matrix and then locate where peaks are clustered together. This process may be thought of as a refined averaging process. Since groups of clustered peaks with similar peak features may then be averaged together. By identifying groups of peaks, it is possible to identify which groups or clusters contribute 
most to the overall evoked potential signal and also gauge the repeatability of the evoked potential by the size of the cluster in these areas.

Data is separately scaled across each variable and grouped into bins. By careful scaling, unitary sized bins may then be used and graphically, a three dimensional grid of bins is formed. Visually, this may be observed in Fig. 3.18. Each peak contributes a single value to a single bin. The accumulation of peak parameters into bins facilitates the identification of localised higher concentrations of similar peaks.

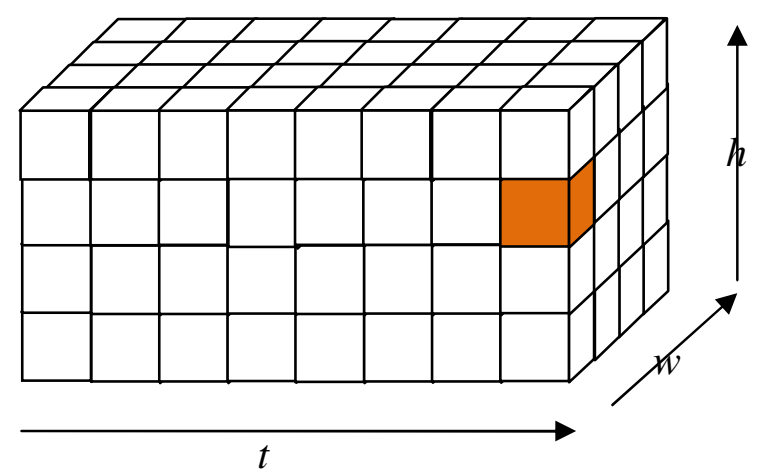

Fig. 3.18: A sample block cluster matrix. Peak variables are scaled over the range of boxes and each peak is assigned to a single cluster block (such as the orange coloured block). Once all peaks have been assigned the location of high areas of concentration indicate the presence of repeated peak shapes.

Block clustering has some drawbacks with regards to resolution. The amount of variation that discriminates peaks at the lower end of the scale (in width and height dimensions in particular) is much less that the amount of variation that discriminates peaks at the larger end. Non-linear scaling (in particular logarithmic scaling) can help this problem. Of more concern is the fact that neighbouring blocks may contain peaks that are very close to a particular block. These adjacent blocks do not contribute to the particular block and so a distortion of the concentration matrix is possible. This problem is similar to the 'belongedness' problem described for K-means clustering.

\subsubsection{Spread Clustering}

Spread clustering works on a slightly different premise. Again the variables are separately scaled but uniform matrix bins are not used; instead a 'spread space' is defined for each peak. This spread space defines the extent of the peak in each axis based on the parameter size. The centre of the spread space is directly located at the position of the scaled parameters. Smaller variables intrinsically will have a smaller spread while conversely large variables will have a larger spread. The entire spread space is 
incremented by 1 for each peak in that space. This method overcomes the border problems of block clustering as the spread space provides a broader border for identifying an exact area of highest concentration. The area of highest concentration is then found by locating the maximum value of the concentration matrix. Once this location is identified, the individual peaks that are contained in this maximal concentration location may be examined and averaged separately from the remaining dataset. The drawback with spread clustering is that once the highest concentration location is selected, the concentration matrix must be recalculated before the next highest peaks may be chosen. This is due to the fact that the peaks that contributed to the highest concentration must be removed as they may also contribute to other areas of the matrix and thus skew the results. However, this drawback only extends the computational requirement. It has the advantage that the result is independent of starting conditions and repeatable when re-run, similar to QT clustering.

By creating a 3-dimensional matrix covering the full range of values, it is possible to categorise each peak into a unique region of this matrix. This categorization is then applied for all peaks and areas of higher concentration are identified. Since in theory Evoked Potentials arise from the presence of repeated time-locked stimuli responses, such a mechanism should favour identification of evoked potential signals. However, this analysis technique is blind to the presence of (or lack of) evoked potentials since it does not discriminate between types of peak but simply categorizes these peaks into ascribed bins in the concentration matrix. This is an advantage of this technique as no apriori conditions regarding the expected response are necessary.

\subsubsection{Average Cluster}

In typical background EEG, certain peak shapes occur more often than others. This generates a typical non-uniform distribution of peak quantities across all height and width bins. The latency exhibits a truly stochastic distribution but height and width exhibit a characteristic profile for background EEG. We may subtract this 2-dimensional average width and height profile from all time slices. We wish to identify the time-locked peaks so in order to separate background EEG from time-locked Evoked Potential signals, the mean across all time bins for each width and height bin is calculated and subtracted across each time bin location. The first true cluster is identified by choosing the location that has the highest concentration of peak. The location of this true cluster in terms of 
latency, height and width is recorded together with the specific peaks that form the cluster. Then these peaks are removed from the dataset and concentration matrix is recalculated and the next highest cluster maximum identified. The process is repeated until the maxima concentration reaches zero. This yields a concentration profile for bin locations in terms of number peaks present above the mean expected number of peaks preset. Since the mean expected concentration levels for each width and height position have been subtracted from the concentration matrix, any concentration locations above zero represent locations of peaks that occur more than expected. Conversely, any concentration levels below zero occur less than expected. We wish to focus on the former to identify those peaks that occur most often.

\subsubsection{Spatial Discrimination}

Our initial analysis techniques have so far been in two real dimensions - time space and frequency or scale space. The wavelet transform was chosen for its superior performance in these domains. As mentioned previously, however, there is not enough information in these two domains to fully describe the EEG activity. Other sources of information must be added. One technique used, which has already been discussed is averaging where multiple repeated samples are taken to highlight the required response at the expense of background noise signals. In this section another approach is mentioned where the response signal of adjacent EEG sites may be analysed and compared to each other. In this case we are looking to identify signals that do not originate at the EEG site under investigation.

This method is beyond the scope of this research, but is discussed as an area of future research and is the next logical progression for the methods developed during this research. Up to now, information from temporal, spectral and epoch domains have been utilized. These methods yield an output which fits in the 'Epoch Decomposition' segment of the Edwards Venn diagram shown in Fig. 2.10. Adding spatial discriminations would shift the analysis into the full decomposition box shown in Fig. 2.10 which would be the only identified technique retaining an output perspective in all four identified domains.

An array of EEG sites surrounding the site of interest is measured during experimentation. The same evoked potential data is measured for all sites which may 
also be analysed individually; however we now discuss techniques that can relate the peaks together in novel ways.

In this analysis method, we are essentially trying to identify only the EEG response which we believe emanates from directly under the site of interest. Peaks which propagate from other sites but extend under the site of interest are removed. This leaves us with a clearer picture of the brain response at the site of interest only and not taking in a larger area. It is hoped that this can show a focussing effect or sharpening effect of the EEG. One of the difficulties of EEG is that the skull has a smearing effect of the EEG; here we are investigating a technique which may improve the EEG resolution greatly.

\subsection{SUMMARY}

In this chapter, the various theoretical aspects involved in this research are described. They may be summarized as follows:

- Wavelets, wavelet transform, splines and the inverse wavelet transform are introduced.

- Arising from this wavelet background is the theory of modulus maxima discrete wavelet transform and singularity detection described by Mallat and presented as the basis from which the this research is built upon.

- The concept of peaks with EEG signals is described and a correspondence between peaks and pairs of singularities is drawn.

- An outline for an algorithm to decompose Evoked Potential data into sets of separated peaks is described.

- Independent peak characteristics are identified that may be used to compare and contrast different peaks.

- Some clustering techniques are described and two clustering techniques more honed to the problem at hand are described.

- An outline for an algorithm to cluster peaks into meaningful similar groups is described. 


\section{CHAPTER 4 MATERIAL AND METHODS}

\subsection{OVERVIEW}

7 his chapter is broken up into two sections - Experimental Methods and Analytical Methods. The Experimental section is further split into three areas.

Lirstly, the area of investigation and preparation is described - the early phase of the research where different techniques were investigated and a picture of the challenges involved was established and also describes the necessary preparatory steps that had to be put in place before formal testing could begin. Next the formal experimental design is described including a description of the experimental test procedure and the parameters and setting. Finally, in the last experimental methods section, the outcome of the experiments is described.

In the analytical methods sections the process by which the measured data was analysed is described in detail.

\subsection{EXPERIMENTAL METHODS}

\subsubsection{Investigation and Preparation}

In this research, it was important to follow a number of preliminary steps in order correctly focus the research in the right direction. These steps have had a major effect on the outcomes of the research and are described herein.

\subsubsection{Choice of Evoked Potential Type}

There are three main types of evoked potential signal which may be measured under normal laboratory conditions. These are:

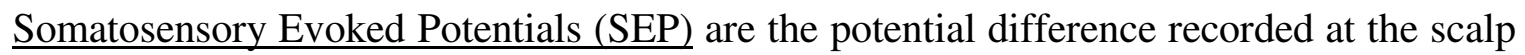
above the somatosensory cortex due to stimulation of the peripheral nerves.

Visual Evoked Potentials (VEP) are the potential difference recorded at the occipital region of the scalp in response to visual stimuli. There are two commonly used types of VEP: flash VEP is recorded in response to a flash of light presented to the eye and pattern reversal VEP (PRVEP) is recorded in response to an alternating black and white checkerboard pattern display presented to the subject. 
Auditory Evoked Potentials (AEP) are the potential difference recorded at scalp above the auditory cortex due to the stimulation of the ear by auditory signals such as clicks, tones or white noise.

All three were investigated to determine which would be most appropriate for this research. In choosing a particular type of Evoked Potential, the location of the expected response was important. It was preferred that the primary evoked potential generators should occur at or near the surface of the scalp and not deep within the folds of cortex. The reason for this is that spatial discrimination is expected to be simpler for generators on the surface as opposed to those deeper within the cortex. Auditory Evoked Potentials were quite quickly eliminated from investigation. The primary auditory cortex is located in the superior temporal lobe and is largely hidden within the lateral fissure. Investigation was undertaken to examine the suitability of Somatosensory Evoked Potentials to the research goals. Preliminary experiments were designed and carried out to measure Somatosensory Evoked Potential. Here a small electrical pulse was repeatedly applied at the wrist and the response was measured at the scalp above the somatosensory cortex area. However, the magnitude of the evoked potential response when averaged was particularly small and so Visual Evoked Potentials were then investigated.

Both types of VEP signal - pattern reversal and flash - give larger potential difference amplitudes relative to the AEP and SEP signal, which is an advantage. In clinical terms, the pattern reversal Visual Evoked Potential is more useful. It has less inter-individual and intra-individual variability [79]. In a clinical setting, it is usually a specific latency which is of most interest and pattern reversal VEP provide both a more precise and repeatable estimate of latency. However, in this research, we are more interested in the peak components of evoked potentials and as such the flash VEP was chosen for further detailed examination. The main reason for this is that the Flash VEP is known to be made up of 6 overlapping response peaks which interact with each other. This overlapping and interaction provides a fertile ground for examination of the techniques proposed herein.

\subsubsection{Equipment Selection}

The university had a choice of two EEG systems - the Biopac system and the Mindset MS-1000 system. The Biopac system had the advantage of having a specific evoked potential module which easily integrated with the EEG modules to tie the stimulus and 
response in time. However, the system at the university only had four EEG channels which were considered insufficient for the purposes of this research. The Mindset system was a full 16-channel EEG system designed for clinical application. It had no evoked potential channel and so one of the 16 channels was used to detect the stimulus to allow exact time correlation between stimulus and response. Hence the Mindset MS-1000 system was selected for use in this research.

The detection of the stimulus signal required careful design of a photosensor circuit that could detect a fraction of the stroboscope light without interfering with other EEG channels. The final design consisted of a photodiode in parallel with a $2.2 \mathrm{k} \Omega$ resistor connected directly across the mindset terminals with as short a length as possible. During experiments, the Mindset equipment was orientated in relation to the stroboscope to ensure that the short-lead photodiode would detect the stroboscope flash. Testing was undertaken to ensure that the stroboscopic flash was accurately detected by the photodiode arrangement and that there was no interference on any other electrodes.

\subsubsection{Bipolar or Referential Measurement}

EEG measurements are given as a potential difference between two different electrodes. There are generally two different montage types that may be chosen - bipolar and
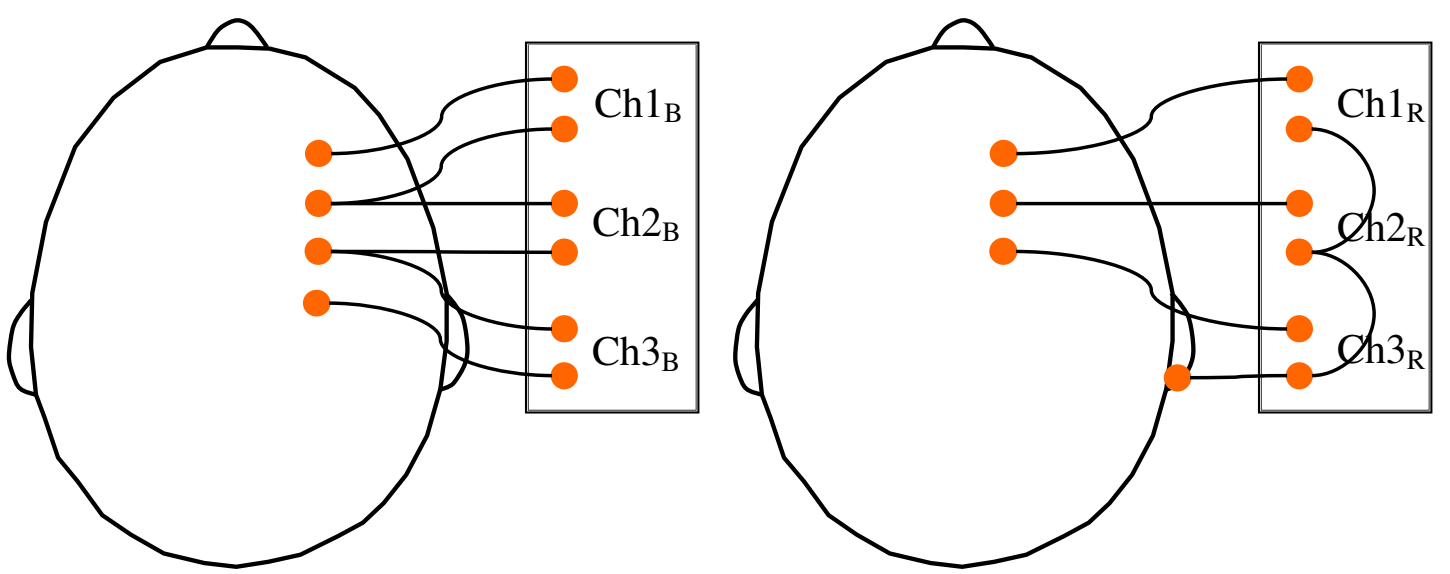

Fig. 4.1: Comparison of Bipolar (left) and Referential (right) montage arrangements. Bipolar measurements may be interpreted from the referential measurements by calculating the difference between referential sites. Therefore, $\mathrm{Ch}_{\mathrm{B}}=\mathrm{Ch}_{\mathrm{R}}-\mathrm{Ch} 2_{\mathrm{R}}$.

referential montages. Fig. 4.1 illustrates examples of both a bipolar and referential montage. Here it is seen that the bipolar montage measures potential difference between adjacent electrode pairs, while the referential montage has a single reference electrode, usually further away, to which all other electrodes are measured against. The referential montage system has the advantage of having a single referential to which all other sites may be compared. This allows each of these other sites to be compared to each other 
more directly and hence it is easier to visualize. The bipolar montage gives a more local representation of electrical activity and is more immune to distant EEG signal as the circuit loop is intrinsically smaller. In our experiments, referential measurements were taken with all electrode sites references to the linked-ears. The benefit of this method is that the bipolar measurements may be calculated by a simple subtraction between two measurements from different sites.

\subsubsection{Cap Design}

The standard head cap design for EEG is based on the 10-20 system formalised by the American Electroencephalographic Society guidelines [79]. The montage is a general arrangement of electrodes proportional to individual heads based on a roughly equispaced arrangement. Traditionally, the amount of effort and minor discomfort required to attach each electrode together with the computing complexities of analysing large amounts of channel data have limited the number of electrodes used. More recent simplification in electrode application procedures and increased computing power has made high-resolution EEG analysis more attainable.

However, for this research, our interest is really on a small localised area of the brain. A standard alternative montage for Pattern Reversal Visual Evoked Potentials is known as the Queen Square System:

"In the Queen Square System, the electrodes are labelled and positioned as follows:

MO Midoccipital, in midline $5 \mathrm{~cm}$ above inion

LO and RO Lateral occipital, $5 \mathrm{~cm}$ to the left and right of MO

MF Midfrontal, in midline $12 \mathrm{~cm}$ above nasion

A1/A2 At ear or mastoid, left and right

Ground At vertex" [79]

For flash VEP this scheme is modified since the Midfrontal reference is more likely to be contaminated with electroretinographic (ERG) activity.

"A simple four-channel recording montage would be:

Channel 1: Left occipital to reference: LO-Reference.

Channel 2: $\quad$ Midoccipital to reference: $\quad M O-$ Reference.

Channel 3: Right occipital to reference: RO-Reference.

Channel 4: Vertex to reference: V-Reference.

The reference can be single or linked ears/mastoids." [79] 
This standard was chosen as the basic measurement scheme although it does not measure sufficient electrode sites to perform a spatial analysis between adjacent electrodes. Hence four additional electrodes spaced $2.5 \mathrm{~cm}$ from $\mathrm{MO}$ position were also measured. Two of these were lateral exactly halfway between MO and LO and also between MO and RO. The other two electrodes were taken $2.5 \mathrm{~cm}$ from $\mathrm{MO}$ in the superior and inferior direction.

In most montage schemes such as the 10-20 system, the inter-electrode distance is variable and based on the overall skull size. The modified Queen Square system is preferable in our case since the inter-electrode distance is fixed at $2.5 \mathrm{~cm}$. This distance is important as it has been shown that this electrode distance is sufficiently large to detect different EEG events but not too large for adjacent electrode measurements to be unrelated [6].

Once the montage was described the next step involved examining how to apply this montage to subjects under experimental conditions. Investigations with a standard adjustable headcap proved awkward and tedious. Also it was difficult to locate all sites at both the correct separation and alignment. So work was undertaken to try to design something more apt for the purpose. Various alternative schemes evolved from this process but an illustration of the final design is shown in Fig. 4.2.

The framework for this headcap is a series of square (and triangular) rubber spacers. Each electrode space has a precise carved niche for the electrode to sit together with a hole drilled through to the back of the spacer. This hole provided access for electrode gel to be carefully injected through space and onto electrode-scalp surface without disturbing the position of the electrode.

The spacers are cut precisely to ensure the inter-electrode distance is $2.5 \mathrm{~cm}$ between horizontal and vertical electrodes. The scheme is connected together by elastic rope threaded through the spacers horizontally. There are also two elastic ropes holding the spacers in the vertical direction. The horizontal ropes extend from the spacers at each end and are attached to plastic O-ring on either side which are each attached to a Velcro strip. Each electrode is fitted into its electrode spacer and the cap is fitted to the midsagittal occipital region of the scalp with electrodes at the scalp surface. The Velcro strips are wrapped around the head over the ears and join each other comfortably on the forehead. 


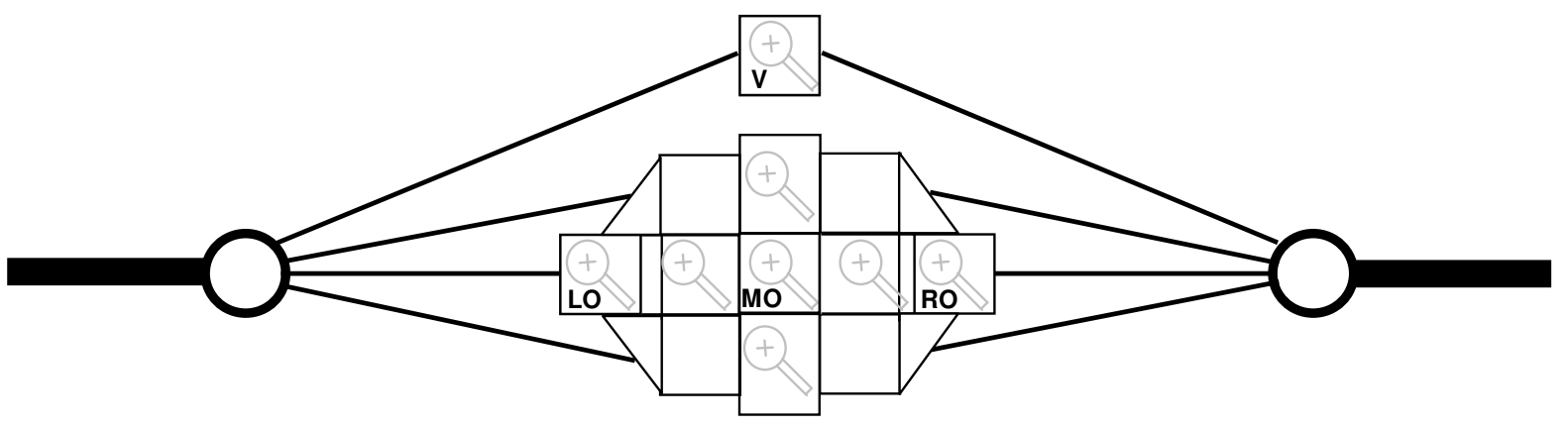

Fig. 4.2: Finalised Headcap design. The combination of rubber spacers and elastic ties is flexible enough to provide a contoured fit to the head yet retains the important $2.5 \mathrm{~cm}$ separation between electrodes in both horizontal and vertical directions. In the Test Procedure it is referred to as the VEP Custom Cap.

\subsubsection{Ethics Approval}

At RMIT University, all research involving human subjects must apply to the RMIT Human Research and Ethics Committee (HREC) for approval prior to any subject testing commencing. Two applications were submitted during the course of this research. The first submission in 2002 requested approval for the performance of Somatosensory Evoked Potential testing on a number of subjects. This application received HREC approval. The second submission occurred in 2004 and requested approval for the performance of Visual Evoked Potential testing on a number of subjects. This application also received HREC approval. All concerns and risks were highlighted within the applications and the committee was satisfied with the safeguards that were put in place. EEG is a passive electronics system where electrical potentials are being measured and so inherently quite safe. However, as with any electrical system, safeguards for equipment malfunction were put in place. The noted risk with the first submission pertained to the use of an electrical stimulator which applying a short repeated electrical pulse to the subjects wrist. A standard stimulator designed for this purpose was used and all safety precautions as per manufacturer's instructions were followed. For the second submission, which involved flash light stimulation presented to the eye, the flash rates used were much lower than those that might induce photic epilepsy [80].

\subsubsection{Faraday Cage}

The location of the Biomedical Laboratory at RMIT University is in a large multi-storey building with lots of standard electrical fittings and PC's operating at any one time. This was noted as a noisy background and $50 \mathrm{~Hz}$ noise was significant. RMIT do have an electrically quiet chamber. This chamber while not an anechoic chamber did provide significantly less background noise. The drawback with this solution was that all 
electrical equipment would have to be located outside the chamber which would require longer electrode leads which was not preferable. Instead, it was decided, for this and other research projects, to design and build a Faraday cage to be located in the Biomedical Laboratory. The cage dimensions were approximately $3.5 \mathrm{~m} \times 2 \mathrm{~m} \times 2 \mathrm{~m}$ allowing enough room for a subject to be seated or horizontal on a bed. The cage had a square gap size of $2 \mathrm{~cm}$ and when grounded prevented interference signals up to the high kilohertz range from penetrating its surface. Measurement equipment was located on the outside of the cage close to its surface. This included both the stroboscope and EEG device and detector. The only items located in the cage were the subject and cap and electrodes and chair.

\subsection{EXPERIMENTAL DESIGN}

\subsubsection{Experimental Aim}

Having completed the preliminary investigations, it was necessary to decide on the exact experiment that was to be performed and the exact data that was to be measured. The main aim of the experiments was to perform a standard Flash Visual Evoked Potentials measurement on a number of subjects using the American Clinical Neurophysiological Society guidelines [79]. The specific guideline most appropriate to this experiment was Guideline 9B Visual Evoked Potentials. This ensured that our experimental procedure would be both comparable and repeatable. Furthermore some additional electrode sites were measured which would allow future analysis of the spatial interrelations between sets of decomposed evoked potential peaks.

\subsubsection{Experimental Parameters}

Table 4.1 outlines all specific parameters within the experimental regime, their setting for the purposes of the experiments herein and where appropriate the rationale for the setting.

\begin{tabular}{|l|l|l|}
\hline 01 & Description & Setting \\
\hline \multirow{2}{*}{01} & Light Source Selection & Stroboscope \\
\cline { 2 - 3 } & $\begin{array}{l}\text { Rationale: The choices for a light source for Flash VEP are a Ganzfeld } \\
\text { photostimulator in the form of a stroboscope was available and adequate for the } \\
\text { purposes of the experiment. The stroboscope produces brief flashes of light via a } \\
\text { discharging Xenon light tube. The discharge rate was controllable. To stimulate } \\
\text { the entire visual field a Ganzfeld stimulator would be required. This produces truly }\end{array}$ \\
\hline
\end{tabular}




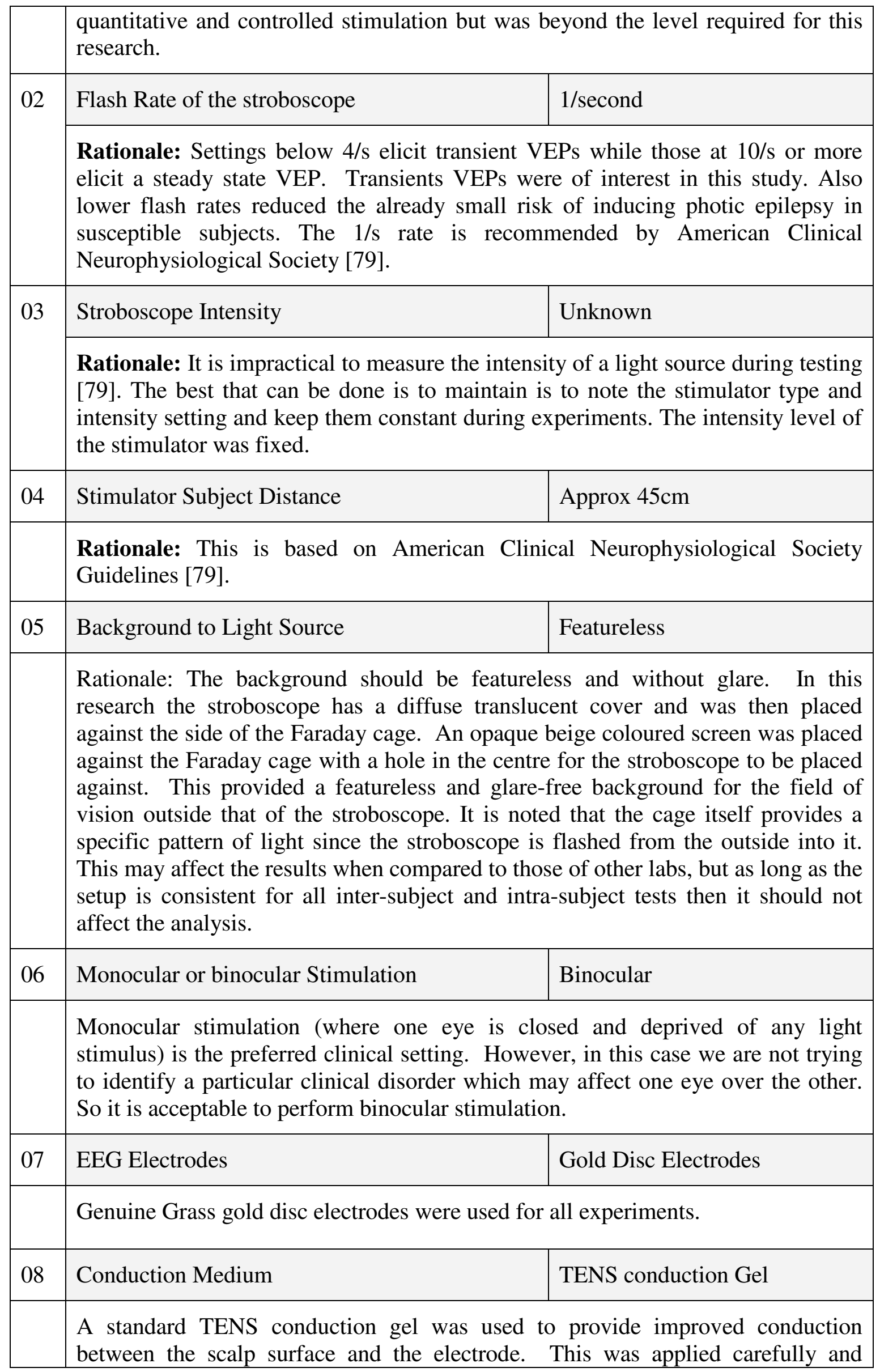




\begin{tabular}{|l|l|l|}
\hline & \multicolumn{3}{|l|}{$\begin{array}{l}\text { sparingly to prevent spreading which might electrically link adjacent electrode } \\
\text { should a conductive path be formed between electrodes by the gel. }\end{array}$} \\
\hline 09 & EEG Settings & MindMeld File 8CHEP.em2 \\
\hline \multirow{4}{*}{$\begin{array}{l}\text { The MindSet EEG system comes with software called MindMeld which performs } \\
\text { the capture and some basic analysis functions. This software was used for all data } \\
\text { collection. Within this program the parameters for data collection are set and then } \\
\text { stored in a file known as a 'scheme'. The scheme parameters are defined below } \\
\text { and were stored as a scheme file named } 8 \text { CHEP.em2. This scheme was used in all } \\
\text { formal testing. }\end{array}$} \\
\cline { 2 - 3 } & Host Adaptor & 0 \\
\cline { 2 - 3 } & SCSI ID & 1 \\
\cline { 2 - 3 } & Sampling Rate & $512 \mathrm{~Hz}$ \\
\cline { 2 - 3 } & Block Size & $512 \mathrm{Bytes}$ \\
\hline
\end{tabular}




\subsection{EXPERIMENTAL PROCEDURE}

The next step involved writing a test procedure to be followed before, during and after all testing. This test procedure consisted of an ordered checklist of all steps that had to be followed to successfully complete a formal VEP test. A blank copy of the checklist used is attached as Appendix A. This checklist went through a number of iterations and revisions before the final checklist was ready. The following comments refer directly to each section of the Test Procedure in Appendix A and provide a deeper understanding of the methodology.

\subsubsection{A. Equipment List}

This section details a complete inventory required to perform the experiments and includes a general layout illustration for information purposes.

Measuring tape is required to ensure that the subject is placed $45 \mathrm{~cm}$ from the stroboscope. This measurement is recommended in Guideline 9B of the American Clinical Neurophysiology Society Guidelines [79].

Ear plugs are used because the stroboscope was noted to give a very low audible click with each flash. This could have induced an auditory evoked potential time locked to the stimulus which may have interfered with the results. The click was inaudible with use of the 'putty-like' earplugs.

\subsubsection{B. Equipment Setup}

This section details the setup required to prepare the laboratory and equipment prior to performing the experiments. The checklist format ensured that there were no variances in setup from one subject to the next which might affect the consistency of the results.

The stroboscope, Mindset EEG equipment and light detector are all tested and operations prior to any formal testing.

Sections A and B were performed prior to the participants arrival to avoid subject fatigue.

\subsubsection{Participant Preparations}

This section prepares the subject for the experiment. Firstly, the subject was made comfortable and the project was discussed in general terms and also the specifics of what 
would happen during testing. The plain language statement and consent form were coread and understood and the latter signed by both parties.

A sequential participant number incremental from P01, P02 and so on, was assigned and written onto both the consent form and the Test Procedure. No recording of the subjects name or identity was recorded on the Test Procedure to ensure privacy and anonymity.

The steps for attaching the electrodes were then carefully followed and the subject was seated in the Faraday cage. Figure 4 of the Test Procedure was used for guidance of the location of the test sites. Figure 3 illustrates the electrode location of the 10/20 System (in black) and the Queen Square system (in red).

The Electrode cables are fed through the Faraday cage as a plaited bundle to reduce the chance of differential voltage pickup and then plugged into Mindset using Table 1 and Figure 3 of Test Procedure for guidance. The seven recorded EEG channels are arranged in a referential montage where the reference electrode is the average at the left and right earlobe.

\subsubsection{Experimental Procedure}

Before taking formal test results, it was important to confirm a good contact with each of the electrodes to the scalp. This was performed using Mindset by observing the frequency spectrum of each electrode and in particular the $50 \mathrm{~Hz}$ pickup. It is known that if there is a high input impedance at the electrode-scalp interface, there will be more $50 \mathrm{~Hz}$ pickup so by observing the continuous FFT spectrum, electrodes with large $50 \mathrm{~Hz}$ component were reattached to the scalp either by careful abrasion or additional gel to improve the contact.

The formal testing now begins and 3 sets of $2 \frac{1}{2}$ minutes of evoked potentials data were taken for each subject. Where time permitted some additional tests were understand with some minor variations in setup such as eyes open and eyes shut.

\subsubsection{E. Record of Data. Settings and Results}

The records of actual tests and details of each test are recorded on this log sheet. The filenames for each test are logged together with observations, comments and any additional tests or deviations from the Test Procedure. The data files themselves are collects on computer using these filenames and stored for later analysis. 


\subsection{METHODS}

Ostensibly this research examines an existing method Averaged Evoked Potentials and looks to add to the understanding within this modality. Hence, it is not necessary to test large number of participants in order to prove the results. The results need to be proved over a number of participants to show that it will work generically; however, each dataset may be compared to the average evoked potential with that dataset. Also multiple datasets may be examined from within a single participant. Hence, final formal testing was performed on three subjects with a minimum of three repeated tests on each subject.

\subsection{ANALYTICAL METHODS}

This section describes the processing that was applied to the datasets measured during experiments. The analytical process is split into three distinct stages. The first describes the pre-processing or data conversion stage of analysis, the second describes the peak detection algorithms in detail and the third describes the peak analysis algorithms. Each stage may be run separately or all together. The output of each stage is a file containing all the variables that form the input of the next stage in the chain.

All analyses were performed using the Mathworks Matlab software suite Release 12.1. Fig. 4.3 illustrates an overview of the main modules that make up the analysis techniques that have been developed during the research. Detailed flowcharts are described in Appendix $\mathrm{C}$ and full source code listings are provided in Appendix D and also on the attached CD.

\subsubsection{Data Conversion}

The purpose of the Data Conversion stage is to take the raw EEG files that are produced by the Mindset EEG amplifier during formal experiments and convert them into a set of discrete Evoked Potential epochs that are filtered and ready to be used in the next processing stage - the Peak Detection algorithm. 


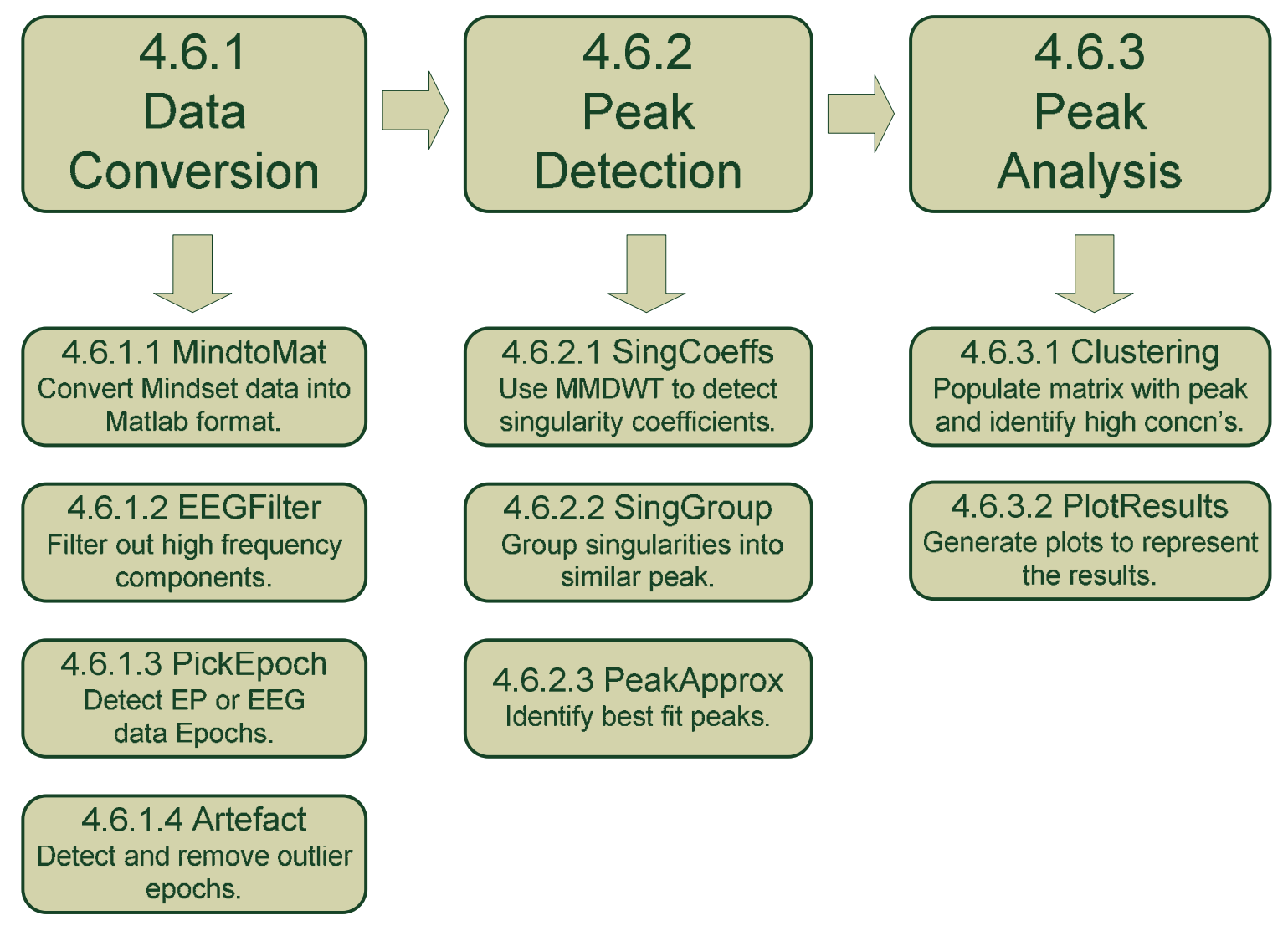

Fig. 4.3: Data analysis flowchart summary: The three major software modules of Data Conversion, Peak Detection and Peak Analysis are broken down into their major constituent components.

The step performed in this module are converting from Mindset to Matlab format, filtering out higher frequency components, select epochs based on stimulus channel timing, remove partial peaks at the beginning and end of each epoch and finally removing epochs that are overly contaminated with extraneous artefact. A graphical representation of what is happening to the input signal is provided in Fig. 4.4. An estimation of the information content required to encode the signal at each stage is included. 


\subsubsection{Peak Detection Method}

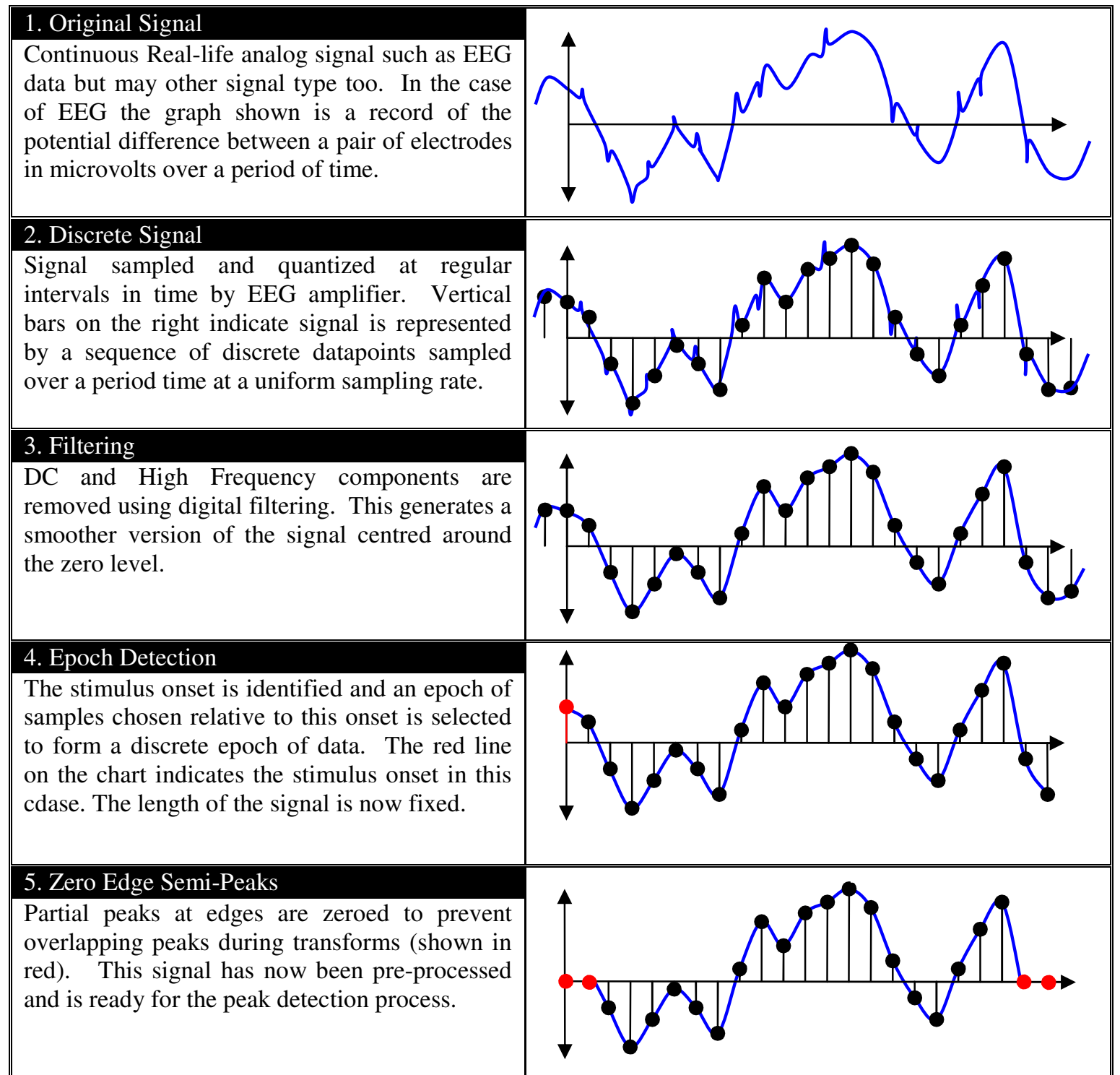

Fig. 4.4: Summary of the effect of Data Conversion processing of EEG data.

\subsubsection{Mindset EEG Format to Matlab File Format}

The output of each test during the experimental phase was a raw EEG file recorded over 8 channels with channels 1 to 7 recording the specific EEG are particular sites and channel 8 recording the timing of the stimulus detection. Mindset data is recorded in a proprietary binary format. There is no automatic way to import this data into Matlab. Instead, the Mindset software developer was contacted and he passed on the byte format for the EEG files. I then wrote a Matlab program to read these bytes and assign suitable variables to represent the EEG and parameters that are contained in the file. This program automates the import process taking a filename.BIN file as its input and outputting a set of variables representing all the salient EEG parameters to a filename.MAT file. This file format is 
the standard date format for Matlab. The parameters and their format as imported into Matlab are listed in Table 4.2.

\begin{tabular}{|c|c|c|c|}
\hline Description of variable & Name & Matrix Dimensions & Unit \\
\hline Number of samples taken & totsamples & $1 \times 1$ & - \\
\hline Time scale ( $\mathrm{x}$-axis) in seconds & time & $\begin{array}{c}1 \\
\text { totsamples }\end{array}$ & $\mathrm{s}$ \\
\hline $\begin{array}{l}\text { Number of channels of } \\
\text { recorded }\end{array}$ & channels & $1 \times 1$ & - \\
\hline $\begin{array}{l}\text { EEG data (y-axis) from each } \\
\text { channel }\end{array}$ & EEG & $\begin{array}{l}\text { channels } \\
\text { totsamples }\end{array}$ & $\mu \mathrm{V}$ \\
\hline $\begin{array}{l}\text { Number of samples taken per } \\
\text { second }\end{array}$ & samplerate & $1 \times 1$ & $\mathrm{~Hz}$ \\
\hline $\begin{array}{l}\text { Date stamp of when recording } \\
\text { began }\end{array}$ & starttime & $8 \times 1$ & $\begin{array}{l}\text { (1) Year 2Month } \\
\text { 3Day 4Date } \\
\text { 5Hour 6Min }\end{array}$ \\
\hline $\begin{array}{l}\text { Date stamp of when recording } \\
\text { finished. }\end{array}$ & stoptime & $8 \times 1$ & 7 $\mathrm{Sec} \mathbf{8} \mathrm{mS}$ \\
\hline
\end{tabular}

Table 4.2: List of parameters extracted from raw EEG file and imported into Matlab for further analysis.

\subsubsection{Filtering}

The first signal processing stage consisted of filtering out both DC components and high frequency components. The American Encephalographic Society recommendations for general EEG were followed:

"For Standard recordings, the low frequency filter should be no higher than $1 \mathrm{~Hz}(-3 \mathrm{db})$ corresponding to a time constant of at least $0.16 \mathrm{~s}$. The high frequency filter should be no lower than $70 \mathrm{~Hz}(-3 \mathrm{db})$." [79]

High frequency filtering is performed first. A Chebyshev Type II digital low-pass filter was applied to the full channel record set. The filter has order 9 and a cut-off determined by a $-3 \mathrm{~dB}$ fall-off a $70 \mathrm{~Hz}$. The Chebyshev Type II filter has no ripple in the passband (below $70 \mathrm{~Hz}$ in this case) and is equiripple in the stopband. The upper graph of Fig. 4.5 illustrates the frequency response of this filter as implemented by the Matlab cheby2.m command.

The phase variation which is apparent in the passband (lower graph in Fig. 4.5) was cancelled out by applying the filter to the channel data in both the forward and backward direction yielding a net phase variation of 0 degrees. Because this process can cause edge effects, the filtering was applied prior to the EEG signal being split into sets of discrete epochs. 
Frequency Response Curve for Chebyshev Type II Filter (order $\mathrm{n}=9$ and cut-off frequency at $-3 \mathrm{~dB}=70 \mathrm{~Hz}$ )
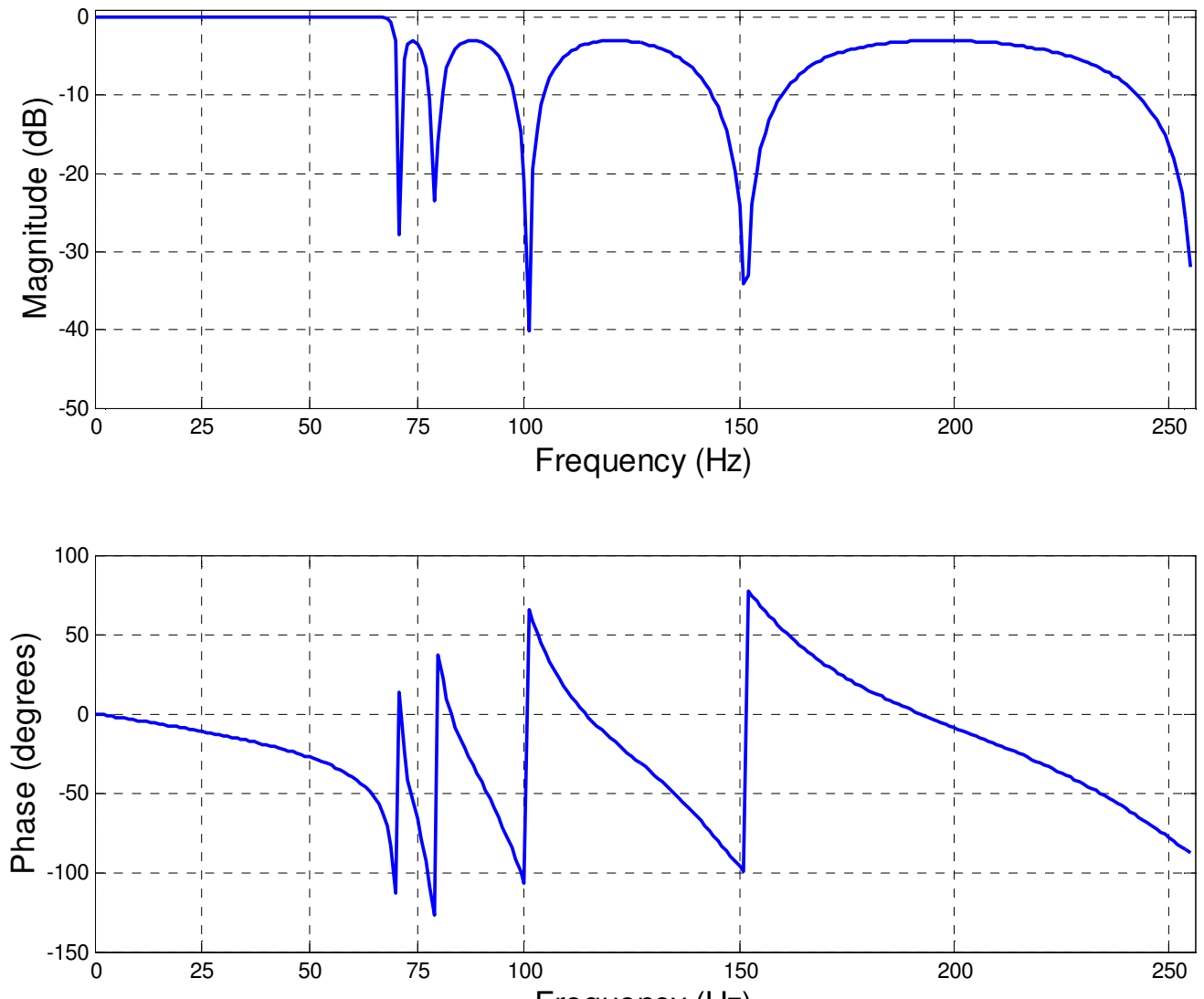

Fig. 4.5: Frequency response curve for Chebyshev Type 2 Filter. This lowpass filter was used to eliminate frequency components above $70 \mathrm{~Hz}$. The phase distortion was dealt with by processing the EEG signal with this filter in the forward and backward direction. This yields a net phase shift of zero degrees.

Next DC filtering was performed. It was observed in experiments that the magnitude of the DC component was small and so rather than applying a digital filter which would attenuates low frequencies above DC; it was decided to calculate the mean of each channel and offset the entire channel by this mean amount. The overall mean of each channel is therefore $0 \mathrm{mV}$.

\subsubsection{Pick Epoch}

The next step was to select epochs of data based on user defined criteria. Epochs are chosen in two ways. Firstly, they may be linked to a stimulus channel trigger. All Visual Evoked Potential data is extracted in this way. The algorithm scans the stimulus channel and a characteristic stimulus response is identified from which the stimulus onset is calculated. This time location is the stimulus onset time and the epoch is extracted relative to this position. Typical extraction settings might request $100 \mathrm{~ms}$ pre-stimulus 
followed by $400 \mathrm{~ms}$ post-stimulus yielding a 500ms epoch where the stimulus occurs after $100 \mathrm{~ms}$. This process is repeated across the entire stimulus channel dataset to extract all epochs associated with stimuli.

A second epoch extraction process has also been developed. This allows for a set of epochs to be extracted from an ongoing EEG dataset. This is used to give a representative sample of background EEG without any evoked potentials stimuli or response present. This is required (and explained further) in the Peak Analysis section

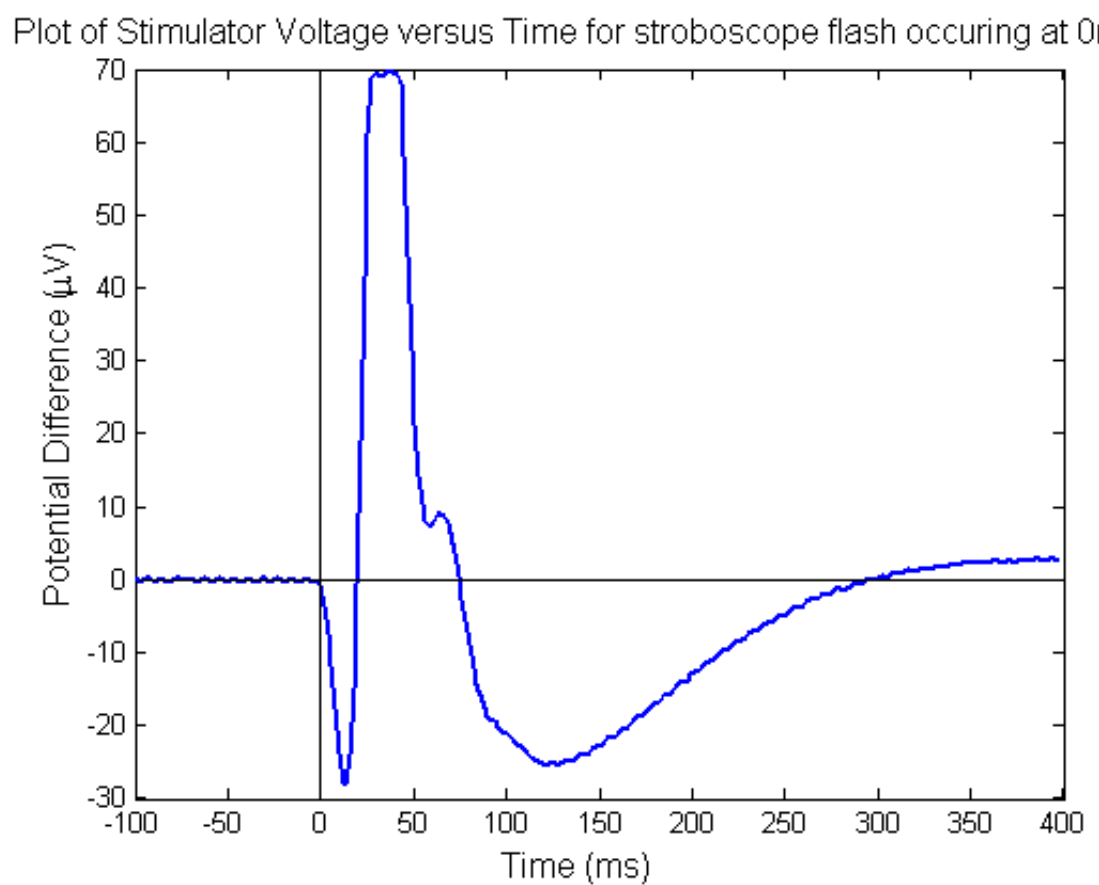

Fig. 4.6: Plot of the observed potential difference recorded by EEG device connected to photodiode circuit when detecting a stroboscope flash occurring at $0 \mathrm{~ms}$.

(Section 0 ) later in this chapter. Typically a 500ms epoch would be extracted at a random position within each 1 second period of the EEG data.

These two extraction processes yielded identically formatted epoch data that formed the input to the peak detection algorithm. The only difference being that one reflects the evoked potentials (temporally triggered) and the other discrete epochs of EEG data (temporally untriggered).

In the Visual Evoked Potential experiments, channel 8 was assigned as the stimulus channel. The photodiode response is illustrated in Fig. 4.6. The waveform response shows saturation and a slow discharge of photodiode voltage. The EEG amplifier amplifies voltages in the order of micro volts and so the photodiode response to the flash reaches channel limits very quickly. The shape of the waveform is less important than the 
onset time of the waveform. The onset time is taken as the position marked 0ms in Fig. 4.6.

This first section describes the pre-processing stage of analysis where Evoked Potential data was manipulated into a form that could be analysed in Matlab. There were several stages in this process which are outlined below.

\subsubsection{4 $\underline{\text { Artefact }}$}

It is common in EEG recording to occasionally pick up spurious signals not caused by real EEG but by some external condition. Such signal interference may be caused my muscle movement, eye movement or elsewhere and is known as signal artefact. Typically these signals are much larger than normal EEG and so are relatively easy to identify. Epochs of data contaminated with this artefact noise were removed for datasets prior to analysis. The EEG was assumed to have a normal distribution about some mean value close or equal to zero. The standard deviation for the EEG data gives a measure of the spread of this distribution around its mean. Normal EEG data would fit into the distribution which is spread up to 3 times the standard deviation around the mean. Occasional EEG outliers might lie outside this limit. However any datapoints in the distribution located beyond 6 standard deviations from the mean were considered artefact and data epochs with these artefact points were removed from the study. A record was kept of how many artefacts were removed as too high a number of artefacts may indicate a problem with the experimental setup.

The last step in the signal pre-processing stage consisted of removing partial peaks from the beginning and end of each epoch. The Peak Detection algorithm detects singularities and major changes in signal. It is apparent that at the beginning and end of each signal, there will be an edge. Typically this will be a disjoint break in the signal and hence will generate a large singularity. Since such peaks will be incomplete and more importantly outside the area of interest for the signal response they may be deleted prior to beginning processing. This is achieved by identifying the peak that is both complete and nearest the edge and setting all datapoints from here to the edge to zero. An example of this process is illustrated in Fig. 4.7. 


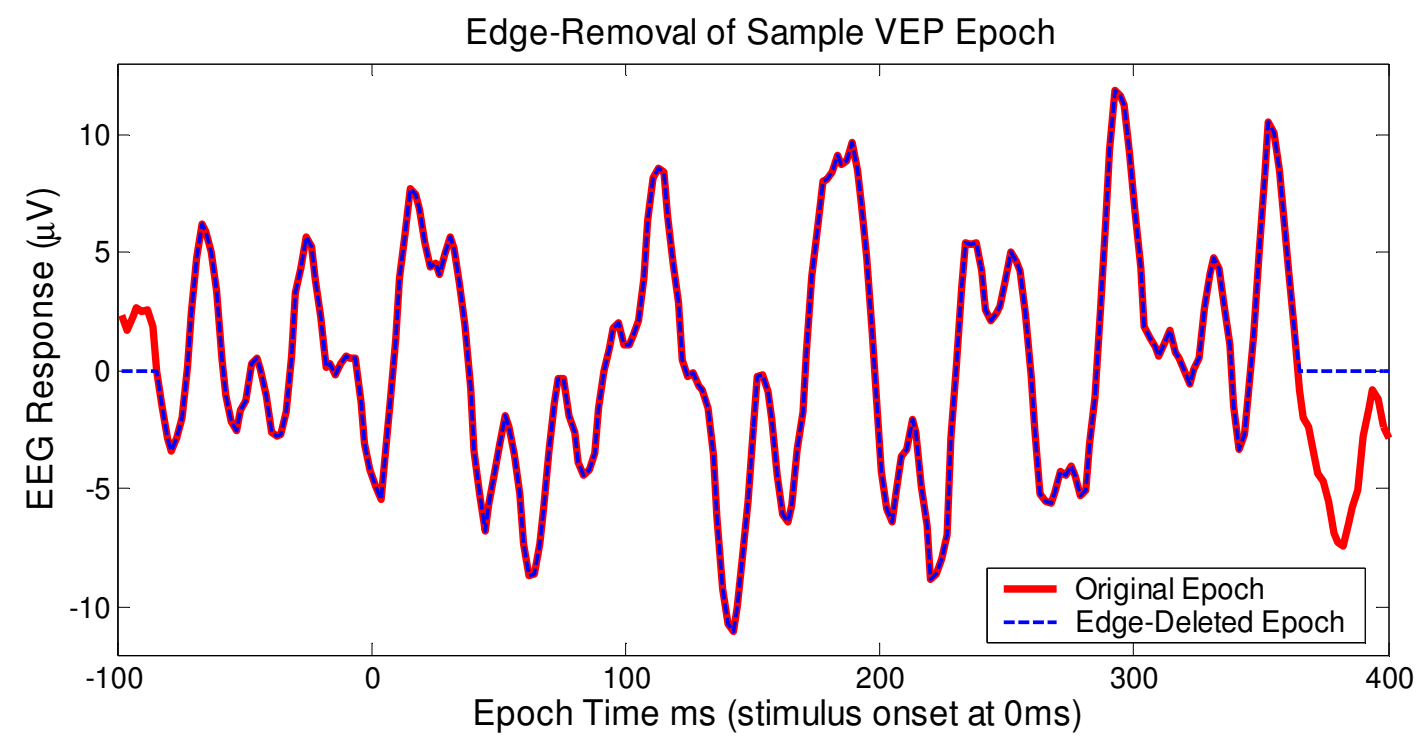

Fig. 4.7: Example of edge deletion of individual evoked potential epochs. The first and last incomplete peaks are eliminated as shown by the flat blue line at the beginning and end of each epoch. Datapoints in-between are unaffected.

The output of all these pre-processing steps is a set of artefact-free epochs that have been converted, filtered and centred and have partial edge peaks deleted. This dataset is now ready to be used as the input to the Peak Detection algorithm. Its format is shown in Table 4.3.

\begin{tabular}{|l|c|c|c|}
\hline Description of variable & Name & Matrix Dimensions & Unit \\
\hline Number of epochs detected/chosen & numepoch & $1 \times 1$ & - \\
\hline Length of each data epoch & epoch & $1 \times 1$ & $\mathrm{~s}$ \\
\hline Size of pre-stimulus record & prepos & $1 \times 1$ & $\mathrm{~s}$ \\
\hline Evoked Potential/EEG data epochs & $\mathrm{sch} 1$ & samples* $\times$ numepoch & $\mu \mathrm{V}$ \\
from each channel $(1,2, \ldots, x)$ & $\operatorname{sch} 2$ & & \\
& $\ldots$ & & \\
& $\operatorname{sch} x$ & & \\
\hline
\end{tabular}

Table 4.3: List of additional parameters that define extracted epoch data. These parameters are in addition to those shown in Table 4.2.* Note that the number of samples in each epoch is calculated from the length of each epochs and the samplerate i.e. samples $=$ epoch $x$ samplerate e.g. $0.5 \mathrm{~s} \times 512 \mathrm{~Hz}=256$ samples.

\subsubsection{Peak Detection Method}

The heart of this research is the Peak Detection Method. It is implemented as a set of Matlab algorithms that take the conditioned EEG epoch signals described in section 4.6.1 as its input and produce a concise set of wavelet coefficients that reflect the singularity properties of the signal. These singularities are grouped into pairs from which a representation for separated discrete peaks may be found. Summing theses peaks yields an accurate represent of the original epoch signal. This process is completely automated and so is readily performed across multi-channel and multi-epoch data. It evolved over 
the 4 year period of the doctoral studies and was subject to regular revision, improvement and refinement.

Fig. 4.9 graphically illustrates the effect on the signal due to the steps that occur during the Peak Detection Algorithm transformation process. The signal format in Fig. 4.9(1.) is the same as the output of Fig. 4.4(5.) and the Peak Detection algorithm is described in terms of singularity detection. Fig. 4.9 charts the conversion from the original converted signal to set of singularities, from which, peaks are interpreted through the wavelet transform detail and approximation coefficients, Finally, the peak representation coefficients are inverse transformed back to separate temporal peaks which may be added together to form a representation of the original signal.

The PDA.m Matlab file instigates and performs the entire peak detection algorithm. The process flowchart is given in Appendix $\mathrm{C}$ together with all relevant sub-process flowcharts. The associated program listings are presented in Appendix D. The major functional elements of these processes are described in subsequent sections. In PDA.m the input variables for the peak detection process are inputted - these include filename of the converted epoch data, the channels which are to be analysed and the epochs which are to be analysed. The defaults are all channels (excluding stimulus channel) and all epochs. Next, each epoch of each channel is iterated through the peak detection steps. Firstly, the epoch is split into positive datapoints and negative datapoints. The reason for this was discussed in section 3.5.4. This process is illustrated in Fig. 4.9.

It is noted that splitting signals at the zero level will on occasion cause some peaks to be split potentially in an artificial manner into two separate peaks. Fig. 3.13 illustrates this point well. However, it should also be noted that the averaging process is not sensitive to such peaks in any case and so this does not represent a loss of resolution. However, it does represent a limitation to this method. 


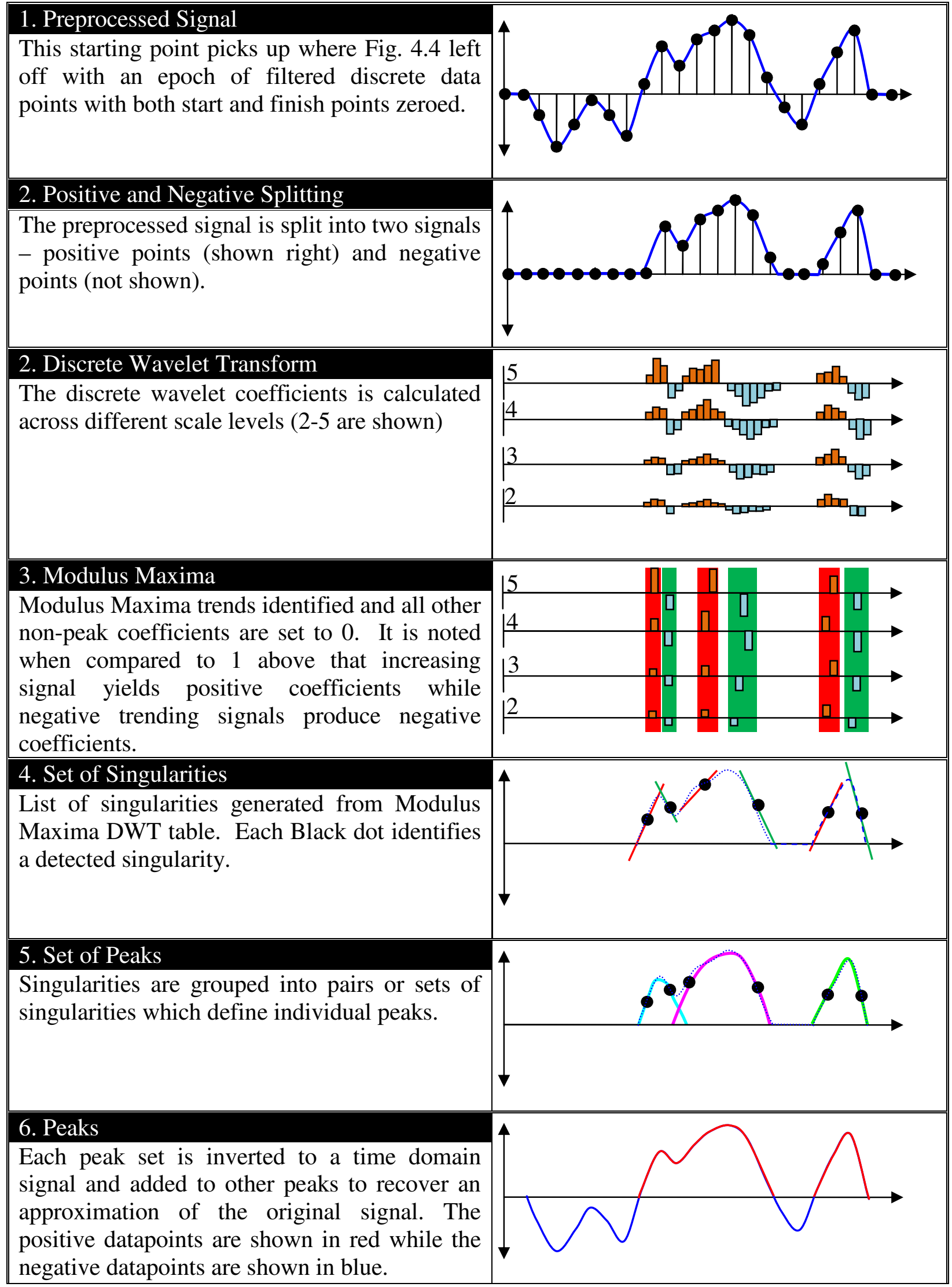

Fig. 4.8: Flowchart for transforming signals into set of peaks. For illustrative purposes the signals are not split into separate positive and negative signals. Thus positive and negative signal processing is shown together in this figure. In the peak detection algorithm they are split initially and processed separately and then ad the end the reconstructed positive and negative peaks are added together. 
Once the signal is split, the singularity coefficients are calculated for both the positive signal and the negative signal using the routine SingCoeffs.m. Then the set of coefficients for positive and negative peaks are grouped together using the SingGroup.m algorithm which identifies similar and matching properties within the singularity coefficient sets that would favour particular pairs of singularities. Finally, the approximation coefficient of the grouped singularities is calculated by comparing the resultant peak to the original peak using the PeakApprox.m routine. In this way the best fitting peak is identified. Once all peaks are identified this process is iterated once more to find sub-peaks that remain after the first iteration. Using Fig. 3.14 as an example, the first iteration would be expected to identify the blue peak in Fig. Fig. 3.14(c) and the second iteration would be expected to identify the red peak in Fig. 3.14(c). This is an important technique for separating overlapping peaks into constituent simple peaks. It is noted that depending on the nature and position of the overlap, not all overlapping peaks are separated. In some cases, the dominant peak alone is identified.

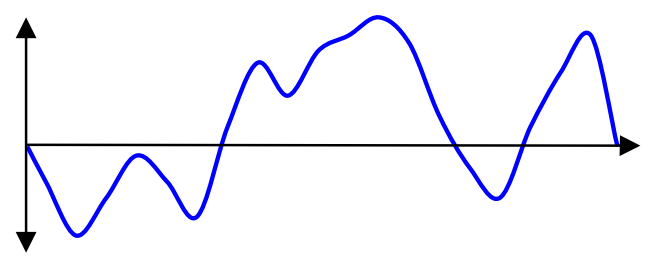

Epoch signal
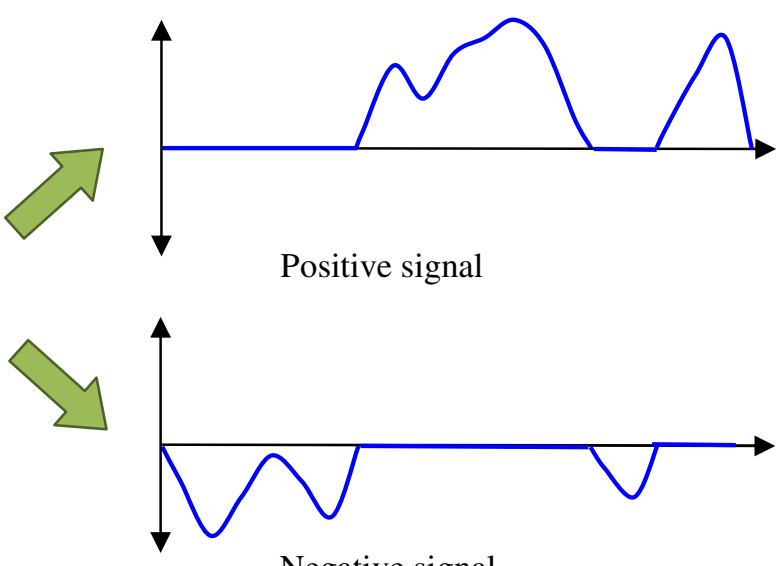

Negative signal

Fig. 4.9: Splitting of Epoch signal into positive and negative signals which are then analysed separately.

Once all peaks are identified, the output is stored in a Matlab variable file. There are two variables that completely define the set of identified peaks. There are called allsing and allvars. Their variable format is described in Table 4.4. All channels and all epochs and all peaks are stored in these two variables alone for each EEG dataset. Columns 11, 22 and 7 of allvars allow unique identification of any peaks based on the Channel, Epoch and Peak Number respectively. Note that any particular peak is uniquely defined by a pair of rows in allvars together with the corresponding pair of rows in allsing. This tabular format is suited to the Peak Analysis techniques that follow the Peak Detection algorithm. 
Each entire row of allsing and each row of allvars from column 1 to column 5 encode data for each singularity in the peak. Each peak is made up of two singularities; the first row encoding the left hand side peak information and the second row encoding the right hand side peak information. Columns 8,9 and 10 record the approximation data that is associated with the peaks. Columns 13 to 19 are metrics from the reconstituted peak. They are calculated during the Peak Detection algorithm but used later in the Peak Analysis algorithm. They provide descriptors that uniquely characterise the peak that has just been decomposed.

To understand this nomenclature further let us examine a single peak from Table 4.4. If we take the peak that is uniquely characterised by row 1 and 2 of allsing and allvars, then what can be said about this peak is that it is Channel 1, Epoch 1, Peak Number 1. Column 2 of allvars informs us that this is a negative peak. Column 3 reaffirms this since row 1 equals -1 indicating a negative sloped singularity followed by row 2 which is +1 i.e. a positive slope singularity. (For a positive peak this order would be reversed). This peak has scale 3 (column 8 of row 1 of allvars) and so only columns 1 to 6 of allsing are used in regenerating the peak. Higher level data in allsing is unused in this case and could be deleted. In terms of the data that is required to reconstruct the original peak that is characterised in this example, only that data highlighted in the two red boxes in Table 4.4 are required. The metrics associated with this peak are located in columns 13 to 19 . Hence we can say this peak has a lower y-intercept at the $15^{\text {th }}$ sample as per column 13 , row 1 or allvars. In an epoch of 256 samples and a sampling rate of $512 \mathrm{~Hz}$, the $15^{\text {th }}$ sample equates to a time position given by:

$$
\text { time }=\frac{15}{512} \times 1000=29.29 \mathrm{~ms}
$$

The upper $y$-intercept for this peak is at the $24^{\text {th }}$ sample. The difference between these two numbers is 9 which gives an indication of the scale of width of the peak. The midpoint between these two values is 20 which is the centre-point of the peak. The area under the curve is 15.55 (where time is measured in number of samples). The maximum value of the peak is $2.38 \mu \mathrm{V}$ and it is located at the $18^{\text {th }}$ sample. These metrics will be used in the Peak Analysis but first we examine the techniques employed to extract these peaks. 


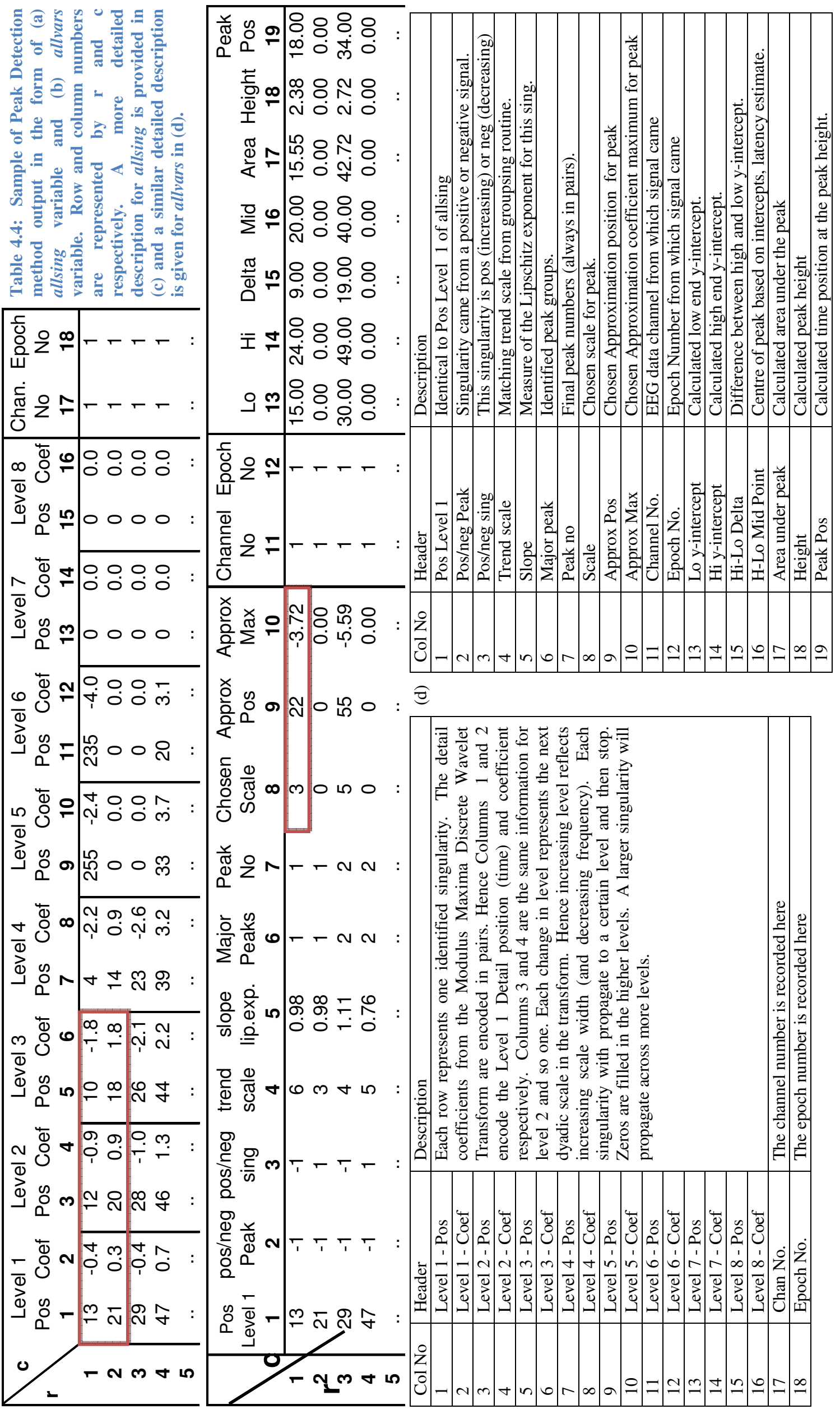




\subsubsection{Singularity Coefficients (SingCoeffs)}

There are four major steps involved in calculating the singularity coefficients of an input signal. These steps are identified in Fig. 4.10 .

This routine starts by calculating the modulus maxima discrete wavelet transform (MMDWT) of the input signal. This process implemented in Wavelab [2]. This is the only coding in the whole of the research which was not written by the author. Wavelab is a freely distributed set of Matlab functions used to implement a variety of algorithms related to wavelet analysis. The Wavelab routines that are used in this research are:

FWT_ATrou.m: This routine performs a fast dyadic wavelet transform using the implementation described by Mallet in A Wavelet Tour of Signal Processing [32].
Sing

Coeffs

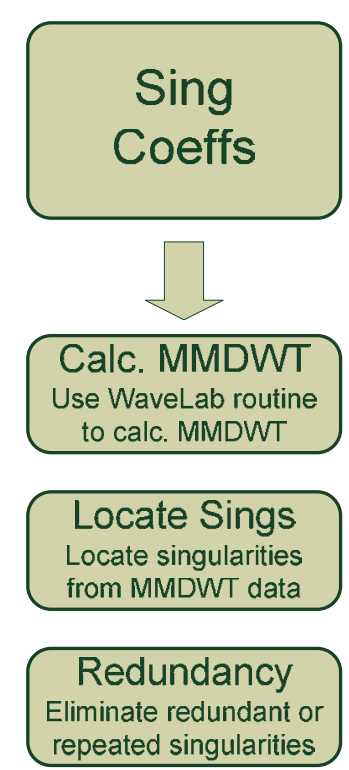

Fig. 4.10: Steps involved in calculating the singularity coefficients of an input signal.

MakeATrouFilter.m: This routine defines the coefficients for the quadratic spline filter that is described in Fig. 3.7.

MM_DWT.m: This routine examines the fast dyadic wavelet transform coefficients and identifies the maxima and minima coefficients by generating a matrix of ones only at these maxima/minima positions.

IMM_DWT.m: The inverse fast dyadic wavelet transform takes a set of coefficients in a format similar to the output of FWT_ATrou.m and inversely iterates an approximation of the signal based on the coefficients.

Once the MM_DWT has been calculated, the next step is to identify a discrete, concise set of singularities. This is performed by scanning through all the time positions of the set of coefficients and examining the trend a cross scales. Genuine singularities will have increasing coefficient values across a number of scales starting with scale 2 up to at least scale 4. According to Mallet, this trend for increasing coefficient values across increasing scale is constrained within an ever broadening range for each increasing scale. The range within a singularity may effect is called the cone of influence.

Once all the singularities have been identified and defined in terms of a set of coefficient pairs, the next step reviews the singularity list for redundancy. This is due to the fact that 
the singularity detection algorithm can pick up slightly different coefficient sets as being the same singularity. If a pair of singularity sets differ differs by only one coefficient then the set with the larger coefficient value is chosen and the other singularity set is deleted. Thus the output of this process is a concise set of singularities.

\subsubsection{Group Singularities (SingGroups)}

The singularities detected in the previous section must now be compared to neighbouring singularities and a set of rules drawn up to automatically match them into pairs which will form a peak.

Cone of Influence: After removing repeated singularities that are nearly identical (i.e. only level 2 scale coefficients differ across all scales), the first task is to calculate a cone of influence over which a matching singularity may occur. Mallat's singularity detection theory states that the trend of coefficients values increase across scales for increasing scales. For larger singularities (reflective of larger peaks), this singularity pattern extends further into the scale information, therefore such a singularity will have a larger cone of influence over which a matching singularity may be found. This cone yields a time domain range inside which the matching singularity will occur.

Singularity Direction: Other information about the signal is also useful in narrowing down the choice of match singularity. Remember that positive and negative peaks are being processed

Group Sing

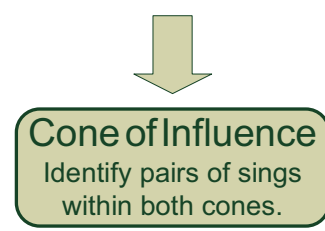

within both cones.

Sing Direction Coefficient sign dictates match direction

Sing Magnitude Matching magnitudes indicates sing pairs

Lipschitz Vars Calculate the Lipschitz exponent for each sing

\section{Sing Order Largest sings matched first, then iterated.}

Fig. 4.11: Steps involved in calculating the singularity coefficients of an input signal. separately; hence it is known which is being looked for at any one time. For example, a singularity which is in the positive peak group has positive coefficient, then it is a rising edge of a positive peak hence it's matching partner must be located at a time point greater than this singularity location. Therefore, inherent in the singularity coefficient set is the singularity direction and the sought after matching singularity must have an opposite singularity direction to form a peak.

Singularity Magnitude: The cone of influence yields an approximate distance limit within which the matching peak should occur. We examine all possible matching singularities within this range which leads to a small subset of singularity possibilities. From this range of peaks, the singularity whose coefficient magnitudes most closely 
match the original singularity as it trends to higher relevant scale levels is chosen as the matching singularity that together with the first singularity will form a peak.

Lipschitz Exponent: The Lipschitz exponent of the possible matching singularities is investigated and only those singularities with Lipschitz exponents greater than zero can form a match. So those that are less than zero are eliminated.

Singularity Order: This grouping is performed in singularity magnitude order. Hence the largest coefficient singularity is chosen first and its partner found. Then these two singularities are removed from the dataset and the next highest coefficient singularity is matched and so on until all singularities are matched. The pair of singularities formed by investigation of the cone of influence 


\subsubsection{Peak Approximation (PeakApprox)}

At this point the detail coefficients for the left-hand and righthand singularities are known together with an estimate of the required scale decomposition level. The components remaining to be found in order to have a complete peak representation in the wavelet domain are the exact decomposition level and the approximation coefficient(s) at this level. In detail decomposition we could use the trend across scales to pick out the singularity coefficients. However, the approximation level yields a single set of coefficients and suffers from the fact that adjacent peaks will influence the size of the approximation coefficients for the peak in question. Therefore, a technique was developed to extract the required approximation coefficient(s) without interference from adjacent peaks.

Firstly, the known singularity pair is used to effective crop the

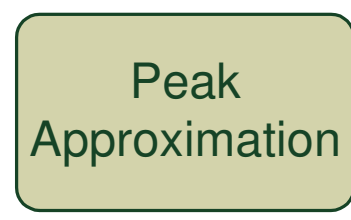

PeakSiglet.m Crop signal using location of singularities

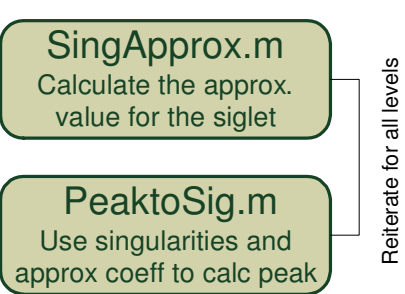

Pick Best Match Pick peak with min. error compared to orig. signal

Fig. 4.12: These steps are required to calculate the approximation coefficients for each peak. original signal as near to the bottom of the peak as possible. This technique uses the slope of the singularity to estimate the y-axis intersection of the peak as shown in Fig. 4.13. This signal (known here as a siglet) is transformed (using the DWT) to the first possible scale level and the modulus maxima approximation value within the singularity pair range is recorded. A peak is generated in the time domain defined by the known singularity coefficients and the approximation coefficient for this particular scale level. Next this process is repeated for every possible level yielding a peak representation for each level. Finally, the peaks across all scale levels are compared to the peak siglet signal and the peak with the smallest difference error to the peak siglet is chosen as the best match peak. Now the level and the approximation coefficient are known and the peak representation is in terms of wavelet coefficients is

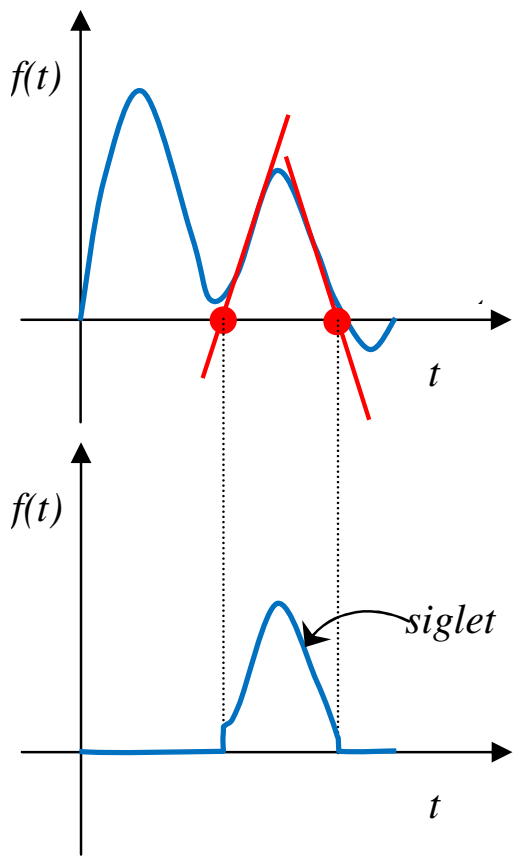
complete. It turns out that single peak approximation

Fig. 4.13: Slope of the singularities is used to estimate y-intersection of the signal and an estimate of the peak required is taken by setting all signal values outside this range to zero. 
coefficient value (together with its location in time) is sufficient to represent the required approximation information for the peak. This is similar to the detail coefficients where only a single detail coefficient (per level) is required. Also, it is chosen using the same modulus maxima coefficient technique that was described my Mallat for detail coefficients. Table 4.5 outlines the number of wavelet coefficients required to yield a decomposition of individual peaks.

\begin{tabular}{|c|c|c|c|c|}
\hline $\begin{array}{c}\text { Scale } \\
\text { Level }\end{array}$ & $\begin{array}{c}\text { Left Singularity } \\
\text { Detail }\end{array}$ & Approximation & $\begin{array}{c}\text { Right Singularity } \\
\text { Detail }\end{array}$ & $\begin{array}{c}\text { Total } \\
\text { Coefficients }\end{array}$ \\
\hline 2 & 2 & 1 & 2 & 5 \\
\hline 3 & 3 & 1 & 3 & 7 \\
\hline 4 & 4 & 1 & 4 & 9 \\
\hline 5 & 5 & 1 & 5 & 11 \\
\hline
\end{tabular}

Table 4.5: Table of wavelet coefficients required to characterise a single peak. The number of coefficients required is dependent on the scale level which best characterises the peak.

It is now a simple matter to take these coefficients together with their location and populate and empty wavelet coefficient matrix and apply the inverse wavelet transform to generate a time domain representation of the peak. This analysis is then performed for all peaks in the signal.

The peak parameters described in section 3.6.2 may now be calculated directly from the peak. These are peak latency measured from the start of the signal to the centre of the peak, peak width which is measured as the distance between the two y-intercepts of the peak and peak height which is measured from the 0 level up to the maximum peak value. The area under the curve is also calculated using the adaptive Simpson quadrature [81]. Quadrature is a numerical method that may be used to determine the area under a curve and is implemented in a Matlab routine. This formula calculates an estimate of the area under the curve using piecewise linearisation of the signal followed by summation of trapezoidal area calculation. Their inter-relation and interpretation are discussed further in the next section and the results section. 


\subsubsection{Peak Analysis}

Block clustering and spread clustering were described in Section 3.6.5 and 3.6.6 respectively. Both methods take 3 independent parameters as 3 separate dimensions and generate a peak concentration matrix (herein called the concentration matrix) which correlates areas of higher concentration to larger clusters of peaks. The intention being that time-locked nature of the evoked potential response will tend to occur more often and thus be identified by these clustering methods.

Initial testing of the clock clustering method mapped peaks onto a 1:1 basis, thus any one peak could only belong to any one block or bin. It became clear that this technique was sensitive to the positioning of the block edges and that unless the block size neatly fitted the spread of a set of peaks then, concentrations tended to be split over a number of bins making identification of the highest concentration block problematic and unclear.

Thus spread clustering was preferred for all the analysis in this research and is solely described in the following sections.

The spread clustering technique overcomes this problem

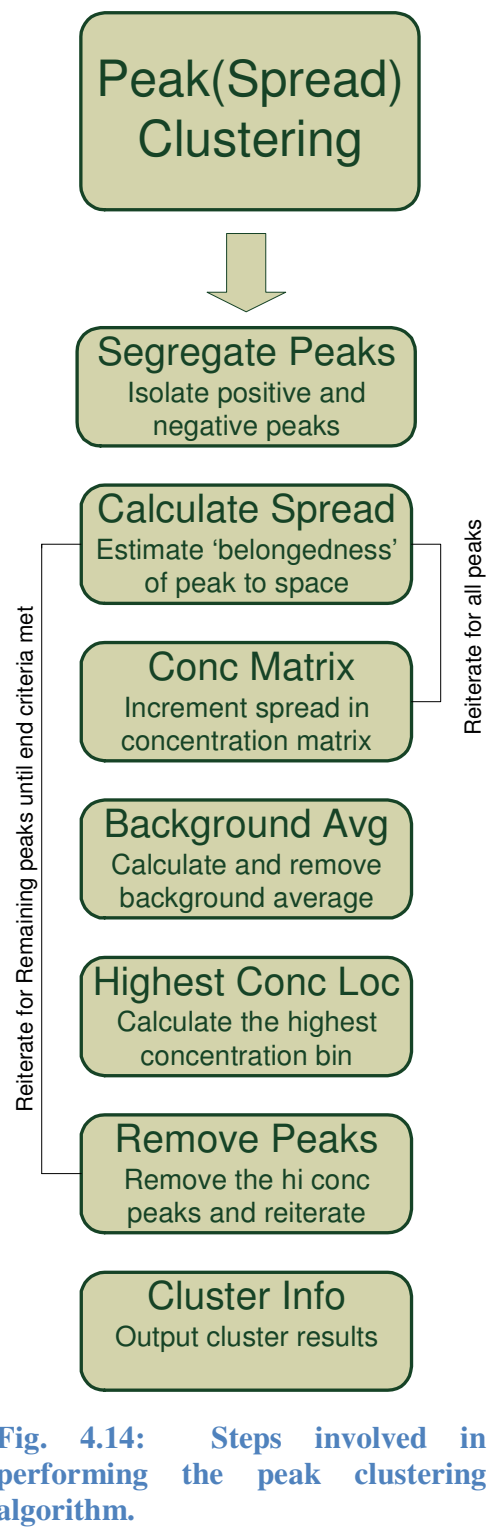
by calculating a range in each dimension over which a peak could belong. Across 3dimensions, this spread generates a volume space over which this peak could belong to a particular cluster. Calculating this 'belongedness' measure removes the need to measure Euclidian distances associated with more traditional clustering methods. A high level flowchart for the process flow of the Peak (Spread) Clustering technique is given in Fig. 4.14. The rest of this section summarises the steps followed and refers to the following section in areas where more detail is required.

The factors determining the size of the concentration matrix and the peak spreads are described in the next section, 4.6.4.1. Once identified, the spread volume within the 
concentration matrix is simply incremented by 1 and the process is repeated for all remaining peaks.

When all peaks are processed in this fashion, the concentration matrix becomes fully populated yielding a 3-dimensional matrix array with areas of higher value reflecting area of higher numbers of 'similar' peaks. Similar peaks have similar latencies, similar widths and similar heights. It is this similarity and belongedness which is used to perform the clustering process.

Before examining the matrix results, one further factor must be considered. In ongoing background EEG, inherently some peak shapes tend to occur more regularly. The peak width and peak height are not purely random distributions. The latency distribution is essentially random because any peak may occur any particular time across the epoch. Our intention is to de-emphasize the background EEG and emphasize the evoked response. One simple and effective method to do this is to calculate the mean concentration matrix across all time latencies yield a 2-dimensional mean array (across peak width and peak height) and then subtract this 2-d array from all latency locations in the concentration matrix. This concept is discussed further in section 4.6.3.2.

Now the concentration matrix is populated and the background EEG average has been offset, the location of the highest peak shape concentration is simply the location of the maxima of the concentration matrix. Once located, the latency, width and height information associated with its position is recorded. Next these peaks are removed from the peak dataset and the whole process is repeated again to identify the next highest concentration location and so on until some end criteria is met.

\subsubsection{Range, Scale and Spread}

The dimensions of the concentration matrix are scaled to minimize redundancy and speed processing. So for example, although typical epoch length and thus latency range is 256 samples, this is scaled by a factor of 2 to give a latency length of 128 in the concentration matrix. Essentially a gap of 1 between latencies is too small to be important and thus they may be grouped together.

It is also possible to reduce the concentration matrix size by limiting the range of the concentration matrix and in particular the upper limit. By examination of the peak width distribution and the peak height distribution, it is clear that at a certain point the distribution tail becomes extremely low and a very few upper limit values are present. 
Thus where the distribution is low, we may crop the concentration matrix upper limit to exclude these values as they are too low to form any meaningfully sized peak cluster. The latency upper limit cannot be cropped as it has a purely random nature across its distribution as described in the previous section.

The final parameter to calculate is the spread. Let us examine the amount of variation we wish to allow any particular peak so that that any other peak located within this variation made constitute part of the same cluster. In thinking about this, it is clear that the latency variation will be related to the width of the peak. If the latency variation is half the peak width then there is no overlap between the peaks and so it is asserted that this spread is too large and outside the cluster for this peak. As the spread is reduced it eventually comes to pass that the peak spread is close enough to another peak at this spread to be considered a similar peak and thus the peak spread values have been determined empirically. It was found that a base 2 logarithmic scale yields a good estimate to the amount of allowable spread. This creates a nonlinear variation where the higher parameter values have proportionately higher spread.

\begin{tabular}{|l|c|c|c|c|c|c|c|}
\cline { 2 - 8 } \multicolumn{1}{c|}{} & $\begin{array}{c}\text { Typical } \\
\text { Full Range }\end{array}$ & Scaling & $\begin{array}{c}\text { Range } \\
\text { Limits }\end{array}$ & $\begin{array}{c}\text { Conc. } \\
\text { Matrix } \\
\text { Limits }\end{array}$ & Spread & $\begin{array}{c}\text { Sample } \\
\text { Peak }\end{array}$ & $\begin{array}{c}\text { Bin } \\
\text { Range }\end{array}$ \\
\hline $\begin{array}{l}\text { Latency, } \\
t\end{array}$ & $1-256$ & $\times \frac{1}{2}$ & None & $1-128$ & $\pm 3 \log _{2}$ w & 81 & $38 \rightarrow 42$ \\
\hline Scale, $w$ & $2-60$ & None & $1-36$ & $1-36$ & $\pm 3 \log _{2}$ & 18 & $14 \rightarrow 22$ \\
\hline Height, $h$ & $1-25$ & None & $1-18$ & $1-18$ & $\pm \log _{z} h$ & 10.1 & $8 \rightarrow 12$ \\
\hline Sign, $s$ & $+1--1$ & None & None & $+1--1$ & none & +1 & \\
\hline
\end{tabular}

Table 4.6: Summary of the scaling of peak information into concentration matrix. The latency information is scaled by one half and the spread is given by $\log 2$ of the width. There is no scaling for width or height and peaks outside the maxima of 36 and 18 respectively are not used. These are outliers and will not contribute to high bin areas. Yet their exclusion greatly reduces the concentration matrix size and speeds processing times considerably. The spread for width and height is given by one-fifth of the respective size.

\subsubsection{Background Peak-Shape Average}

Certain peak shapes (i.e. peak width and peak heights) occur more regularly than other during ordinary EEG analysis. This means that these more common peaks will skew the concentration matrix towards these peak shapes. These distributions may vary from person to person or from test to test on the same person. We wish to focus on time locked peaks hence the concentration matrix average distribution is calculated across all latency values. This yields a 2 dimensional matrix of peak width against peak height average 
concentrations. This distribution is subtracted from the entire concentration matrix along the latency axis. Refer to Fig. 4.15 for a graphical representation.
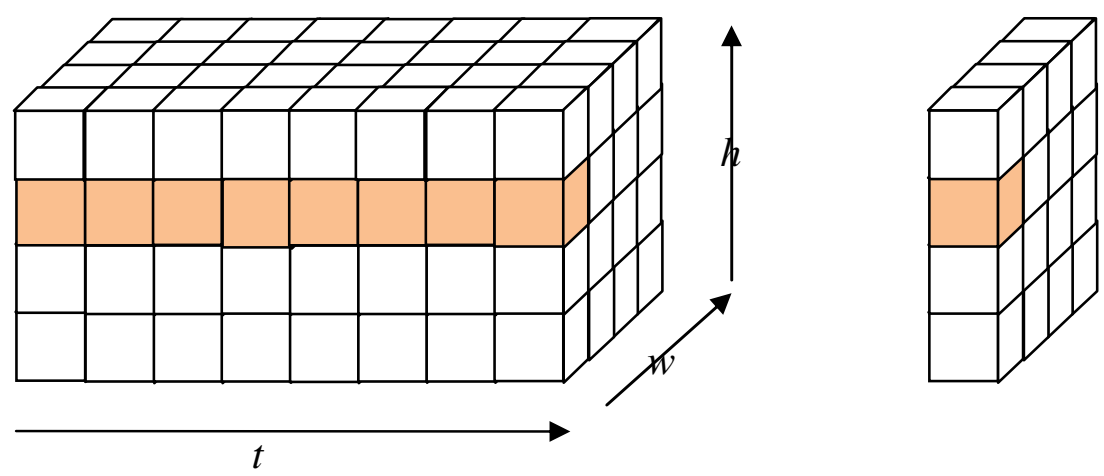

Fig. 4.15: Background Peak-Shape averaging is performed by averaging along the latency dimension. In the above example the Concentration matrix (a) is averaged along the orange points yielding a single value in the Peak-Shape average distribution in (b), also orange. This is repeated for all width and height position in the concentration matrix yielding the full Peak-Shape average matrix in (b). After this is calculated, the Peakshape average distribution is subject from each latency position along the concentration matrix.

The concentration matrix is now has a range where a matrix value of 0 represents the average amount of peaks are presents for any particular width and height position. We examine concentration matrix and identify the largest peak concentration matrix values since the high values indicate a higher concentration of particular width and height peaks and a particular time location. Once the highest concentration position is identified, together with its location in terms of latency, width and height, it is possible to determine which peaks contribute to this high concentration and graph them to estimate the contribution of these peaks to the original averaged evoked potential signal. It is also possible to examine the distribution of this peak set to arrive at a average peak shape in terms of position, width and height and calculate a single peak shape which is the average peak shape that contributes to a certain amount (hopefully large) to the overall averaged evoked potential. Once the highest concentration location is identified and associated peaks extracted, these peaks are then removed from the concentration matrix and the next highest concentration location is identified. Repeating this method against the next highest concentration level allows further evoked potential components to be decomposed; leading to an accumulation of sub-peak sets whose contribution towards the overall averaged evoked potentials is measurable in terms of peak shape and number of epochs that contribute. Each of these high concentration locations together with the peaks associated with each location are referred to a Peaksets.

\subsubsection{Plot Results}


There is now three ways to represent peaksets which provide different viewpoints of the same thing:

1. Peakset Average: This is the sum of all peaks in a peakset divided by the total number of peaks in the peakset. It is scaled version of the standard averaging VEP but is not identical since the number of peaks (or epochs) in the denumerator varies.

2. Mean Peak Shape: Peaks in a peakset vary across latency, width and height. By taking the average of each of these parameters, we can calculate the average latency, average, width and average height of peaks within the peakset. Next we can use this information to manufacture a peak to these dimensions. This manufactured peak is implemented in Matlab using a scaled and transformed Hann window. This routine takes the calculated mean peak shape per bin and generates a standard peak shape that exactly fits the average latency, width and height. It gives an indication of the 'average' individual peak shape within the peakset.

3. Equivalent Peak Average: This is calculated by adding all the peaks within a single peakset and dividing by the total number of epochs. This parameter gives a direct measure of how much a particular bin contributes to the overall VEP response and so is directly related to the standard average VEP signal.

\subsubsection{Non-Evoked Potential Comparison}

A further comparison technique was used to verify that the presence of significant clusters was due to the time locked evoked potential and not due to some other EEG effect not account for thus far. In order to differentiate between ongoing EEG peaks and evoked potential peaks, this analysis was repeated for a similar number of random epochs of EEG from the same subject taken at the same time at the evoked potential signals. There is no time-locking to a stimulus in this case. The EEG Epoch results represent an expected concentration matrix for ongoing background EEG. This allowed the EP+EEG analysis to be superimposed over the EEG analysis and hopefully delineating the contribution of each component to the total peakset distribution.

\subsubsection{Significance Measure}


The standard averaging approach does not discriminate between the influence of the number of peaks and the size of a particular peak. This lack of specificity is explained in Fig. 4.16. However, in the algorithms developed here, it is possible to distinguish between the contribution of the size of the peak and the number of peaks. We have used peak clustering to detect similar peaks that occur most often. We wish to decompose the standard average results and understand how its components are made up. In order to get a closer representation or decomposition of exactly what makes up the standard evoked potential, we must take into account that standard averaging favours large peaks for any given number of peaks present. By multiplying the number of peaks present by the area under the peak, we get an interpretation that focuses on the same factors as averaging.

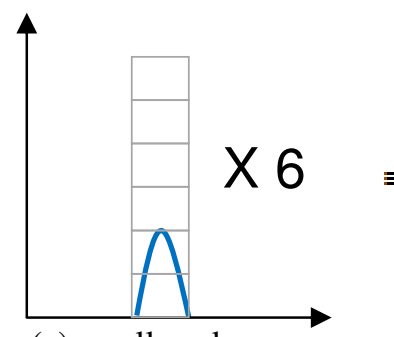

(a) small peak, occurring often

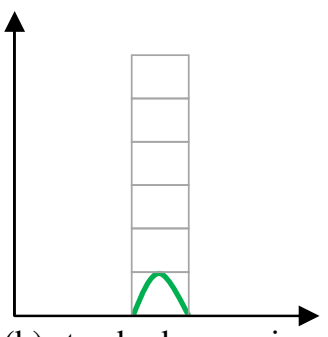

(b) standard averaging for 12 epochs

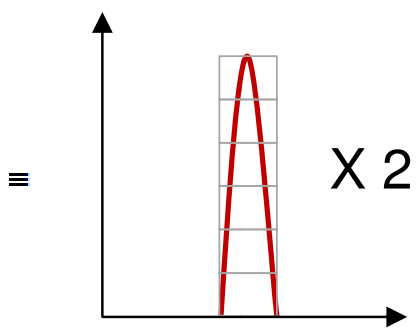

(c) large peak, occurring rarely

Fig. 4.16: Demonstration of how standard averaging cannot distinguish peak shape origin. Let us take a simple evoked potential dataset of 12 epochs. The small peak (a) occurs 6 times in the trial. Its contribution to the standard averaged evoked potential is shown in (b) where the sum of 6 (a) peaks averaged over the 12 epochs yields the green signal. Now if instead the dataset consisted of 2 larger peaks shown in (c) and the dataset size was still 12, it is apparent that the contribution of the red peak to the standard average is also the green line shown in (b), since 2 red epochs averaged over 12 epochs still yields the same green line. Standard averaging does not yield any indication into which is really present since it is the green line in (b) that is calculated and (a) or (c) are unknown.

Therefore, we can order out clustered peaks in two ways. These two ways are described in Table 4.7 in terms of significance. This provides two viewpoints from which to view the data which is explored further in the results.

\begin{tabular}{|c|c|}
\hline If the following is Significant ... & Then order peaksets based on ... \\
\hline $\begin{array}{c}\text { relating peaksets directly to the } \\
\text { standard averaged response }\end{array}$ & No. of Peaks X Areas under Peak \\
\hline $\begin{array}{c}\text { identifying the peaksets that } \\
\text { occur most often }\end{array}$ & No. of Peaks \\
\hline
\end{tabular}

Table 4.7: How significance affects the analysis method that you choose. 


\section{CHAPTER 5 RESULTS}

\subsection{OVERVIEW}

his chapter presents the results recorded from Visual Evoked Potential trials
using the experimental procedure in Appendix A. It also reports the results from
the peak detection algorithm and peak clustering algorithm using the same VEP data. The major sections of this chapter are as follows: firstly, a summary of the VEP data recorded is presented. The next section presents the results of analysing a single evoked potential dataset measured on a single channel for a single subject. The peak detection and clustering results are described step-by-step for this dataset and some descriptive explanation is included to aid comprehension. Then the last section outlines the results for all other datasets. The graphs associated with these results are presented in Appendix B. Analysis and observations across all results are reserved for the next chapter.

\subsection{SUMMARY OF VEP RECORDINGS}

There were three participants who undertook the experimental protocol. The results from each participant were assigned the following unique non-identifying labels - P01, P02 and P03. Three suitable datasets from each participant were recorded and visually checked. Each dataset was made up of 7 recording channels and 1 stimulus channel and were at least $2 \frac{1}{2}$ minutes in length. Pre-processing was performs using method described in section 4.6. A summary of the recorded datasets is presented in Table 5.1.

\begin{tabular}{|c|c|c|c|c|c|c|c|c|}
\hline \multirow{2}{*}{$\begin{array}{l}\text { Part- } \\
\text { ici- } \\
\text { pant }\end{array}$} & \multirow{2}{*}{$\begin{array}{l}\text { Record } \\
\text { Order }\end{array}$} & \multirow{2}{*}{$\begin{array}{l}\text { Record } \\
\text { Length }\end{array}$} & \multicolumn{3}{|c|}{ Number of Stimuli } & \multirow{2}{*}{$\begin{array}{l}\text { No. of } \\
\text { Channels }\end{array}$} & \multirow{2}{*}{$\begin{array}{l}\text { VEP Dataset } \\
\text { Reference }\end{array}$} & \multirow{2}{*}{$\begin{array}{l}\text { EEG Dataset } \\
\text { Reference }\end{array}$} \\
\hline & & & Total & $\begin{array}{l}\text { Artefact- } \\
\text { free }\end{array}$ & PDA & & & \\
\hline P01 & First & $160.6 \mathrm{~s}$ & 146 & 144 & 141 & $7+1$ (stim.) & P01VEP04 & P01EEG04 \\
\hline $\mathrm{P} 01$ & Second & $156.8 \mathrm{~s}$ & 148 & 146 & 143 & $7+1$ (stim.) & P01 VEP06 & P01EEG06 \\
\hline P01 & Third & $154.4 \mathrm{~s}$ & 144 & 144 & 141 & $7+1$ (stim.) & P01VEP07 & P01EEG07 \\
\hline $\mathrm{P} 02$ & First & $180.3 \mathrm{~s}$ & 166 & 160 & 155 & $7+1$ (stim.) & P02VEP02 & P02EEG02 \\
\hline $\mathrm{P} 02$ & Second & $184.7 \mathrm{~s}$ & 171 & 169 & 168 & 7+1(stim.) & P02VEP03 & P02EEG03 \\
\hline $\mathrm{P} 02$ & Third & $181.4 \mathrm{~s}$ & 168 & 165 & 164 & $7+1$ (stim.) & P02VEP04 & P02EEG04 \\
\hline $\mathrm{P} 03$ & First & $180.7 \mathrm{~s}$ & 167 & 163 & 153 & $7+1$ (stim.) & P03VEP05 & P03EEG05 \\
\hline $\mathrm{P} 03$ & Second & $160.6 \mathrm{~s}$ & 147 & 146 & 140 & $7+1$ (stim.) & P03VEP07 & P03EEG07 \\
\hline $\mathrm{P} 03$ & Third & $161.3 \mathrm{~s}$ & 148 & 146 & 141 & $7+1$ (stim.) & P03VEP08 & P03EEG08 \\
\hline
\end{tabular}

Table 5.1: Summary of VEP recordings. Experiments followed the procedure given in Appendix A. Preprocessing followed the method shown in section 4.6. The number of stimuli used for analysis is given in the PDA (Peak Detection Algorithm) column. EEG datasets were extracted randomly from the same datasets and the number of EEG stimuli was chosen to match the VEP stimuli shown in the PDA column. 
The total number of full epochs identified and the number of epochs that were gross artefact-free are shown as the 'Total' and 'Artefact-free' columns.

All datasets were descriptively labelled for easy identification using the notation $\mathrm{P} 0 x Y Y Y 0 z$ where $x$ referred to the participant number, $Y Y Y$ refers to whether the data was genuine VEP (stimulus-locked) or background EEG (non-stimulus-locked) and $z$ referred to the sequence of dataset recorded from a particular participant.

\subsection{SINGLE DATASET RESULTS}

The processing of a single evoked potential dataset involves four distinct steps described in the next four sub-sections. Firstly, the standard averaging technique is applied to the pre-processed dataset. Secondly, the output of the Peak Detection algorithm is presented. Thirdly, the individual peaks are characterised into distinct descriptive components or parameters. Finally, these descriptive components are examined using a peak clustering technique to identify higher concentrations of particular peaks being indicative of evoked potential activity. At each step comparison of the output is made with reference to the standard averaging results.

All of the results within this section and subsections therein are based on the analysis of data from Participant 02, dataset recording number 2 (referred to as P02VEP02) and channel 2 only within this dataset. Channel 2 represents the Left Occipital (LO) electrode position. The EEG Epochs are randomly selected from the same dataset and channel and are referred to as P02EEG02.

\subsubsection{Standard Averaging Processing}

It is noted from Table 5.1 that the P02VEP02 record length was 180.3 seconds. There were 166 VEP epochs detected within this dataset. Pre-processing in accordance with section 4.6 was performed and 6 Epochs were judged to contain artefact and removed. Fig. 5.1 presents (a) a sample data epoch and (b) the standard Visual Evoked Potential response signal averaged over the remaining 160 epochs, shown for both the original signal (in blue) and the pre-processed signal (in red). These results are for channel 2 only.

The epoch length was 500ms with $100 \mathrm{~ms}$ pre-stimulus length. Generally, flash VEP responses are observed in the first 250ms after stimulation [79]. The pre-processed signal forms the input to the peak detection algorithm. It also forms the input to the standard 
VEP averaging process. Throughout all the remaining results and analysis, the standard averaged VEP signals have been calculated using the pre-processed signal as their input and not the original or raw signal.

Fig. 5.2 provides the equivalent results for the non-stimulus-locked EEG dataset, P02EEG2, channel 2. Note the absence of a clear EP response at 100-150ms in Fig. 5.2(b) when compared with Fig. 5.1(b), as expected.

(a) A single sample epoch (P02VEP02, channel 2, epoch \#1)

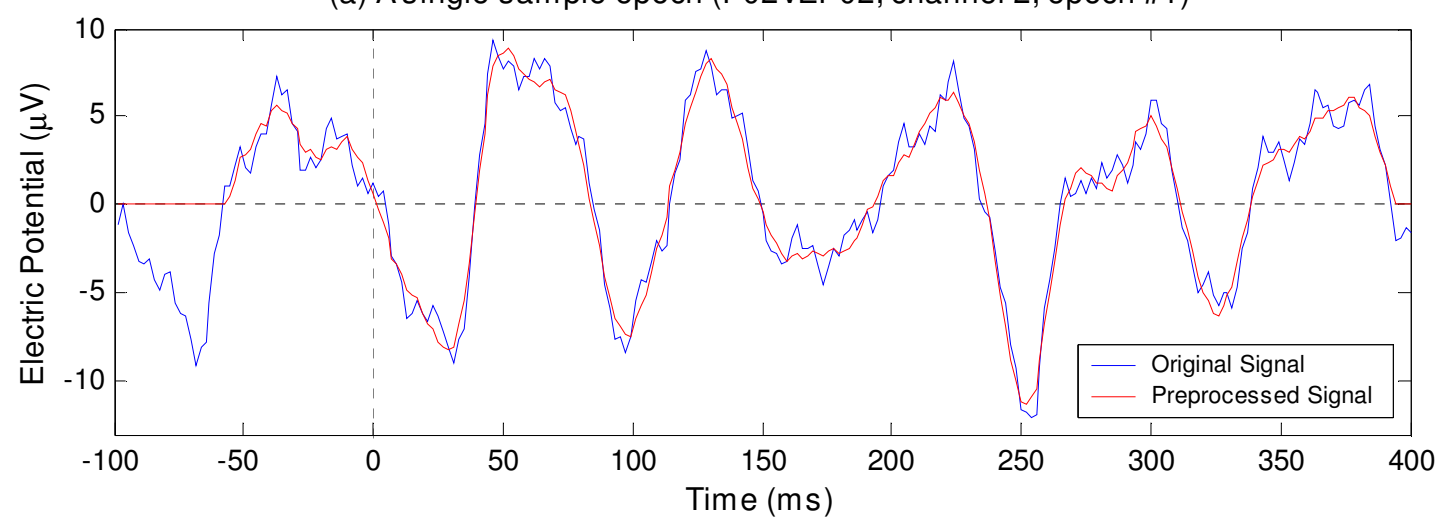

(b) Averaged VEP for P02VEP02 channel 2

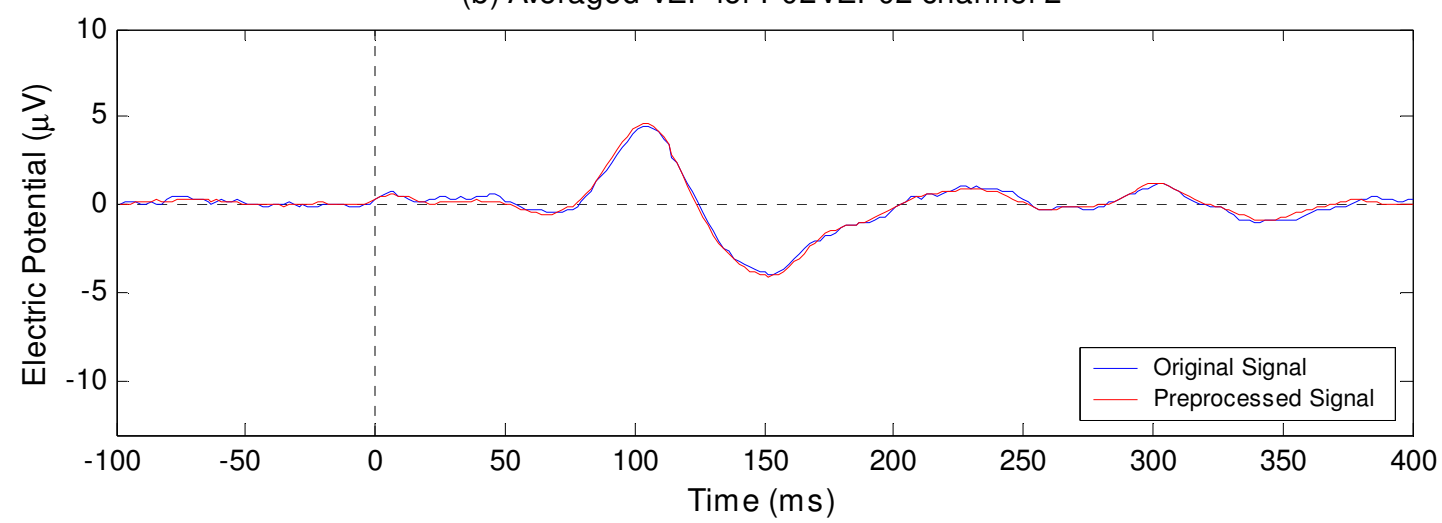

Fig. 5.1: (a) A sample visual evoked potential epoch is shown - epoch \#1 of 160. The blue line shows the original signal recorded from the EEG amplifier. The red line shows the pre-processed signal in accordance with method in section 4.3.1. (b) The standard average of the original signal and the standard average of the pre-processed signal are compared. 
(a) A single sample epoch (P02'ËĒḠō, channel 2, epoch \#9)

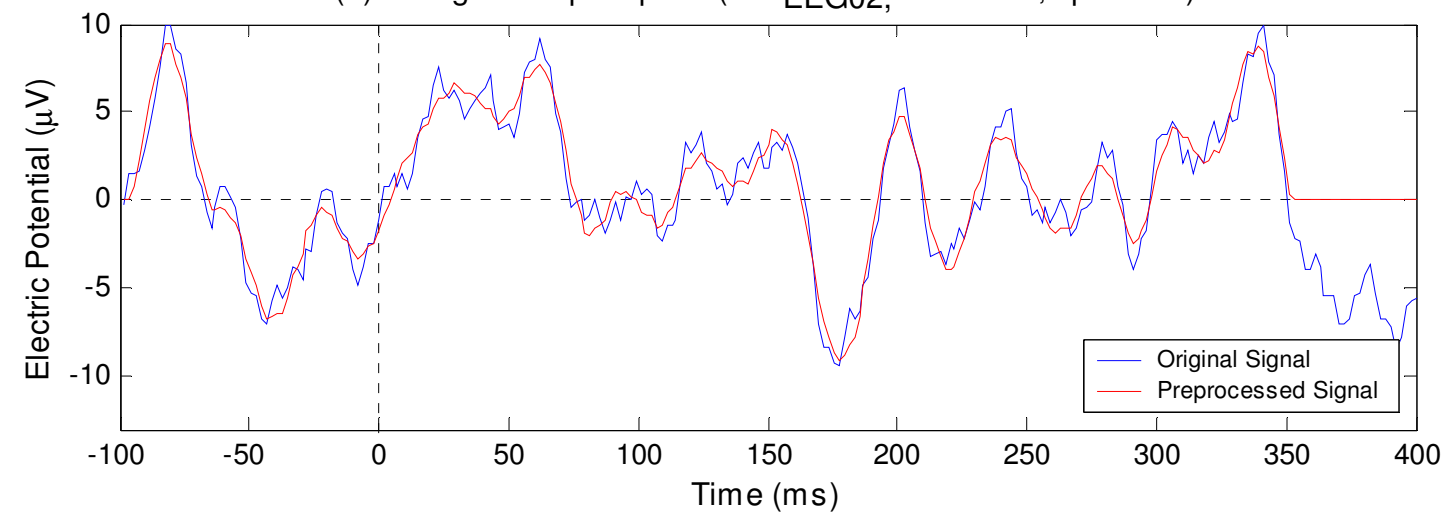

(b) Averaged VEP for P0Ż̇̇ĒḠōē, channel 2

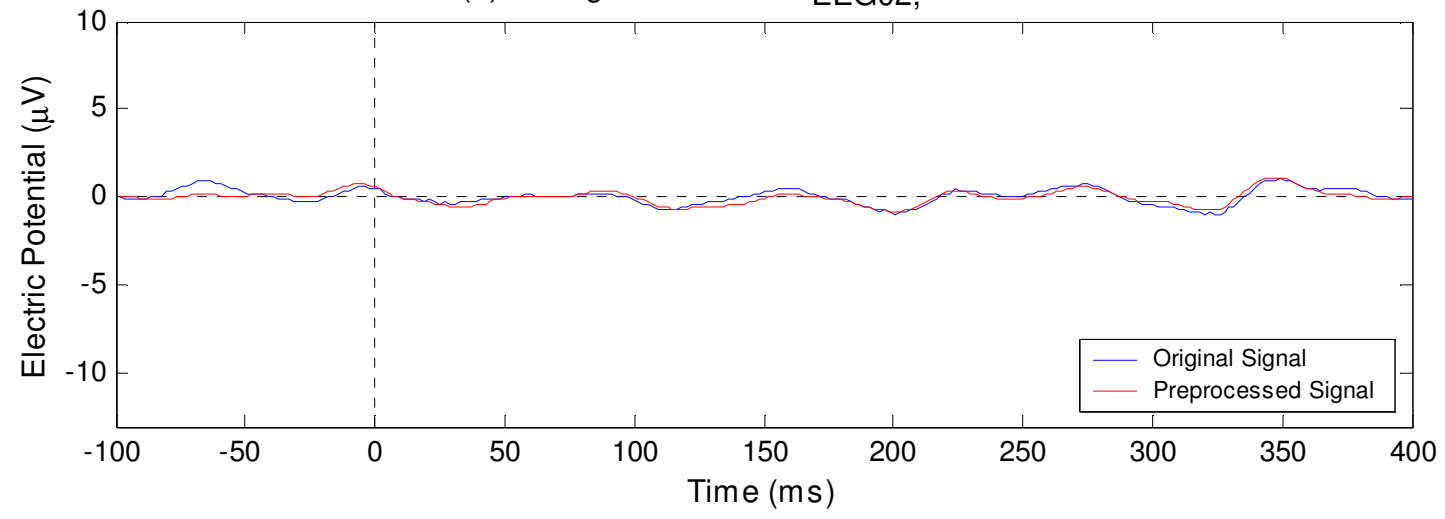

Fig. 5.2: (a) A sample EEG epoch is shown - epoch \#9 of 160. The blue line shows the original signal recorded from the EEG amplifier. The red line shows the pre-processed signal. (b) The standard average of the original signal and the standard average of the pre-processed signal are compared.

\subsubsection{Peak Detection Results}

The peak detection algorithm decomposes each VEP or EEG epoch into a discrete set of peaks that, when summed together, closely represent the original epoch signal. The results for a sample epoch are shown in Fig. 5.3. The pre-processed signal for a single epoch (\#1) is shown in black and each detected peak is separately coloured for illustrative purposes. The Pearson correlation coefficient between the pre-processed epoch signal and the sum of the (in this case 12) peaks is 0.997 indicating a very close fit. This peak detection process is repeated for each individual epoch yielding similar sets of peaks that describe all epochs in the dataset.

The Pearson correlation coefficient between each epoch and the set of decomposed peaks was calculated for all epochs in this dataset. This was expressed as a histogram of Pearson correlation coefficients shown in Fig. 5.4. There were five epochs which lie below the chosen limit of 0.96. These represented five epochs where the peak detection algorithm was unable to accurately capture a significant peak (or peaks) and so there is a drop in the 


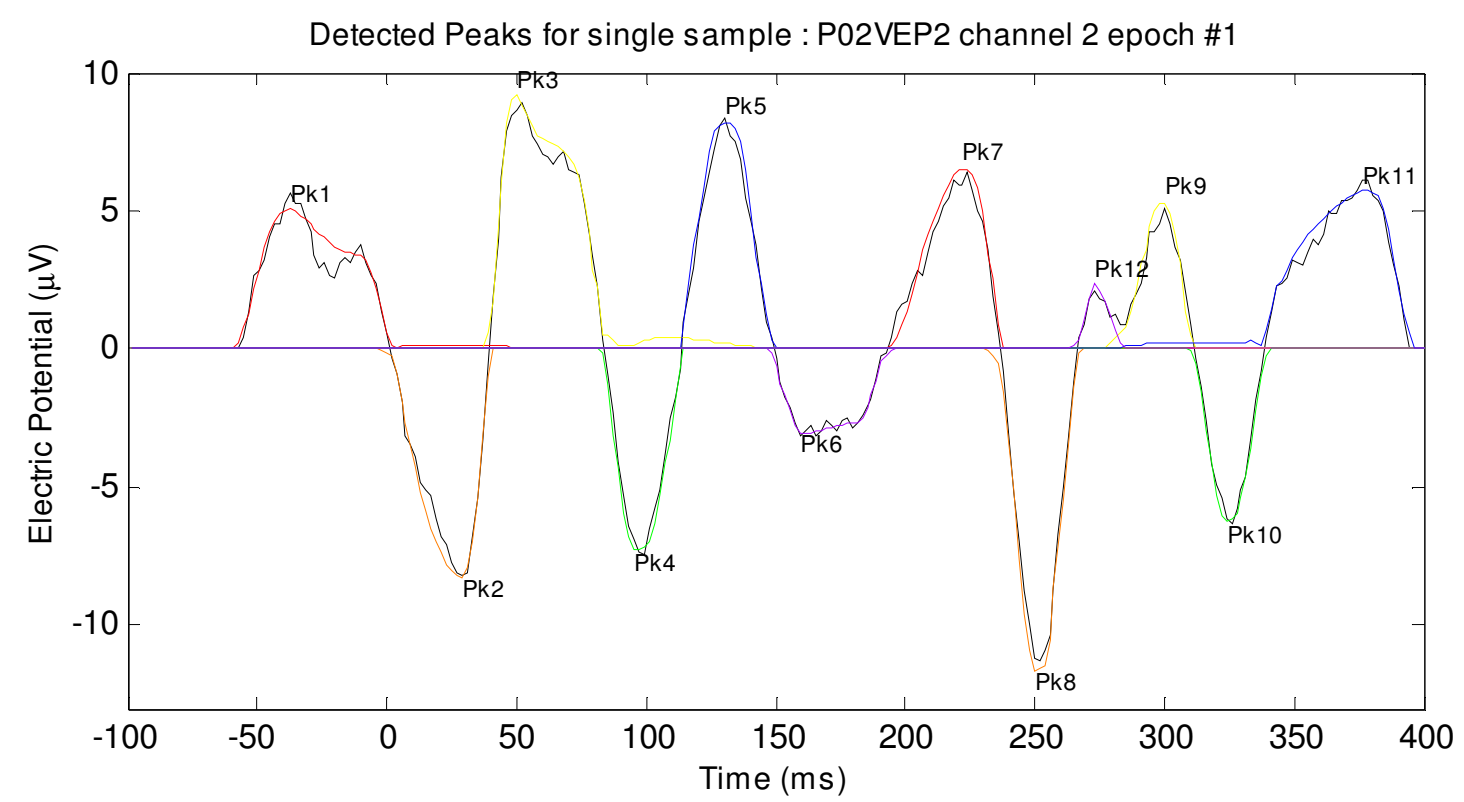

Fig. 5.3: Detected peaks for a sample epoch. The peak labels indicate the order in which peaks are detected. The peak labeled Pk12 was not detected on the first iteration of the algorithm but was detected on the second iteration after it was satisfactorily separated from the adjacent Pk9.

correlation coefficient. These five epochs were deleted from the dataset leaving 155 epochs with a correlation coefficient mean centred at 0.994 and a standard deviation of 0.0036. Each of these 155 epochs had a correlation coefficient greater than or equal to 0.96 .

Across these 155 epochs, there were a total of 1985 peaks detected yielding an average of just under 13 peaks per epoch.

The detected peaks were summed to form epochs that closely approximate the original pre-processed signal. Next, these summed detected peaks were averaged and compared to the average of the pre-processed epochs. This is illustrated in Fig. 5.5. The Pearson correlation coefficient between these two averaged signals is 0.9996 . 


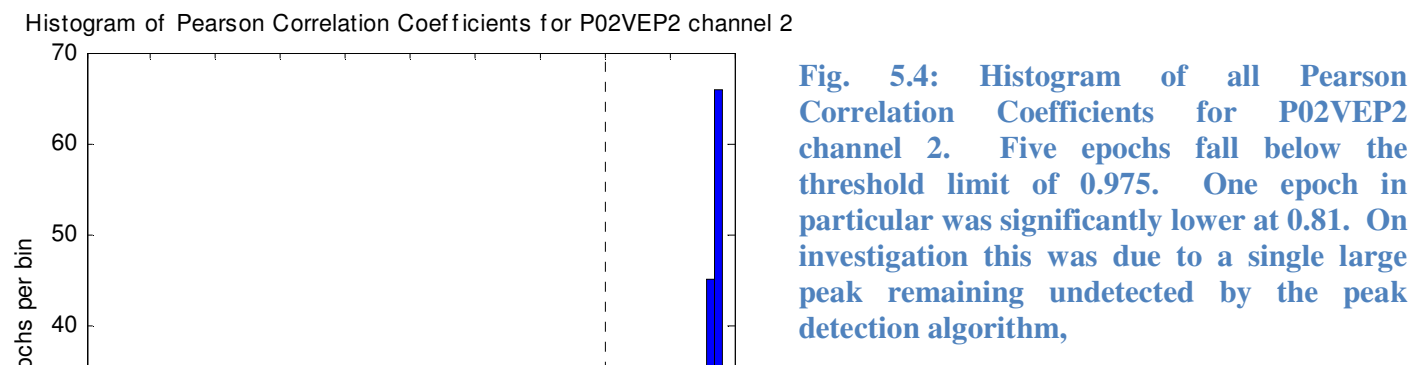

This completes the first results stage. A set of VEP epoch data has been decomposed into sets of discrete peaks and these peaks when summed together give a close approximation to the original epoch data.

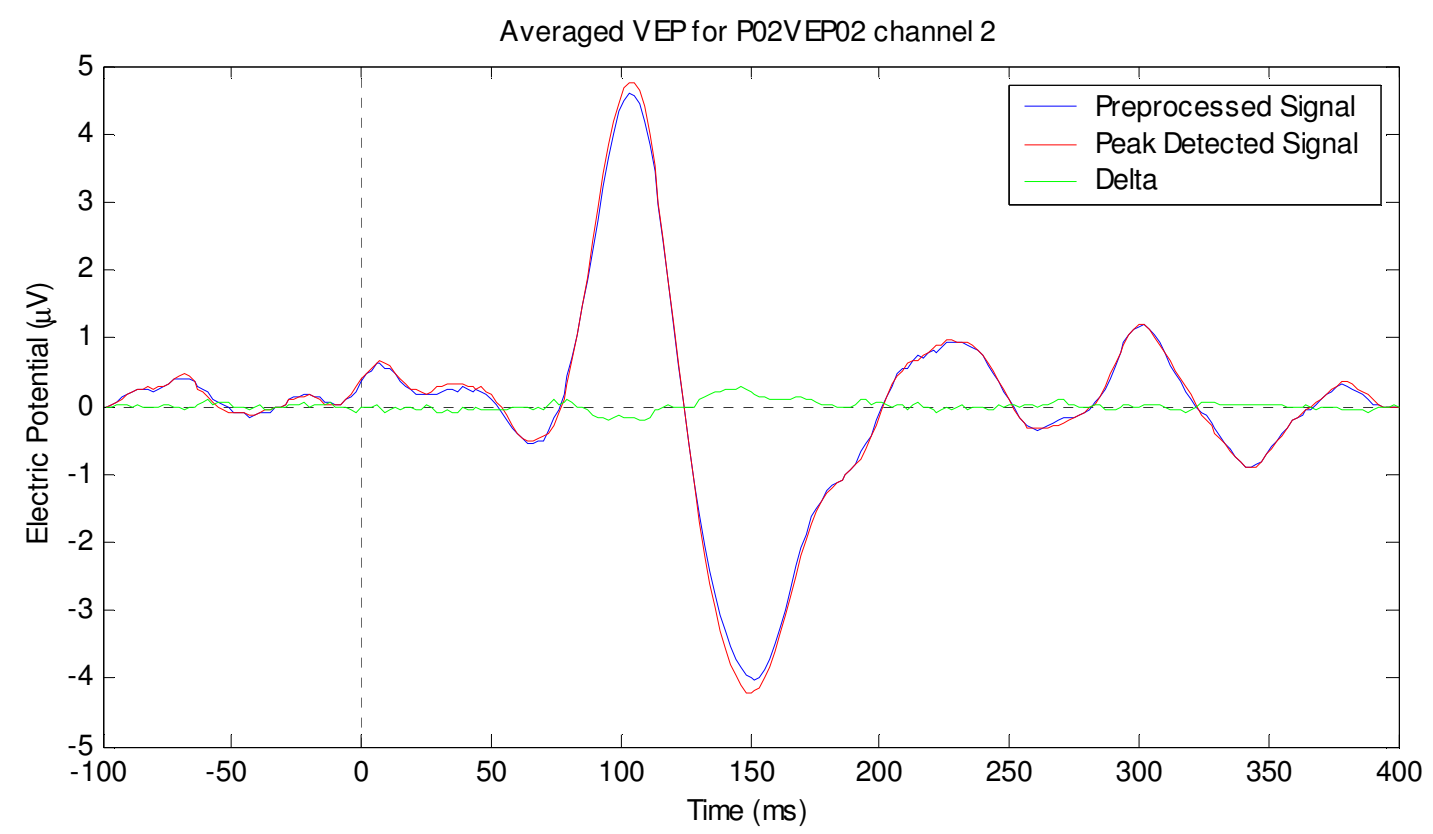

Fig. 5.5: Comparison of the pre-processed average VEP response and the Peak Detected average VEP response. The Pearson correlation coefficient is 0.9996 indicating a very close match.

\subsubsection{Peak Characterisation Results}

Since each epoch has been decomposed into a set of peaks, one may characterize these peaks individually. This is distinct from the standard averaging technique where peaks can only be analysed as part of an entire epoch. The characteristics chosen are discussed in section 3.6.2 and are either intrinsic (i.e. a standard output parameter from the wavelet transform based peak detection process) or calculated from the final peak signal shape 
itself. The parameters are listed in Table 5.2 along with the range of each parameter for the P02VEP02 channel 2 dataset.

\begin{tabular}{|l|c|c|c|c|c|}
\hline Parameter Name & Label & Type & Minimum & Maximum & Units \\
\hline orientation or sign & $\mathrm{s}$ & Intrinsic & -1 & +1 & - \\
\hline approximation position & $\mathrm{A}_{\text {pos }}$ & Intrinsic & -80 & 400 & $\mathrm{~ms}$ \\
\hline approximation maximum & $\mathrm{A}_{\max }$ & Intrinsic & -48 & 57 & - \\
\hline approximation scale level & $\mathrm{A}_{\mathrm{lvl}}$ & Intrinsic & 2 & 6 & - \\
\hline peak time centre & $\mathrm{t}$ & Calculated & -82 & 394 & $\mathrm{~ms}$ \\
\hline peak width or scale & $\mathrm{w}$ & Calculated & 4 & 103 & $\mathrm{~ms}$ \\
\hline peak height or maximum & $\mathrm{h}$ & Calculated & 0.9 & 26 & $\mu \mathrm{V}$ \\
\hline area under peak & $\mathrm{a}$ & Calculated & 2 & 735 & $\mathrm{nVs}$ \\
\hline
\end{tabular}

Table 5.2: Peak parameters, label, type and range of values found for P02VEP2 channel 2 dataset

\subsubsection{VEP Calculated Parameters}

Calculated parameters were evaluated by direct examination of the output peak signal calculated from the inverse discrete wavelet transform of the coefficients that characterized each particular peak. The calculated parameters were time location, $t$, peak width, $w$, peak height, $h$ and area under each peak, $a$. Each parameter is calculated by

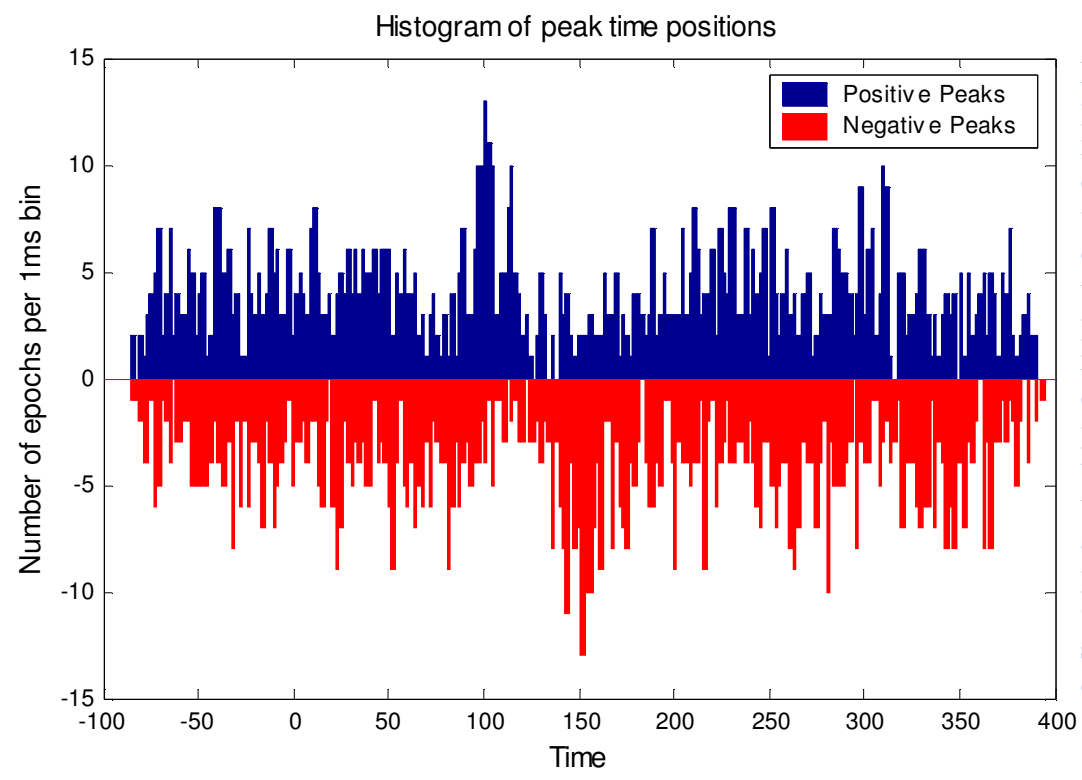

examination of

Fig. 5.6: Histogram of time position for all positive and negative peaks for P02VEP2, channel 2 - 1985 time position values. The distribution tails off at the beginning and end due to the zeroing of partial edge peaks. There is a prominent increase in positive peaks and coincident reduction in negative peaks at around $100 \mathrm{~ms}$. Also, there is a prominent increase in the amount of negative peaks and coincident reduction in positive peaks at around $150 \mathrm{~ms}$. Otherwise although there is some variation, there are no obvious patterns .

the individual

reconstructed peaks such as those shown in Fig. 5.3. For the dataset P02VEP2, channel 2 there are 1985 peaks, hence there are 1985 values for each calculated parameter chosen. Let us examine the distributions within these parameters in Fig. 5.6 to Fig. 5.11. A summary of points of note is included in the caption for each figure. 


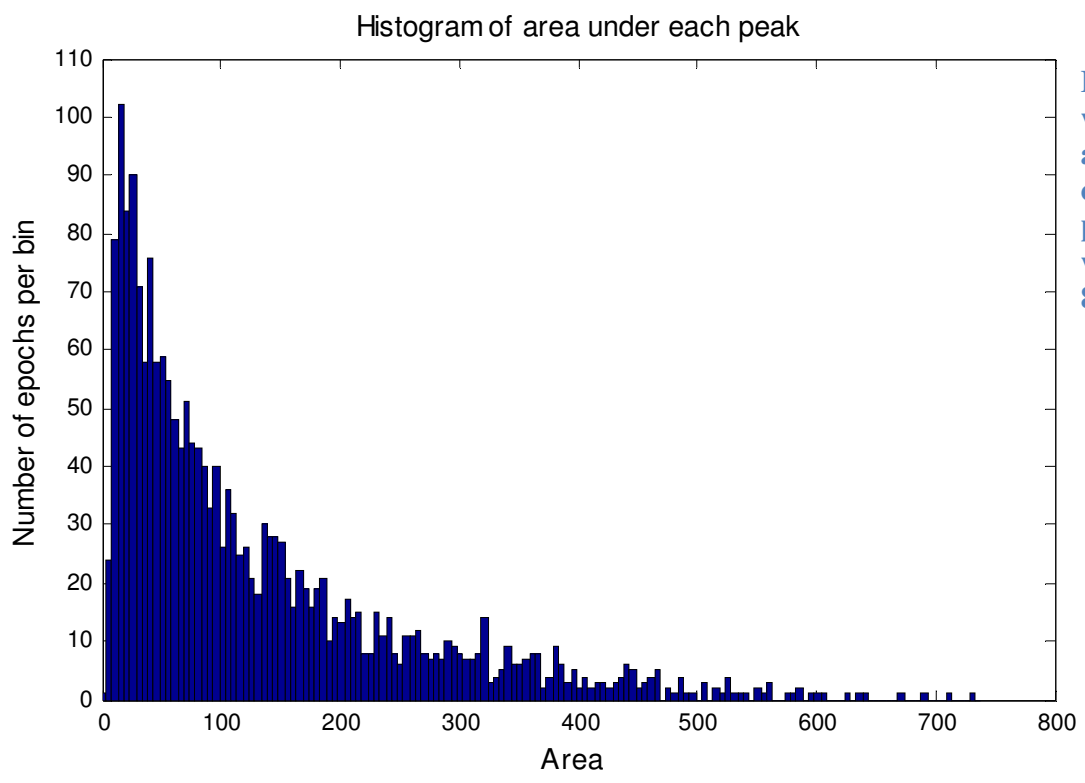

Fig. 5.7: Histogram of peak widths for all peaks (positive and negative) for P02VEP2, channel 2. The majority of peaks have a maximum peak width located in the $8 \mathrm{~ms}$ to $80 \mathrm{~ms}$ range.

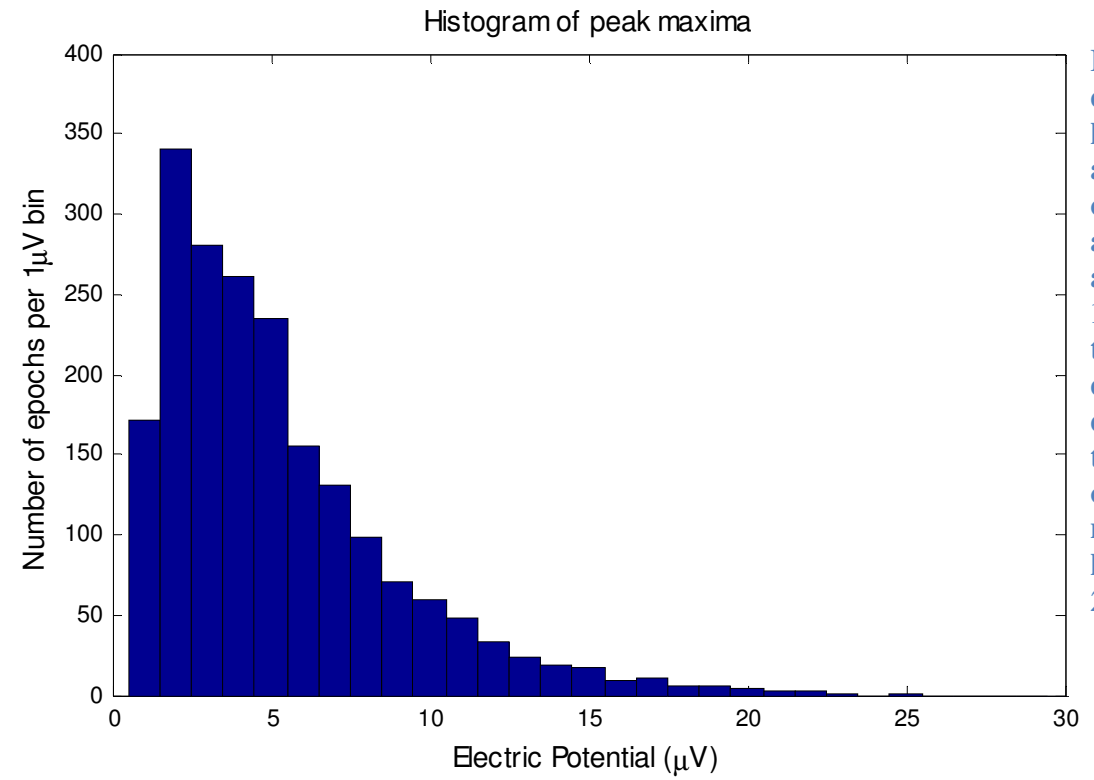

Fig. 5.8: Histogram of peak electric potential maxima (peak height) for all peaks (positive and negative) for P02VEP2, channel 2. The distribution appears roughly Gaussian with a noticeable reduction at the $1 \mu \mathrm{V}$ level. This is attributable to some small peaks being eliminated from the peak detection process as they are too small to be either detected or significant. The great majority of peaks have a peak height located in the $1 \mu \mathrm{V}$ to $20 \mu \mathrm{V}$ range.

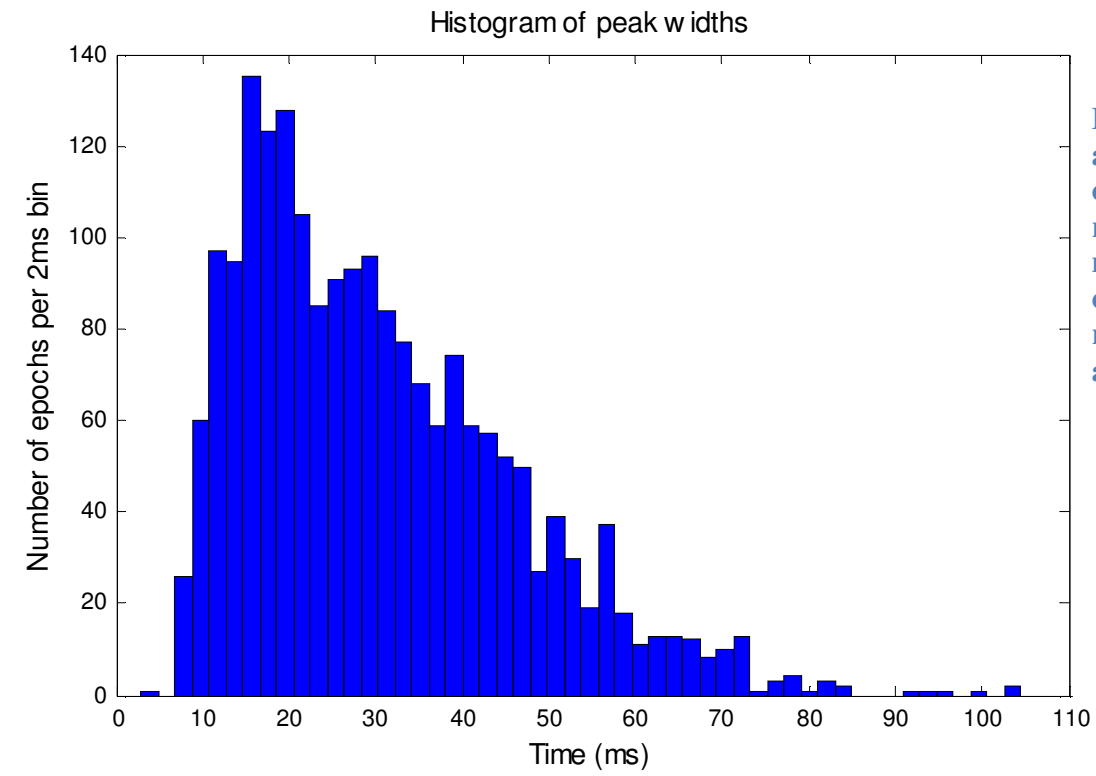

Fig. 5.9: Histogram of the area under each curve calculated from the reconstructed positive and negative peaks for P02VEP2, channel 2. This parameter is related to both the peak width and the peak height. 


\subsubsection{VEP Intrinsic Parameters}

Intrinsic parameters are taken from the discrete wavelet transform process used to identify particular peaks. Again for P02VEP2, channel 2 there were 1985 peaks and so each of the following distributions shown in Fig. 5.10 and Fig. 5.11 is based on a 1985 samples.

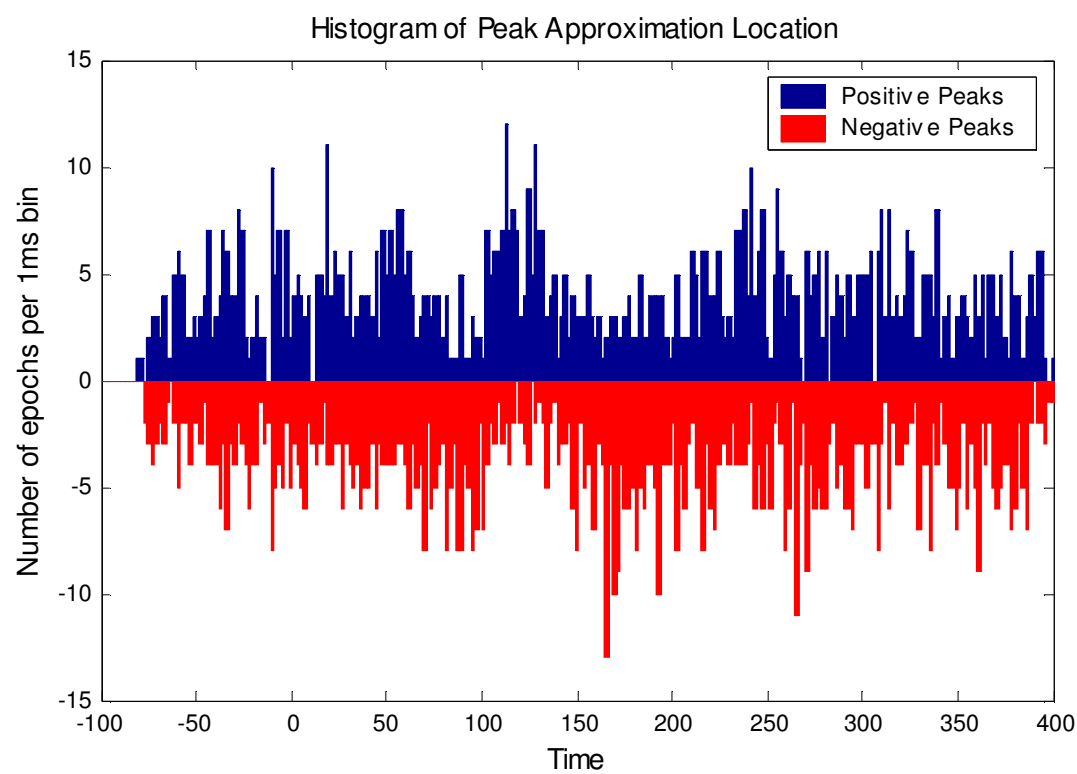

Fig. 5.10: Histogram of peak approximation location for P02VEP2, channel 2. The distribution shows a similarity to Fig. 5.6.

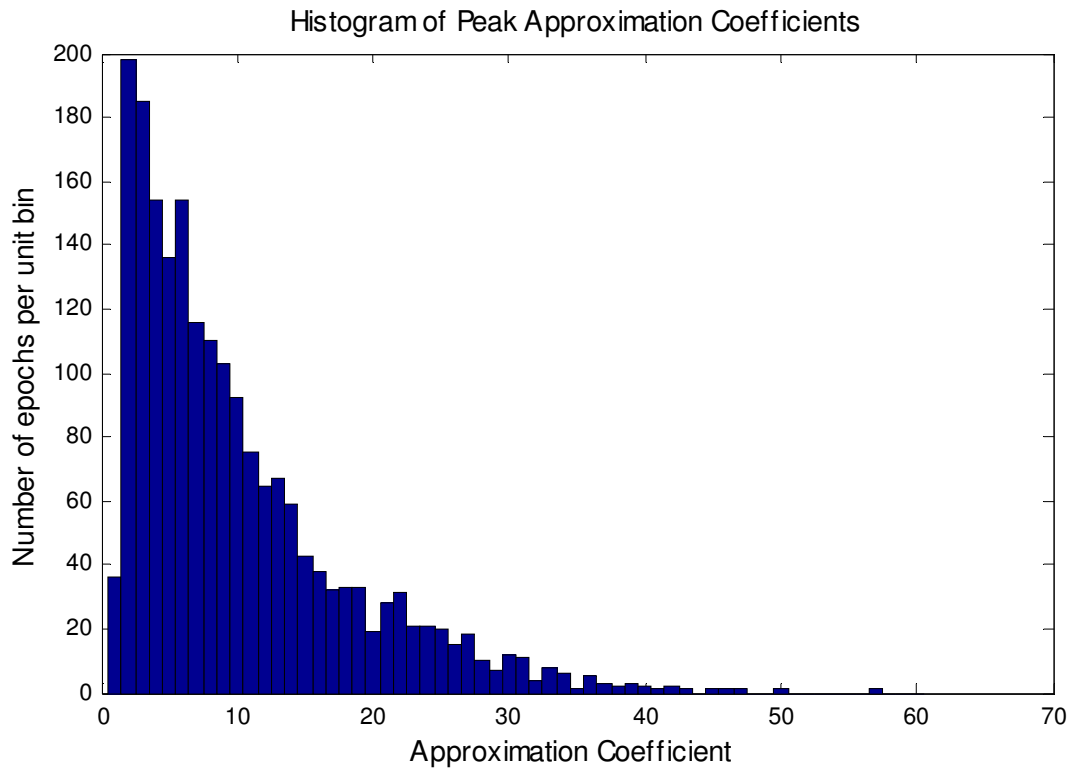

Fig. 5.11: Histogram of modulus of peak approximation values for all positive and negative peaks for P02VEP2, channel 2. Approximation values are sign sensitive i.e. negative approximation indicates a negative peak. However, the modulus of all approximation coefficients is shown here.

\subsubsection{EEG Calculated Parameters}

The EEG dataset P02EEG2, channel 2 was applied to the peak detection algorithm from which the total number of peaks across all 155 epochs was 1987. For comparison, the distributions of the calculated parameters for these peaks are presented as Fig. 5.12, Fig. 5.13 and Fig. 5.14. 


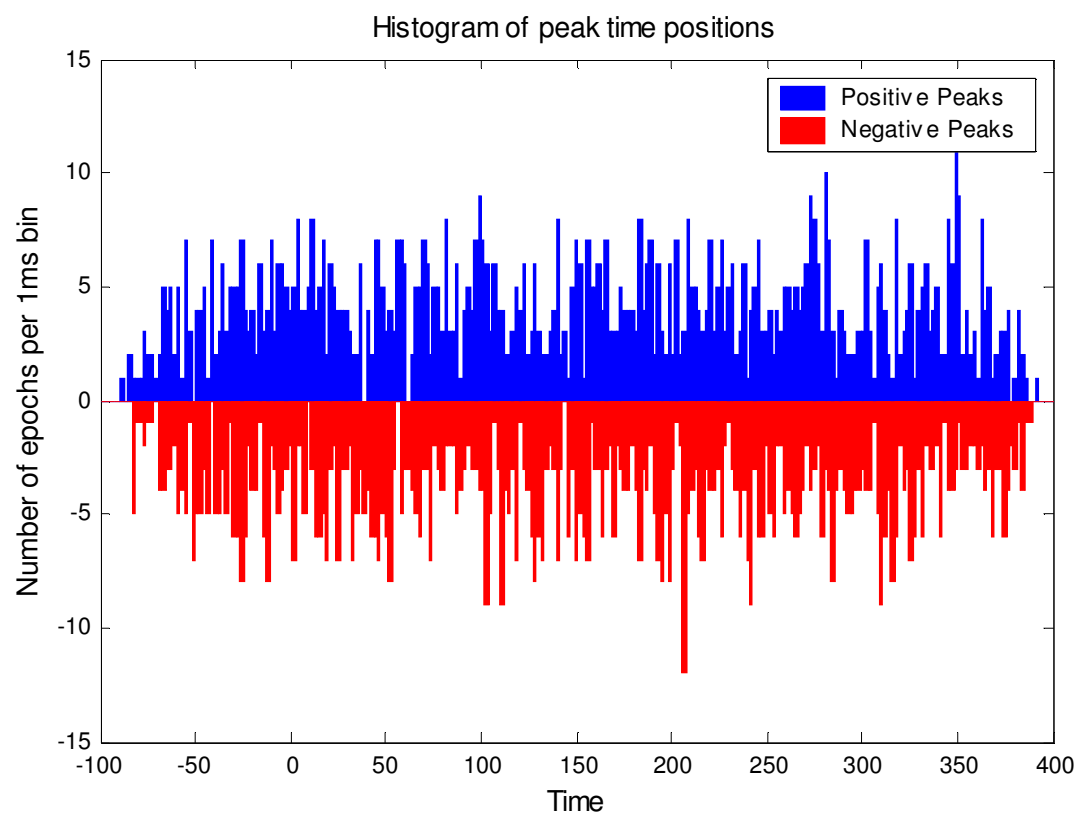

Fig. 5.12: Histogram of the time position of all positive and negative peaks for P02EEG2, channel 2 . The general distribution is similar to that seen in Fig. 5.6 but there are no discernible prominent features.

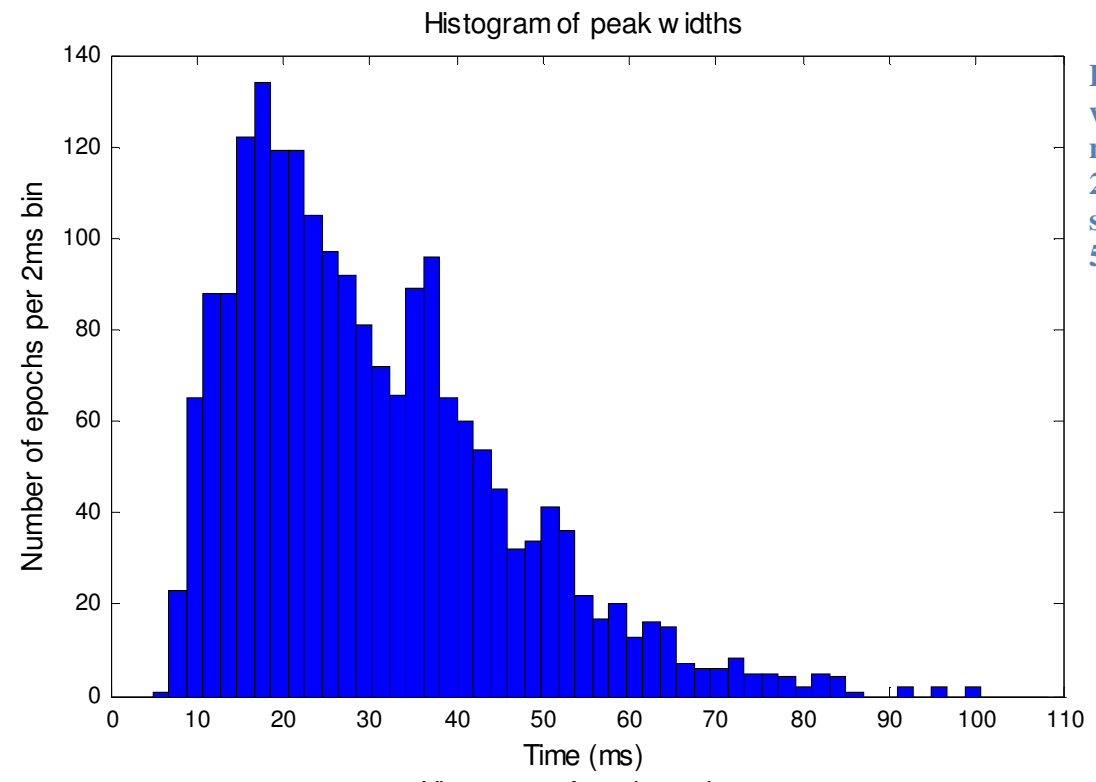

Fig. 5.13: Histogram of peak widths all peaks (positive and negative) for P02EEG2, channel 2. The distribution appears very similar to that observed in Fig. 5.7.

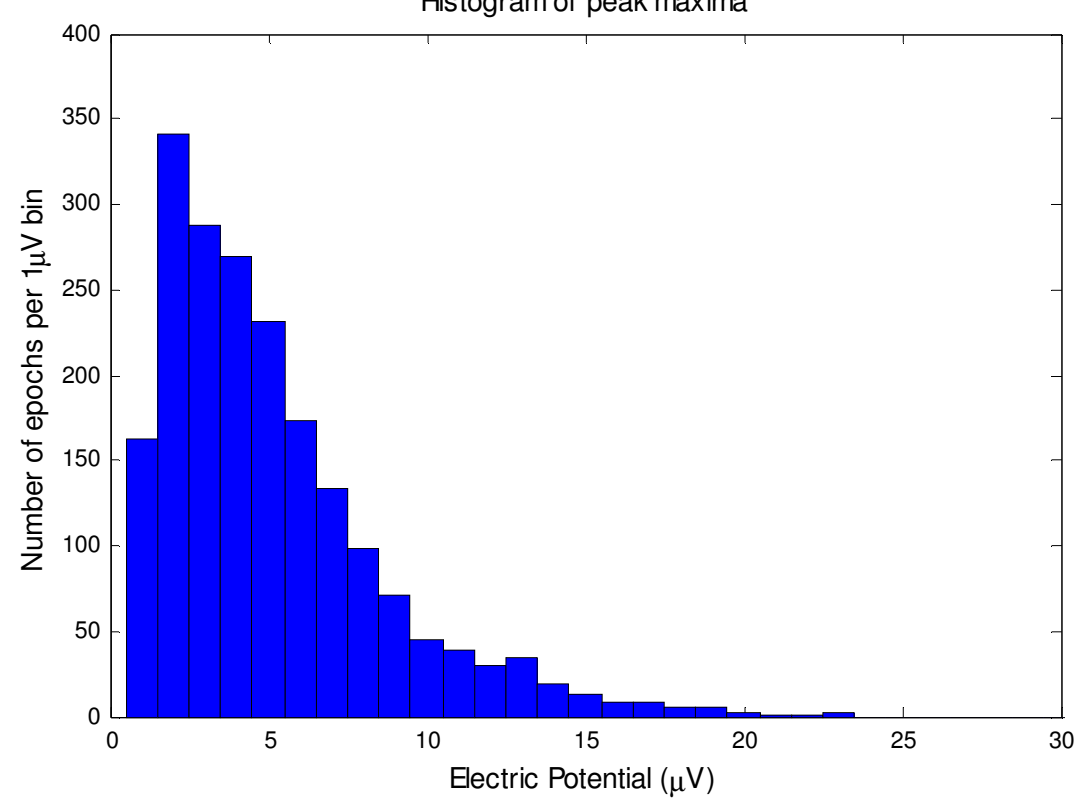

Fig. 5.14: Histogram of peak electric potential maxima for all peaks (positive and negative) for P02EEG2, channel 2. The distribution appears very similar to that observed in Fig. 5.8 . 


\subsubsection{Peak Clustering Results}

Having examined distributions of individual parameters in the previous section, multiple parameters are now examined simultaneously. This is achieved using the clustering technique, described in section 0 , to examine the following 3 parameters: peak time position $(t)$, width of the peak $(w)$ and height of the peak $(h)$ - illustrated in Fig. 5.6, Fig. 5.8 and Fig. 5.7 respectively. Following pre-processing of these parameters, a threedimensional matrix with dimensions $(t, w, h)$ was populated. Each peak fills a specific volume of the matrix determined by the size of each of the 3 parameters used. The result is a concentration matrix where high concentration areas represent a large number of peaks present.

The mean of this matrix across all time locations (but still as a function of width and height) was calculated and subtracted from the entire concentration matrix to better identify the highest concentration of peaks due to stimulus response and not just background EEG. Fig. 5.15 illustrates the mean concentration profile across all time locations in width and height dimensions for P02VEP2, channel 2.
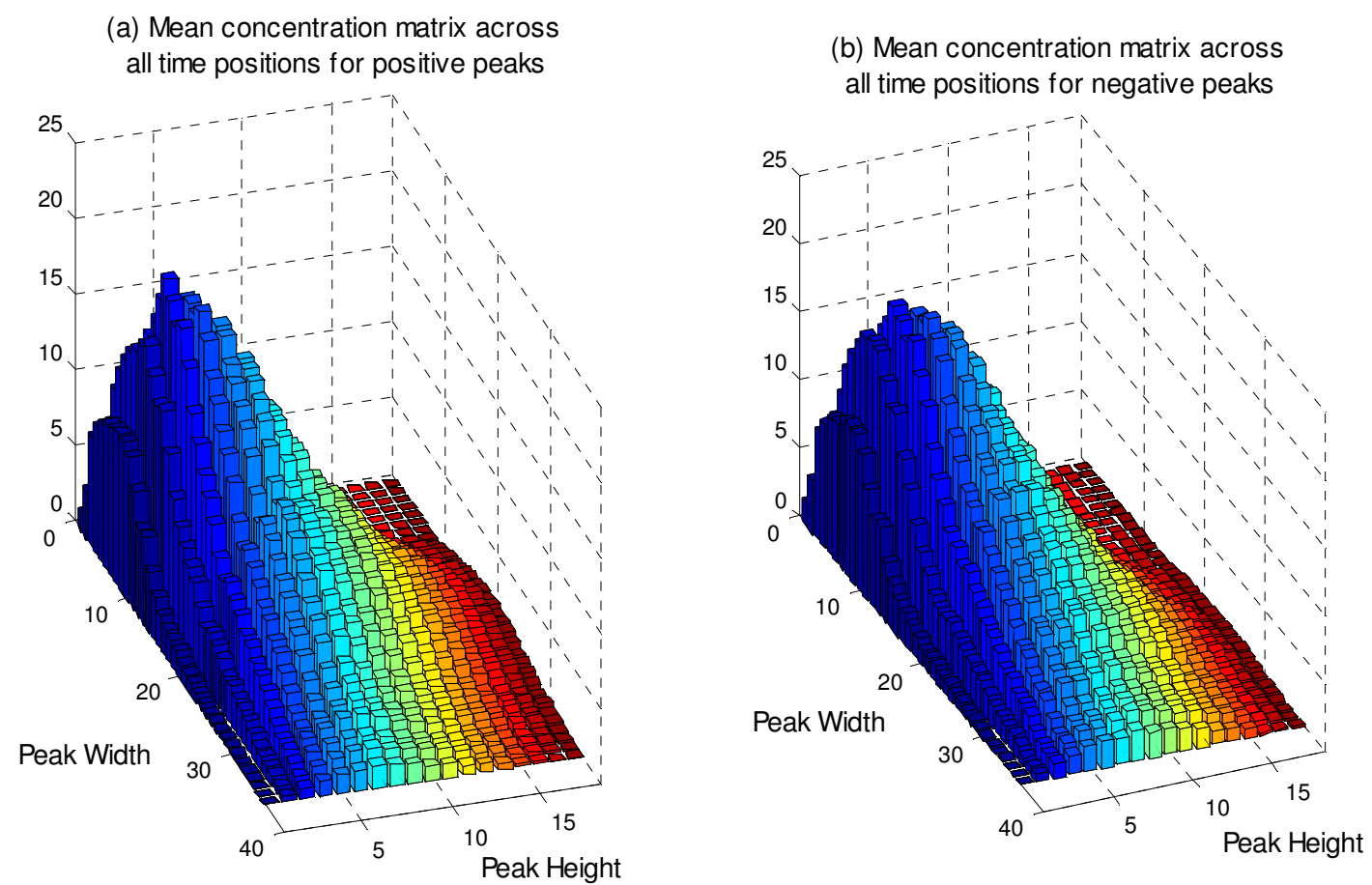

Fig. 5.15: The mean concentration matrix across all time positions for (a) positive peaks and (b) negative peaks for P02VEP2. This distribution is subtracted from each time location slice so that bin locations across time, width and height are comparable.

This clustering technique is applied to channel 2 of both P02VEP2 and P02EEG2. In Fig. 5.16 , the peak concentration profile is illustrated by plotting the highest concentration 
bins in sequential order (highest concentration first). Fig. 5.16(a) is ordered according to highest peak concentration per bin above the mean concentration for that bin. Fig. 5.16(b) is ordered according to the significance metric. Significance is a calculated parameter that reflects the scaled product of number of peaks and average area under all peaks in a particular bin. It more accurately reflects the standard averaging process. The deviation between the blue and red lines in Fig. 5.16 is an indication of the presence of Visual Evoked Potentials. The first two bins of Fig. 5.16 (a) and Fig. 5.16 (b) are much higher than the rest of the distribution (almost twice the number of peaks present).

Fig. 5.17 and Fig. 5.18 illustrate the peaks that form first two bins, Bin\#1 and Bin\#2 respectively. In (a) each of the peaks that has been characterised into the bin is superimposed upon each other to roughly illustrate the range of peak sizes within this bin. The bin sizes of 48 and 47 correlate directly to the peak concentration (above the mean) of 39.1 and 38.2 respectively. The difference is due to the average expected bin size for these two bins. Hence in Bin\#1 there were 8.9 epochs expected here on average. In this particular bin there were actually 48 epochs which is 39.1 epochs above average which is plotted in Fig. 5.16(a). In (b) all bins are sorted by the significance factor which alters some bin ordering but the first two bins are the same as that in (a).

Examining these bins more closely, the individual peaks that are part of each bin were plotted in Fig. 5.19 together with a comparison of the mean of these peaks with the standard average VEP response. As well as averaging the peaks contained in particular bins, we can also use the distribution of peaks within any bin to determine the average characteristic peak shape for that bin. Table 5.3 illustrates the bin parameters for Bin\#1 and Bin\#2.

\begin{tabular}{|c|c|c|c|c|c|c|c|}
\hline \multirow[b]{2}{*}{ Bin } & \multirow[b]{2}{*}{ No. of Epochs } & \multicolumn{2}{|c|}{$\begin{array}{l}\text { Concentration } \\
\text { Location }\end{array}$} & Matrix & \multicolumn{3}{|c|}{$\begin{array}{l}\text { Mean over } \\
\text { all peaks per bin }\end{array}$} \\
\hline & & Time & Width & Height & Time & Width & Height \\
\hline$\# 1$ & 48 & 65 & 25 & 9 & $129.3 \mathrm{~ms}$ & $25.3 \mathrm{~ms}$ & $9.2 \mu \mathrm{V}$ \\
\hline$\# 2$ & 47 & 52 & 23 & 10 & $104.2 \mathrm{~ms}$ & $22.6 \mathrm{~ms}$ & $9.8 \mu \mathrm{V}$ \\
\hline
\end{tabular}


(a) Highest peak concentration profile

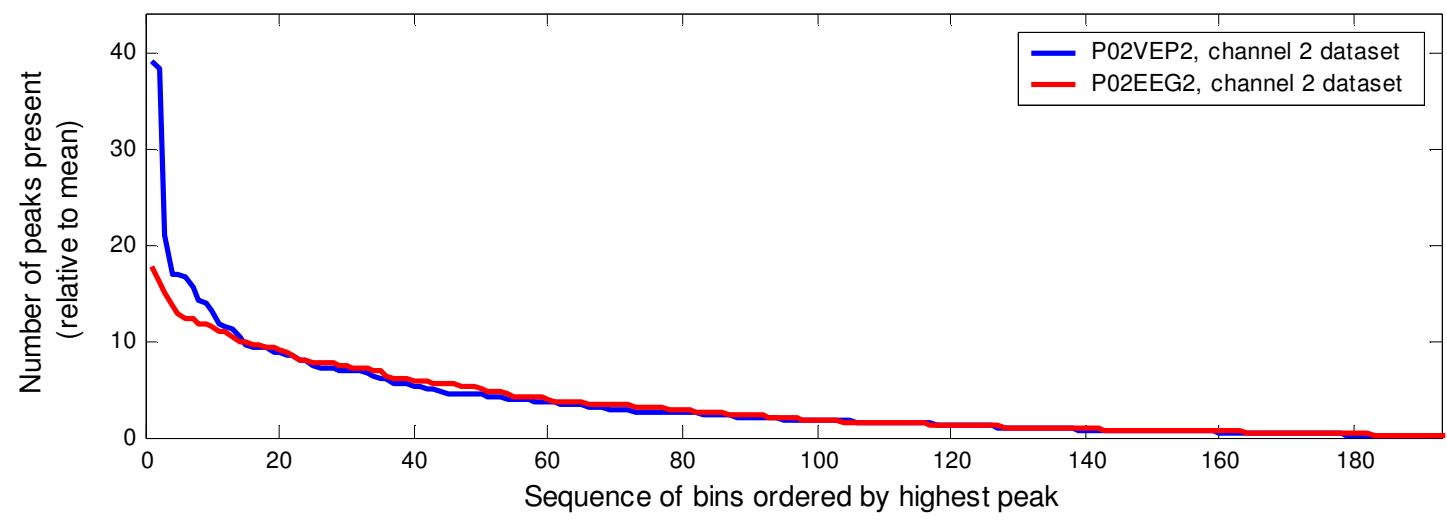

(b) Significance (Peak Concentration X Peak Area) Profile

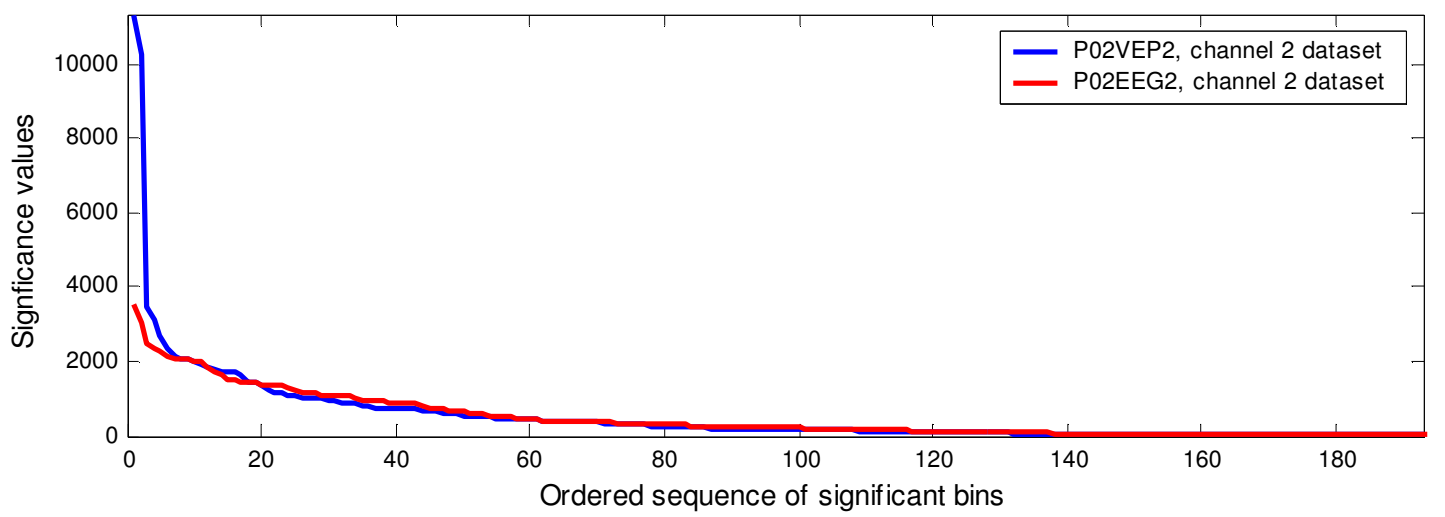

Fig. 5.16: Peak concentration profiles. (a) Relative bin concentrations are ordered and plotted against the number of peaks in each bin (relative to mean). So for P02VEP2 the first bin has 39.1 more peaks than the average for that particular height and width. The sequence of bins is plotted down to zero numbers of peaks. At this level average bin concentration levels appear. Continuation of axis would yield negative numbers of peaks present indicating bins that have below average bin concentration. However, we are interested in the above behaviour and in particular the deviation from the background EEG (P02EEG2) behaviour. This is evident in the first two peaks. (b) By examining the significance factor rather than the number of peaks, we focus on the types of peaks that will contribute most towards the standard VEP average profile. Overall, the profile shapes in (b) are similar to (a) as the number of peaks is the most evident factor of variation. However, some local rearrangement is evident after the first 2 bins. 
(a) plot of all peaks within selected bin for P02VEP2 ch 2

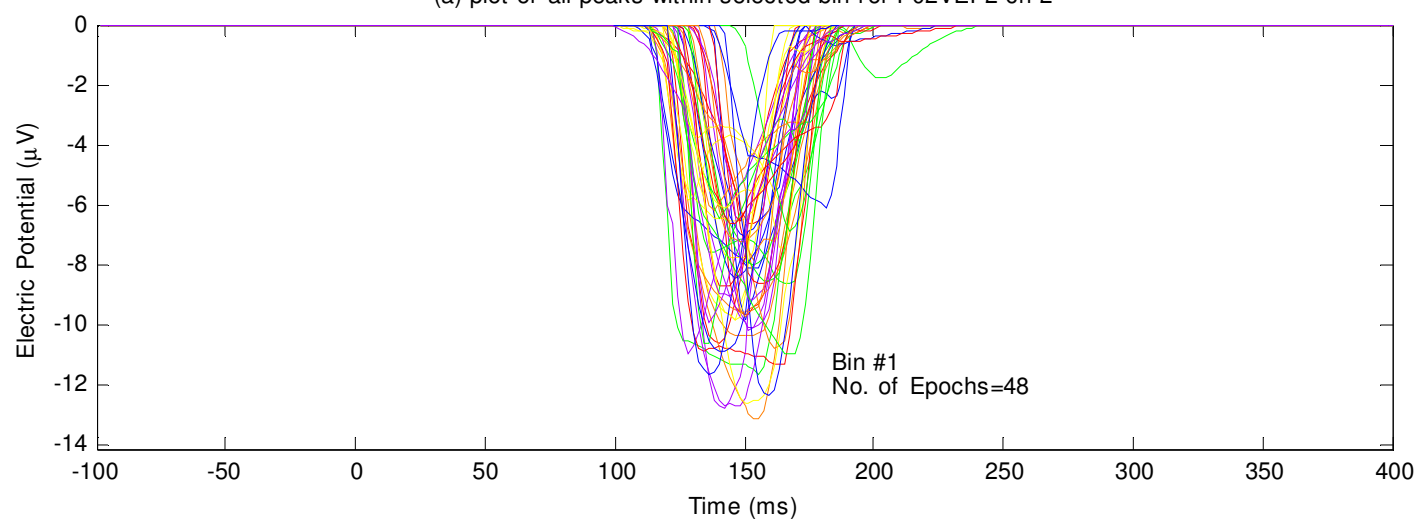

(b) plot of av eraged signals for P02VEP2 ch 2

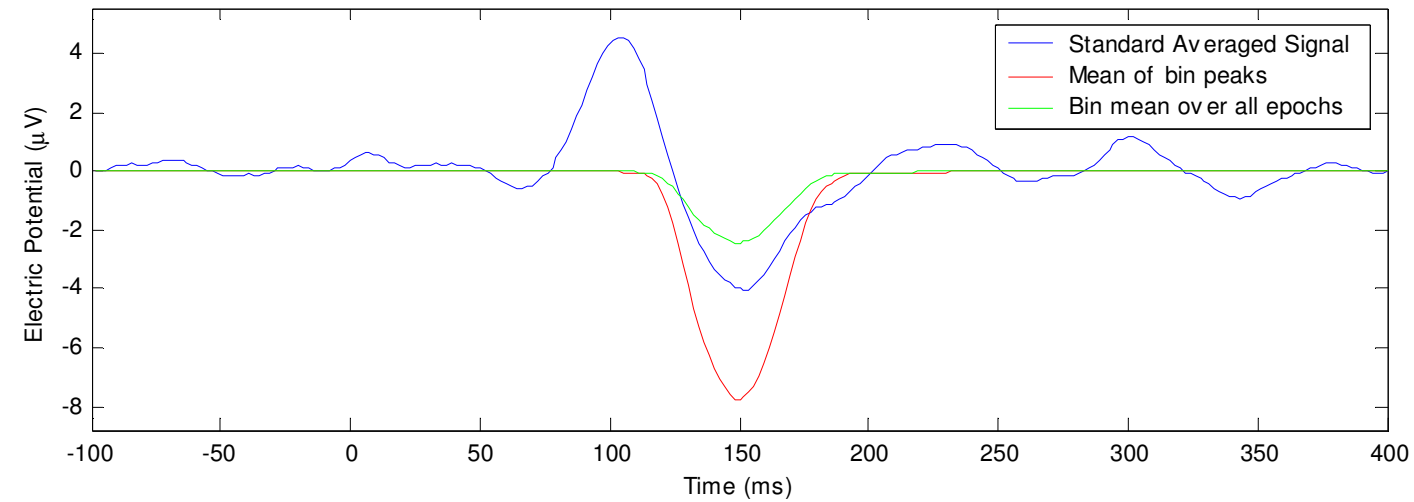

Fig. 5.18: (a) Plot of Bin\#1 peaks - superimposed to demonstrate level of variation within bin. (b) Comparison of standard average for P02VEP2 shown in blue versus the average of Bin\#1 peaks shown in red. (peaks are added together and divided by number of peak (or epochs) which is 48 . Also shown is the average of Bin \#1 peaks divided by total number of peaks which is 155 for P02VEP2. Hence the green curve represents the contribution of Bin \#1 directly towards the averaged signal in blue.

Fig. 5.17: (a) Plot of Bin\#2 peaks. (b) Comparison of standard average for P02VEP2 shown in blue versus the average of Bin\#2 peaks shown in red (divided by 48 ). The average of Bin \#2 peaks divided by 155 , the total number of peaks. The green curve represents the contribution of Bin \#2 directly towards the averaged signal in blue.

(a) plot of all peaks within selected bin for P02VEP2 ch 2

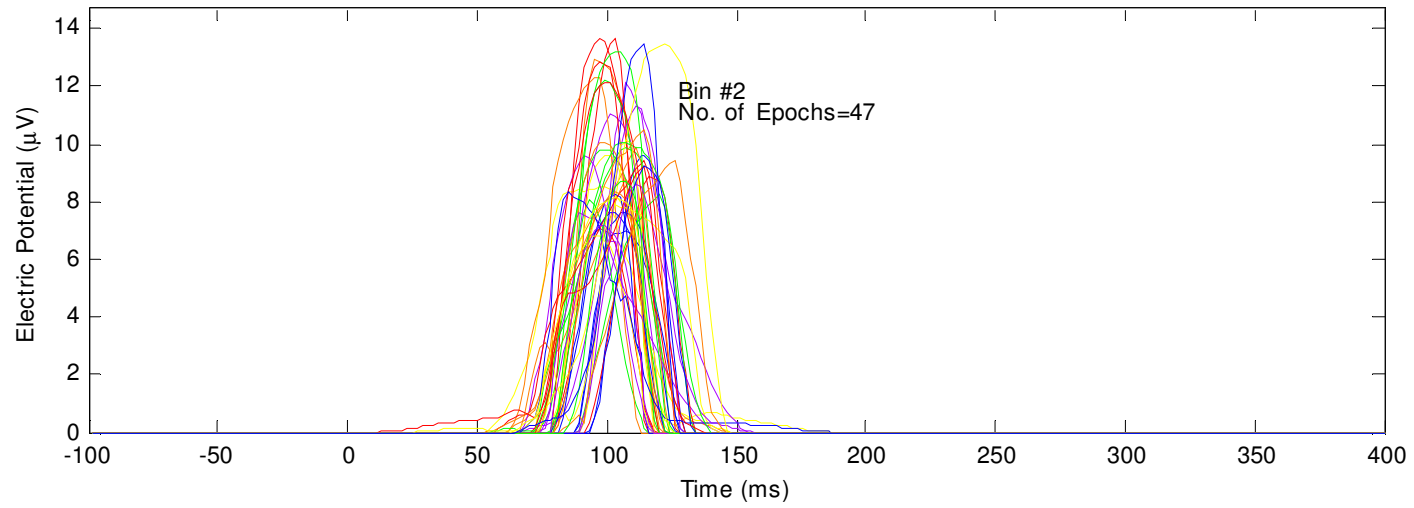

(b) plot of av eraged signals for P02VEP2 ch 2

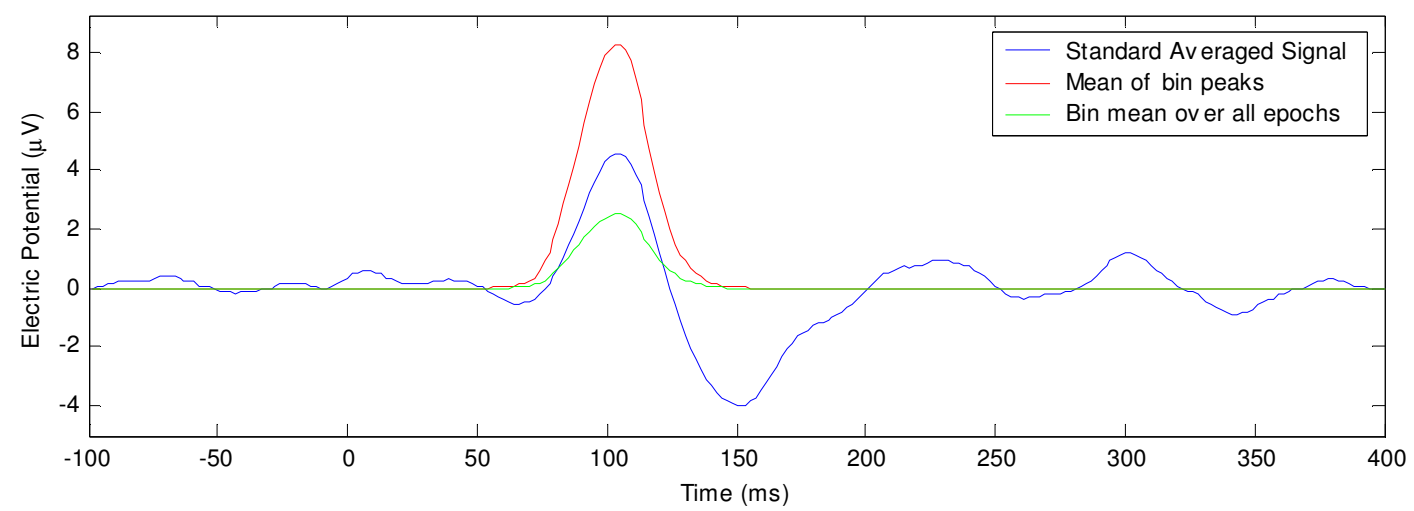


As well as averaging the peaks contained in particular bins, we can also use the distribution of the peak concentration profile to determine the average characteristic peak shape. The bin average, mean peak shape and equivalent peak average are shown in Fig. 5.19 for Bin \#1 and Bin \#2 together.

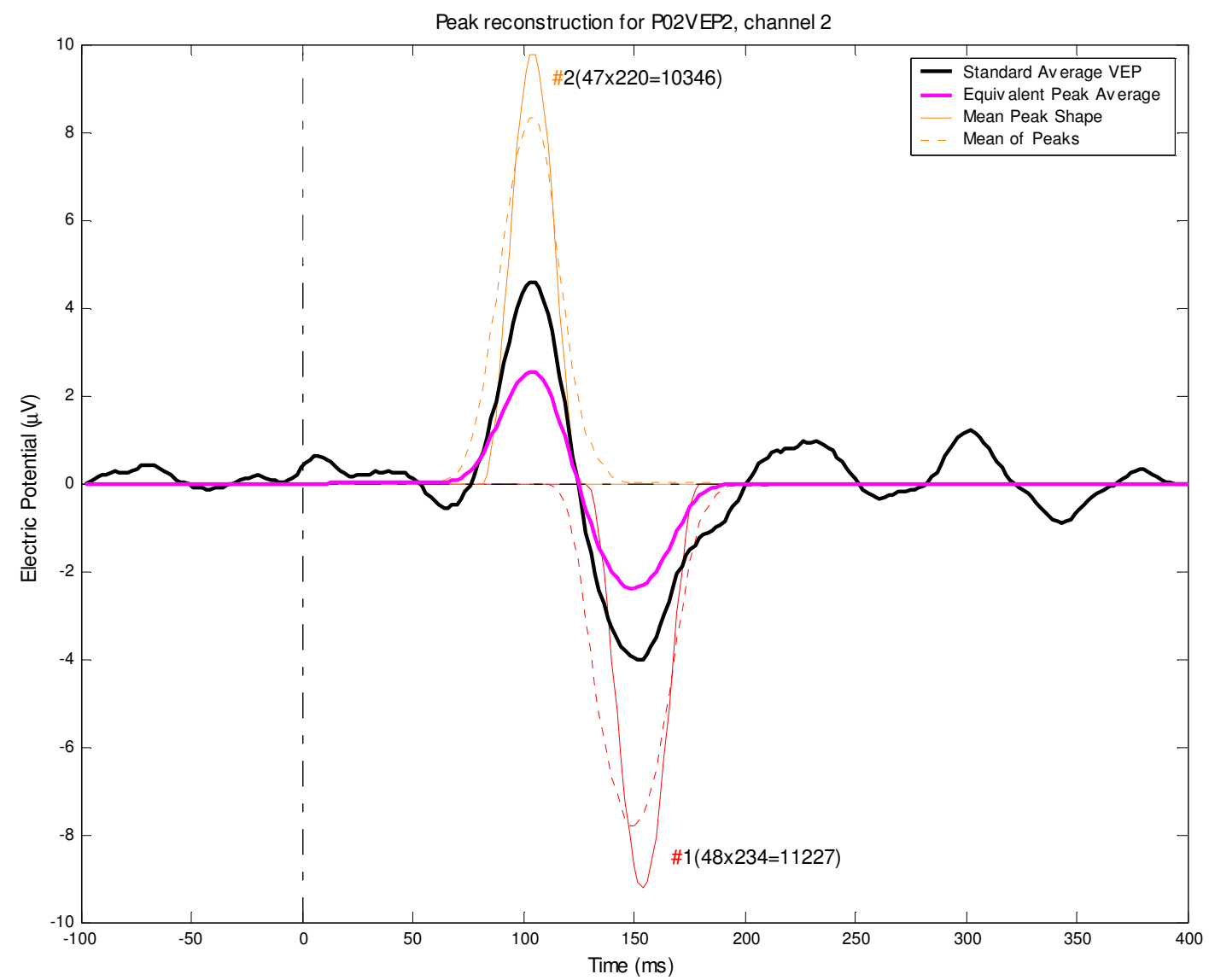

Fig. 5.19: Reconstructed peaks for Bin\#1 and Bin\#2. The ordering of bins is based on the number of peaks multiplied by average area under curve. So in Bin\#1, there are 50 epoch peaks with an average area of 120. This gives a factor of 5987. Bin \#2 has slightly lower factor of 5431 and so on. The bold black line is the standard average VEP. The dashed coloured lines represent the average of the peaks in the particular bin alone (i.e. in Bin\#1 the average of 50 epoch peaks). The non-dashed thin coloured line represents the peak shape calculated from the mean of the concentration matrix variables. Hence the peak in Bin\#1 is centered at $130.6 \mathrm{~ms}$ with a width of $23.3 \mathrm{~ms}$ and a height of $9.1 \mu \mathrm{V}$. By adding all the peaks from Bin\#1 and Bin\#2 and then dividing by the total number of epochs (160 for this dataset), you get the magenta bold line. Comparing the black and magenta lines gives an indication of how close the peaks of Bin\#1 and Bin\#2 match the averaged signal. These signals are directly comparable since they are both averaged by the total number of epochs. The Pearson Correlation coefficient between these two lines is calculated as 0.95.

By adding more bins and evaluating the correlation coefficient of the cumulative peaks against the standard average VEP signal, the set of bins or peaksets more closely approximate the standard averaged VEP signal. It is a trade-off between the number of bins chosen and the preferred level of correlation. Essentially, the aim is to represent the averaged VEP signal using as few bins as possible. If too few bins are chosen then there 
will not be enough resolution to pick out subtle features. If too many bins are chosen then the visual inspection task, that is a necessary follow-on, becomes onerous and confused. Fig. 5.20 plots the peak profiles for the first 22 bins. 22 bins were chosen as this number gives a good trade-off between getting the best match to the standard averaged VEP signal and still being of a manageable size for analyzing.

Thus the standard average VEP signal for P02VEP2 channel 2, has been decomposed into 22 peaks. Each of these peaks may be represented by the average peak shape in the local area (the coloured thin continuous lines in Fig. 5.20). The peak may also be represented by the mean of the peaks within a particular bin (the coloured dashed thin line in Fig. 5.20). Fig. 5.21 illustrates the Pearson Correlation Coefficient between averaged VEP signal and the equivalent peak mean over increasing numbers of added bins (or peaksets). The circled point represents the local maximum correlation coefficient that occurs after 22 peaks as shown in Fig. 5.20 which is 0.9835 . The correlation coefficient has levelled off by Bin \#22 with only a marginal improvement in correlation with added bins beyond this point.

\subsubsection{Effect of Bin Ordering}

Table 5.4 presents a table format version of the peak reconstruction graph of Fig. 5.20. Each line in the table represents one bin. This table is ordered by the significance metric in column 11 (total no. of peaks $X$ area under peak). However by reordering the full bin matrix (not just the 22 bins shown) using column 5 (total no. of peaks above average) a truer representation of the most repetitious peaks in the dataset is found. This is illustrated in Table 5.5 where the first 13 bins are ordered this way. The first two bins remain the same but otherwise the order of bins is quite different. Also there are 5 bins that did not appear in the first 22 bins shown in Table 5.4 and Fig. 5.20. The peak reconstruction is illustrated in Fig. 5.23.

The equivalent mean average (magenta) does not match the average signal as well as it did in Fig. 5.20. This is shown more clearly in Fig. 5.22 where the trend of the correlation coefficient after the first 13 bins is about 0.95 . This is lower than that achieved in Fig. 5.21 which was of the order of 0.98 . 


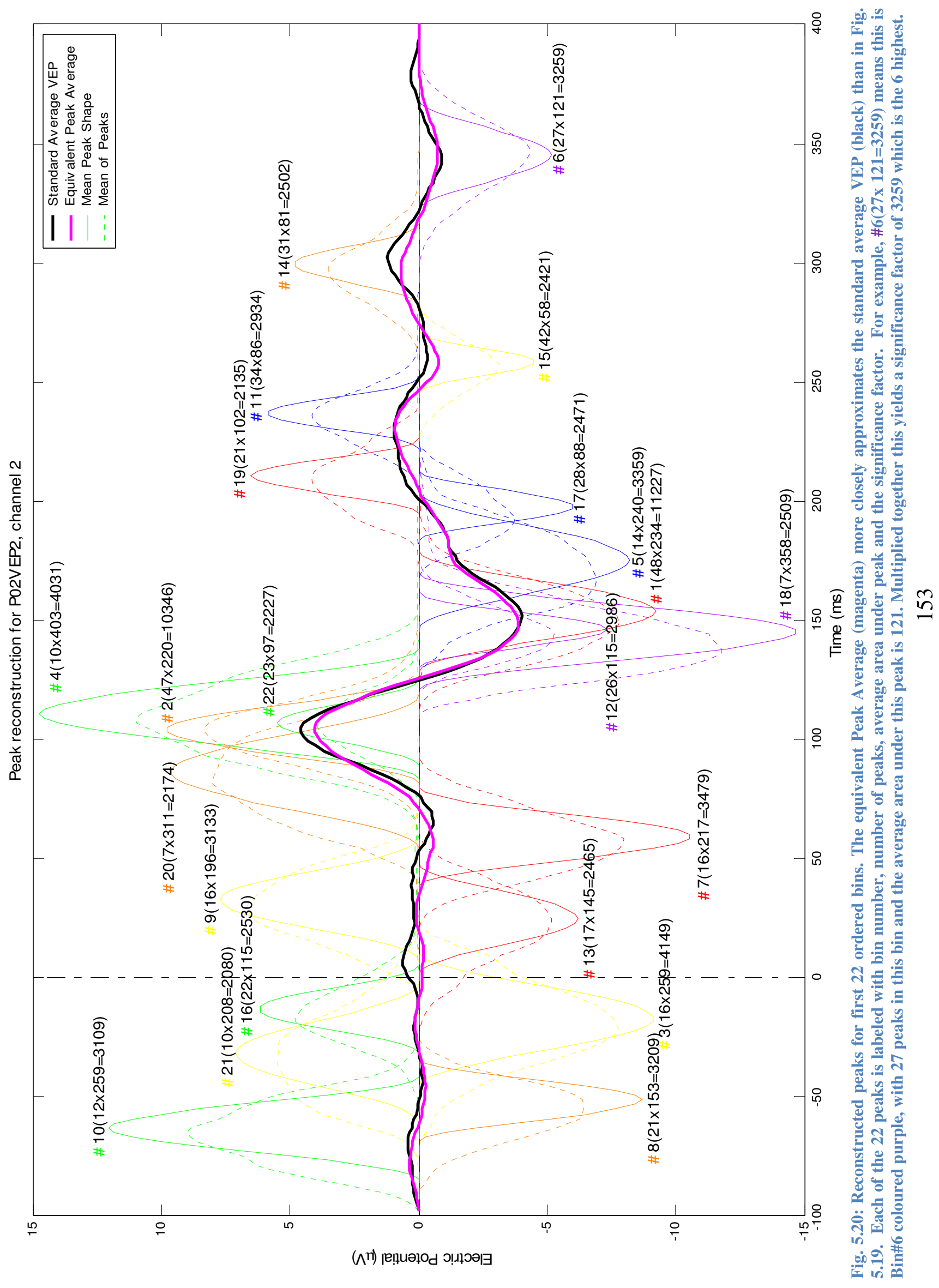




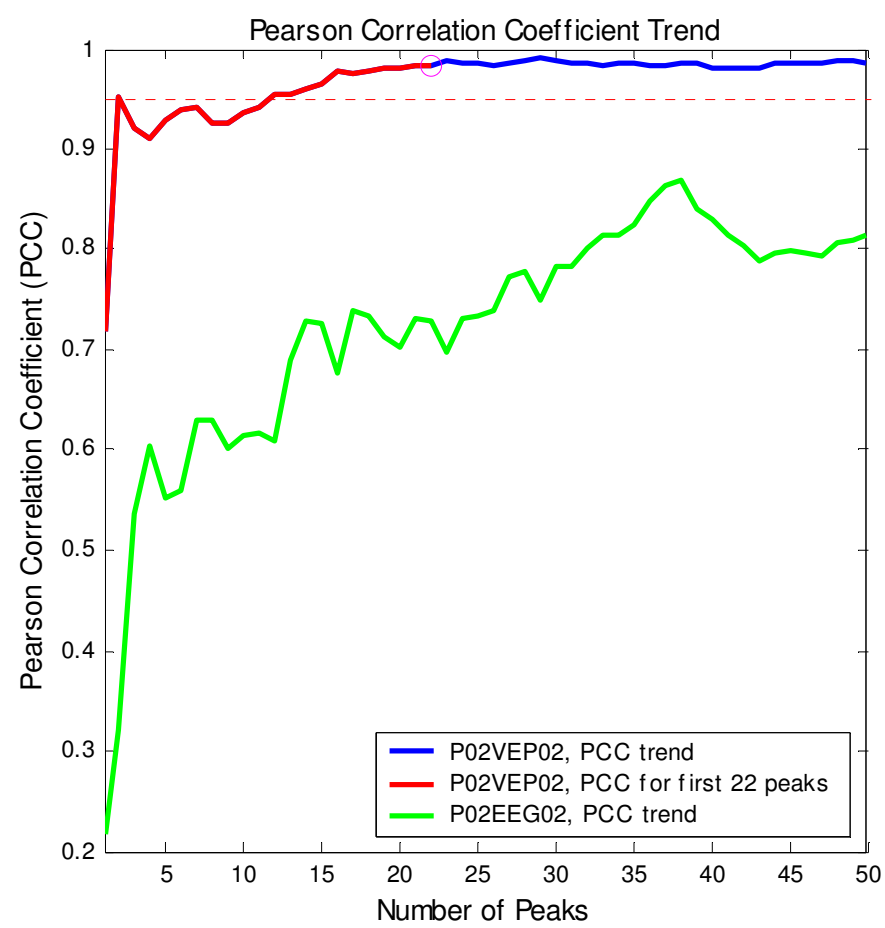

Fig. 5.21: Pearson Correlation Coefficients profile between the standard averaged VEP signal and the equivalent peak average based on the number of bins chosen for dataset P02VEP2.

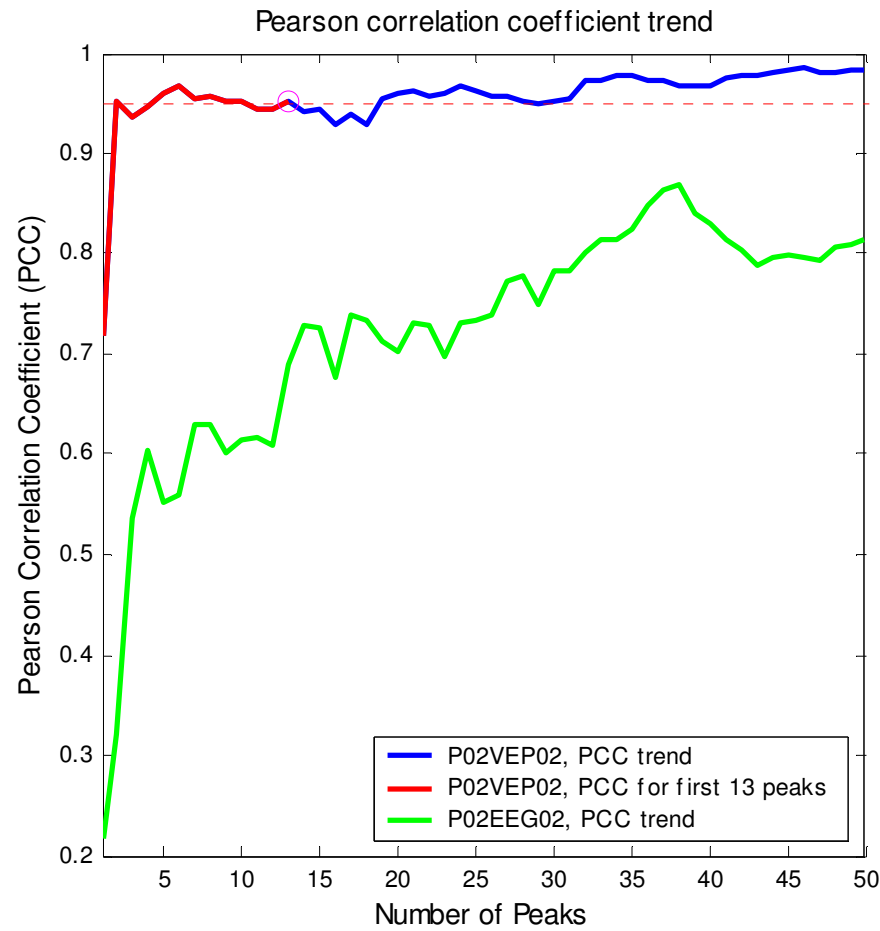

Fig. 5.22: Pearson Correlation Coefficients profile between the standard averaged VEP signal and the equivalent peak average based on the number of bins chosen using the maximum number of peaks above the bin average. 


\begin{tabular}{|c|c|c|c|c|c|c|c|c|c|c|c|c|c|c|c|c|c|c|c|c|c|c|c|c|c|}
\hline$\underset{\sim}{\infty}$ & & D & $\stackrel{r}{\sim}$ & $\stackrel{\circ}{i}$ & $\stackrel{m}{\sim}$ & $\stackrel{\sim}{\sim}$ & $\stackrel{\sim}{\sim}$ & ᄂ & 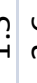 & ְ. & $\stackrel{r}{\sim}$ & $\stackrel{0}{i}$ & $\stackrel{m}{\sim}$ & - & ? & i & ָָ & ri & ت & تِ & - & ז. & $\stackrel{r}{\sim}$ & $\stackrel{m}{\sim}$ & ت. \\
\hline ન્ & & $\begin{array}{l}i \\
i^{n} \\
3\end{array}$ & $\hat{m}$ & $\begin{array}{l}\dot{r} \\
\dot{m}\end{array}$ & $\stackrel{\text { In }}{\forall}$ & $\stackrel{\sim}{\sim}$ & $\stackrel{\varphi}{\forall}$ & 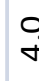 & $\stackrel{f}{f}$ & ?ִ & 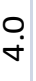 & $\begin{array}{l}\varphi \\
\dot{m}\end{array}$ & $\begin{array}{l}\infty \\
\dot{m}\end{array}$ & $\dot{m}$ & $\hat{i}$ & $\stackrel{\varphi}{\dot{\gamma}}$ & $\begin{array}{l}\infty \\
\dot{m}\end{array}$ & $\begin{array}{l}\varphi \\
\dot{m}\end{array}$ & $\begin{array}{l}\varphi \\
\dot{m}\end{array}$ & $\stackrel{N}{m}$ & $\hat{m}$ & $\hat{m}$ & $\stackrel{\nabla}{\sim}$ & $\stackrel{\text { ? }}{\mathrm{N}}$ & $\stackrel{\rho}{m}$ \\
\hline$\underset{-1}{0}$ & & is & $\begin{array}{l}m \\
m\end{array}$ & $\begin{array}{l}\varphi \\
\dot{m}\end{array}$ & ґ & : & $\stackrel{m}{m}$ & 9 & $+f$ & $\begin{array}{r}\mathrm{y} \\
\mathrm{f}\end{array}$ & $\begin{array}{l}\varphi \\
\dot{m}\end{array}$ & $\begin{array}{l}\infty \\
\dot{m}\end{array}$ & $\stackrel{\gamma}{\sigma}$ & $\begin{array}{l}\infty \\
\dot{m}\end{array}$ & $\begin{array}{l}\varphi \\
\dot{m}\end{array}$ & $\hat{m}$ & $\stackrel{\sim}{\sim}$ & $\begin{array}{l}\varphi \\
\dot{m}\end{array}$ & $\stackrel{\infty}{m}$ & $\stackrel{r}{\sigma}$ & $\begin{array}{l}\text { ? } \\
\text { ? }\end{array}$ & $\stackrel{r}{\sigma}$ & $\stackrel{m}{\sim}$ & $\stackrel{r}{\sigma}$ & $\stackrel{\sigma}{m}$ \\
\hline$\stackrel{n}{\sim}$ & & ع & $\stackrel{N}{a}$ & $\stackrel{\infty}{\sigma}$ & ஸे & $\underset{+}{+}$ & $\stackrel{N}{\infty}$ & 5 & tr) & •ُمْ & $\hat{\infty}$ & $\hat{r}$ & $\underset{\sim}{\stackrel{-}{\sim}}$ & क? & $\stackrel{ナ}{r}$ & $\stackrel{\sim}{0}$ & $\stackrel{\sigma}{\sigma}$ & $\stackrel{\stackrel{n}{\gamma}}{\dot{\gamma}}$ & $\stackrel{\sim}{6}$ & $\stackrel{\circ}{0}$ & $\underset{+}{\stackrel{f}{-}}$ & 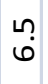 & $\begin{array}{l}\varphi \\
\sigma\end{array}$ & $\stackrel{r}{r}$ & 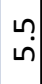 \\
\hline$\underset{\sim}{+}$ & & $\sum_{3}^{\infty 00}$ & $\stackrel{⿱ 亠 凶}{\sim}$ & $\begin{array}{l}0 \\
\stackrel{N}{N}\end{array}$ & $\begin{array}{l}m \\
\infty \\
\sim\end{array}$ & $\begin{array}{l}\infty \\
\dot{\varphi} \\
N\end{array}$ & $\begin{array}{l}\infty \\
\infty \\
\sim\end{array}$ & $\grave{n}$ & $\hat{h}$ & ְn. & 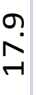 & $\begin{array}{l}\infty \\
\stackrel{d}{N}\end{array}$ & $\stackrel{m}{\stackrel{m}{N}}$ & 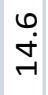 & $\hat{n}$ & $\stackrel{\varphi}{\stackrel{n}{N}}$ & $\begin{array}{l}\text { ? } \\
\qquad \\
\end{array}$ & $\begin{array}{l}0 \\
\dot{m} \\
\sim\end{array}$ & $\begin{array}{l}\infty \\
\infty \\
-1\end{array}$ & 뭄 & $\stackrel{\circ}{\stackrel{+}{N}}$ & $\begin{array}{l}N \\
ٌ \\
-1\end{array}$ & $\stackrel{+}{\stackrel{+}{m}}$ & $\stackrel{m}{\text { กุ }}$ & ז. \\
\hline$\stackrel{m}{\rightarrow}$ & & 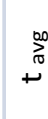 & $\begin{array}{l}n \\
\stackrel{n}{\sim} \\
\sim \\
r\end{array}$ & 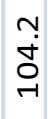 & $\begin{array}{l}\sigma \\
\dot{\sigma}\end{array}$ & $\begin{array}{l}N \\
\\
\text { o } \\
\end{array}$ & \begin{tabular}{l}
+ \\
\multirow{্}{+}{}
\end{tabular} & $\sqrt{2}$ & ș & $\begin{array}{l}\infty \\
\stackrel{\infty}{\infty}\end{array}$ & $\begin{array}{l}\stackrel{0}{\dot{d}} \\
\stackrel{\sim}{N}\end{array}$ & $\hat{\imath}$ & $\begin{array}{l}m \\
\infty \\
\sim\end{array}$ & $\begin{array}{l}0 \\
\\
\end{array}$ & 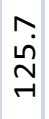 & $\hat{\tilde{n}}$ & $\begin{array}{l}0 \\
\text { ñ } \\
\stackrel{N}{N}\end{array}$ & $\begin{array}{l}n \\
\sim \\
\infty \\
-1 \\
-1\end{array}$ & $\begin{array}{r}\dot{\nabla} \\
\stackrel{\nabla}{ }\end{array}$ & $\begin{array}{l}0 \\
\stackrel{0}{-} \\
\text { in } \\
r\end{array}$ & $\begin{array}{l}0 \\
\stackrel{\sim}{\sim} \\
\underset{\sim}{\mid}\end{array}$ & $\begin{array}{l}\overrightarrow{-} \\
\infty \\
n \\
\sim\end{array}$ & ڤึ & 官 & $\begin{array}{l}9 \\
\text { กิ } \\
\text { O- }\end{array}$ \\
\hline$\underset{\sim}{\sim}$ & 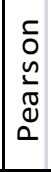 & \begin{tabular}{l}
4 \\
\multirow{4}{0}{} \\
0 \\
0 \\
$\vdots$ \\
0 \\
0
\end{tabular} & $\begin{array}{l}\infty \\
\stackrel{\infty}{+} \\
\hat{n} \\
0\end{array}$ & $\begin{array}{l}\text { ก๊ } \\
\text { กุ } \\
0\end{array}$ & $\begin{array}{l}\text { N } \\
\text { ஸे }\end{array}$ & $\begin{array}{l}\circ \\
\circ \\
\circ \\
\circ\end{array}$ & مે & $\begin{array}{l}\sigma \\
\text { o } \\
0\end{array}$ & గn: & Ş & 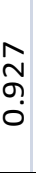 & $\begin{array}{l}\stackrel{\text { N}}{\sigma} \\
\text { }\end{array}$ & $\begin{array}{l}\infty \\
\text { m } \\
\sigma \\
\vdots\end{array}$ & 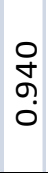 & $\begin{array}{l}\text { กी } \\
\text { ดุ } \\
0\end{array}$ & 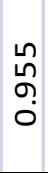 & 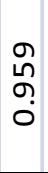 & $\begin{array}{l}\text { ठே } \\
\text { ڤุ } \\
0\end{array}$ & $\begin{array}{l}\hat{n} \\
\hat{\sigma} \\
0\end{array}$ & $\begin{array}{l}\stackrel{0}{\hat{n}} \\
\stackrel{\operatorname{c}}{0}\end{array}$ & $\begin{array}{l}\text { ñ } \\
\text { ดे } \\
0\end{array}$ & $\begin{array}{l}N \\
\infty \\
\sigma \\
0\end{array}$ & $\begin{array}{l}0 \\
\infty \\
\sigma \\
0\end{array}$ & $\begin{array}{l}n \\
\infty \\
\sigma \\
0\end{array}$ & 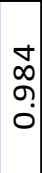 \\
\hline$\stackrel{-}{-}$ & & $\begin{array}{l}\frac{\pi}{d} \\
\frac{1}{\alpha} \\
x \\
\frac{n}{2} \\
\frac{v}{\alpha}\end{array}$ & $\stackrel{r}{m}$ & $\begin{array}{l}\stackrel{\varphi}{m} \\
m\end{array}$ & $\stackrel{\circ}{\stackrel{+}{\sim}}$ & $\begin{array}{l}\underset{r}{\sim} \\
\underset{-}{0}\end{array}$ & $\underset{r}{\stackrel{m}{r}}$ & $\frac{m}{7}$ & $\begin{array}{r}n \\
-7\end{array}$ & تُ & $\begin{array}{l}\hat{0} \\
0 \\
-1\end{array}$ & $\begin{array}{l}0 \\
0 \\
0 \\
-1\end{array}$ & बे & $\begin{array}{l}\infty \\
\dot{\sigma}\end{array}$ & $\hat{\sigma}$ & $\begin{array}{l}\infty \\
\infty\end{array}$ & $\begin{array}{l}\infty \\
\infty\end{array}$ & $\begin{array}{l}\infty \\
\infty\end{array}$ & $\stackrel{\sim}{\infty}$ & $\stackrel{\circ}{\infty}$ & $\stackrel{\infty}{N}$ & $\stackrel{ナ}{\wedge}$ & $\stackrel{n}{n}$ & $\stackrel{n}{n}$ & $\stackrel{N}{N}$ \\
\hline 암 & & $\begin{array}{l}\overrightarrow{b 0} \\
I \\
\times \\
\tilde{v} \\
\frac{v}{a}\end{array}$ & $\underset{\infty}{\infty}$ & $\stackrel{-}{\infty}$ & 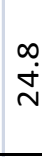 & 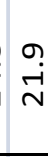 & $\stackrel{m}{\sim}$ & $\stackrel{n}{n}$ & مُ & הُ & $\stackrel{r}{\sim}$ & 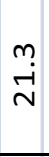 & $\begin{array}{l}0 \\
\stackrel{\sim}{N}\end{array}$ & $\begin{array}{c}n \\
\infty \\
\sim\end{array}$ & $\stackrel{+}{\stackrel{\sim}{N}}$ & $\stackrel{0}{\stackrel{0}{r}}$ & $\hat{\mathrm{N}}$ & 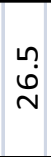 & $\stackrel{N}{\sim}$ & $\stackrel{-r}{\stackrel{-}{N}}$ & $\begin{array}{l}n \\
6 \\
-1\end{array}$ & $\stackrel{\text { חִ }}{\stackrel{1}{\sim}}$ & 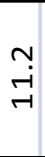 & $\begin{array}{l}0 \\
\stackrel{-}{-} \\
\rightarrow-1\end{array}$ & $\begin{array}{l}m \\
\infty \\
+\end{array}$ \\
\hline$\sigma$ & ${ }^{\infty 00}$ & 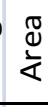 & 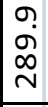 & $\begin{array}{l}\tilde{n} \\
\hat{\sigma} \\
\end{array}$ & 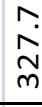 & 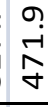 & $\begin{array}{l}+ \\
\text { N } \\
\mathrm{m}\end{array}$ & {$\left[\begin{array}{l}5 \\
6 \\
0\end{array}\right.$} & $\begin{array}{l}n \\
\overbrace{}^{2} \\
\overbrace{}^{\prime}\end{array}$ & ve & 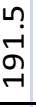 & $\begin{array}{l}\stackrel{-}{\infty} \\
\stackrel{+}{N}\end{array}$ & 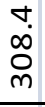 & 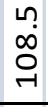 & $\begin{array}{l}0 \\
\dot{0} \\
+ \\
+1\end{array}$ & $\begin{array}{l}\vec{H} \\
\text { n̊ } \\
\sim\end{array}$ & $\begin{array}{l}\tilde{n} \\
\stackrel{0}{0} \\
\stackrel{-}{\sim}\end{array}$ & $\begin{array}{l}m \\
\infty \\
\infty\end{array}$ & 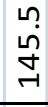 & 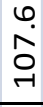 & $\begin{array}{l}n \\
\dot{\theta} \\
\dot{\gamma} \\
\gamma\end{array}$ & $\begin{array}{l}\stackrel{n}{-} \\
\stackrel{n}{m} \\
\sim\end{array}$ & & $\begin{array}{l}\text { ạ } \\
\stackrel{\sim}{N} \\
\end{array}$ & $\begin{array}{l}0 \\
\\
-1 \\
-1\end{array}$ \\
\hline$\infty$ & $\underset{⿱}{\gtrless}$ & $\begin{array}{l}\frac{\tilde{c}}{00} \\
\cdot \bar{d} \\
\underline{T}\end{array}$ & $\stackrel{\infty}{\infty}$ & $\stackrel{m}{\infty}$ & $\stackrel{\infty}{\infty}$ & & $\begin{array}{l}\stackrel{9}{0} \\
\varphi\end{array}$ & 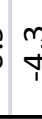 & $\begin{array}{l}n \\
\dot{f} \\
1\end{array}$ & o. & $\begin{array}{l}\stackrel{t}{0} \\
\dot{\varphi}\end{array}$ & $\hat{\theta}$ & $\stackrel{\circ}{\circ}$ & $\stackrel{\sim}{\sim}$ & $\begin{array}{l}m \\
\\
\end{array}$ & חִ & $\stackrel{\text { ஸे }}{m}$ & $\stackrel{n}{p}$ & $\stackrel{\infty}{\dot{\tau}}$ & $\stackrel{\infty}{\dot{m}}$ & $\begin{array}{l}\infty \\
\stackrel{-}{-} \\
\rightarrow\end{array}$ & $\stackrel{\sim}{\sim}$ & $\stackrel{\circ}{\infty}$ & $\begin{array}{l}\text { In } \\
\text { เึ? }\end{array}$ & $\stackrel{\circ}{\circ}$ \\
\hline$\Lambda$ & & in & $\overrightarrow{1}$ & $r$ & $\neg$ & -1 & 7 & 4 & & י1 & r & -1 & $r$ & $r$ & $\neg$ & $\nearrow_{1}$ & -1 & 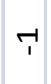 & $r$ & $r_{1}$ & 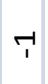 & -1 & $r$ & $r$ & $r$ \\
\hline 6 & $\mid \begin{array}{l}4 \\
0 \\
0 \\
2\end{array}$ & $\begin{array}{l}\tilde{v} \\
\frac{v}{\pi} \\
\mathbb{D} \\
0\end{array}$ & $\stackrel{\infty}{+}$ & $\hat{\sigma}$ & $\underset{-1}{6}$ & 암 & $\stackrel{+}{-}$ & $i$ & & $\underset{-1}{0}$ & $\stackrel{-}{\sim}$ & $\underset{\sim-1}{0}$ & $\underset{\sim}{\sim}$ & $\stackrel{+}{m}$ & $\stackrel{\bullet}{N}$ & $\hat{\imath}$ & $\stackrel{-1}{m}$ & $\stackrel{\mathcal{F}}{\sim}$ & $\tilde{N}$ & $\stackrel{\infty}{\sim}$ & $\lambda$ & $\stackrel{-1}{N}$ & $\Lambda$ & $\stackrel{0}{\sim}$ & $\stackrel{n}{N}$ \\
\hline Ln & $\begin{array}{l}\tilde{y} \\
\frac{v}{\tilde{d}} \\
\tilde{\alpha} \\
\dot{0} \\
\tilde{z}\end{array}$ & 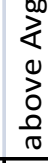 & $\stackrel{-1}{m}$ & $\begin{array}{l}m \\
\infty \\
m\end{array}$ & ণे & $\stackrel{m}{r}$ & $\stackrel{\infty}{6}$ & $\frac{0}{2}$ & با & $\begin{array}{l}\infty \\
\infty \\
\infty\end{array}$ & 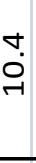 & $\begin{array}{l}\infty \\
\omega^{\circ}\end{array}$ & חִ & $\begin{array}{l}\infty \\
\dot{\omega} \\
-\end{array}$ & $\begin{array}{l}\stackrel{?}{m} \\
\stackrel{m}{\sim}\end{array}$ & $\stackrel{\circ}{\wedge}$ & $\vec{\omega}$ & $\stackrel{\overrightarrow{-}}{\vec{n}}$ & $\begin{array}{l}0 \\
\infty\end{array}$ & $\begin{array}{r}-r \\
- \\
-\end{array}$ & $\stackrel{\sim}{n}$ & $\hat{\theta}$ & & $\underset{6}{n}$ & $\hat{\infty}$ \\
\hline$\nabla$ & 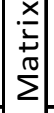 & ع & $\sigma$ & $\stackrel{0}{\circ}$ & $\sigma$ & $\stackrel{+}{+}$ & $\infty$ & 15 & & $\underset{r}{r}$ & $\sigma$ & $\wedge$ & $\stackrel{\sim}{\sim}$ & 6 & $\infty$ & in & $\nabla$ & $\nabla$ & 6 & 6 & $\stackrel{6}{-1}$ & $\Lambda$ & $\sigma$ & $\Lambda$ & $\nabla$ \\
\hline$m$ & 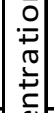 & 3 & $\stackrel{\sim}{\sim}$ & $\stackrel{m}{N}$ & શิ & $\stackrel{\infty}{\sim}$ & શ & $\lesssim$ & $\vec{v}$ & ? & $\stackrel{\infty}{\rightarrow}$ & $\stackrel{\bullet}{\sim}$ & $\stackrel{\mathscr{n}}{\sim}$ & $\stackrel{+}{-}$ & $\stackrel{+}{\sim}$ & $\stackrel{\searrow}{\sim}$ & $\hat{ન}$ & $\stackrel{m}{\sim}$ & $\stackrel{\sigma}{-}$ & $\stackrel{\varphi}{\sim}$ & $\stackrel{\bullet}{\sim}$ & $\stackrel{\sim}{\sim}$ & $\stackrel{\llcorner}{m}$ & $\stackrel{N}{m}$ & A \\
\hline$N$ & $\mid \begin{array}{l}\mathrm{u} \\
\mathrm{c} \\
\mathrm{U} \\
\end{array}$ & + & $\stackrel{\Omega}{6}$ & กN & $\stackrel{-1}{\sim}$ & i & 옷 & 7 & 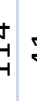 & $\begin{array}{r}y \\
y\end{array}$ & $\underset{\sim}{\sim}$ & $\stackrel{m}{m}$ & $\sigma$ & $\begin{array}{l}6 \\
\infty\end{array}$ & $\tilde{\omega}$ & $\stackrel{N}{m}$ & ำ & ă & $\stackrel{N}{N}$ & $\stackrel{\varphi}{\wedge}$ & $\tilde{\omega}$ & I & $\hat{\gamma}$ & $\stackrel{\infty}{\rightarrow-1}$ & $m$ \\
\hline-1 & $\therefore \frac{c}{\infty}$ & $\frac{0}{0}$ & $\#$ & $\#$ & $\#$ & $\#$ & 坚 & 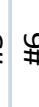 & & \# & $\begin{array}{l}\infty \\
\#\end{array}$ & \$ & 荓 & $\underset{ت}{\vec{H}}$ & $\underset{7}{\stackrel{N}{\#}}$ & $\stackrel{m}{\underset{7}{\#}}$ & $\underset{\#}{\stackrel{\nabla}{\#}}$ & 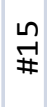 & $\begin{array}{l}6 \\
\underset{7}{\#}\end{array}$ & $\underset{\vec{F}}{\vec{F}}$ & $\begin{array}{l}\infty \\
\underset{\#}{\#}\end{array}$ & $\underset{\nexists}{\stackrel{9}{\#}}$ & $\stackrel{\text { N }}{\#}$ & $\underset{+}{\mathbb{N}}$ & $\underset{N}{\mathbb{N}}$ \\
\hline
\end{tabular}

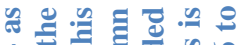

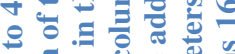
N

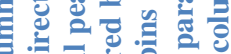

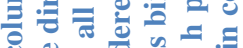

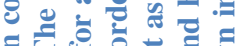

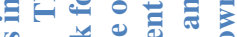
ํ. อ。 2 的

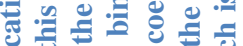

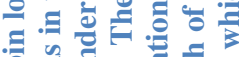
范

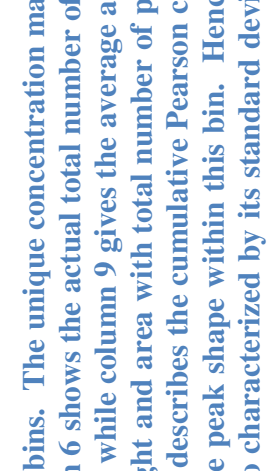

ง ํㅡㄹ

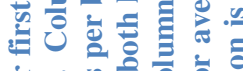
อี

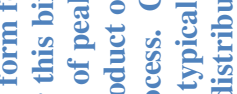$$
\text { . }
$$ \\ ,}

政 


\begin{tabular}{|c|c|c|c|c|c|c|c|c|c|c|c|c|c|c|c|}
\hline & 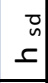 & $\stackrel{i}{i}$ & $\stackrel{\circ}{i}$ & $\stackrel{+}{r}$ & $\stackrel{n}{r}$ & - & ָָ & $\stackrel{\text { n? }}{r}$ & $\stackrel{0}{\circ}$ & $\stackrel{9}{r}$ & $\stackrel{n}{\sim}$ & i & r- & $\begin{array}{l}0 \\
-1 \\
\end{array}$ & 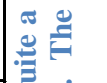 \\
\hline & $\begin{array}{l}0 \\
0 \\
3\end{array}$ & $\hat{m}$ & $\stackrel{\nabla}{\dot{m}}$ & $\begin{array}{l}\varphi \\
\dot{m}\end{array}$ & $\stackrel{0}{\dot{\sigma}}$ & $\vec{m}$ & $\stackrel{\infty}{m}$ & $\stackrel{\varphi}{i}$ & r & $\hat{\sim}$ & $\begin{array}{l}\varphi \\
\dot{m}\end{array}$ & $\hat{\sim}$ & $\stackrel{+}{\sim}$ & & 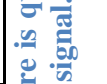 \\
\hline & $\begin{array}{r}0 \\
+ \\
+\end{array}$ & $\begin{array}{l}4 \\
\text { a }\end{array}$ & $\stackrel{\varphi}{\dot{m}}$ & $\begin{array}{l}\varphi \\
\dot{m}\end{array}$ & $\stackrel{+}{+}$ & $\stackrel{\infty}{m}$ & $\stackrel{\sim}{\forall}$ & $\dot{\varphi}$ & $\stackrel{\infty}{m}$ & $\stackrel{\varphi}{\dot{m}}$ & ñ & $\stackrel{\sim}{\sigma}$ & $\stackrel{N}{m}$ & $\stackrel{r}{+}$ & 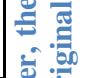 \\
\hline & ع & 2 & $\stackrel{\infty}{\sigma}$ & $\stackrel{\text { In }}{\sigma}$ & İ & बุ & $\stackrel{\sigma}{\sigma}$ & $\stackrel{+}{\dot{\sigma}}$ & $\stackrel{9}{m}$ & $\stackrel{+}{\Lambda}$ & $\stackrel{\varphi}{\dot{\sigma}}$ & $\stackrel{N}{1}$ & $\stackrel{N}{\sigma}$ & $\begin{array}{l}0 \\
0\end{array}$ & \\
\hline & 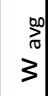 & $\stackrel{\sim}{\sim}$ & $\stackrel{6}{N}$ & $\begin{array}{l}0 \\
\dot{m} \\
-1\end{array}$ & $\hat{i}$ & $\underset{\dot{\sigma}}{-}$ & $\begin{array}{l}q \\
0 \\
-1\end{array}$ & $\stackrel{\check{r}}{\stackrel{r}{r}}$ & ă & in & $\begin{array}{l}0 \\
\dot{m} \\
-1\end{array}$ & $\underset{m}{m}$ & o̊ & تُ & 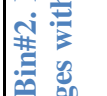 \\
\hline & $+\stackrel{\pi}{\pi}_{+\infty}^{\infty}$ & $\begin{array}{l}n \\
\stackrel{n}{\sim} \\
\text { r }\end{array}$ & $\underset{\sim}{\sim}$ & 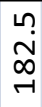 & กิ & $\begin{array}{l}0 \\
\stackrel{1}{N} \\
\end{array}$ & $\begin{array}{l}0 \\
\dot{m} \\
\stackrel{\nu}{N}\end{array}$ & $\stackrel{m}{\stackrel{m}{N}}$ & $\begin{array}{l}0 \\
\infty \\
\stackrel{m}{N}\end{array}$ & 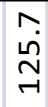 & ă & $\stackrel{i}{\stackrel{i}{N}}$ & $\begin{array}{l}\infty \\
\dot{\sigma} \\
\stackrel{-}{-1}\end{array}$ & & 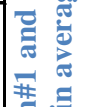 \\
\hline 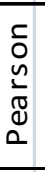 & $\begin{array}{l}\frac{4}{4} \\
0 \\
0 \\
0 \\
\vdots \\
0 \\
0\end{array}$ & $\begin{array}{l}\infty \\
\stackrel{1}{1} \\
0 \\
0\end{array}$ & $\begin{array}{l}\text { Nิ } \\
\text { กิ } \\
0\end{array}$ & $\begin{array}{l}0 \\
m \\
0 \\
0\end{array}$ & \begin{tabular}{l}
0 \\
\multirow{+}{\sigma}{} \\
0
\end{tabular} & $\begin{array}{l}\text { ⿵⺆ } \\
\text { nิ } \\
0\end{array}$ & $\begin{array}{l}0 \\
\emptyset \\
0 \\
0\end{array}$ & $\begin{array}{l}\text { ஸे } \\
\text { क़े } \\
0\end{array}$ & $\begin{array}{l}\hat{n} \\
\text { ọ } \\
0\end{array}$ & $\begin{array}{l}\text { กิ } \\
\text { ด़ } \\
0\end{array}$ & $\begin{array}{l}m \\
\text { ñ } \\
0 \\
0\end{array}$ & \begin{tabular}{l}
$m$ \\
\multirow{\sigma}{\sigma}{} \\
0
\end{tabular} & $\begin{array}{l}\text { ñ } \\
\text { ọ } \\
0\end{array}$ & ஸี & 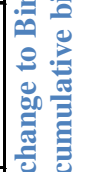 \\
\hline & 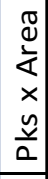 & & $\stackrel{\sim}{m}$ & $\stackrel{\infty}{\infty}$ & $\stackrel{m}{-}$ & $\stackrel{\infty}{\sigma}$ & $\infty$ & $\stackrel{\varphi}{\varphi}$ & $\hat{m}$ & $\hat{\sigma}$ & $\stackrel{\circ}{N}$ & $\hat{\theta}$ & $\stackrel{\infty}{\dot{\sigma}}$ & $\begin{array}{l}0 \\
\infty\end{array}$ & 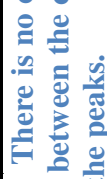 \\
\hline & $\begin{array}{l}\breve{b} \\
\underline{x} \\
\times \\
\tilde{v} \\
\frac{v}{a}\end{array}$ & $\begin{array}{l}\infty \\
\dot{N}\end{array}$ & $\stackrel{+}{\infty}$ & $\stackrel{\stackrel{n}{0}}{\stackrel{\leftrightarrow}{N}}$ & $\stackrel{\stackrel{n}{n}}{N}$ & $\begin{array}{l}m \\
\infty \\
\sim\end{array}$ & $\hat{i}$ & ㅁ. & $\begin{array}{l}0 \\
- \\
- \\
-1\end{array}$ & $\stackrel{+}{\stackrel{\sim}{N}}$ & $\begin{array}{l}\varphi \\
\stackrel{\leftrightarrow}{N}\end{array}$ & $\begin{array}{l}\stackrel{n}{\infty} \\
\stackrel{\infty}{-1}\end{array}$ & $\begin{array}{l}9 \\
\varphi \\
-1\end{array}$ & & 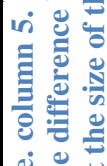 \\
\hline$\stackrel{000}{\gtrless}$ & $\frac{\mathbb{8}}{\frac{1}{4}}$ & 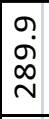 & $\stackrel{n}{\hat{v}}$ & $\begin{array}{l}m \\
\infty\end{array}$ & 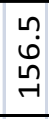 & $\begin{array}{l}n \\
\infty \\
0 \\
0\end{array}$ & $\begin{array}{l}\stackrel{n}{0} \\
\stackrel{0}{0} \\
\stackrel{-}{r}\end{array}$ & ?ุ. & $\begin{array}{l}\infty \\
0 \\
0\end{array}$ & $\begin{array}{l}0 \\
\dot{0} \\
+ \\
-1\end{array}$ & $\stackrel{m}{N}$ & $\stackrel{+}{\dot{\delta}}$ & $\stackrel{\infty}{\text { in }}$ & 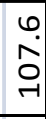 & 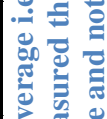 \\
\hline$\stackrel{\infty}{<}^{\infty}$ & $\begin{array}{l} \pm \\
\frac{1}{00} \\
\cdot \frac{0}{1} \\
\pm\end{array}$ & $\stackrel{\infty}{i}$ & $\stackrel{m}{\infty}$ & $\stackrel{n}{m}$ & $\stackrel{m}{\dot{q}}$ & $\stackrel{\sim}{\forall}$ & $\stackrel{n}{n}$ & $\stackrel{9}{\sim}$ & $\stackrel{-1}{\sim}$ & mִ & $\begin{array}{l}0 \\
\dot{m}\end{array}$ & $\ddot{m}$ & $\stackrel{r}{i}$ & $\begin{array}{l}\infty \\
\dot{p} \\
\dot{1}\end{array}$ & 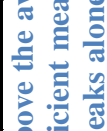 \\
\hline & $\frac{c}{00}$ & $\overrightarrow{1}$ & -1 & 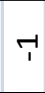 & $\neg$ & $r$ & $r$ & $r$ & $r$ & 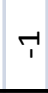 & $\rightarrow$ & 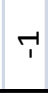 & 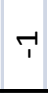 & $\neg$ & s \\
\hline $\begin{array}{l}4 \\
0 \\
0 \\
2\end{array}$ & $\begin{array}{l}\frac{\tilde{v}}{\tilde{\pi}} \\
\stackrel{d}{0} \\
0\end{array}$ & $\stackrel{\infty}{+}$ & $\hat{\sigma}$ & $\stackrel{\mathcal{F}}{\sim}$ & $\hat{\sim}$ & $\stackrel{+}{m}$ & $\stackrel{-1}{m}$ & $\stackrel{\ln }{m}$ & $\grave{N}$ & $\stackrel{\bullet}{\sim}$ & $\stackrel{+}{m}$ & $\stackrel{\circ}{m}$ & $\vec{m}$ & $\stackrel{\infty}{\sim}$ & 范 \\
\hline $\begin{array}{l}\frac{\tilde{v}}{\pi} \\
\Phi \\
0 \\
\dot{0}\end{array}$ & $\begin{array}{l}00 \\
20 \\
0 \\
0 \\
0 \\
\frac{0}{0} \\
\end{array}$ & aे & $\begin{array}{l}m \\
\infty \\
m\end{array}$ & $\stackrel{r}{\stackrel{H}{N}}$ & ํ. & $\begin{array}{l}\infty \\
\dot{0} \\
-1\end{array}$ & $\overrightarrow{0}$ & $\begin{array}{l}\stackrel{+}{6} \\
\stackrel{1}{-1}\end{array}$ & 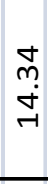 & $\begin{array}{l}\sigma \\
\stackrel{m}{-}\end{array}$ & $\begin{array}{l}\infty \\
0 \\
m \\
\sim\end{array}$ & $\begin{array}{l}6 \\
6 \\
-1 \\
-1\end{array}$ & $\begin{array}{l}\stackrel{\sigma}{6} \\
\text { - } \\
-1\end{array}$ & $\stackrel{-1}{-}$ & 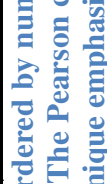 \\
\hline 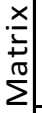 & ـ & $\sigma$ & 악 & $\nabla$ & Ln & 6 & $\nabla$ & $\nabla$ & $\nabla$ & $\infty$ & 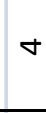 & Ln & $\nabla$ & 6 & م. \\
\hline 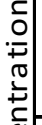 & 3 & $\stackrel{\stackrel{n}{N}}{\sim}$ & $\stackrel{m}{N}$ & $\stackrel{m}{r}$ & 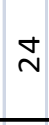 & $\stackrel{+}{-}$ & ને & $\stackrel{0}{\sim}$ & $\Lambda$ & $\stackrel{+}{\sim}$ & $\stackrel{m}{\rightarrow}$ & $\stackrel{-}{-}$ & $\stackrel{\circ}{\sim}$ & $\underset{-1}{6}$ & ]ٕ \\
\hline பิ & + & ט & กี & ন & $\stackrel{+}{\stackrel{\sim}{-}}$ & $\underset{\infty}{\bullet}$ & $\begin{array}{l}N \\
0 \\
-1\end{array}$ & $\stackrel{9}{m}$ & 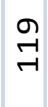 & ๓ึ & $\stackrel{\varphi}{q}$ & $\stackrel{-}{-}$ & a & $\stackrel{0}{N}$ & 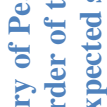 \\
\hline$\frac{.}{\infty}$ & $\begin{array}{l}\frac{1}{0} \\
\frac{0}{0} \\
0\end{array}$ & $\#$ & \# & $\stackrel{n}{\beth}$ & \# & $\stackrel{-1}{7}$ & $\underset{7}{\#}$ & & & $\underset{\sim}{\stackrel{N}{*}}$ & & & & 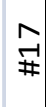 & E \\
\hline $\begin{array}{l}\frac{\tilde{v}}{\tilde{\pi}} \\
\stackrel{0}{\alpha}\end{array}$ & $\begin{array}{l}\frac{1}{0} \\
\frac{0}{0} \\
\frac{1}{0}\end{array}$ & -1 & $N$ & $m$ & $\nabla$ & Ln & 6 & $\Lambda$ & $\infty$ & $\sigma$ & $\stackrel{ }{\circ}$ & $\stackrel{-}{-}$ & $\underset{\sim}{\sim}$ & $\stackrel{m}{r}$ & 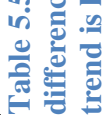 \\
\hline
\end{tabular}




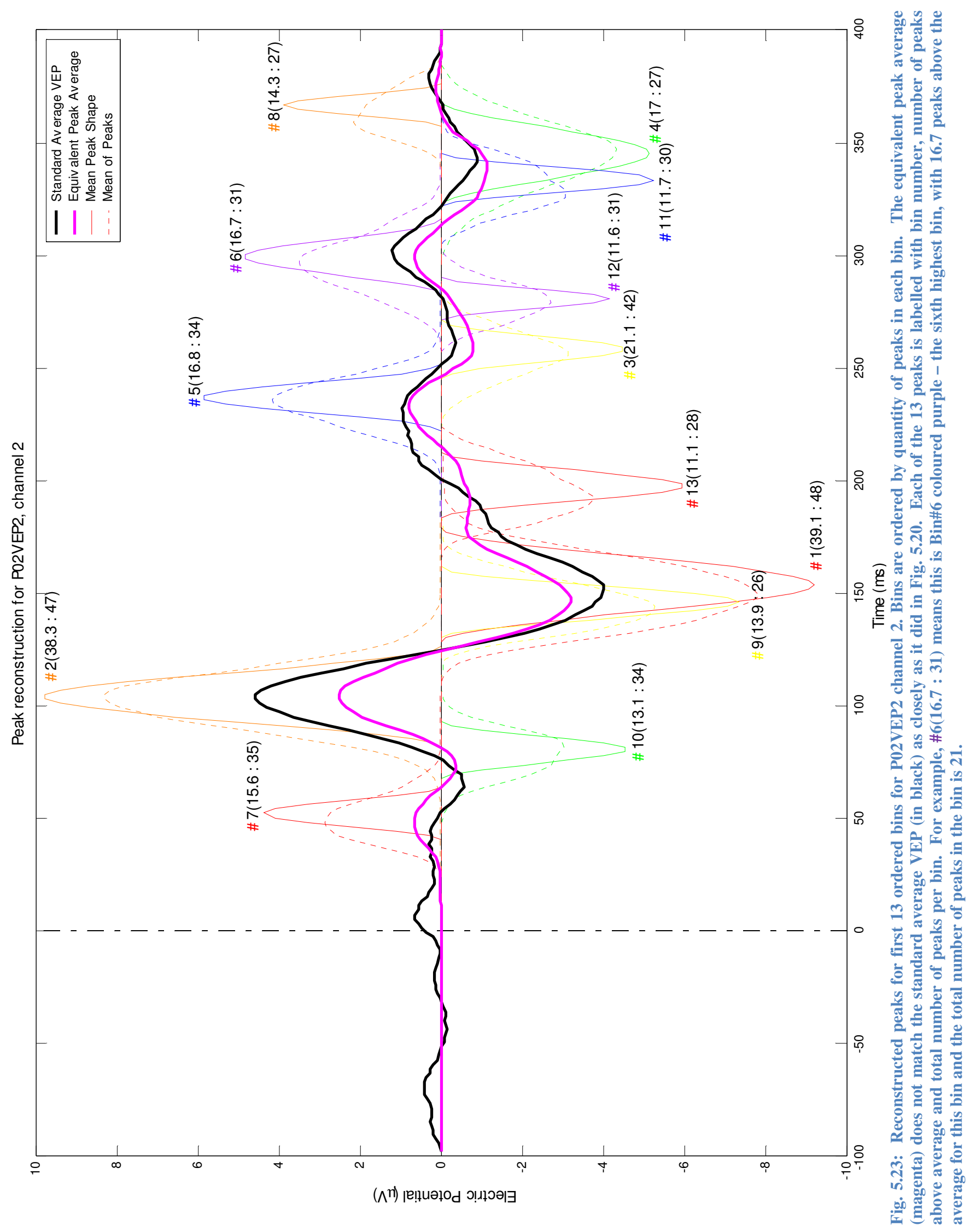




\subsection{ALL PARTICIPANT RESULTS}

Visual Evoked Potential results on three different participants were measured and a summary of the results is presented in the following subsections with associated illustrations recorded in Appendix B.

\subsubsection{Participant 01}

There were three sequential VEP tests performed where approximately $2 \frac{1}{2}$ minutes of EEG data was recorded with strobe light flashes presented at approximately 1 flash per second in accordance with the test protocol of Appendix A. The datasets were labelled P01VEP4, P01VEP6 and P01VEP7. A summary of the results listed by figure reference is given in Table 5.6.

\begin{tabular}{|l|l|l|}
\hline Reference & Dataset & Description \\
\hline Fig. B.1(a) & P01VEP4 & Average VEP and peak detected VEP \\
\hline Fig. B.4 & P01VEP4 & Peak concentration Profile \\
\hline Fig. B.12 & P01VEP4 & Reconstructed Peaks - ordered by significance factor \\
\hline Fig. B.15 & P01VEP4 & Reconstructed Peaks - ordered by total peaks above average \\
\hline Fig. B.29(a) & P01VEP4 & Pearson Correlation Coefficient Trend \\
\hline Fig. B.1(b) & P01VEP6 & Average VEP and peak detected VEP \\
\hline Fig. B.5 & P01VEP6 & Peak concentration Profile \\
\hline Fig. B.13 & P01VEP6 & Reconstructed Peaks - ordered by significance factor \\
\hline Fig. B.16 & P01VEP6 & Reconstructed Peaks - ordered by total peaks above average \\
\hline Fig. B.29(b) & P01VEP6 & Pearson Correlation Coefficient Trend \\
\hline Fig. B.1(c) & P01VEP7 & Average VEP and peak detected VEP \\
\hline Fig. B.6 & P01VEP7 & Peak concentration Profile \\
\hline Fig. B.14 & P01VEP7 & Reconstructed Peaks - ordered by significance factor \\
\hline Fig. B.17 & P01VEP7 & Reconstructed Peaks - ordered by total peaks above average \\
\hline Fig. B.29 & P01VEP7 & Pearson Correlation Coefficient Trend \\
\hline
\end{tabular}

\subsubsection{Participant 02}

There were three sequential VEP tests performed where approximately $2 \frac{1}{2} 2$ minutes of EEG data was recorded with strobe light flashes presented at approximately 1 flash per second in accordance with the test protocol of Appendix A. The datasets were labelled P01VEP4, P01VEP6 and P01VEP7. A summary of the results listed by figure reference is given in Table 5.7 . 


\begin{tabular}{|l|l|l|}
\hline Reference & Dataset & Description \\
\hline Fig. B.2(a) & P02VEP2 & Average VEP and peak detected VEP \\
\hline & P02VEP2 & Peak concentration Profile \\
\hline Fig. 5.20 & P02VEP2 & Reconstructed Peaks - ordered by significance factor \\
\hline Fig. 5.23 & P02VEP2 & Reconstructed Peaks - ordered by total peaks above average \\
\hline & P02VEP2 & Pearson Correlation Coefficient Trend \\
\hline Fig. B.2(b) & P02VEP3 & Average VEP and peak detected VEP \\
\hline Fig. B.7 & P02VEP3 & Peak concentration Profile \\
\hline Fig. B.18 & P02VEP3 & Reconstructed Peaks - ordered by significance factor \\
\hline Fig. B.20 & P02VEP3 & Reconstructed Peaks - ordered by total peaks above average \\
\hline Fig. B.28(b) & P02VEP3 & Pearson Correlation Coefficient Trend \\
\hline Fig. B.2(c) & P02VEP4 & Average VEP and peak detected VEP \\
\hline Fig. B.8 & P02VEP4 & Peak concentration Profile \\
\hline Fig. B.19 & P02VEP4 & Reconstructed Peaks - ordered by significance factor \\
\hline Fig. B.21 & P02VEP4 & Reconstructed Peaks - ordered by total peaks above average \\
\hline Fig. B.28(c) & P02VEP4 & Pearson Correlation Coefficient Trend \\
\hline
\end{tabular}

\subsubsection{Participant 03}

There were three sequential VEP tests performed where approximately $2 \frac{1}{2} 2$ minutes of EEG data was recorded with strobe light flashes presented at approximately 1 flash per second in accordance with the test protocol of Appendix A. The datasets were labelled P03VEP5, P03VEP7 and P03VEP8. A summary of the results listed by figure reference is given in Table 5.8.

\begin{tabular}{|l|l|l|}
\hline Reference & Dataset & Description \\
\hline Fig. B.3 (a) & P03VEP5 & Average VEP and peak detected VEP \\
\hline Fig. B.9 & P03VEP5 & Peak concentration Profile \\
\hline Fig. B.22 & P03VEP5 & Reconstructed Peaks - ordered by significance factor \\
\hline Fig. B.25 & P03VEP5 & Reconstructed Peaks - ordered by total peaks above average \\
\hline Fig. B.30(a) & P03VEP5 & Pearson Correlation Coefficient Trend \\
\hline Fig. B.3(b) & P03VEP7 & Average VEP and peak detected VEP \\
\hline Fig. B.10 & P03VEP7 & Peak concentration Profile \\
\hline Fig. B.23 & P03VEP7 & Reconstructed Peaks - ordered by significance factor \\
\hline Fig. B.26 & P03VEP7 & Reconstructed Peaks - ordered by total peaks above average \\
\hline Fig. B.30 & P03VEP7 & Pearson Correlation Coefficient Trend \\
\hline Fig. B.3(c) & P03VEP8 & Average VEP and peak detected VEP \\
\hline Fig. B.11 & P03VEP8 & Peak concentration Profile \\
\hline Fig. B.24 & P03VEP8 & Reconstructed Peaks - ordered by significance factor \\
\hline Fig. B.29 & P03VEP8 & Reconstructed Peaks - ordered by total peaks above average \\
\hline Fig. B.30(c) & P03VEP8 & Pearson Correlation Coefficient Trend \\
\hline
\end{tabular}




\section{CHAPTER 6 ANALYSIS}

\subsection{OVERVIEW}

$\mathrm{O}$

bservations from the results and specific analyses are described in this chapter.

Where possible, reference is made to the results (in Chapter 5 and Appendix B)

and where necessary additional graphs and tables are presented herein. The analyses and observations are grouped into specific topics below.

\subsubsection{Pre-processing}

From examination of Fig. B.1, Fig. B.2 and Fig. B.3, the following points are noted:

- In (a), the pre-processed signal is observed to be attenuated at the beginning and end of the signal due to the zeroing of partial peaks which is part of the preprocessing routine.

- The pre-processed signal is a smoother representation of the original signal due to the high-frequency filtering.

\subsubsection{Standard VEP Averaging}

From examination of Fig. B.1, Fig. B.2 and Fig. B.3, the following points are noted:

- A positive visual evoked potential response is visible whose maximum occurs at approximately $100 \mathrm{~ms}$. This is labelled the P100 response. This response is observable for all participants and all datasets.

- A negative visual evoked potential response is visible whose maximum occurs at approximately $150 \mathrm{~ms}$. This is labelled the N150 response. This response is observable for all participants and all datasets.

- In the sample epoch in Fig. 5.1, the range of values in (a) is twice the size of the final evoked potential signal observed at P100 and N150 in (b).

- For all participants and all datasets, there is no clear evoked potential component before $50 \mathrm{~ms}$ or after $350 \mathrm{~ms}$. Between $50 \mathrm{~ms}$ and $100 \mathrm{~ms}$ and between $175 \mathrm{~ms}$ and $350 \mathrm{~ms}$, there appears to be some variable activity which is not distinct enough to clearly identify as evoked potential but is also slightly larger than background EEG signal. 
- The shape of the P100 and N150 components remains the same for datasets for the same participants. However there is considerable inter-participant variability with regard to these component peak shapes. Using standard averaging, the latency of the P100 and N150 has been measured and shown to be repeatable (Table 6.3); however there is little other information that may be gleaned from observation of the averaging results.

\subsubsection{Standard EEG Averaging}

From examination of Fig. 5.2, the following points are noted:

- The averaged EEG dataset shows no apparent evoked potential response as expected. This is confirmed by similar examination of other datasets (not presented).

- For the averaged EEG dataset, the typical background EEG noise ranges about the $\pm 1 \mu \mathrm{V}$ signal level.

- When this noise level is compared to the VEP signal in Fig. 5.2, it is observed that adding such a noise level will only minimally affect the P100 and N150 components since there are of significantly higher magnitude than the background EEG. However, outside these particular components any particular EP component is of the same magnitude as the background EEG noise and so detecting EP components is much more difficult.

\subsubsection{Peak Detection Efficacy}

- The automatic peak detection algorithm was applied to all datasets for all participants. Any peak detected epochs that did not match the original epoch well ( $>0.96$ correlation coefficient) were eliminated from the dataset. Adding the total for selected peaks across all datasets from Table 5.1, we see that the number of non-detected epochs is 37 out of a possible total of 1383 which means that approximately $2.5 \%$ of epochs had some peak or peaks undetected, resulting in removal of the epoch from the dataset. Therefore the peak detection algorithm performs at $97.5 \%$ efficiency across all datasets.

\subsubsection{Peak Characterisation - Calculated Time Position}

From the peak characterisation results in section 5.3.3, it is observed that: 
- The time position distribution for positive and negative peaks (Fig. 5.6) is evenly distributed for VEP data with the following two notable exceptions.

- The first exception is a marked increase in the positive peak distribution around the $100 \mathrm{~ms}$ mark. Coinciding with this is marked decrease in the negative peak distribution around the $100 \mathrm{~ms}$. This dual distribution shift in positive and negative peaks is a direct consequence of presence of the evoked potential P100 component.

- The second exception is a marked increase in the negative peak distribution around the $150 \mathrm{~ms}$ mark. Coinciding with this is marked decrease in the positive peak distribution around the $150 \mathrm{~ms}$. This dual distribution shift in positive and negative peaks is a direct consequence of presence of the evoked potential N150 component.

- The time position distribution for positive and negative peaks (Fig. 5.12) is evenly distributed for EEG with no exceptions. This reaffirms that the exceptions for the VEP dataset were due to the EP component - the EEG dataset has an even distribution and no components (Fig. 5.2).

\subsubsection{Peak Characterisation - Calculated Peak Width}

From the peak characterisation results in section 5.3.3, it is observed that:

- The peak width distributions for both the VEP (Fig. 5.7) and EEG (Fig. 5.13) datasets are almost identical to one another. This is an important consideration for the validity of comparing the defined VEP and EEG datasets. The peak width distributions for VEP and EEG should be very similar since the component of difference we wish to emphasize is the time position only.

- The peak width distributions for VEP and EEG approximate a chi-square distribution.

- It is believed that the distribution near the 0 axis position is reduced to some extent by the pre-processing filtering process which filters out higher frequency (narrower width) peaks. Although the distribution is affected, it does not adversely affect the results of the clustering process since peaks in this range are too small to be evoked potentials. 


\subsubsection{Peak Characterisation - Calculated Peak Height}

From the peak characterisation results in section 5.3.3, it is observed that:

- The peak width distributions for both the VEP (Fig. 5.8) and EEG (Fig. 5.14) datasets are almost identical to one another. Again, this is an important consideration for the validity of comparing the defined VEP and EEG datasets. The peak height distributions for VEP and EEG should be very similar since the component of difference we wish to emphasize is the time position only.

- The peak width distributions for VEP and EEG approximate a lognormal distribution.

\subsubsection{Peak Characterisation - Area under the Peak}

From the peak characterisation results in section 5.3.3, it is observed that:

- The area under the peak distribution for the VEP dataset (Fig. 5.9) approximates an exponential distribution.

- It is believed that the distribution near the 0 axis position is reduced slightly by the pre-processing filtering process which filters out higher frequency (narrower width - smaller area) peaks.

\subsubsection{Peak Characterisation - Interrelatedness}

All the calculated parameters and the intrinsic parameters were plotted against each other to determine the independence between parameters.

- All 6 identified parameters (calculated - time position, peak width, peak height, area under peak and intrinsic - peak approximation location, peak approximation coefficient) were pair-wise independent with the exception of the following cases.

- The calculated time position distribution and the intrinsic peak approximation location are related. This is intuitively hinted at by comparing Fig. 5.6 and Fig. 5.10 - where distribution shifts in P100 and N150 are seen in both distributions. Plotting these parameters against each other yields a linear relationship dependent on the wavelet transform level of the associated peak as demonstrated in Fig. 6.1. 


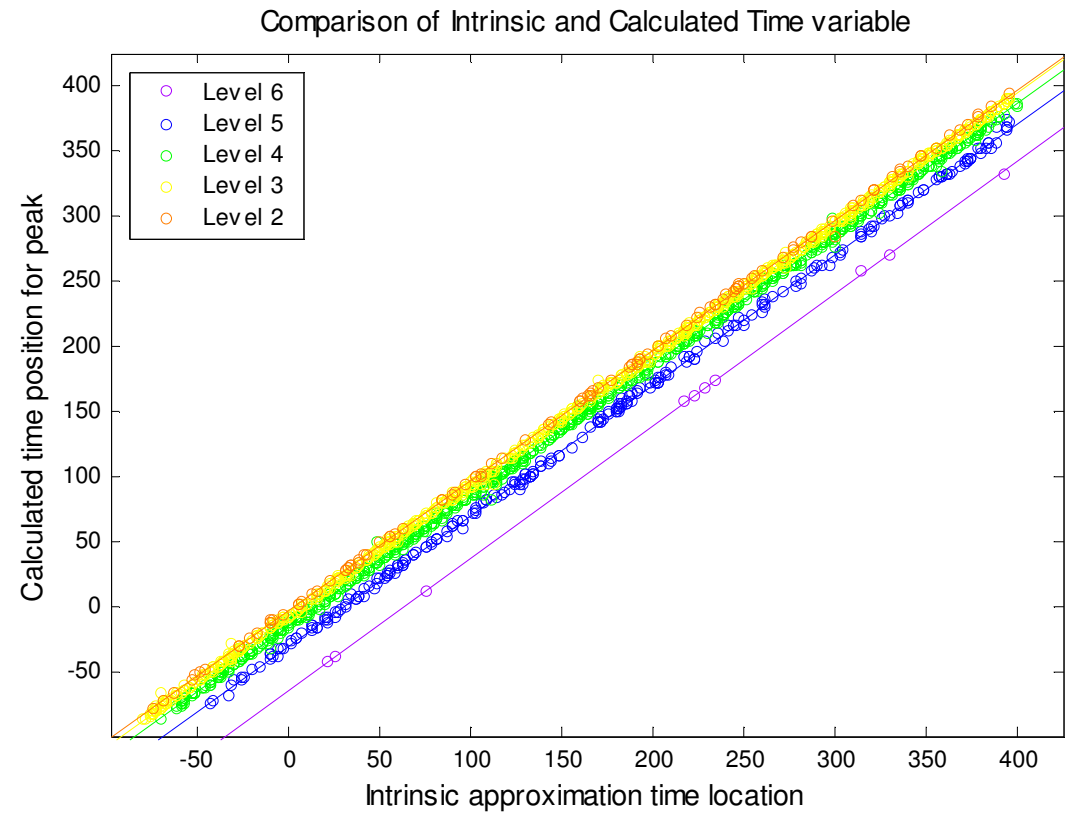

Fig. 6.1: Comparison of the calculated time position and the peak approximation location for P02VEP2, channel 2. A linear relationship is apparent with a scalar shift dependency based on the level (or scale) of the peak.

- The calculated area under the peak distribution and the intrinsic peak approximation coefficient are related. Plotting these parameters against each

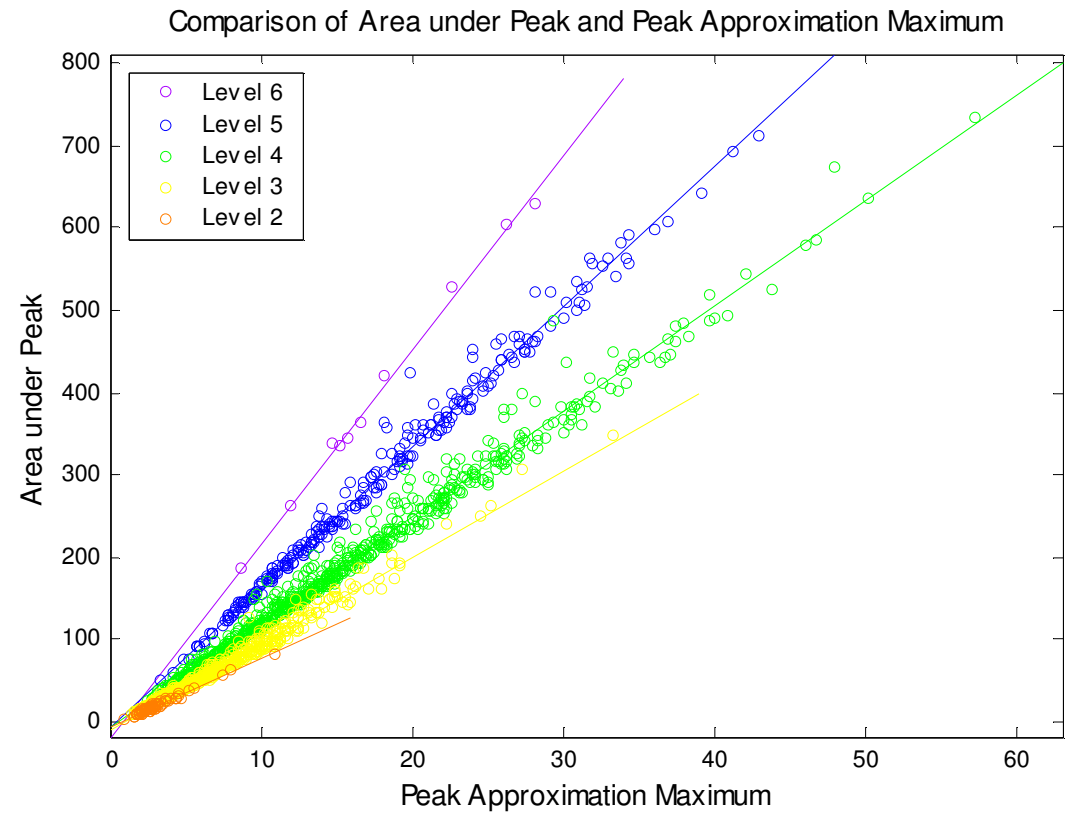

Fig. 6.2: Comparison of the peak approximation coefficient (or maximum) and the area under the peak for P02VEP2, channel 2. A linear relationship is apparent where the slope of the relationship is dependent on the level (or scale) of the peak.

other yields a linear relationship where the slope is dependent on the wavelet transform level of the associated peak as demonstrated in Fig. 6.2.

- The calculated peak height distribution and calculated area under the peak coefficient are loosely related. Plotting these parameters against each other yields a linear relationship where the slope is dependent on the wavelet transform level of the associated peak as demonstrated in Fig. 6.3. This is expected since area of a peak is directly related to the height. 


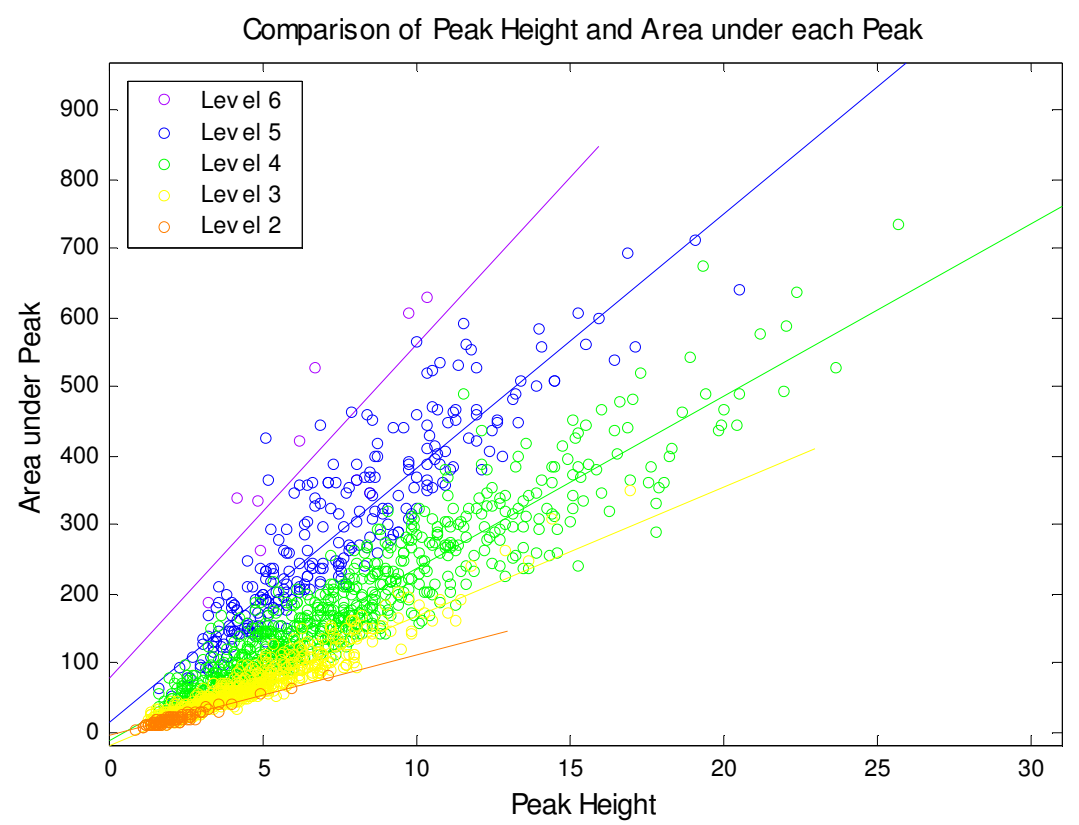

Fig. 6.3: Comparison of peak height, $h$ and area under peak, for P02VEP2, channel 2. There is a loose correlation between these two parameters.

\subsubsection{Peak Clustering - Mean Concentration Matrices}

- The mean concentration matrix distributions are smooth and very similar between positive and negative peaks. The peak concentration level observed in Fig. 5.15(a) is 21 peaks which occurs at bin location given by width=10 and height=3. The peak concentration level observed in Fig. 5.15(b) is also 21 peaks which occurs at bin location given by width $=13$ and height $=4$.

- These results were very repeatable across all dataset and also across all EEG datasets. Table 6.1 presents a summary of this data. It is observed that there is very little variation between EEG and VEP datasets which is desirable as these results are time independent. 


\begin{tabular}{|c|c|c|c|c|c|c|}
\hline $\begin{array}{l}\text { Participant, } \\
\text { Dataset }\end{array}$ & \multicolumn{2}{|c|}{$\begin{array}{l}\text { Max Conc. } \\
\text { for VEP }\end{array}$} & \multicolumn{2}{|c|}{$\begin{array}{l}\text { Max Conc. } \\
\text { for EEG }\end{array}$} & \multicolumn{2}{|c|}{$\begin{array}{l}\text { Max Delta Range } \\
\text { VEP-EEG }\end{array}$} \\
\hline & Pos. & Neg. & Pos. & Neg. & Pos. & Neg. \\
\hline P01, DS4 & 15.8 & 17.5 & 16.3 & 16.0 & {$[-1.8,+1.5]$} & {$[-0.9,+1.4]$} \\
\hline P01, DS6 & 17.9 & 18.2 & 16.4 & 18.1 & {$[-2.7,+1.6]$} & {$[-2.6,+1.4]$} \\
\hline P01, DS7 & 16.7 & 16.72 & 17.6 & 17.6 & {$[-2.0,+1.7]$} & {$[-2.2,+1.7]$} \\
\hline $\mathrm{P} 02, \mathrm{DS} 2$ & 20.9 & 21.7 & 20.9 & 22.6 & {$[-1.9,+0.8]$} & {$[-1.7,+1.6]$} \\
\hline $\mathrm{P} 02, \mathrm{DS} 3$ & 28.1 & 26.1 & 29.3 & 28.6 & {$[-2.3,+2.5]$} & {$[-1.4,+1.7]$} \\
\hline P02, DS4 & 23.1 & 23.1 & 21.3 & 22.7 & {$[-1,4,+1.5]$} & {$[-2.0,+2.4]$} \\
\hline P03, DS5 & 19.6 & 20.8 & 19.7 & 19.7 & {$[-1.7,+1.1]$} & {$[-1.7,+1.8]$} \\
\hline P03, DS7 & 16.7 & 16.9 & 17.5 & 16.7 & {$[-1.1,+1.3]$} & {$[-1.6,+2.7]$} \\
\hline P03, DS8 & 19.8 & 19.5 & 20.0 & 20.6 & {$[-1.6,+1.9]$} & {$[-1.9,+1.5]$} \\
\hline
\end{tabular}

Table 6.1: Summary of mean concentration matrices maxima and range across all dataset. The maximum concentration per bin is shown for the VEP and EEG signal and for positive and negative peaks. The variation between the VEP and EEG dataset is very small as demonstrated by the relatively small maximum delta variations for both positive and negative peaks.

- The consequence of subtracting the mean concentration matrices from the overall peak concentration matrices is to provide some separation between the evoked potential response and the background EEG and to reduce the background EEG

\subsubsection{Peak Clustering - Concentration Matrices}

- The peak concentration profile results confirm that the P100 and N150 components are far and away the most repeated peak type present. All datasets from all participants identified the P100 and N150 components as the most dominant components present. In terms of significance, these two components had always at least twice the significance value of the highest EEG bin. Compare the bins \#1 and \#2 of the blue and red signals part (b) of Fig. B.4 - Fig. B.11. These high concentration bins have a range of between 30 to 50 peaks. This equates to a peak being detected at most between 30-50 epochs - about one third of all possible epochs.

- Taking the example of the P02VEP2 channel 2 dataset in Fig. 5.16, the N150 peak is practically completely described by selecting three concentration bins - \#1 \#12 and \#18. The total number of peak (or epochs) represented by these 3 bins is $48+26+7=81$ out of a total of 155 or just over half. The remaining 74 epochs have no net effect on the N150 component. 


\subsubsection{Peak Clustering - Peak Magnitude}

- It is apparent from comparing all standard average peaks and reconstructed peaks that reconstructed peaks have significantly higher magnitudes. This is due to the fact that reconstituted peak are derived from averaging over a target subset of peaks known to contribute to a particular component. In contrast, the standard averaging process averages over all epochs whether or not there is any contribution by an epoch at a particular time.

- Historically, peak magnitude has had limited importance, yes, when the magnitude is high, it can be a clear indicator of the presence of an evoked response (such as P100 and N150). However, too often, its level is close to the background EEG noise and so is hard to discriminate.

- Reconstructed peaks are an average of the genuine observed peak response for the particular bin. The peak has been separated into peak shape and number of epochs - two very use parameter for understanding the nature of evoked responses.

\subsubsection{Peak Clustering - VEP Decomposition}

- The peak detection and peak clustering techniques allow a VEP to be decomposed into constituent sets of peaks. These peak sets are characterised by peak shape and number of epochs in which they are present. They are also summable and can be directly compared to the standard VEP signal. This yields a lot of additional information over the standard VEP technique. Examining Fig. 5.20, we see that the effect of overlapping components is better understood by studying adjacent peaksets:

- The N150 component is made up of 3 different types (Bin \#1, \#12,\#18). Of these Bin \#1 occurs most often - 48 peaks/epochs. Bin \#18 is very large in size but only occurs 7 times.

- The P225 component does not appear to be a single positive peak but decomposes to two overlapping positive peaks (Bin \#11 centred at 240ms and Bin \#19 centred at $210 \mathrm{~ms})$.

- In contrast, the N350 component which is similar in shape to the P225 component looks to be made of a single wide peakset (Bin \#6). 


\subsubsection{Peak Clustering - Vertical Stacking}

- It is clear from examining the P100 and N150 components across all datasets that there tends to be multiple peaksets that characterize each of these components. Hence in Fig. 5.20, the P100 component is made up of 3 peaksets (Bin \#2, \#22 and \#4). These peaks are vertically stacked having almost identical time location. The most common component is the middle peakset (Bin \#2) which has 47 peaks. The other bins are either side of this peakset.

- In thinking about an evoked response superimposed on background EEG signal, the above orientation makes sense. It is conjectured that Bin \#2 more closely represents the real evoked response and Bin\#4 and \#22 represent Bin \#2 with added positive and negative background EEG components respectively. Hence, Bin \#2 is tending towards the real evoked potential response to the provided stimulus. It cannot be said that is represents the response exactly as it is still itself an averaged signal. However, it is a much more targeted average than can be achieved with and the standard averaging process.

\subsubsection{Peak Clustering - Importance of Absence}

- Outside the P100 and N150 components there is less certainty regarding the presence or absence of evoked potential components primarily because the peak concentration levels are nearer to typical background noise levels. This is observed in Fig. B.4 - Fig. B.11, where after the initially high VEP levels in blue, the trend very quickly follows the background EEG level.

- However, examining the Peak reconstruction graphs which are plotted in significance order, such as Fig. 5.20, it is noted that between $-100 \mathrm{~ms}$ and $75 \mathrm{~ms}$, the positive and negative peaksets are relatively evenly spaced out resulting in a flattish averaged signal while the peakset after $75 \mathrm{~ms}$ are all directional, that is there are peaks that are either positive or negative not both positive and negative peaks at the same time. The later component peakset sizes are significantly smaller than the P100 and N150 components. Hence they will be more susceptible to the variations of background noise. If an evoked response is present at a particular time it is clear that it will skew the background EEG trend in either the positive or negative direction. This will lead to more peaks in the direction of the evoked response and equally importantly less peaks in the 
opposite direction. It is proposed that this is the effect observed in the peak reconstruction graphs such as Fig. 5.20.

- However some prudence is necessary as these graphs do not plot all concentration peaksets - only the highest observed. However it is still an important trend and provides additional information not regarding where peaksets are located but rather where they are not located.

\subsubsection{Peak Clustering - Confidence Factor}

- Separating evoked potential components into an average peak shape and number of peak repetitions, provides a lot of additional information over standard averaging. We have seen in 6.1.12 that the peak shape and cumulative peaks shapes help to separate evoked potential components. However, the number of repetitions (or peaks or epochs) also provides a measure of the confidence in the genuine presence of the peak shape. Hence if for a dataset of 150 epochs, if a peakset concentration is 150 then it is apparent that this peak occurs in every epoch as the exact same shape at the same time. This does not happen in real EEG systems. The background EEG signal ensures that there will be significant variation from epoch to epoch. The P100 and N150 components are large in size and so are more immune to background EEG than smaller components. Hence, they have a bin concentration of 30-50 epoch/peaks. There is no doubt that adjacent bins will be elevated which is observed in 6.1.14. The components after N150 are smaller in size and so are less immune to background EEG. Hence they appear at lower peak concentration levels and by implication at lower confidence levels.

\subsubsection{Peak Clustering - Pearson Correlation Coefficient Trends}

- It is noted in the Pearson Correlation Coefficient trend charts (Fig. 5.21 and Fig. B.29 - Fig. B.30) That the first two bins provide capture over $90 \%$ of the VEP signal and these two bins are by far the most prominent sources of variation within the VEP signal.

- The EEG correlation trend shown on the same graphs has a much slower and gradual rise due primarily to the lack of the EP component in the EEG signal. 
- Some caution is advised when considering these charts as adding some peaksets can reduce the correlation coefficient.

\subsubsection{Peak Clustering - Data Reduction}

- It is observed that the peak detection and peak clustering techniques can significantly reduce the size of the dataset used to calculate an evoked potential.

- Examination of the reconstructed peak graphs in Fig. 5.20 and Fig. B.12 - Fig. B.14 and Fig. B.18 - Fig. B.19 and finally Fig. B.22 - Fig. B.24, demonstrates that a small set of high concentration peaksets can accurately represent the Visual Evoked Potential response (Pearson correlation coefficient $>0.95$ ). This data reduction may be measured by comparing the number of peaks used to generate the reconstructed peak representation in each figure to the total number of peaks in the dataset. This total number is also the number used to generate the standard average VEP signal. This comparison yields a ratio that is an indicator of the data reduction property of the peak detection and peak clustering algorithms as a whole.

- For example, in Fig. 5.20, the total number of peaks detected for P02VEP2 channel 2 is 1985. Summing the number of peaks for the first 22 bins in Fig. 5.20, the total is 495 . Therefore the fraction of total peaks used to generate the equivalent peak average is:

$$
\begin{aligned}
\mathrm{f}_{\mathrm{epa}} & =\frac{\text { Num peaks Bin } \# 1-\# 22}{\text { Total Num Peaks }}=\frac{495}{1985}=0.249 \\
& =25.0 \%
\end{aligned}
$$

- A summary of this result for all datasets is provided in Table 6.2 along with figure reference, number of bins chosen, Pearson correlation coefficient at the is bin level and number of peak information.

- It is important to note that the same number of epochs must be measured however, since only partial components of each epoch are used. 


\begin{tabular}{|l|l|l|l|l|l|l|l|}
\hline & Figure & PCC & $\begin{array}{c}\text { No of } \\
\text { Bins }\end{array}$ & $\begin{array}{c}\text { Recon- } \\
\text { structed } \\
\text { Peaks }\end{array}$ & $\begin{array}{l}\text { Total } \\
\text { Peaks }\end{array}$ & Ratio & $f_{\text {epa }}$ \\
\hline P01VEP4 & & 0.986 & 22 & 446 & 1730 & $446 / 1730$ & $25.8 \%$ \\
\hline P01VEP6 & & 0.955 & 21 & 442 & 1716 & $442 / 1716$ & $25.8 \%$ \\
\hline P01VEP7 & & & 23 & 492 & 1676 & $492 / 1676$ & $29.4 \%$ \\
\hline P02VEP2 & Fig. 5.20 & & 22 & 495 & 1985 & $495 / 1985$ & $25.0 \%$ \\
\hline P02VEP3 & & 0.943 & 12 & 319 & 2686 & $\mathbf{1} / 2686$ & $11.8 \%$ \\
\hline P02VEP4 & & & 15 & 308 & 2285 & $\mathbf{1} / 2285$ & $13.4 \%$ \\
\hline P03VEP5 & & & 19 & 453 & 1707 & $453 / 1707$ & $26.5 \%$ \\
\hline P03VEP7 & & & 17 & 390 & 1548 & $390 / 1548$ & $25.1 \%$ \\
\hline P03VEP8 & & & 15 & 365 & 1690 & $365 / 1690$ & $21.5 \%$ \\
\hline
\end{tabular}

Table 6.2: Reduction in data is expressed as $f_{\text {epa }}$, the fraction of reconstructed peaks versus total peaks per dataset. For each dataset, the figure number locates where the data is found. For the number of bins chosen, PCC represent the Pearson correlation coefficient. Reconstructed peak total is given by the sums of the peaks in the chosen bins. The total peaks column represents all peaks in all epochs which when added and averaged yield the standard averaged VEP signal. The ratio of these last two values yields the fraction of peaks used to generate the peak reconstructed signal.

- Take the following, simplified example based on Table 6.2. A dataset of 150 repeated stimuli or epochs, each $0.5 \mathrm{~s}$ in duration has approximately 12 peaks per epoch. The total number of peaks per dataset is $150 \times 12=1800$ peaks. The clustering algorithm can reconstruct a close approximation of the average VEP response using one-quarter of these peaks, i.e. 450 peaks. Typically, this equates to approximately 20 different peaksets (or bins) each containing approximately 22.5 peaks $(=450 \div 20)$ on average. While this representation is not as simple as the standard VEP response, it contains a lot more useful information regarding position of peaks, shape of peaks and repeatability of peaks. Also it can still be directly related to the standard VEP response.

\subsubsection{Peak Clustering - P100 and N150 Components}

- The observed latency and magnitude of the P100 and N150 components is recorded in Table 6.3 for all datasets and participants. It is observed that the latency and magnitude is very consistent for intra-participant datasets but more variable for inter-participant datasets. This is due to normal variation between participant responses. 


\begin{tabular}{|c|c|c|c|c|c|}
\hline & \multicolumn{2}{|c|}{ P100 EP component } & \multicolumn{2}{|c|}{ N150 EP component } \\
\hline & & Latency & Magnitude & Latency & Magnitude \\
\hline \multirow{3}{*}{$\begin{array}{l}\text { Participant } \\
01\end{array}$} & P01VEP4 & $112.9 \mathrm{~ms}$ & $3.3 \mu \mathrm{V}$ & $142.2 \mathrm{~ms}$ & $-5.1 \mu \mathrm{V}$ \\
\hline & P01VEP6 & $110.9 \mathrm{~ms}$ & $2.9 \mu \mathrm{V}$ & $142.2 \mathrm{~ms}$ & $-5.1 \mu \mathrm{V}$ \\
\hline & P01VEP7 & $112.9 \mathrm{~ms}$ & $2.9 \mu \mathrm{V}$ & $142.2 \mathrm{~ms}$ & $-5.3 \mu \mathrm{V}$ \\
\hline \multirow{3}{*}{$\begin{array}{l}\text { Participant } \\
02\end{array}$} & P02VEP2 & $103.1 \mathrm{~ms}$ & $4.6 \mu \mathrm{V}$ & $151.9 \mathrm{~ms}$ & $-4.0 \mu \mathrm{V}$ \\
\hline & P02VEP3 & $103.1 \mathrm{~ms}$ & $4.5 \mu \mathrm{V}$ & $155.9 \mathrm{~ms}$ & $-4.5 \mu \mathrm{V}$ \\
\hline & P02VEP4 & $101.1 \mathrm{~ms}$ & $4.0 \mu \mathrm{V}$ & $163.6 \mathrm{~ms}$ & $-4.1 \mu \mathrm{V}$ \\
\hline \multirow{3}{*}{$\begin{array}{l}\text { Participant } \\
03\end{array}$} & P03VEP5 & $107.0 \mathrm{~ms}$ & $3.7 \mu \mathrm{V}$ & $146.1 \mathrm{~ms}$ & $-3.4 \mu \mathrm{V}$ \\
\hline & P03VEP7 & $107.0 \mathrm{~ms}$ & $3.6 \mu \mathrm{V}$ & $150.0 \mathrm{~ms}$ & $-2.7 \mu \mathrm{V}$ \\
\hline & P03VEP8 & $107.0 \mathrm{~ms}$ & $3.8 \mu \mathrm{V}$ & $150.0 \mathrm{~ms}$ & $-3.0 \mu \mathrm{V}$ \\
\hline
\end{tabular}

\subsubsection{Peak Clustering - Cross-dataset Correlations}

Ordering bins by the number of peaks above the average for that bin and without taking account of the peak size is the best method for focussing on the most repeated peaks due to the presence of an evoked potential signal. Let us examine the peaks detected for Participant 02 shown in Fig. 5.23, Fig. B.18 and Fig. B.19. These are the highest concentration peaks ordered by number of peaks above the average. The peaks that are coincident in most or all datasets are selected and illustrated in Fig. B.31 - Fig. B.33. Table 5.9 shows the sequence of detected components in terms of time and peak size in a tabular form. Peaks are chosen that appear consistent across all datasets. Several new peaks are apparent which are not distinguishable from the standard average VEP. Also the peak magnitude is higher for the reconstructed peaks.

(a) Reconstructed peak maxima location and size - based on number of peaks above average

\begin{tabular}{|l|l|l|l|l|l|l|l|l|l|l|}
\hline Dataset & N75 & P100 & N150 & P190 & N210 & P240 & N260 & N280 & P310 & N340 \\
\hline P02VEP2 & & +9.8 & -8.0 & & -6.0 & +6.0 & -4.4 & -4.3 & +4.2 & -5.5 \\
\hline P02VEP3 & & +9.8 & -10.8 & +4.0 & -2.5 & +5.8 & -4.3 & & +4.3 & -7.2 \\
\hline P02VEP4 & & +10.4 & -9.8 & +4.2 & -4.4 & +7.3 & & -5.1 & +5.2 & -4.8 \\
\hline
\end{tabular}

(b) Peak maxima location and size for standard averaged VEP signal.

\begin{tabular}{|l|l|l|l|l|l|l|l|l|l|l|}
\hline Dataset & $\mathrm{N} 75$ & $\mathrm{P} 100$ & $\mathrm{~N} 150$ & $\mathrm{P} 190$ & $\mathrm{~N} 210$ & $\mathrm{P} 240$ & $\mathrm{~N} 260$ & $\mathrm{~N} 280$ & $\mathrm{P} 300$ & $\mathrm{~N} 340$ \\
\hline P02VEP2 & & +4.6 & -4.0 & & & +1 & -0.8 & & +1.2 & -1.4 \\
\hline P02VEP3 & & +4.5 & -4.5 & & & +1.5 & -1.1 & & & -0.6 \\
\hline P02VEP4 & & +4.0 & -4.1 & & & +2.1 & -0.6 & & +0.8 & -0.4 \\
\hline
\end{tabular}

Table 5.9: The detected standard average peaks (b) are compared to the peaks elicited by choosing the highest concentration bins above the average level - reconstructed peaks (a). These reconstructed peaks have a much higher magnitude and there are also additional peaks identified that are not observed in the averaged VEP.

- The same process is applied to datasets for Participant 02 which is shown in Fig. B.32 and also for Participant 03 which is shown in Fig. B.33. 


\section{CHAPTER 7 DISCUSSION \& CONCLUSION}

\subsection{INTRODUCTION}

7 his research set out to investigate a one-to-one stimulus-response paradigm for Evoked Potential EEG signals. Decoding these neurological messages would L have far reaching implications in many fields such as neurology, cognitive psychology and the brain-computer interface as well as providing a powerful technique for examining efficacy of many therapeutic modalities including acupuncture or reflexology. However, as yet, no method has been discovered to directly relate a single stimulus to a single response in the brain.

Investigation into this paradigm has been, and continues to be, an area of major scientific investigation. A review of different analysis techniques demonstrates that different methods emphasize the information content of different domains - temporal, spatial, spectral or epoch. Thus, by applying particular transforms to raw evoked potential data, it is possible to highlight the information content in one domain at the expense of information content in other domains Analysis across different domains demonstrates that each domain offers insights into the nature of evoked potential signals. The difficulty is that each analysis technique includes some domains and excludes others. This research endeavours to take a more holistic approach by attempting to characterise evoked potential activity in such away that information in multiple domains is retained throughout the analysis. It is hypothesized that such a technique would offer new insights into evoked potentials not obtainable with other techniques.

This led to the idea of investigating multi-domain analysis to determine whether additional useful information could be gleaned using such techniques. The EEG or Evoked Potential signal is made up a series of positive and negative deflections or peaks. The focus of this research is to take these deflections and characterise them discretely across all domains. The definition of these positive and negative deflections as peaks is an intuitive and commonsense representation since current evoked potential responses are defined in terms of peaks [82]. The process of decomposition of Evoked Potential signals into sets of discrete peaks or 'Peak Detection' became the first major focus of this research. 
The next major step called 'Peak Characterisation' involves taking the detected peaks and characterising them into discrete, independent parameters which characterise the peaks across different domains.

The last major focus involves developing an analytical framework by which the characterising parameters may be scrutinised and areas of higher concentration of parameters across all domains identified. This is the 'Peak Clustering' phase whose output constitutes an entirely new technique for presenting and observing evoked potential signals.

Finally the results of the peak clustering technique are reviewed against the original aims of the research. The following discussion is divided up into these three areas of study and finishes with possible applications and future research directions for this research.

\subsection{PEAK DETECTION}

The peak detection algorithm developed by the author meets all of the required design criteria. It decomposes individual epochs of evoked potential data into a simple set of peaks. Each peak is represented by a set of discrete wavelet transform coefficients. This set is made up of left-hand singularity detail wavelet coefficients, right-hand singularity detail wavelet coefficients and approximation wavelet coefficients. Taken together, these coefficients may be inverse transformed into an accurate representation of the local peak. The peaks of an epoch are then summable to reconstruct a close approximation of the epoch signal. Unlike the approach of Rodionov et al [60] or Arikidis et al [65], this representation is complete - peak characteristics lead to selection of pertinent peaks instead of leading to a selection of pertinent epochs. It is the ability to define individual peaks that give the Peak Detection method is analytical advantage over other methods analysing individual peaks instead of whole epochs is a big step forward. The validity of the representation is fully tested by inverse transforming the coefficients to form a regenerated signal which is then compared to the original epoch.

All EEG analysis was performed on time-varying non-stationary signals that are encoded with the sum of EEG activity detected at a particular electrode. By observation, the basic blocks of these signals are peaks. A peak represents the summed response of detected activity at a particular electrode. This research set out to decompose epochs of evoked potential EEG into set of discrete peaks where each peak could then be uniquely 
characterised. To this end, an effective peak detection algorithm has been developed and tested which runs fully automatically. This technique takes a modulus maxima discrete dyadic wavelet transform and identifies signal singularities. Then the Lipschitz exponent is calculated for the singularities and used to group singularities into pairs known as peaks. The sum of these peaks over an epoch represents the original epoch data very well. By selecting only those peaksets that yield a Pearson correlation coefficient 0.96 or higher, $97.5 \%$ of all epochs were decomposed into sets of peaks (refer to 6.1.4).

The representation of a non-stationary signal as a set of peaks provides a framework for various types of analysis. The one analysis approach used in this research is to characterise these peaks and use clustering to focus on areas of interest. However, there are other methodologies that could be applied to this signal representation for differing applications. For example, the peak detection method provides an efficient coding mechanism for signal compression. A 256 sample EEG epoch is typically represented by a time start position, time sampling rate (or time interval) and one potential difference value for each of the samples yielding $2+256=258$. The peak detection method however can represent the same signal over an average of 12 peaks with an average of 19 points to represent each peak. This yields a total of 228 which reduces the dataset size by $10 \%$. This dataset size reduction is made significantly larger by implementing a selective peak analysis technique.

The peak detection technique executes automatically using singularity detection and Lipschitz exponent theory to identify peaks from their Discrete Wavelet Transform coefficient patterns. The concept of applying modulus maxima DWT to determine the detail coefficients of singularities is well known [32], however the application of the modulus maxima (MM) approach to the approximation coefficient appears to be a novel but effective technique to determine the shape of the peak between the singularities. Typical MM DWT analysis interprets the singularity coefficients alone, however in this research the MM DWT concept has been furthered to use these coefficients to regenerate the separable peak shape which may then be further analysed. This generates a powerful 'wholeness' to the representation allowing complete forward and inverse transformability. This gives flexibility in the analysis - thus for a brain-computer interface application, it is possible to now focus on a particular evoked response shape and not just on the time a particular response occurs. 


\subsection{PEAK CHARACTERISATION}

The characterisation process is simply an intermediate stage where the output of the peak detection process is examined to provide the most suitable input to the peak clustering process. In other words, the characteristics of the peaks are compared to each other and the most independent variables are chosen to uniquely describe the output. In choosing the calculated parameters of time position, height and width (together with epoch) the most independent choice is being made. Using these parameters involves a higher computational load than choosing the intrinsic parameters however the exactness and independence yield better peak segregation results (refer to section 0 ).

While Rodionov et al [60] used peak time location to highlight difference in peak concentration between positive and negative peaks, our research uses the time position distribution but also expands this decomposition further by introducing the width and height as additional sorting criteria. Rodionov et al used this characterisation to select a focussed subset of all epochs and re-averaged using this subset. However, using our approach only certain identified peaks need to be selected for recalculation of the average. This has the effect of targeting the evoked potential to a set of individual peaks rather than targeting a set of individual epochs.

Another similarity to that of Rodionov et al. is the description of time domain data into separate positive and negative sets of peaks and the observation of relative shifts in concentration between these opposite signed peaksets. This is similar to the time location graphs of Fig. 5.6, Fig. 5.10 and Fig. 5.12 that show where similar shifts occur.

Interrelations between calculated and intrinsic parameters have been noted. The calculated time position and approximation location are directly related and the latter could easily be substituted for the former in all our analysis. It has also worth noting that this approximation coefficient is directly related to the area under the peak (refer to Fig. 6.2). However, the separate width and height parameters provide better peak segregation than the single area under the peak parameter. 


\subsection{PEAK CLUSTERING}

The peak clustering algorithm provides a simple but powerful method of categorising individual peaks in terms of peak orientation (positive or negative), peak time location, peak width and peak height, while retaining the record of which epoch the peak belongs to. This categorisation leads to a pair of 3-dimensional concentration matrices that can easily be analysed to find areas of higher concentration. The results (Fig. 5.20 and Fig. B.12 to Fig. B.14, Fig. B.18 to Fig. B.19 and Fig. B.22 to Fig. B.24) demonstrate that the proposed technique can identify the averaged VEP signal using only a fraction of the standard dataset. Table 6.2 illustrates that the dataset size has been reduced by an average of nearly $75 \%$ across all datasets. The number of epochs required to be measured is still the same. However, one major outcome of this technique is that it provides a subset of data in the form of selected peaks across all these epochs can be used to generate very similar results as the standard VEP response.

Moreover, this technique offers a new understanding of the relationship between the peak magnitudes and number of epochs in evoked potential signals. The proposed technique presents a new representation of the evoked potential response where the number of epochs is emphasized and the magnitude of peaks de-emphasized. This approach has the potential to improve the interpretation of evoked responses by providing a clearer breakdown of the components that make up each evoked response.

Not only is the same information available from a substantially reduced dataset but much additional information is also available due to the clustering process. Firstly, the 'equivalent peak average' signal can be decomposed into a manageable set of peaksets. This differs from standard VEP averaging where signal response can only be broken down by the experienced eye of an expert. Expressing a VEP signal as a sum of, say, 20 different peaksets is a very useful representation. It is now much easier to understand how particular peaksets occur. Secondly, the individual peaksets are decomposed into a peak-shape and the number of times (or epochs) it occurs. Discrimination between VEP components that are small but occur frequently versus larger components that occur less often is possible. This is not possible with standard averaging.

However, it is noted that the peak reconstruction process does not give an exact or definitive breakdown of all VEP components. It is telling that the P100 and N150 components in our results have a much higher peak concentration level that all other 
peaks. These two components obviously stand-out. They also stand-out in the standard averaging VEP response. The peak concentration levels for other possible components are much smaller and only just above the range of the background EEG level. This means that particular peaksets at this level cannot categorically be ascribed as VEP components. They must be examined in the context of adjacent peaks and their position and perhaps a lack of opposite signed peaks at the same time position. Further analysis between particular responses and peaksets must be manually interpreted by an expert in the field. Based on the observations from the decomposed EEG signal into peaks that represent pulse activity of the brain, it is evident that the decomposed signals will provide useful information to the neurological experts. At these lower concentration levels, the peak clustering technique tends to emphasize evoked potential responses but does not definitively discriminate between evoked potential responses and background EEG signals as both have similar concentration levels.

\subsubsection{Peakset Expression}

The expression of an averaged VEP response in terms of peaksets may be viewed as an improvement in the signal resolution. The time position of events is more refined or accurate as more distinct and separate peaks are identified as being important (refer to Fig. 5.20). Also, the proposed technique presents three different expressions for each peakset reconstruction that provide three different viewpoints.

- The first is a simple average over the number of peaks in the particular peakset. This gives a good expression of the spread of the peakset and is a scaled replica of how much this peakset adds to the standard averaged VEP response (refer to the dashed line in Fig. 5.20).

- The second gives a representation of the 'average peak shape'. The parameters that define the elements of the peakset are the time position, peak width and peak height. By averaging each individual parameter and using the results to create an 'average peak shape', it is possible to present an average peak shape which gives a visual representation of what a real evoked potential component may look like. The 'average peak shape' is narrower and taller than the peakset average. In this way, the proposed technique overcomes some of the inherent smoothing effects typical of standard VEP averaging. 
- The third peakset expression gives a measure of how much the peakset contributes to the comparable standard averaged evoked response. It is an average of the peaks within a peakset but instead of being averaged over the number of peaks in the peakset as in the first expression, it is averaged over all epochs in the dataset. This expression is illustrated in Fig. 5.20 as 'the equivalent peak average'. It is not shown individually but as a cumulative sum of all peaks added together so that comparison with the standard averaged signal is possible.

\subsubsection{VEP Representations}

The extraction criteria for clustering may be tuned to perceive different aspects of EP activity. Primarily, there are two such criteria of interest and ordering by these criteria yields slightly different but significant results. The two criteria types are discussed in the following sub-sections.

\subsubsection{Total Number of Peaks X Area under Peak}

This representation most closely approximates the process of standard averaging (refer to Fig. 5.20 and Fig. B.12 to Fig. B.14, Fig. B.18 to Fig. B.19 and Fig. B.22 to Fig. B.24). In standard averaging peak shape and number of such peaks are equally important. For example, the peak shape may be expressed as an area under the peak given in terms of time (msec) multiplied by peak magnitude $(\mu \mathrm{V})$ having units $\mathrm{nVs}$ (nano-Volt-seconds). Therefore, with standard averaging a peak of shape 100nVS occurring 50 times would appear identical to a larger peak of area $500 \mathrm{nVs}$ occurring only 10 times. Hence, in order to identify peaksets that will most closely approximate the standard average VEP signal, concentration bins are ordered in terms of the product of these two parameters yielding a set of peaksets that closely approximate the standard averaged VEP response. This representation can be directly interpreted as a decomposition of the standard averaged VEP response providing invaluable information of the location, shape and importance of peaks that sum to form the averaged VEP response.

\subsubsection{Net Peaks above Average}

The purpose of averaging is to emphasize the repeated stimulus response alone (refer to Fig. 5.23 and Fig. B.15 to Fig. B.17, Fig. B.20 to Fig. B.21 and Fig. B.25 to Fig. B.27). However, with averaging, larger peaks inherently contribute more to the overall evoked 
response than smaller peaks - which is a drawback of this technique. It is preferable to identify the most repeated peaks irrespective of size. This is not possible with the standard VEP averaging approach. However it is possible with the proposed peak detection/peak clustering technique.

A further refinement is also possible. Certain background EEG peaks are more common than others i.e. there is a profile or range of values (across the peak height and peak width domain) that occurs (refer to section 5.3.4). We wish to identify activity that is associated with the evoked response only, rather than the evoked response and the background EEG. The influence of background EEG on our results is reduced by calculating the average across all time location bins of the width and the height concentration levels and subtracting this average distribution from the peak concentration matrices. Background EEG is not time locked. Hence when the average is removed from all time position, those time position that have time-locked (or evoked potential) activity stand-out. This average background EEG distribution is presented for the P02VEP2 dataset in Fig. 5.15.

\subsection{APPLICATIONS}

The techniques and algorithms described in this research provide potentially valuable additional information to all clinicians who measure and interpret any type of evoked potential signal. This technique is not limited to Visual Evoked Potentials but may equally be applied to Somatosensory Evoked Potentials, Auditory Evoked Potentials or, more broadly, to Event-Related Potentials. In each area, the same decomposition of the evoked response is performed to identify groups of peaks that contribute most to the particular observed components. Up to now, this process has been performed intuitively by clinicians - but by providing more detail regarding the dominant shape of particular peaks as wells as yielding the number of time such shapes are present across all evoked responses, clinicians have considerable additional data from which to interpret the observed responses.

The variability of evoked potentials responses both between subjects and within individual subjects has been a barrier to confident interpretation of smaller evoked response components. This research illustrates how smaller perceived evoked responses are difficult to separate from certain coherent background EEG signals. However, separation of such components into shape and number of peaks present offers a useful 
technique for distinguishing the genuine evoked response from mere coherent background signals.

The technique requires no apriori estimate of where the evoked potential response is expected. Hence the reconstruction signals are generated independently of, but are still comparable to the standard VEP response. The fact that this technique is compatible with the standard averaging VEP technique makes it a valuable additional tool for neurological experts; A tool that presents the data in a more detailed form than is currently available.

This research is most suited to applications relating to the brain-computer interface primarily because if offers the opportunity to interpret the data in new and innovative ways. In BCI applications, often repeated stimuli or sensory information is required in order to reach a decision about a particular message [83-86]. However, by interpreting individual messages more effectively, it is possible to use fewer repetitions and thus react more quickly to brain messages.

Another potential application in BCI includes analysis of spatial data to interpret a single epoch from multiple adjacent sites to localise the origin of a particular signal. Thus it may be possible to filter out signals permeating across the surface of the scalp and focus on those signals originating at a particular central point of interest. This method together with the methods applied to a single site in this research has the potential for cumulative reductions in number of samples required to interpret a message. This localisation scheme would be quite different from other localization methods such as source localisation since it is not dependent on a whole head model to which the data must 'fit' but provides a truly local interpretation of spatial data.

\subsection{LIMITATIONS}

Zero splitting of positive and negative peaks means that determining interrelatedness between different adjacent components is not possible. For example, it may be that the P100 and N150 represent a single complex component or two separate components. The peak detection and peak clustering algorithms do not provide any understanding regarding the relatedness of these 2 components. They are analysed separately and thus must be presented separately. It is worth noting that the standard averaging evoked potential response also has the same limitation. 
Further work is required to understand the decomposition for a much wider range of circumstances before this technique could be applied for detecting single responses to tactile stimulation modalities such as acupuncture or reflexology. Somatosensory evoked responses tend to have a smaller magnitude response than visual evoked responses which may reduce the effectiveness of peak detection.

The peak detection and peak clustering techniques provide a valuable additional analytical tool to all standard evoked potential testing. It is equally applicable to somatosensory, audio and visual evoked potentials without modification. It is a technique that is very orientated to the neurological expert's viewpoint - providing a simple but indepth analysis of the major peak shapes that make up the evoked potential response. The fact that it is directly comparable to the standard evoked potential response also aids the integration of this method into standard evoked potential procedures. Using Matlab and a modern personal computer, it currently takes 10 minutes to perform a full analysis on a single channel of data. This would be greatly improved by writing a dedicated program in a $\mathrm{C}++$ or similar environment. This processing time saving needs to be offset by the expectation that the number of channels to be analysed would be greater. Hence, this technique may be reserved for offline analysis after all results have been taken. It has a computational overhead that prevents its use during experimentation.

Another limitation of this technique, as presented in this thesis, is that it only examines single electrode locations. Thus it is unable to provide any spatial interpretation. However, there is considerable scope for developing this technique further analysing across multiple sites and relating adjacent peaks to one another.

The goal of achieving a one-to-one stimulus-response paradigm has not been achieved in this research. However new and valuable insights into the nature of an evoked potential signal have been identified. There is still a strong dependence on the repeated stimuli information, however a more accurate picture of what individual peak shape may look like and how often they are present in a dataset has been achieved.

\subsection{FUTURE RESEARCH}

There are several directions in which this research could advance. 


\subsubsection{Algorithm Improvement}

It is proposed to improve the peak detection and peak clustering algorithms by increasing the peak detection ratio from $97.5 \%$ to $100 \%$. Also, rewriting the algorithms as a dedicated software package with a more user-friendly graphical interface would be important. Broad distribution of these algorithms would be best served by linking up with EEG software providers and incorporating these algorithms into existing EEG analysis software.

\subsubsection{Further Testing}

A better understanding of the range of benefits of the peak detection and peak clustering techniques would be attained by applying the techniques across a broad range of evoked potentials types - such as Somatosensory Evoked Potential, Auditory Evoked Potentials as well as Visual Evoked Potentials. These techniques could also be applied to Electrocardiogram data. It is proposed to link up with other research and clinical institutions and apply these techniques to a broad range of datasets for further interpretation.

Furthermore, as more and more datasets are analysed with this method, a better understanding of the inter-participant variations and peaksets will become more apparent. It is outside the scope of this research to identify this inter-participant correlation.

\subsubsection{Spatial analysis.}

This research has investigated the relationship between the temporal, spectral (scale) and epoch domains and retained viewpoints from each domain. However, the spatial domain has not been analysed. Incorporating this perspective into the peak detection and peak clustering algorithms would provide additional information from which to decode the evoked potential. This would be a large undertaking and was beyond the scope of this research yet is the next logical area of investigation for this technique.

\subsection{CONCLUSION}

This research has identified and developed a multi-domain evoked potential analysis technique - retaining perspective in the temporal, spectral and epochal domains. This meets the first of our research aims. This technique retains a view of single-trial data throughout the analysis. 
A comprehensive set of algorithms to decompose evoked potential data into sets of peaks has been developed and tested. These sets of peaks then form the basis for a subsequent clustering analysis which can identify sets of localised peaks that contribute the most towards the standard evoked response. The technique is quite novel as no closely similar work in research has been identified.

New and valuable insights into the nature of an evoked potential signal have been identified. Although the number of stimuli required to calculate an Evoked Potential response has not been reduced, the amount of data contributing to this response has been effectively reduced by $75 \%$. Therefore better examination of a small subset of the evoked potential data is possible. Furthermore, the response has been meaningfully decomposed into a small number (circa 20) of constituent peaksets that are defined in terms of the peak shape (time location, peak width and peak height) and number of peaks within the peak set. This representation retains a perspective in temporal (time location), spectral (peak width which is a measure of scale) and epoch (number of peaks within a peakset) which addresses the first aim of the research.

The nature of the output of this technique is quite different to that of standard averaging. It has been shown that the larger clustering of certain peak shapes (evident in the P100 and N150 components in the results) accurately represents the averaged signal. This leads to the supposition that these peaks represent a much closer approximation of a genuine evoked potential response. This represents a considerable advance in our understanding of what a single response to an evoked potential looks like. It is not the single-stimulussingle-response paradigm but it does offer a better understanding of what a single response may look like - by identifying the most common peak responses. In this way, this technique brings us closer to the single-stimulus-single-response understanding. Thus the second research aim has been addressed.

The question of why some evoked potential components appear more strongly than others is probed by this technique. Delineation between individual peak sizes and how often they occur is for the first time possible and this representation helps to provide an understanding of how particular evoked potentials components are made up.

A major advantage of this techniques is the there are no pre-conditions, constraints or limitations to the technique. 
The techniques developed during this research have real-world applications as a useful additional tool to current techniques in both clinical and research EEG environments. This research set out to find an EEG analysis technique that would be more suited to the one-to-one stimulus response paradigm and cited one particular application in the detection of a response in the brain to therapeutic modalities such as acupuncture and reflexology. Employing the techniques of this research results in a parsimonious representation of the evoked potentials signal that can be identified using approximately $25 \%$ of the dataset. This is a massive reduction on the evoked response dataset dependency.

The techniques of this research are highly relevant to all evoked potential modalities and have the potential to offer insights in all these areas. It is a focus of future research to apply these methods to other evoked potential areas. The other area of future research relates to the incorporation of the spatial domain which has the potentials to further expand our understanding of evoked responses. Examination of peak geometries relative to their physical locations opens up the avenue of peak selection based on relative peak strength between electrode locations.

Overall, a novel evoked potential technique has been described and tested. The results provide new insights into the nature of evoked potential peaks with application across various evoked potential modalities. 


\section{CHAPTER 8 PUBLICATIONS}

During the course of this research, four papers were submitted, approved and presented at internationally recognized conferences. They provided an intermediate report on progress of the research at the time. The citations are listed below and a copy of each paper is included in Appendix D.

\subsection{EMBC 2007}

- McCooey, C., Kant Kumar, D, "Automated Peak Decomposition of Evoked Potential Signals using Wavelet Transform Singularity Detection," presented at IEEE Engineering in Medical Biology Conference, Lyon, France, 2007.

\subsection{ICINCO 2005}

- McCooey, C., Kant Kumar, D, "Characterising evoked potential signals using wavelet transform singularity detection," presented at 2nd International Conference on Informatics in Control in Automation and Robotics (ICINCO), Barcelona, Spain, 2005.

\subsection{EMBC 2005}

- McCooey, C., Kant Kumar, D, Cosic, I, "Decomposition of Evoked Potentials using Peak Detection and the Discrete Wavelet Transform," presented at IEEE Engineering in Medical Biology Conference, Shanghai, China, 2005.

\subsection{WCMPBE 2003}

- McCooey, C., Kant Kumar, D, "Characterising evoked potential signals using wavelet transform singularity detection," presented at World Conference on Medical Physics and Biomedical Engineering, Sydney, Australia, 2003. 


\section{CHAPTER 9 REFERENCES}

[1] "Wikipedia, The Free Encyclopedia," http://en.wikipedia.org/, 2007.

[2] D. L. Donoho, A. Maleki, M. Shahram, and e. al, "Wavelab," Version 850 ed, 2006.

[3] J. Pinel, "A Colorful Introduction to the Anatomy of the Human Brain," Allyn and Bacon, 1998.

[4] "Microsoft Clipart," Microsoft Coproration, 2007.

[5] S. Mallat and W. L. Hwang, "Singularity Detection and Processing with Wavelets," Ieee Transactions on Information Theory, vol. 38, pp. 617-643, 1992.

[6] P. Nunez, Electric Fields of the Brain, 2nd ed: Oxford University Press, 2006.

[7] M. Vetterli, "Wavelet Transforms and Applications to Signal Processing and Communications," presented at AVIPAC (Audiovisual Information Processing and Communications) Workshop, Melbourne, 2003.

[8] P. M. Barnes, E. Powell-Griner, K. McFann, and R. L. Nahin, "Complementary and Alternative Medicine use among Adults: United States," CDC Advance Data REport \#243, 2004.

[9] "Manipulative and Body-Based Practices: An Overview, Backgrounder," National Centre for Complementary and Alternative Medicine Dec 20042004.

[10] H. Moffat, "How might Acupuncture Work? A systemic review of physiologic rationales from clinical trials.," Biomed Central, 2006.

[11] "Acupuncture, Get the Facts," National Centre for Complementary and Alternative Medicine, 2004.

[12] A. F. Leuchter, I. A. Cook, E. A. Witte, M. Morgan, and M. Abrams, "Changes in Brain Function of Depressed Subjects During Treatment With Placebo," Am J Psychiatry, vol. 159, pp. 122-129, 2002.

[13] A. Khan, H. A. Warner, and W. A. Brown, "Symptom Reduction and Suicide Risk in Patients Treated With Placebo in Antidepressant Clinical Trials: An Analysis of the Food and Drug Administration Database," Arch Gen Psychiatry, vol. 57, pp. 311-317, 2000.

[14] S. G. Mallat, "A theory for multiresolution signal decomposition: the wavelet representation," Pattern Analysis and Machine Intelligence, IEEE Transactions on, vol. 11, pp. 674-693, 1989.

[15] J. Malmivuo, Plonsey, R, Bioelectromagnetism: Principles and Applications of Bioelectric and Biomagnetic Fields: Oxford University Press, 1995.

[16] K. Misulis, Essentials of Clinical Neurophysiology: Butterworth Heinemann, 2003.

[17] K. Kooi, Visual Evoked Potentials in Central Disorders of the Visual System, 1st Ed ed: Harper \& row, 1979. 
[18] W. Truccolo, K. H. Knuth, A. Shah, S. L. Bressler, C. E. Schroeder, and M. Ding, "Estimation of single-trial multicomponent ERPs: Differentially variable component analysis (dVCA)," Biological Cybernetics, vol. 89, pp. 426-438, 2003.

[19] R. Quian Quiroga, "Obtaining single stimulus evoked potentials with wavelet denoising," Physica D: Nonlinear Phenomena, vol. 145, pp. 278-292, 2000.

[20] D. Iyer and G. Zouridakis, "Single-trial evoked potential estimation: Comparison between independent component analysis and wavelet denoising," Clinical Neurophysiology, vol. 118, pp. 495-504, 2007.

[21] E. A. Bartnik, K. J. Blinowska, and P. J. Durka, "Single evoked potential reconstruction by means of wavelet transform," Biol Cybern, vol. 67, pp. 175-81, 1992.

[22] T. Demiralp, A. Ademoglu, M. Schurmann, C. Basar-Eroglu, and E. Basar, "Detection of P300 Waves in Single Trials by the Wavelet Transform (WT),," Brain and Language, vol. 66, pp. 108-128, 1999.

[23] H. Berger, "On the Electroencephalogram of Man," Electroencephalogr Clin Neurophysiol, vol. Suppl 28, pp. 37+, 1969.

[24] H. M. Freeman WJ, Burke BC, Vanhatalo S, "Spatial spectra of scalp EEG and EMG from awake humans," Clinical Neurophysiology, vol. 114, pp. 1053-1068, 2003.

[25] P. L. Nunez, R. Srinivasan, A. F. Westdorp, R. S. Wijesinghe, D. M. Tucker, R. B. Silberstein, and P. J. Cadusch, "EEG coherency. I: Statistics, reference electrode, volume conduction, Laplacians, cortical imaging, and interpretation at multiple scales," Electroencephalogr Clin Neurophysiol, vol. 103, pp. 499-515, 1997.

[26] A. F. Leuchter, I. A. Cook, I. Mena, J. J. Dunkin, J. L. Cummings, T. F. Newton, O. Migneco, R. B. Lufkin, D. O. Walter, and P. A. Lachenbruch, "Assessment of cerebral perfusion using quantitative EEG cordance," Psychiatry Research: Neuroimaging, vol. 55, pp. 141-152, 1994.

[27] M. C. Pascual-Marqui RD, Lehmann D, "Low Resolution Electromagnetic Tomography: a new method for localizing electrical activity in the brain," International Journal of Pschophysiology, vol. 18, pp. 49-65, 1994.

[28] F. Perrin, O. Bertrand, and J. Pernier, "Scalp Current Density Mapping: Value and Estimation from Potential Data," Biomedical Engineering, IEEE Transactions on, vol. BME-34, pp. 283-288, 1987.

[29] F. Perrin, J. Pernier, O. Bertrand, and J. F. Echallier, "Spherical splines for scalp potential and current density mapping," Electroencephalography and Clinical Neurophysiology, vol. 72, pp. 184-187, 1989.

[30] H. J. Heinze, G. R. Mangun, W. Burchert, H. Hinrichs, M. Scholz, T. F. Munte, A. Gos, M. Scherg, S. Johannes, H. Hundeshagen, M. S. Gazzaniga, and S. A. Hillyard, "Combined spatial and temporal imaging of brain activity during visual selective attention in humans," vol. 372, pp. 543-546, 1994.

[31] D. Gabor, "Theory of Communication," J. IEE, vol. 93, pp. 429-457, 1946.

[32] S. Mallat, A Wavelet Tour of Signal Processing, 2nd ed: Academic Press, 1999. 
[33] A. Grossmann and J. Morlet, "Decomposition of Hardy Functions into Square Integrable Wavelets of Constant Shape," SIAM Journal on Mathematical Analysis, vol. 15, pp. 723-736, 1984.

[34] I. Daubechies, Ten Lectures on Wavelets: Society for Industrial and Applied Mathematics, 1992.

[35] Z. Cvetkovic and M. Vetterli, "Discrete-Time Wavelet Extrema Representation Design and Consistent Reconstruction," Ieee Transactions on Signal Processing, vol. 43, pp. 681-693, 1995.

[36] M. Unser and A. Aldroubi, "A review of wavelets in biomedical applications," Proceedings of the IEEE, vol. 84, pp. 626-638, 1996.

[37] V. J. Samar, "Wavelet Analysis of Neuroelectric Waveforms," Brain and Language, vol. 66, pp. 1-6, 1999.

[38] N. V. Thakor, X. R. Guo, Y. C. Sun, and D. F. Hanley, "Multiresolution wavelet analysis of evoked potentials," IEEE Trans Biomed Eng, vol. 40, pp. 1085-94, 1993.

[39] O. Bertrand, J. Bohorquez, and J. Pernier, "Time-frequency digital filtering based on an invertible wavelet transform: an application to evoked potentials," IEEE Trans Biomed Eng, vol. 41, pp. 77-88, 1994.

[40] R. Quian Quiroga, "Single-trial event-related potentials with wavelet denoising: method and applications," International Congress Series: Unveiling the Mystery of the Brain: Neurophysiological Investigation of the Brain Function, vol. 1278, pp. 429-432, 2005.

[41] E. John, Brain Evoked Potentials. Acquisition and analysis: Academic Press, 1973.

[42] J. Yordanova, V. Kolev, O. A. Rosso, M. Schurmann, O. W. Sakowitz, M. Ozgoren, and E. Basar, "Wavelet entropy analysis of event-related potentials indicates modality-independent theta dominance," Journal of Neuroscience Methods, vol. 117, pp. 99-109, 2002.

[43] C. Shannon, "A Mathematical Theory of Communication," Bell Syst Technol J, vol. 27, pp. 379-423; 623-656, 1948.

[44] T. Inouye, K. Shinosaki, H. Sakamoto, S. Toi, S. Ukai, A. Iyama, Y. Katsuda, and M. Hirano, "Quantification of EEG irregularity by use of the entropy of the power spectrum," Electroencephalography and Clinical Neurophysiology, vol. 79, pp. 204-210, 1991.

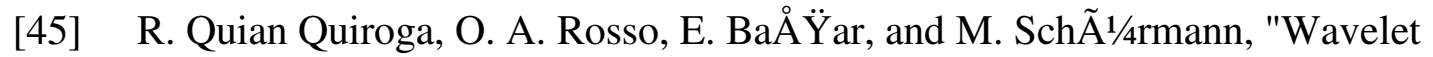
entropy in event-related potentials: a new method shows ordering of EEG oscillations," Biological Cybernetics, vol. 84, pp. 291-299, 2001.

[46] J. Raz, L. Dickerson, and B. Turetsky, "A Wavelet Packet Model of Evoked Potentials,," Brain and Language, vol. 66, pp. 61-88, 1999.

[47] G. D. Dawson, "A summation technique for the detection of small evoked potentials," Electroencephalography and Clinical Neurophysiology, vol. 6, pp. 6584, 1954. 
[48] E. Glaser, Ruchkin, DS, Principles of Neurobiological Signal Analysis. New York: Academic Press, 1976.

[49] C. Shagass, Evoked Potentials in Psychiatry. New York: Plenum, 1972.

[50] r. Spehlmann, Misulis, KE, Fakhoury, T, Spehlmann's Evoked Potential Primer: Butterworth-Heinemann, 2001.

[51] E. Donchin, "A multivariate approach to the analysis of average evoked potentials," IEEE Trans Biomed Eng, vol. 13, pp. 131-139, 1966.

[52] J. c. d. a. r. Kayser and C. E. Tenke, "Trusting in or breaking with convention: Towards a renaissance of principal components analysis in electrophysiology," Clinical Neurophysiology, vol. 116, pp. 1747-1753, 2005.

[53] C. C. Wood and G. McCarthy, "Principal component analysis of event-related potentials: Simulation studies demonstrate misallocation of variance across components," Electroencephalography and Clinical Neurophysiology/Evoked Potentials Section, vol. 59, pp. 249-260, 1984.

[54] W. Collet, "Doubts on the adequacy of the principal component varimax analysis for the identification of event-related brain potential components: A commentary on Glaser and Ruchkin, and Donchin and Heffley," Biological Psychology, vol. 28, pp. 163-172, 1989.

[55] J. Dien, D. J. Beal, and P. Berg, "Optimizing principal components analysis of event-related potentials: Matrix type, factor loading weighting, extraction, and rotations," Clinical Neurophysiology, vol. 116, pp. 1808-1825, 2005.

[56] L. Zhukov, D. Weinstein, and C. Johnson, "Independent component analysis for EEG source localization," Engineering in Medicine and Biology Magazine, IEEE, vol. 19, pp. 87-96, 2000.

[57] A. Hyvarinen and E. Oja, "Independent component analysis: algorithms and applications," Neural Netw, vol. 13, pp. 411-30, 2000.

[58] R. N. Vigario, "From principal to independent component analysis of brain signals," presented at Engineering in Medicine and Biology Society, 2001. Proceedings of the 23rd Annual International Conference of the IEEE, 2001.

[59] A. K. Barros, R. Vigario, V. Jousmaki, and N. Ohnishi, "Extraction of eventrelated signals from multichannel bioelectrical measurements," Biomedical Engineering, IEEE Transactions on, vol. 47, pp. 583-588, 2000.

[60] V. Rodionov, C. Goodman, L. Fisher, G.-Z. Rosenstein, and H. Sohmer, "A new technique for the analysis of background and evoked EEG activity: time and amplitude distributions of the EEG deflections," Clinical Neurophysiology, vol. 113, pp. 1412-1422, 2002.

[61] A. C. W. Ian McDonald, Gilles Edan, Donald Goodkin, Hans-Peter Hartung, Fred D. Lublin, Henry F. McFarland, Donald W. Paty, Chris H. Polman, Stephen C. Reingold, Magnhild Sandberg-Wollheim, William Sibley, Alan Thompson, Stanley Van Den Noort, Brian Y. Weinshenker, Jerry S. Wolinsky,, "Recommended diagnostic criteria for multiple sclerosis: Guidelines from the international panel on the diagnosis of multiple sclerosis," Annals of Neurology, vol. 50, pp. 121-127, 2001. 
[62] R. M. Chapman, G. H. Nowlis, J. W. McCrary, J. A. Chapman, T. C. Sandoval, M. D. Guillily, M. N. Gardner, and L. A. Reilly, "Brain event-related potentials: Diagnosing early-stage Alzheimer's disease," Neurobiology of Aging, vol. 28, pp. 194-201, 2007.

[63] J. Aunon, McGillem CD, Childers DG, "Signal processing in evoked potential research: averaging and modeling," Critical Reviews in Bioengineering, vol. 5, pp. 323-367, 1981.

[64] J. I. Aunon, "Evoked potentials research," Engineering in Medicine and Biology Magazine, IEEE, vol. 11, pp. 67-68, 1992.

[65] N. S. Arikidis, E. W. Abel, and A. Forster, "Interscale wavelet maximum - a fine to coarse algorithm for wavelet analysis of the EMG interference pattern," Biomedical Engineering, IEEE Transactions on, vol. 49, pp. 337-344, 2002.

[66] H. Weyl, The THeory of Groups and Quantum Mechanics. New York: Dutton, 1931.

[67] S. Mallat and S. Zhong, "Characterisation of Signals from Multiscale Edges," IEEE Trans Pattern Analysis and Machine Intelligence, vol. 14, pp. 710-732, 1992.

[68] R. A. Carmona, "Extrema reconstruction and spline smoothing: variations on an algorithm of Mallat and Zhong," in Wavelets and Statistics, A. Antoniadis and G. Oppenheim, Eds. Berlin: Springer-Verlag, 1995.

[69] K. Grochenig, "Irregular sampling of wavelet and short-time Fourier Transforms," Constr. Approx., vol. 9, pp. 283-297, 1993.

[70] Z. Cvetkovic and M. Vetterli, "Consistent reconstruction of signals from wavelet extrema/zero crossings representation," IEEE Trans Signal Processing, 1995.

[71] Z. Berman and J. S. Baras, "Properties of the multiscale maxima and zero crossings representation," IEEE Trans Signal Processing, vol. 41, pp. 3216-3231, 1993.

[72] I. Daubechies and W. Sweldens, "Factoring wavelet transforms into lifting steps," J. Fourier Analysis and Applications, vol. 4, pp. 245-267, 1998.

[73] K. Grochenig, "Acceleration of the frame algorithm," IEEE Trans Signal Processing, vol. 41, pp. 3331-3340, 1993.

[74] Y. Meyer, Wavelets: Algorithms and Applications: SIAM, 1993.

[75] Z. Berman and J. S. Baras, "Properties of the multiscale maxima and zerocrossings representations," Signal Processing, IEEE Transactions on [see also Acoustics, Speech, and Signal Processing, IEEE Transactions on], vol. 41, pp. 3216-3231, 1993.

[76] T. C. Hsung, D. P. K. Lun, and W. C. Siu, "Denoising by singularity detection," Ieee Transactions on Signal Processing, vol. 47, pp. 3139-3144, 1999.

[77] J.-H. Zhang, K. Janschek, J. F. Bohme, and Y.-J. Zeng, "Multi-resolution dyadic wavelet denoising approach for extraction of visual evoked potentials in the brain," Vision, Image and Signal Processing, IEE Proceedings-, vol. 151, pp. 180186, 2004. 
[78] L. J. Heyer, S. Kruglyak, and S. Yooseph, "Exploring Expression Data: Identification and Analysis of Coexpressed Genes 10.1101/gr.9.11.1106," Genome Res., vol. 9, pp. 1106-1115, 1999.

[79] R. Gilmore, "American Electroencephalographical Society Guidelines in Electroencephalography, Evoked Potentials, and Polysomnography," Journal of Clinical Neurophysiology, vol. 11, pp. 1-150, 1994.

[80] G. Harding, "Photosensitivity: a vestigial echo? The first Grey Walter Lecture," Int J Psychophysiol, vol. 16, pp. 273-9, 1994.

[81] I. The Mathworks, "Matlab," Release 12.1 ed, 2001.

[82] J. V. Odom, M. Bach, C. Barber, M. Brigell, M. F. Marmor, A. P. Tormene, G. E. Holder, and Vaegan, "Visual evoked potentials standard (2004)," in Documenta Ophthalmologica, vol. V108, 2004, pp. 115-123.

[83] Lee, P.-L., et al., Visual evoked potential actuated brain computer interface: a brain-actuated cursor system. Electronics Letters, 2005. 41(15): p. 832-834.

[84] Lebedev, M.A. and M.A.L. Nicolelis, Brain-machine interfaces: past, present and future. Trends in Neurosciences, 2006. 29(9): p. 536-546.

[85] Sellers, E.W. and E. Donchin, A P300-based brain-computer interface: Initial tests by ALS patients. Clinical Neurophysiology, 2006. 117(3): p. 538-548.

[86] Middendorf, M., et al., Brain-computer interfaces based on the steady-state visual-evoked response. Rehabilitation Engineering, IEEE Transactions on [see also IEEE Trans. on Neural Systems and Rehabilitation], 2000. 8(2): p. 211-214. 

Appendix A VEP TEST PROCEDURE 



\section{Procedure for Measuring Flash Visual Evoked Potentials}

\section{Formal Experiment}

\section{A. Equipment List:}

The following equipment is required and must be functional and calibrated where necessary:

- MindSet MS-100 Electroencephalograph Equipment with 16 Channels

- Mindset Box

- Power Lead and DC converter.

- SCSI cable to PC

- SCSI Terminator

- PC configured for Mindset, Matlab \& File conversion

- Variable Montage Selector (37 pin D connector with common linked b channels ch1-ch7)

- VEP Custom Cap Type (7 Channel EEG array + Reference)

- Light Detector

- Conductive adhesive pads, crocodile clips, 1 rubber spacer

- Exfoliant clenser, electrode gel, cotton buds, wet wipes, tissues.

- Measuring Tape.

- Ear Plugs (cleaned and boiled).

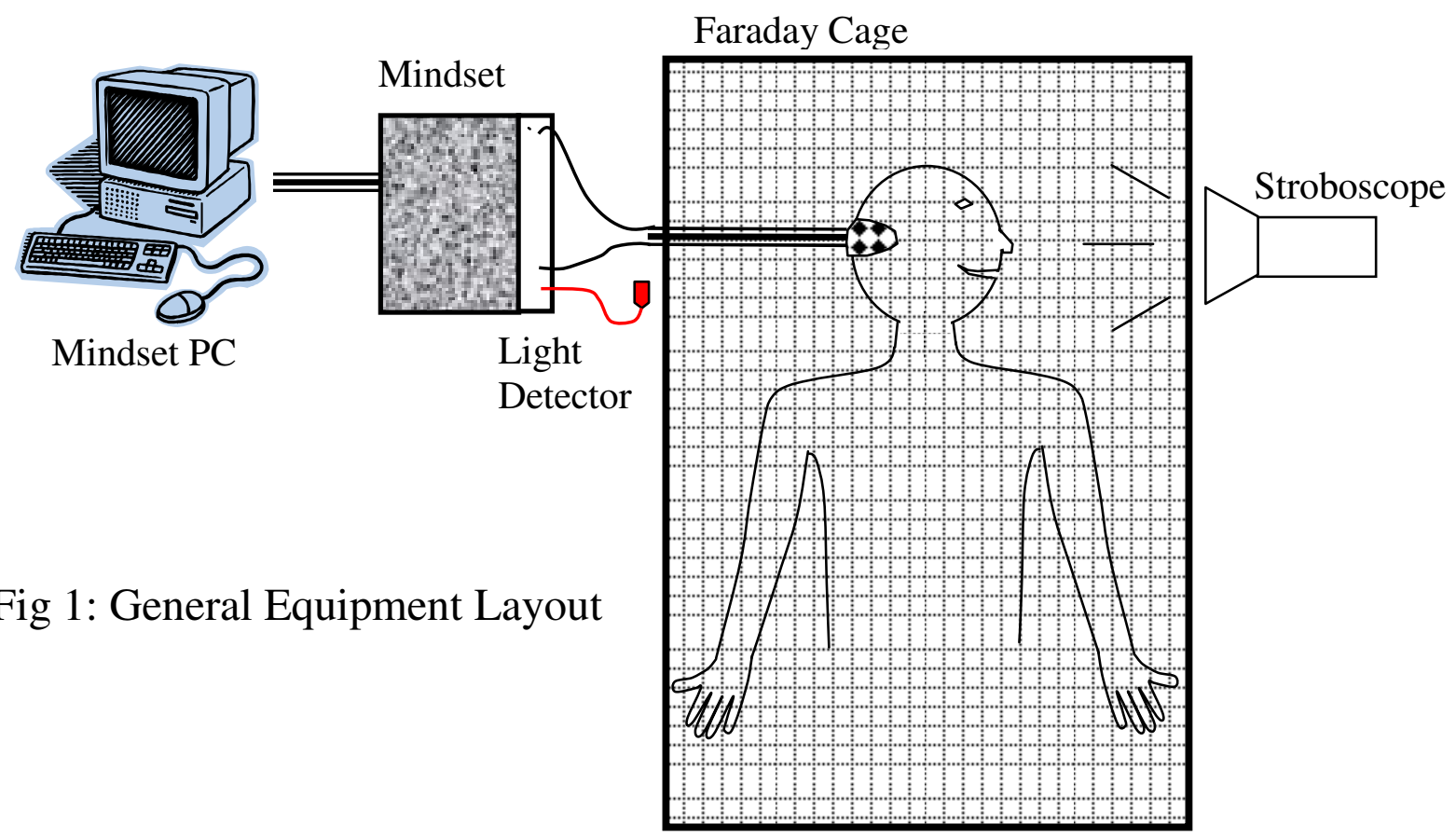




\section{B. Equipment Setup:}

1. Mindset Setup

- Connect Mindset (Bottom SCSI connection) to PC with SCSI cable

- Attach SCSI Terminator to Mindset (Top SCSI Connection).

- Confirm Montage Connector jumpers are correct and connect to front port.

- Place front of Mindset flush against outer Faraday Cage wall inside equipment shield box

- Switch Mindset on and power up PC and login

- Load Mindset setup file 8CHEP.

- Change output file name to relevant participant number. P??VEP.bin

2. Electrode Setup

- Ensure all electrodes are properly cleaned....

- Arrange chair inside Faraday Cage and ensure environment is tidy.

- Connect electrodes to Mindset as per Figure 2

3. Stroboscope Setup

- Place plain material over inside of each door of faraday cage with hole in material

- Setup stroboscope at seated eye level in front of cage door directed through hole

- Set stroboscope to lowest rate and test.

- Place plain background partition behind stroboscope.

- Attach Light Detector to Mindset from inside cage and point towards stroboscope

4. Functional Test

- Run Mindset \& then switch on Stroboscope. Verify the following.

- Light detector output is visible on Mindset for each flash, flash rate is approx $1 / \mathrm{s}$

- There is no interference on other mindset channels.

- Stop both devices. Record data on datasheet, verifying files have been recorded 


\section{Appendix A}

\section{Participant Preparations:}

1. Introduction

- Record start time of this portion of experiment

- Discuss the project with the participant in general terms

- Discuss details of what will happen and discuss any safety concerns

- Give the participant the plain language statement and consent form to read and sign

- Assign Participant Number and note here and on consent form

- Seat participant comfortably in Faraday Cage.

2. Electrode Location (Refer to Fig. 3 for exact locations)

- Measure distance from nasion to inion along the midline NIN

- From front to back, use labels to mark $\mathrm{Cz}$ and $\mathrm{Oz}$ points

- Mark MO as $5 \mathrm{~cm}$ above $\mathrm{Oz}$.

- Measure distance between two preauricular points through $\mathrm{Cz}$ A12

- Precisely mark $\mathrm{Cz}$

3. Electrode Placement

- Carefully attach Electrode cap to head with centre electrode at MO, vertex electrode at Cz..

- Prepare each electrode site as follows:

- Lift each rubber spacer and clean using exfoliant cleaner rubbed with a cotton bud

- Wipe off excess cleaner

- Once each site is prepared, finalise each electrode site as follows:

- Slowly inject electrode gel into hole in rubber spacer above electrode.

- Avoid over injecting gel and causing spreading of gel to other sites .

- Attach Channel $1 \& 15$ reference electrode to left \& right ears as A1 and A2.

- Attach Channel 14 electrode to Erb's point located on the shoulder.

- Check all electrodes are connected correctly and securely

4. Final Checks

- Ask participant to place earplugs in their ears

- Ensure participant is comfortable.

- Ensure Faraday cage door is closed

\section{Useful Definitions:}

NASION: The bridge of the nose directly under the forehead.

INION: The bony protuberance in the middle of the back of the head.

PREAURICULAR POINT: The depression of bone in front of the ear canal.
Fp - Frontopolar
P - Parietal
F - Frontal
O - Occipital
MO - Mid Occipital
C - Central
T - Temporal
LO - Left Occipital
P - Parietal
V - Vertex
RO - Right Occipital 


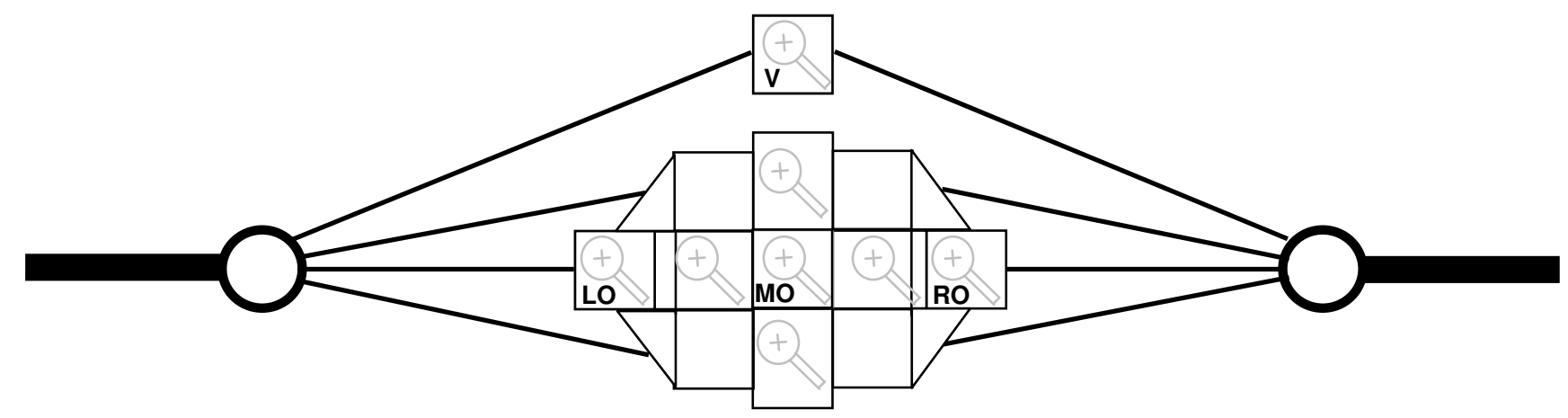

Fig 2: Spatial Cap Electrode Arrangement (Top)

\begin{tabular}{|l|l|l|l|l|l|l|}
\hline Label & \multicolumn{2}{|l|}{ Colour } & Type & From & To Mindset & Note \\
\hline A & White & $\square$ & Grass & LO (MO-5cm) & $1 \mathrm{a}$ & \\
\hline B & Grey & $\square$ & Grass & (MO-2.5cm horiz) & 2a & \\
\hline C & Green & $\square$ & Grass & MO & $3 \mathrm{a}$ & \\
\hline D & Orange & $\square$ & Grass & (MO+2.5cm horiz) & $4 \mathrm{a}$ & \\
\hline E & Blue & $\square$ & Grass & RO & $5 \mathrm{a}$ & \\
\hline F & Red & $\square$ & Grass & (MO+2.5cm vert) & $6 \mathrm{a}$ & \\
\hline G & Yellow & $\square$ & Grass & (MO-2.5cm vert) & $7 \mathrm{a}$ & \\
\hline H & & $\square$ & Grass & CZ (V Vertex) & Ground & \\
\hline I & Purple & $\square$ & Grass & A1 Left Ear Mastoid & $1 \mathrm{~b}$ & \\
\hline J & Lt.Brown & $\square$ & Grass & A2 Right Ear Mastoid & $7 \mathrm{~b}$ & \\
\hline K & Cream & $\square$ & Device & + Photodiode Cable & $8 \mathrm{a}$ & \\
\hline L & Cream & $\square$ & Device & - Photodiode Cable & $8 \mathrm{~b}$ & \\
\hline & & & & & & \\
\hline & & & & & & \\
\hline
\end{tabular}

Table 1: Mindset Electrode Assignment

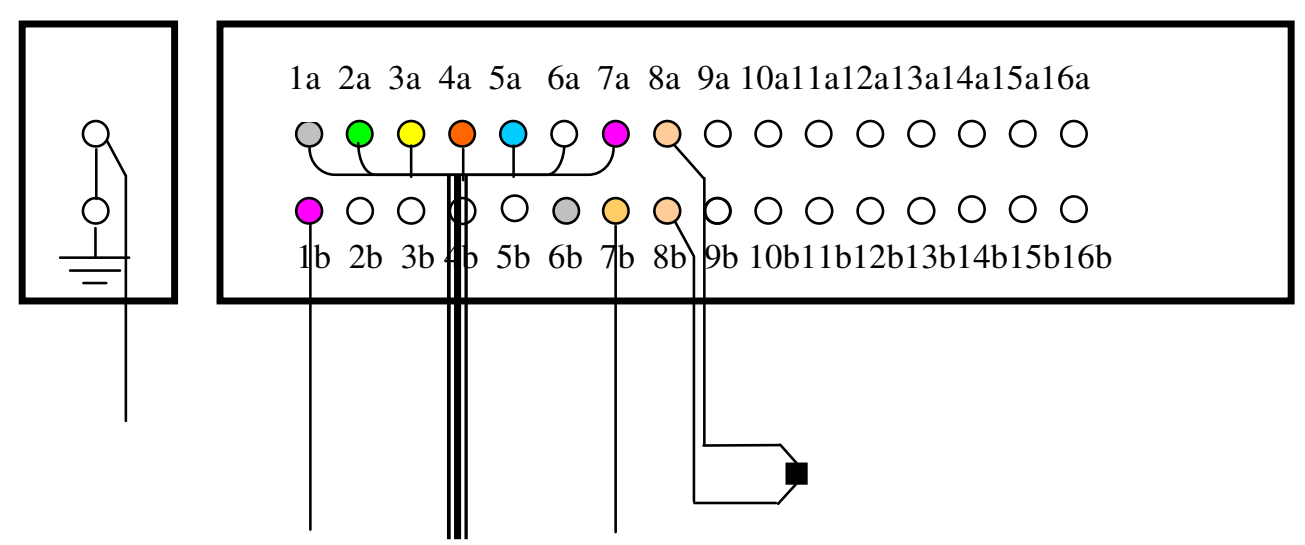

Fig 3: Mindset Electrode Arrangement 


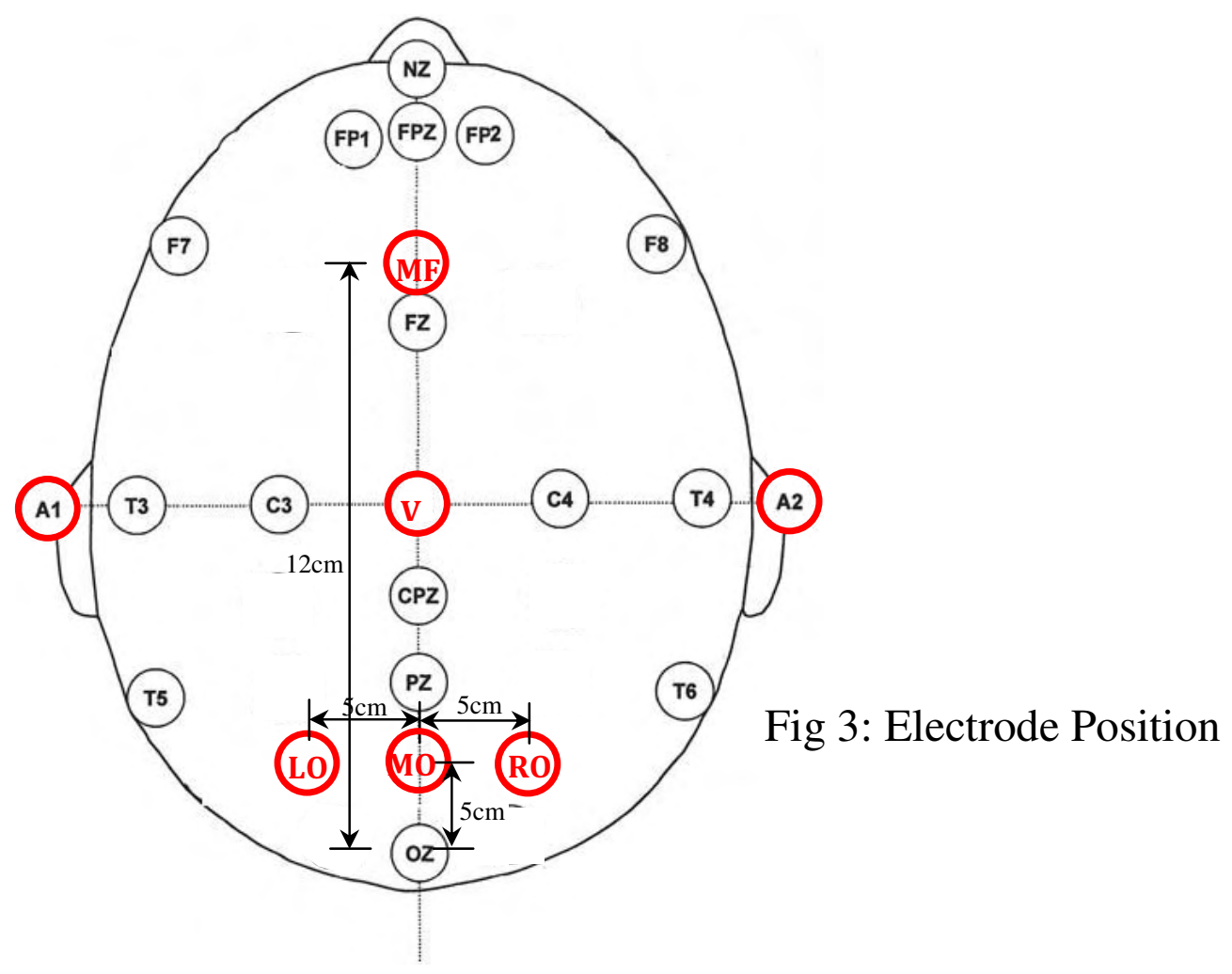

\section{Experimental Procedure:}

1. Preliminary Testing

- Note start time

- Request Participant to be comfortable, relax and focus on stroboscope (eyes open)

- Run Mindset to ensure all channels are reading within range

- Switch on stroboscope and record 10 seconds of data from Mindset.

- Verify artifacts, noise and interference within normal range.

- Identify any channel with high impedance (visible as $50 \mathrm{~Hz}$ noise on the channel)

2. Formal Testing

- Confirm participant is comfortable and record 3 sets of $2 \frac{1}{2} 2$ min data with 2 minute interval

- If an additional tests are outlined on record sheet, then perform these.

3. Completion Steps

- Request participants rates the audibility of the stroboscope

- Carefully remove all electrodes and ear plugs, provide wipes for participant.

- Seat the participant outside cage for a few minutes while tidying up, offer glass of water.

- Review record sheet and ensure all requirements are filled in

- Copy all files to network and compare filename to checklist.

- Ensure all equipment is switched off and power points are switched off.

- Wash all electrodes carefully and return all equipment to proper storage location

- Note the finish time for experimentation, sign-off record sheet 


\section{Procedure for Measuring Flash Visual Evoked Potentials}

\section{Record Sheet}

\section{E. Record of Data, Settings \& Results:}

1. Personal Details:

\begin{tabular}{|l|l|l|}
\hline Subject No: & $\mathrm{P}_{-}$ & \\
\hline Sex: & $\square$ male $\square$ female & childhood febrile convulsion \\
\hline Handedness: & $\square$ left $\square$ right & Epilepsy \\
\cline { 2 - 2 } PLS signed: & $\square$ yes $\square$ no & \\
\hline Known strobe aversion & $\square$ yes $\square$ no & If yes, then do not proceed \\
\hline
\end{tabular}

2. Timing

- A: Record Date:

- A: Start Time:

- C1: Start time with subject

- D1: Commencement time of Experiment

- D3: Completion Time of the Experiment

3. Measurement

- C2:Nasion-Inion distance NIN $\mathrm{cm} \ldots$

- C2:Pre-auricular distance A12 $\mathrm{cm} \ldots$

- D3: Audibility of stroboscope (circle as appropriate):
Inaudible
Faint
Distinct
Clear
Distracting
Loud

4. Log of Data Recorded

\begin{tabular}{|l|l|l|l|}
\hline Section & Filenames & Length(sec) & Comments \\
\hline B4 & & & Equipment Functional test \\
\hline D1 & & & EEG \& stroboscope Functional Test \\
\hline D2a & & & Main Flash VEP Test \#1 \\
\hline D3b & & & Main Flash VEP Test \#2 \\
\hline D4c & & & Main Flash VEP Test \#3 \\
\hline \multicolumn{2}{|l|}{} \\
\hline Other Tests & & & \\
\hline & & & \\
\hline & & & \\
\hline
\end{tabular}

5. Notes

Signature of Completion:

Date:

6. Detailed Notes 


\section{Appendix A}

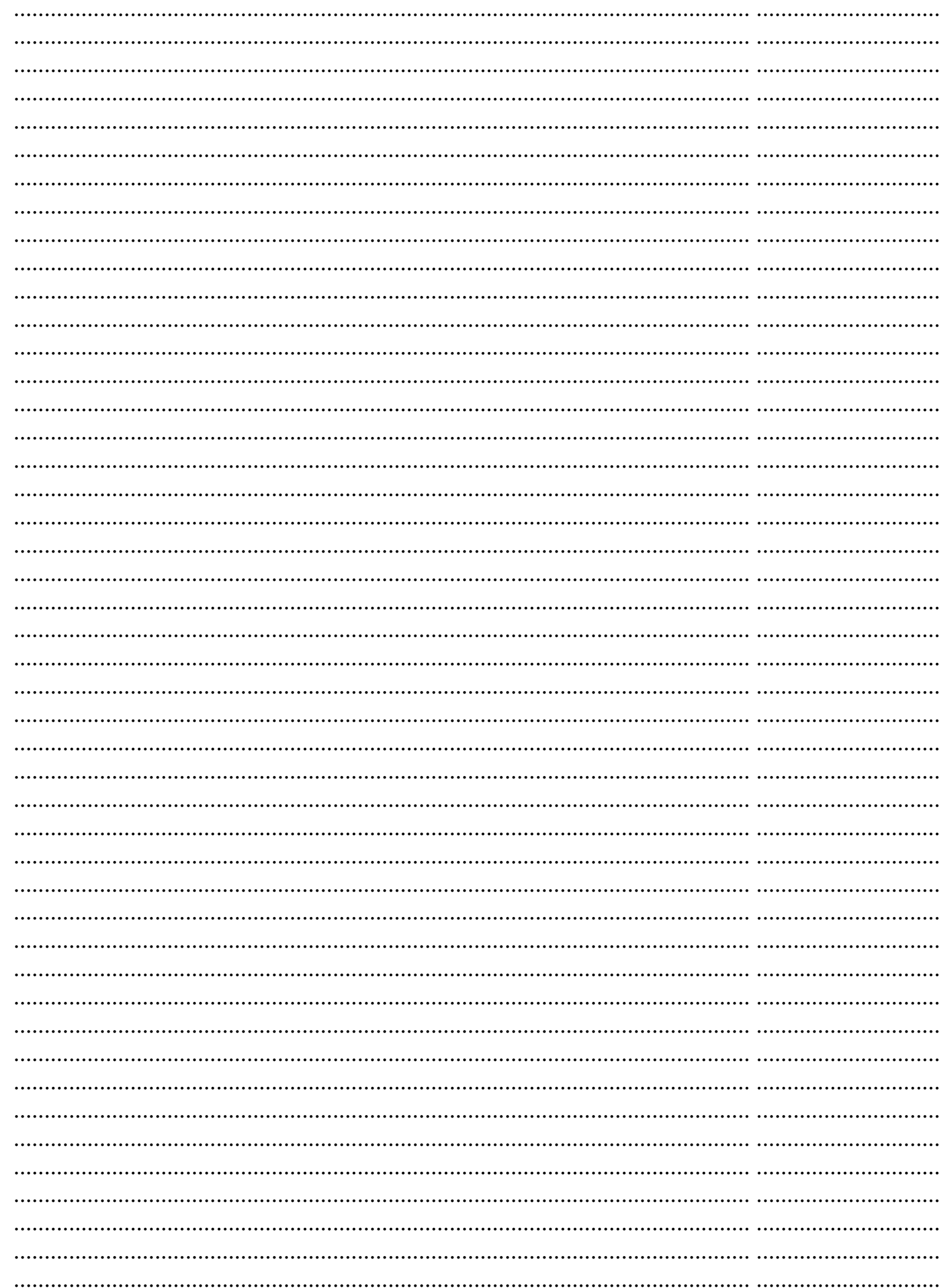


RMIT HUMAN RESEARCH ETHICS COMMITTEE

Prescribed Consent Form For Persons Participating In Research Projects Involving Tests and/or Procedures

\section{FACULTY OF}

SCHOOL OF

Name of Participant:

Participant Number:

Project Title:

Name(s) of investigator:

Name of Supervisor:

\section{ENGINEERING}

ELECTRICAL AND COMPUTER ENGINEERING

\begin{tabular}{lll}
\hline Characterising Evoked Potential Signals using Wavelet Transform \\
\hline Singularity Detection & \\
\hline Conor McCooey & Phone: & $($ (03) 99259576 \\
Dr. Dinesh Kant Kumar & Phone: & $(03) 99251954$ \\
&
\end{tabular}

1. I have received a statement explaining the tests/procedures involved in this project - PLS Form E1.

2. I consent to participate in the above project, the particulars of which - including details of tests or procedures have been explained to me.

3. I authorise the investigator or his or her assistant to use with me the tests or procedures referred to in 1 above.

4. I acknowledge that:

(a) The possible effects of the tests or procedures have been explained to me to my satisfaction.

(b) I have been informed that I am free to withdraw from the project at any time and to withdraw any unprocessed data previously supplied.

(c) The project is for the purpose of research and/or teaching. It may not be of direct benefit to me.

(d) The confidentiality of the information I provide will be safeguarded. However should information of a confidential nature need to be disclosed for moral, clinical or legal reasons, I will be given an opportunity to negotiate the terms of this disclosure.

(e) The security of the research data is assured during and after completion of the study. The data collected during the study may be published, and a report of the project outcomes will be provided to School of Electrical and Computer Systems Engineering, RMIT. Any information, which will identify me, will not be used.

Participants Consent :

Name:

$$
\text { (Participant) }
$$

Name:

$$
\text { (Witness to signature) }
$$

Where participant is under 18 years of age:

I consent to the participation of in the above project.

Date:
$(1)$
(2)

Date:

Date:

Name:

$$
\text { (Witness to signature) }
$$

Participants should be given a photocopy of this consent form after it has been signed.

Any complaints about your participation in this project may be directed to the Secretary, RMIT Human Research Ethics Committee, University Secretariat, RMIT, GPO Box 2476V, Melbourne, 3001. The telephone number is (03) 99251745. 
RMIT SCHOOL OF ELECTRICAL \& COMPUTER ENGINEERING

Plan Language Statement For Persons Participating In Research Projects Involving Tests and/or Procedures

\section{Characterising Evoked Potential Signals using Wavelet Transform Singularity Detection}

Investigator: Conor McCooey s3029091@student.rmit.edu.au (03) 99259576

Supervisor: Dr. Dinesh Kant Kumar (03) 99251954

1. The messages linking the body and brain are gradually becoming more understood but many areas remain shrouded in mystery. This research concentrates on the link between the bodies sensory system and how the brain interprets these sensations. We are developing new analytical techniques to examine the signals that arise in the brain when the optic nerves sense a change in light intensity observed in the eyes.

2. In order to do this, we flash a strobe light about $60 \mathrm{~cm}$ in front of you. This stimulus is repeated and the electrical activity in the brain is measured by an array of electrodes at the back of the head. This is the area of the brain where the information about the flash relayed by the optic nerve is processed. The electrodes pick up the tiny electric signals emanating from the skull, commonly known as brain waves.

3. The procedure is very safe and contains a number of safeguards to protect the subject. Firstly the EEG electrodes are isolated from the mains current in the EEG equipment. Secondly, the strobe flash rate is only approximately 1 flash per second and so is not uncomfortable. Thirdly, if you know that you are photosensitive to light flashes then please let the investigator know and as a precaution, you will not be able to take part in this experiment. People who have epilepsy or a history of seizure of febrile convulsion in infancy are also excluded.

4. The skin or scalp shall be swabbed clean at the point where an electrode is applied. A small amount of conductive gel is used to get a better connection. This is easily washed off afterwards.

5. In order to measure the tiny EEG signal due to the strobe flashes, several hundred measurements (flashes) shall be taken. However these are automatically repeated and the recording portion shall last less than 20 minutes. One of the aims of this research is to reduce the number of stimuli required to a few samples or even 1 sample. The whole process should take less than 1.5 hours.

6. The possible benefits of this research are:

- Simplified clinical Neurological testing of Evoked Potential pathways.

- Individual responses to stimuli are not measurable to researchers currently - only averaged responses. Being able to examine individual responses would allow researchers in several Neurological fields such as Alzheimer's disease, Epilepsy and Multiple Sclerosis to better interpret the signals observed from the brain and in the future possibly, provide better diagnosis.

- Another area of possible investigation for this one sample technique is Complementary Medicine where tactile therapies such as Reflexology or Acupuncture may be investigated on a more scientific basis.

7. Any personal details (such as Name and Phone Number) shall be kept strictly confidential. A reference number shall be assigned to you, which references any data collected. Any publication arising from this data shall not bear any personally identifying marks.

8. Participation in this research is voluntary and you are at liberty to withdraw at any time before or during the test. Thank you for your interest in this project. Please feel free to discuss this project with the investigator at any stage before, during or after testing.

Participants should be given a photocopy of this plain language statement for their information.

Any complaints about your participation in this project may be directed to the Secretary, RMIT Human Research Ethics Committee, University Secretariat, RMIT, GPO Box 2476V, Melbourne, 3001. The telephone number is (03) 99251745. 

Appendix B DETAILED RESULTS 



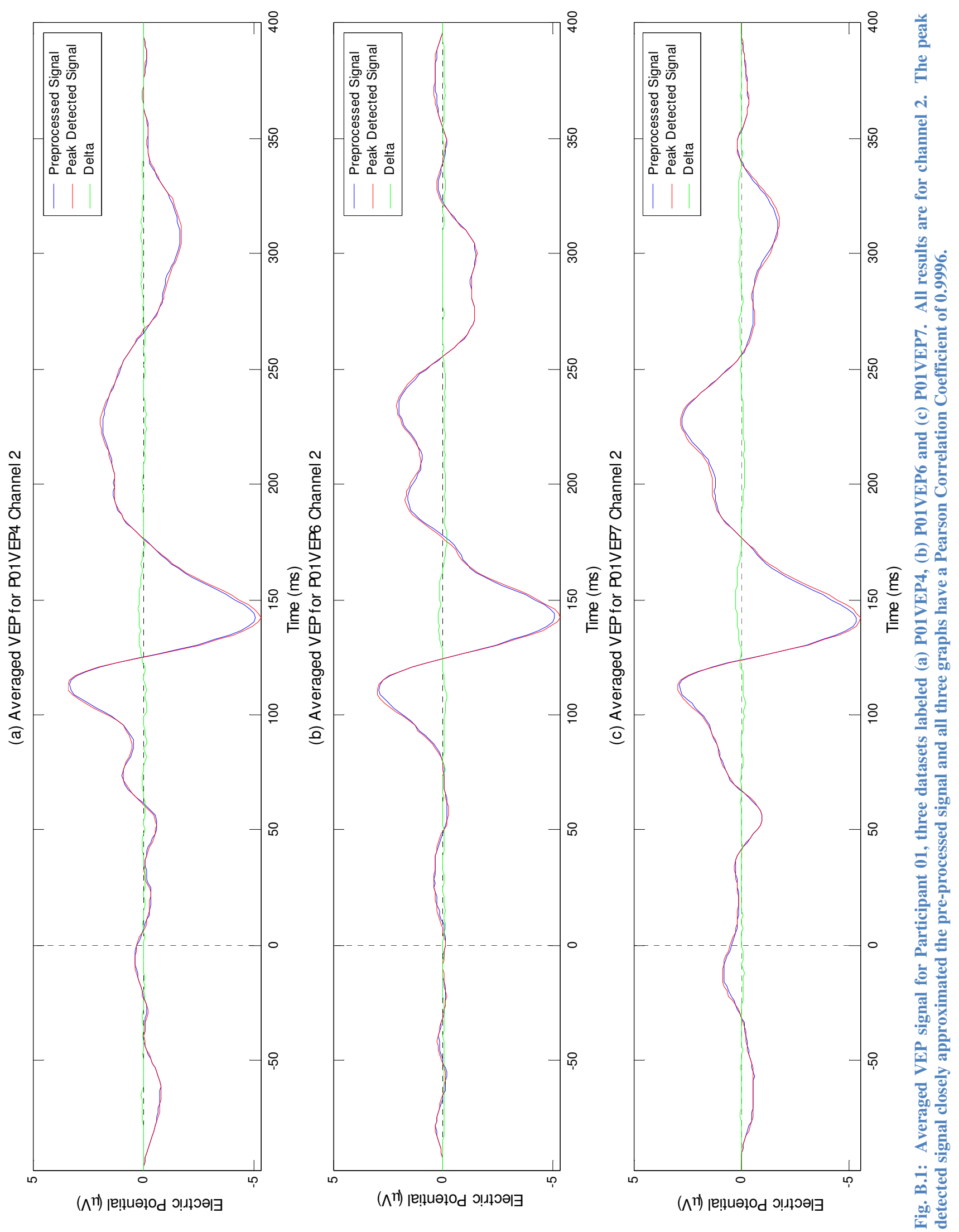



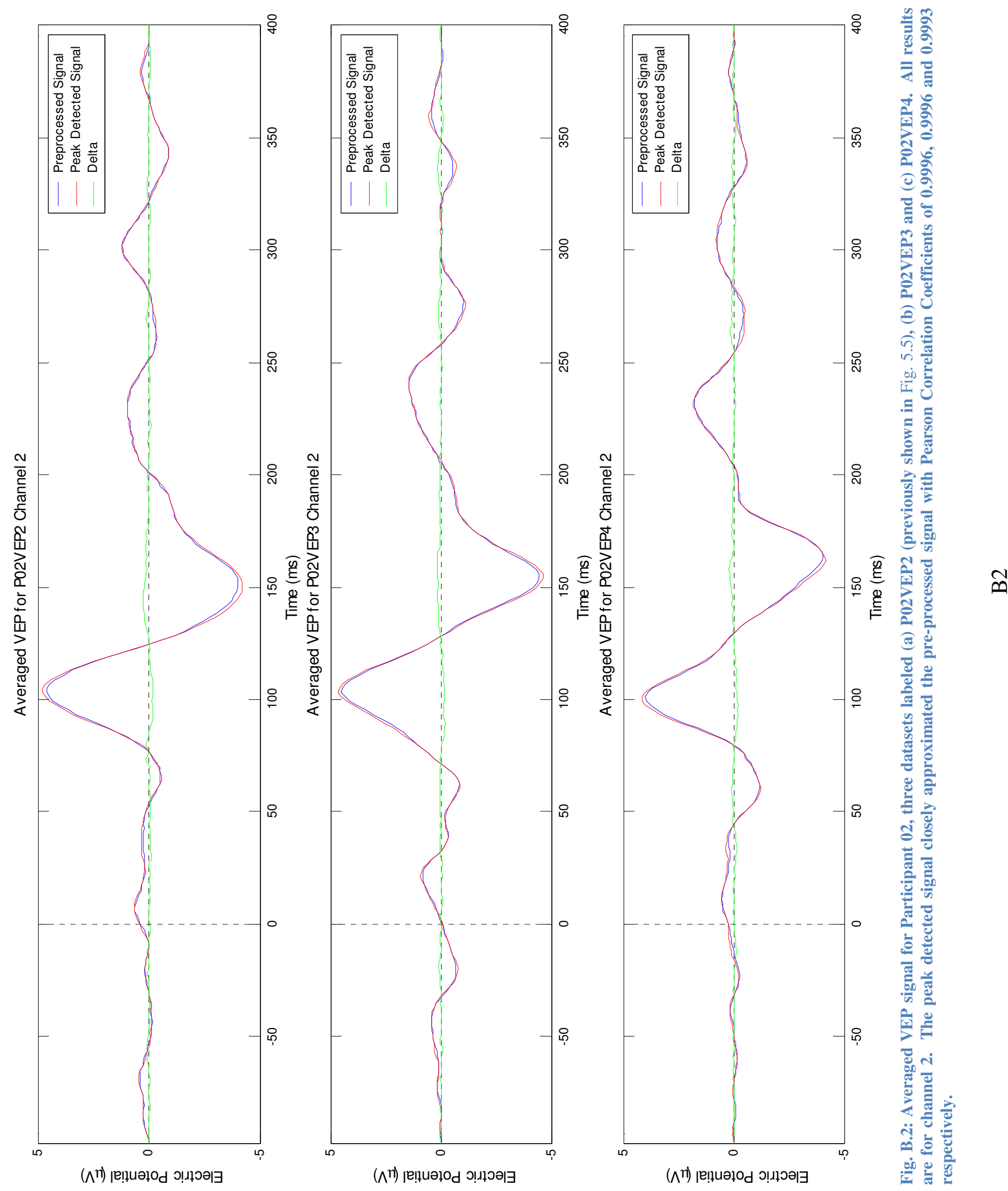


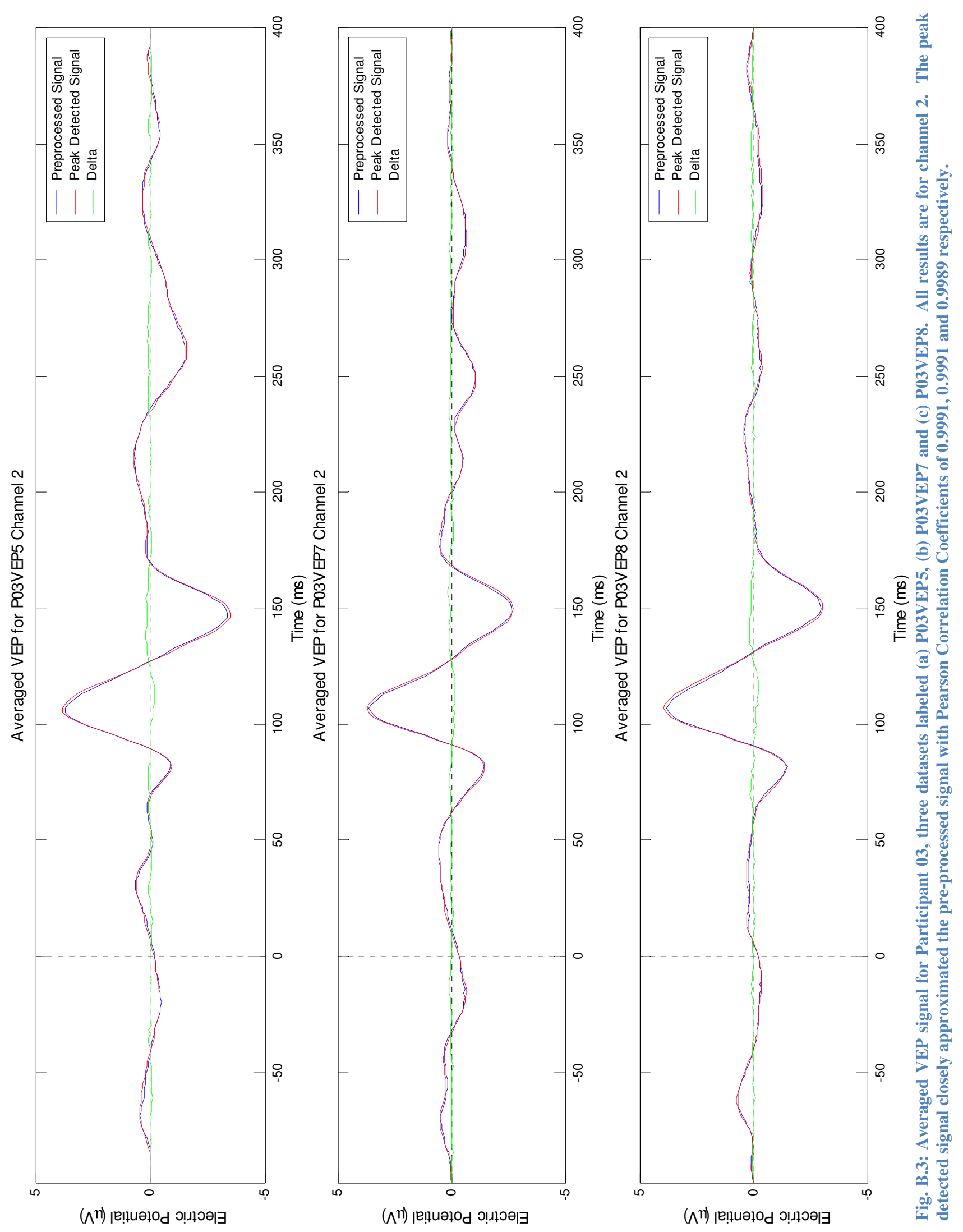


(a) Highest peak concentration profile

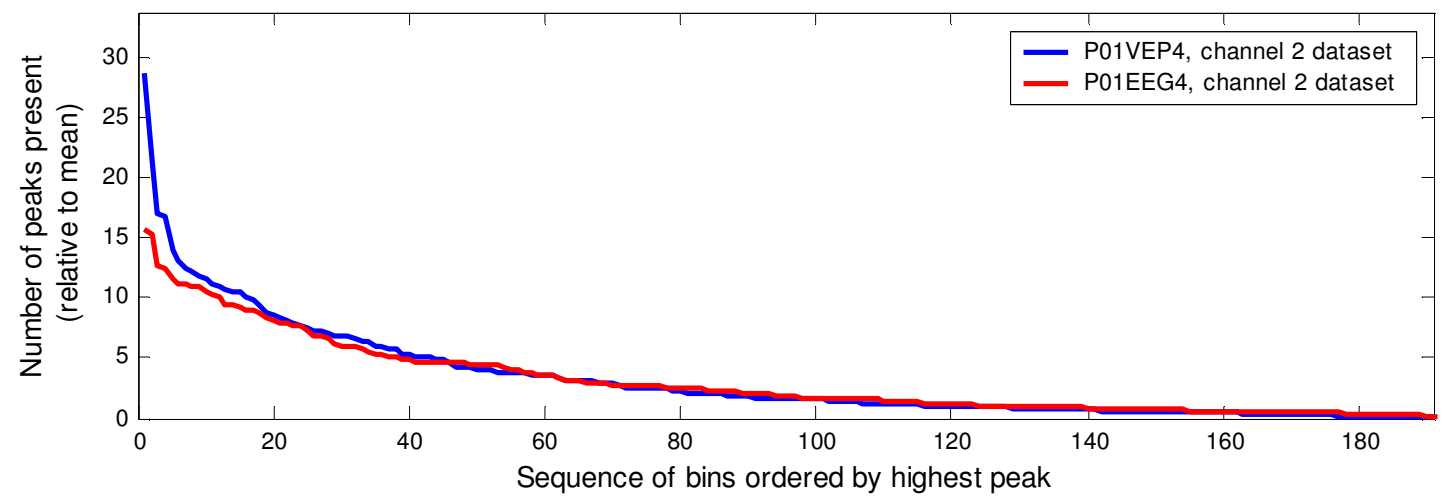

(b) Significance (Peak Concentration X Peak Area) Profile

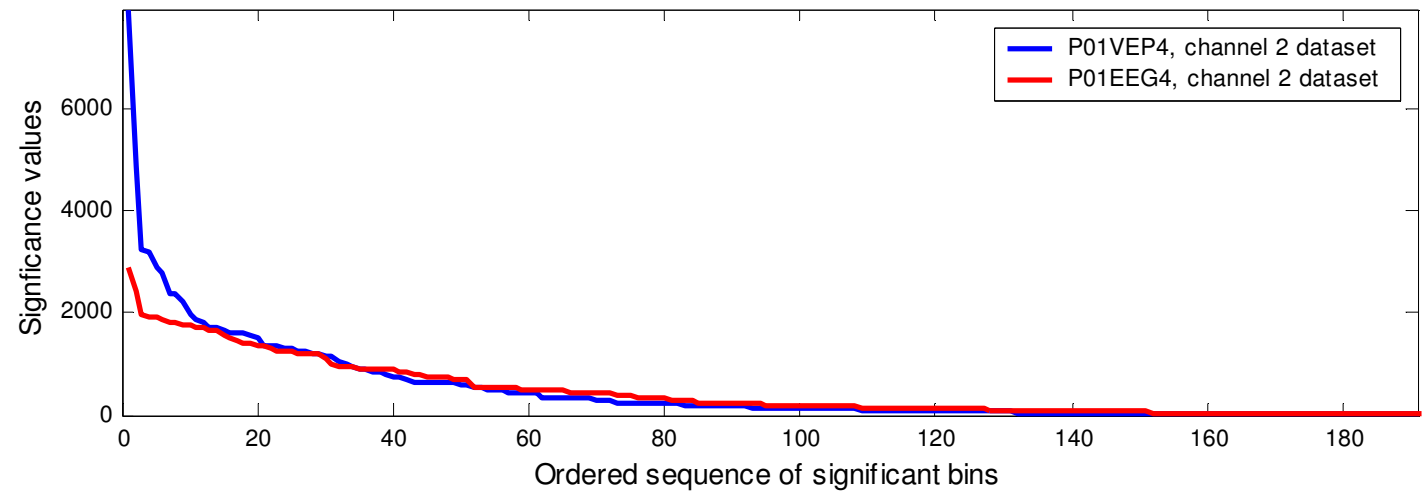

Fig. B.4: Peak concentration profiles for P01VEP4. (a) Relative bin concentrations are ordered and plotted against the number of peaks in each bin. (b) Concentration matrix is ordered by the significance factor (peak concentration peak area) rather than the number of peaks.

(a) Highest peak concentration profile

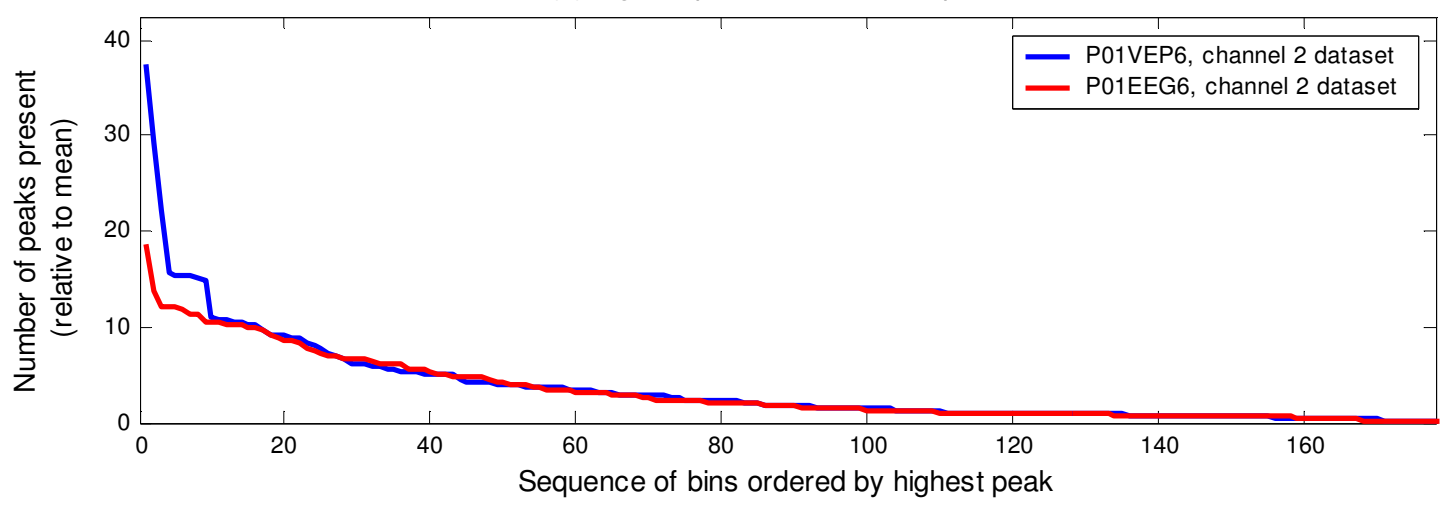

(b) Significance (Peak Concentration X Peak Area) Profile

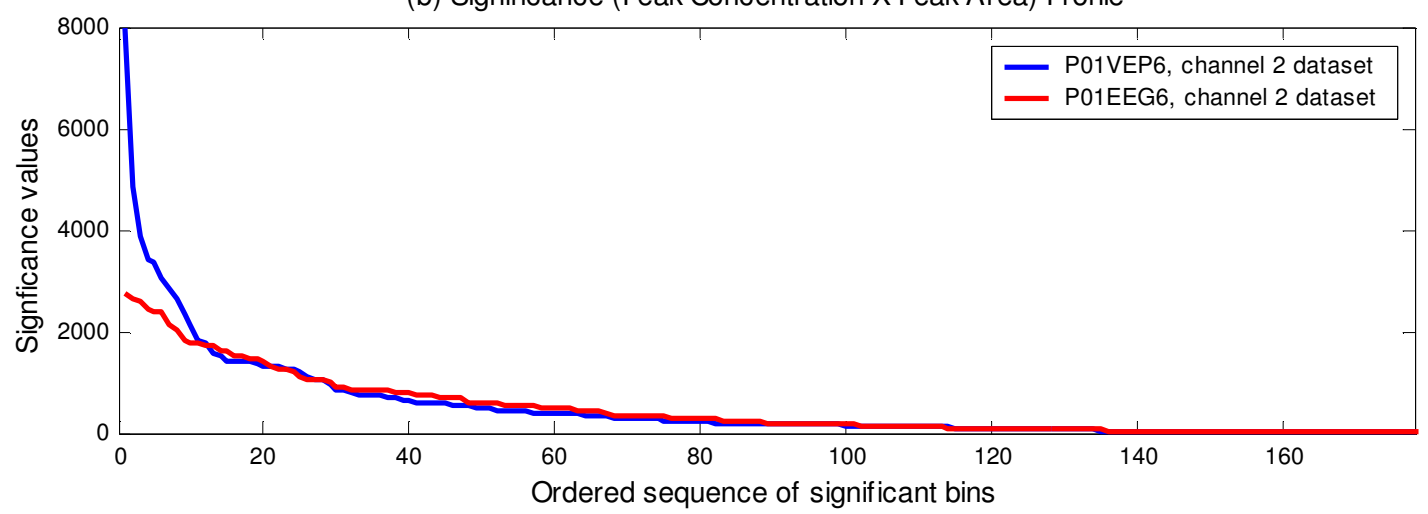

Fig. B.5: Peak concentration profiles for P01VEP6. (a) Relative bin concentrations are ordered and plotted against the number of peaks in each bin. (b) Concentration matrix is ordered by the significance factor (peak concentration peak area) rather than the number of peaks. 
(a) Highest peak concentration profile

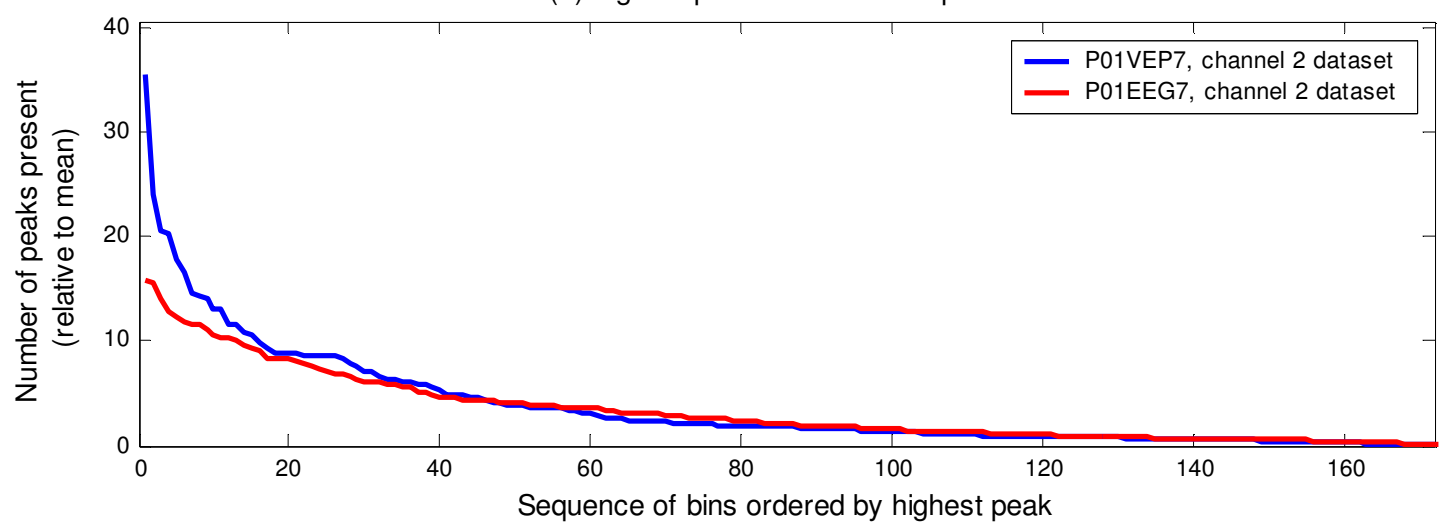

(b) Significance (Peak Concentration X Peak Area) Profile

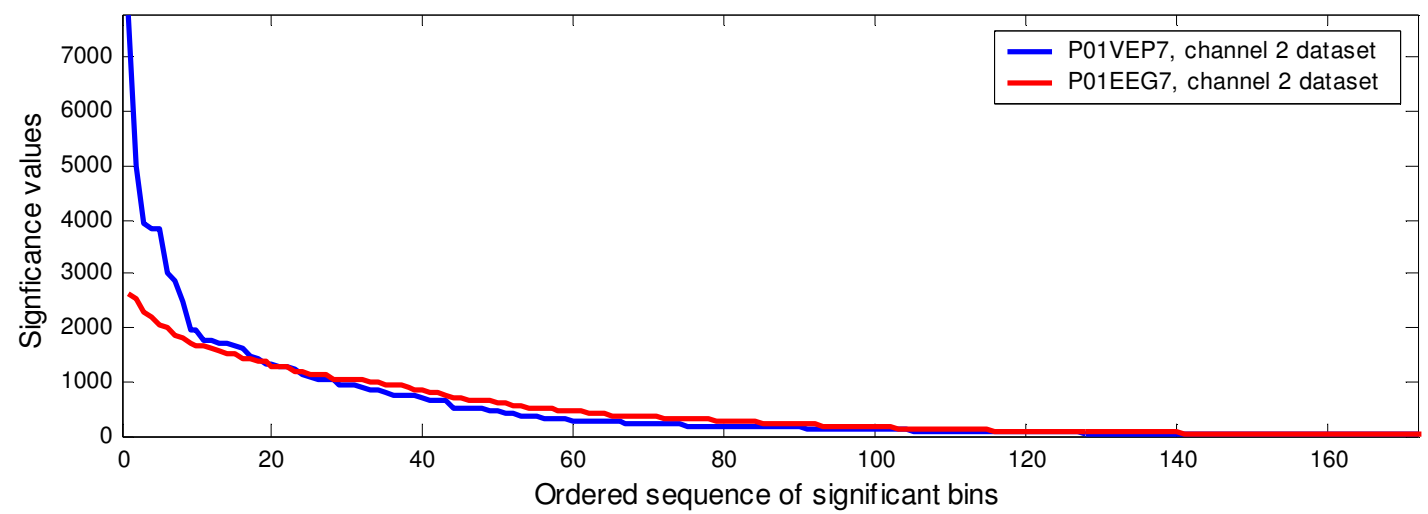

Fig. B.6: Peak concentration profiles for P01VEP7. (a) Relative bin concentrations are ordered and plotted against the number of peaks in each bin. (b) Concentration matrix is ordered by the significance factor (peak concentration peak area) rather than the number of peaks.

(a) Highest peak concentration profile

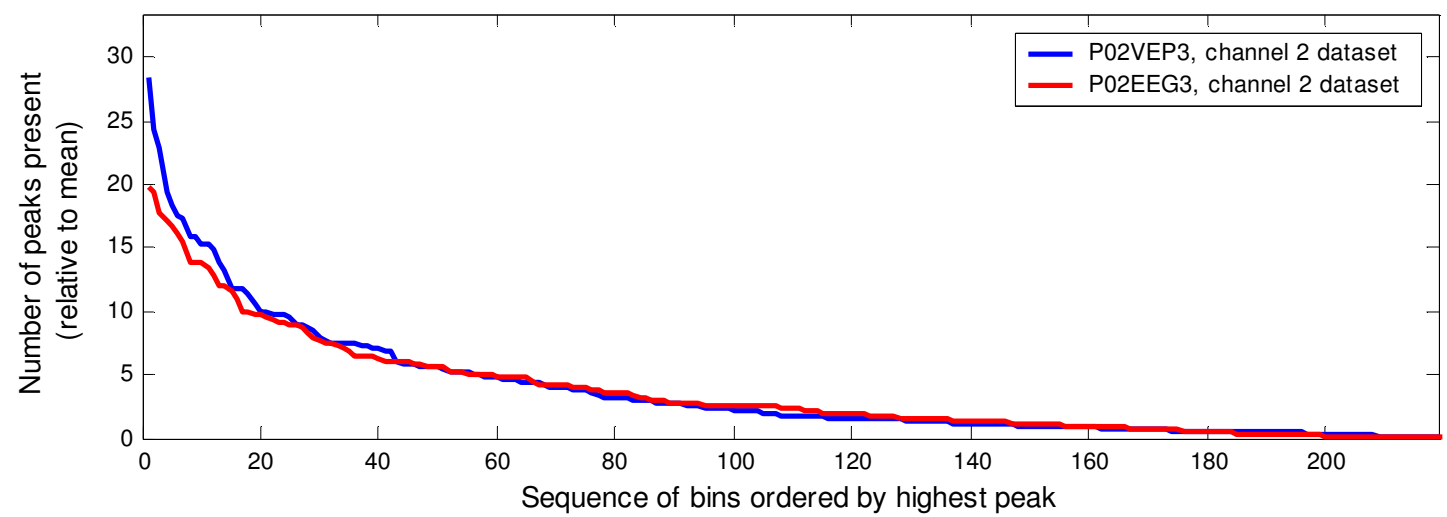

(b) Significance (Peak Concentration X Peak Area) Profile

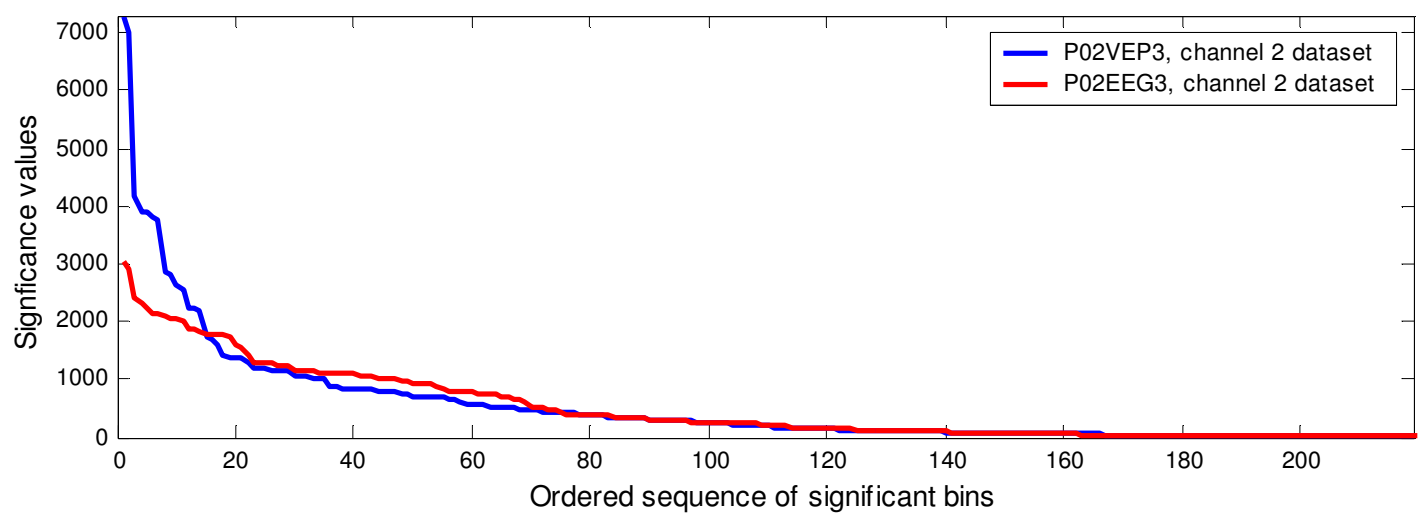

Fig. B.7: Peak concentration profiles for P02VEP3. (a) Relative bin concentrations are ordered and plotted against the number of peaks in each bin. (b) Concentration matrix is ordered by the significance factor (peak concentration peak area) rather than the number of peaks. 
(a) Highest peak concentration profile

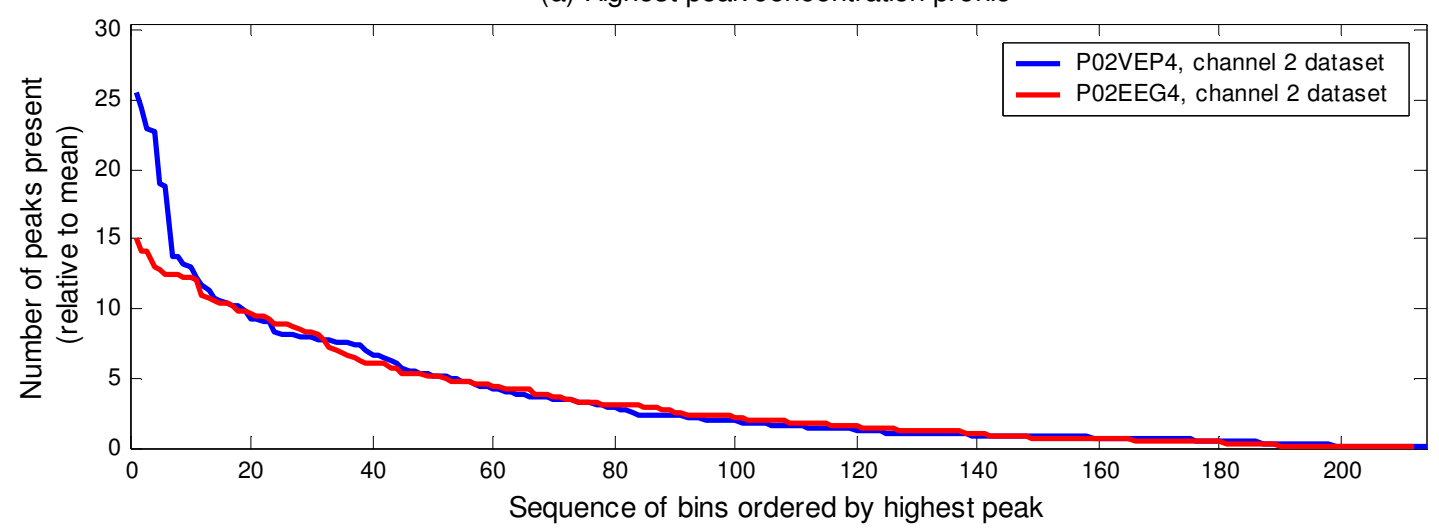

(b) Significance (Peak Concentration X Peak Area) Profile

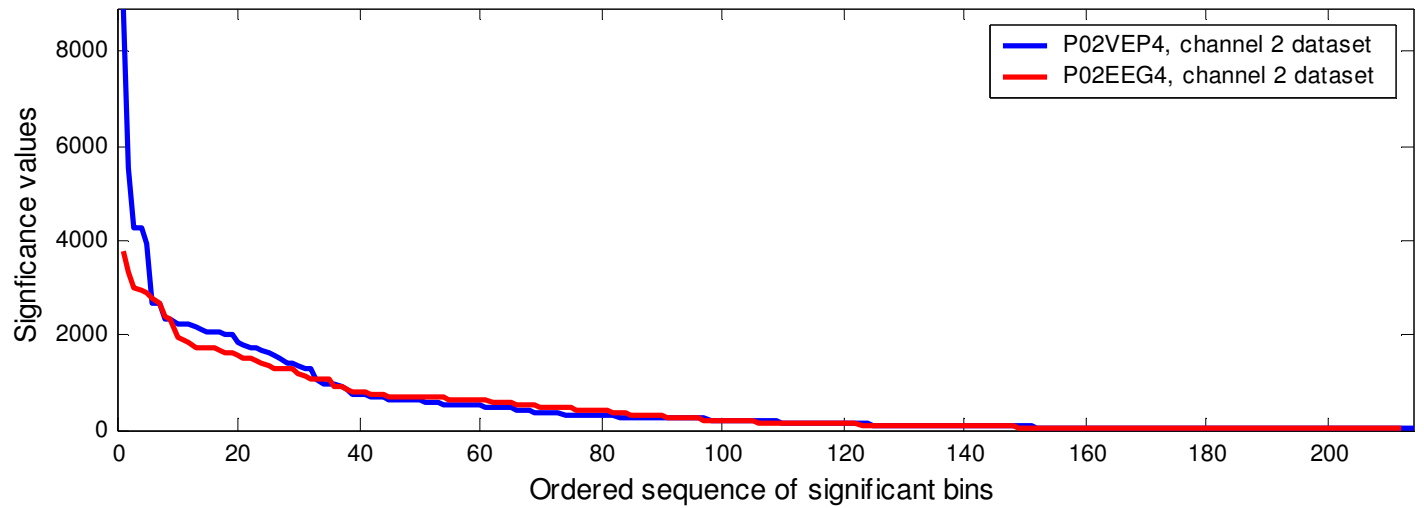

Fig. B.8: Peak concentration profiles for P02VEP4. (a) Relative bin concentrations are ordered and plotted against the number of peaks in each bin. (b) Concentration matrix is ordered by the significance factor (peak concentration peak area) rather than the number of peaks.

(a) Highest peak concentration profile

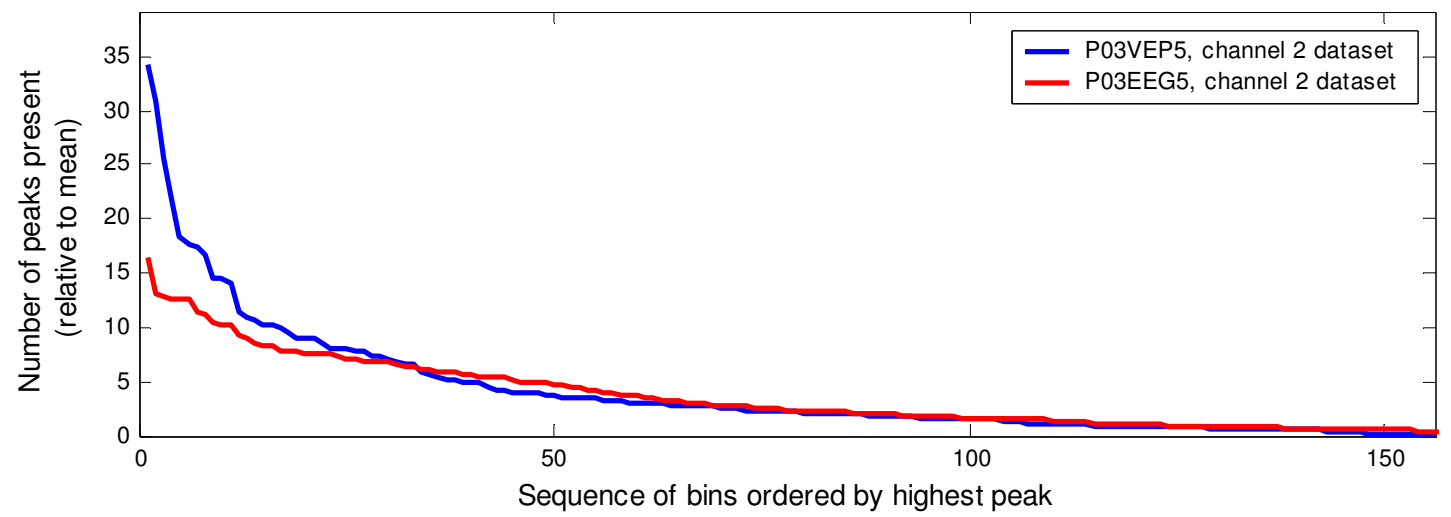

(b) Significance (Peak Concentration X Peak Area) Profile

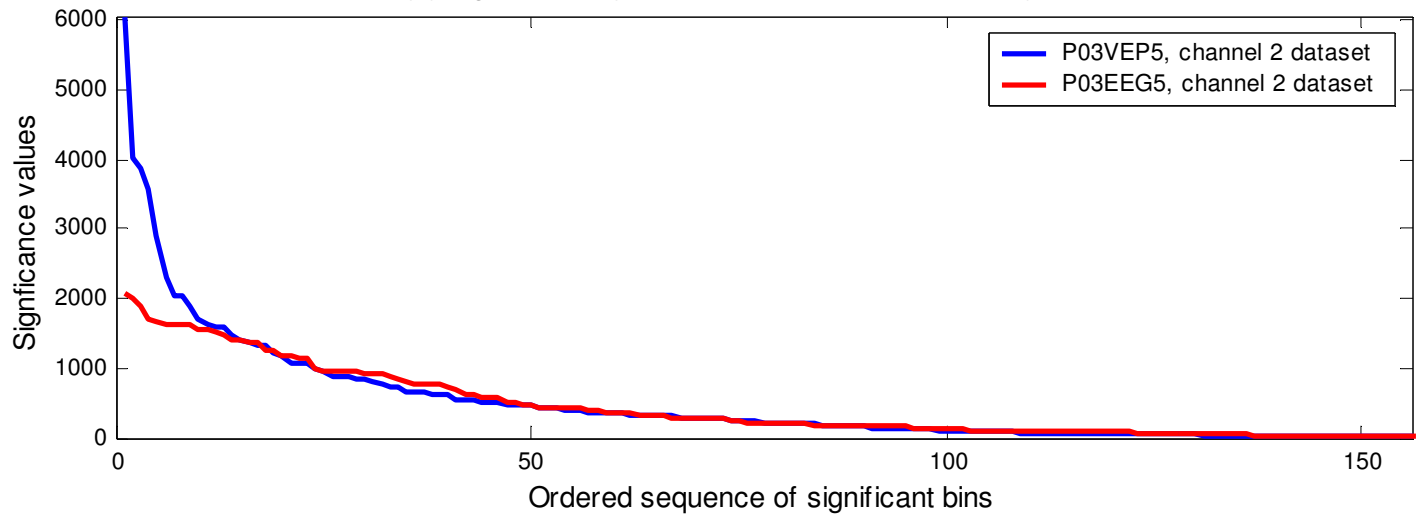

Fig. B.9: Peak concentration profiles for P03VEP5. (a) Relative bin concentrations are ordered and plotted against the number of peaks in each bin. (b) Concentration matrix is ordered by the significance factor (peak concentration peak area) rather than the number of peaks. 
(a) Highest peak concentration profile

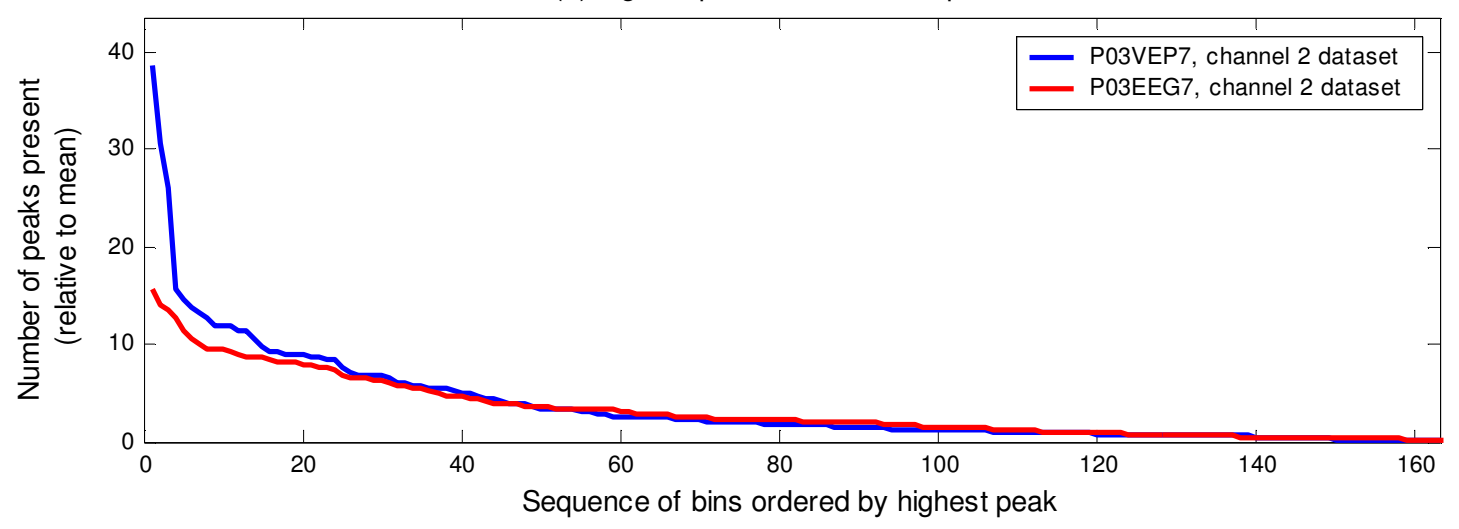

(b) Significance (Peak Concentration X Peak Area) Profile

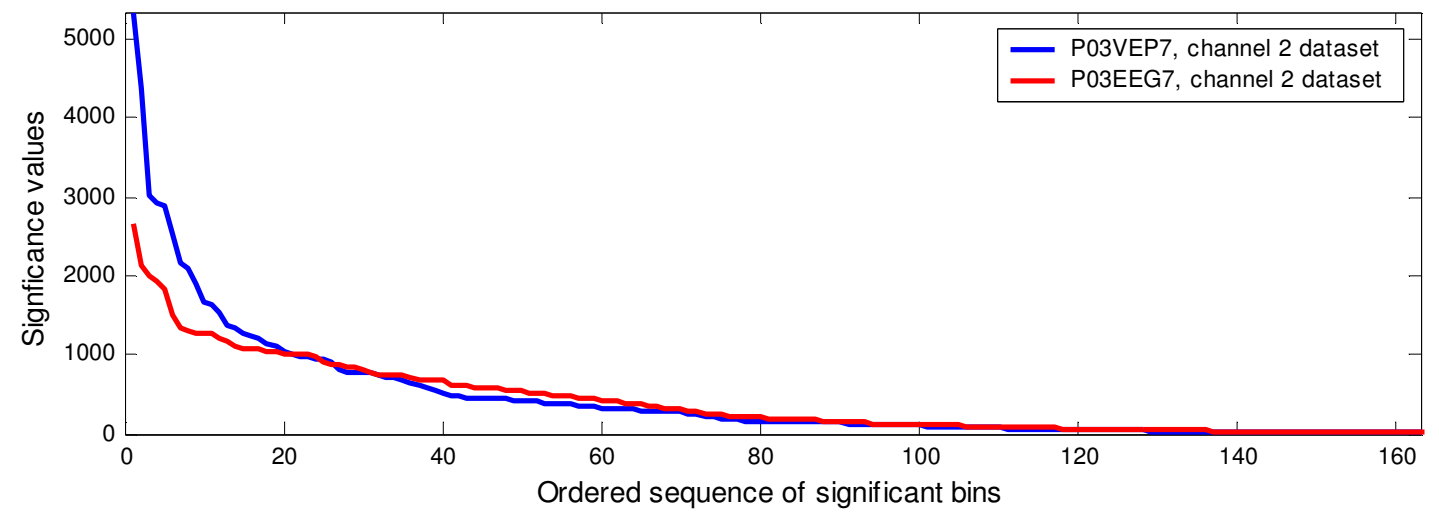

Fig. B.10: Peak concentration profiles for P03VEP7. (a) Relative bin concentrations are ordered and plotted against the number of peaks in each bin. (b) Concentration matrix is ordered by the significance factor (peak concentration peak area) rather than the number of peaks.

(a) Highest peak concentration profile

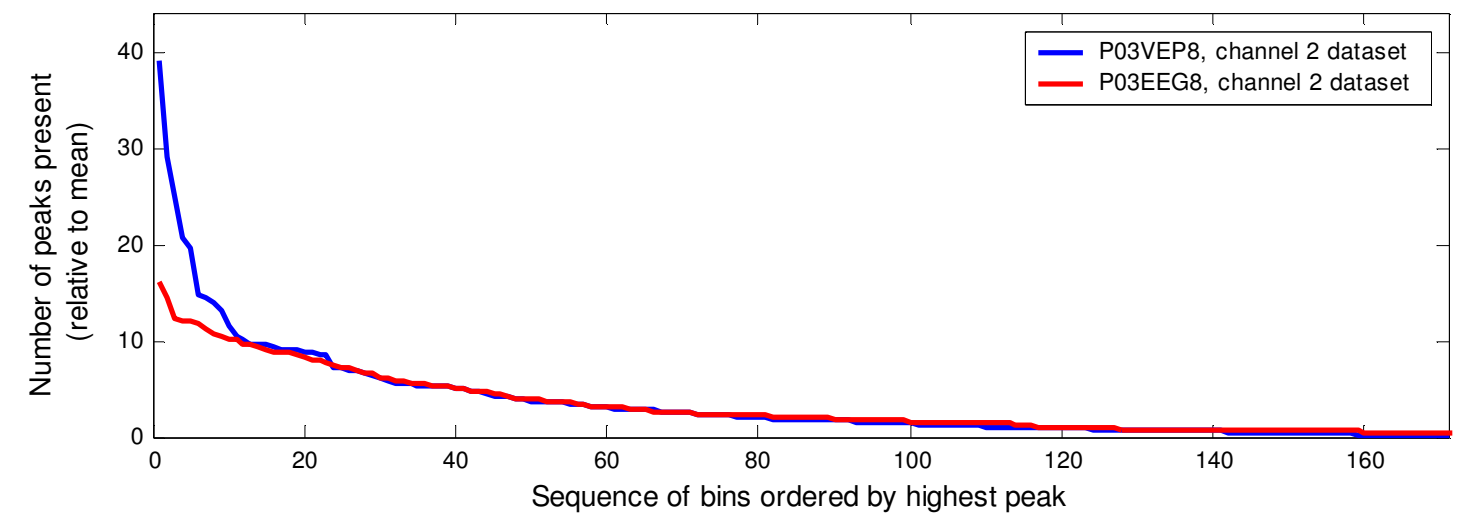

(b) Significance (Peak Concentration X Peak Area) Profile

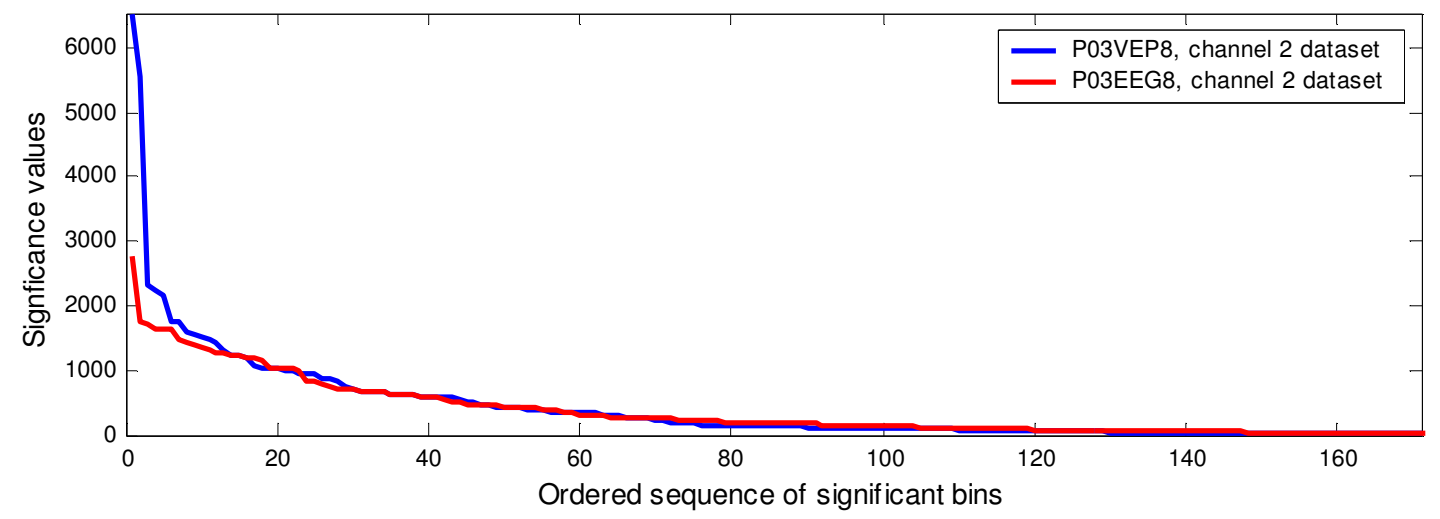

Fig. B.11: Peak concentration profiles for P03VEP8. (a) Relative bin concentrations are ordered and plotted against the number of peaks in each bin. (b) Concentration matrix is ordered by the significance factor (peak concentration peak area) rather than the number of peaks. 


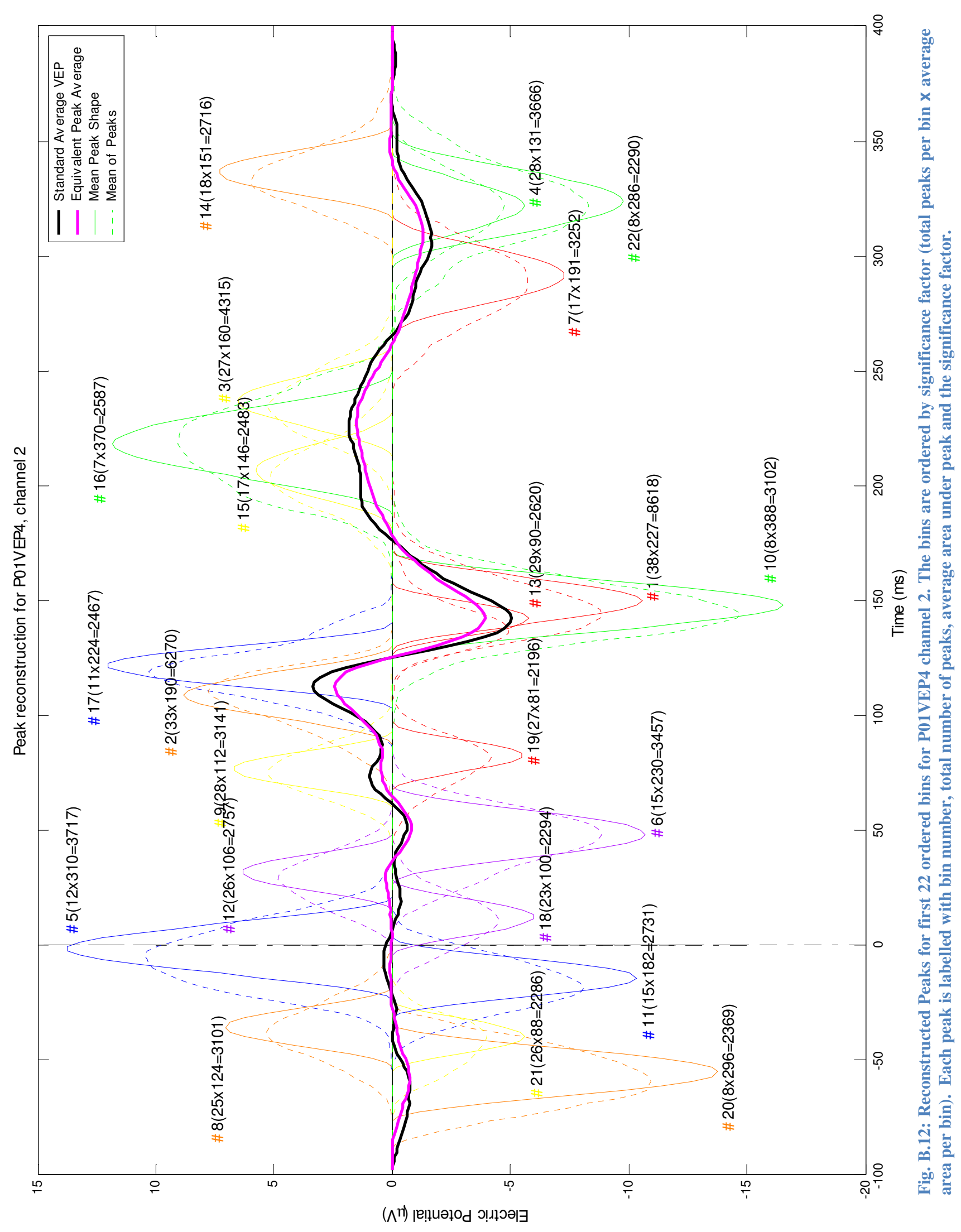




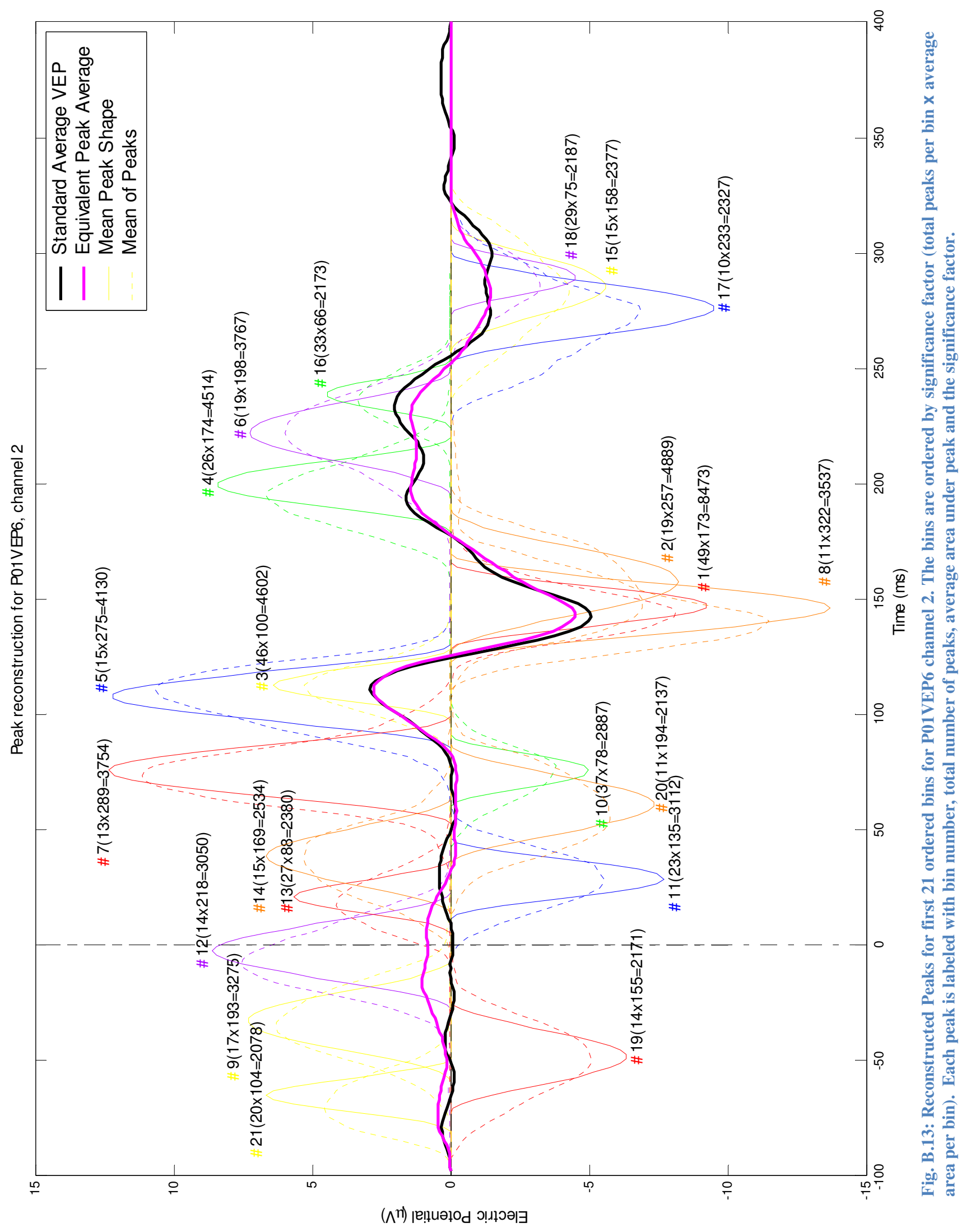




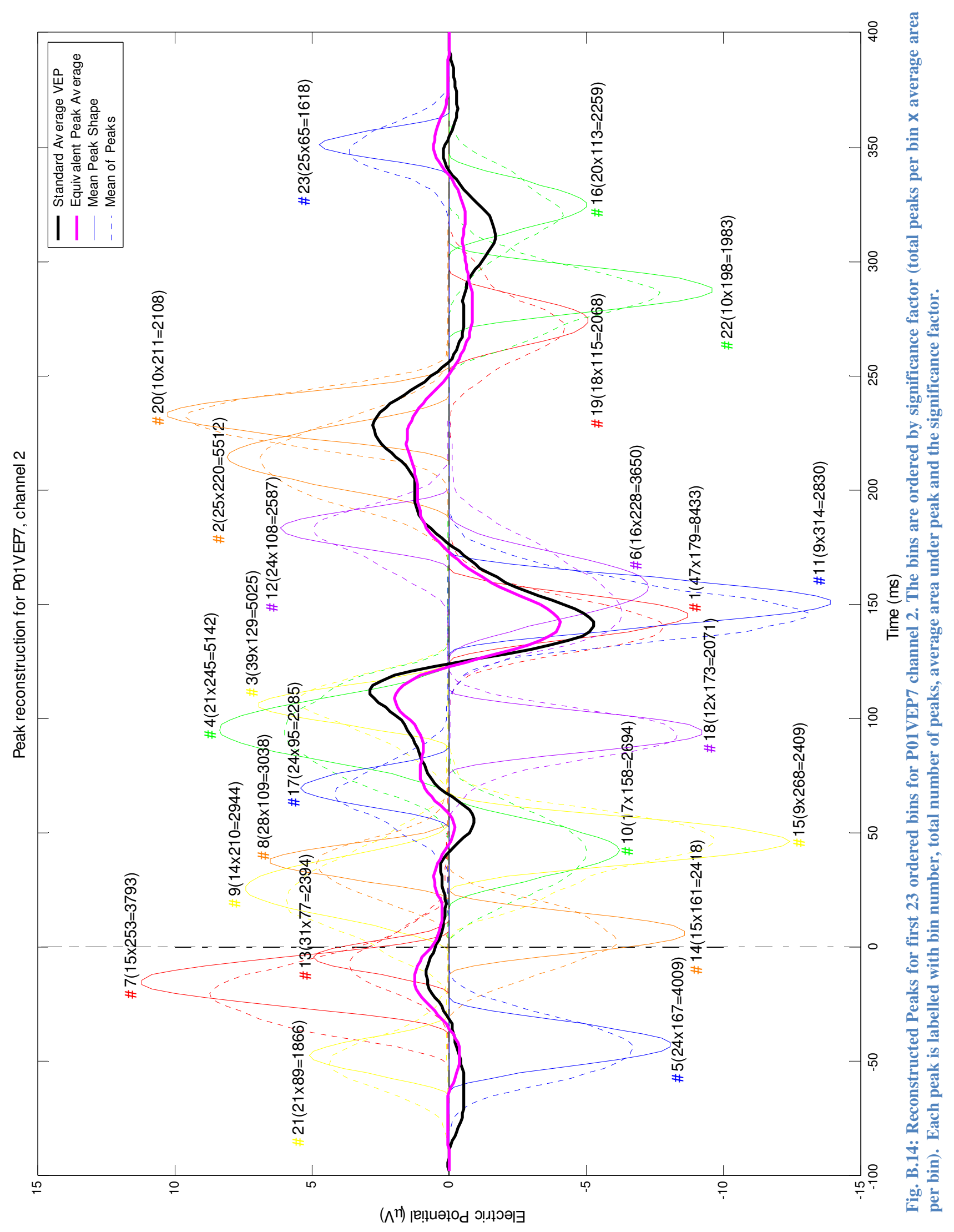

$\frac{0}{n}$ 


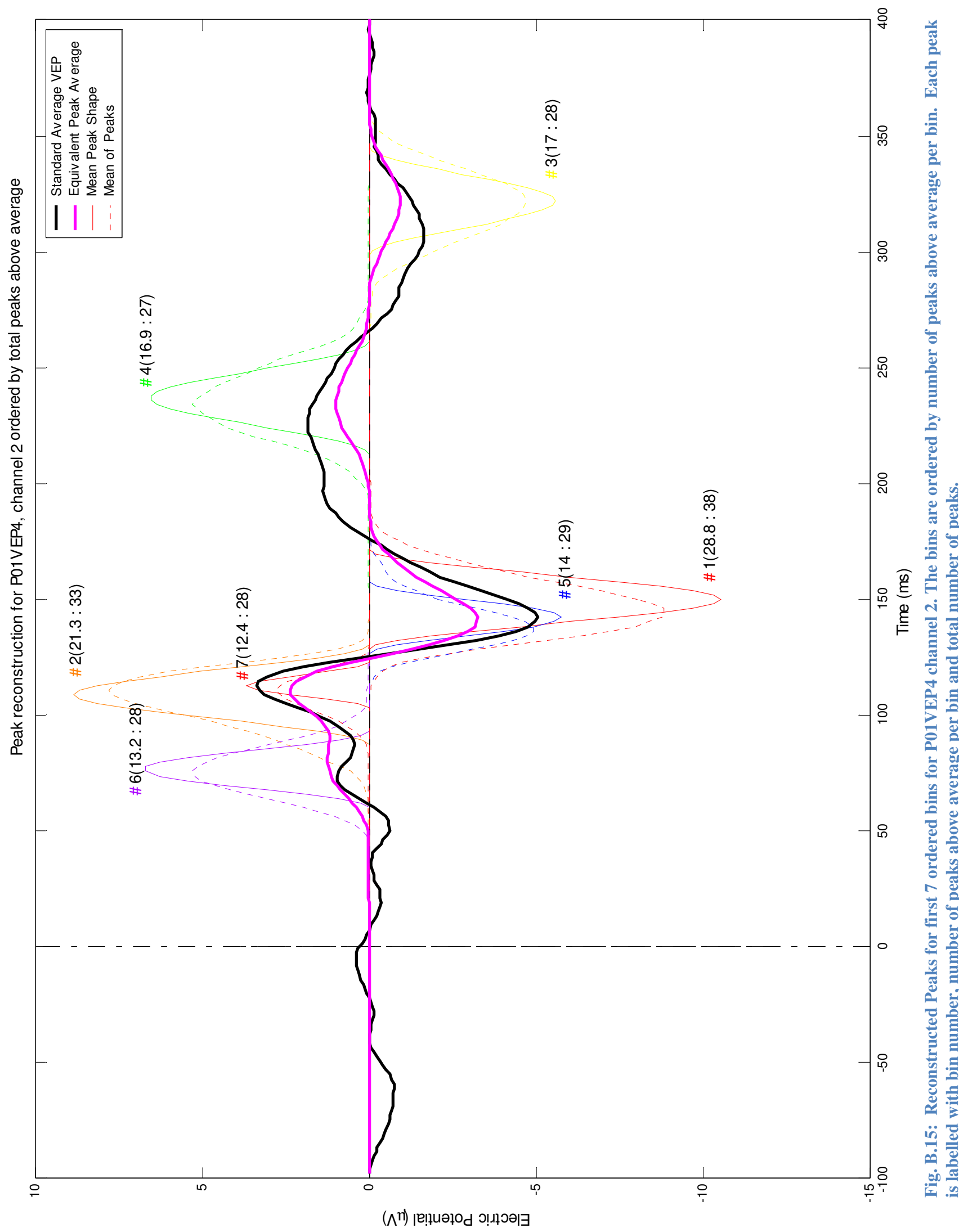

$\overline{7}$ 


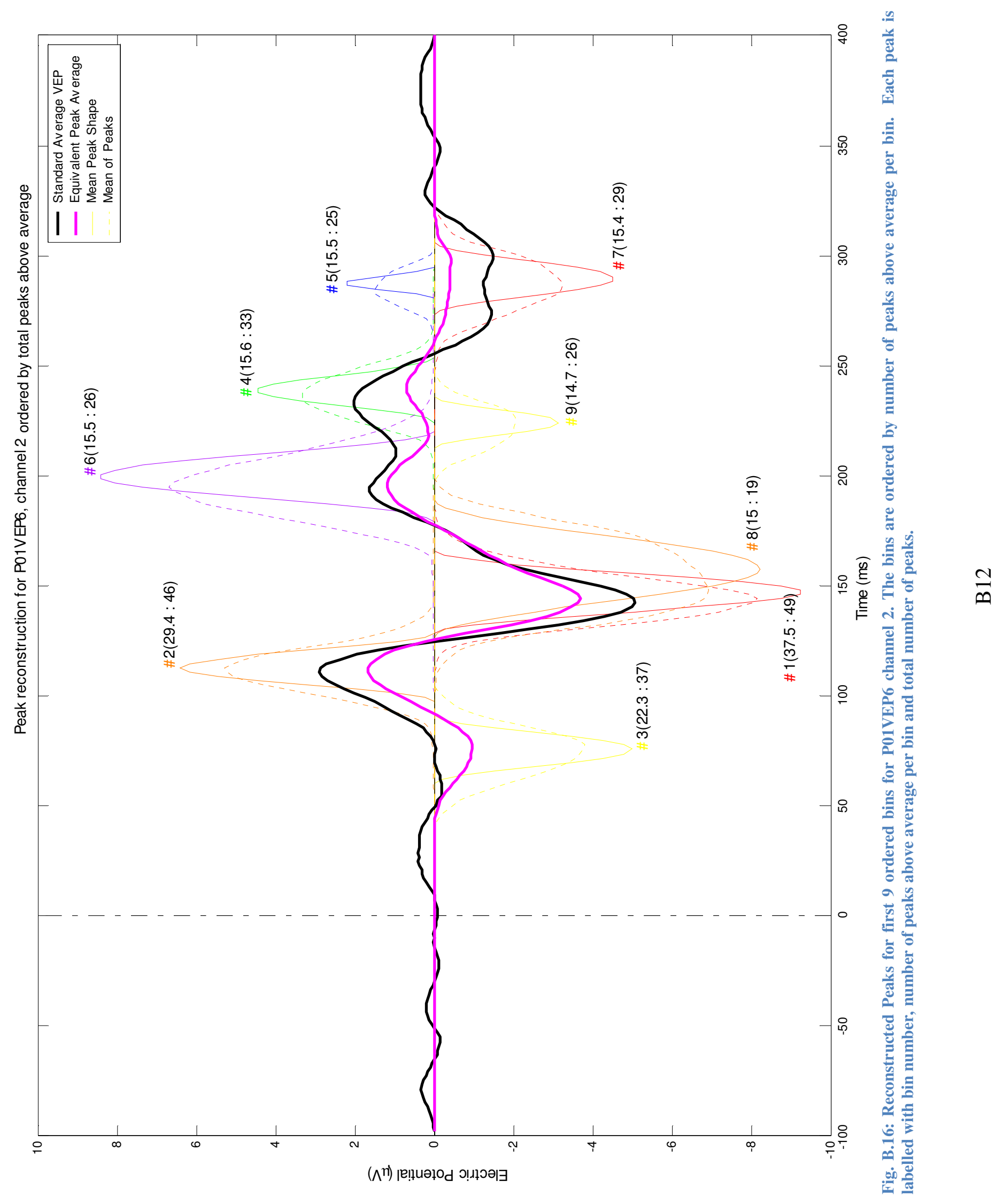




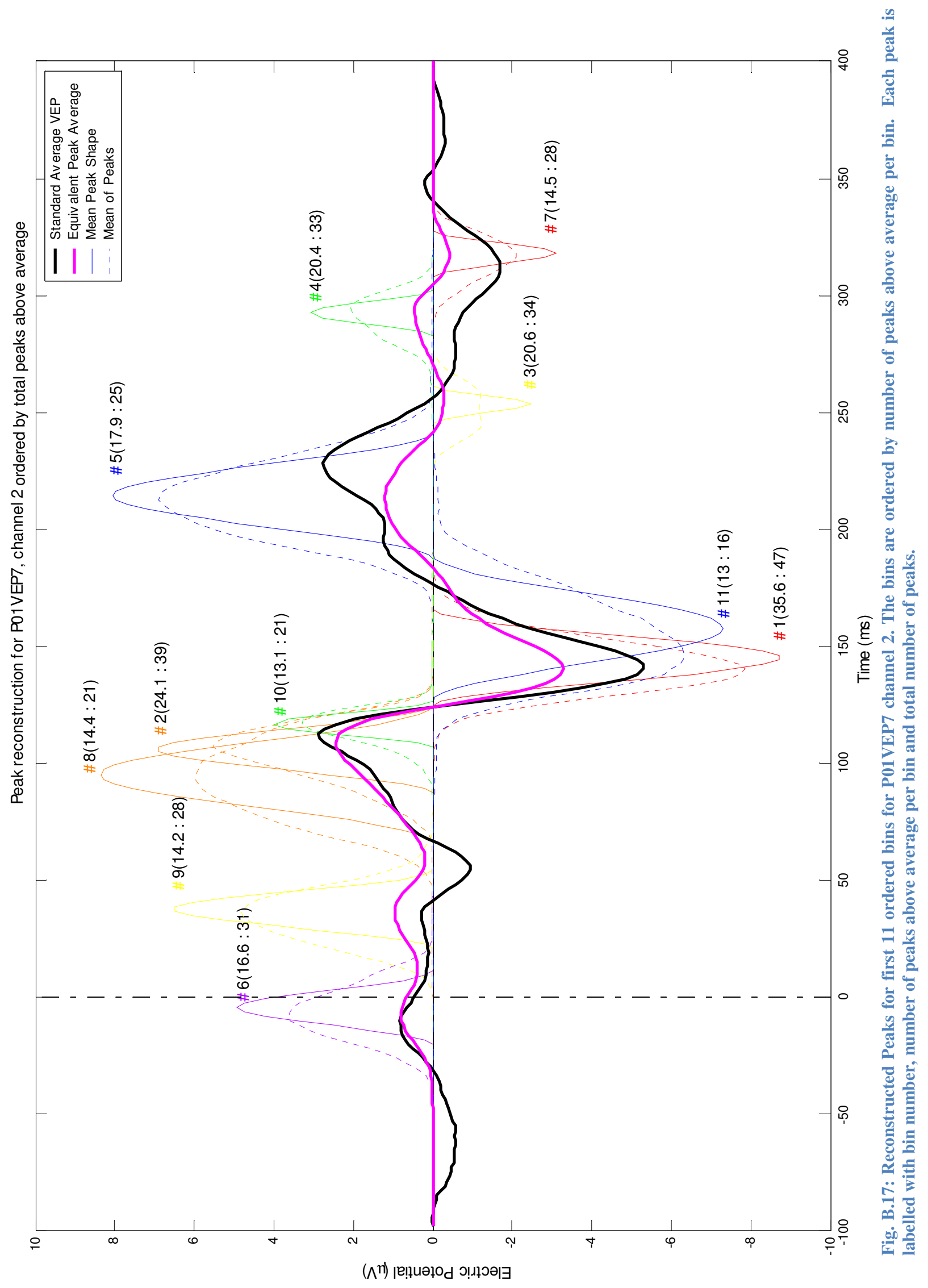

$\frac{m}{n}$ 


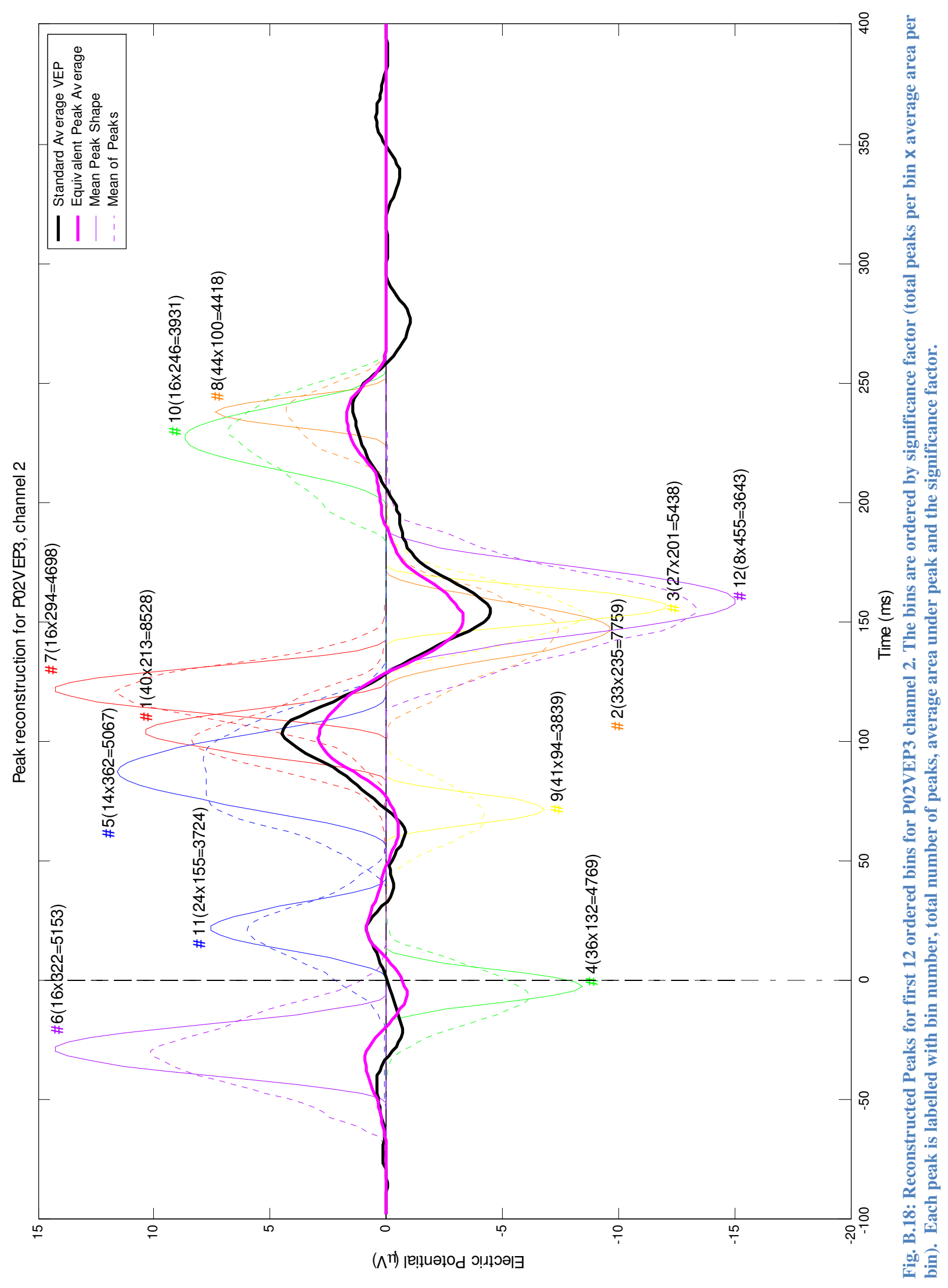

$\frac{ \pm}{n}$ 


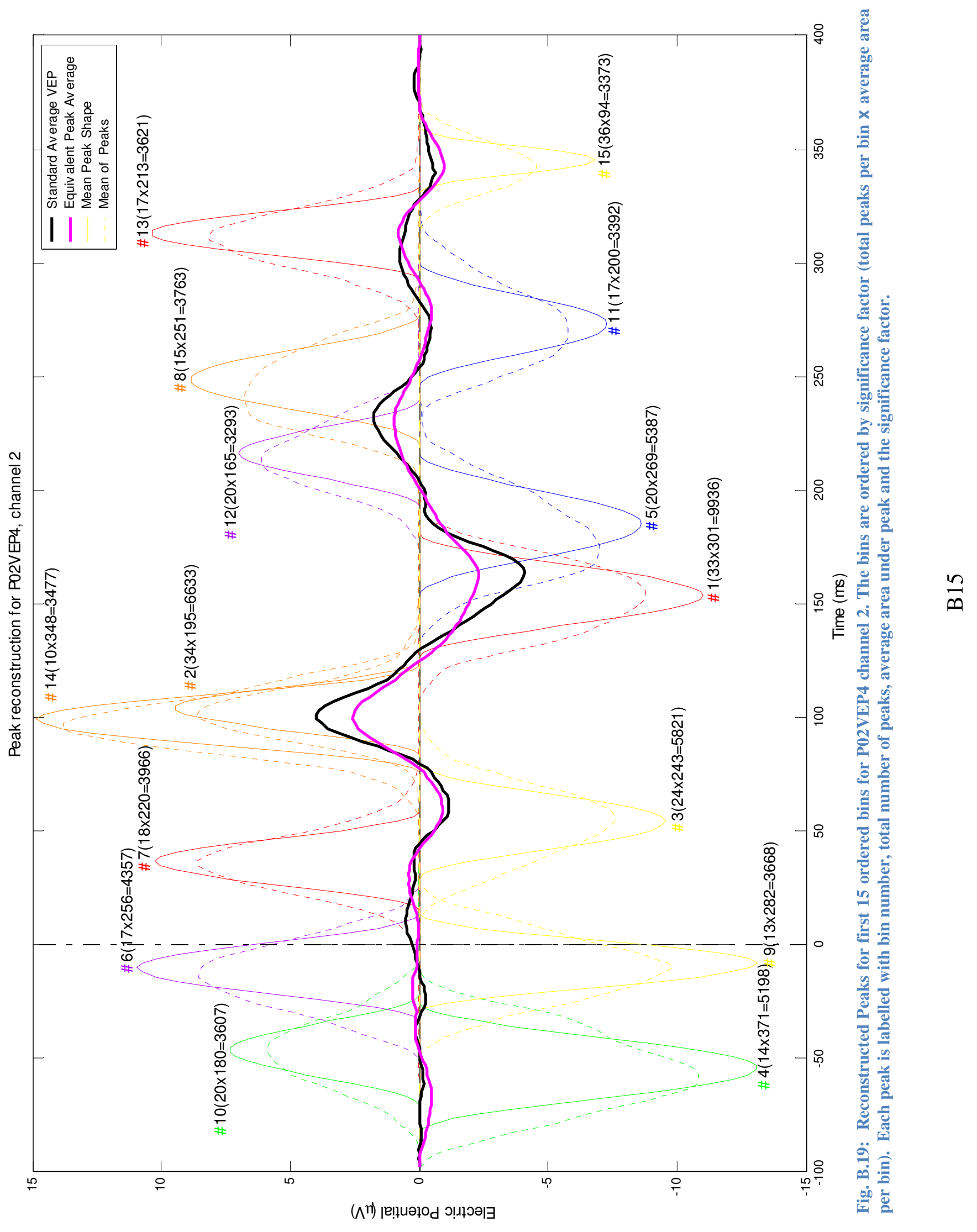




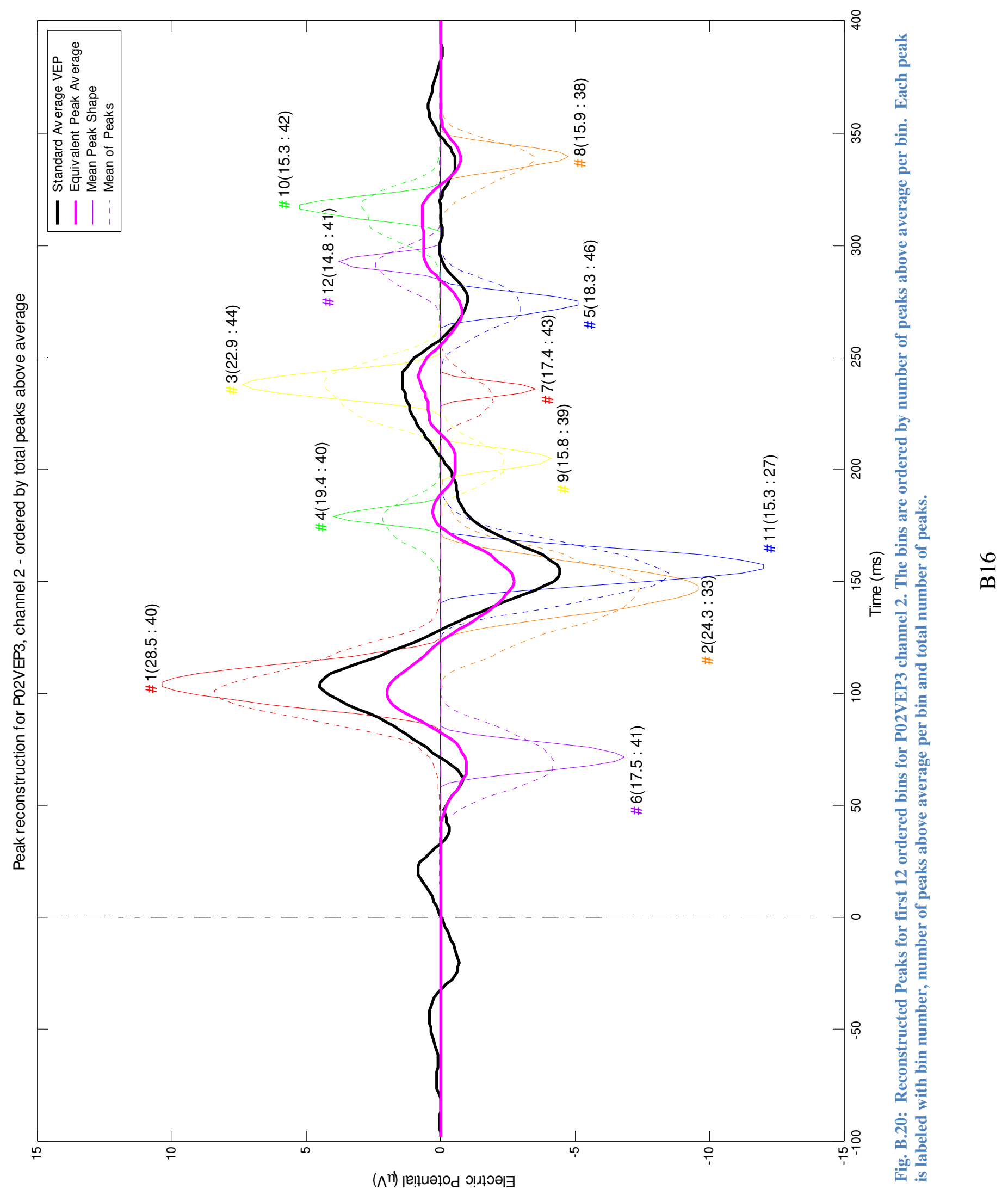




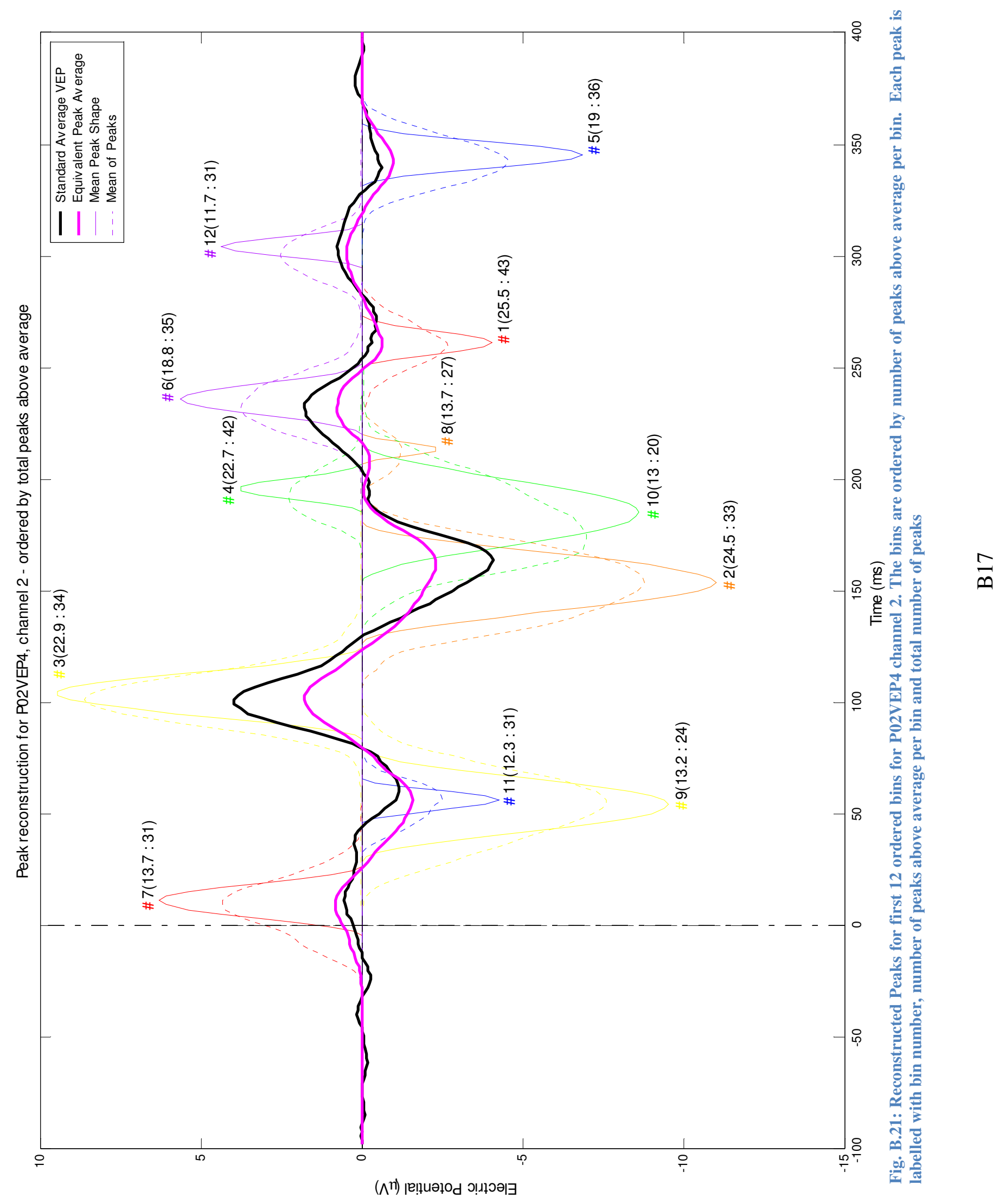




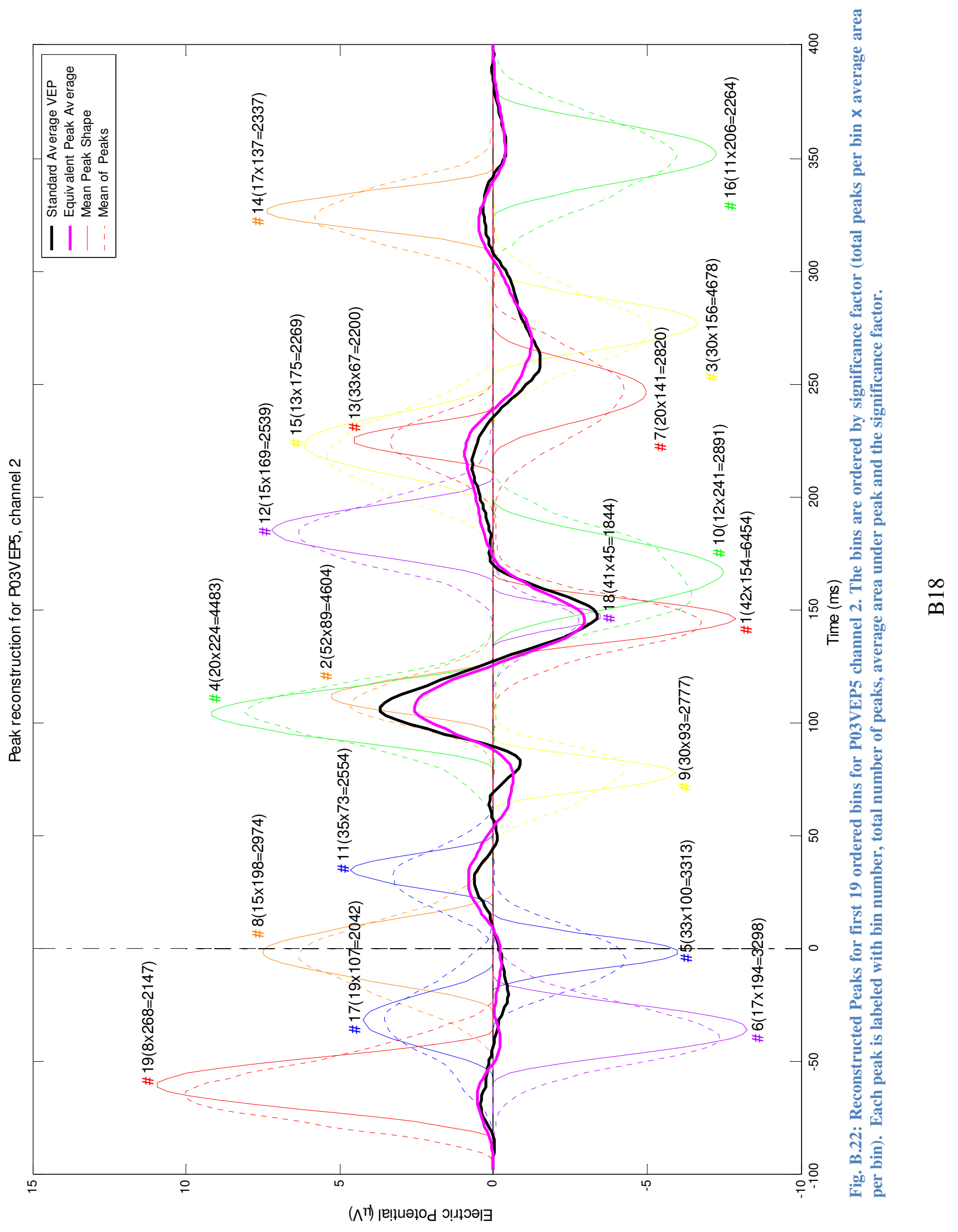




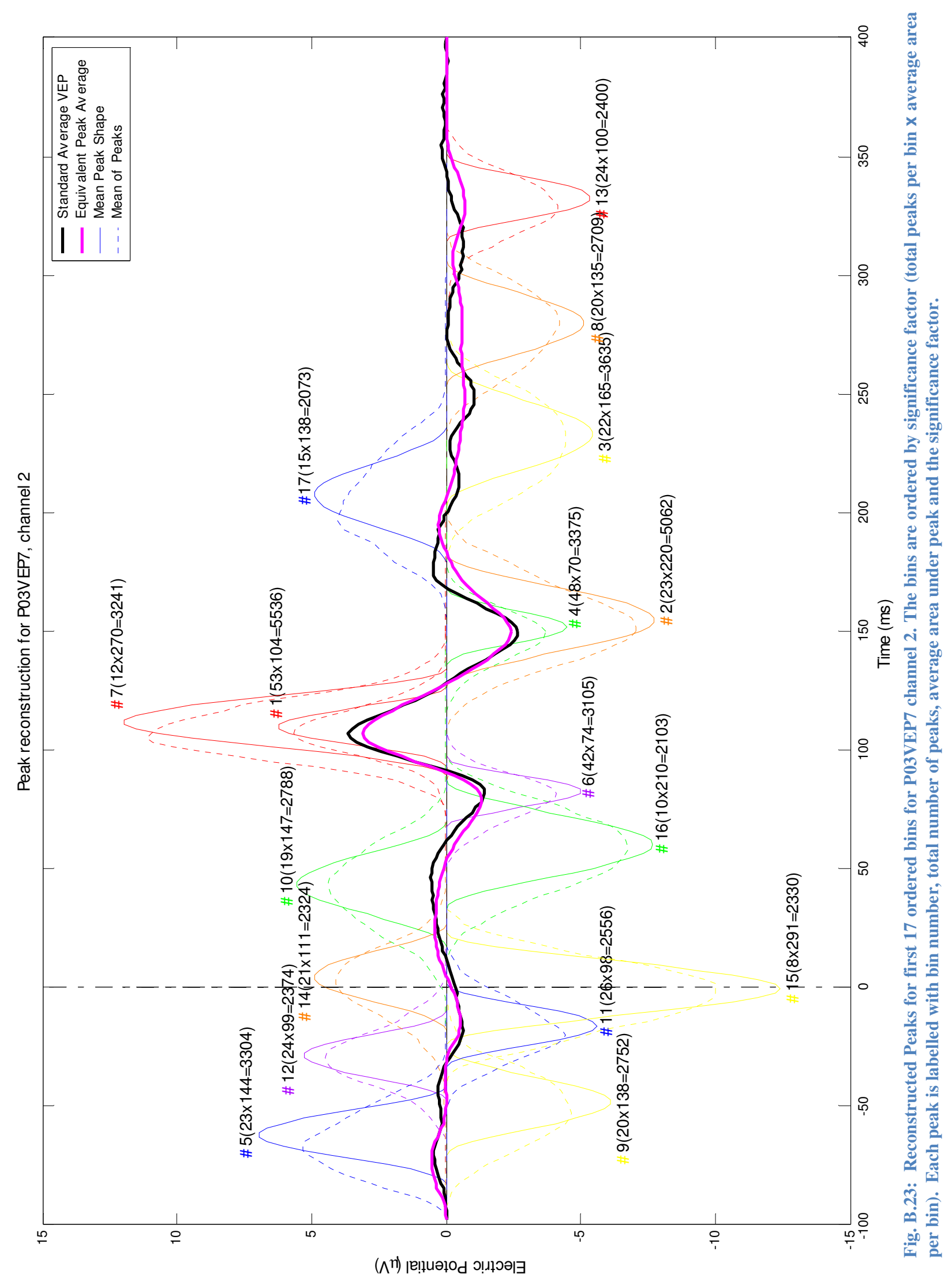

$\frac{a}{n}$ 


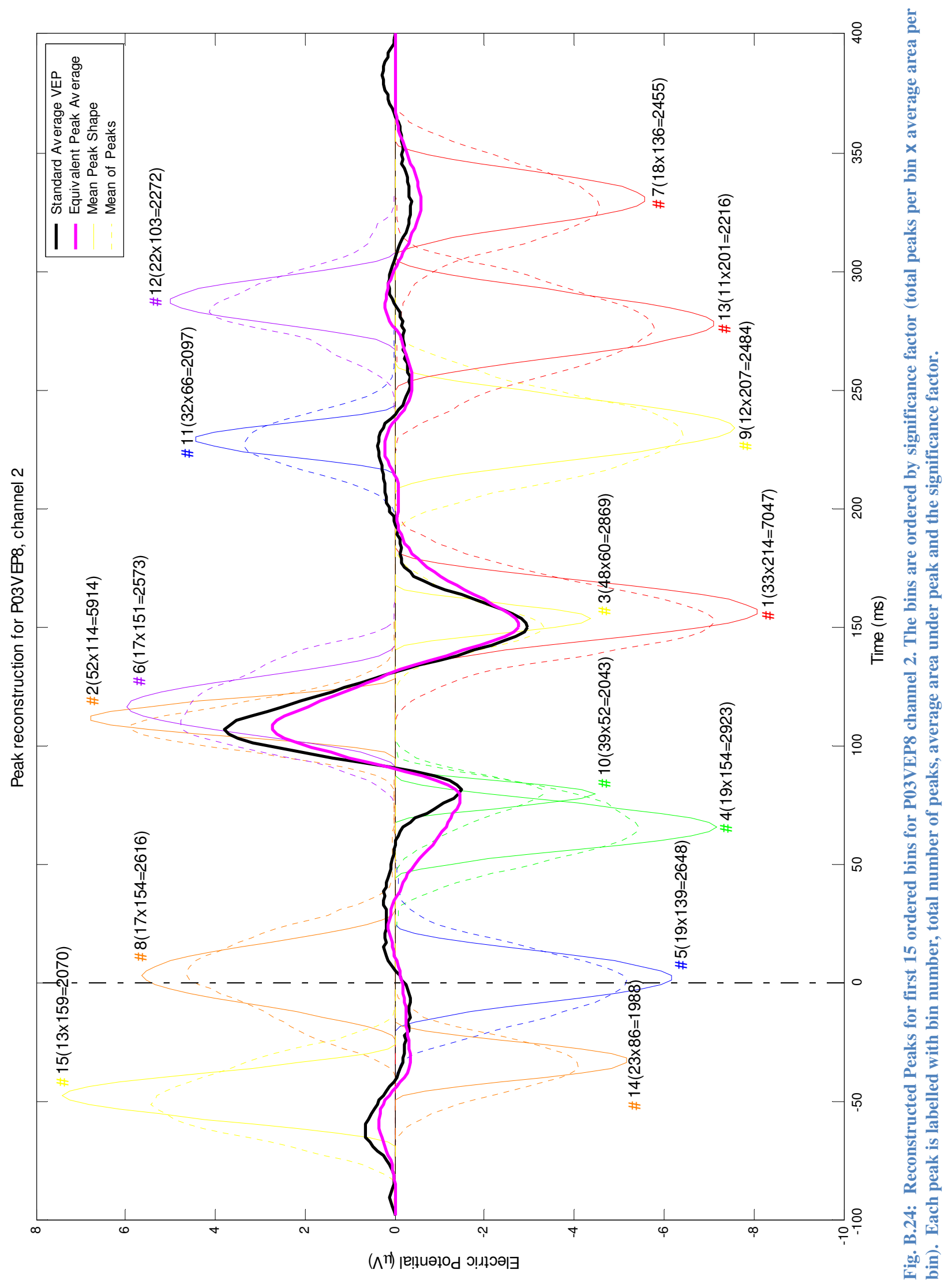

กิ 


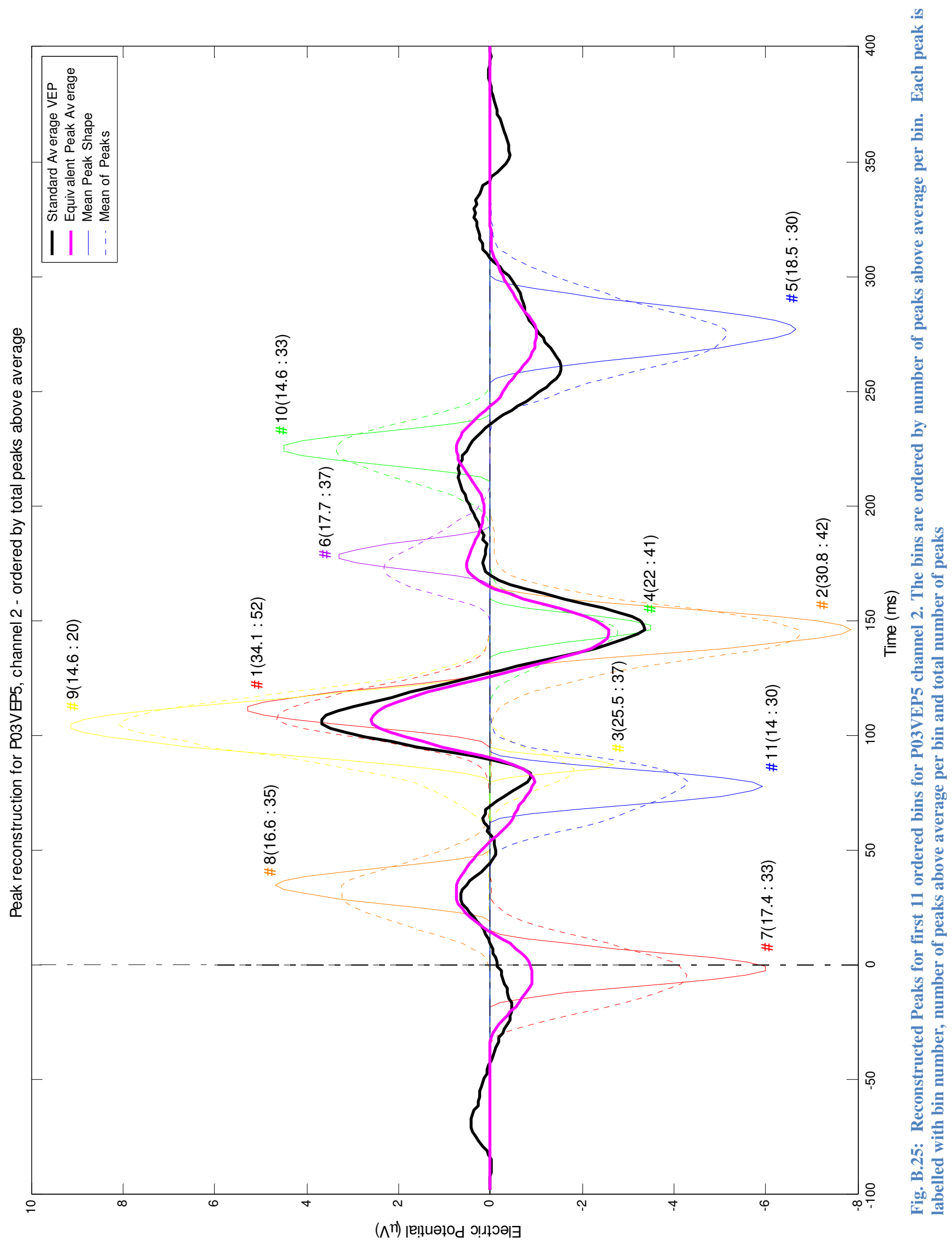

กิ 


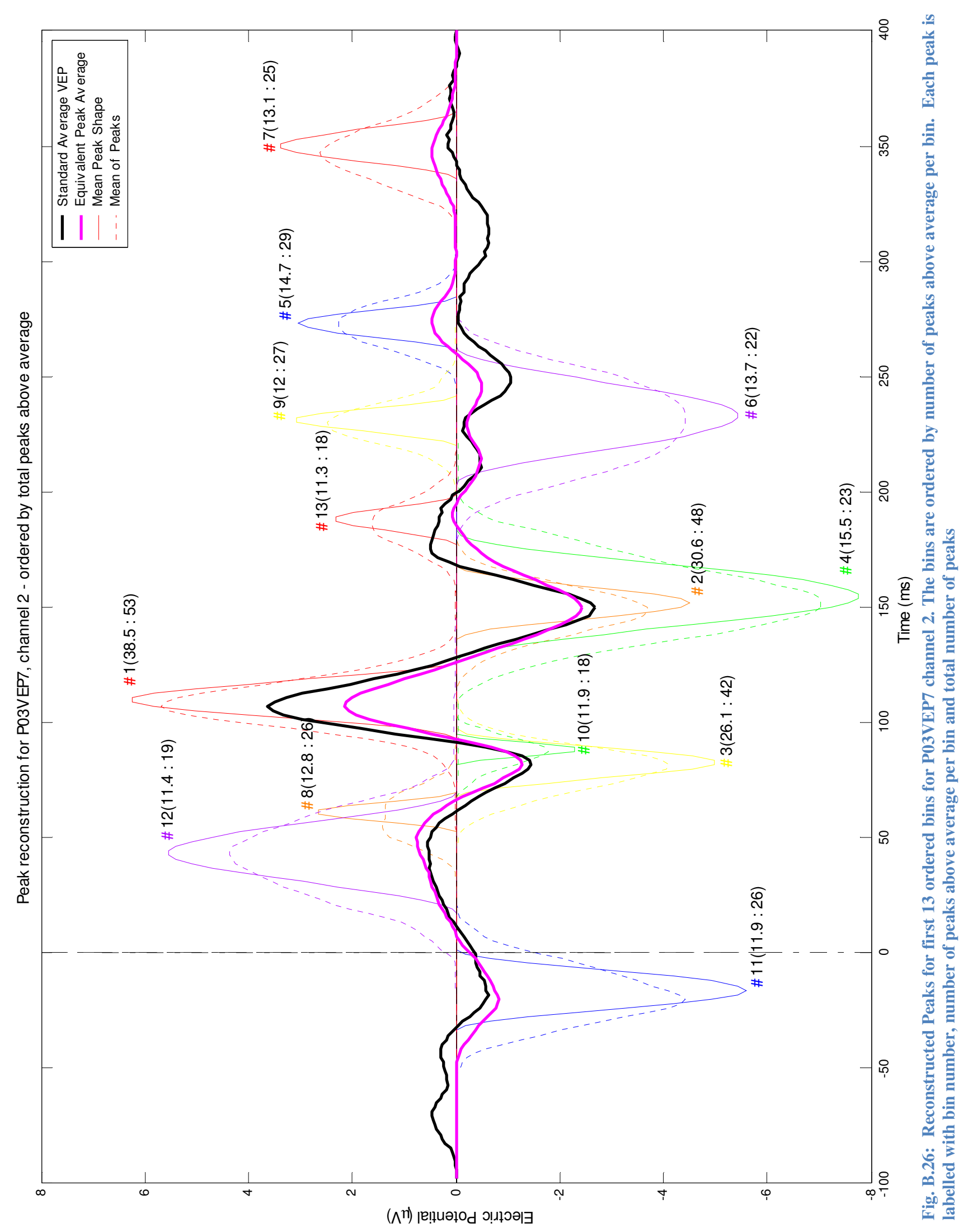




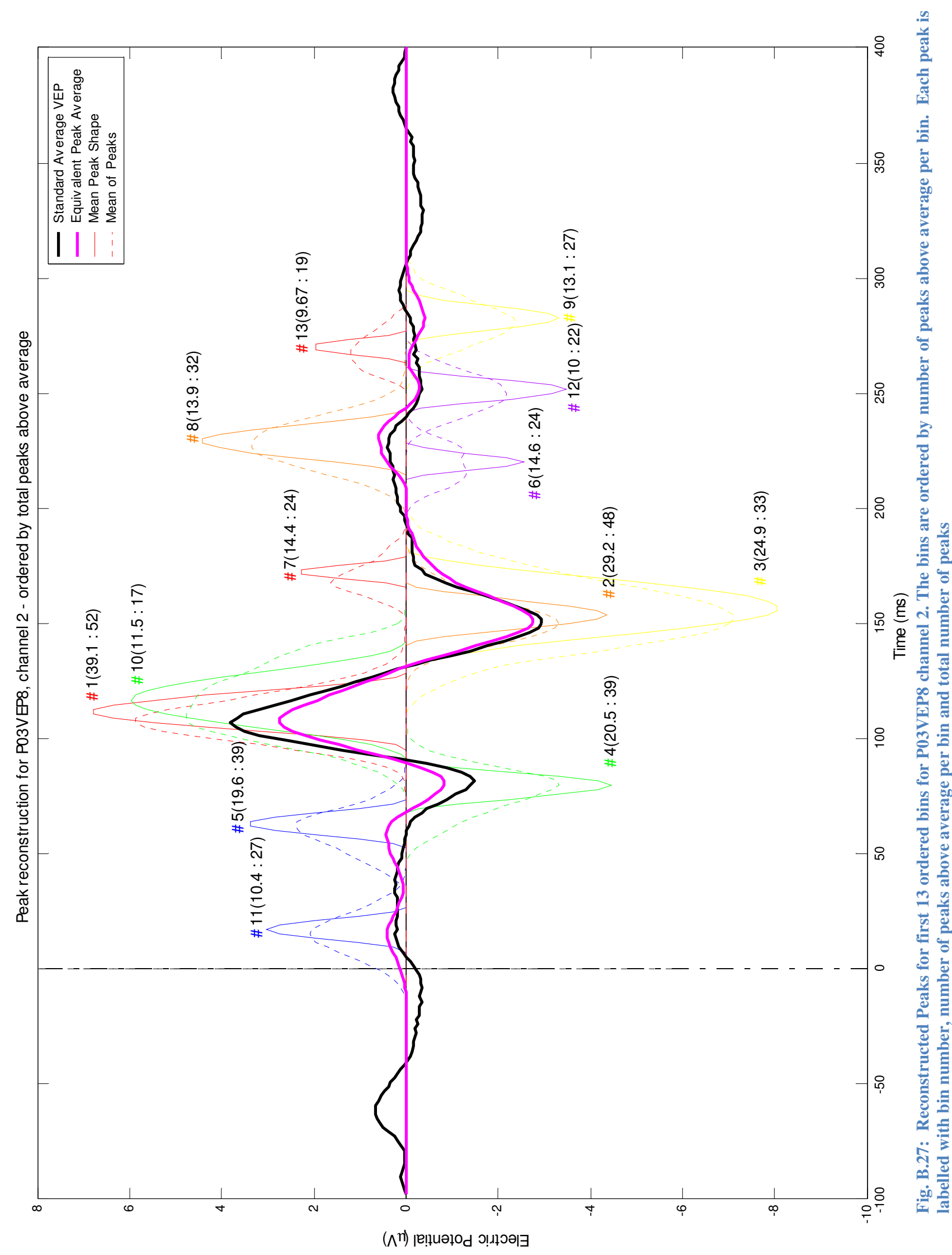

กิ 
(a) Pearson Correlation Trend

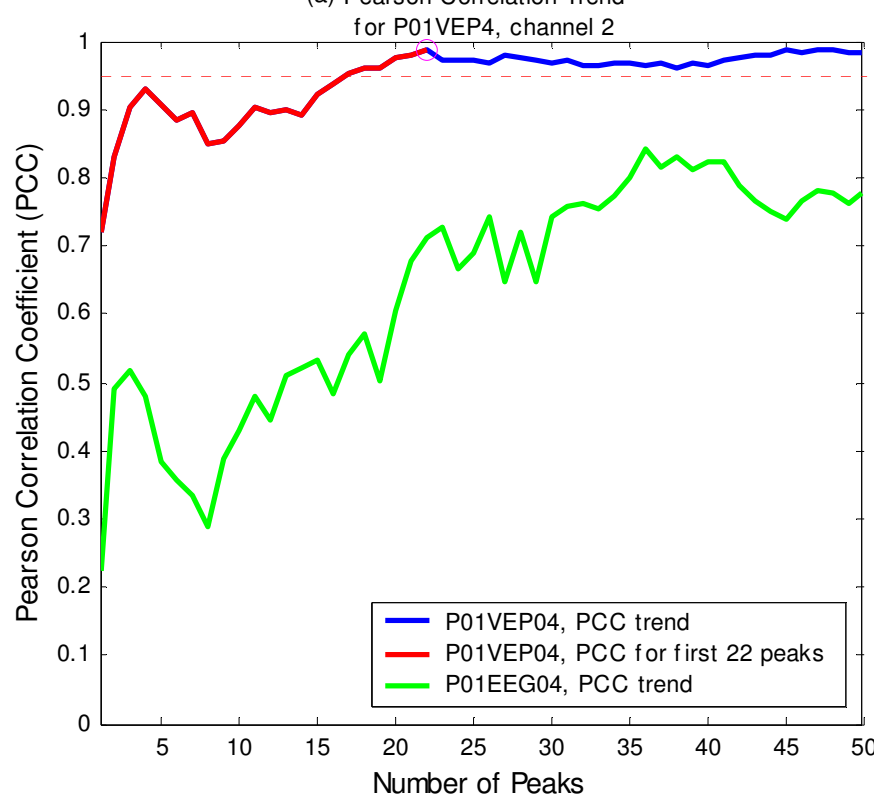

(c) Pearson Correlation Coefficient Trend

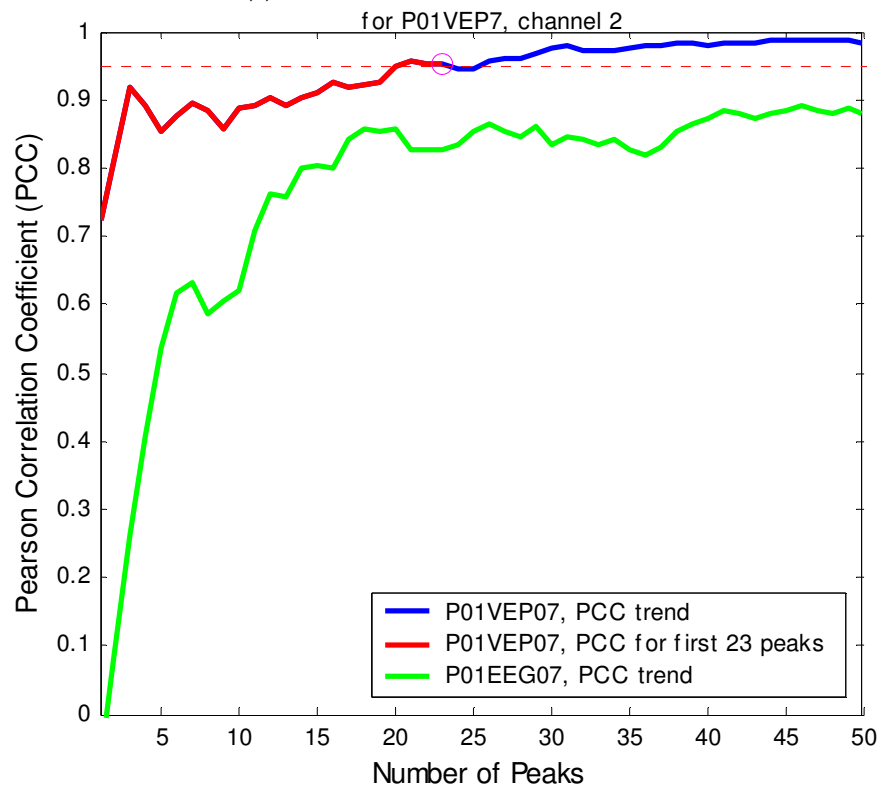

(a) Pearson Correlation Coefficient Profile

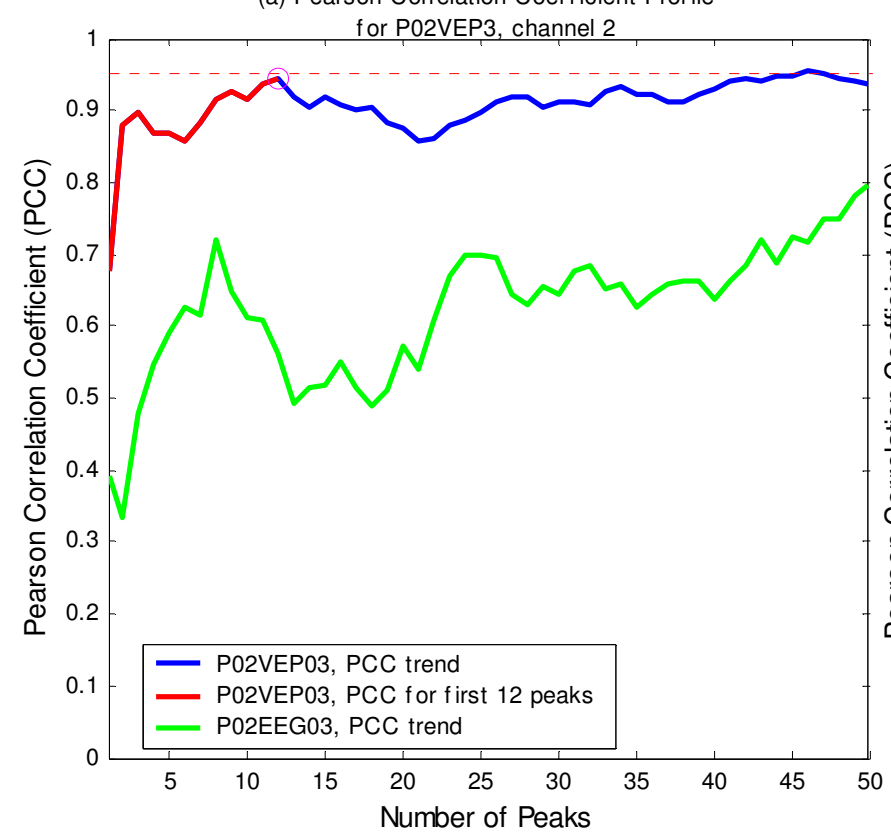

(b) Pearson Correlation Coefficient Trend

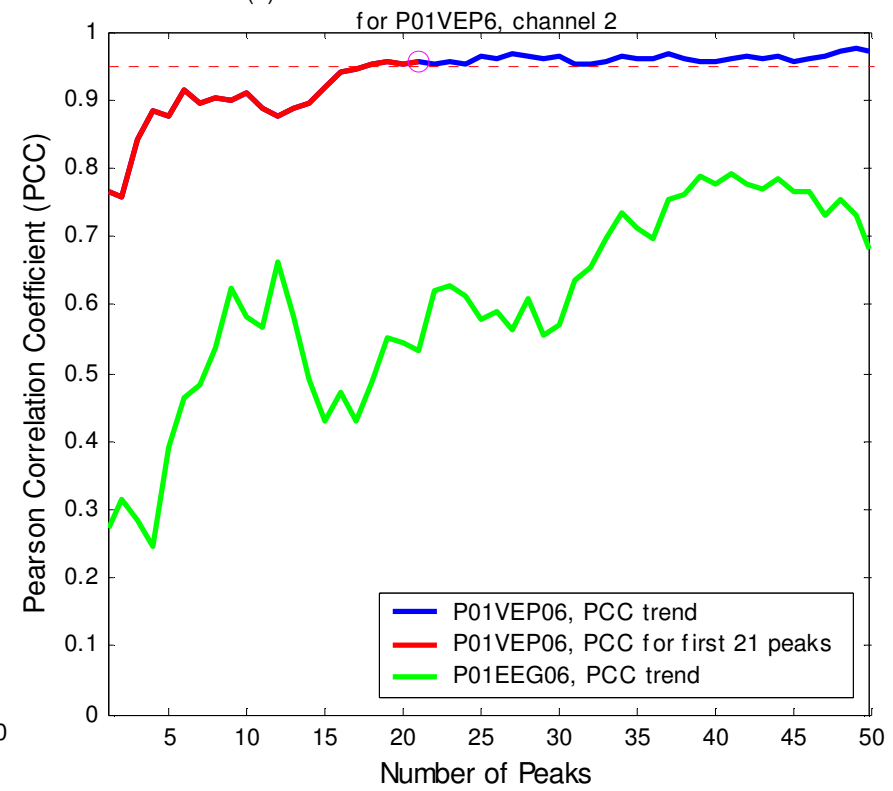

Fig. B.29: (above and left) Pearson correlation coefficient trend for Participant 01, three separate datasets (a) P01VEP4, channel 2, (b) P01VEP6, channel 2 and (c) P01VEP7, channel 2. Each graph shows the VEP trend when bins are ordered by significance (red and blue). The magenta circle indicates the chosen stop position.

Fig. B.28: (below) Pearson correlation coefficient trend for Participant 02, two separate datasets (a) P02VEP3, channel 2 and (b) P02VEP4, channel 2. Each graph shows the VEP trend when bins are ordered by significance (red and blue). The magenta circle indicates the chosen stop position.

(b) Pearson Correlation Correlation Trend

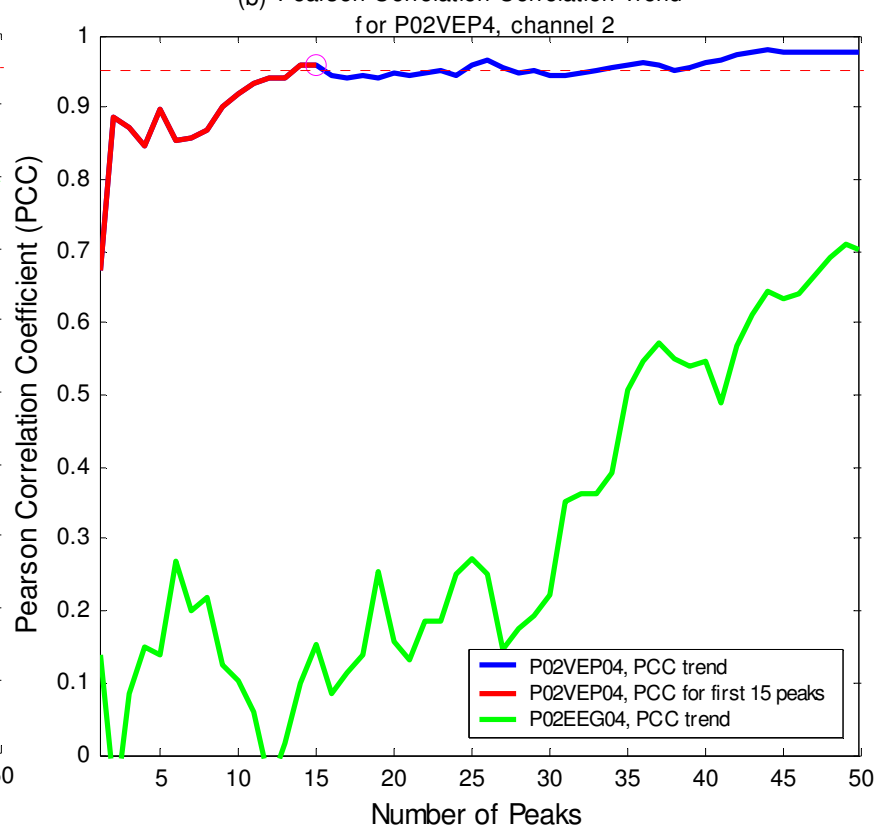


(a) Pearson Correlation Coef ficient Trend

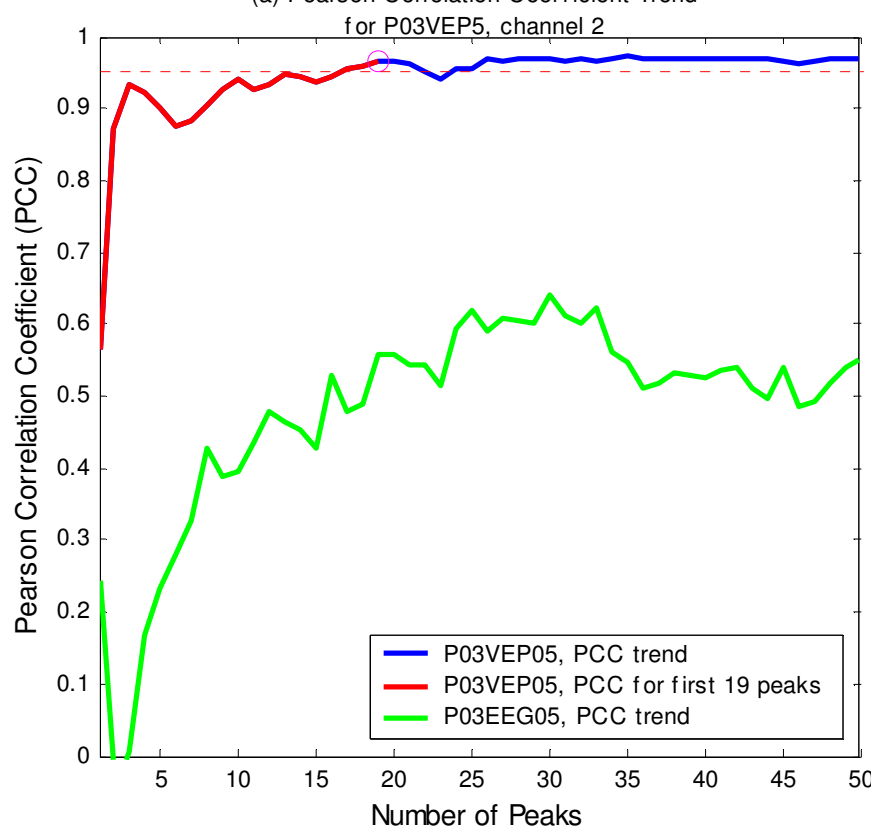

(c) Pearson Correlation Coefficient Trend

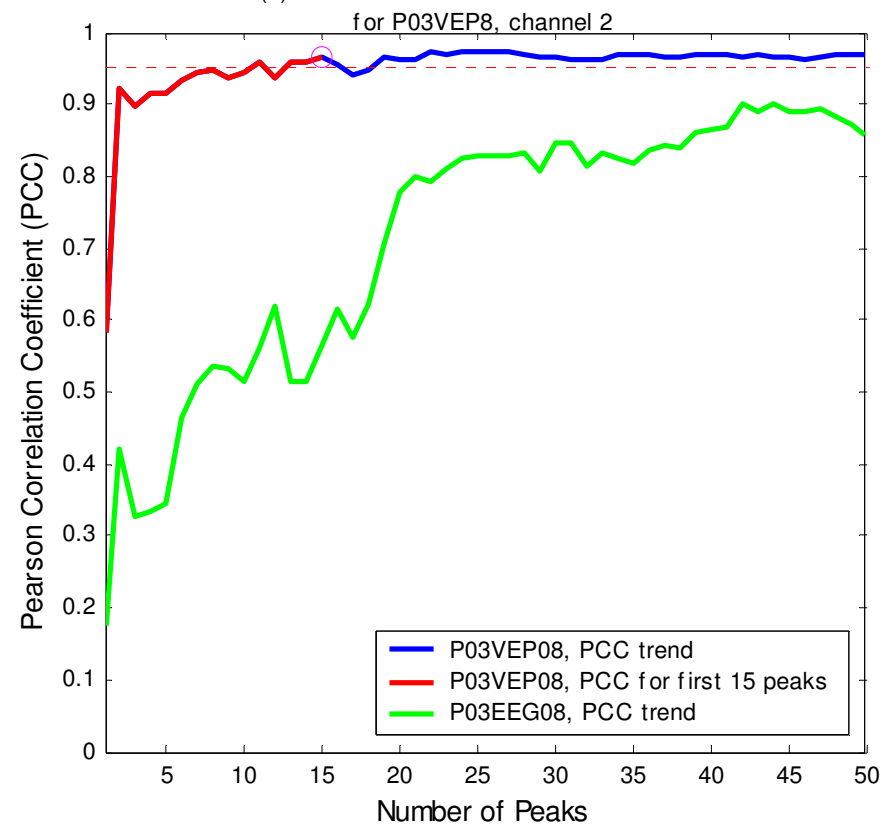

(b) Pearson Correlation Coefficient Trend

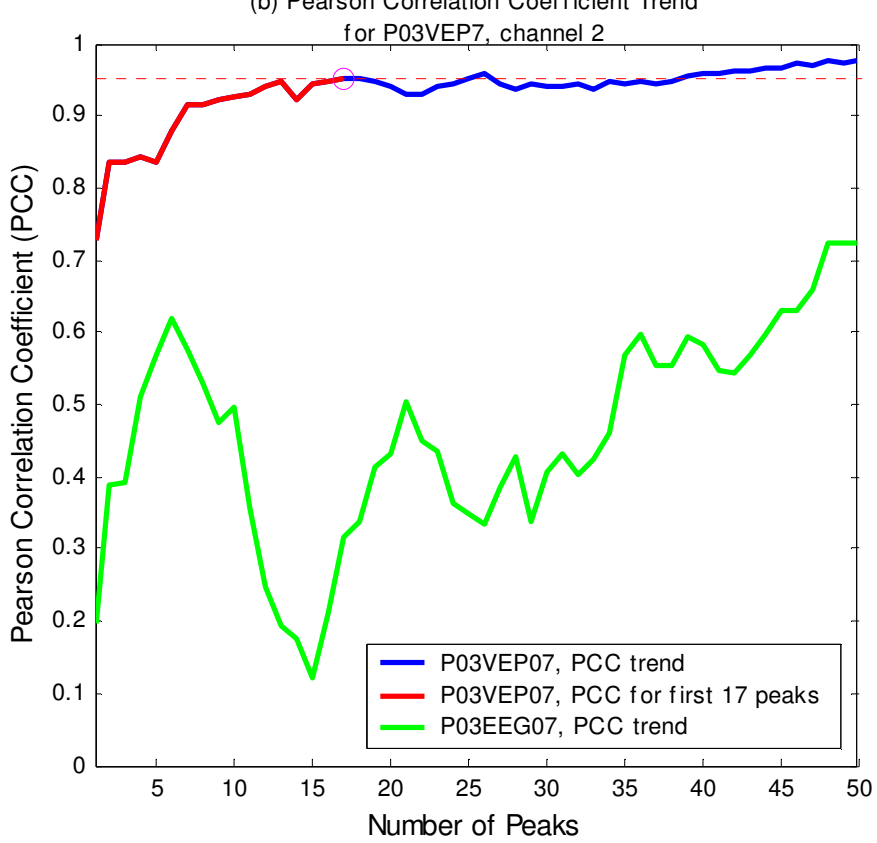

Fig. B.30: (above and left) Pearson correlation coefficient trend for Participant 03, three separate datasets (a) P03VEP5, channel 2, (b) P03VEP7, channel 2 and (c) P03VEP8, channel 2. Each graph shows the VEP trend when bins are ordered by significance (red and blue). The magenta circle indicates the chosen stop position. 


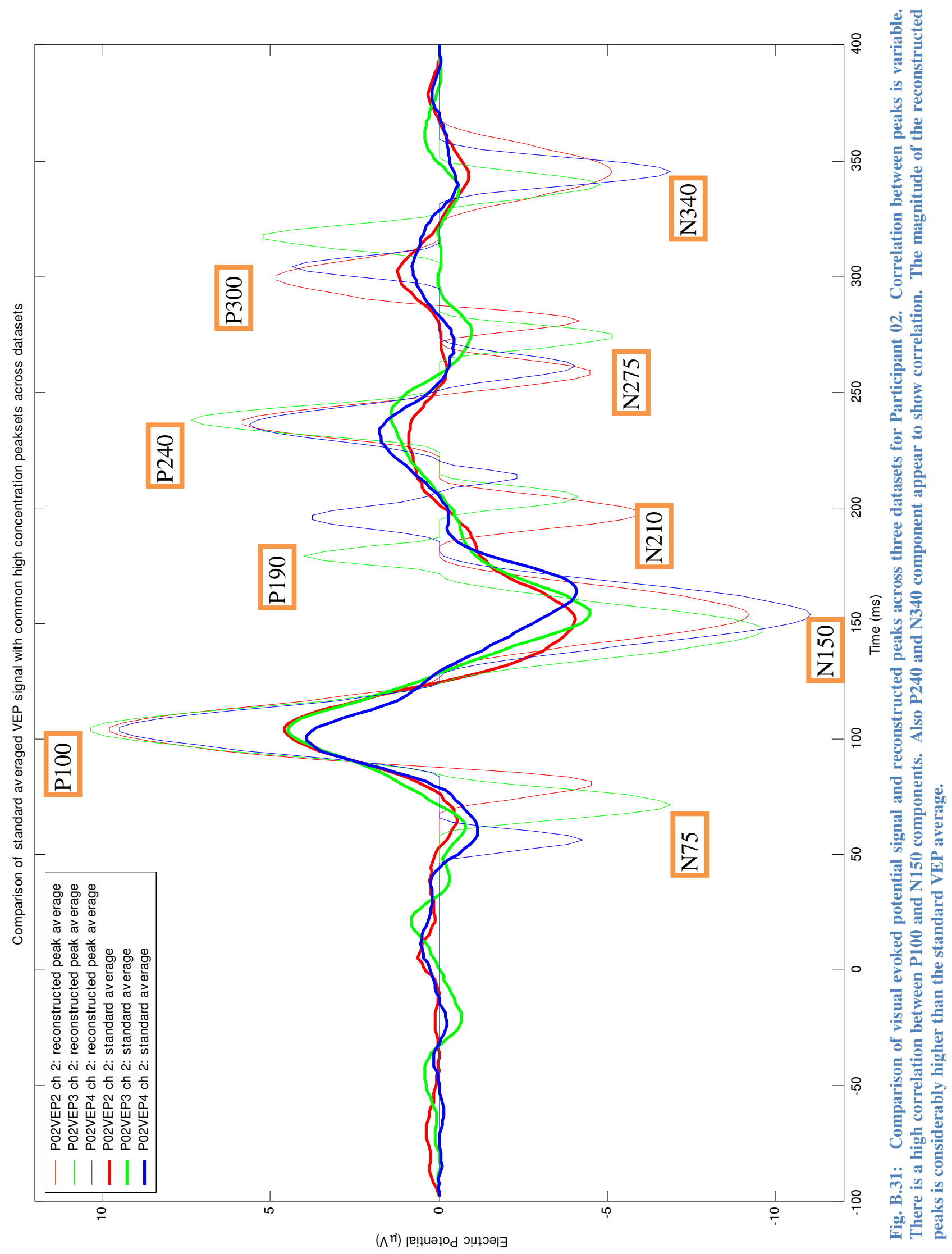

กิ 


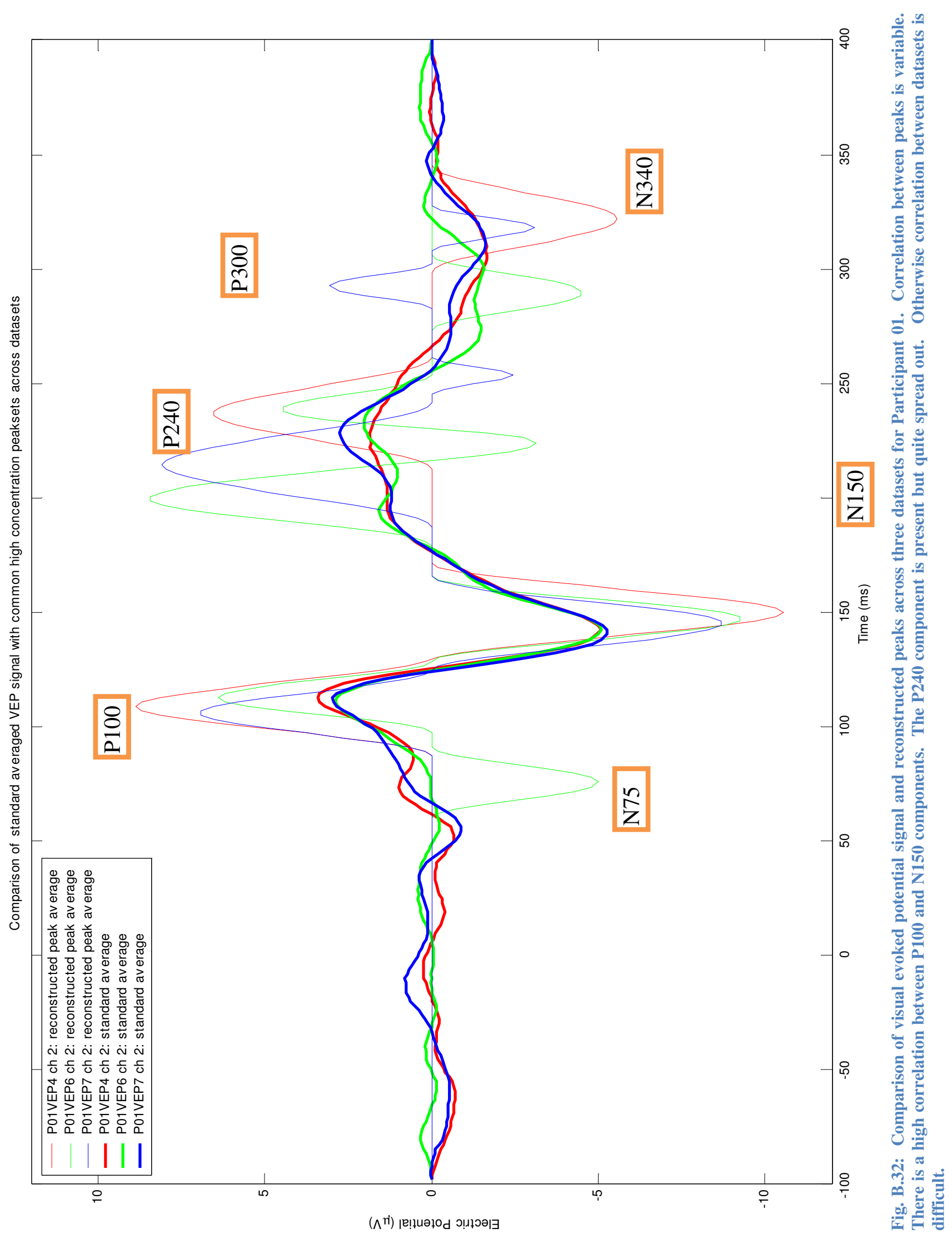




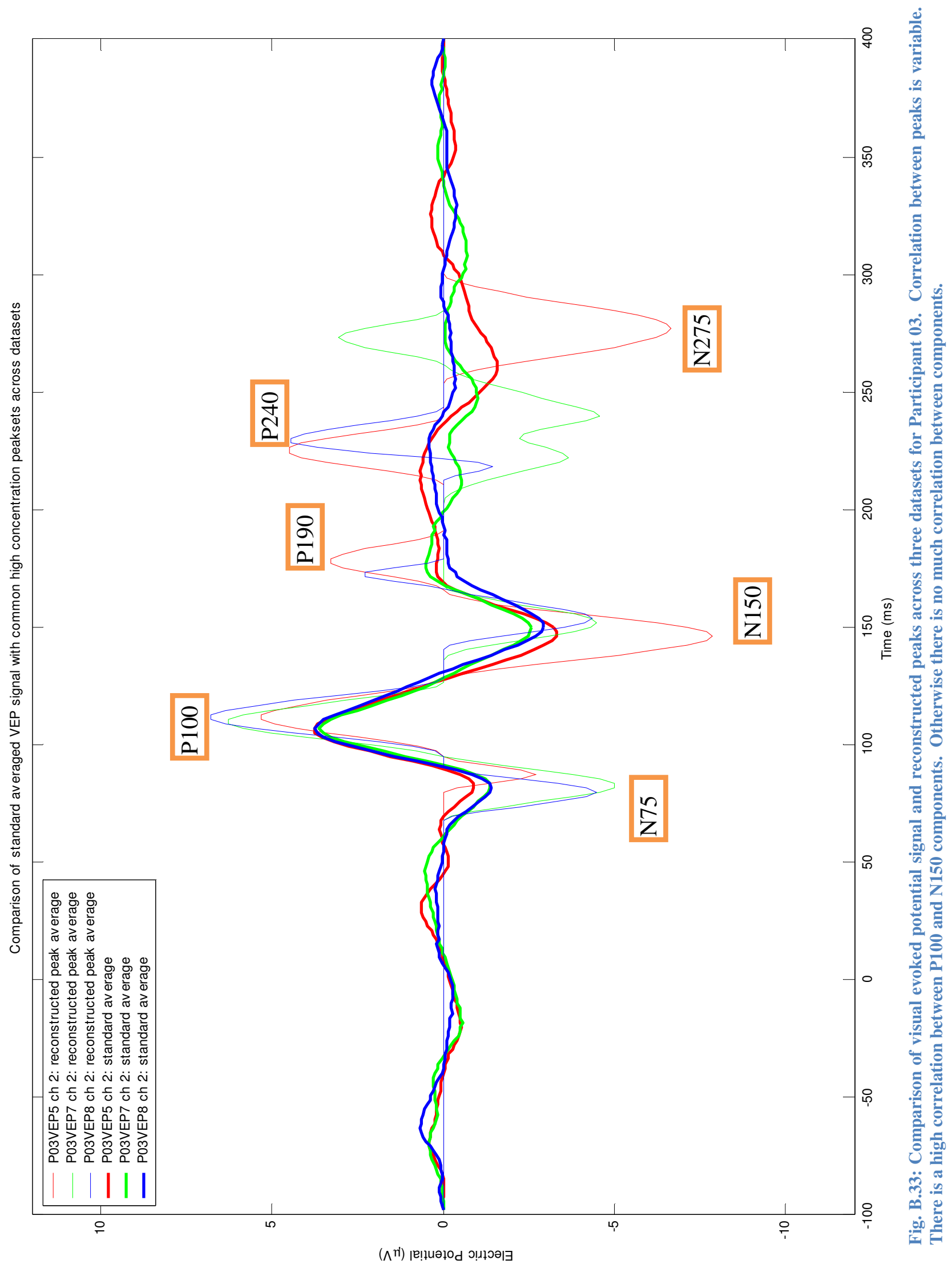

$\stackrel{\infty}{\sim}$ 
Appendix C $\sim$ PROGRAM FLOWCHARTS 

Appendix C

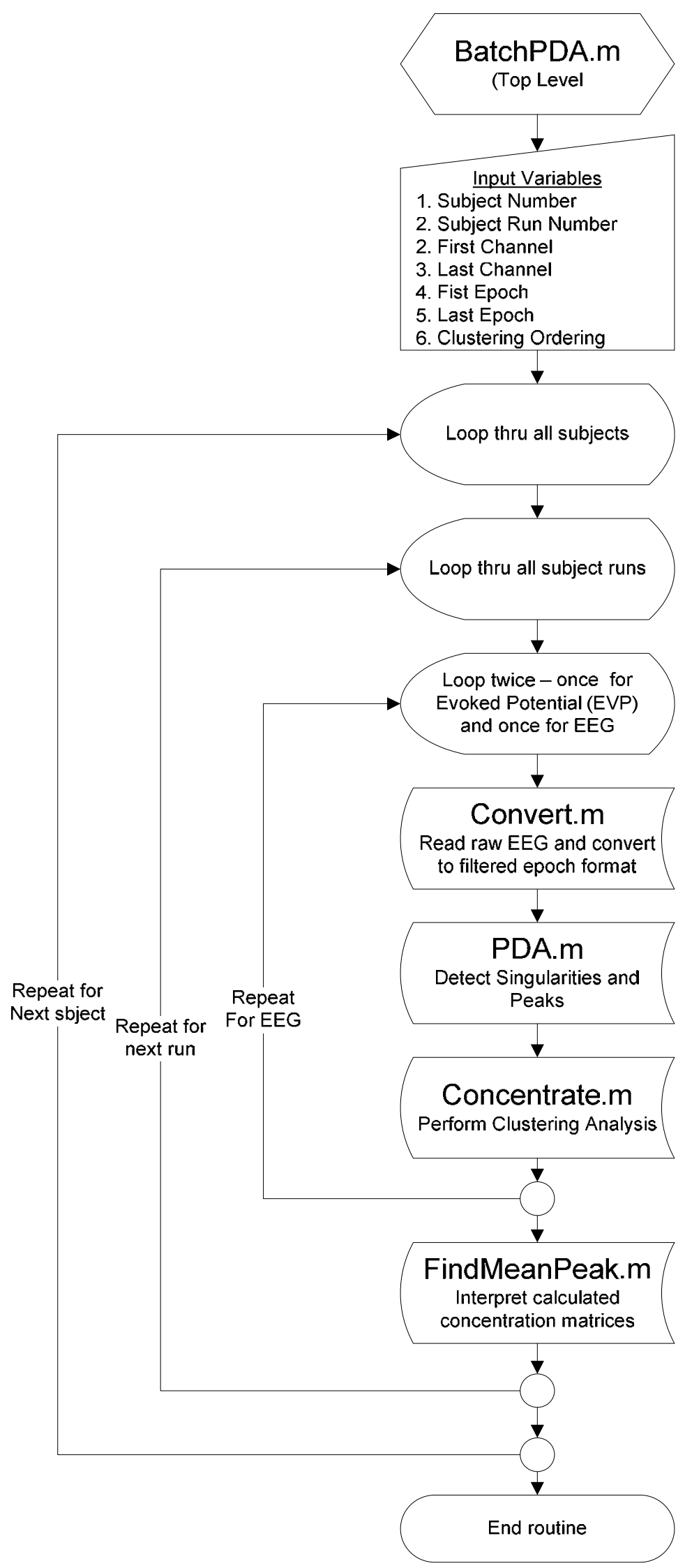




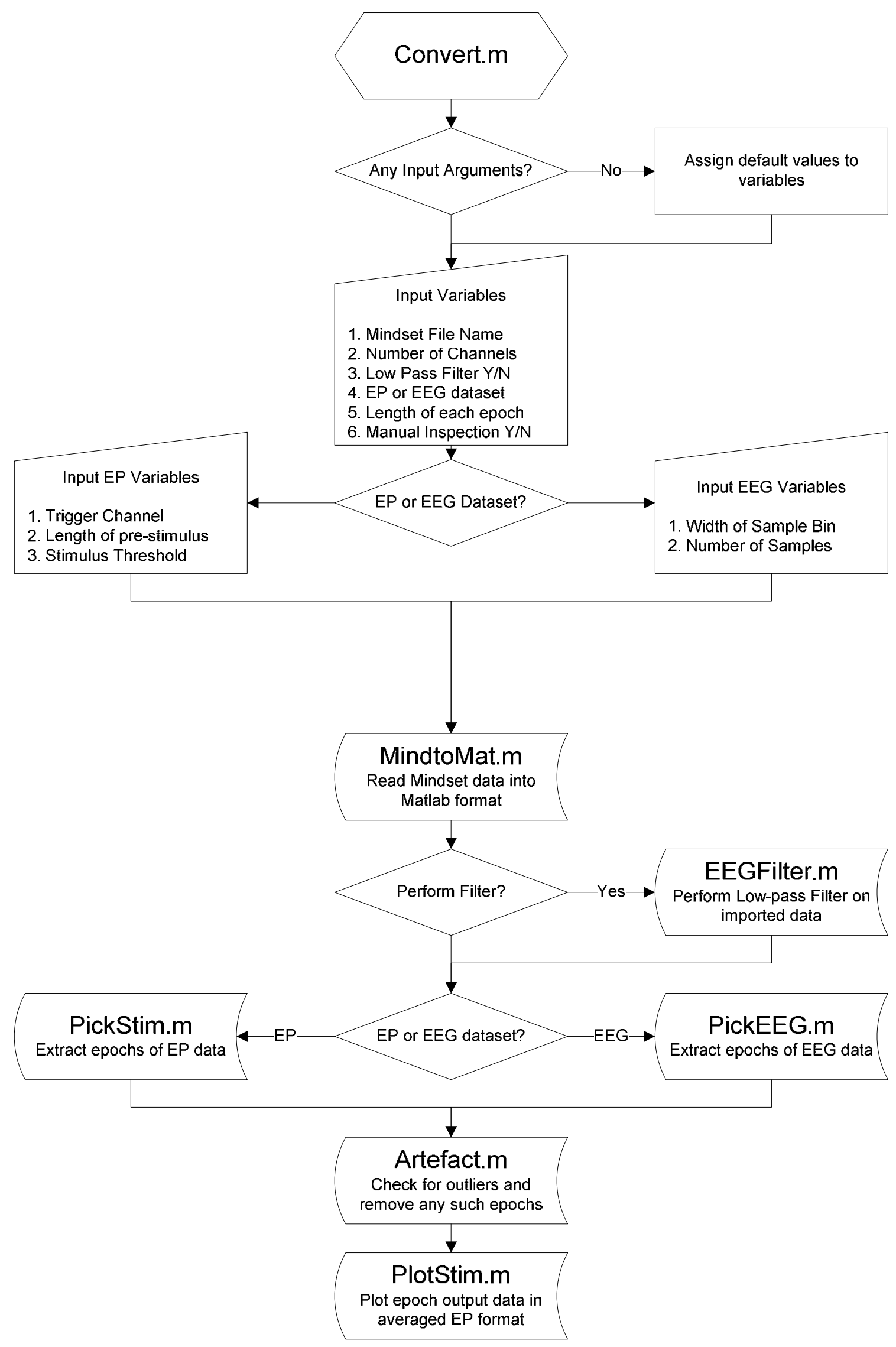


Appendix C

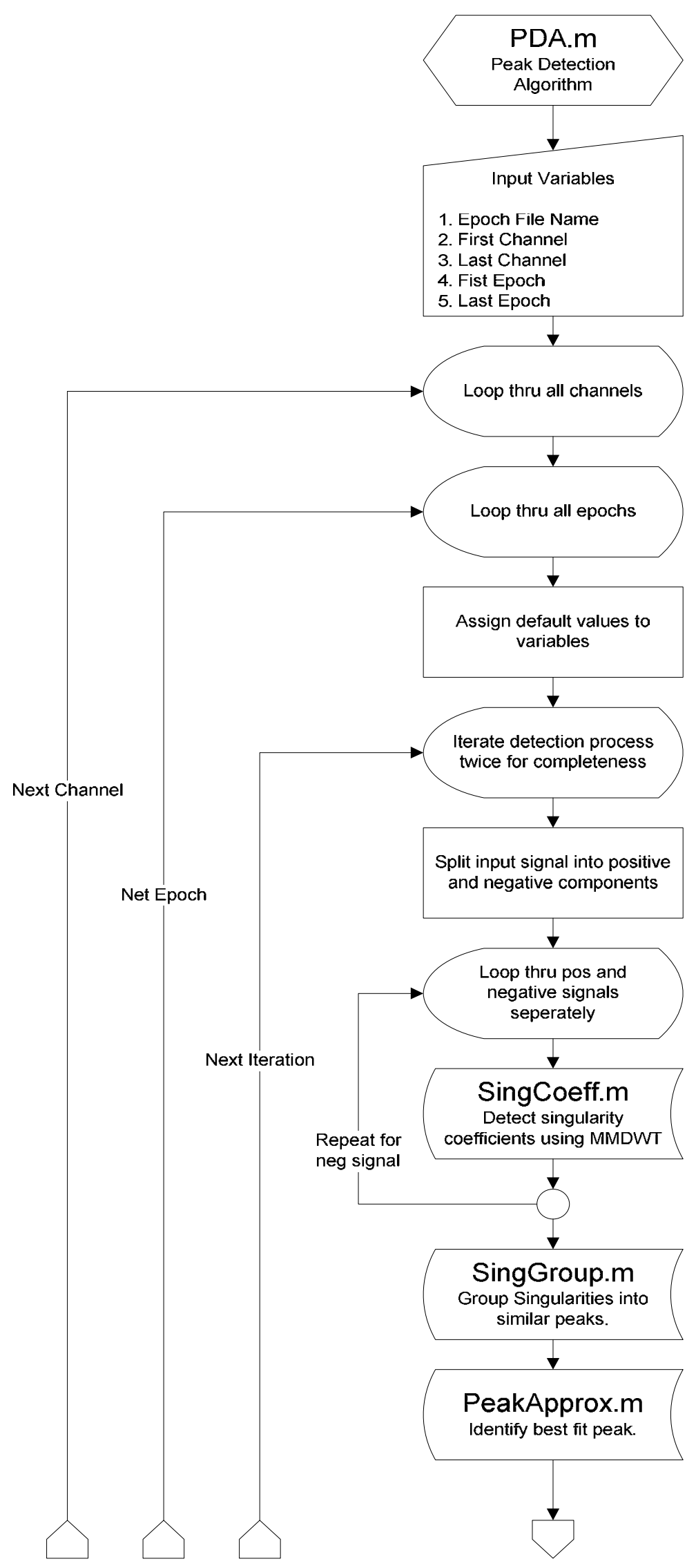




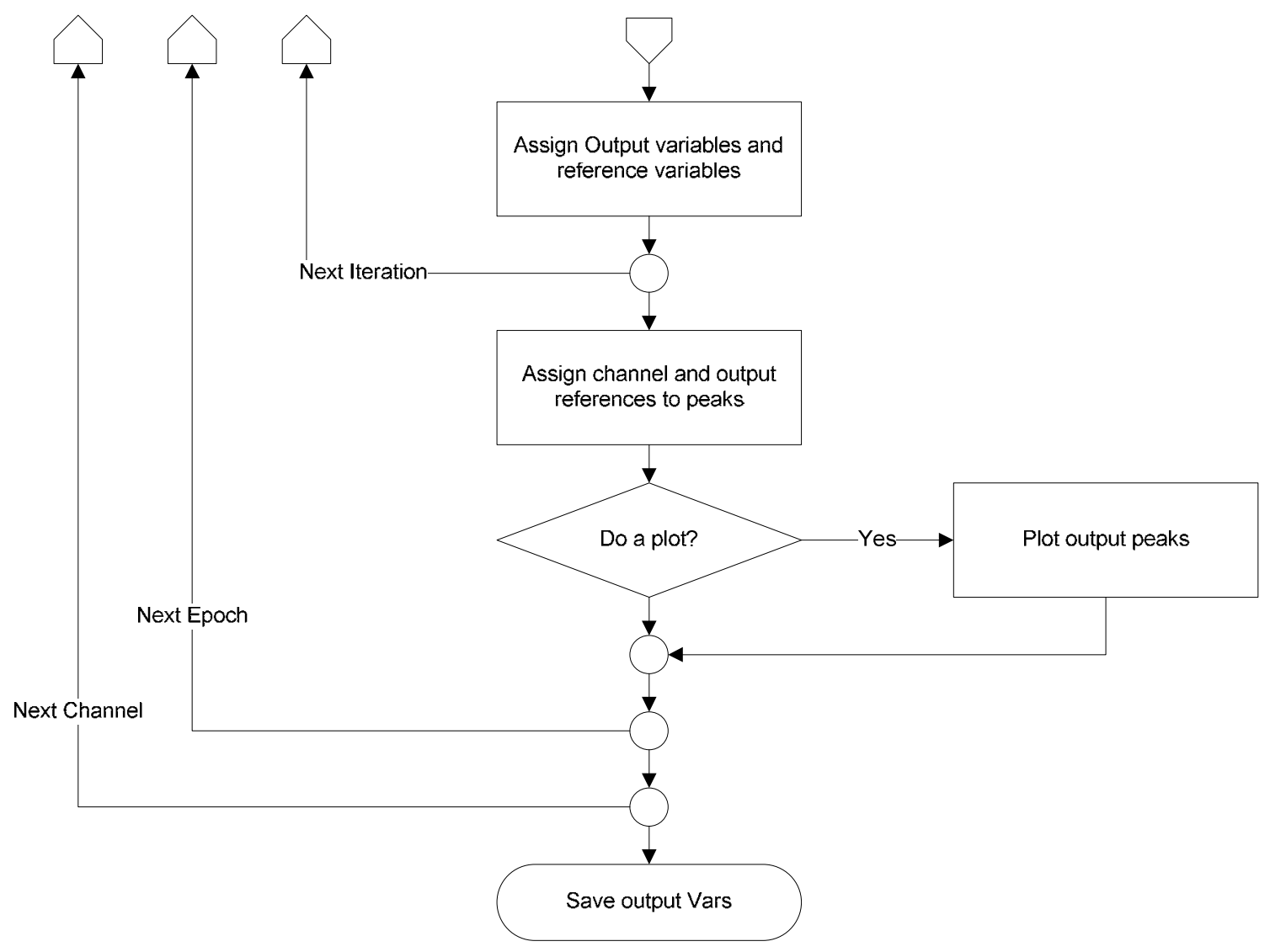


Appendix $\mathrm{C}$

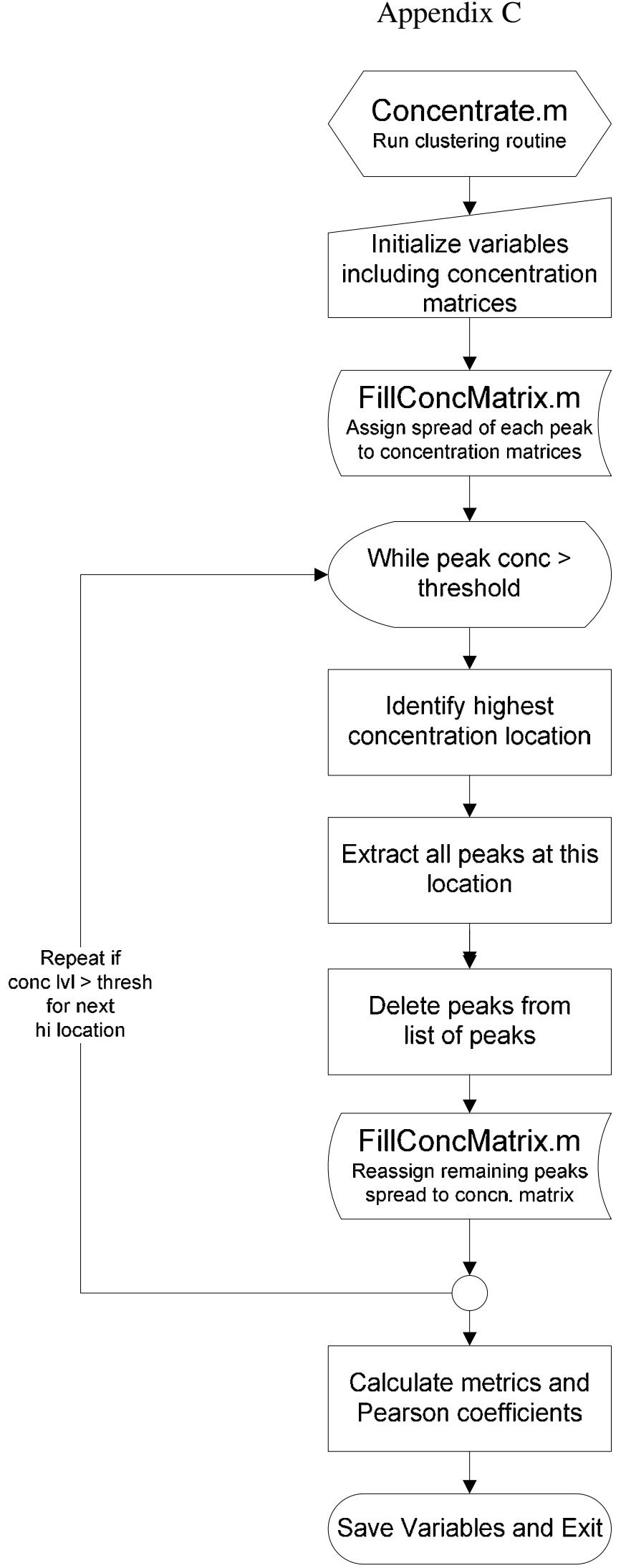




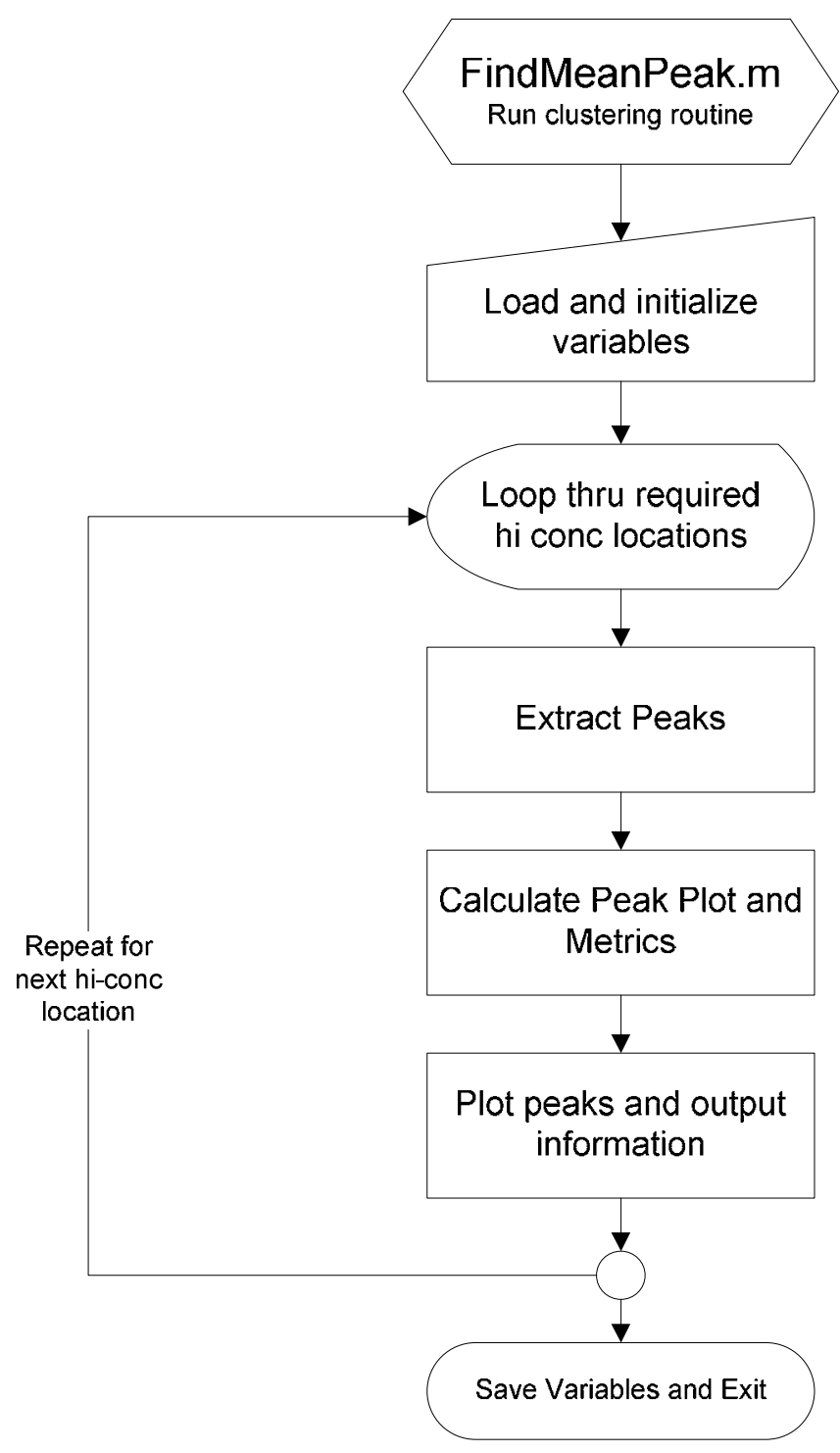


Appendix D PUBLISHED PAPERS 



\title{
Automated Peak Decomposition of Evoked Potential Signals using Wavelet Transform Singularity Detection
}

\author{
Conor G. McCooey, and Dinesh Kumar, Member, IEEE
}

\begin{abstract}
Averaged evoked potential (EP) signals are automatically decomposed into sets of peaks that may be characterised using sparse Wavelet Transform coefficients. The Wavelet Transform singularity detection technique is employed to convert Electroencephalograph (EEG) data into sets of singularities. A peak detection algorithm matches singularity pairs into sets of peaks. A single EEG epoch is then represented by a separable set of peaks. The approximation parameters classify the size and shape of the peak.
\end{abstract}

\section{INTRODUCTION}

$\mathrm{E}$ voked potential signals measure the EEG response to a specific external stimulus. The nature of the EEG generators that trigger the observed response is complex and not fully determinable. Typically, the signal of interest is embedded in similar-sized or larger ongoing background EEG signals. Ensemble averaging is used to detect the Evoked Potential and, in the case of visual evoked potentials (VEP), requires 100 to 200 repeats of the stimulus to obtain an observable response. Averaging of these epochs reduces the uncorrelated background EEG signals which tends towards a zero level as it is considered random in nature, while the stimulus response is timelocked to the stimulus and so averages to a particular voltage profile.

The standard averaging approach is simple in its application but limited in its usefulness. In clinical neurological application it may be used to verify the integrity of the neurological pathway under examination and may also yield a latency measure. In event related potentials, applications tend to be limited to determining a correlation between stimulus and response.

Other authors have proposed different methods to elicit additional insight into evoked potential studies. These include AutoRegressive Moving Averaging (ARMA), Time-Frequency Analysis, Factor Analysis (Bayesian), Independent Component Analysis and Wavelet Transform. One shortcoming of each of these methods is that they emphasize one domain at the expense of others. The method described herein is invertible to the original form at any stage. It is possible to trace the effect that any particular peak in any particular epoch at any time.

Manuscript received June 22, 2007.

C. G. McCooey is a $\mathrm{PhD}$ candidate at Royal Melbourne Institute of Technology (RMIT) University, Melbourne, VIC 3001, Australia (phone: +4428 9042 7357; e-mail: s3029091@student.rmit.edu.au).

Assoc. Prof. D. Kumar is a lecturer at RMIT University, Melbourne, VIC 3001, Australia (e-mail: dinesh (armit.edu.au).

\section{Singularity Detection}

A wavelet is a time domain wave which is localized, oscillatory and with zero average. The Wavelet Transform yields a set of coefficients in the time and scale (or frequency) domain that correlate the shape of the signal with the scaled and dilated wavelet. The type of wavelet used here is a quadratic spline.

Mallet and Hwang [1] and Mallet [2] introduced a practical filter bank implementation of the discrete wavelet transform using a dyadic scale $\left(2^{j}\right)$ called the "algorithme à trous". Each dyadic scale yields a subdivided detail component and for any particular scale level a remainder called the approximation component. The detail component records the higher scale correlation between signal and wavelet. The approximation records the lowest scale level correlations between signal and wavelet.

Singularities are discontinuities in a signal where there is a large displacement over a short period. In applications such as image processing singularities may be present as an edge. In EEG analysis, the rise and fall of particular peaks edges may be described as singularity type behaviour.

Mallet and Hwang described how singularities may be detected by observing the decay of the detail component across scales. By selecting only the coefficients that fit this pattern and indeed only the modulus maxima coefficients, they demonstrated that this small subset of coefficients could give a good approximation of the original signal. This led to applications such as signal denoising, edge detection in image processing and signal compression using this singularity detection technique.

\section{PeAK Detection MethoD}

The peak detection method has been developed to take identified singularities and automatically match singularity pairs into groups of linearly independent peaks and troughs that sum together to form the original EEG signal. This method was previously described in previous papers by the authors $[3,4,5]$. However, previous algorithms struggled to automatically detect peaks due difficulties in training the algorithm to cope with all circumstances that arise during processing. This shortcoming has now been 
Appendix D.1 EMBC 2007

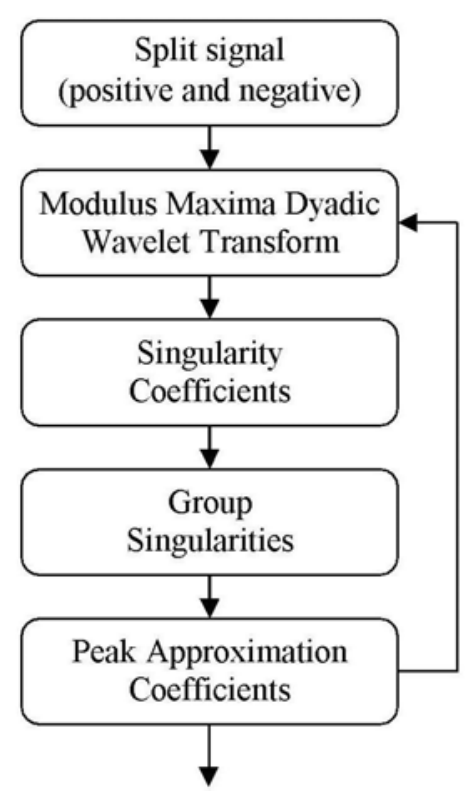

Fig. 1. Flowchart for the peak detection method. The upwards arrow indicates that the whole process is iterated once.

overcome and full automatic processing has been achieved. The parameters which describe the signal are then examined in more detail to evaluate their usefulness in further processing.

Fig. 1 illustrates the flowchart used to evaluate the peaks. The zero baseline was taken as the true zero position and therefore all peaks were calculated relative to this baseline. In order to process an EEG signal it was first split into two along the zero line yielding a set of positive only datapoints and a set of negative only datapoint. Each of these sets was processed separately to locate and characterise its peaks.

The next step in this characterization process is to calculate the modulus maxima dyadic wavelet transform for each dataset. This yields a sparse representation of the dyadic wavelet transform. Using Mallet and Hwang singularity detection technique, the increasing trend across increasing detail levels was observed. Fig. 2. illustrates a sample signal and the arrows indicate position of the two singularities in this case. The first singularity will have positive coefficients and the second will have negative coefficients. The extent to which the singularity is observable in terms of detail coefficient level is dependent on the regularity of the singularity. This regularity is obtained by calculating the Lipschitz exponent. For grouping singularities, the maximum detail level to which detail coefficients are deemed to propagate determine the extremity of where a matching singularity can be found

The singularity grouping routine takes each singularity and determines all possible other singularities that could form a match with it. In cases where more than one other singularity could form a match, the Lipschitz regularity

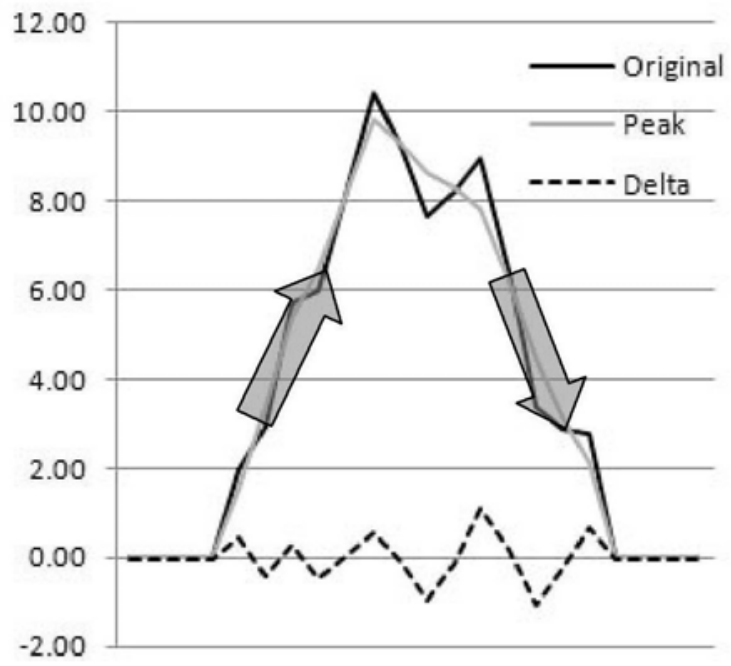

Fig. 2. Sample peak is shown as original signal. The arrows indicate positions where a positive and negative singularity have been detected by Peak Detection Algorithm. This pair of singularities form the Peak signal which is a close representation of the original signal. The Delta signal indicates the error between these two signals.

measure is used to match more similarly regular singularities together.

Once pairs of singularities have been grouped, the approximation coefficients must now be found. In order to better represent the peak, the approximation value is calculated not using the signal itself but with a subset of the signal made up of the signal located within the peak limits. If the original signal were used, then adjacent peaks would interfere with the peak under investigation and skew the output signal.

\section{RESUlts}

\section{A. Experimental Setup}

Flash Visual Evoked Potential signals were taken for a single subject. Ethics approval for the experimental procedure was obtained from the local university Human Research Ethics Committee. Repeated strobe light flashes were presented to the subjects visual field at a distance of about one from the eye at a rate of about $1 / \mathrm{sec}$. The response was measured at the Mid-Occipital (MO) location on the scalp, which is located at a point $5 \mathrm{~cm}$ directly above the inion [6]. An epoch length of 500ms was recorded synchronously with the strobe flash. The data was normalised to give a zero mean across all data. Visual inspection was performed to remove gross artifacts. Partial peaks occurring at the edges of each epoch were removed to eliminate border effects from the subsequent DWT processing. A total of 144 epochs were measured. 


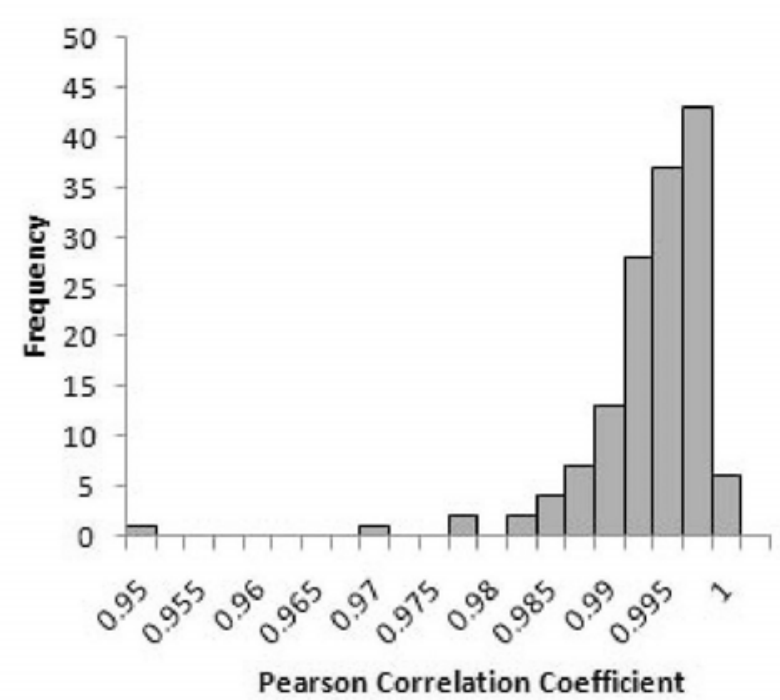

Fig. 3. Histogram of correlation coefficients for all 144 epochs of data. A correlation coefficient of 1 indicates an identical match. All peaks are in a distribution close to this point.

\section{B. Peak Detection Results}

The peak detection method automatically calculated the peaks of all 144 epochs of data. In order to assess the accuracy of the peak detection, the Pearson correlation coefficient value was calculated for each epoch and plotted as a histogram. The results are shown in Fig. 3. The correlation coefficient value is calculated from the following formula:

$$
r=\frac{\sum_{n}\left(A_{n}-\bar{A}\right)\left(B_{n}-\bar{B}\right)}{\sqrt{\left(\sum_{n}\left(A_{n}-\bar{A}\right)^{2}\right)\left(\sum_{n}\left(B_{n}-\bar{B}\right)^{2}\right)}}
$$

A correlation coefficient of 1 represents a perfect match between signals. The mean correlation coefficient of these 144 samples was 0.9754 and the standard deviation was 0.01537. In our previous results [3], the correlation coefficient mean for 103 epochs was 0.9560 and the standard deviation was 0.0211 . This represents an almost $2 \%$ improvement in the accuracy of the peak detection. Also, the previous algorithm was semi-automatic in that each epoch needed reviewing and a few required a manual adjustment to the coefficients. This algorithm in contrast was totally automated.

\section{Averaged Evoked Potentials}

The averaged evoked potential signal is practically identical to the averaged evoked potential taken by averaging all the peaks together. Fig. 4. illustrates both these signal. It is observable that the peak average signal is slight smoother due to the minor denoising effect of the peak detection method where very small peaks are not detected and hence are removed.

\section{Peak Parameters}

In order to assess how well the approximation values of each peak represent the overall shape of that peak, the centre of each peak and the area under the peak were compared to the approximation detail value and the approximation detail location. A linear relationship between the between the position of the peak and the approximation peak position is shown in Fig. 5. A slightly more complex linear relationship between the approximation value and the area under the peak is illustrated in Fig. 6. In this case there is a linear relationship but it is dependent on the level at which the approximation is taken. Positive and negative peaks are separated in Fig. 6 and levels 2,3,4 and 5 are shown.

These peak parameters can be used to characterise the peaks and identify similar peaks across different epochs. This is the subject of further study by the authors. It is foreseen that this classification mechanism will allow localized sub-average peaks to be selected that focus on the evoked potential signal at the expense of the background noise.

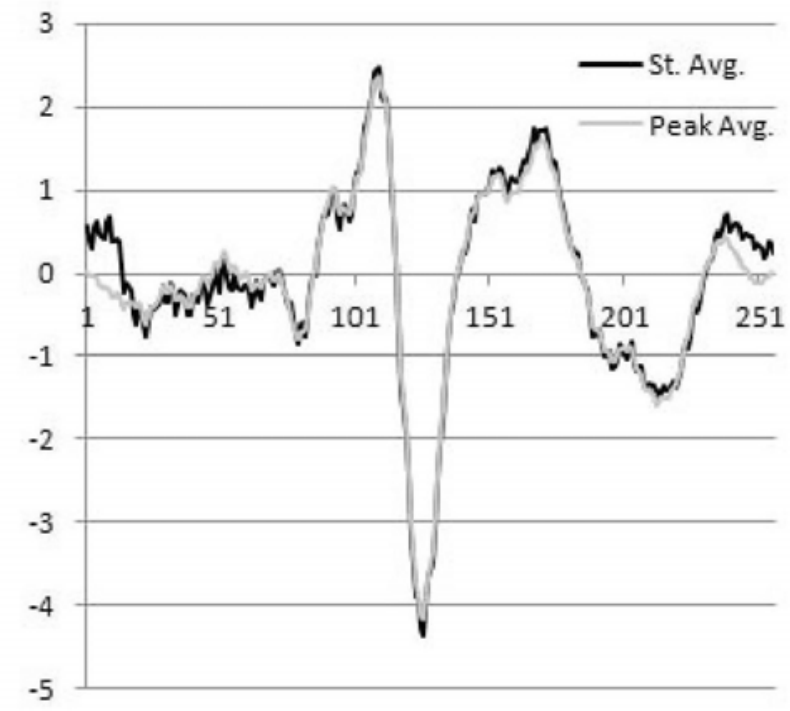

Fig. 4. Comparison of standard ensemble VEP average and sum of peak detected ensemble averaging. The darker line represents the original signal. The lighter line represents the peak detected ensemble average which closely resembles the standard average. More variation exists at the edges due to removal of partial peaks at the edge of the epoch as part of the Peak Detection algorithm. 


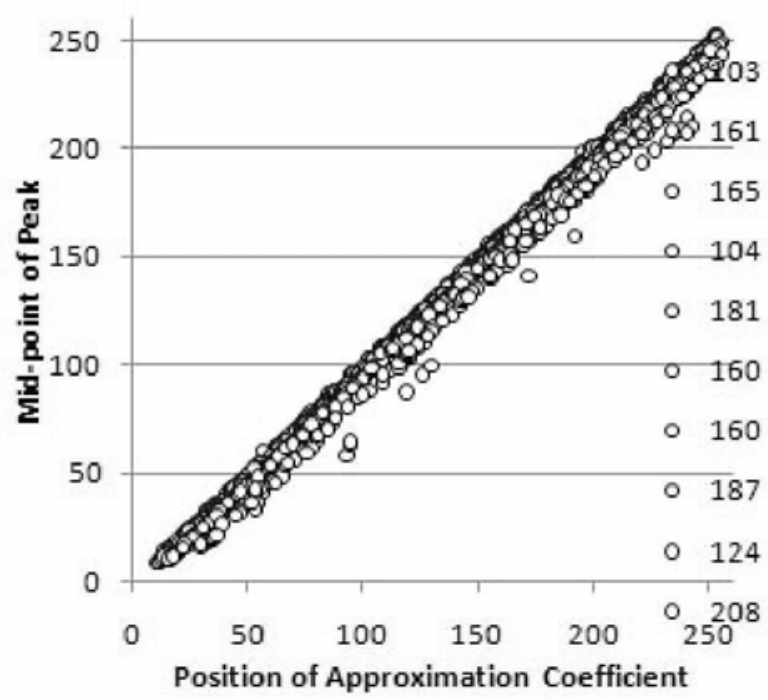

Fig. 5. There is a linear relationship between the position of the approximation location and the centre point of the peak. The above figure is not scaled by sampling rate and so represents each of 256 samples in time.

\section{CONClusion}

This paper reports the successful completion of an automated peak detection method for evoked potential signals and asserts that the approximation location and coefficient for each peak may be used to characterise the peak. It is hoped that further work will decompose the ensemble averaged evoked potential signal into more basic constituent parts that may provide additional information to neurologists. Decomposing Evoked Potential into constituent parts would provide additional clinical information not currently observable with standard averaging Evoked Potentials. This includes detection of opposite sign peaks that currently cancel each other out and also yielding a measure of how repeatable a certain peak is. This extra resolution is achieved while retaining the averaged Evoked Potential information which can be compared for reference.

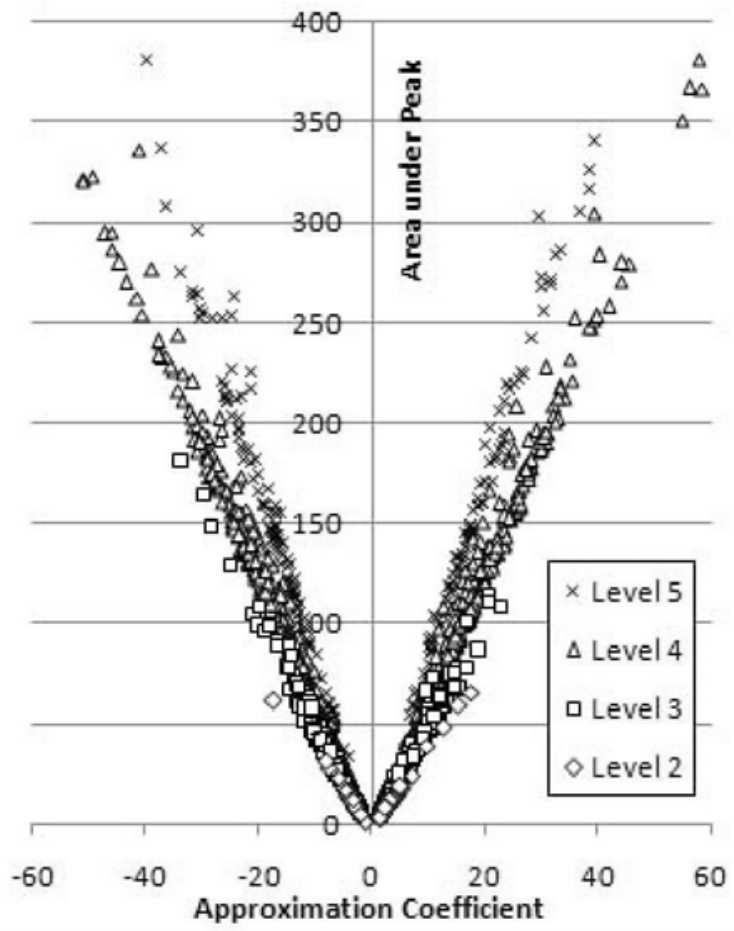

Fig. 6. There is a linear relationship between the approximation coefficient (positive and negative $\mathrm{x}$-axis) and the calculated area under the peak (y-axis). The slope of the relationship is dependent on the particular scale level of the approximation coefficient

\section{REFERENCES}

[1] S. G. Mallat, W. L. Hwang. "Singularity detection and processing with wavelets," IEEE Trans. on Info. Th., vol. 38, pp. 617-643, March 1992.

[2] S. G. Mallet, "A Wavelet Tour of Signal Processing", Second Edition, Academic Press, 1999.

[3] C. McCooey, D. Kant Kumar, "Characterising evoked potential signals using wavelet transform singularity detection", World Congress on Medical Physics and Biomedical Engineering, Sydney, 2003.

[4] C.McCooey, D. Kant Kumar, "Characterising evoked potential signals using wavelet transform singularity detection", ICINCO Conference, Barcelona, 2005.

[5] C.McCooey, D. Kant Kumar, "Decomposition of Evoked Potentials using Peak Detection and the Discrete Wavelet Transform", Engineering in Medicine and Biology Conference 2005, Shanghai, 2005.

[6] K. E. Misulis, "Spehlmann's Evoked Potential Primer", Second Edition, Butterworth-Heinemann, 1994. 


\title{
Decomposition of Evoked Potentials using Peak Detection and the Discrete Wavelet Transform
}

\author{
Conor McCooey, Student Member, IEEE, Dinesh Kant Kumar, Member, IEEE, \\ and Irena Cosic, Member, IEEE \\ RMIT University \\ PO Box 2476V, \\ Melbourne, VIC 3000, Australia
}

\begin{abstract}
A new method of viewing evoked potential data is described. This method, called the peak detection method, is based on singularity detection using the Discrete Wavelet Transform. The peaks and troughs of raw Visual Evoked Potential data are identified and characterized using the algorithms of this method, resulting in a linear decomposition of the recording into sets of individual peaks. The individual peaks are then added together, averaged and compared to the ensemble average signal. The peak detection method correlates strongly to the ensemble average showing that this method retains the same evoked potential signal profile.
\end{abstract}

Index Terms-Averaging, Discrete Wavelet Transform, EEG, Singularity Detection, Visual Evoked Potentials,.

\section{INTRODUCTION}

Evoked Potentials (EP) are Electroencephalograph (EEG) recordings in response to an external stimulus, where the response is not triggered endogenously by the natural processes of the brain but exogenously by an external trigger to one of the senses. Flash Visual Evoked Potentials (VEP) represent the EEG response to stimulation triggered by a series of flashes presented to a human subject's eye. The EEG response to a single stimulus is not directly observable. The response is masked by other ongoing background EEG events. Typically ensemble averaging of a few hundred epochs of repeated flash stimuli is required to obtain an observable VEP. The background EEG activity, which is assumed to be random in nature, tends towards a zero level as the number of epochs averaged increases. Meanwhile, the stimulus response is treated as time-locked to the stimulus and omnipresent in each epoch and so averages to a particular voltage profile. The latency at which these profile events occur is helpful for clinicians to determine the time delay of the VEP and assess the integrity of the sensory system involved.

From literature, it is evident that there is lack of precise understanding of what exactly EP signals represent. There is no widely acceptable model that explains the details of the generation of this signal. While averaging of EEG is a useful means of extracting some important features of the response, it is known that some information is lost in the process [1]. Reference [2] describes averaged EEG activity as being made up of 3 parts:

"1) a signal which is correlated in time with the stimulus,

2) some signal variations which are less correlated in time with the stimulus, and

3) ongoing activity not related to the stimulus."

The current technique of ensemble averaging has proven efficacy for separating the signal that has temporal correlation (part 1) from the background activity (part 3). However, semi-correlated activity (part 2) may erroneously add to the final VEP depending on its magnitude. EPs are non-stationary and are known to vary from trial to trial [1]. It is very difficult to analyze the spatial origin of EP components because individual events are no longer separable, once averaged.

Furthermore, there has been debate over the origin of EP responses with opinion divided between those who believe it results from stimulus-evoked brain events and those who believe it results from stimulus-induced changes in ongoing brain activity [3]. Finally, EPs require a large number of experiments to be conducted which may not always be appropriate or comfortable for the patient and may itself introduce another source of variation into the VEP due to subject fatigue.

Electroencephalograph (EEG) signals represent a summation of a number of spatially and temporally separated electrical events. Each of these events may be characterized by sharp, irregular non-stationary transitions that are known as singularities. Reference [4] states "Singularities and irregular structures often carry the most important information in signals". Thus, the singularities of EEG recordings may contain useful information regarding the response to the stimulus. This research aims to determine the singularity features of EEG in order toovercome shortcomings of the averaging process. Towards this aim, it is essential that the proposed technique should retain the evoked potential information that is currently available using the ensemble averaging method. This paper tests this by comparing the evoked potential generated using averaging of the singularities and the standard ensemble averaging technique for flash VEP data. The paper also reports analysis of singularities of each of the epochs.

The authors' previous work [5] has examined the use of 
modulus maxima singularity detection of the DWT [4] but the shortcoming in the algorithm reported in the paper was that it did not generate a linearly independent set of peaks. This paper reports an enhanced technique where this limitation has been overcome. Here, the singularities are grouped together to identify the individual peaks that would be the basic building block of the EEG recordings. The method is designed to decompose each EP epoch into a linearly independent set of peaks.

The advantage of this decomposition technique is that it allows significantly more flexibility in how averaging may be performed. This is a necessary first step towards an alternative analysis method where selective peaks may be chosen towards the averaging process.

The separation of signals that represent different events can have applications in determining the underlying events that sum up to make the EEG recordings. It is envisaged that this could lead to better understanding of the recordings itself and lend itself to separation of sources and events that generate the EP signals. It may then be possible to identify the response to the stimulus with minimal repetitions.

\section{PEAK DEtection MethoD}

This technique requires the detection and identification of peaks in the recordings. As a first step, singularities in the EEG recordings are identified using the DWT based modulus maxima singularity detection method [4]. Expressed using array based logic and analyzed using the peak detection method (PDM) (described in section II B), these singularities are selectively grouped to make a set of discrete peaks that describe the original recording. These linearly independent peaks are recombined for each epoch and compared to the original signal using Pearson's correlation coefficient formula to verify that a close fit is achieved. All peaks across each of the epochs are averaged. This result is compared with the evoked potential generated using the ensemble average method. This comparison is performed to determine whether or not the evoked potential information embedded in the recording is corrupted by the technique. For a better understanding of the technique, a brief of the major concepts is provided below.

\section{A. Wavelets and Singularity Detection}

A wavelet is a time domain wave which is localized, oscillatory and with zero average. A wavelet, $\psi(t)$ may be expressed in terms of a scale or dilation factor, $s$, which when varied compresses or expands the wavelet shape and a translation factor, $u$ that physically shifts the wavelet along the axis (in this case the time axis). The quadratic spline wavelet [4] was used in this research. Wavelets are observed to have similar properties to the peaks we wish to detect insofar as they have large amplitude changes over narrow time changes.

The output of the wavelet transform is a set of coefficients along the time axis for each scale factor [4]. Coefficient values are larger where the signal more closely approximates the particular scaled wavelet at the particular time. Equally, where there is little or no 'fit', coefficient values tend towards zero.

Using a dyadic scale $\left(s=2^{j}\right.$ where $\left.j=1,2,3, \ldots\right)$, a practical implementation of the DWT using a filter bank algorithm called the "algorithme à trous" may be implemented [6]. It generates a detail component (similar to a low pass filter) at each $2^{j}$ scale up to the maximum calculated scale, together with a remaining approximation component.

By selecting a mother wavelet function that is the derivative of a smoothing function, such as the quadratic spline wavelet of degree 2, Reference [4] proved that by selecting the modulus maxima DWT coefficients that had a certain evolution across scales, one could detect local singularities and in the process, demonstrated that a large set of wavelet coefficients could be simplified into a much smaller discrete set of coefficients grouped per singularity. This is a powerful step forward in the characterization of a signal into a group of singularities. An inverse discrete wavelet transform using the selected singularity coefficients was demonstrated to approximate the original signal well.

\section{B. Peak Detection}

Singularity detection using the discrete wavelet transform (DWT) described by Reference [4] is the starting point for the theory outlined in this paper. Singularities characterize areas where large magnitude change in the signal over a short period occurs i.e. points where there is a large slope. A peak may be considered to be a set of two or three singularities that record a simple shift away from and back to a particular baseline. The position and value of both the detail and approximation components are recorded in a single line of an array which defines the singularity. The singularities are matched into pair or triplets that identify distinct peaks within the data. Sets of singularities are then identified that combine to form peaks using the following set of rules:

i. A singularity characterizing a bi-phasic transition is split into two singularities - one characterizing the positive transition and the other the negative transition. This allows a bi-phasic peak to be divided into a pair of mono-phasic peaks.

ii. A pair of singularities is more likely to form a peak if the absolute magnitudes of the detail components at the highest scale level for each singularity are similar to one another.

iii. Singularities that increase with time have positive coefficients and those that decrease with time have negative coefficients. A peak must have both.

iv. Singularities that match to form a peak should have similar maximum detail scale level $(s)$ within \pm 1 scale factor of each other.

v. The gap between the position of the first detail component of each singularity in a pair is related to the dyadic scale factor fit the following rule: 


$$
2^{s j}<\operatorname{pos}_{1}\left(s_{2}\right)-\operatorname{pos}_{1}\left(s_{1}\right)<2^{s+1}
$$

where $\operatorname{pos}_{1}\left(s_{x}\right)$ represents the position of the first coefficient value for singularity $\left(s_{x}\right)$ (pos 1 in Table 1)

The PDM algorithm matches the array of singularities using these rules to yield a single peak. The process is then repeated for all remaining singularities in the epoch until all singularities are matched into a set of linearly independent peaks. Inverting (inverse DWT) and adding together these peaks yields a close approximation of the original signal.

\section{REsUlts}

\section{A. Experimental Setup}

Ethics approval for the experimental procedure was obtained from the local human research ethics committee. Flash Visual Evoked Potential signals were recorded for a single subject. Repeated strobe light flashes were presented to the subject's visual field at a distance of about 1 meter from the eye at an approximate rate of 1 flash per second. The EEG response was measured from the MidOccipital(MO) position, which is located $5 \mathrm{~cm}$ directly above the inion [7]. An epoch length of $500 \mathrm{~ms}$ was recorded synchronously with the strobe flash where the flash occurred at 0 seconds in each epoch. The sample rate was 512 per second. Data was normalized to give a zero mean across all data. Partial peaks occurring at the edges of each epoch were removed to eliminate border effects from the subsequent DWT processing. Visual inspection was performed to remove gross artifacts. A total of 104 epochs were initially used for analysis.

\section{B. Preliminary Results}

For a better understanding to the peak detection process, an example consisting of analysis of a representative peak is given in Fig 1. The original signal is a small section of the recording extracted, for illustrative purposes, using a temporal window of size $25 \mathrm{~ms}$ and corresponding to a single peak. The modulus maxima DWT coefficients of this signal are shown in Table 1 as Singl and Sing2. Sing 1 characterizes the upward slope of the original signal at 20$30 \mathrm{~ms}$ and Sing 2 characterizes the downward slope of the original signal at $35-45 \mathrm{~ms}$. The peak has been characterized by considering both, Sing 1 and Sing 2 and an inverse DWT is performed, resulting in the reconstructed signal shown as the PDM signal in Fig. 1.

The DWT generates coefficients that are made up of detail and approximation components. In Table 1, the $l v l$ column indicates the $\max$ scale used to characterize singularities or peaks. A $l v l$ of 4 indicates all detail coefficients from 1 to 4 are used to characterize the peak. The coefficients, $A_{l v}, A_{p}, A_{a}$, give the correct approximation information in terms of level, coefficient

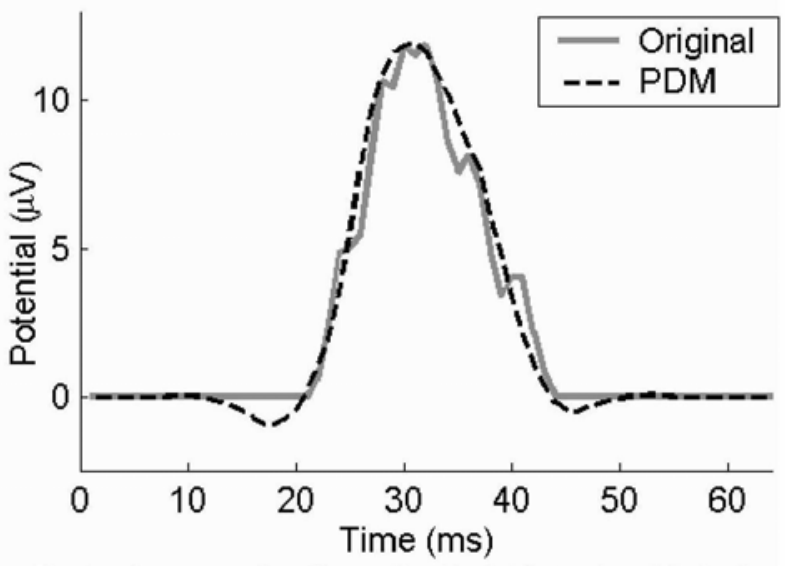

Fig 1. An example of how the Peak Detection Method signal (PDM) approximates an EEG signal (Original).

position and coefficient magnitude. $A_{l v l}=0$ means that this approximation coefficient is not used to regenerate the peak.

\section{Epoch Results}

The PDM was applied to each epoch to identify and characterize the major peaks. The results from each epoch were manually inspected and occasional scale $(l v l)$ adjustment was required to ensure accurate peak reconstruction. This process shall be automated in future research into this method.

Pearson's correlation coefficient, $r$, was calculated for each epoch signal, $A$ and its corresponding PDM signal, $B$ using:

$$
r=\frac{\sum_{n}\left(A_{n}-\bar{A}\right)\left(B_{n}-\bar{B}\right)}{\sqrt{\left(\sum_{n}\left(A_{n}-\bar{A}\right)^{2}\right)\left(\sum_{n}\left(B_{n}-\bar{B}\right)^{2}\right)}}
$$

where $\bar{A}, \bar{B}$ represent signal means and $n$ is the number of data points.

Fig 2. illustrates a histogram of correlation coefficients for all 104 epochs. A correlation coefficient of 1 represents a perfect match between signals. Three epochs were classified as outliers with correlation coefficients less than 0.86 and were removed from further analysis leaving 101 epochs from a total of 104. The mean correlation coefficient of these is 0.9560 with a standard deviation of 0.0211 .

\section{Averaging Results}

The standard ensemble average and the PDM average were calculated for the 101 epochs and the results are shown in Fig 3. It is noted that the PDM average gives an excellent approximation of the ensemble average signal with a correlation coefficient of 0.9948 , indicating that decomposing the evoked potential response into a set of peaks has retained the information that is observable by ensemble averaging.

TABLE 1

COEFFICIENT SETS OR SINGULARITIES FOR THE PEAK SHOWN IN FIG 1. DETERMINED BY THE PEAK DETECTION METHOD.

\begin{tabular}{|c|c|c|c|c|c|c|c|c|c|c|c|c|c|c|}
\hline Sing & lvl & pos $_{1}$ & det $_{1}$ & pos $_{2}$ & det $_{2}$ & pos $_{3}$ & det $_{3}$ & pos $_{4}$ & det $_{4}$ & pos $_{5}$ & det $_{5}$ & $A_{\text {lvl }}$ & $A_{p}$ & $A_{a}$ \\
\hline 1 & 4 & 22 & 1.7 & 21 & 3.0 & 21 & 7.4 & 16 & 12.8 & 0 & 0 & 0 & 14 & 0.4 \\
\hline 2 & 4 & 36 & -1.6 & 35 & -2.8 & 32 & -5.3 & 30 & -11.5 & 0 & 0 & 4 & 39 & 24.0 \\
\hline
\end{tabular}




\section{CONCLUSION}

The peak detection method is described and tested for flash VEP data taken from a single subject. The results indicate a high level of correlation between the signals generated using PDM and the original EEG recording. Excellent correlation is also demonstrated when comparing the results with evoked potential generated using ensemble averaging, demonstrating that this technique gives results that are not inferior to the conventional averaging process.

The PDM may be used to characterize EP data into a set of linearly independent peaks that closely approximate the original signal. Applications for this method are not described herein. The next step is to develop a classification mechanism to identify peaks more likely to be associated with EP signals and less likely to be background EEG. This is the topic of further study by the authors.

\section{REFERENCES}

[1] J. Zhang, C. Zheng, "Extracting Evoked Potentials with the Singularity Detection Technique", IEEE Engineering in Medicine and Biology, pp 155-161, Sept. 1997.

[2] C. Kooi, "Visual evoked potentials in central disorders of the visual system," Harper \& Row, $1^{\text {st }}$ edition, 1979.

[3] S. Makeig, M. Westerfield, T.-P. Jung, S. Enghoff, J. Townsend, E. Courchesne, T. J. Sejnowski, "Dynamic Brain Sources of Visual Evoked Responses," Science, vol. 295, pp. 690-694, 2002.

[4] S. G. Mallat, W. L. Hwang. "Singularity detection and processing with wavelets," IEEE Trans. on Info. Th., vol. 38, pp. 617-643, March 1992.

[5] C. McCooey, D. Kant Kumar, "Characterising evoked potential signals using wavelet transform singularity detection", World Congress on Medical Physics and Biomedical Engineering, Sydney, 2003.

[6] S. G. Mallet, "A Wavelet Tour of Signal Processing", Second Edition, Academic Press, 1999.

[7] K. E. Misulis, "Spehlmann's Evoked Potential Primer", Second Edition, Butterworth-Heinemann, 1994.

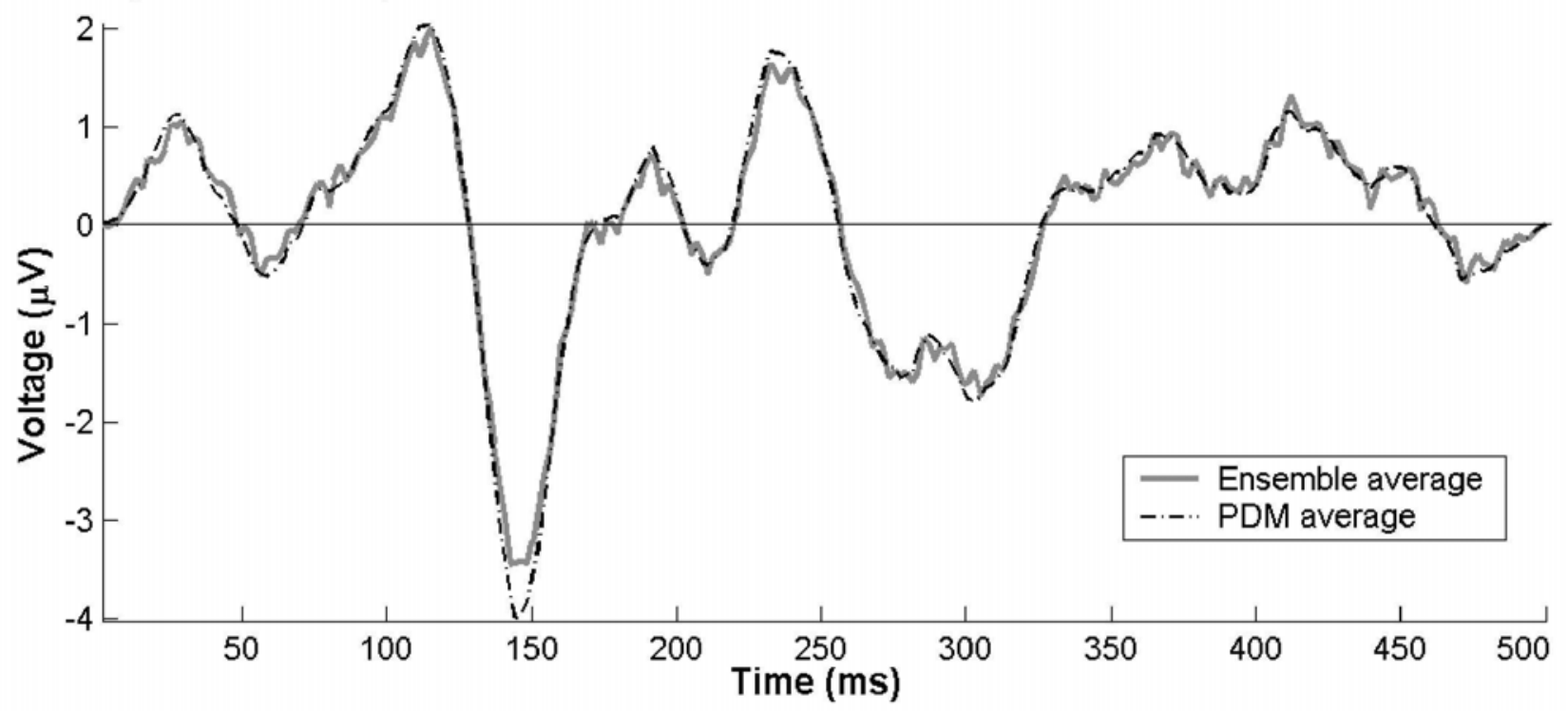

Fig 3. Comparison of standard ensemble average VEP and average of peaks detected for all epochs using the PDM.

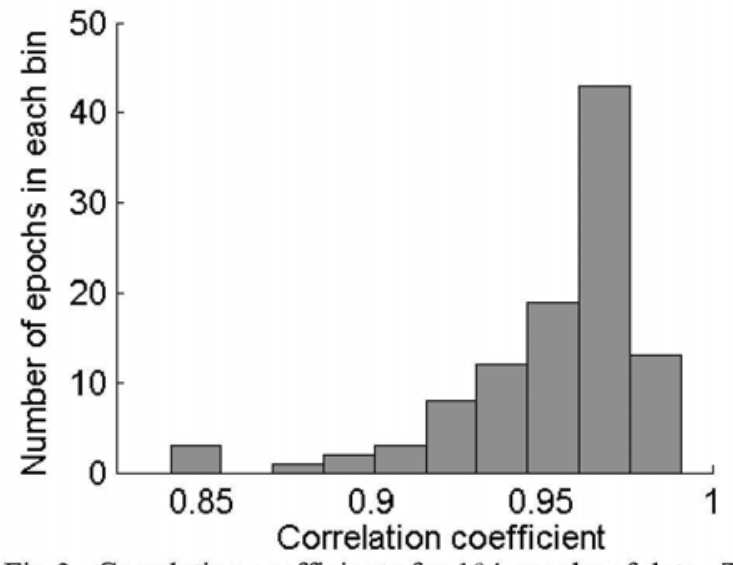

Fig 2. Correlation coefficients for 104 epochs of data. The three epochs below 0.86 were removed leaving 101 epochs. 


\title{
CHARACTERISING EVOKED POTENTIAL SIGNALS USING WAVELET TRANSFORM SINGULARITY DETECTION
}

\author{
Conor McCooey, Dinesh Kant Kumar \\ School of Electrical \& Computer Engineering, RMIT University, PO Box 2476V, Melbourne,VIC 3000, Australia \\ Email:s3029091@student.rmit.edu.au,dinesh@rmit.edu.au
}

Keywords: $\quad$ EEG, Visual Evoked Potentials, Discrete Wavelet Transform, Averaging

Abstract: A new method of viewing evoked potential data is described. This method, called the peak detection method, is based on singularity detection using the discrete wavelet transform. The peaks and troughs of an EEG recording are identified and characterised using the algorithms of this method, resulting in a linear decomposition of the data into sets of individual peaks. Then, by classifying the peaks in terms of latency (time), magnitude (voltage potential) and width (scale), the locations of higher concentrations of similar peaks are identified. These are grouped and sub-averaged, yielding sets of sub-averaged peaks that characterise the shape and give a measure of the repeatability of particular sub-averages within the visual evoked potential ensemble average signal. Linearity is maintained allowing the ensemble average to be generated by adding the sub-averaged peaks together. This method highlights evoked potential features that may be obscured or cancelled out with standard ensemble averaging. This is demonstrated for visual evoked potential data taken from a single subject.

\section{INTRODUCTION}

Electroencephalography (EEG) is the electrical recording of brain activity and is generated by the summation of spatially and temporally separated action potentials. It is a complex signal and even though it appears noisy, is very useful for identifying certain brain conditions. For many years the frequency spectrum of EEG has been examined and usefully interpreted for clinical and research purposes.

The Fourier transform uses time invariant sine waves and effectively gives a measure of the presence of a particular range of frequencies of a given time course signal. In the process of performing the transform the time location of the frequency is lost. The wavelet transform (Grossman and Morlet, 1984) uses time bound waves (wavelets) and may be thought of as an extension of the Fourier transform where the frequency content and time location are recorded simultaneously. Wavelet representations are more efficient than the traditional Fourier representation where the signal is non-stationary such as EEG. Such a method is able to localise the time when changes in the signal properties occur.

Evoked Potentials (EPs) are EEG recordings in response to visual, auditory or somatosensory sensory stimulation. EPs are used by clinicians to study specific neurological disorders. Due to the presence of background activity and noise, a single stimulus response is not detectable, and so multiple stimuli triggered recordings are taken. Then, statistical averaging is performed to reduce the background activity. This research aims to determine the singularity features of EEG recordings in order to overcome some of the limitations in the analysis of evoked potentials by averaging. This paper describes a method where the singularities are grouped together to identify the individual peaks that are the basic building block of the EEG recordings.

The method is designed to decompose each EP epoch into a linearly independent set of peaks, which are then characterised and classified to locate groups of peaks that have similar characteristics across multiple EP epochs. In this way, an alternative 
expression of the EP signal is presented - as a combination of multiple sub-averaged peaks.

\section{EPANALYSIS}

In natural systems such as EEG or EPs, the generators underlying the observed signals are complex and not fully determinable. Typically, the signal of interest is embedded in similar-sized or larger ongoing background EEG signals. Ensemble averaging is used to detect the Evoked Potential and in the case of visual evoked potentials (VEP) requires 100 to 200 repeats of the stimulus to obtain an observable response. Averaging of these epochs reduces the uncorrelated background EEG signals which tends towards a zero level as it is considered random in nature, while the stimulus response is time-locked to the stimulus and so averages to a particular voltage profile.

The individual response characteristics are lost in the averaging process. Kooi (1979) describes EEG activity in response to an external stimulus in terms of three parts:

"1) a signal which is correlated in time with the stimulus,

2) some signal variations which are less correlated in time with the stimulus, and

3) ongoing activity not related to the stimulus."

If the EP recording consisted purely of the time-locked stimulus response and the background activity was purely Gaussian in nature, then there would exist a high degree of repeatability across sequential tests on individual subjects. Experience under such conditions shows that, while strong similarities show through the EP, significant changes from one test to the next are observed.

There is also ongoing debate over the significance of evoked potential responses generated using the averaging technique, with opinion divided between those who believe it results from stimulusevoked brain events and those who believe it results from stimulus-induced changes in ongoing brain activity (Makeig et al 2002). Standard ensemble averaging cannot be used to resolve these questions.

Obtaining single-trial evoked potential reconstruction would greatly assist in addressing some of the unanswered questions regarding evoked potential activity. Researchers have attempted to develop several methods to discern the sought-after signal from the background activity. These include time-frequency digital filtering (Demiralp et al 1999), Matched Filtering (Maclennan and Lovely 1995), Wavelet Denoising (Quian Quiroga 2000) and Wavelet Thresholding.

One important property of EEG recordings is the presence of singularities and peaks. EEG signals are characterised by sharp, irregular non-stationary transitions, which are known as singularities (Mallet \& Hwang 1992). The singularities of EEG have been investigated using a different method called fragmentary decomposition (Melkonian, Blumenthal $\&$ Meares 2003) with the same goal of eliciting a better understanding of the EEG recording using discrete peaks. This paper reports research conducted to extract and use these singularities for the purpose of analysing the EEG response to external stimuli and overcome some of the limitations currently faced for EP analysis.

\section{THEORY}

A wavelet is a time domain wave which is localized, oscillatory and with zero average. Figure 1 shows a sample wavelet - the quadratic spline (Mallet \& Hwang 1992). A wavelet, $\psi(t)$ may be expressed in terms of a scale or dilation factor, $s$, which as it is varies compresses or expands the wavelet shape and a translation factor, $u$ that shifts the wavelet along the time axis. Wavelets have narrow time and frequency support (ideally) and are suitable for singularity identification and peak detection.

The output of the wavelet transform is a set of coefficients along the time axis for each scale factor (Mallat 1989). These coefficients indicate the correlation between the signal and the wavelet at the specific scale and translation. These are larger when the signal at the temporal location of the wavelet has spectral properties similar to the wavelet scale.

Using a dyadic scale (2), Mallat and Hwang (1992) describe a practical implementation of the dyadic wavelet transform using a filter bank algorithm called the "algorithme à trous". It generates a detail component (similar to a low pass filter) at each $2^{j}$ scale up to the maximum calculated scale, together with a remaining approximation component. By selecting the wavelet function to be the derivative of a smoothing function, such as the quadratic spline wavelet of degree 2 , the evolution

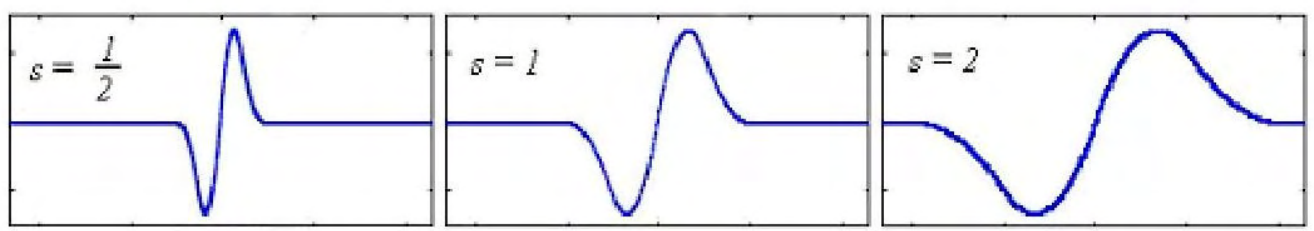

Figure 1: a quadratic spline wavelet with a scale, $s$ equal to $1 / 2,1$ and 2 . 
of the local maxima of the wavelet transform modulus across scales can be used to detect local singularities. The detail components, taken from the "algorithme à trous" method, yield a set of coefficients for each scale.

By selecting only the modulus maxima values that fitted a linear relationship across scales, Mallet and Hwang simplified the large set of wavelet coefficients into a much smaller discrete set of coefficients grouped per singularity. This is a powerful step forward in the characterization of a signal into a group of singularities. Mallet and Hwang did not further analyze individual singularities but did demonstrate an inverse discrete wavelet transform that showed that the selected modulus maxima singularity coefficients alone still approximated the original signal well.

The technique reported in this paper detects singularities using the Discrete Wavelet Transform (DWT) (Mallat and Hwang 1992). Singularities are grouped into peaks within the signal and the EEG recording is decomposed into a linearly independent set of peaks that sum to approximate the original signal. This is a sparse representation of the original signal. This paper demonstrates an approach to extract specific information from these peaks. Figure 2(a) illustrates an example of peak detection.

Once identified, each peak is then described by its characterising features - time latency, width of peak and height of peak, refer to Figure 2(b). Each identified peak is classified using these characteristics to populate a pair of threedimensional matrices - one for positive peaks and the other for negative peaks. The local area within the matrices as defined by the peak characteristics is incremented. Performed across all peaks and all epochs for the full EEG recording, this leads to a pair of concentration matrices where regions with higher peak concentration are readily identified. Based on these concentration matrices, the peaks occurring at the regions of higher concentration may be locally averaged to give a measure of temporally localised activity. This paper demonstrates that the concentration matrices used in conjunction with the ensemble averaging may be used to decompose the EP peaks.

This paper reports the use of the technique for flash visual evoked potentials (VEP). The authors do not see any reason why this could be extended to other stimulation techniques. Other possible applications include denoising, data compression and all applications where nonstationary signal activity is important.

\section{METHOD}

\section{E. Peak Detection}

Singularity detection using the discrete wavelet transform (DWT) described by Mallet and Hwang (1992) is the starting point for the theory outlined in this paper. Singularities characterise areas of large magnitude change in the signal over a short period i.e. points where there is a large slope. A peak may be thought of as a pair (or triplet) of singularities that record a simple shift away from and back to a particular baseline. These singularity coefficient sets are matched into pairs (or triplets) that identify distinct peaks within the data. The position and value of both the detail and approximation components are recorded in a single line of an array for each singularity.

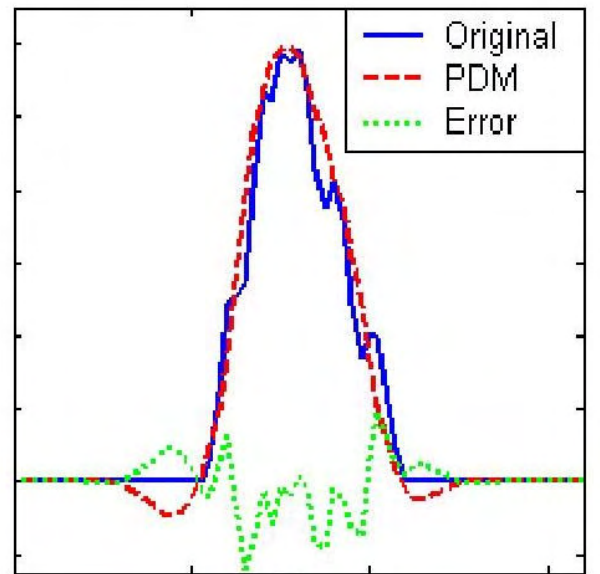

(a)

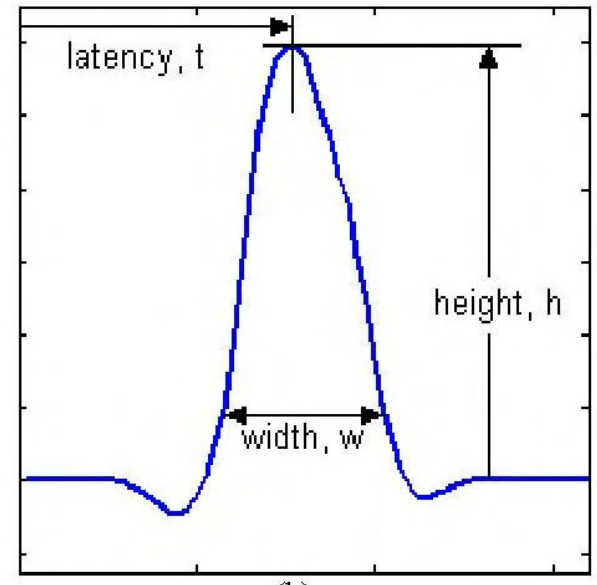

(b)

Figure 2: (a) An example of how the Peak Detection Method signal (PDM) approximates an EEG signal (Original) and the difference between these two signals (Error). (b) The peak characteristics for classification are identified latency of peak maximum height of peak from zero line to maximum and width of peak at $10 \%$ of the height. 
Using these arrays, sets of singularities are made that would combine to make peaks using a set of rules listed below:

i. A pair of singularities is more likely to form a peak if the absolute magnitudes of the detail components at the highest scale level for each singularity are similar to one another.

ii. Singularities that increase with time have positive coefficients and those that decrease with time have negative coefficients. Therefore a peak must be made up of both positive and negative coefficient singularities.

iii. Singularities that match to form a peak should have similar maximum detail scale level ( $s$ ) within \pm 1 scale factor of each other.

iv. The gap between the position of the first detail component of each singularity in a pair is related to the dyadic scale factor fit the rule in equation (1).

$$
2^{\mathrm{s}}<\mathrm{p}_{1}\left(\mathrm{~s}_{2}\right)-\mathrm{p}_{1}\left(\mathrm{~s}_{1}\right)<2^{\mathrm{s}+1}
$$

where $p_{l}\left(s_{x}\right)$ represents the first coefficient value for singularity $\left(s_{x}\right)$.

The PDM algorithm matches the singularities to generate singularity sets using these rules that form discrete peaks. Using the inverse DWT and only the coefficients of each singularity set, a signal representation for each identified peak is generated. These peaks were added together to yield a close approximation of the original signal. All peaks were defined as starting at the zero baseline, deviating either positively or negatively and returning to the baseline. Bi-phasic peaks are divided into 2 monophasic peaks - one positive and one negative. The features of all the detected peaks were identified. The result of this is the decomposition of the original signal into a linear set of separable peaks, which can be recombined to reconstruct the original signal.

\section{F. Classification of Peaks}

To identify whether the peaks represent the signal, features of the signal, and the peaks representing the signal, were extracted. The features of each of the peaks detected in section 4.2 were identified based on the following criteria and illustrated in Figure 2 .

i. The direction of the peak (positive or negative).

ii. latency or the time between stimulus onset (zero time) and the max peak magnitude

iii. height or maximum magnitude of the peak

iv. width of the peak which is measured not where the peak cuts the baseline but where the peaks cuts $10 \%$ of the max height from the baseline

These criteria were calculated from the peak signals generated by the inverse transform of the singularity coefficient sets. However, it is envisaged that in future, these criteria will be calculated directly from the singularity coefficient sets themselves.

\section{G. Concentration Matrix}

For further analysis, these criteria were used to populate two matrices, each of three dimensions, representing the properties of the peaks. These matrices identify the region of concentration of peaks. One matrix was for positive and the other for negative peaks, and the dimensions of each matrix correspond to the latency, height and width parameters. The local area within the matrix corresponding to the associated temporal latency, height and width location was incremented by a single value 1 for each identified peak. This was repeated for every peak of every epoch and the result was a concentration matrix where there was a cluster around areas of higher matrix value indicating regions where there were multiple similar type peaks.

The highest concentration region was identified and the corresponding value was computed and thus all peaks associated with that concentration value were identified. Local average or sub-averaging of the highest concentration region was calculated to give the trend of the local peak shape and characteristics. After obtaining the trend, all peaks related to this region were removed from the concentration matrices and the process repeated for the next highest concentration region. This process was repeated iteratively until both concentration matrices became empty.

\section{H. Sub-Averaging}

Examination of the concentration matrix has identified peaks with similar characteristics and grouped them. Each group of peaks is sub-averaged to give a representative signal for that group. To determine the quality and usefulness of the technique, sub-averages were compared to the standard ensemble average by two visual tools.

The first measure consisted of plotting and summing all the sub-averaged peaks in a region and comparing to the standard ensemble average signal. Peaks that appear less often get reduced while those peaks that occur more often get relatively amplified. This is then compared to the standard averaged signal. This gives a genuine representation of the sub-average magnitude and shape.

For the second measure, a slightly different subaverage is measured where the group of peaks are added and then divided by the total number of all peaks. In this way, the contribution of each sub-average to the standard ensemble average may be compared, as the denumerator in the averaging calculation is the same. 


\section{RESULTS}

\subsection{Experimental Setup}

Flash Visual Evoked Potential signals were taken for a single subject. Ethics approval for our experimental procedure was obtained from the local Human Research Ethics Committee. Repeated strobe light flashes were presented to the subject's visual field at a distance of about one metre from the eye at an approximate rate of 1 per second. The response was measured Mid-Occipital (MO) location, which is located at a point $5 \mathrm{~cm}$ directly above the inion (Misulius, 1994). An epoch length of 500ms was recorded synchronously with the strobe flash. The data was normalised to give a zero mean across all data. Visual inspection was performed to remove gross artefacts. Partial peaks occurring at the edges of each epoch were removed to eliminate border effects from the subsequent DWT processing. A total of 104 epochs were measured.

\subsection{Correlation}

The Peak Detection Method was applied to each epoch to identify and characterise the major peaks. Pearson's correlation coefficient, $r$, given in equation (2), was calculated for each epoch signal and its corresponding PDM signal:

Figure 3 illustrates a histogram of correlation coefficients for all 104 epochs. A correlation coefficient of 1 represents a perfect match between signals. Three epochs had a correlation coefficient below 0.86 and these were discarded from further analysis leaving 101 epochs. The mean correlation coefficient of these 101 epochs is 0.9560 with a standard deviation of 0.0211 . This suggests that using the PDM, it was possible to approximate the original signals well.

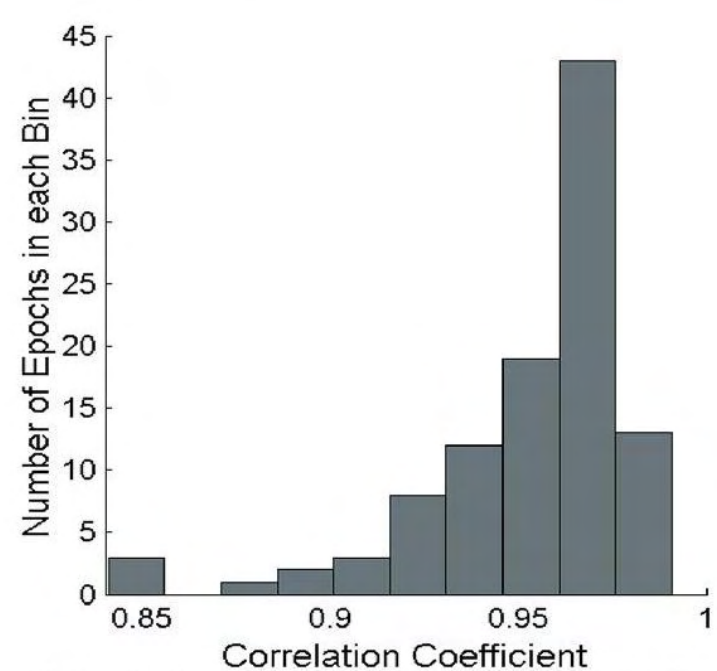

Figure 3: Correlation coefficients for 104 epochs of data. Three epochs below 0.86 were removed.

\subsection{Ensemble Average Comparison}

$$
r=\frac{\sum_{n}\left(A_{n}-\bar{A}\right)\left(B_{n}-\bar{B}\right)}{\sqrt{\left(\sum_{n}\left(A_{n}-\bar{A}\right)^{2}\right)\left(\sum_{n}\left(B_{n}-\bar{B}\right)^{2}\right)}}
$$

The comparison of the VEP signal generated using ensemble averaging and the sub-average from all the peaks using peak detection method for all the 101 epochs is in Figure 5. It is observed that the PDM average gives a good approximation of the ensemble average signal. This indicates that decomposing the evoked potential response into a set of peaks has retained the information that is observable by ensemble averaging.

\subsection{Concentration Matrices Results}

The concentration matrices were generated from all the peaks across the 101 epochs used. From these matrices the location of the highest value in either matrix was identified. Reading the matrix parameters for this location identifies that the highest repetition was of a negative peak located at a latency of $144.5 \mathrm{~ms}$, with a height of $8 \mu \mathrm{V}$ and a width of $31.25 \mathrm{~ms}$. Of the 101 epochs, 31 contain a peak with similar characteristics to this. Figure 6(a) illustrates the 31 individual peaks together with the average of these 31 peaks defined by equation (3).

$$
\text { First sub-average (a) }=\frac{\sum_{i=1}^{31} \text { peak }}{31}
$$

First sub-average (a) gives a picture of the actual average,

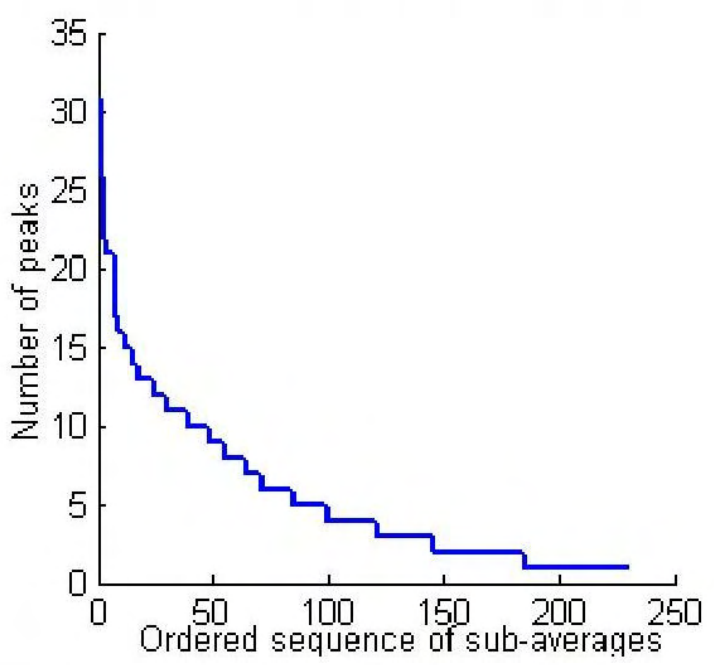

Figure 4: Ordered sequence of sub-average peaks versus number of peaks within each sub-average. 
referred to as sub-peak. Figure 6(b) illustrates the average of the 31 peaks defined by equation (4).

$$
\text { First sub-average }(\mathrm{b})=\frac{\sum_{i=1}^{31} \text { peak }_{i}}{101}
$$

The first sub-average (b) is directly comparable with the Ensemble Average since these are all averaged over the 101 epochs. It is a scaled version of first sub-average (a). This comparison gives a good indication of how the first sub-average peak compares to the ensemble average peak in local area.

After calculating the first sub-average, the subsequent sub-averages were calculated. The number of peaks identified in each sub-average group is charted in Figure 4. The summation of all 231 sub-averages (a) yields the same PDM average illustrated in Figure 5.

Figure 6 demonstrates that some sub-averages are more significant than others. Based on this, the first 15 sub-averages are presented in Figure 6. The others had a very low frequency and were ignored, representing the high frequency background noise. signal has been decomposed into the PDM and the discarded error signal has a high level of high frequency components. This effect is visible in Figure 4 where the PDM average signal is observably smoother than the ensemble average signal.

It is also observed that the cumulative of the 15 subaverages in Figure 7(b) retains the major features of the ensemble average. These 15 sub-averages represent a total of just 283 peaks used out of a total of 1278 peaks originally identified in the 100 epochs of data. Hence only $22 \%$ of the total Evoked Potential raw data is used to generate this averaged evoked potential. It is also observed that the cumulative sub-average of Figure 7(b) tracks the ensemble average. The notable exception is the appearance of a negative peak at $82 \mathrm{~ms}$, which is not apparent in the Ensemble Average signal. This could be an advantage of this system over the typical ensemble average system where such a peak is not evident and could result in loss of information.

Separating overlapping peaks is an important and difficult component of any singularity detection technique (Melkonian, Blumenthal \& Meares, 2003). On occasion, some detected peaks do not have a close fit to the original signal (the lower correlation coefficients in Figure 3). However, it may also be seen here that the vast majority of

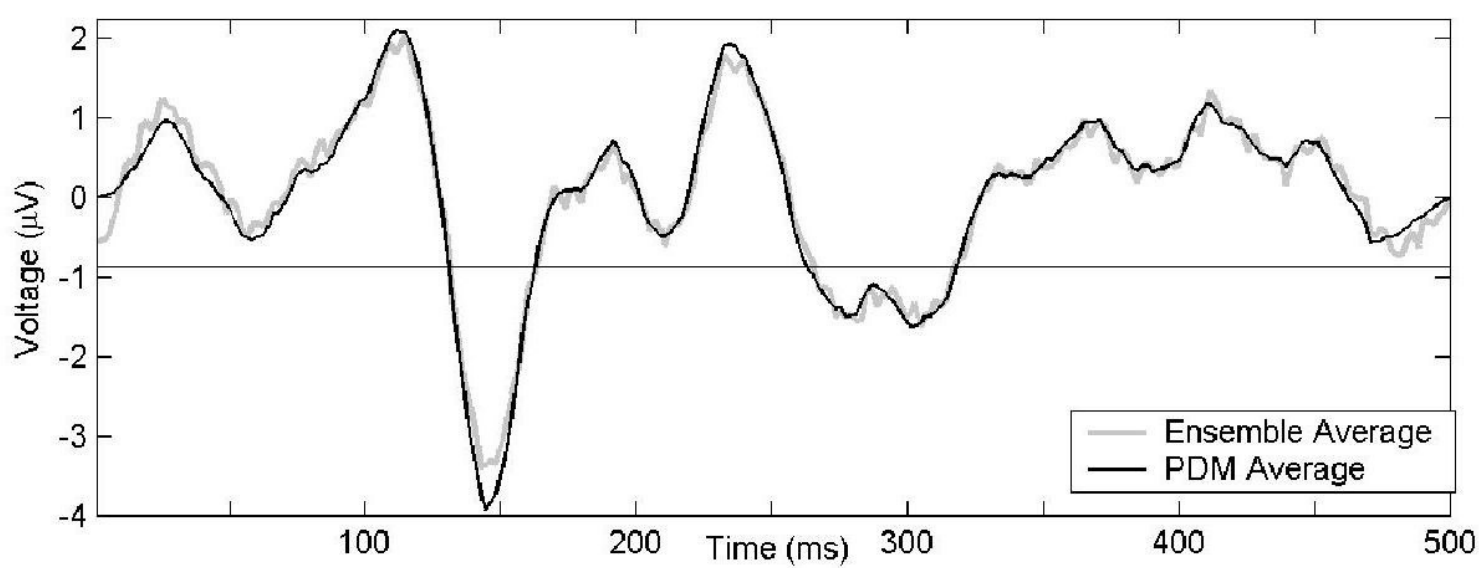

Figure 5: Comparison of ensemble VEP average and sum of peak detected with PDM divided by total number of peaks

epochs do give an excellent fit to the original signal.

\section{OBSERVATIONS}

It is observed that the Peak Detection Method is effectively a high frequency filter, an inherent property of singularity detection techniques where coefficients that don't migrate to higher scales are not chosen. High frequency components are found only on the low scale levels and hence are effectively filtered out. This may be seen in the example of Figure 2(a) where the original

\section{DISCUSSION}

A new method of peak detection for evoked potentials has been presented. Using an example of VEP based EEG data generated using 104 experiments, this peak detection method is shown to retain the same evoked potential information as the ensemble averaging technique. The individual peaks are then characterised by latency, width 
and height and a classifying scheme is implemented where higher concentrations of similar type peaks are identified. This scheme yields an ordered list of locations where peaks are clustered. The peaks within each location are sub-averaged to give a local peak of interest. The ensemble average may now be viewed as a summation of all these sub-averages and because linear independence has been maintained throughout, a subset of the subaverages may be selected which have a higher incidence and so are more likely to represent real evoked potential responses.

It is envisaged that this tool could help interpret the visual evoked potential in two ways. Firstly, the higher concentration locations indicate a more repeatable evoked potential signal and hence give a reliability factor to the peak that is being viewed. Secondly, when cancellation of positive an negative peaks occurs, the make-up of that cancellation may be examined in terms of size of peak and number of times it occurs. Further testing and analysis of this technique is being undertaken to broaden its application and to verify the results more generally.

\section{REFERENCES}

Demiralp, T., Yordanova, J., Kolev, V., Ademoglu, A., Devrim, M., Samar, V. J., 1999, Time-Frequency analysis of single-sweep event-related potentials by means of fast wavelet transform. In Brain and Language, vol 66(1), pp 129-145.

Grossman, A., Morlet, J., 1984. Decomposition of Hardy functions into square integrable wavelets of constant shape. In SLAM J. Math., vol. 15, pp723-736.

Kooi, C., 1979. Visual evoked potentials in central disorders of the visual system, Harper \& Row, $1^{\text {st }}$ edition.

Makeig, S., Westerfield, M., Jung, T.-P., Enghoff, S., Townsend, J., Courchesne, E., Sejnowski, T. J., 2002. Dynamic Brain Sources of Visual Evoked Responses. In Science, vol 295, pp 690-694.

Maclennan, A. R., Lovely, D. F., 1995. Reduction of evoked potential measurement by a TMS320 based adaptive matched-filter. In Medeical Engineering \&

(a) First Sub-Average signal determined by highest concentration matrix value

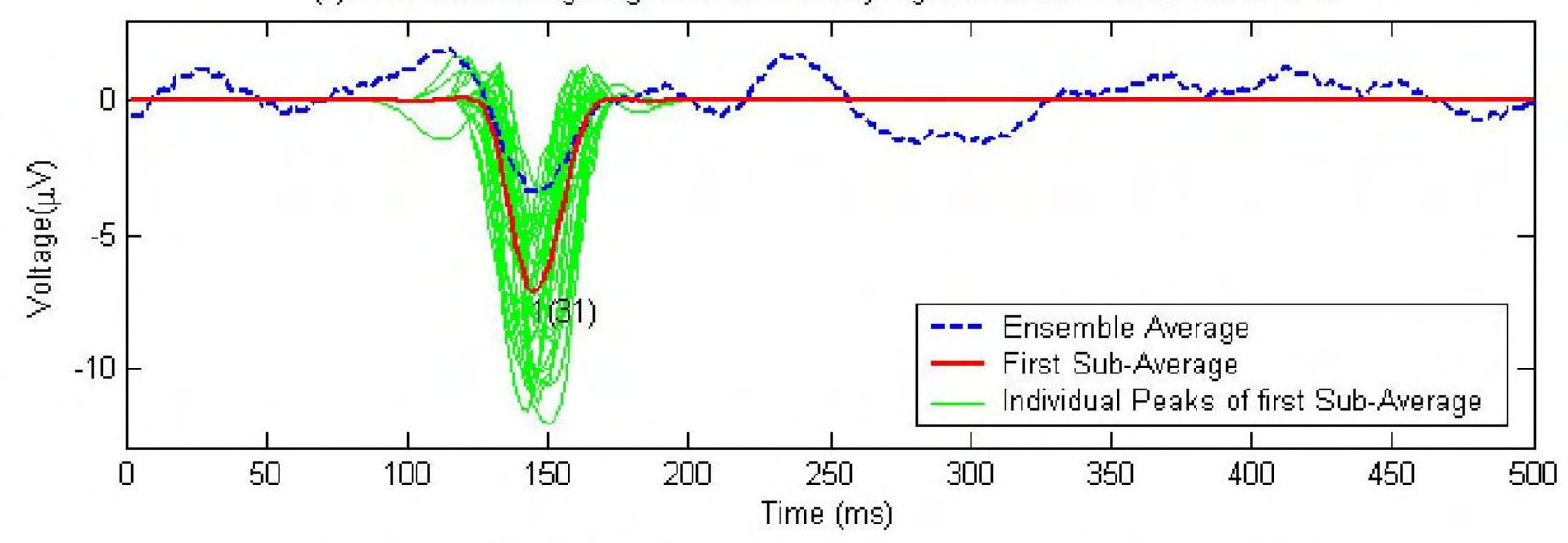

(b) Comparison of First Sub-Average signal and Ensemble Average signal

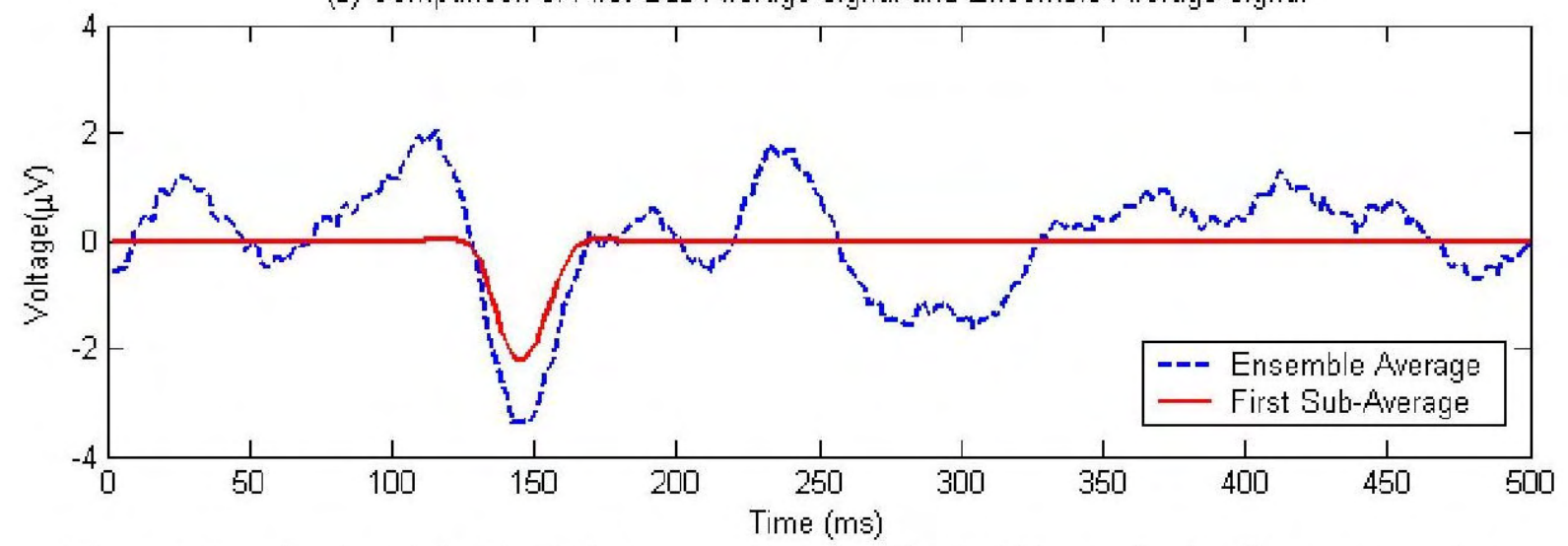

Figure 6: Examination of the First Sub-Average determined by the highest value in either concentration matrix representing the similar peaks that occurs most often. In this case, there are 31 peaks out of a possible 101 epochs. 
Physics, vol 17(4), pp 248-256.

pp 141-153.

Mallet, S., 1989, A theory for multiresolution signal decomposition: the wavelet representation. In IEEE Trans. on Pattern Analysis and Machine Intelligence, vol 11(7), pp 674-693.

Mallat, S., Hwang W. L., 1992. Singularity detection and processing with wavelets. In IEEE Transactions on Information Theory vol. 38, no 2 pp 617-643, IEEE.

Melkonian, D., Blumenthal, T. D., Meares, R., 2003, High Resolution Fragmentary Decomposition - a model based method of non-stationary electrophysiological signal analysis. In Journal of Neuroscience Methods, vol 131, pp. 149-159.

Misulis, K. E., 1994. Spehlmann's Evoked Potential Primer, Second Edition, Butterworth-Heinemann.

Quiroga, R. Q., van Luijtelaar, E. L. J M., 2001,

Habituation and sensitization in rat auditory evoked potentials: a single-trial analysis with wavelet denoising. In Int. Jour. Of Psychophysiology, vol 43,

(a) Cumulative sum of first 15 sub-average peaks
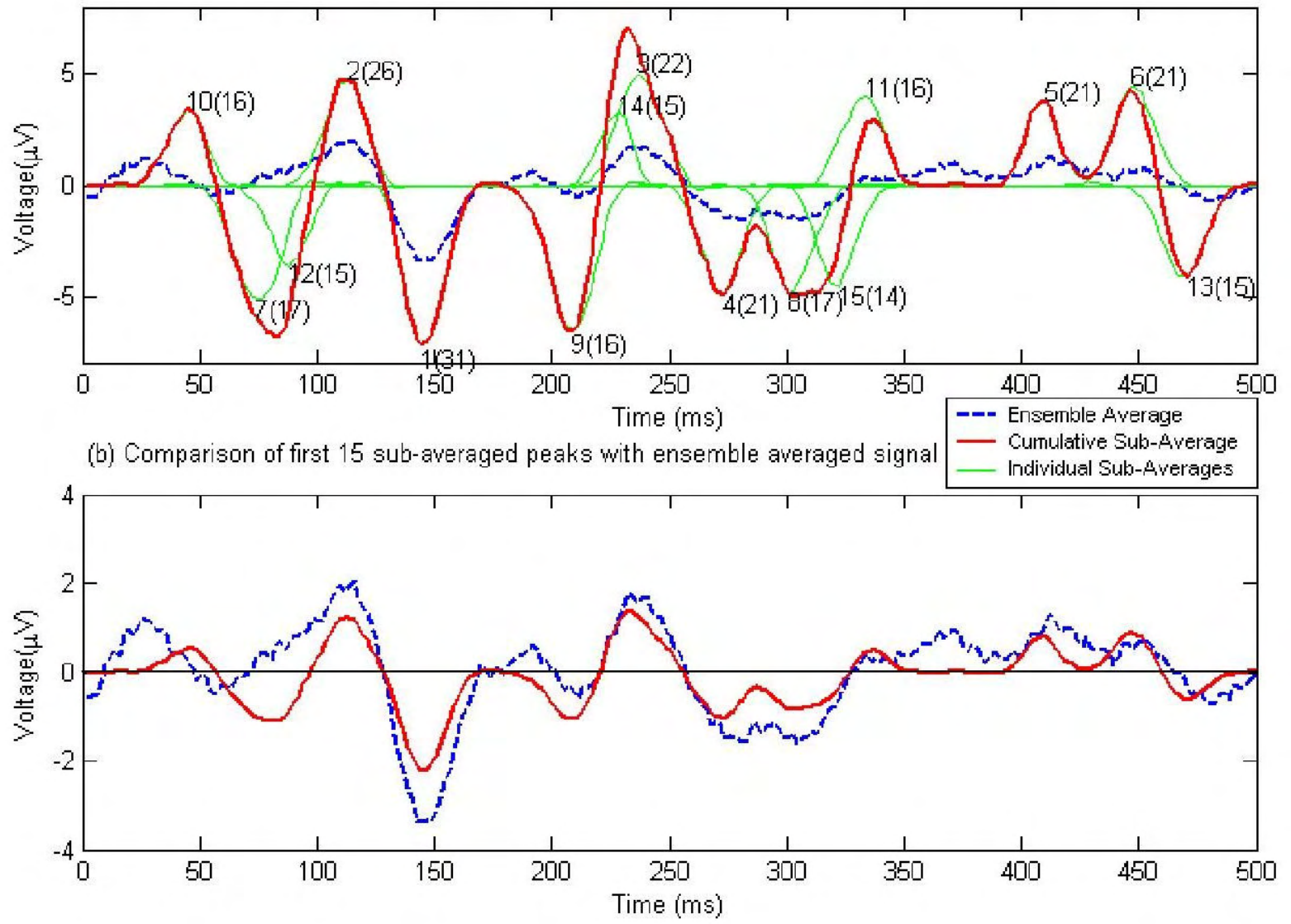

Figure 7: Examination of the first 15 Sub-Average signals. Indices in (a) are annotated as $\mathrm{x}(\mathrm{y})$ where $\mathrm{x}$ is the order number for the sequence of sub-averages (1-15) and $y$ is the number of peaks in that subaverage (when $\mathrm{x}=1, \mathrm{y}=31$ ). 


\title{
Characterising Evoked Potential Signals using Wavelet Transform Singularity Detection
}

\author{
1. Author \\ C. McCooey, RMIT University, Melbourne, Victoria, Australia. \\ D. Kant, RMIT University, Melbourne, Victoria, Australia.
}

\section{Introduction}

Electroencephalography (EEG) is the measurement of the brain's electrical potential at various points on the surface of the scalp. Evoked Potentials (EPs) may be defined as EEG activity occurring in response to an external stimulus (typically visual, auditory or somatosensory). Both EEG and EP's are characterised by small signal amplitudes $(<100 \mu$ Volts), localised function and complicated, overlapping and interfering patterns. These potentials arise as part of the brain's normal functioning - the triggering and resetting of neuronal signals to and from the brain.

Stimulus-synchronised averaging of many repeated trials is necessary to pick out the evoked response from the ongoing background EEG activity that obscures it. On average, the background EEG activity cancels, as it behaves like random noise, while the time-locked evoked response, if present, averages to a certain distinct visible pattern called the evoked potential. This evoked potential may then be clinically interpreted based on the latency, amplitude or absence of the evoked potential components and this has neurological applications in intraoperative monitoring, detecting spinal cord lesions ${ }^{10}$ and diagnosis of demyelinating diseases.

There are limitations to the averaging technique and applications for which it is not suitable. The merit of a meaningful interpretation of single sample evoked responses has been argued before ${ }^{1,2,3}$. During surgery, evoked potentials may be used to monitor the integrity of particular neurological pathways being operated upon. With fewer samples necessary to give a reading, a surgeon would be informed more quickly of a change in the patient's condition. Also, there are applications where repeated stimuli to the extent currently required for averaging purposes, would be uncomfortable for the subject - applications such as observing the effects of acupuncture or reflexology on the brain.

The aim of this research is to establish a wavelet-based technique to determine the evoked potential from a single evoked response trial. This paper reports our efforts in developing a wavelet-based peak detection method and demonstrates that it is comparable with averaging. This peak detection method decomposes an evoked response signal into a sequence of peaks together with a small residual signal. It is shown that the residual signal may be considered as noise and that averaging the sequence of peaks (without the residual signal) gives improved signal strength over standard averaging.

Future research shall identify correlations between individual peaks across a given sample set. This will allow a more selective averaging to take place with the effect of reducing the 
required number of trials necessary to obtain the evoked potential. By understanding the underlying mechanisms that generate the evoked potential and reducing the number of trials necessary to calculate it, the overall objective is to develop a method to extract an evoked potential from a single trial.

\section{3. $\underline{\text { Theory }}$}

In mathematics, a singularity is a point at which a function is not differentiable although it is differentiable in a neighbourhood of that point ${ }^{5}$. Singularities record large signal changes over very short time changes. Peaks may be thought of as a smoother version of singularities where large signal changes occur over slightly broader time changes. Mallat and $\mathrm{Hwang}^{4}$ developed a comprehensive theory for examining the singularities of functions using the wavelet transform, which has been applied here to identify and characterise the peaks that make up the evoked response. But first, a brief background on wavelets.

A wavelet is a time domain wave which is localised, oscillatory and with zero average. Figure 1 shows a sample wavelet - the quadratic spline ${ }^{7}$. A wavelet, $\psi(t)$ may be expressed in terms of a scale or dilation factor, $s$, which when varied compresses or expands the wavelet shape and a translation factor, $u$ that physically shifts the wavelet along the axis (in this case time axis). Wavelets are observed to have similar properties to the peaks we wish to detect insofar as they have large amplitude changes over narrow time changes.

The continuous wavelet transform of $f(t) \in \mathbf{L}^{2}(\mathbb{R})$ at translation $u$ and scale $s$ may be defined in terms of the inner product of $f$ and $\psi$.

$$
\begin{aligned}
W f(u, s)=\left\langle f, \psi_{u, s}\right\rangle & =\int_{-\infty}^{+\infty} f(t) \psi_{u, s}^{*}(t) d t \quad * \text { complex conjugate of } \psi \\
& =\int_{-\infty}^{+\infty} f(t) \frac{1}{\sqrt{s}} \psi^{*}\left(\frac{t-u}{s}\right) d t
\end{aligned}
$$

Using a dyadic scale and expressing as a convolution product gives

$$
W f\left(u, 2^{j}\right)=f * \bar{\psi}_{2^{j}}(u)
$$

where $\bar{\psi}_{2^{j}}(t)=\psi_{2^{j}}(-t)=\frac{1}{\sqrt{2^{j}}} \psi\left(\frac{-t}{2^{j}}\right)$

The output of the wavelet transform is a set of coefficients dependant on translation, $u$ and scale $s\left(=2^{j}\right)$, which give a measure of how the properties of the chosen wavelet 'fit' the function at the particular translation and scale. Mallat $^{7}$ describes a practical implementation of the dyadic wavelet transform using a filter bank algorithm called the "algorithme à trous". It generates a detail component (similar to a low pass filter) at each $2^{j}$ scale up to the

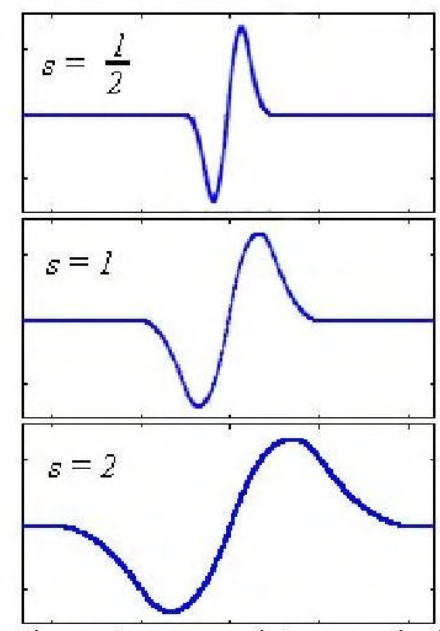

Figure 1: a wavelet - quadratic spline with a scale of 1 (middle). The same wavelet with scale factor of $1 / 2$ (top). Same wavelet with scale factor of 2 (bottom). 
maximum calculated scale, together with a remaining approximation component. For a thorough background on this area the reader is referred to references $4 \& 7$.

By choosing a wavelet that was the derivative of a smoothing function, such as the quadratic spline wavelet of degree 2, Mallat and $\mathrm{Hwang}^{4}$ proved that the evolution of the local maxima of the wavelet transform modulus across scales could be used to detect local singularities. The detail components, taken from the "algorithme à trous" method, yield a set of coefficients for each scale. At a point of singularity, there is a linear relationship between $\log _{2}$ of the modulus maxima response and its associated scale $j$. We apply this property to an evoked response signal, yielding singularity features, which record areas or rapid rise and fall along the signal. A rapid rise is matched to a rapid fall to identify a peak, using the following matching criteria:

1. Opposite sign (rising signals give positive modulus maxima coefficients while falling signals give negative modulus maxima coefficients).

2. The maximum scales, $j$, at which the singularity is present shall be the same

3. The distance between the singularities must be within the cone of influence ${ }^{4}$ i.e. $\Delta u \leq$ $2^{j+1}$.

The most pronounced peak is identified, calculated as a separate signal and subtracted from the original signal. This subtracted signal is then fed back into the algorithm to find the next most pronounced peak. In this way, the original discrete signal, $f[n]$, is decomposed into a summation of $I$ individual peaks, $s_{i}[n]$. ( $n$ is the total number of samples in the original signal.). As the algorithm continues, there is a point where peaks become too small to be detected and so there is a residual factor, $r[n]$, which must be added to the summated singularities to yield the original signal.

$$
f[n]=\sum_{i=1}^{I} s_{i}[n]+r[n]
$$

\section{Method}

A block diagram of the peak detection algorithm is illustrated in Figure 2. The fast dyadic wavelet transform developed by Mallat and Hwang ${ }^{4}$ is first performed and the modulus maxima across each scale are identified. This is performed utilising Wavelab ${ }^{8}$ standard routines running in Matlab

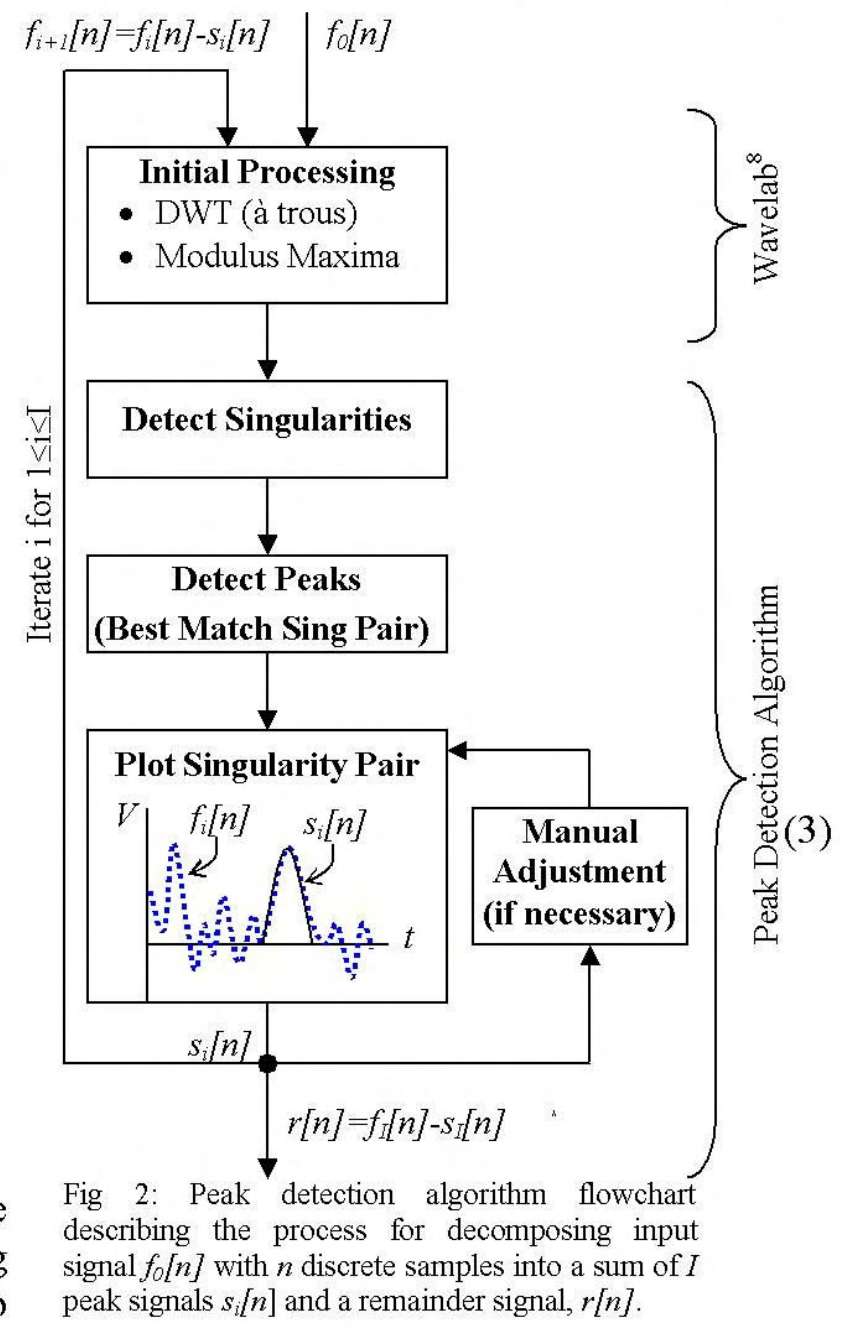


software. Next, the trend of the scale exponent factor $j$, versus $\log _{2}$ of the modulus maxima coefficients across increasing scales is examined. A linear trend indicates the location of possible singularities. The sequence of coefficients and their position are recorded to encode the singularity.

Peaks are then detected by matching pairs of singularities. All peaks are detected and then ordered in terms of largest scale and largest approximation value, which gives the most pronounced peak. Plotting the most pronounced peak against the original signal allows a manual comparison and, in a small number of cases, an adjustment to be made. Further development of the peak detection algorithm will overcome this limitation, yielding an automated extraction process. The chosen peak is removed from the original signal and the process is repeated until all significant peaks are extracted leaving only a residual signal.

An Event Related Potential dataset of standard Visual Evoked Potential (VEP) data, based on target stimulation with an oddball paradigm, was applied to the peak detection algorithm. The data constituted thirty artifact-free recordings from the left occipital electrode (O1) with linked earlobes reference for a single subject. There were 512 data points with 256 prestimulation and 256 post-stimulation with a sampling frequency of $250 \mathrm{~Hz}$. Data was prefiltered in the range $0.1-70 \mathrm{~Hz}$. See Reference 9 for more details of the experimental setup. The entire dataset was adjusted by subtracting the overall mean $(=8.33 \mu \mathrm{V})$ of the prestimulus data $(<0 \mathrm{sec})$ to give a zero centered baseline for the data.

\section{Results}

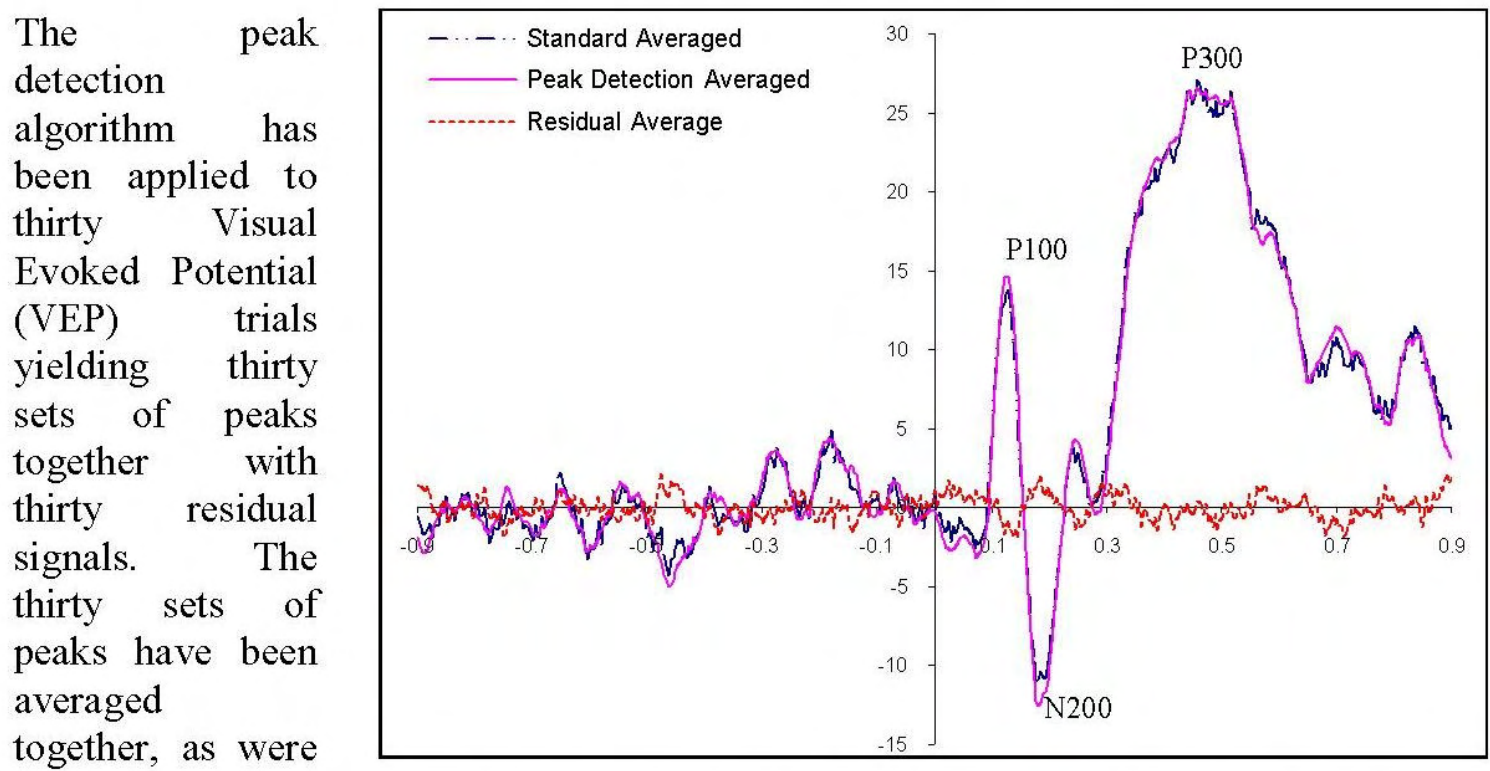

the residual signals. Figure 3 shows these results.

Figure 3: The averaged VEP signal using the peak detection method is comparable to the standard averaged method. The P100 peak and the N200 peak are slightly larger in amplitude and smoother indicating the peak detection method has had a noise reduction effect. This is further evidenced by the fact that the residual average signal at these points is concentrated in the opposite direction as the evoked potential signal. (Note: PD Avg + Residual = St. Avg.)

There are 3 
primary peaks of interest which have a typical interpretation based on the paradigm used. At $100 \mathrm{~ms}$, a positive peak (called P100) closely followed by

\begin{tabular}{|l|c|c|c|c|c|}
\cline { 2 - 6 } \multicolumn{1}{c|}{} & $\operatorname{Begin}\left(n_{1}\right)$ & $\operatorname{End}\left(n_{2}\right)$ & $\mathrm{V}_{\text {rms }}($ St.Avg) & $\mathrm{V}_{\text {rms }}$ (PD Avg) & Delta (\%) \\
\hline Total Signal & $-0.900 \mathrm{~s}$ & $0.90 \mathrm{~s}$ & $9.871 \mu \mathrm{V}$ & $9.990 \mu \mathrm{V}$ & +1.2 \\
\hline PreStimulus & $-0.900 \mathrm{~s}$ & $0.000 \mathrm{~s}$ & $2.650 \mu \mathrm{V}$ & $3.296 \mu \mathrm{V}$ & +12.0 \\
\hline P100-N200 & $0.092 \mathrm{~s}$ & $0.224 \mathrm{~s}$ & $8.840 \mu \mathrm{V}$ & $9.722 \mu \mathrm{V}$ & +10.0 \\
\hline P300 & $0.292 \mathrm{~s}$ & $0.656 \mathrm{~s}$ & $30.083 \mu \mathrm{V}$ & $30.351 \mu \mathrm{V}$ & +0.9 \\
\hline
\end{tabular}

Table 1: Comparison of Vrms for standard averaging (St.Av) method and peak detection method (PDM). An increase in signal strength is shown in all areas.

a negative peak

(called N200) are present in both the target data (shown) and the non-target data (not shown). The P100-N200 component is a sensory related response to the presentation of a pattern (either pattern). The subject was instructed to pay attention to the target stimuli and this attention is reflected in the P300 peak, which is present in the target data only and so is related to the cognitive event of recognizing the target.

A comparison of the standard averaging and peak detection method averaging is shown in Table 1 where the signal strengths at particular epochs of interest are calculated using:

$$
V_{r m s}=\sqrt{\frac{1}{N} \sum_{n=n_{1}}^{n=n_{2}}\left|x_{n}\right|^{2}}
$$

\section{Discussion}

It is observed from both Table 1 and Figure 3 that the peak detection method has a noise reduction effect by increasing the signal strength. This is most apparent for the P100-N200 component where the $10 \%$ increase in signal strength may be attributable to concentrating of detecting peaks. The P300 component, which is of most interest, has the smallest increase, which may be due to the P300 being made up of several overlapping components not separable with the averaging techniques here. Further research shall examine this area.

\section{Conclusions}

The preliminary results demonstrate that evoked response signals can be decomposed into a finite set of distinct simple peaks without any loss of overall information, when averaged across all signals. Averaging these peaks is at least as good as standard averaging, if not marginally better. The peak detection method has generated a set of peaks that are characterised by position, scale and amplitude. This allows them to be analysed in more refined ways than just averaging which will be the subject of further research.

\section{Acknowledgements}

Thank you to Martin Schuermann and Rodrigo Quian Quiroga for providing the VEP data. 


\section{References}

1. Bartnik A., Blinkowska K. \& Durka P., Single Evoked Potential Reconstruction by means of Wavelet Transform, Biological Cybernetics, 1992, Springer-Verlag.

2. Zhang J \& Zheng C, Extracting Evoked Potentials with the Singularity Detection Technique, IEEE Engineering in Medicine and Biology, September/October, 1997

3. Heinrich H, Dickhaus H, Rothenberger A, Heinrich V \& Moll G, Single-Sweep Analysis of EventRelated Potentials by Wavelet Networks - Methodological Basis and Clinical Application, IEEE Transactions on Biomedical Engineering, Vol 46, No. 7, July 1999.

4. Mallat, S. and W.L. Hwang, Singularity Detection and Processing with Wavelets. IEEE Transactions on Information Theory, 1992. 38(2): p. 617-643.

5. Collins Concise Dictionary, Fourth Australian Edition, HarperCollins Publishers, 1999.

6. Holschneider M., Kronland-Martinet R, Morlet J, and Tchamitchian P, Wavelets, Time-Frequency Methods and Phase Space, chapter A Real-Time Algorithm for Signal Analysis with the Help of the Wavelet Transform, pages 289-297, Springer-Verlag, Berlin, 1989.

7. Mallat, S., A Wavelet Tour of Signal Processing. 1999, London: Academic Press.

8. Wavelab Version 802, http://www-stat.stanford.edu/ wavelab

9. Quian Quiroga R \& Shuermann M, Functions and sources of event-related EEG alpha oscillations studied with the Wavelet Transform, Clin. Neurophysiol. 1999, 110: 643-655.

10. Toga A \& Mazziotta J, Brain Mapping - The Methods, Academic Press, 1996. 
Appendix E ORIGINAL DATA AND SOFTCOPIES 